Portland State University

PDXScholar

Winter 3-6-2020

\title{
Detecting Geomorphic Change and Stream Channel Evolution on the Sandy River, Oregon, Using Lidar Following Dam Removal in 2007
}

Lowell Henry Anthony

Portland State University

Follow this and additional works at: https://pdxscholar.library.pdx.edu/open_access_etds

Part of the Geomorphology Commons

Let us know how access to this document benefits you.

\section{Recommended Citation}

Anthony, Lowell Henry, "Detecting Geomorphic Change and Stream Channel Evolution on the Sandy River, Oregon, Using Lidar Following Dam Removal in 2007" (2020). Dissertations and Theses. Paper 4844. https://doi.org/10.15760/etd.6720

This Thesis is brought to you for free and open access. It has been accepted for inclusion in Dissertations and Theses by an authorized administrator of PDXScholar. Please contact us if we can make this document more accessible: pdxscholar@pdx.edu. 
Detecting Geomorphic Change and Stream Channel Evolution on the Sandy River, Oregon, Using Lidar Following Dam Removal in 2007

by

Lowell Henry Anthony

A thesis submitted in partial fulfillment of the requirements for the degree of

\author{
Master of Science \\ in \\ Geography
}

\author{
Thesis Committee: \\ Martin Lafrenz, Chair \\ Scott Burns \\ David Banis
}

Portland State University

2019 
(C) 2018 Lowell Henry Anthony 


\begin{abstract}
Following the removal of Marmot Dam on the Sandy River, Oregon, several Lidar flights were flown over the area of the former reservoir. The resultant sequential DEMs permitted calculation of reach-scale volumetric erosion and aggradation following dam removal. This allows for change detection across the entire affected reach of the former impoundment rather than just at several cross sections. In the first year there was a net loss of blank sediment in the dewatered reach. Subsequent flights show continued degradation of $145,649 \mathrm{~m}^{3}$ as well as aggradation of $6,232 \mathrm{~m}^{3}$. Sediment transport reached quasiequilibrium in 2012 with a net change of $65 \mathrm{~m}^{3}$. In addition, this technique allows the extraction of cross-section information which shows that the channel continues to be active migrating in some areas while also being constrained by bedrock features from past volcanism in some reaches. This study further shows the capability of lidar to measure rates of aggradation and degradation for an entire river system instead of reach specific extrapolations and that repeat lidar flights can more than adequately assess the changing nature of entire stream reaches more rapidly and more cost effectively than traditional field techniques. In addition:
\end{abstract}

- The utility of Lidar to do river management with repeat returns, having successive lidar acquisitions run on the watershed level will help us to gain insight into the correlation to precipitation events and geomorphological change in a given reach.

- Lidar can be used to assess the validity of channel evolution models. Sequential runs of lidar can be used to adjust the overall effectiveness of current CEM's and create new ones that consider reach specific geomorphology.

- Dam removal projects should incorporate initial lidar flights prior to removal and follow acquisitions based on known CEM's for the region and overall regionspecific physiography.

- Sequential lidar should be used for hazard mitigation and geohazards analysis with an acquisition timeframe that is appropriate for the region's physiography, geology, geomorphology and the return interval of the hazard being monitored. 


\section{Dedication}

I wish to express special thanks to all those that have made this endeavor possible and put up with me while I went through it. To my parents Lawrence L. and Mollie P. Anthony, for their unwavering support and encouragement throughout my adventures in higher education, and life. To my children Ariana and Nicholas who added their own adventures on top of this one and yet helped me to learn more about myself in the process. To everyone that believes in themselves and that they can change their life for the better irrespective of past mistakes. And most especially to my wife, Heather, for her unwavering support, love and encouragement while I struggled to finish this endeavor, that patience and understanding came when I needed it the most.

It's never too late to be what you might have been. George Elliot 


\section{Acknowledgements}

I wish to express special thanks to Professors Martin Lafrenz and Scott Burns for all their help throughout my course study and in preparation for this thesis. The influence of Scott Burns throughout my geologic studies at Portland State University helped shape my knowledge of the region and pique my curiosity to understand more of the underlying forces at work all around us.

The influence and patience of Martin Lafrenz both as my advisor and as a field assistant has been pivotal to helping me accomplish this manuscript and for keeping me focused when the task at hand seemed to become more daunting and my confidence in what I was doing was waning; he always challenged me to look at things from varied perspectives.

In addition, special thanks are due to my numerous colleagues at the Oregon Department of Geology for their support and help throughout this process, most especially Clark Niewendorp, Ian Madin, Jed Roberts, Yumei Wang, Bill Burns, and John English. Each of you helped me gain a deeper understanding of the geologic and geomorphic principles at work in the region and through the work done on our many projects over the years, have helped to expand my knowledge and appreciation of what we do. And to Deb Schueller, for helping me be a better writer and understanding the ins and outs of map and manuscript layout and keeping me from losing my mind fighting with Word and Adobe Illustrator, your knowledge and patience with me over the years has been most appreciated. 


\section{Table of Contents}

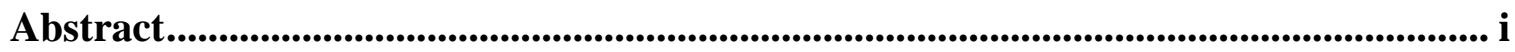

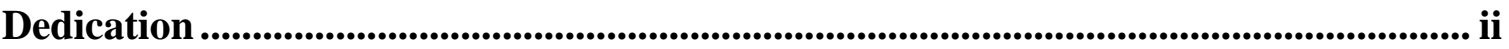

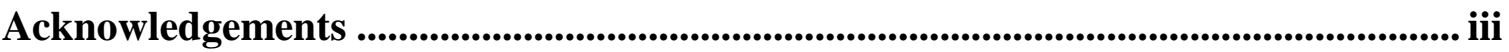

List of Tables ....................................................................................................................... vi

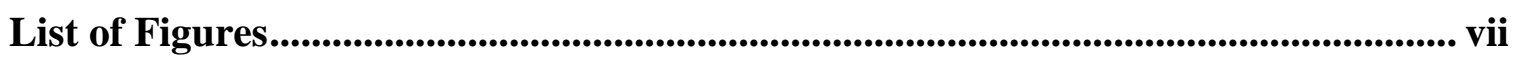

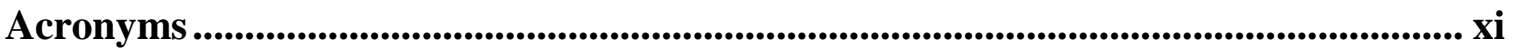

Definitions ...................................................................................................................................... xii

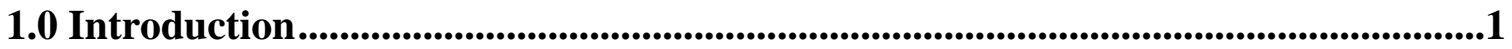

1.1 Study Area - Marmot Dam .......................................................................

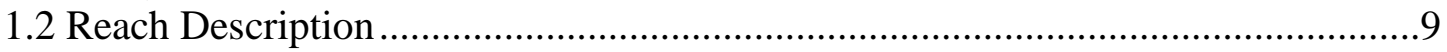

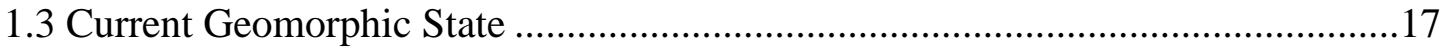

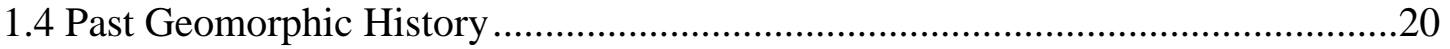

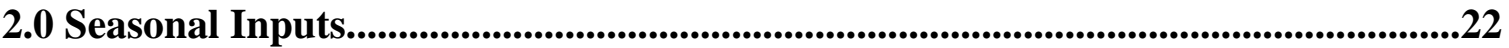

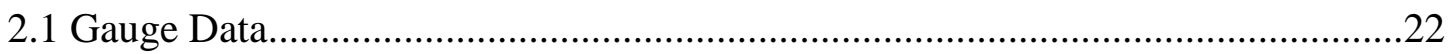

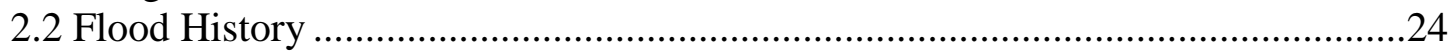

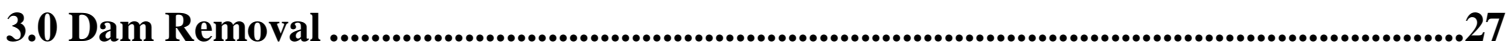

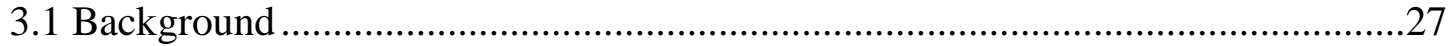

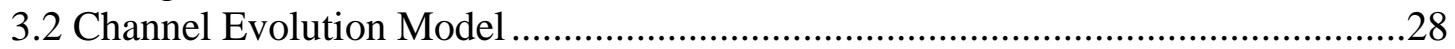

4.0 Lidar..................................................................................................................................446

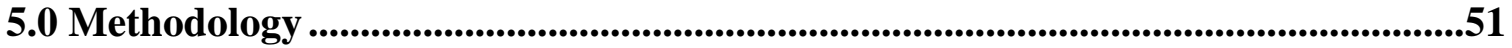

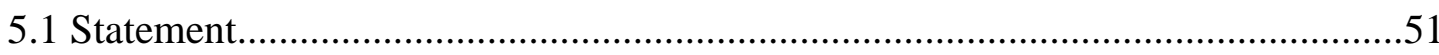

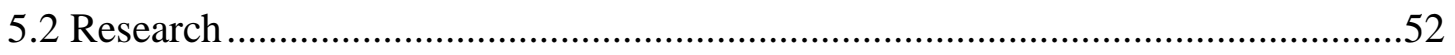

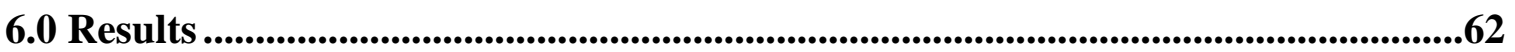

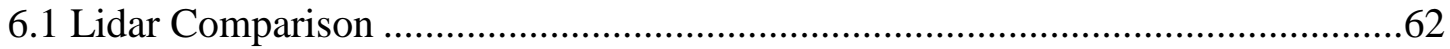

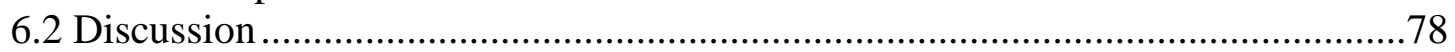

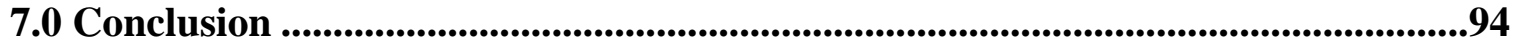

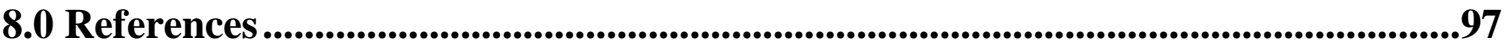

9.0 Appendix A: Differential Correction File........................................................100

10.0 Appendix B: Transect Tables ..........................................................................................102

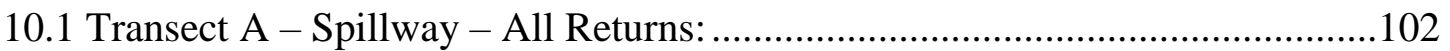

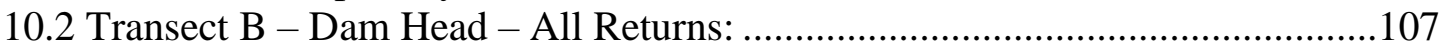

10.3 Transect C - Gravel Bar - All Returns:.......................................................112

10.4 Transect D - Lahar Dike - All Returns: .......................................................117

10.5 Transect E - End of Rapids/Pool Bank - All Returns:....................................124

10.6 Transect F - Crib Structure North Points - All Returns:...................................131

10.7 Transect G - Crib Structure South Points - All Returns: .................................138

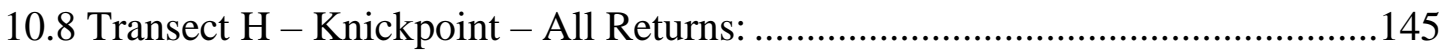


11.0 Appendix C: Stream Gradient Profiles............................................................151

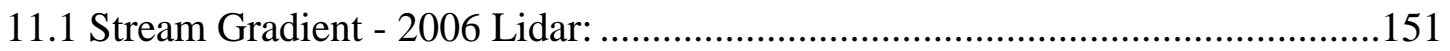

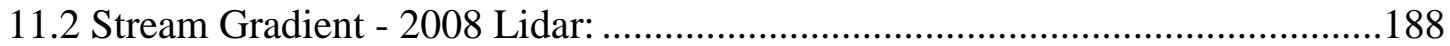

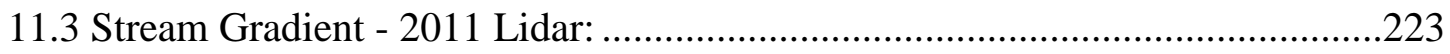

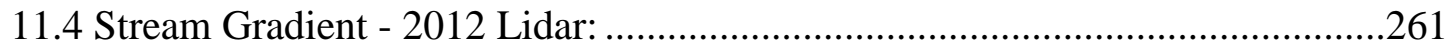




\section{List of Tables}

Table 1. Top ten peak flow events at Sandy River Marmot gauge (\#14137000). Abbe et

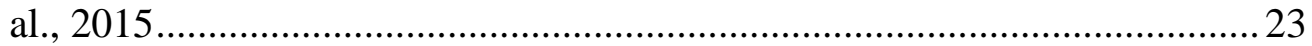

Table 2. Lidar accuracy and mean flow for date of acquisition................................53

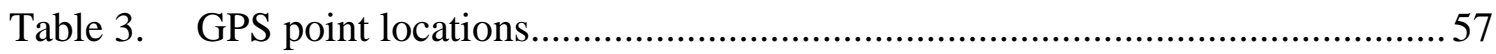

Table 4. Total lidar derived active channel margin change from 2006 - 2012. Note: Areas of degradation and aggradation are only for changes in the active channel margins. Note that in the 2011 to 2012 returns the data shows the reach

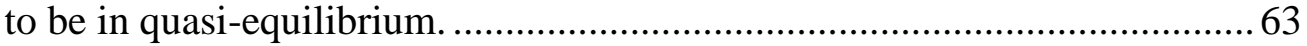

Table 5. Total impoundment area change 2006-2012. Note: Average overall net change of the study area over the course of six years. .............................................6 64 


\section{List of Figures}

Figure 1. Plate 1 Marmot Dam study area showing active channel margins mapped from the four separate lidar flights. .................................................................... 2

Figure 2. Cross-sectional view of the hyporheic zone in a river catchment. The hyporheic zone (HZ) is a fundamental part of a river ecosystem. It is the zone of interaction between groundwater and channel water (Biddulph, 2015)..... 5

Figure 3. Reverse sorting of sediment with large cobbles intermixed throughout the gravel bar, approximate location of Transect C. Photo by Lowell Anthony, Oregon Department of Geology, October 13th, 2014 (left) at 294 cfs and July 15 th, 2018 at 410 cfs (right).

Figure 4. Large woody debris accumulation atop brecciated lahar dike, approximate location of Transect D. Photo by Lowell Anthony, Oregon Department of Geology, October 13th, 2014 (left) at 294 cfs facing south and July 15th, 2018 at $410 \mathrm{cfs}$ in channel facing north (right).....

Figure 5. Channel migration induced slope failure. Note the exposed sediment layers which show Troutdale formation overlaying older flood deposition (left) 2018 photo shows the same location from a different angle the exposure is less evident. Photo by Lowell Anthony, Oregon Department of Geology, October 13th, 2014 (left) at 294 cfs and July 15th, 2018 at 410 cfs (right).

Figure 6. Location of the old Marmot Dam and beginning of the spillway looking upstream from the footbridge. Photo by Lowell Anthony, Oregon Department of Geology, October 13th, 2014 (left) at 294 cfs and July 15th, 2018 at 410 cfs (right).

Figure 7. 'Crib' structure mid-rapid above the old Marmot Dam location. Photo by Lowell Anthony, Oregon Department of Geology, October 13th, 2014 (left) at 294 cfs and July 15th, 2018 at 410 cfs (right).... 15

Figure 8. Brecciated lahar dike north of the crib structure at the end of the riffle/run. Photo by Lowell Anthony, Oregon Department of Geology, October 13th, 2014 (left) at $294 \mathrm{cfs}$ and July 15th, 2018 at $410 \mathrm{cfs}$ (right).

Figure 9. Reverse gradient foreset deposit (left) and foreset deposits exposed in slope failure (right). Photo by Lowell Anthony, Oregon Department of Geology, October 13th, 2014 (left) at 294 cfs and July 15th, 2018 at 410 cfs (right)... 17 
Figure 10. Resistant lahar dike, reverse sorted gravel bar, and channel migration induced slope failure. Photo by Lowell Anthony, Oregon Department of Geology, October 13th, 2014 (left) at 294 cfs and July 15th, 2018 at 410 cfs (right)... 19

Figure 11. Daily rainfall accumulation compared to peak flows from October 1, 2009 through December 31, 2014. (Abbe et al., 2015).

Figure 12. Channel Evolution Model stages in profile view. The channel profile view (c) shows each stage as it relates to equilibrium development in the reservoir impoundment. (Doyle et al., 2003b)

Figure 13. Stage Progression of a channel evolution model showing channel development in a reservoir after the removal of a dam: Stages A to C. Channel degradation begins quickly once the structure is removed. (Doyle et al., 2003b).

Figure 14. Stage Progression of a channel evolution model showing channel development in a reservoir after the removal of a dam: Stages E to D. As degradation continues, the channel widens and begins to aggrade downstream of the knickpoint eventually reaching quasi equilibrium. (Doyle et al., 2003b)...... 34

Figure 15. Study area map Cannatelli and Curran, (2012).

Figure 16. Modified channel evolution model. Stages A-E illustrate the progression of the reservoir from pre-removal in Stage A through channel evolution in Stages C-E. (Cannatelli and Curran, 2012).

Figure 17. Modified channel evolution model with addition of vegetation modeling. CEM deviates from previous models to incorporate flow and vegetation data; Stage $F$ shows the channel state corresponding to the seasonal flow regime. (Cannatelli and Curran, 2012). 43

Figure 18. Degradation and widening and aggradation and narrowing based on seasonal flow variability. Seasonal flow regime shown as the high-flow season (HFS), low-flow season (LFS), and seasonal transitions: $\gamma$ or $\delta$ correspond to channel degradation and widening in Stage $\mathrm{F} ; \alpha$ or $\beta$ correspond to channel aggradation and narrowing in Stage F; the channel is in the quasi-equilibrium state in Stage F; (g) channel proceeds from Stage F to a stable channel at Stage G only after vegetation has established and banks are stabilized. (Cannatelli and Curran, 2012). 44

Figure 19. Crib structure used in GPS survey. Photo by Lowell Anthony, Oregon Department of Geology, October 13th, 2014 (left) at $294 \mathrm{cfs}$ and July 15th, 2018 at $410 \mathrm{cfs}$ (right) 58 
Figure 20. Head of gravel bar location of GPS points G, H, and I. Photo on the left is looking upstream photo on the right is also upstream but from the north flank of the gravel bar just past the end of the long run. Photo by Lowell Anthony, Oregon Department of Geology, October 13th, 2014 (left) at 294 cfs and July 15 th, 2018 at $410 \mathrm{cfs}$ (right).

Figure 21. Water surface elevation longitudinal profile gradient. While bedform is not something that can be mapped at this point using lidar, inferences in bedform activity can be made by comparing the downstream difference between profile years. The highpoint shown in the 2006 line just downstream from Transect B is the Marmot Dam prior to removal. 65

Figure 22. Transect $\mathrm{H}$ is located at the uppermost limit of the reservoirs influence....... 67

Figure 23. Transect $\mathrm{G}$ follows the southern edge of the crib structure. 2011 is the first indication of it on the lidar profile. .68

Figure 24. Transect $\mathrm{F}$ follows the northern edge of the crib structure. 2011 is the first indication of it on the lidar profile. 70

Figure 25. Transect E crosses the end of the active channel in 2011/2012 and traverses the northern portion of the gravel terrace head. .71

Figure 26. Transect D traverses the exposed lahar dike that influences the current stream pattern in the reach. .73

Figure 27. Transect $\mathrm{C}$ traverses the gravel bar and sandy beach terrace that has formed downstream of one of the lahar dikes. 75

Figure 28. Transect B is located just above the location for the head of the Marmot Dam. .76

Figure 29. Transect A is just downstream of the location of the Marmot Dam spillway.

Figure 30. Geomorphic features present in the 2006 lidar acquisition. Note the long bar of sediment in line with the lahar intrusions visible now.

Figure 31. Geomorphic features present in the 2008 lidar acquisition. Note the lahar intrusions is just becoming visible now.

Figure 32. Active channel change between 2006 and 2008. The influence of the lahar in channel pattern is evident here. 
Figure 33. Channel morphology shown in the 2011 hillshade DEM, the lahar intrusion is a driving factor the shape of the reach and influence on the aggradation of the gravel bar marked by GPS point $\mathrm{H}$.

Figure 34. Active channel change between 2008 and 2011. We see the bar being built

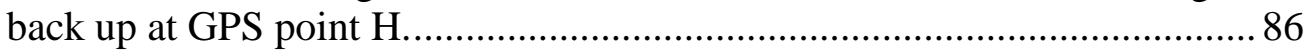

Figure 35. Channel morphology shown in the 2012 hillshade DEM, the minor adjustments to the morphology from 2011 shows a channel close to

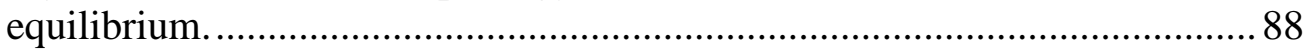

Figure 36. Active channel change between 2011 and 2012. Very little overall change in the channel thalweg, further degradation of the channel has occurred.......... 90

Figure 37. Overall active channel pattern changes between 2006 and 2012. Morphological differences in the overall configuration of the channel position

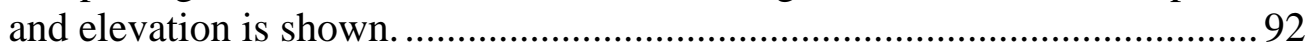




\section{Acronyms}

$\begin{array}{ll}\text { CEM } & \text { Channel evolution models } \\ \text { CFS } & \text { Cubic feet per second } \\ \text { CMS } & \text { Cubic meters per second } \\ \text { CMZ } & \text { Channel migration zone } \\ \text { DO } & \text { Dissolved oxygen } \\ \text { DOGAMI } & \text { Department of Geology and Mineral Industries (State of Oregon) } \\ \text { ESA } & \text { Endangered Species Act } \\ \text { FERC } & \text { Federal Energy Regulatory Commission } \\ \text { GIS } & \text { Geographic Information System } \\ \text { GPS } & \text { Global Positioning System } \\ \text { HZ } & \text { Hyporheic zone } \\ \text { LWD } & \text { Large woody debris } \\ \text { NAIP } & \text { National Agriculture Imagery Program } \\ \text { NOAA } & \text { National Oceanic and Atmospheric Administration } \\ \text { OLC } & \text { Oregon Lidar Consortium } \\ \text { RTK } & \text { Real-time kinematic } \\ \text { SFM } & \text { Structure from Motion } \\ \text { TIN } & \text { Triangulated Irregular Network } \\ \text { UAV } & \text { Unmanned Aerial Vehicle } \\ \text { USACE } & \text { U.S. Army Corps of Engineers } \\ \text { USGS } & \text { U.S. Geological Survey }\end{array}$




\section{Definitions}

Active Channel - A short-term geomorphic feature formed by prevailing stream discharges, is narrower than the bankfull channel and is defined by a break in bank slope that also typically is the edge of permanent vegetation.

Aggradation - The process by which a stream's gradient steepens due to increased deposition of sediment.

Bathymetry - The study of the "beds" or "floors" of water bodies, including the ocean, rivers, streams, and lakes.

Channel Migration - Channel migration is the natural process by which streams move laterally over time. It is typically a gradual phenomenon that works over many years to effect significant migration. In some cases, usually associated with flood events, significant migration can happen rapidly.

Competence - A streams ability to transport sediment. The measure of the maximum size of particles a stream can transport.

Degradation - The process by which a stream's gradient becomes less steep, due to the Erosion of sediment from the Stream bed. Such Erosion generally follows a sharp reduction in the amount of sediment entering the Stream.

Fluvial geomorphology - Examination of the processes that operate in river systems and the landforms which they create or have created.

Geomorphology - Study of landforms, the processes that created them, and the history of their development.

Headcut - Channel bottom erosion progressing upstream indicating a readjustment of slope, discharge, and sediment.

Incised Channel - Channel that is not hydraulically connected with its floodplain due to erosion.

Knick Point - Location in the thalweg where there is an abrupt change in elevation.

Lahar - A flow of material comprised from a mixture of hot or cold water, pyroclastic material, and rock fragments flowing down the slopes of a volcano typically within a river valley.

Lidar - A remote sensing technology that measures distance by illuminating a target with a laser and analyzing the reflected light. Lidar is popularly used as a technology to make high-resolution maps. 
Orthophoto - An aerial photograph that has been geometrically corrected or 'orthorectified' such that the scale of the photograph is uniform and utilized in the same manner as a map. An ortho-photograph can be used to measure true distances of features within the photograph.

Pools - Are the deepest locations of the reach. Water surface slope of pools at below bankfull flows is near zero. Pools are often located at the outside of meander bends.

Riffles - The sections of the bed with the steepest slopes and shallowest depths at flows below bankfull. Riffles typically occur at the cross over locations and have poorly defined thalweg.

Riverine - Of or produced by a river. Riverine floodplains have readily identifiable channels.

Susceptibility - Degree of proneness to natural hazards that is determined based on physical characteristics that are present.

Thalweg - Longitudinal outline/trace/survey of a deepest part of riverbed from source to mouth (upstream/downstream). Line of steepest descent along the stream.

Tinning - Areas where lidar returns are sparsely recorded; this decreased point spacing results in a tinning pattern inherent in the extrapolation between points in a triangulated irregular network. 


\subsection{Introduction}

\subsection{Study Area - Marmot Dam}

In this study I examine the geomorphic change on a small reach of the Sandy River, Oregon after the removal the Marmot Dam in 2007 (Figure 1). Dam removal presents researchers with a remarkable opportunity to gain a better understanding of the complex process of stream morphology channel adjustment. Dam removal is often as contentious a proposition today as the building of the original dams was during the 20th century. Advocates for dam removal focus on the benefits to ecology through the removal of structures and the subsequent reestablishment of the 'natural' flow of the stream reach. This restoration of the natural process benefits all facets of the watershed and the various species that reside in the reaches themselves, while providing benefits to several endangered species that were essentially cut off from the watershed and areas upstream of the dam. While many studies of dam removal focus just on the immediate reach where the dam was removed, more comprehensive studies are being made that investigate the effects of dam removal on the watershed and full-scale impacts on the environment from the release of trapped sediments.

Accumulated sediment builds up behind the dam structure, this, however, can be mitigated through dredging of the material, slow draw down of the dam reservoir level, removal of trapped sediments, or with the rapid "blow and go" method that quickly disperses much of the trapped sediments back in to the reach. While studies into the various methods are still in progress, there is evidence that the sediment impact to downstream habitat is minimal. With the advent of Light Detection and Ranging (lidar) technology, we can gain a better understanding of the morphological process intervals (changes in channel 


\section{Sandy River, Oregon Marmot Dam Study Area}
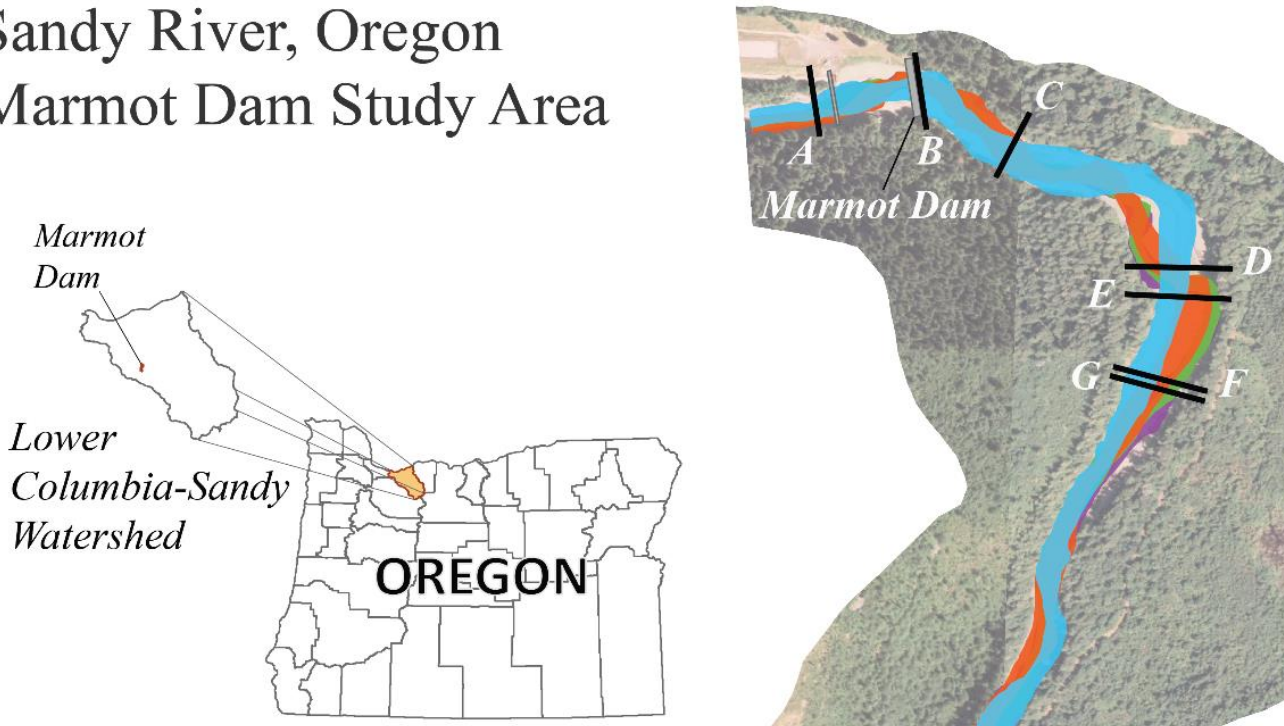

Plate 1

Channel positions for all return years of lidar for the Marmot Dam in Clackamas County, Oregon. Lidar was used to derive channel positions included in this map plate and was subject to interpretation of the lidar features by the author.

\section{Channel Positions}

2006 Active Channel
2008 Active Channel
2011 Active Channel
2012 Active Channel
Transects

\section{Data Sources:}

Basemap: Oregon Department of Geology and Oregon Lidar Consortium (2006)

Ortho: National Agriculture Imagery Program (2005)

Projection: NAD 1983 HARN Oregon Statewide Lambert (Intl Feet)

Software: Esri $\left.{ }^{(}\right)$ArcMap 10, Adobe $\left.{ }^{(}\right)$Illustrator CS6

Cartography by: Lowell H. Anthony, 2018

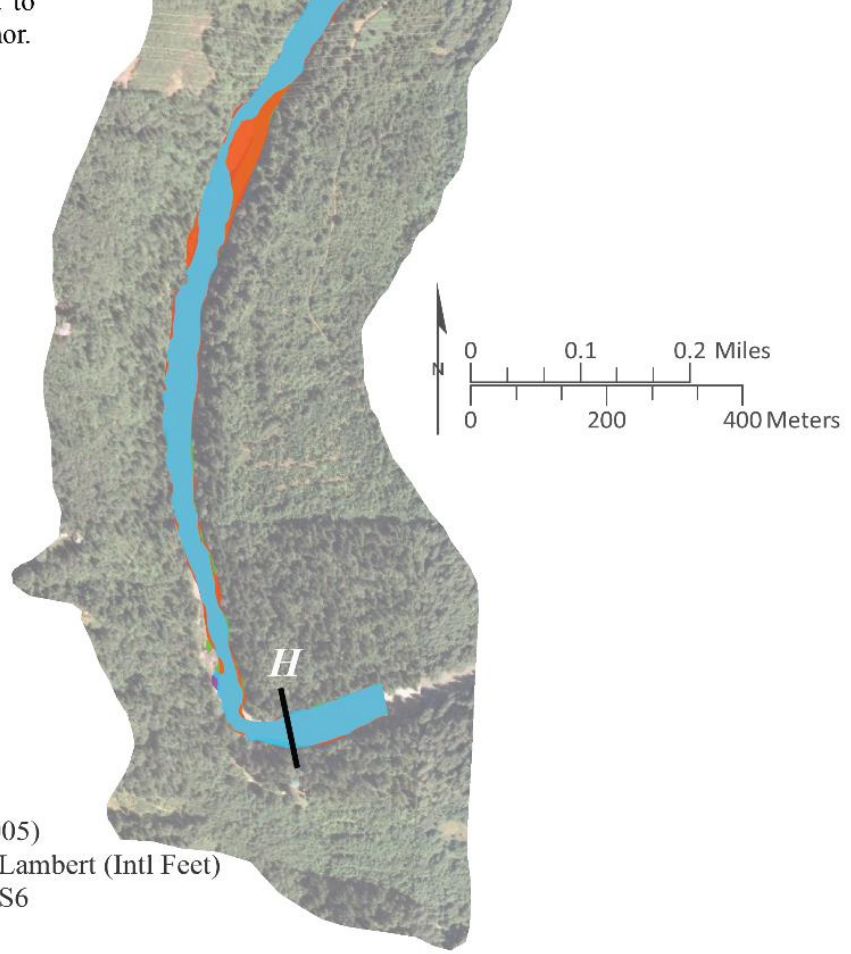

Figure 1. Plate 1 Marmot Dam study area showing active channel margins mapped from the four separate lidar flights.

position; changes in channel gradient, etc.) over time and how they correlate to the flow of the reach and the current geologic conditions. Having successive lidar acquisitions run on 
the watershed level will help us to gain insight into the correlation to precipitation events and geomorphological change in a given reach. The study of river morphology using lidar is just starting to gain traction and as lidar sensors improve the quality of the data gathered continues to improve giving us more insight in the bedform morphology and channel specific dynamics in a given reach. After constructing thousands of dams in the 20th Century, the past several decades have seen the removal of some aging dams. These removals provide the fluvial geomorphologist the opportunity to observe how stream channels respond to these sudden changes in hydrology. Most of these studies have documented these changes by examining stream cross-sections to monitor, for instance, changes in the position of the thalweg. However, this technique necessarily acts as only an estimate of the total amount change in stream plan form as well as the position of all geomorphic features in the channel. In this study I use repeat flights of remotely sensed lidar imagery to map and quantify changes in channel evolution of an entire reach of a river following dam removal over a period of 6 years. This technique allows for an additional account of sediment movement, which allows river managers the opportunity to better predict, monitor, and mitigate the effects of dam removal on both downstream and upstream ecosystems.

Fluvial geomorphology is the study of the development of valley and channel landforms by moving water, such as rivers and streams. It is the form and function of the river system that fluvial geomorphologists study to better understand the processes at work in the fluvial system (Schumm 1977). By studying how moving water shapes the landforms we gain perspective on the active processes at work and how our own actions can affect the system (Endreny, 2003). We can see an example of how our interactions have negatively affected 
the system through the decade's long process of channelization and dam building to control stream flow. These engineering techniques were considered the most effective way to control critical flows (floods) and the loss of bank material through the process of erosion. These projects have disrupted the fluvial system's hydrology, altered water temperatures, and seriously disturbed biological and ecological aquatic systems (Babbitt, 2002; Higgs, 2002).

What we have come to realize is that the control of the natural river process (erosion, deposition, and meandering) often does more harm than good; for instance, the accumulation of sediment behind dams decreases the available habitat that is critical to the river's species diversity (Endreny, 2003). Streams are classified as one of three types including straight, meandering, and braided. The form and function of the river system is such that it rarely runs in a straight channel; even at the headwaters in steep mountain channels there is some lateral movement that occurs in the system (Schumm, 1977). As the angle of the slope lessens, the waterway starts to wander laterally across the channel. This lateral movement produces a meandering course on a moderate slope.

Classic meandering streams have many meanders or bends and are a stable system, although they will shift and move across the floodplain through time. Meandering streams contribute to a healthy riverine environment and create diverse habitat types. They also disperse the potential hydraulic energy of the reach through turbulent eddies and the process of scour (Endreny, 2003). Other features of meandering streams that are important to the understanding of the form and function of the system is the pool-riffle complex. The pool-riffle complex makes up the deep and shallow portions of meandering stream beds, with the pools forming near the outer edge of the meander bends, while the riffles form in 
short, shallow, wider portions of the stream between the meander bends. The pool-riffle sequence occurs at repeating intervals within the meanders of $\sim 5$ to 7 widths of the channel (Charlton, 2008). The pool-riffle sequence provides dissolved oxygen (DO) to the river system as water flows into and out of the hyporheic zone (HZ) (Figure 2), which is the zone of interaction between groundwater and channel water. This movement of water through the $\mathrm{HZ}$ occurs through changes in hydraulic pressure at multiple scales. This further contributes to habitat diversity in the system (Winter et al., 1998).

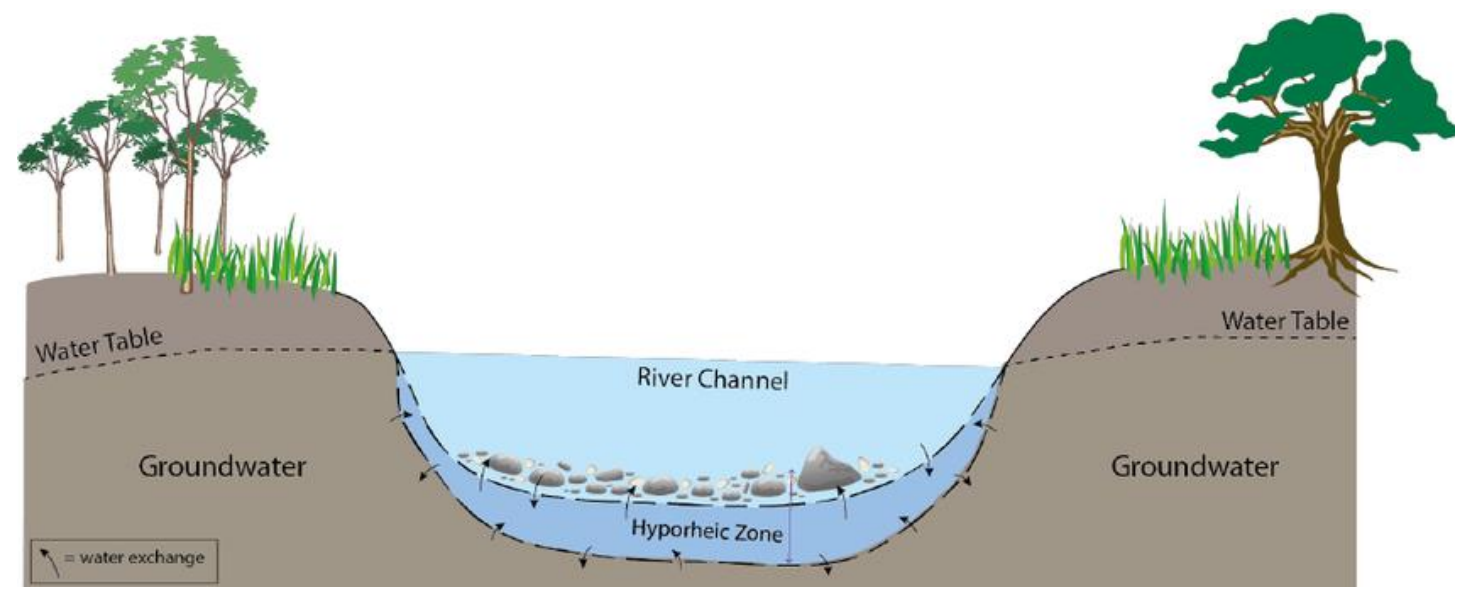

Figure 2. Cross-sectional view of the hyporheic zone in a river catchment. The hyporheic zone (HZ) is a fundamental part of a river ecosystem. It is the zone of interaction between groundwater and channel water (Biddulph, 2015).

Additionally, the pool-riffle sequence provides transport of sediment materials, which are important for salmon spawning habitat; while there is erosion and deposition occurring in both the pools and the riffles, the coarser material in the riffle region is what salmon prefer. It is in this deposition region of the riffle that salmon lay their eggs; it is also the region where the smaller macroinvertebrates reside which provides a nutrient rich region for the young salmon. Fundamentally, the relationship of pools and riffles to bed form structures is a key attribute to understanding functions of riverine ecology (Endreny, 2003). 
This initial mobility of trapped sediments is the first factor to consider in the geomorphic response to the removal of a dam, yet there are other factors that need to be explored. It is important to consider the initial channel response and subsequent channel migration/morphology and the reestablishment of key instream habitat structures such as pools and riffles. The effects of dams and reservoirs create an imbalance to the natural system and have been shown to have adverse effects on native fish populations, even when fish ladders have been installed to offset the presence of the dam (Neave et al., 2009). The consideration given to threatened native fish species and the restoration of their natural habitat have been discussed in the dam removal process but have not been the key factor cited for the removal which in many cases have been the initiation of Federal Energy Regulatory Commission (FERC) relicensing and the high expense of implementing changes in line with the Endangered Species Act (ESA) as was the case with the Marmot Dam. The introduction of new technologies has made the study of the geomorphic response to dam removal over large areas more feasible. Lidar is one such breakthrough that allows researchers to view changes over time and apply watershed and geologic reach similarities to disturbance regime models which would prove beneficial in dam removal impact assessments.

The removal of the Marmot provided an opportunity to run repeat lidar acquisitions over a 6-year period. Oregon Lidar Consortium (OLC) and the Oregon Department of Geology and Mineral Industries (DOGAMI) gathered a group of stakeholders to fund the multiple flights and study various geohazards in the region (English, 2008). The Sandy River is highly competent as are many of the streams the come out of the Cascades (Abbe et al., 2015). The competence of the Sandy lends it to the mobilization of boulders and has 
resulted in a reverse sorting due to the extreme pulses on the stream (Figure 3); this has led to the formation of well armored terraces and islands which have not seen flow levels sufficient enough to mobilize them. Past avulsion/channel migration paths are visible in the terraces and the fact that the river has shifted laterally and incised vertically in its current path lends to my theory that the current stream position is highly stable given current conditions and has reached the quasi-equilibrium stage of the process.

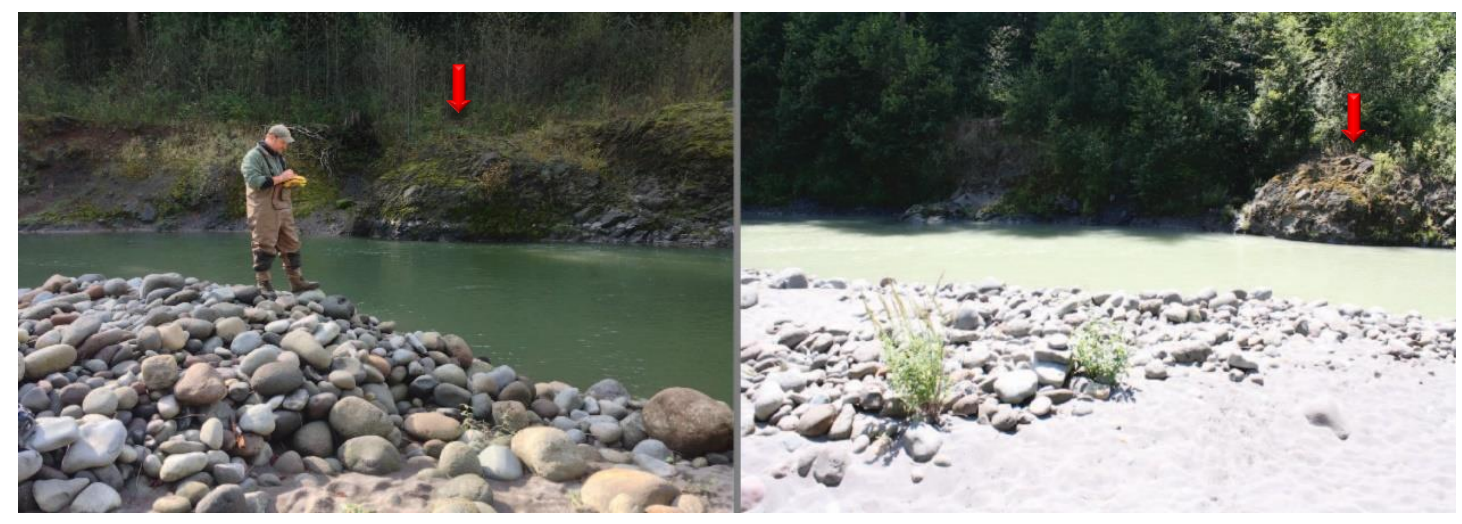

Figure 3. Reverse sorting of sediment with large cobbles intermixed throughout the gravel bar, approximate location of Transect C. Photo by Lowell Anthony, Oregon Department of Geology, October 13th, 2014 (left) at 294 cfs and July 15th, 2018 at 410 cfs (right).

However, lack of any significant revegetation on the bars would indicate significant flows during the winter months which would continually scour the bars and further readjust their position. Since the removal of the Marmot Dam the channel has gone through various changes but is currently in a condition of quasi-equilibrium and the 'flashy' nature of flooding on the Sandy lends to the reverse sorting seen on the bars, during high quick flows there is enough discharge to mobilize the large boulders, which in turn end up falling out much quicker when the flows start to slacken leaving very well armored bars that would need significant flow over a stretch of time to see further mobilization or significant change in the lateral profile of the reach. Well consolidated and resistant bedrock flows also limit the lateral migration potential of the Sandy in this area and is probably one of the driving 
factors behind choosing the site for the dam. Flow is diverted around these resistant lahar tufts and an accumulation of large woody debris (LWD) is noticeable atop one of the formations (Figure 4) downstream of the crib structure mid-riffle, which in turn forms a pool before sliding around the bend into another riffle.

The resistant lahar tufts that extend into the river margin currently preclude further migration potential, but also lead to sweeping meander arcs that present the potential for slope failure; channel migration induced landslides can occur when the outside arc of the meander bend cuts into the margin of the bank enough to undermine the angle of repose for the bank. This in turn leads to instances of channel blockage and avulsion potential for the river. Landslides are downhill movements of rock, debris, or soil. While there are many different types of landslides in Oregon.

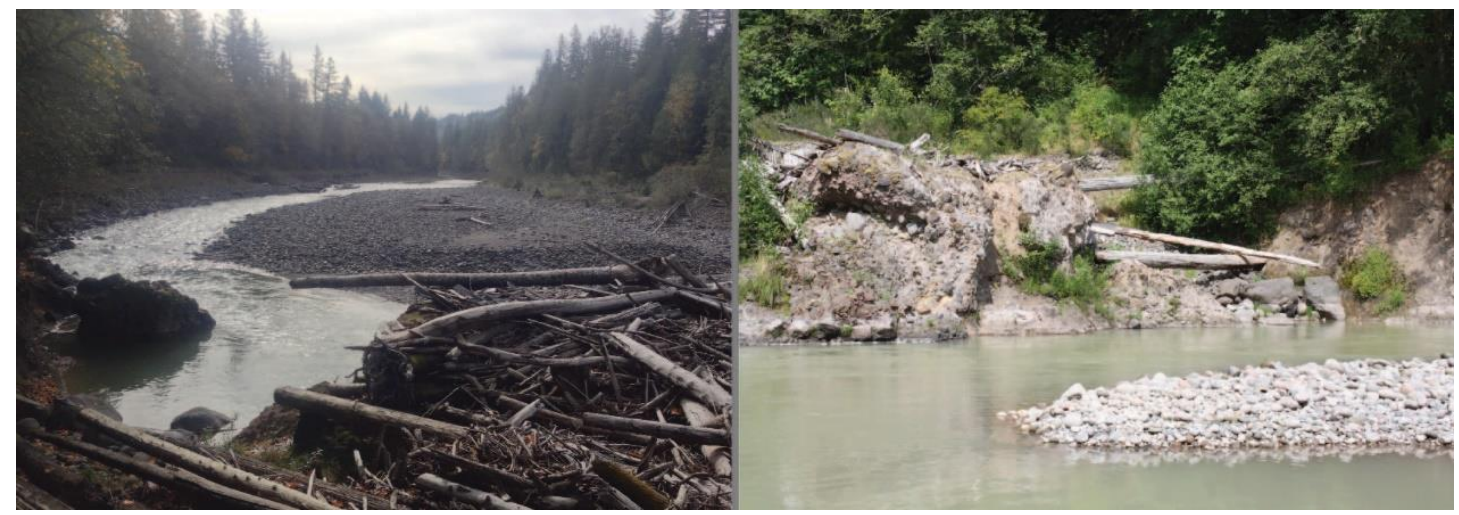

Figure 4. Large woody debris accumulation atop brecciated lahar dike, approximate location of Transect D. Photo by Lowell Anthony, Oregon Department of Geology, October 13th, 2014 (left) at 294 cfs facing south and July 15th, 2018 at 410 cfs in channel facing north (right).

In the Lower Columbia-Sandy watershed where the Sandy River is located, the most common are debris flows and shallow- and deep-seated landslides. Landslides can occur in many sizes, at different depths, and with varying rates of movement. Given the Sandy Rivers competence and mobility along it course there is a potential for channel migration induced landslides in the area. Many triggers can cause a landslide: intense rainfall, 
earthquakes, or human-induced factors like excavation along a landslide toe or loading at the top or channel migration induced action (Figure 5). Landslides can cause severe damage to buildings and infrastructure. Fast-moving landslides may pose life safety risks and can occur throughout Oregon (Burns et al., 2016).

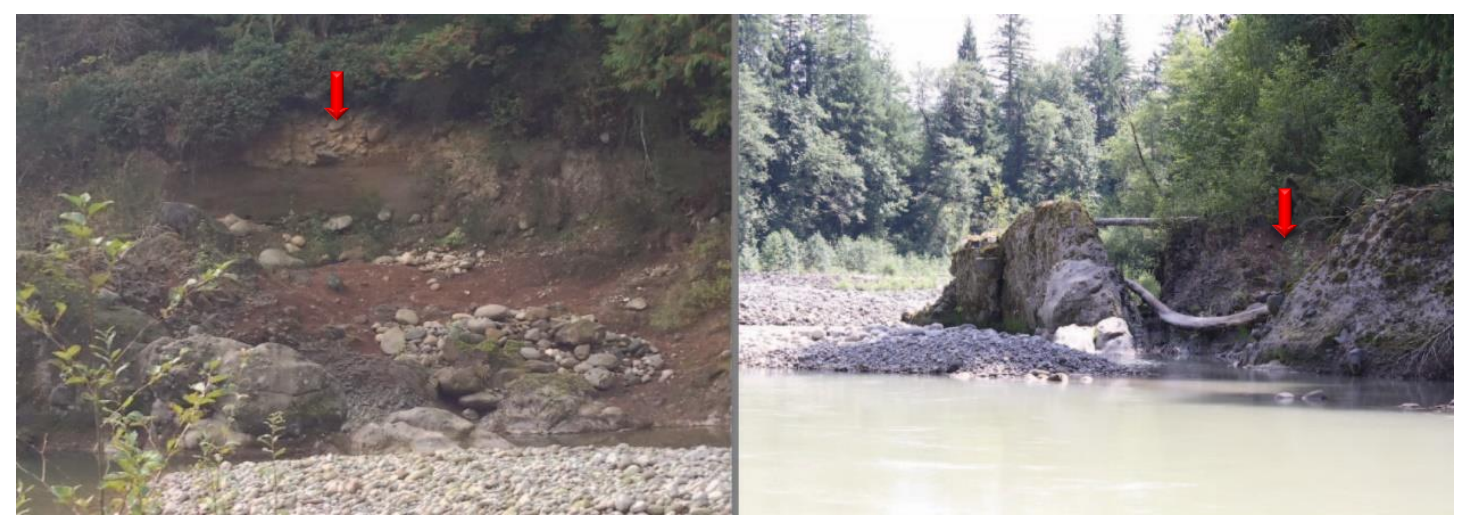

Figure 5. Channel migration induced slope failure. Note the exposed sediment layers which show Troutdale formation overlaying older flood deposition (left) 2018 photo shows the same location from a different angle the exposure is less evident. Photo by Lowell Anthony, Oregon Department of Geology, October 13th, 2014 (left) at 294 cfs and July 15th, 2018 at 410 cfs (right).

\subsection{Reach Description}

The study area is the located at the site of the removed Marmot Dam, a portion of the Sandy River, Oregon, which is a 90-kilometer tributary of the Columbia River originating on the southwest flanks of Mount Hood from the Reid Glacier. The Sandy River then flows through Clackamas and Multnomah County ending at the Columbia River near Troutdale Oregon. Increased interest in the Sandy River's hydraulics and geomorphology has come from the decommissioning of the Marmot Dam in July of 2007 and its final removal completed in October of 2007. The removal of the dam provided a unique opportunity to examine channel morphology characteristics in a sediment-rich environment (Major et al., 2012). 
The examination of the old impoundment of the Marmot Dam, a 3.2-kilometer reach, is the area of focus. Using field surveys, known literature of the same region, orthophotos and lidar, I examine whether lidar can be an effective proxy to field work to map geomorphic features and pool-riffle complexes on the river. Previous literature shows that lidar can be effective for extracting water surface features when the angle of incidence for the pulse return is at its nadir and that rougher water surfaces give more return pulses for extraction (English, 2008). The use of lidar for natural resource management and planning has progressed over the past decade and a national U.S. lidar acquisition (https://nationalmap.gov/3DEP/) is expected to be a fact by 2020 (Evans et al., 2009). Lidar has been used to illustrate the geometry and slope of mountain channels in the Pacific Northwest giving researchers the capability to have a reliable and repeatable approach for quickly and effectively sampling and analyzing the physical characteristics within the watershed (Faux et al., 2009). New studies using green wavelength lidar allows for highresolution bathymetric studies of channel depth and bed profiles. My own research is a continuation/addition to the growing use of lidar for resource management and channel morphology studies and allows me to build off previous research methods while still providing a valuable contribution to the field.

The immediate downstream area of the dam has not seen significant changes due to the well armored channel carved out of the river entrenchment downstream of the dam spillway. Figure 6 shows the channel morphology at the spillway looking upstream from the footbridge, notice that there is not significant difference in the channel morphology at this point. The natural constriction formed at this point of the reach would explain the location of the dam. Overall incision rate of the entire Sandy compared to the rivers around 
it from the last lahars flow approximately $1781 \mathrm{CE}$ (Old Maid eruptive period) still puts it at an overall higher elevation than other rivers around it suggesting downward incision still needed to reach equilibrium, the area of the Marmot Dam had the downward incision rate halted and the accumulation of additional sediment from upstream erosion.

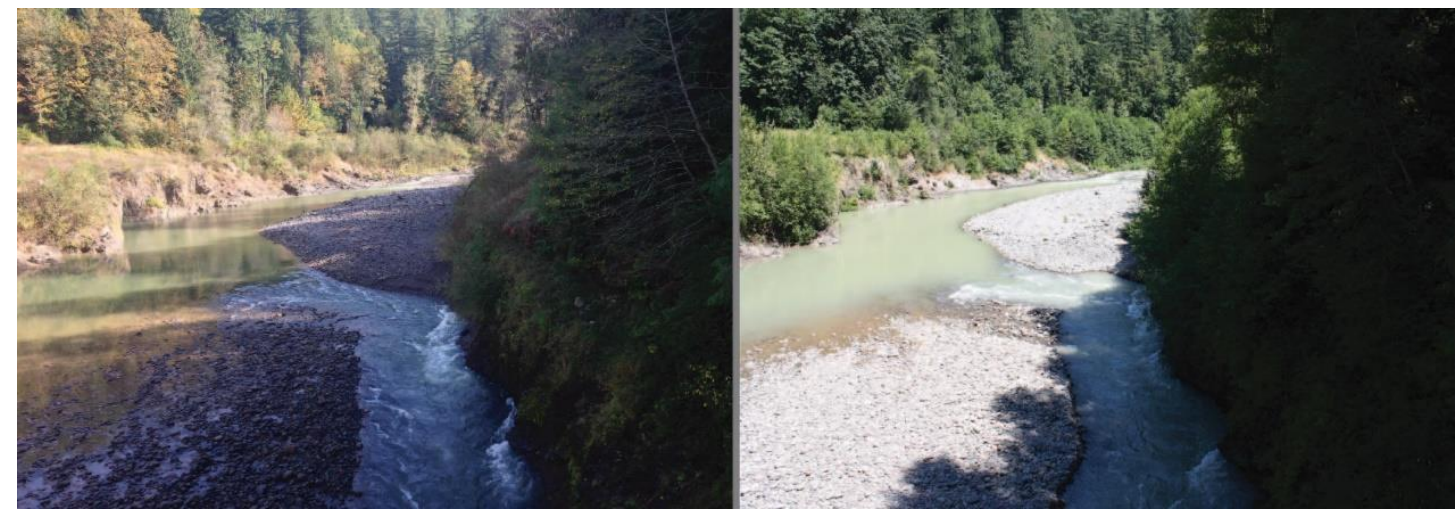

Figure 6. Location of the old Marmot Dam and beginning of the spillway looking upstream from the footbridge. Photo by Lowell Anthony, Oregon Department of Geology, October 13th, 2014 (left) at 294 cfs and July 15th, 2018 at 410 cfs (right).

This coupled with development in the Villages at Mt. Hood area and subsequent channel modifications by the United States Army Corps of Engineers (USACE) has adjusted the Sandy Rivers capability to mobilize the sediment that was left after the Old Maid eruptive period. We know from previous studies of the Mt. Hood region that the Old Maid eruptions added large volumes of sediment to the upper reaches of the Sandy River, overwhelming the highly competent river system, accelerating the sediment loading to the river during peak flow conditions.

The large sediment loads exceeded the Sandy's transport capacity changing the rivers geomorphology downstream of the event (Pierson et al., 2009). Evidence of this volcanic action is still visible in the stream cuts and bank exposures along the old Marmot Dam impoundment. The current position is well entrenched, but further plucking and high flows continue to pick at the margins. 
The initial removal of the dam saw the bulk of accumulated dam sediment quickly mobilized and redistributed with the knickpoint retreat establishing itself at the pre-dam incision point upstream (Major et al., 2012). Local geology constrains the extents of lateral migration with well consolidated dikes of brecciated lahar tufts scattered through the reach and well armored boulder fields that are well defined.

Major et al., (2008) looks at the initial response to the removal of the Marmot Dam which was located approximately 45 kilometers upstream of the Columbia River Confluence. The removal of the dam released approximately 730,000 cubic meters of sediments stored behind the dam. The articles focus was on the initial fluvial response to the removal of the dam in the first 48 hours after the breach of the coffer dam. In that period approximately 100,000 cubic meters of stored sediment was transported downstream by the erosive force of the Sandy River (Major et al., 2008).

The original rock-and-timber-crib structure dam was built in 1913 and was later replaced in 1989 by a 14-meter high and 50-meter wide concrete dam owned and operated by Portland General Electric (PGE) and was used as part of the Bull Run Hydroelectric Project, diverting water from the Sandy River and up to Lake Roslyn, a manmade lake that is no longer in existence after the removal of the dam. Options were evaluated for the decommissioning of the dam, and it was determined that the "blow and go" method would be the most effective given the competency of the river; all that was needed was a sufficient flow (Major et al., 2008).

With this method the dam is removed as quickly as possible with minimal sediment removal taking place before the dam is breached. To facilitate the dam's removal an earthen cofferdam was built on top of impounded sediment approximately 70 meters upstream of 
the 1989 concrete dam, diverting the Sandy River around both the dams and allowing for the pre-removal of close to 20,000 cubic meters of sediment that was located between the two dam structures. While this method is desirable from a low cost and minimal in-stream disturbance perspective, there was still the issue of the 730,000 cubic meters of sediment remaining behind the coffer and dam (Major et al., 2008).

To minimize the impact to seasonal fish runs and any adverse effects to resident and migratory fish, the dam breach schedule was decided in July of 2007 to coincide with the higher flows associated with region which typically begin in mid-October. The Sandy River is an unfettered river with a high-gradient of $0.006-0.009$ meters per meter and peak flows in the winter months above 20,000 cfs (Major et al., 2008). With a heavy rainfall forecasted for October $19^{\text {th }}, 2007$ and a predicted flow of 68 cubic meters per second $(\mathrm{cms})$, a dam breach was initiated at 10:00 am PDT, dewatering pumps were removed around 12:00 pm PDT and by 1:30 pm PDT the first signs of water began seeping through the earth dam, triggering a small mass failure of the dam's downstream face. By 5:00 pm PDT the river was flowing near $50 \mathrm{cms}$ and a small notch was carved in the crest of the cofferdam allowing for the release of the flow (Major et al., 2008). By 5:45 pm PDT the flow was exponentially enlarging the breach which resulted in a flash flow peak of 136 cms as the river rapidly reasserted itself to predam profile, by 6:00 pm PDT the river had completely incised through the cofferdam and by 11:30 pm PDT the river had completely removed it (Major et al., 2008).

As the dam washed away, the Sandy River continued to erode the impoundment of sand and gravel through a combination of lateral erosion and headward cutting, and within 12 hours of the breach, it had incised through the sand and gravel fill in the lower 300 meters 
of the reservoir reach (Major et al., 2008). As the knickpoint retreated upstream the lateral migration of the river swiftly widened the river and eroded the meters high unconsolidated fill which was exposed as the reservoir drained. Preliminary analysis of the initial results indicated approximately 100,000 cubic meters of sediment was eroded in the first 48 hours of the breach at about $50 \mathrm{cms}$ which is a discharge just $30 \%$ greater than the median flow. That sediment load correlates to the approximate mean annual load of the Sandy River at the Marmot Dam (Major et al., 2008). Within 6 months of the breach approximately 300,000 cubic meters of sediment had eroded from the reservoir, and the knickpoint had retreated 1.5 kilometers upstream of the dam to the approximate intersection point of the Sandy River to the upstream influence of the Marmot Dam, the area of reduced discharge where the river transitions from being unfettered to the reservoir (Major et al., 2008).

The removal of the Marmot Dam marked the largest release of a trapped impoundment up to that time and was the first instance in close to 100 years that the Sandy River ran unconstrained from its headwaters to its confluence with the Columbia River (Major et al., 2012). Channel response following the removal of Marmot Dam showed the same geomorphic characteristics described by Doyle et al., (2005); the initial knickpoint progressed rapidly upstream as the high velocity Sandy River eroded the unconsolidated sand-gravel sediment impoundment This quick incision rate and knickpoint migration upstream caused the channel to widen rapidly and mobilize a large portion of the 15-meterhigh sediment banks on either side of the initial breach channel.

The high velocity Sandy River incised to near pre-dam elevations in just 12 hours the upstream geomorphology further adjusted following high velocity stormflows typical of the Sandy River in winter months. Thus, by mid-January close to another 300,000 cubic 
meters of impoundment was removed (Major et al., 2012). The knickpoint retreat stabilized at the upper most end of the original impoundment deltaic zone and the river has migrated laterally a few times over the years since the initial removal but seems to have stabilized in the current channel configuration. My observation correlates to the conclusion drawn by Major et al., (2012) and shows the channel has reached the quasi-equilibrium stage of the removal. As degradation and lateral migration continued a wooden 'crib' structure (Figure 7) emerged from the sediment and has since formed part of the rapid/riffle structure with the resistant lahar dike forming a breakwater that lends to the pool structure at the end of the riffle run (Figure 8).

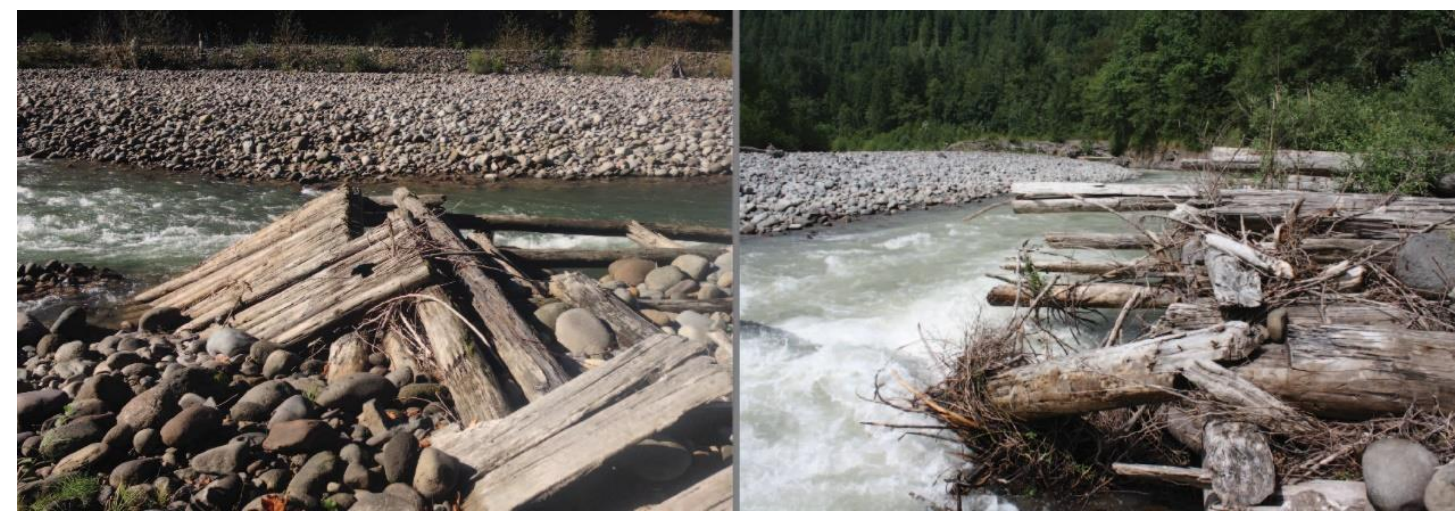

Figure 7. 'Crib' structure mid-rapid above the old Marmot Dam location. Photo by Lowell Anthony, Oregon Department of Geology, October 13th, 2014 (left) at 294 cfs and July 15th, 2018 at $410 \mathrm{cfs}$ (right).

Upstream of this structure, reverse gradient deposition beds can be seen in the bank margin, this shows the accumulation of sediment in the impoundment overtime (Figure 9). The different sediment accumulations can give us a picture of the flow regimes that influenced the river over time.

Different layer accumulations show sediment differences, these differences would correlate to high flow regimes and the type of sediment mobilization from further upstream. Given the nature of the Sandy River and the geologic history of the region, high 
precipitation events dislodge material from further up the reach. This material can be in the form of old lahar deposits, glacial till, and landslide/debris flow material from over saturation and channel migration events. Major et al., (2012) is a comprehensive USGS report on the results of the multi-year study of the dam removal. The report provides a starting point for my own look at this region.

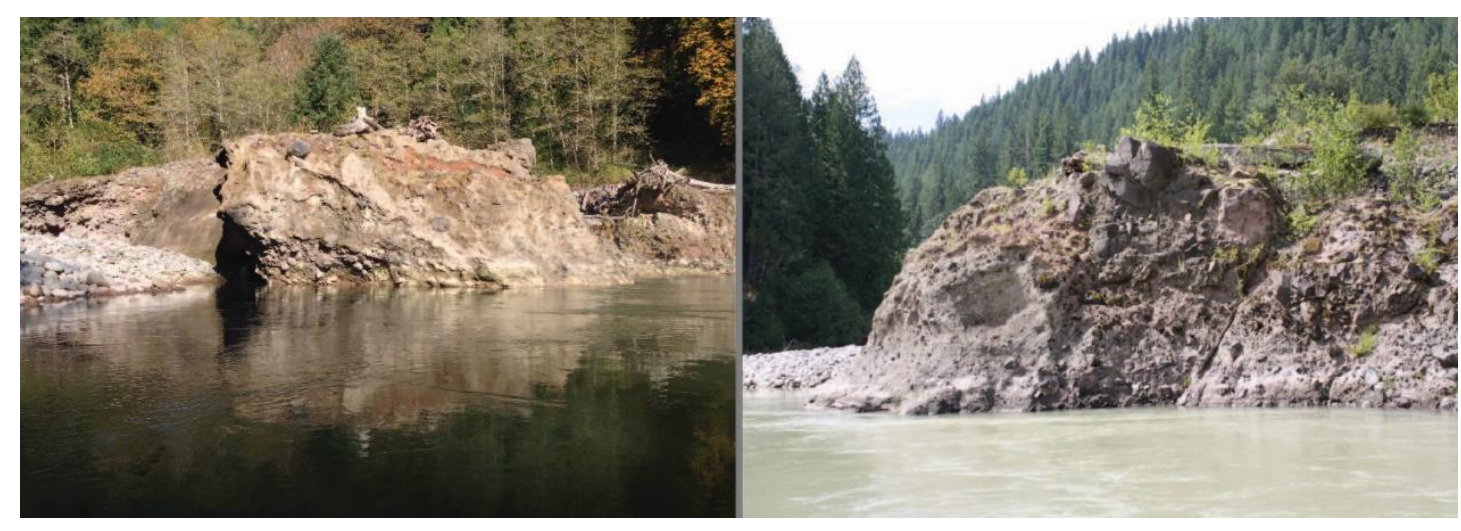

Figure 8. Brecciated lahar dike north of the crib structure at the end of the riffle/run. Photo by Lowell Anthony, Oregon Department of Geology, October 13th, 2014 (left) at 294 cfs and July 15th, 2018 at 410 cfs (right).

By the end of the first year after the breach the slope discontinuity caused by the rapid retreat of the knickpoint and subsequent lateral migration of the channel was nearly indistinct and bed coarsening progressed to near normal conditions for a high velocity high competency stream like the Sandy River. Seasonal variability on the Sandy River plays a significant role in shaping the morphology. Flow conditions on the Sandy are tied to the seasonal precipitation inputs. Precipitation in the system comes as both rain and snow, with the amount of snow affecting the seasonal discharge rates in late spring and through the summer months with lowest discharge rates occurring between late August and early October (Major et al., 2012; Abbe et al., 2015).

As pointed out in previous articles channel bedform watershed geology strongly influence the rate of degradation, aggradation and over transport capabilities of the stream 
reach (Major et al., 2012). Causal correlation exists between upstream geomorphic responses to disturbance regimes such as dam removals. The relationship between the initial head-cut knickpoint creation with rapid upstream migration and the subsequent migration of the channel both lateral and in the incision, rates correlate to the volume of impoundment removed over a given timeframe (Major et al., 2012).

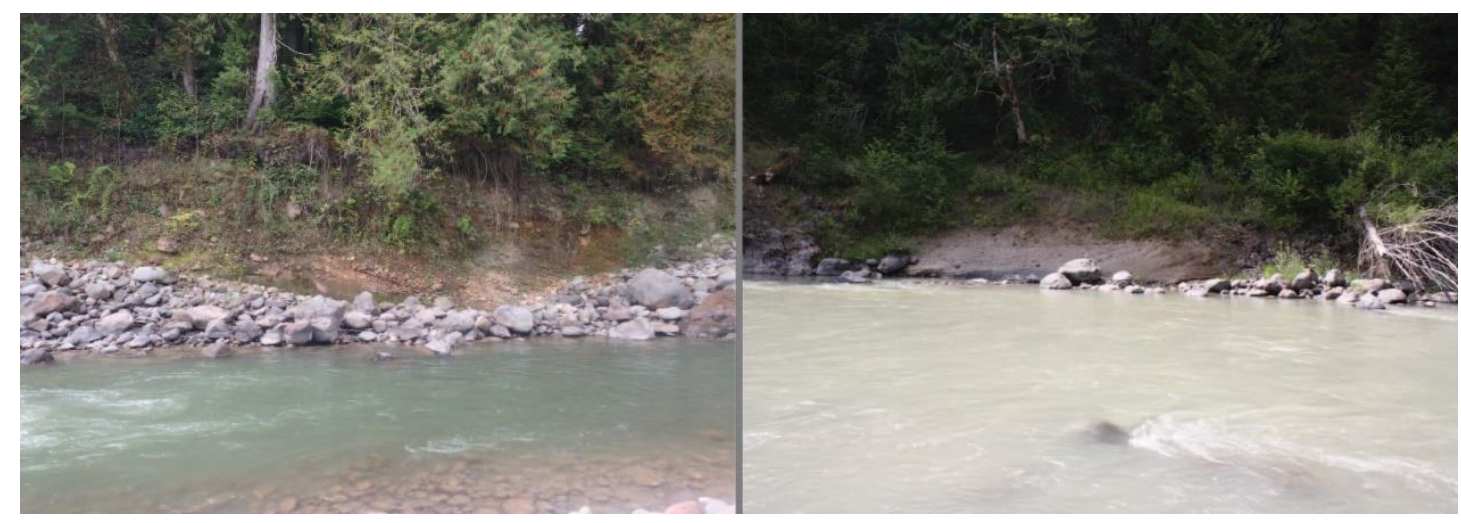

Figure 9. Reverse gradient foreset deposit (left) and foreset deposits exposed in slope failure (right). Photo by Lowell Anthony, Oregon Department of Geology, October 13th, 2014 (left) at 294 cfs and July 15 th, 2018 at $410 \mathrm{cfs}$ (right).

The duration of the fluvial response to dam removal will vary from reach to reach based on the reach boundary conditions and valley form variations. The Sandy River provided the researchers with an opportunity to record geomorphic changes on a high energy stream and put into play various interrelated functions because of dam removal that played out in a relatively short timeframe compared to past studies on the subject (Major et al., 2012).

\subsection{Current Geomorphic State}

The Sandy River is in the Lower Columbia-Sandy watershed, it is subject to numerous natural hazards that pose a potential to alter the current geomorphic conditions, as it has many times in the past. The Lower Columbia-Sandy watershed, which extends from the High Cascades through the Western Cascades and into the Portland Basin, is subject to 
several significant natural hazards, including riverine flooding, earthquake, landslides, volcanic lahars, channel migration, and wildfire (Anthony et al., 2019).

Flooding is a recurring problem for most of the riverine communities along the Sandy River and many buildings within the floodplain are vulnerable to significant damage from flooding and prone to channel migration hazard being located within the mapped channel migration zone (CMZ). While the impact of flooding on residence from a risk prospective is minor this is mainly due to local initiatives that have looked to reduce the risk to infrastructure. The Sandy River is the primary drainage for the watershed, but many secondary tributaries add to the overall mobility of the riverine environment that are prone to flooding (Anthony et al., 2019).

Volcanic activity of Mount Hood has added character to the Sandy River and human activity has further defined its current characteristics, but the Sandy River itself continues to define the region around it due to the yearly flooding that occurs in the upper reaches. This volcanism can be seen in areas of resistant brecciated lahar tufts within the study area, and the large reverse sorted gravel bars with boulder sized rocks sitting on top, and significant areas with large woody debris built up show the power and frequency of the flood events on the Sandy River. Prior to the removal of the dam, orthophotos show large sand bars covering most of the areas now exposed as boulder fields and lahar dikes. Evidence of the past volcanic lahar from Mt. Hood can be seen in the resistant lahar dikes and channel migration induced slope failure (Figure 10) GIS analysis of the longitudinal profile of the stream is in line with previous literature from Leopold and Maddock, (1953), wherein he describes the longitudinal profile of a stream as a product of the overall change in the bed load downstream. 
The correlation to stream gradient and bed load is also taken into consideration as stream competency and overall configuration of the stream bed are a result. In the case of the Marmot Dam, past geologic events have shaped the margins of the stream channel and after the Old Maid approximately 1781 C.E. pyroclastic flows and lahars choked the Sandy River with sand and gravel altering the river valley margins and increasing sediment loads out to the Sandy Delta. The unconsolidated lahar tuffs and breccia are visible in the study area. The angularity of many of the exposed cobbles within the lahar complex lends to the ferocity of the eruptive period resistance to the erosive competency of the Sandy River.

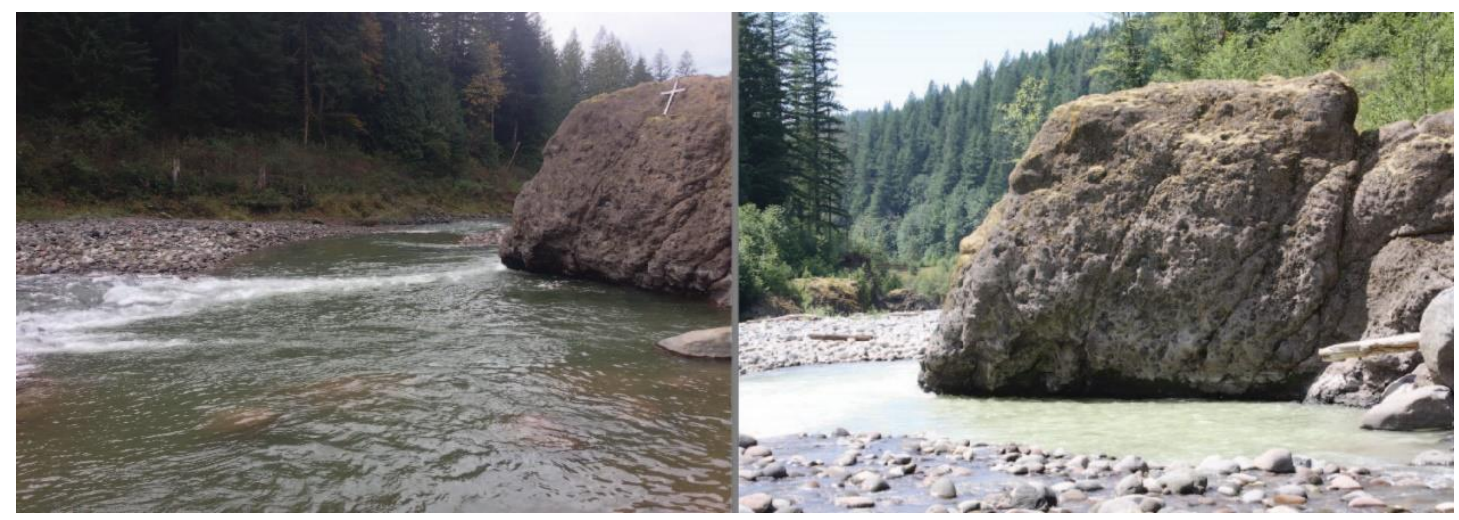

Figure 10. Resistant lahar dike, reverse sorted gravel bar, and channel migration induced slope failure. Photo by Lowell Anthony, Oregon Department of Geology, October 13th, 2014 (left) at 294 cfs and July 15 th, 2018 at $410 \mathrm{cfs}$ (right).

The area has reached a stage of quasi-equilibrium with the channel well defined and bracketed by large gravel bars and resistant lahar deposits. Some area on the west bank are still prone to slope failure given significant enough flows to overtop the gravel bars and current (2014) channel. Most of the gravel/boulder bars have limited amount of sediment accumulated on them, suggesting that the winter flows off Mt. Hood continue to scour them, but do not provide sufficient flows to mobilize the gravel bars themselves.

The reach has a few discernable pool/riffle sequences. The most prominent riffle is located approximately 10 meters upstream of the crib structure before the constriction point 
of the reach and transitions quickly into an approximately 170-meter-long rapid/run before depositing into the first pool at the downstream bend beyond the structure. The resistant lahar tufts that emerge into the channel margin slow the flow of the river at this bend and cause a slackening and deepening of the channel creating a nice pool for salmonids to take advantage of (Charlton, 2008).

\subsection{Past Geomorphic History}

The geomorphology of the Sandy River has been directly affected by past eruptive periods from Mt. Hood followed by successive high flow events that have mobilized the sediment and moved debris from the eruptions down the riverine system. The most recent significant eruption from Mt. Hood was the Old Maid eruptive period which took place over approximately two decades and added significant amount of source sediment to the Sandy River.

Pierson et al., (2009) describe the two most significant eruptive events to come off Mount Hood in the late Holocene period. The Timberline and Old Maid eruptive periods were both considered dome-building eruptions resulting in numerous dome-collapse pyroclastic flows and lahars. These events moved large volumes of sediment and volcanic clasts both into temporary storage near the headwaters, and throughout portions of the entire reach resulting in the resistant lahar exposures visible throughout the Sandy River (Pierson et al., 2009).

These events introduced sediment loads that exceeded the river's transport capacity, leading to channel aggradation, channel widening, and channel morphology change approximately 80 kilometers downstream of Mount Hood (Pierson et al., 2009). Both 
events lead to broad bed-material waves of sediment, creating slurry like conditions that took approximately 2 decades to reach the mouth of the river. The Old Maid event added approximately 23 meters of aggradation from the initial lahar hazard, but the bulk of the aggradation came from transport of sediment and subsequent deposition during normal peak flow conditions on the Sandy River. Over time the supply of sediment in the headwaters diminished enough that the river was able to degrade some areas, incising channels further which are evident today as abandoned terraces with higher avulsion potential and thus exposure to channel migration hazard (Pierson et al., 2009). Since that time the major driver in overall morphology is the variability in seasonal inputs to the system accounting for the high flood hazard for the Upper Sandy River and the overall movement of sediment the Sandy River is capable of (Major et al., 2012; Abbe et al., 2015) 


\subsection{Seasonal Inputs}

Flows on the Sandy are directly correlated to the climate of the region. The typical seasonal outputs for the western Cascades are generally mild, wet winters and relatively cool and drier summers. The annual precipitation average varies from 78 inches down in the lower valley, with 100 inches or more, annually on the slopes of Mount Hood. Most precipitation for the region comes in the form of rain in the valley and transitions to snow in the upper elevations. The elevation variability of the freezing level in the region can account for increased discharges depending on the episodic pulses in the weather pattern (Abbe et al., 2015).

Factors that contribute to the flow regime on the Sandy River vary depending on the level of precipitation, the time of the year, and the level of the snow pack coupled with the time of melt. The streamflow characteristics of the Sandy River are dictated by this variability in precipitation and temperature, leading to low flows in October during the start of the water year, with discharge increasing as the winter storms come in mid-November, continuing to remain high into May. These generally taper off through the summer where they reach minimum flow conditions in August and September (Abbe et al., 2015).

\subsection{Gauge Data}

The active USGS Marmot stream gage \#14137000 has discharge records dating back to 1912 for the Sandy River. The gage is in a confined channel segment near the former Marmot Dam site, comparing daily streamflow stats allows calculation of flood probability and extreme discharge events (Table 1). These data points allow researchers to get a better understanding of the flow regimes on the Sandy River, and for this reach (Abbe et al., 
2015). Seasonal variability on the Sandy is a key factor in the morphological response in my study area, the impact of two key floods provided the most noticeable change in the channel morphology in the lidar comparison and lends credence to having sequential lidar.

Table 1. Top ten peak flow events at Sandy River Marmot gauge (\#14137000). Abbe et al., 2015

\begin{tabular}{ll}
\hline \multicolumn{2}{c}{ USGS Gaging Station \#1413700 } \\
\hline Date & Peak Flow (CFS) \\
\hline January 6, 1923 & 29,200 \\
November 24, 1960 & 27,500 \\
December 22, 1964 & 61,400 \\
January 20, 1972 & 36,200 \\
December 2, 1977 & 34,500 \\
February 23, 1986 & 37,800 \\
February 7, 1996 & 48,100 \\
November 25, 1999 & 34,400 \\
January 2, 2009 & 30,000 \\
January 16, 2011 & 39,000 \\
\hline
\end{tabular}

The largest variability in discharge occurs between November and February with flows that range from $\sim 500 \mathrm{cfs}$, to extremes as much as $\sim 5,000 \mathrm{cfs}$, this extreme variability which is also punctuated by episodic pulses during storm events, lend to the dynamic conditions experienced on the Sandy. Snowmelt runoff contributes to the variability during the late spring months with flows that range from $800 \mathrm{cfs}$ to 3,000 cfs (Abbe et al., 2015). "Extreme streamflow values show that the rainfall-driven and rain-on-snow events in winter can be nearly $100 \%$ greater than the largest flows during April and May. Summer baseflows typically range between 300 and $500 \mathrm{cfs}$ at Marmot, although late season storm events can increase flow to as much as 1,000 cfs in September." (Abbe et al., 2015, page 6). These atmospheric river events contribute to the relationship of the channel characteristics and 
geomorphic features located throughout the length of the river. Many of the communities above the Marmot Dam have increased risk from various hazards that can occur along the Sandy River and being in close proximity to Mt. Hood. Of those hazards the most potentially devastating and regularly occurring are landslides, floods, and channel migration, all three of which are directly related to the seasonal inputs into the system (Anthony et al., 2019). The reworking of soil lithology by the Sandy River lends to the dramatic vistas along the reach and can mobilize very large boulder sized cobbles. This has had devastating impacts to local infrastructure as happened in the 2011 flood event that reworked a portion of the Sandy River and threatened numerous homes and local access roads located within the channel migration hazard zone (Abbe et al., 2015).

\subsection{Flood History}

The results of the seasonal inputs, coupled with the geologic composition of the Sandy River, lends to particularly dramatic flood events. Due to the general conditions that exist in the Sandy Watershed, floods are the threat of greatest concern, especially for those in the upper reaches, above the old Marmot Dam location. The implementation of the dam caused the sediment transport profile of the river to be slowed dramatically, which left the towns along the Mt Hood Village Corridor at risk for dramatic effects from the flood pulses, especially in particularly heavy precipitation years, generally following the El Nino/La Nina cycle. The biggest risk associated with the high sediment loads and extreme atmospheric river storms present is that of avulsions. The unconsolidated lahar fill that dominates the surficial geology of the region is highly mobile, with the over saturated soils becoming viscous and prone to failure in the near bank areas. USGS maintains a gaging 
station near the old Marmot Dam (\#1413700) that provides historical observations of the flood frequency going back to 1987, and both peak streamflow statistics from 1912, and discharge statistics from dating back to 1911 (Abbe et al., 2015).

The robust details available for the region allow flood specialists and hydrologist the ability to create predictive models detailing possible outcomes for the region. Coupled with the advent of high-resolution remote sensing data in the form of lidar, greater detailed hazard mitigation plans, and better planning decisions can be made. The peak flow conditions on the Sandy, generally occur between October and March, with approximately $70 \%$ of the flood peaks occurring during the water rich months from November to January. The largest flood events have coincided with heavy atmospheric river conditions and warmer than usual temperatures (Figure 11). The largest flood on record was the 1964 flood which coupled the heavy precipitation with warmer temperatures, and a thick snowpack that was significantly reduced from the combination of precipitation and warm air, reaching a peak flow of 64,000 cfs on the afternoon of December 22, 1964 (Abbe et al., 2015).

The increase in discharge during the wetter months of November through January lend to the flooding in the region and increase geomorphic change of both the lateral, and downstream profiles of the river. Areas of resistant lahar tuff, breccia, and basalt, further complicate the flow potential of the region, and creating areas of dangerous flow regimes with numerous under currents near resistant outcroppings (Abbe et al., 2015). Since the removal of the Marmot Dam, the Sandy River has experienced two flow events greater than 25,000 cubic feet per second (cfs); in 2009 the flow event was recorded at 30,000 cfs and led to significant damage upstream of the Marmot Dam in region of The Villages at 
Mt. Hood. Again in 2011 a significant flow of 39,000 cfs occurred, damaging more homes and destroying portions of the local road system (English, 2013), which has also led to further change in the morphology in the study reach. While significant, the two events are what would be considered approximately a 15-year (2009) and 33-year (2011) events based on prior records for the region. This is the probability chance that any precipitation event will be matched or exceeded in proceeding years, for comparison the USGS Marmot Dam Gage has a 100-year peak flow of 49,390 cfs (Abbe et al., 2015).

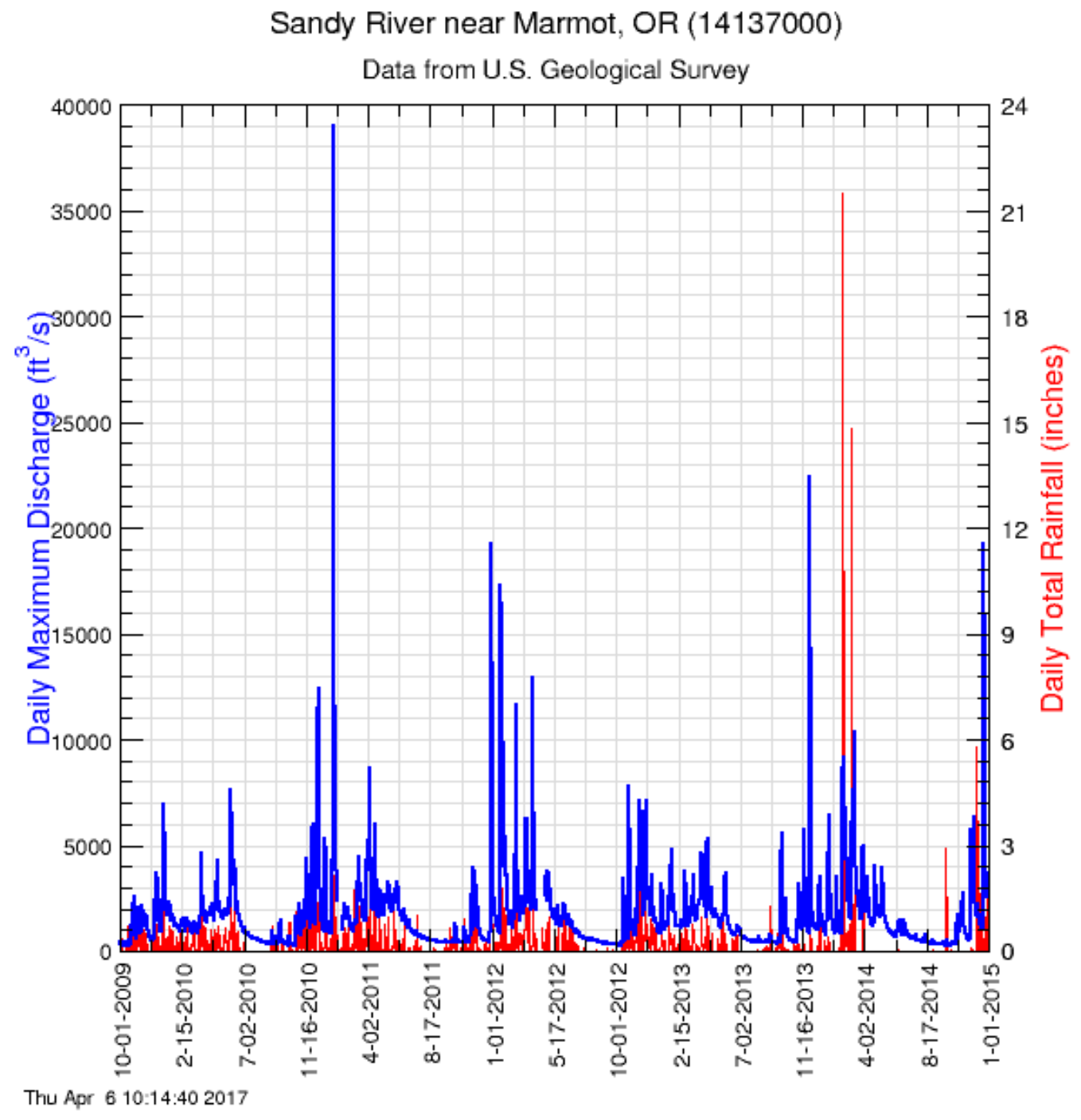

Figure 11. Daily rainfall accumulation compared to peak flows from October 1, 2009 through December 31, 2014. (Abbe et al., 2015). 


\subsection{Dam Removal}

\subsection{Background}

While dam removal has been something of a contentious subject in the past the facts behind the impact's dams have on the natural function of a healthy riverine environment have started to have more of an effect on the conversation (American Rivers, 2018). Research has shown measurable impacts dams have on native fish and other aquatic species by blocking their movement and reducing the necessary flow of fresh water necessary to sustain healthy river systems. With as many as 90,000 dams no longer serving their purpose across the United States, dam removal is fast becoming the default response to a healthy watershed and riverine environment (American Rivers, 2018).

The $21^{\text {st }}$ Century has seen the removal of numerous dams with some of the more highprofile removals garnering years long study. While environmental concerns have been cited as a benefit of removal, the driving factor in most dam removals has been the expense of complying with the ESA for fish passage tied to the renewal of the dams FERC operating license as was the case for the Marmot Dam (Major et al., 2012; Cannatelli and Curran, 2012). While the ESA and FERC costs are driving the bulk of removals, there are numerous other factors related to that added expense that impact the decision. Advances in alternative energy sources such as solar and wind power that have a less invasive impact on the natural system and less cost overhead provide a cost-effective alternative to hydroelectric dams (Cannatelli and Curran 2012).

The driving force behind the environmental concerns are primarily linked to the ESA to provide unobstructed access for native fish to upstream spawning habitats. Some examples cited are the Embry Dam on the Rappahannock River, and the Woolen Mills Dam on the 
Rivanna River in Virginia to open passage for shad migration; the Chiloquin Dam on the Sprague River in Oregon to restore passage for three species of fish; and the Elwha and Glines Canyon dams in Washington to restore spawning habitat and increase the salmon population on the Elwha River (Cannatelli and Curran 2012).

Between 1912 and 2017 the United States has seen the removal of 1,384 dams, given the large number of dams removed to date and those still slated to be removed in the future this highlights the need to have the necessary tools to provide a better understanding behind the temporal and spatial aspects of the morphological response to dam removal. The Klamath River Dam project is slated to begin in 2020 with the removal and four large head dams encompassing more than 300 miles of river habitat. The plan calls for a slow draw down of the reservoirs over a period of two to three months and a detailed restoration plan which aims to return the lands to native habitat (American Rivers, 2018). While environmental impacts have been stated in all removal proposals the overall driver for removal had been the cost of FERC re-certification and the ESA.

\subsection{Channel Evolution Model}

The importance of past research into the impact of dam removal on the downstream habitat should not be overlooked (Babbitt 2002). This is especially necessary because in most cases there were no geomorphic studies prior to and after dam construction. A high level of transparency into the impact of dam removal is necessary given the lack of research in the initial building of the dams. As Babbitt points out, not all dams are good candidates for removal, and the larger the dam the larger the impact; this makes research of the disturbance vital to the project (Babbitt, 2002). 
The size of the dam should have a direct correlation to the scope of research done on the removal. This includes both upstream and downstream impact and geomorphic assessment of the river. The size of the dam also correlates to the size of the geomorphic response of the removal and thus should correlate to the scope of the assessment. The site's geomorphology is a key factor to understanding the response to the disturbance, thus comparing historical data of the reach pre-dam can help researchers to understand geomorphic changes that can occur post-dam.

Martin Doyle examined the upstream geomorphic response to dam removal in a series of articles published over four years. In Doyle et al., (2002) the authors discuss the need for tools useful toward predicting upstream geomorphic changes from dam removal. The examination came at a time when dam removals were occurring more frequently as numerous dams throughout the country were nearing the end of their FERC license; relicensing would require upgrades and retrofits to comply with safety standards and ESA laws that had been implemented since dam construction. As was discussed by Babbitt (2002) it was essential that more research be done concerning the channel morphology and stream biota changes resulting from the removal of these aging dams; at issue was the lack of well-established tools for predicting erosion and deposition rates following dam removal (Doyle, 2002). Of primary interest was the rate of sediment transport following dam removal. In addition, dam impounded sediments contain potential pollutants that could adversely affect downstream structures and biota. Understanding the rates of erosion after removal was essential to gauging the possible impact of trapped sediment on downstream reaches as well as geomorphic changes in upstream reaches. For instance, following dam removal the rate and pattern of upstream migration of the initial knickpoint and the 
subsequent channel migration across the impoundment delta was unknown as the upstream reach seeks to reestablish equilibrium. Thorough understanding of the temporal and spatial scales of dam removal is essential to managing the impacts downstream and understanding the geomorphic changes upstream. Doyle (2002) stated that "understanding the geomorphic processes of channel incision allows prediction of forms and processes, as well as the potential application of documented stabilization techniques already in practice" (Doyle et al., 2002, page 1576). Emphasis needs to be given on pre-removal modeling through scale driven replicas of the impoundment, channel evolution models (CEM), and data collection with equal emphasis given to the post-removal upstream geomorphic changes in the reach (Doyle et al., 2002).

Doyle et al., (2003a) continues the discussion on the importance of good empirical data and the use of CEM's; models used to gain a better understanding of the effects on channel morphology spatially and temporally, developed over time from studies involving channel incision (Doyle et al (2003a). The discussion revolves around predicting the quantity of sediment released from the reservoir impoundment after dam removal. The rates of erosion and deposition are directly correlated to the method of dam removal and the seasonal variations of discharge in the reach (Doyle et al., 2003a). To assist at understanding the rates of change on the impoundment they used a CEM to predict how the channel geometry will respond once the dam is removed. The CEM was adjusted to include bank stability modeling in order to understand changes in the lateral migration of the channel. The rate of knickpoint migration and lateral channel migration will vary from reach to reach dependent upon the geomorphic, geologic, and vegetation characteristics in the impoundment area. For instance, the rate of change closest to the dam is higher as the 
impoundment delta is easily mobilized and unconstrained by bedrock or vegetation (Doyle et al., 2003a). As the knickpoint migrates upstream and the channel migrates laterally across the impoundment, the reach approaches base level and starts to encounter the original channel bedform; this limits the lateral migration of the channel and slows the headward cutting of the knickpoint. Once the channel and knickpoint reach the end of the reservoir constraints there is a decrease in lateral migration due to the stabilizing factors of vegetation and the established channel bedform; it is at this point that the channel reaches equilibrium ending knickpoint migration (Doyle et al., 2003a). It can be concluded that once the dam is removed and equilibrium is reached that the remaining sediment in the impoundment will be relatively stable; it can be further stabilized by planting native plants species that increase bank stability in the region (Doyle et al., 2003a).

In Doyle et al., (2003b) the discussion focuses on the removal of two low-head dams in Southern Wisconsin. The differences in the grain sizes and cohesiveness of the two impoundments determined the rate of changes upstream of each reach. As Doyle has previously stated, both erosion and deposition rates of sediment, the effects of unique watershed hydrology, and geomorphology are key factors to understanding the impact to reestablishing natural stream characteristics post-dam removal. Dam removal provides us with a managed disturbance regime on a given reach, thus allowing researchers to gain a better understanding of the geomorphic response to large scale disturbance. At each of the dam sites a cross section survey was performed to mark the existing bank and reach conditions and to help monitor the geomorphic changes occurring upstream of the breach. Following dam removal, the team ran their previously discussed CEM's for each reach, the results of the dewatering and removal of the dams were consistent with the findings of the 
CEM data (Doyle et al., 2003b). As the water level lowered, an initial head-cut created a knickpoint with high velocity constrained to a narrow, steep channel. As the channel continued to evolve the knickpoint migrated upstream, until it reached the streams intersection with the reservoir upstream limit. As the channel gets closer to equilibrium it becomes wider and slower meandering across the impoundment delta and both eroding sediment on the cut bank and depositing sediment on the point bar regions of the emerging channel. The group classified the changes of upstream geomorphology into six stages with the variability of stage progression (Figure 12) being marked by the velocity of flow, channel slope, seasonality of the dam removal and stratigraphic composition of the impoundment itself (Doyle et al., 2003b).

(c)

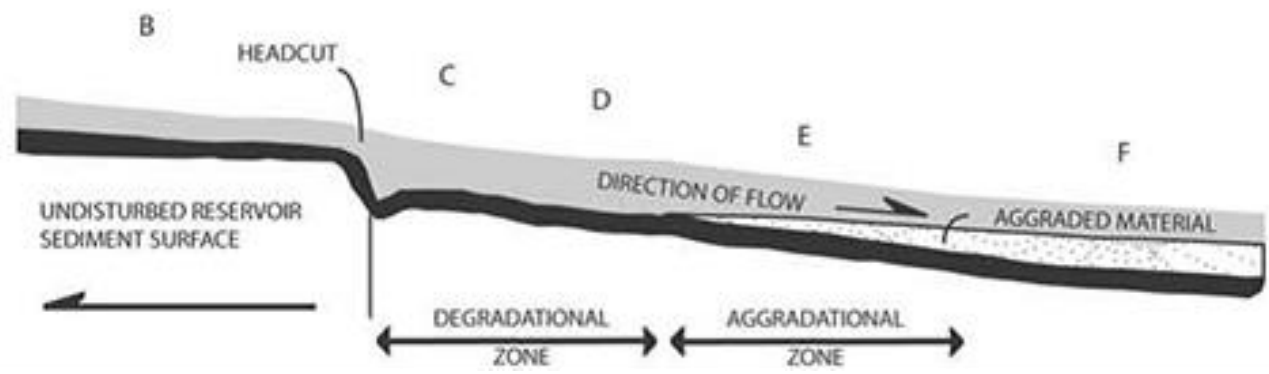

Figure 12. Channel Evolution Model stages in profile view. The channel profile view (c) shows each stage as it relates to equilibrium development in the reservoir impoundment. (Doyle et al., 2003b)

Stage A represents existing condition pre-dam removal, Stage B correspond to the conditions right after the initial removal, Stage $\mathrm{C}$ is characterized by channel degradation (Figure 13). Stage D represents channel widening, Stage E represents continued widening and migration of the channel with combined degradation and aggradation of the channel margins, and Stage F represents further aggradation and channel equilibrium develops (Figure 14). The composition of the sediment in the impoundment dictates the rate of 
upstream channel development and the process of the dam removal itself impacts the rate of change in the reach (Doyle et al., 2003b).

(a)
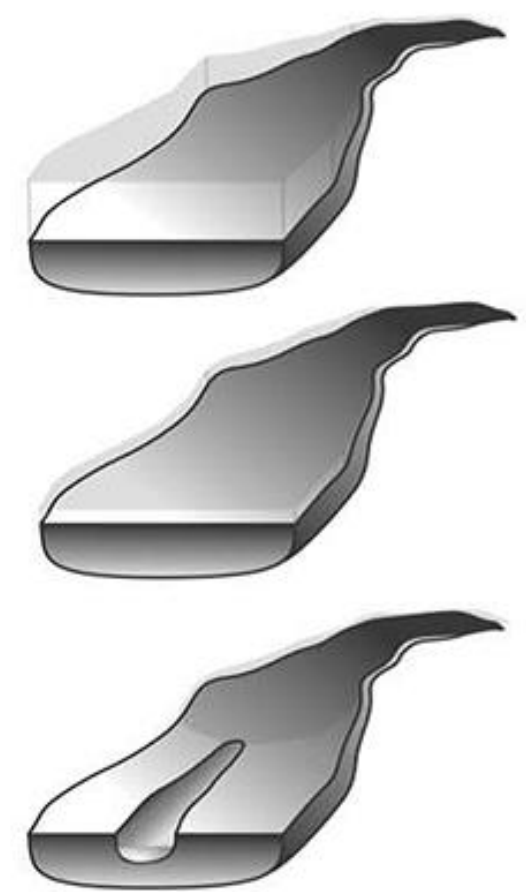

(b)

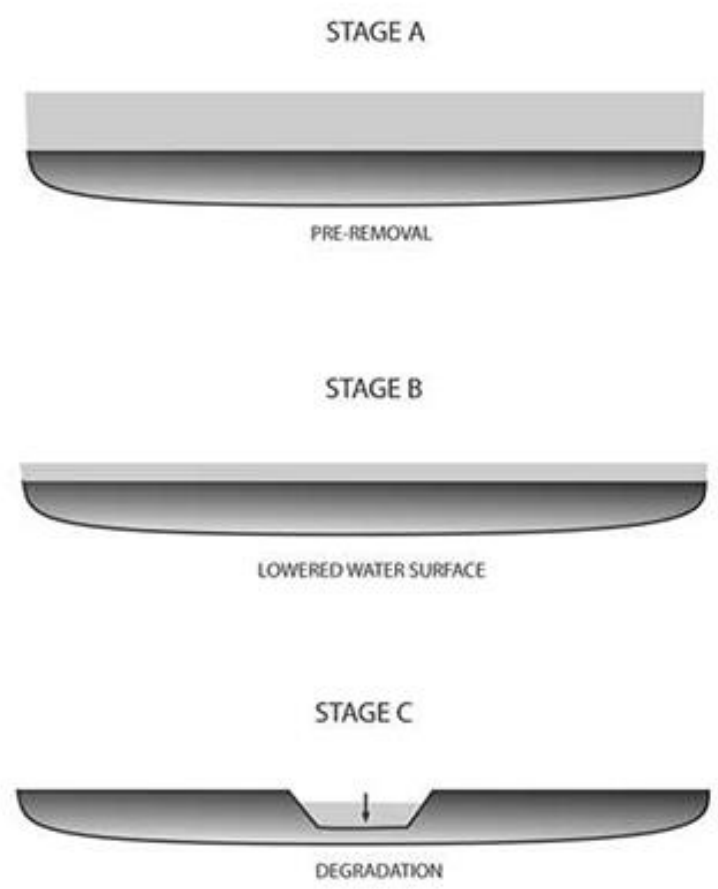

Figure 13. Stage Progression of a channel evolution model showing channel development in a reservoir after the removal of a dam: Stages A to C. Channel degradation begins quickly once the structure is removed. (Doyle et al., 2003b).

In the final Doyle et al., (2005) discussion a series of small dam removals were modeled to understand the effects on upstream channel morphology and downstream effects on key ecosystem attributes. While understanding the upstream effects of dam removal are important to scientific research on the subject, of equal importance is the affect downstream on key stream ecosystems.

The impact downstream on vegetation, fish, macroinvertebrates and nutrient loads should also be considered in the removal of a dam. Understanding the upstream geomorphic response to the removal of a dam can tell us the likely impacts on downstream habitats and allow for mitigation techniques to play a part in the redistribution of trapped 
sediment on downstream habitats. Once again, CEM's were used to establish preliminary responses across a range of trophic conditions (Doyle et al., 2005).
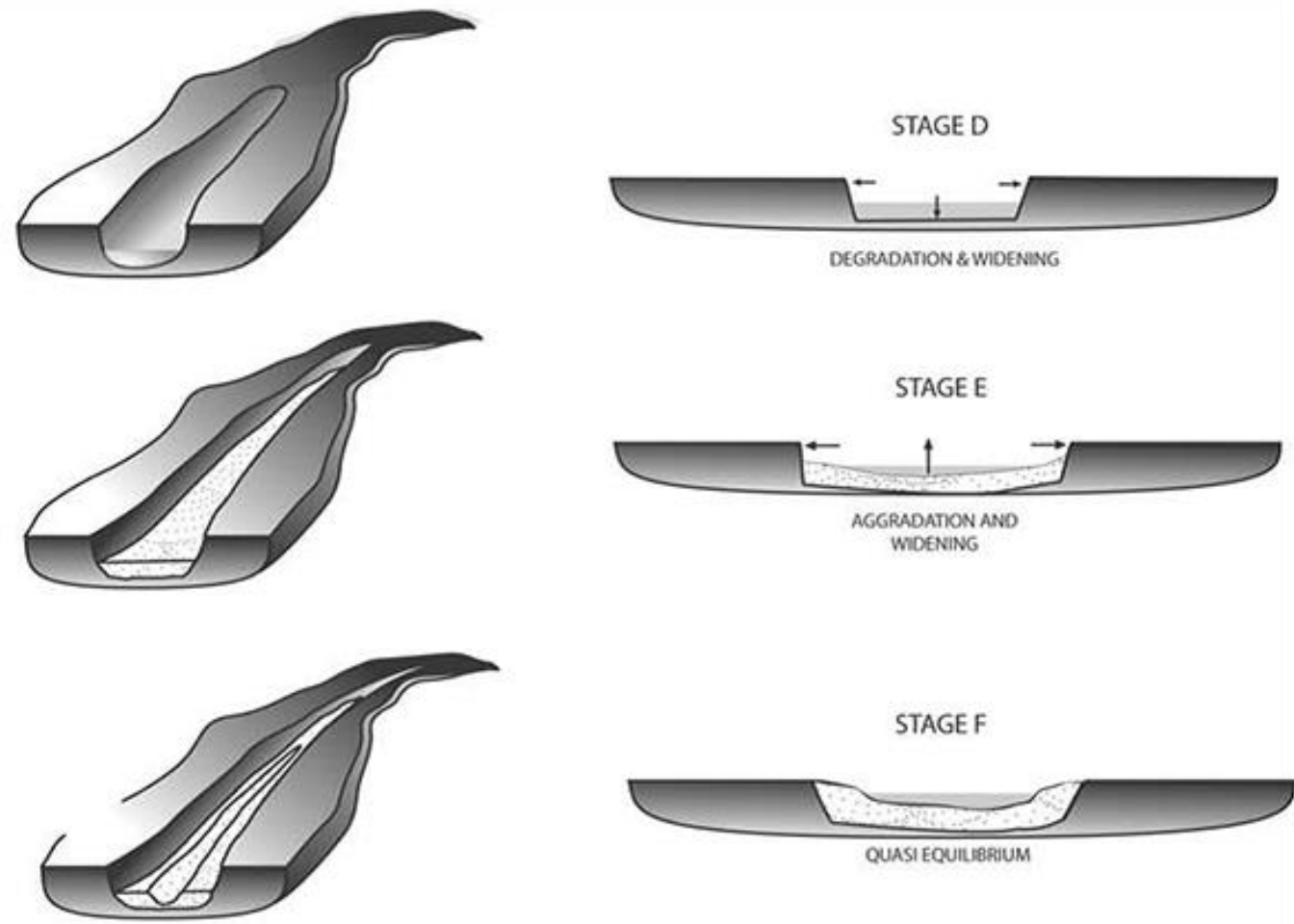

STAGE F

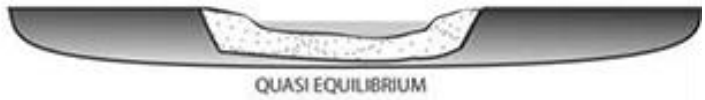

Figure 14. Stage Progression of a channel evolution model showing channel development in a reservoir after the removal of a dam: Stages $\mathrm{E}$ to $\mathrm{D}$. As degradation continues, the channel widens and begins to aggrade downstream of the knickpoint eventually reaching quasi equilibrium. (Doyle et al., 2003b).

As previously stated by Doyle et al., (2002), the geomorphic response to the removal of a dam constitutes a disturbance regime, albeit one that is controlled by those removing the dam. The rates of change upstream and downstream of the dam are controlled by the amount of sediment trapped in the impoundment and the ability of the river to mobilize the sediment and reestablish is base condition. Thus, higher energy streams will be more efficient at redistributing trapped sediment than lower energy streams which directly correlates to the ability of downstream habitats to adjust to changes in sediment loads.

Again, the six stages of channel morphology following dam removal are discussed (AF: Pre-removal, lowered water surface, degradation, degradation and widening, 
aggradation and widening and quasi-equilibrium) and how each of the stages can have different impacts on downstream ecosystems (Doyle et al., 2005). The main point of this discussion was to demonstrate the magnitude of change brought on both upstream and downstream of a dam removal. While the assumption has been made that through the removal of a dam the stream's ecosystem will return to pre-dam conditions, it is important to note that some aspects, such as macroinvertebrates, will return rather quickly to pre-dam conditions but others, like riparian vegetation will likely take decades to realize. This makes it important for decision makers to weigh the impacts of dam removal while maximizing the rate of recovery both upstream and downstream of the all watershed systems (Doyle et al., 2005).

Chang (2008) researched the proposed removal of the Matilija Dam on the Ventura River in Southern California. The project was approved by congress in 2007, but construction costs became an issue and the removal project is still pending. The research on the removal was conducted using a scale model of the impoundment (FLUVIAL-12) to simulate the upstream changes and re-distribution of the sediment trapped in the reservoir (Chang, 2008). Scale models provide an excellent way to examine the upstream changes and sediment distribution properties of a given reach; by recreating the current conditions and running through various dam removal scenarios the researcher can offer solutions to offset environmental concerns and help to control the rate of change in the upstream reach as well as sediment load introduced downstream. When the dam is breached, the first change noted is the creation of a head cutting knickpoint that quickly moves upstream through the sediment impoundment immediately following the breach. The upstream migration of the knickpoint is most pronounced at the delta and rapidly extends upstream 
after the initial breach. The upstream migration of the knickpoint slows after the initial breach as the channel starts reaching a point of pseudo equilibrium and continues to gradually migrate upstream as the channel attempts to reestablish baseline flow. The initial channel width is also rather narrow and steep as the channel reestablishes baseline and only starts to widen and traverse its original flood plain once the original pre-dam bed elevations is reached (Chang, 2008). After the initial burst mobilization of trapped sediment post breach, the impoundment delta slowly undergoes changes, and sediment is slowly redistributed downstream with complete removal of the trapped sediment taking many decades to accomplish. The amount of change in the channel morphology, the rate of knickpoint migration, and the rate of sediment redistribution are affected by the stratigraphy of the reach, the high and low flow rates of the stream and the rate of the initial dam removal. Understanding the rates of erosion and deposition after the removal of a dam are essential to quantifying the upstream geomorphic changes a given reach will undergo (Chang, 2008).

Burroughs et al., (2009) researched the effects on fluvial geomorphic conditions after the removal of the Stronach Dam on the Pine River in Michigan. The study was performed over a 10-year period, both during the slow ("staged") removal of the dam from 1996-2003 and for three years after the dam's removal from 2004-2006. To accomplish this, they established 31 permanent transects in the 10-km study area and monitored the areas for changes in geomorphology. The group's goal was to address questions lacking in the effects on geomorphology through the removal of a dam; the focus was on the scope of the changes and in spatial extent and the length of time the changes would take to happen. The actual response varied slightly from the CEM here in that portions of the upper reach of 
the study area had increased slope steepening that was suggested in the previous papers here as being associated more with the margins closest to where the dam was prior to removal, this suggest that local geology will adjust the expected CEM results and should be considered in the long-term implications.

The scope of the project focused on numerous aspects of fluvial geomorphology, but for my purpose the effects on river morphology attributes have the most import. Documented changes in the slope aspect, thalweg, depth, discharge and riffle-pool composition ("bedform diversity") due to the degree of change in sediment impoundment (Burroughs et al., 2009). The Stronach impoundment was classified by "bedform units of runs, riffles, pools, rapids, or complexes (a designation where more than one category applied)" (Burroughs et al., 2009, page 98). This gave researchers a classification regime that allowed them to document the geomorphic changes occurring in the reach. The impoundment area was classified as a slow-flowing wide run prior to the removal of the dam, while the reach upstream of the impoundment was marked by higher bedform diversity. The upper reach was narrower as is typified of normal stream geomorphology, with faster flow, coarse substrate and "high bedform heterogeneity" (Burroughs et al., 2009). The hypothesized result was that once the dam was removed and the river's geomorphology reached base equilibrium, it would have a classification like the upstream reach with normal variations in the pool-riffle-run-rapid sequence. Burroughs's team used channel evolution models to predict the results and subsequent rate of change in the stream morphology (Burroughs et al., 2009).

The actual changes that took place over the 10-year period showed a significant adjustment to the sediment profile and lateral shift of the stream channel in the 
impoundment while the upstream reach maintained "healthy stream" geomorphology (Burroughs et al., 2009). The highest changes in the impoundment occurred closest to the dam itself with the slope and velocity of the river being faster in the region nearest the dam upstream and downstream and higher velocities recorded in the thalweg, all of which are consistent with normal flow of an unrestricted river with similar bed structure and geology. The methodology created by this research allows estimation of sediment mobilization through dam removal; the outcome of mobilization in this removal was $12 \%$ of the total sediment impoundment, which was consistent with other research done on the subject. Thus, actual sediment mobilization could be estimated prior to the removal of a dam using the equations provided in this paper. Also, of note was the formation of pool-riffle complexes in the impoundment region, although they were not as diverse as the upstream reach new pool-riffle bedform were created through the dam removal. Burroughs et al., (2009) noted that the lack of any significant high velocity events during the study factored in to the lack of more pool-riffle structures in the impoundment reach.

Pearson et al., (2011) looked at geomorphic responses to the removal of the Merrick Village Dam in New Hampshire. The dam impoundment consisted of sand-sized sediment; the mobilization of the impoundment following the initial dam breach was consistent with other studies on reaches with similar composition and rapid initial mobilization of the impoundment (Pearson et al., 2011). Merrick Village Dam was a low-head 4-meter high structure; the accumulated impoundment was composed of sand sized sediment and considered to be highly mobile under high flow conditions. Like other studies pre-dam research was done to determine possible sediment redistribution rates and upstream geomorphic conditions related to the removal of the dam. Building on work by Doyle et 
al., (2003a), Doyle, at al. (2003b), and Major et al., (2008) a modified regional CEM was used to determine rates of incision, degradation and aggradation for the reach (Pearson et al., 2011). The resulting upstream response correlated to that reported in the previous articles. An initial head-cut knickpoint became evident at the breach point and started immediate upstream migration. After the initial deep narrow incision lateral migration of the channel started as the knickpoint reached the upstream end of the reservoir influence and hit the channel bedform structure and thalweg of the river. The differences in the Pearson study reach versus the Doyle Wisconsin reach and the Major et al., (2008) Sandy River study was in the sediment composition itself. While all the research thus far shows similarities in upstream responses to dam removal, the rates at which these responses occur are different. Once again, the driving factors that contribute to the rate of degradation, aggradation, transport and migration are products of bed and valley form structure, sediment composition and regional hydrology (Pearson et al., 2011). In this case the initial geomorphic change was a process-driven exchange because of the removal of the dam itself. This initial change lasted approximately three months and from there event driven exchanges were responsible for driving subsequent mobilization of the impoundment and further lateral and headward migration of the channel (Pearson et al., 2011).

Cannatelli and Curran, (2012) discussed hydrology as a factor of channel evolution post dam removal. The researchers monitored a slow-drained reservoir and used modified CEMs that incorporated local hydrologic conditions to discern the impact on upstream geomorphic condition post dam removal. A survey of cross sections was done at 10 locations within the study area and data over the course of one year. They divided the study area up into three reaches based on the date of drainage (Cannatelli and Curran, 2012). 
Reach 1 is furthest upstream of the dam sitting just outside of the reservoir influence and extending from 0 to $14 \mathrm{~m}$, Reach 2 was the first portion of the creek to drain extending from 14 to $104 \mathrm{~m}$ and Reach 3 was the last portion to drain extending from 104 to $356 \mathrm{~m}$ (Figure 15) (Cannatelli and Curran, 2012).

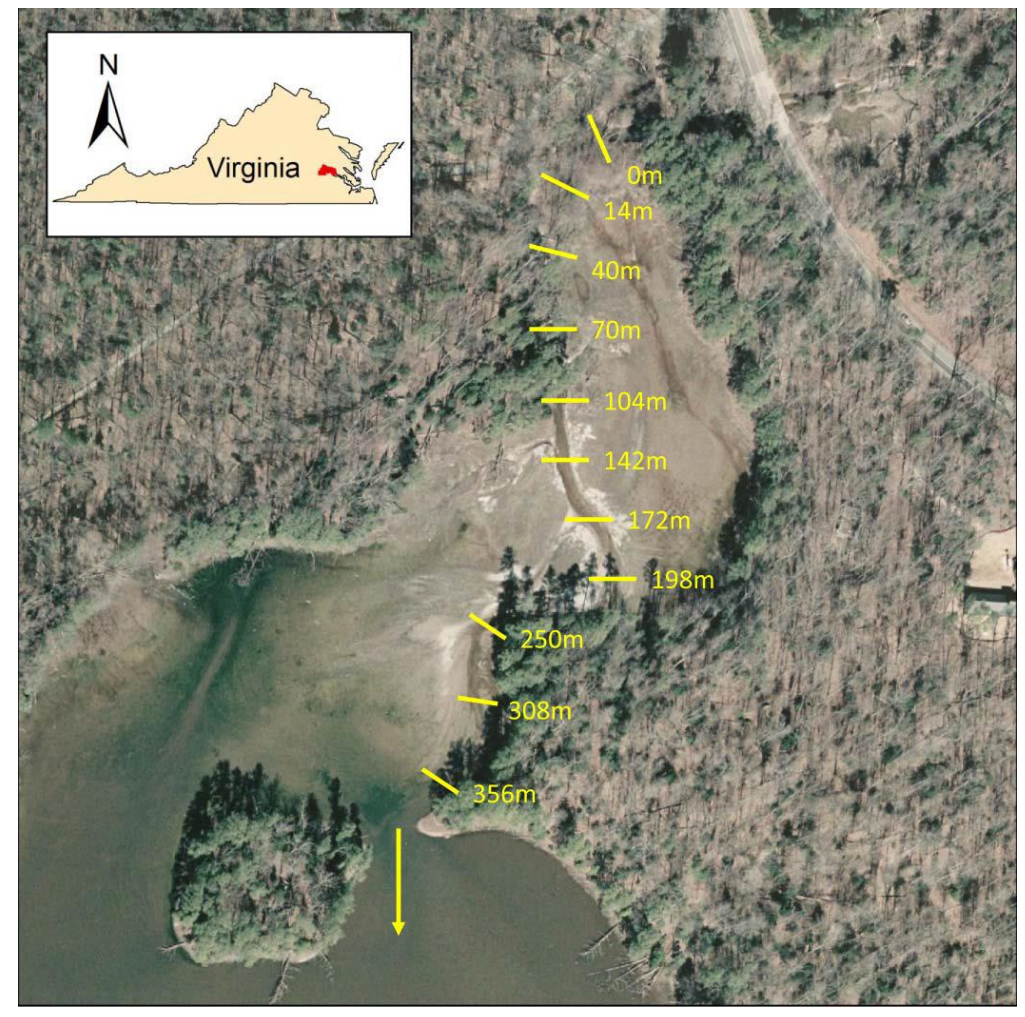

Figure 15. Study area map Cannatelli and Curran, (2012).

As stated by previous researchers there are numerous factors to consider in the removal of a dam. The main factors being the size of the dam, how difficult the removal will be, the effects on downstream reaches through sediment mobilization and downstream impacts through the lack of the dam itself (Cannatelli and Curran, 2012). Building on all the previously discussed articles, the intent of the research was to provide reliable conceptual models that include known regional seasonal variabilities into the upstream geomorphic 
response to the removal of a dam and is considered a key variable in the expected morphological change.

This was accomplished using a modified CEM for the region. The importance of using modified CEM's is logical given you are using the region's known climatic, geologic and geomorphic conditions (Cannatelli and Curran, 2012). Due to the slow-draining aspect of the reach the researchers were able to see the channel evolution and effects of seasonal variability in hydrologic and vegetative regimes over the course of a year. This provided them with the data variables necessary in creating a regional CEM; the variables can be changed to reflect the differences in reach dynamics by regions (Cannatelli and Curran, 2012). Channel changes progressed through the stages of equilibrium (Figure 16) with changes in the channel morphology included marked headward erosion of the knickpoint with lateral changes in the channel and incorporation of vegetation models to modify erosion potential (Figure 17).

An important result from this study was that the lateral migration of the channel was more pronounced on the right bank of Reach 1, which was not protected by established vegetation. Reach 3 was in the center of the original reservoir impoundment and showed the most lateral movement with equally variable shifts left and right during the study. The lack of any established vegetation did not inhibit the channel migration within Reach 3 (Cannatelli and Curran, 2012).

Reach 2 developed a split channel due to high winter flows during the study period; both channels showed equal characteristics becoming narrower and deeper as the ratio between width and depth decreased. 
(a)

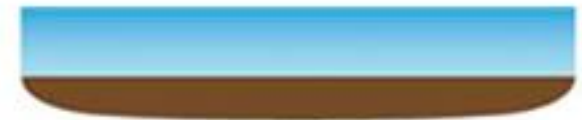

Pre-removal reservoir

(b)

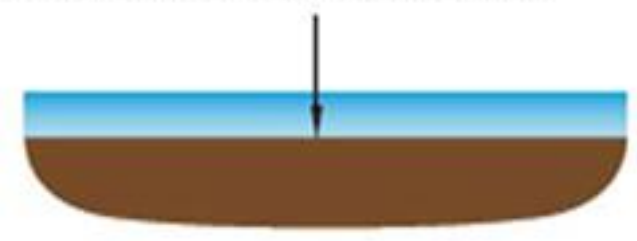

Dewatering immediately following removal

(c)

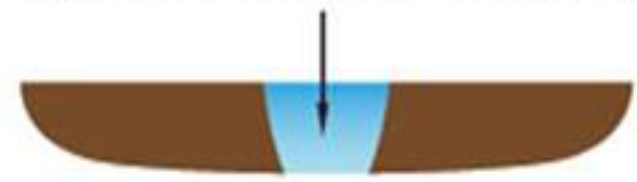

Degradation

(d)

Degradation and Widening

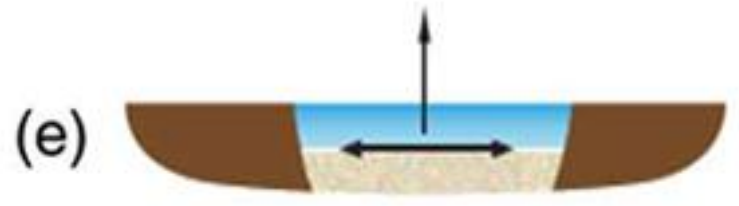

Aggradation and Widening

Figure 16. Modified channel evolution model. Stages A-E illustrate the progression of the reservoir from pre-removal in Stage A through channel evolution in Stages C-E. (Cannatelli and Curran, 2012).

Cannatelli and Curran, propose the active monitoring of cross sections at intervals of less than one year provide more benefit in understanding changes in the geomorphology of the upstream reaches due to seasonal fluctuations (Figure 18). This becomes more evident temporally in the removal of a dam, the types of changes occurring during seasonal variation occur in a much quicker period over a more localized region (2012). 
(f)

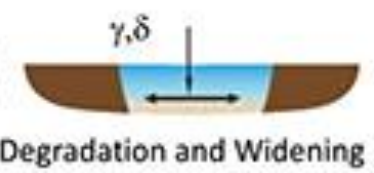

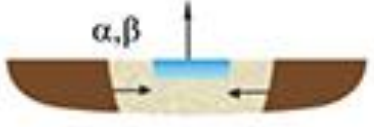

Aggradation and Narrowing

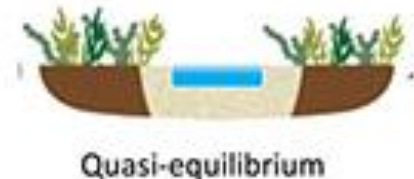

Quasi-equilibrium

(g)

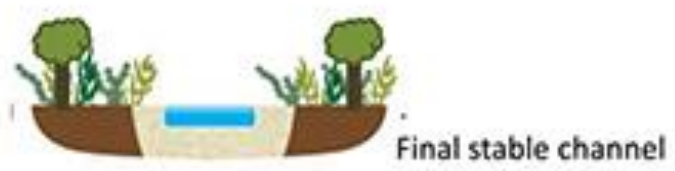

Figure 17. Modified channel evolution model with addition of vegetation modeling. CEM deviates from previous models to incorporate flow and vegetation data; Stage F shows the channel state corresponding to the seasonal flow regime. (Cannatelli and Curran, 2012).

The review of research into the upstream geomorphic responses to dam removal showed six stages of change that were originally classified by Doyle et. al. (2003b). Upon dam removal there is a progressive upstream migration of the knickpoint and the sediment is left as an eroded wedge, which over time may have more removal depending on periodic episodic events driven by variations in the local conditions. It is important to consider the aspects of the removal prior to initiation of a breach. The local conditions are important factors in the mobilization of the sediment impoundment and factors such as underlying bed-form structure, valley-form stratigraphy, sediment stratigraphy and the hydrology and competency of the stream reach itself will all play key roles in the upstream geomorphic changes.

The initial upstream migration of the knickpoint and subsequent channel incision results in a steep narrow channel until the knickpoint reaches the upstream limit of the reservoir thalweg. At this point the knickpoint starts to resolve itself into a low relief riffle complex and we start getting channel widening and continued degradation of the upstream channel. Depending on the sediment composition, bedrock/bedform structure and existing vegetation the next stage progresses to lateral incision rates and further degradation and aggradation cycles of the reach. From here again we start to reach a quasi-equilibrium state 
that will also vary in temporal and spatial scale dependent on the localized regional conditions mentioned before.

While the rates vary the structure formations and upstream geomorphic changes that occur from dam removal remain consistent between the various research articles. The initial sediment push and upstream change will also be affected by the type of dam removal that is implemented. With respect to dam removal technique, a blow and go removal appears to progress through the six stages at a much higher rate than those removed through a slow draw down or even pre-dredging of the impoundment before removal.

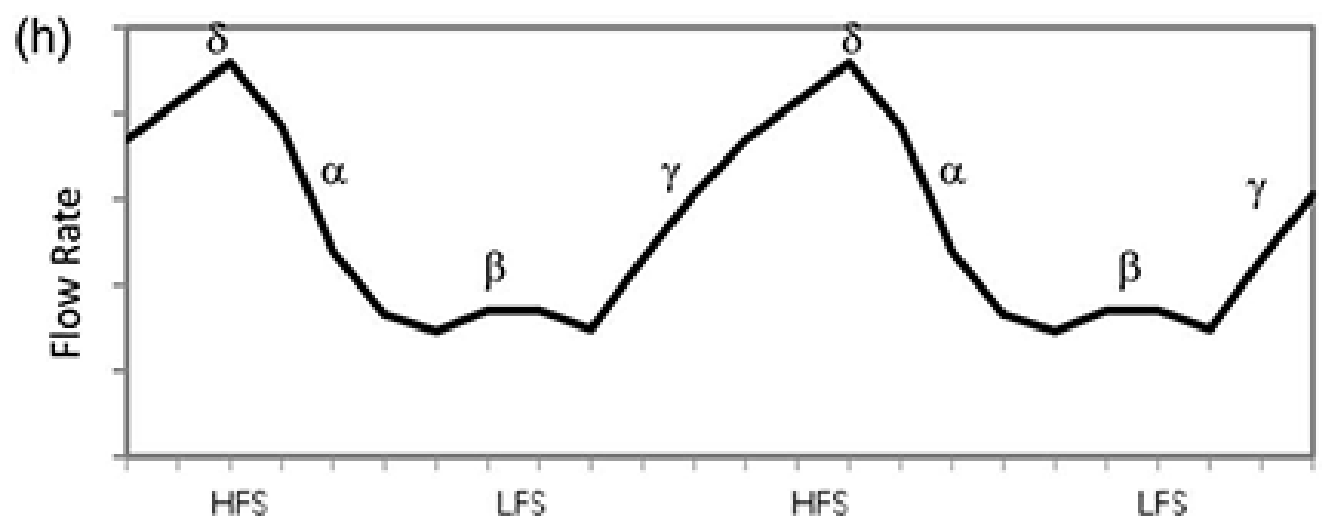

Figure 18. Degradation and widening and aggradation and narrowing based on seasonal flow variability. Seasonal flow regime shown as the high-flow season (HFS), low-flow season (LFS), and seasonal transitions: $\gamma$ or $\delta$ correspond to channel degradation and widening in Stage F; $\alpha$ or $\beta$ correspond to channel aggradation and narrowing in Stage F; the channel is in the quasi-equilibrium state in Stage F; (g) channel proceeds from Stage F to a stable channel at Stage G only after vegetation has established and banks are stabilized. (Cannatelli and Curran, 2012).

Regardless of the dam removal method used, the upstream geomorphic changes that occur from dam removal remain consistent in form while varying temporally. What has been lacking in all these studies is an extensive survey of study reaches affected by dam removal given that each effort relied on an on-the-ground approach, transect style approach. 
Knowledge of the different geomorphic features present and the river's lateral movement potential will give us a better understanding of the forces at work and the types of geomorphic features present. My study examines whether lidar can accurately measure geomorphic features by examining water surface pulse returns in comparison to existing digital elevation models, orthographic/aerial photos and surveyed stream reaches using Geographic Information System (GIS) mapping technology. The use of lidar to detect changes in channel morphology, water surface slope, and floodplain changes has recently become a more common tool. Using lidar, the active channel characteristics extracted include bank profiles, longitudinal profiles, transverse profiles of gullies under forest canopies and water surface slope (English, 2008). Features that are inherent in healthy stream complexes, such as pool-riffle-run portions of a reach, and that are important to the health of river biota would be prime examples of features to identify in lidar extraction. Pool-riffle complexes form in meandering streams that have coarse grain composition with pools being recognizable as areas of lower topography and finer sediment deposition while riffles are characterized by coarser bed material and higher topography (Sawyer et al., 2009). Fluvial geomorphic research has a rich history which has largely been done with field work. Lidar gives us the ability to inform an analysis of geomorphic change using high-resolution, remotely sensed data which allows researchers access too hard to reach areas and provides a tool for analyzing geomorphic changes over a time with sequential acquisitions. 


\subsection{Lidar}

By understanding the function of the river system, we gain a deeper understanding of the process at work in a watershed. The tools used by fluvial geomorphologists to gain this understanding have changed over time and new ideas have helped further researchers' ability to better document the changes over time. In addition to classic methods like matching aerial photos to topographic maps, and field surveys of riverine characteristics (Endreny, 2003), new technologies have provided detailed methods for mapping channel morphology. One example of a new technology that shows great promise for fluvial geomorphic inquiry is lidar. Lidar stands for Light Detection and Ranging and is a remote sensing technique that uses laser pulses to measure the surface variability of the Earth.

The Pacific Northwest is relatively data rich with lidar with the Oregon Lidar Consortium (OLC) and Watershed Sciences/Quantum Spatial group acting as a primary supplier of lidar data and other airborne remotely sensed data, such as thermal imagery and water quality modeling. Lidar has proven to be a cost-effective way to collect highly accurate and detailed terrain surface data over extensive areas. The data is acquired using a laser range finder mounted to either small fixed wing aircraft or on helicopters. These aircraft are precisely navigated to provide the most detailed coverage of the earth's surface dependent on the accuracy of the sensor package at the time of the lidar flight. Sensor packages continue to improve and the accuracy of the lidar acquired. The laser range finder fires a pulse at the earth's surface and records the return pulses from the beam. The laser pulses collect very dense clouds (points) of X-Y-Z coordinates with each return of the beam bringing detail of the surfaces it encounters until the pulse reaches the ground surface. Lidar density involves both the Pulse Spacing and the Pulse Density (Faux et al., 2009). 
Different agencies have suggested different point density needs for accurate mapping of terrain features. The standard recommended by the Oregon Lidar Consortium (OLC) for the best multi-application use in the Pacific Northwest is 8 pulses per square meter with 0.35-meter post spacing. The return pulses enable the datasets to map the features that are on the Earth's surface itself, providing highly detailed representation of the land surface or of the vegetation and buildings on the land. The differences between the first return of the laser pulse (the highest hit) and the last return of the laser pulse (ground cover, or in the case of urban settings impenetrable surfaces such as buildings and roads) provides detail on the amount of vegetation and groundcover in the flight path. Because of its accuracy and detail lidar is fast becoming an important tool for agencies interested in natural resource management and change detection. This tool has become fundamental in helping to map risk assessment scenarios and flood inundation levels for regions along the Oregon Coast. For fluvial geomorphologists this tool has helped to augment current practices used to study channel morphology (Evans et al., 2009).

Lidar has been used to help detect changes in channel morphology by examining the pulse returns from the water surfaces as described in English, (2008), Faux et al., (2009) and English, (2013). Water has a certain refraction point and the pulses will often refract from the surface at angles of incidence that are greater than 53 degrees (English, 2008). Understanding the characteristics of the pulse return aids in the processing of the Log ASCII Standard (LAS) point returns for classification purposes, comparison of hillshade derived Digital Elevation Model (DEM) and current Orthophotos of stream reaches allow a better understanding channel morphology and pool-riffle characteristics (English, 2008). Smooth water surfaces tend to have lower lidar point densities which are indicated by long 
lines of interpolation (lower point density) in the lidar surface while areas of rough water surfaces create more surface variability and higher point return, this difference in water surface returns also correlates closely to pool-riffle sequences on a river (English, 2008).

Beyond what has been done in the Pacific Northwest, lidar is fast becoming a necessary component to fluvial studies and planning efforts. De Rose and Basher, (2011) examined the effectiveness of lidar and aerial orthophotography used in conjunction, for monitoring bank and cliff erosion, and the accuracy of deriving volumetric erosion rates from sequential lidar hillshade DEM's. The discussed the need to be consider resolution differences when using lidar and orthophotography, the resolution differences between sequenced lidar, and the lack of sequential lidar runs in close temporal proximation. The date of acquisition for the orthophotography will play a role in resolution.

The erosion potential measured showed the study reach to be in a state of equilibrium with bank erosion and cliff failure being the contributing factors to sediment fluxes in the system (De Rose and Basher, 2011). Channel migration within the reach drive erosion rates for the reach with $69 \%-88 \%$ of the sediment load being attributed to channel migration induced cliff erosion, but within the study reach itself channel migration potential was low suggesting a fairly stable system and most of the current erosion occurring along the bank margins at the meander bends with minor fluxes in aggradation and degradation noticeable in the sequenced lidar (De Rose and Basher, 2011). With the high positional and vertical accuracies, and the ability to filter out areas of ground clutter, lidar makes it possible to accurately measure the position of the channel and elevation of the banks and water surface to derive volumetric erosion rates on an annual schedule taking into consideration the character of the river and its ability to mobilize sediment (De Rose and Basher, 2011). 
Hilldale and Raff, (2008) look at the accuracy of lidar to map river bathymetry in Washington State. Using a combination of lidar and orthophotography the effectiveness of measuring river bathymetry on shallow regions was assessed. The 3-band color of the orthophotos can be used as another proxy for deriving depth, however resolution of the aerial imagery and time of flight have direct impacts on the accuracy. Specifically, the red band is more sensitive to depth, however, it does not penetrate as deeply into the channel, and differences in substrate, vegetation overhang, and shadows at bank margins introduce error (Hilldale and Raff, 2008). Airborne bathymetry lidar has been developing an improved addition to bathymetric modeling using orthophotos, its ability to penetrate deeper into the water column and account for vegetation reduces and eliminates many of the errors associated with using orthophotography by itself. Another benefit of bathymetric or "Green" lidar is that it is not affected by sun glint off the water surface which can be an issue with orthophotos (Hilldale and Raff, 2008).

Traditionally river bathymetry surveys have been conducted using single beam sonar or acoustic Doppler surveys in conjunction with RTK GPS. This method produces both horizontal position and water surface elevations while depth is derived from the acoustic equipment. This method is both time consuming and costly, in terms of data collection and post processing; accuracy is also an issue in this process and interpolation of surfaces over long distance occurs when GPS coverage is poor. Terrain features and riparian vegetation can also interfere with the reception of the GPS equipment causing poor vertical correlation in the survey method (Hilldale and Raff, 2008).

The results of the research showed that currently (2008) the ability of bathymetric lidar to map riverine environments at the microscale is not possible for either geomorphological 
or numerical modeling analysis due to poor horizontal resolution. Some mesoscale features such as the pool-riffle-glide sequence were well defined from their outputs, but other mesoscale features considered to be integral parts of the overall hydraulic modelling and responsible for critical flow study of the reach, such as large boulders, and other features normally associated with natural obstructions to flow, were not sufficiently rendered (Hilldale and Raff, 2008).

The availability of sequential lidar for the Marmot Dam gave me an opportunity to look at the effectiveness of the lidar to monitor morphological conditions both temporally and spatially. The capabilities of lidar to map channel morphology and changes over an entire reach versus the traditional method using CEM's to predict changes from a small sample size. In addition, the cost effectiveness of lidar allows researchers to get creative in finding new and efficient solutions to managing the riverine environment. 


\subsection{Methodology}

\subsection{Statement}

My research focus involves detecting the changes in geomorphic features and channel morphology of a study reach that has an unusually high number of lidar return flights over a relatively short period of time both before and after dam removal. I show how lidar data can be used to accurately map changes in channel morphology characteristics thus contributing to established field research of stream reaches. The high level of detail allows the detection of water surface characteristics and can extract slope and features such as pool-riffle complexes, which are valuable for mapping and understanding changes in channel morphology. The removal of Marmot Dam has provided researchers the opportunity to examine channel morphology changes that normally occur over longer time periods. The rapid changes in channel morphology that occur as the river re-establishes it base level add to the understanding of river dynamics and the features that are indicators of channel change.

Measurements of the height of water surface with lidar can give researchers a safer, expansive, more cost-effective approach to mapping channel morphology characteristics than field surveys. This is especially important in areas that are inaccessible due to terrain constraints and river reaches that would not be safe to survey using traditional field methods. Oregon Lidar Consortium (OLC) requires that the vertical accuracy of all the data collected be less than or equal to $9.25 \mathrm{~cm}$ accuracy, the vertical accuracy is $3.88 \mathrm{~cm}$ in Oregon, averaged over 72 lidar collections (https://www.oregongeology.org/lidar/DASQuantum_7525Amendment7.pdf). The research will help augment existing techniques for understanding channel morphology and mapping flood plain profiles for use in planning 
and resource management. Lidar accuracy continues to improve, and studies continue to push the limits of what can be derived from the data. The uses already implemented at many levels of natural resource research and management has proven its validity as an augmentation to traditional field methods CITE (Faux et al., 2009).

\subsection{Research}

Using field surveys, known literature of the same region, orthophotos and sequential lidar, I examine channel evolution characteristics on the former Marmot Dam impoundment area. Traditionally, studies of geomorphic features are done at low-flow conditions on small, wadeable streams, which can hinder the examination of channel morphology dynamics during higher flows and on larger stream systems.

Lidar allows for characterizing of different geomorphic changes on larger, faster streams that would preclude traditional stream survey due to the unsafe conditions (Faux et al., 2009). The research is intended to provide evidence supporting the use of lidar to detect geomorphic changes from water surfaces and show the accuracy of lidar as a detection tool comparable to field based approaches and to augment studies for conditions that preclude normal survey techniques and to effectively examine stream reaches over larger scales. Lidar has been used to illustrate the geometry and slope of mountain channels in the Pacific Northwest giving researchers the capability to have a reliable and repeatable approach for quickly and effectively sampling and analyzing the physical characteristics within the watershed. New studies using green wavelength lidar allows for high-resolution bathymetric studies of channel depth and bed profiles (Faux et al., 2009). My own research is a continuation/addition to the growing use of lidar for resource management and channel 
morphology studies and allows me to build off previous research methods while still providing a valuable contribution to the field.

Sequential lidar was used for the study area with the initial pre-dam removal flight used at baseline for the study. Comparison of the subsequent year's lidar flights was used to show changes in channel structure and geomorphic features over time. Using sequenced lidar I examined the reach before dam removal, immediately after dam removal and the current conditions. Lidar acquisition from 2008, year 1 after removal, 2011 year 4 after removal and 2012 year 5 after removal were compared to show the changes in the reach over time based on the degradation and aggradation of bars in the reach.

The 2012 lidar acquisition was done using the newer green lidar and has a bathymetric component to the flight that I used to better visualize the current channel dynamics but was not used to compare differences between flight years for feature extraction given that the other flights did not have the bathymetric component. All flights contained elevation values in meters (NAVD88), values in the report have been left in meters but have their feet equivalents shown with them. Lidar accuracy is different between acquisition years as technology improved Table 2 compares pulse density resolution and vertical accuracy with mean daily cfs provided for the time of flight.

Table 2. Lidar accuracy and mean flow for date of acquisition.

\begin{tabular}{lccc}
\hline Date & $\begin{array}{c}\text { Pulse Density } \\
\text { Resolution }\end{array}$ & $\begin{array}{c}\text { Vertical } \\
\text { Accuracy }\end{array}$ & $\begin{array}{c}\text { Mean Flow (CFS) USGS } \\
\text { Marmot Gage }\end{array}$ \\
\hline October 22, 2006 & $\geq 4$ points $/ \mathrm{m}^{2}$ & $5.9 \mathrm{~cm}$ & 311 \\
October 1, 2008 & 8.04 points $/ \mathrm{m}^{2}$ & $5.1 \mathrm{~cm}$ & 424 \\
September 3, 2011 & 8.80 points $/ \mathrm{m}^{2}$ & $3.6 \mathrm{~cm}$ & 418 \\
September 22, 2012 & 9.57 points $/ \mathrm{m}^{2}$ & $3.7 \mathrm{~cm}$ & 360 \\
\hline
\end{tabular}


Using sequenced lidar studies I compared features between acquisitions to show how valuable lidar is for identifying geomorphic features that are key indicators of a dynamic river system. Changes in pool-riffle placement in the reach, channel migration, and changes in channel morphology attributed to the geomorphic structure of the reach can all be identified using lidar and monitored for change over time with sequential runs. This is especially useful for highly competent and mobile river systems such as the Sandy River, which is known for its high flows and potential for avulsions and channel migration in certain portions of the rivers floodplain.

Further examination of the region's orthophotos were used to support the data interpretation of existing conditions in the lidar to ascertain if the channel morphology changes detected in the reach were visible. Orthophotos have not always been flown with lidar; more recent flights have co-acquired orthophotos which allow for better visualization of the features in the lidar, none of the flights for this study had co-acquired orthophotography. I used the best available imagery from the National Agriculture Imagery Program (NAIP) acquired in 2005, 2009, 2011, and 2014. While they do not all match the acquisition years of the lidar, they are still useful for visualizing the current conditions and those prior to the removal of the dam and can be used to look at channel morphology above and below the impoundment. Also, the coarse level of detail in older orthophotos does not allow for clear feature extraction. The 2014 NAIP imagery was also compared to the 2012 lidar flight and found that conditions in the reach are relatively stable given minimal changes in the channel features shown. Based on the photos taken in the field myself in 2014 and 2018 the data shown in the 2012 lidar flight shows that the river reach is in equilibrium. 
Examination of sequential years of lidar acquisitions shows changes in the geomorphic features within the study reach allowing for characterization of channel morphology changes as they pertain to the features such as active channel margins, floodplain margins and abandoned terraces. Lidar derived stream centerlines were created for each year of delivery, placing the centerline in the perceived channel using a combination of lidar derived bare earth hillshade and orthophotos. Some limitations are inherent in this process as lidar does not capture the water surface elevation unless the returns strike the water surface at a specific angle of nadir or in rapid complexes where the turbulence of the water allows for more refraction of the lidar returns (English, 2008). Also, given that bare earth digital elevation models (DEMs) must extrapolate for the removal of vegetation and other feature where lidar returns are sparsely recorded; stream channels and areas of large bodies of water result in less pulse returns because they are not refracted by water. This decreased point spacing results in a tinning pattern inherent in the extrapolation between points in a triangulated irregular network (TIN) (English, 2008).

Older versions of lidar did not have the resolution capabilities of today, and the newest lidar has 6-inch orthophotos co-acquired at the time of acquisition which provides a reliable secondary source to verify stream position. Lidar derived stream features are used to show channel adjustments over time; these include lateral migration of the channels, channel patterns, gradient changes over time, and terrace/bar realignment within the active river margin. The 2006 lidar acquisition is used as the baseline for the study. This flight was done prior to the removal of the dam and thus, we can compare the changes to the active channel margins for this reach since that removal date. As this data was acquired before 
the removal of the dam, the initial comparison between the remaining year's runs produced a negative elevation change due to the level of the reservoir prior to removal.

This was compared against each successive year to show the overall change in active channel size and shape as well as the geomorphic change that has occurred in the reach during this timeframe. A volumetric comparison was made between the baseline elevation of the reservoir and that of each year's stream profile to compare differences in the lateral and downstream profile of the reach. A second comparison was made between each of the three lidar flights run on the reach since the removal of the dam; and used to compare active changes in the lateral and downstream profiles as well as approximate accumulation and degradation of the deposition regime for the reach.

Further comparisons of the lidar was used to document changes in the geomorphic regime that predate the dam structure itself, looking at the surficial geology and bedrock for the reach to show areas of potential migration, and those that are more resistant material overall. Ground truth field work of the reach was conducted to ascertain current conditions. Current lidar data was compared to the features found from aerial photos, field work photos and GPS points. GPS points were successfully collected on October 13, 2014 using a Trimble GeoXT with sub meter vertical accuracy (Appendix A: Appendix A: Differential Correction File). The GPS point locations are shown in Table 3, each point was selected for ease of access and features that I knew would show up in the lidar.

Point A was collected at the approximate location of the monitoring camera placed above the southern edge of the reservoir. This was accessed by using the trail on the southern side of the foot bridge located on site. Point B was collected at the northwestern corner of the eastern bank gravel bar accessed by a small trail leading from the parking lot 
to the southwest. Point $\mathrm{C}$ was located on the foreset bed of the gravel bar approximately 90 meters southwest of Point B. Points D - G are all located on the margins of the wooden crib structure (Figure 19) that emerged from the sediment between 2009 and 2011. Point F was collected near the center of the gravel bar on the western bank opposite the channel from the wooden structure and in the approximate location between the existing channel and the abandoned channel from approximately 2008 (this channel sees activity in winter flows). Points $\mathrm{G}-\mathrm{I}$ are located across the lip of the gravel bar, directly south from the large lahar dike shown in Figure 20.

Table 3. GPS point locations.

\begin{tabular}{ccccccc}
\hline GPS Point & ID & GPS Time & $\begin{array}{c}\text { Height } \\
\text { (meters) }\end{array}$ & $\begin{array}{c}\text { Vertical } \\
\text { Precision }\end{array}$ & $\begin{array}{c}\text { Horizontal } \\
\text { Precision }\end{array}$ & $\begin{array}{c}\text { Standard } \\
\text { Deviation }\end{array}$ \\
\hline Camera Spot & A & $11: 42: 39 \mathrm{pm}$ & 227.414 & 0.7 & 0.4 & 0.725875 \\
Gravel Bar & B & $12: 15: 14 \mathrm{pm}$ & 217.146 & 0.9 & 0.4 & 0.512149 \\
Foreset Bed & C & $12: 28: 24 \mathrm{pm}$ & 217.984 & 0.8 & 0.5 & 0.263750 \\
CS - Front South Corner & D & $02: 11: 34 \mathrm{pm}$ & 221.888 & 0.7 & 0.5 & 0.331413 \\
CS - Rear South Corner & E & $02: 18: 09 \mathrm{pm}$ & 224.666 & 0.9 & 0.6 & 1.322486 \\
CS - Rear North Corner & F & $02: 20: 29 \mathrm{pm}$ & 222.772 & 0.5 & 0.4 & 0.442280 \\
CS - Front North Corner & G & $02: 22: 42 \mathrm{pm}$ & 222.019 & 0.5 & 0.4 & 0.457460 \\
Stump & H & $02: 38: 39 \mathrm{pm}$ & 222.963 & 0.7 & 0.4 & 0.268551 \\
Left Pool Bank & I & $02: 44: 29 \mathrm{pm}$ & 226.295 & 1.0 & 0.5 & 0.853633 \\
Mid Pool Bank & J & $02: 46: 19 \mathrm{pm}$ & 222.166 & 0.8 & 0.5 & 0.153974 \\
Right Pool Bank & K & $02: 48: 24 \mathrm{pm}$ & 220.560 & 0.8 & 0.5 & 0.237230 \\
\hline
\end{tabular}

A comparison was made against the 2006 base flight to determine rates of morphologic change in the reach. Oregon Department of Geology and Mineral Industries (DOGAMI) and the Oregon Lidar Consortium (OLC) made additional lidar flights of the Sandy in 2008, 2011, and 2012, to track changes in the reach and sediment distribution regimes after dam removal.

The method of change detection shown in Oregon Department of Geology Open File Report O-13-01 (English, 2013) was used to compare the rates of aggradation and 
degradation in the stream reach. To look at overall channel morphologic changes the runs of the reach done after the removal of the Marmot Dam were compared against each other. This was accomplished using raster math, subtracting the bare earth DEM return of one year's run from that of a prior year and comparing the overall change difference between them. Due to noise in the lidar which can come from low vertical accuracy relative to horizontal sample distance and various atmospheric and biotic variables, values between 2.5 feet and 2.5 feet are considered unreliable and so a threshold of values greater than \pm 2.5 feet were used for comparison (English, 2013).

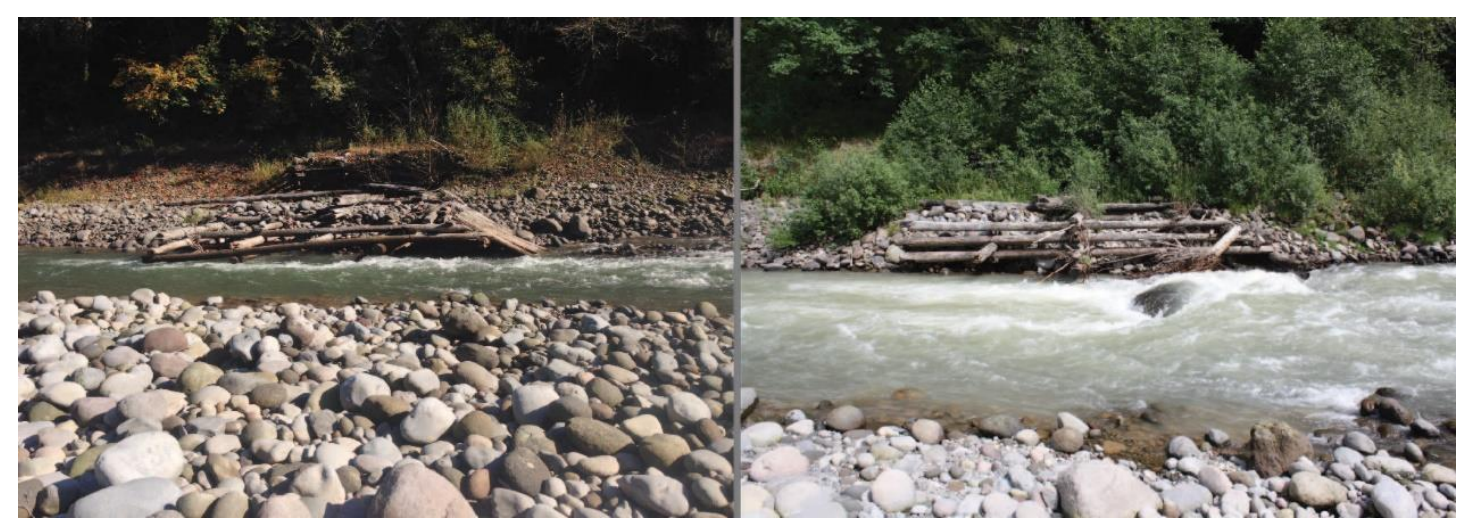

Figure 19. Crib structure used in GPS survey. Photo by Lowell Anthony, Oregon Department of Geology, October 13th, 2014 (left) at 294 cfs and July 15th, 2018 at 410 cfs (right).

I created polygons for the impoundment based on the 2006 lidar and active channel polygons for each of the four lidar flights. I used the same \pm 2.5 feet threshold to account for noise in the lidar. Polygons were digitized at a 1:1000 scale to obtain the most accurate bankfull margins for the reach relative to the resolution of the original data. Elevation values were queried from the DEM and the mean change in elevation for each polygon was calculated. Total impoundment differences were calculated between years and active channel change between years, with attention paid to areas of aggradation and degradation. 


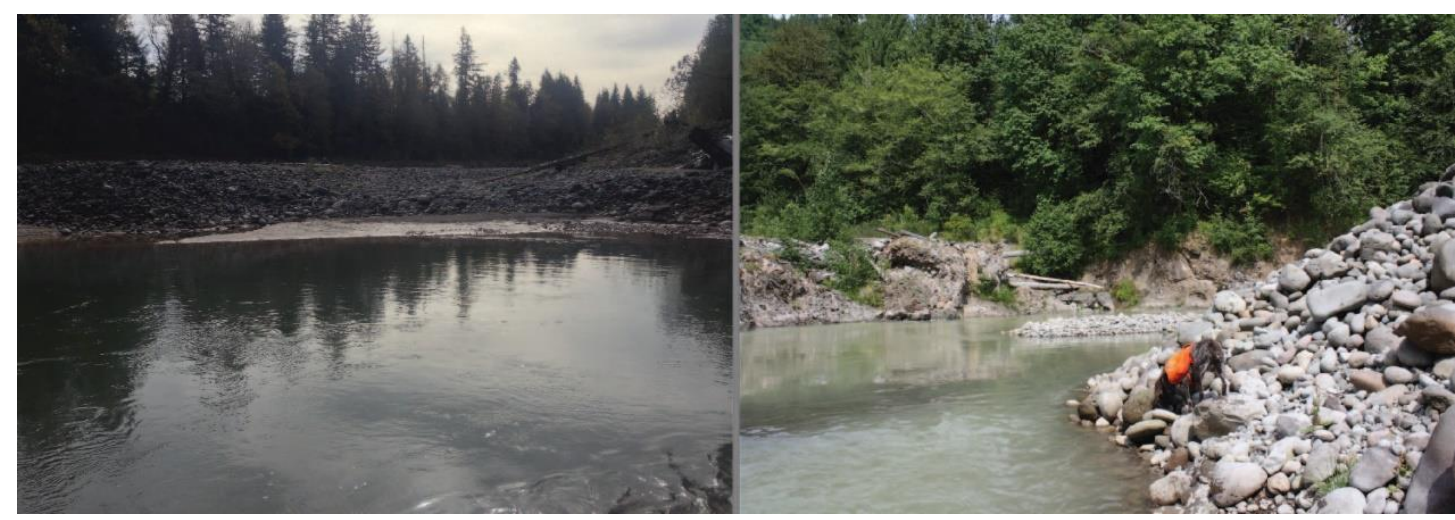

Figure 20. Head of gravel bar location of GPS points G, H, and I. Photo on the left is looking upstream photo on the right is also upstream but from the north flank of the gravel bar just past the end of the long run. Photo by Lowell Anthony, Oregon Department of Geology, October 13th, 2014 (left) at 294 cfs and July 15 th, 2018 at 410 cfs (right).

Using the polygons for each return year I clipped out the difference between active channels to show areas of change in the reach discarding polygons with an area $\leq 20 \mathrm{~m}^{2}$. Using the polygons clipped DEM was created for each lidar flight. Using raster math, a depth variation was created by subtracting the area of each return from that of the subsequent year to show areas of change across the reach. Channel migration pattern within the reach was compared using bankfull polygons of each lidar year. Polygons were digitized to represent the approximate bankfull extent of the reach for a given lidar acquisition. Features were interpreted by colorizing a stack of DEM raster. A colorized DEM was used as the base layer using a terrain shade color ramp and setting the output to generate based on the current elevation extent. A slope shade with a $60 \%$ transparency was stacked on top of the colorized terrain DEM with a hillshade derived DEM as the final layer using a $70 \%$ transparency. Inferences were made in areas of tinning to maintain cohesive bank margins based on knowledge of the ground conditions and familiarity with extrapolating stream features using lidar. Rates of change were compared by measuring the difference between each polygon. Each year's polygon is overlaid on another, the newer lidar acquisition was subtracted from the old acquisition and the remaining areas of the 
polygon were calculated for area. This allows a quick visualization of channel morphology change over time by showing areas of aggradation and degradation within the reach.

Comparison of channel profile gradient for each lidar year was also quantified. A digitized centerline was used for each lidar acquisition; using the Stack Profile tool in ArcMap each of the centerlines were given elevation attributes spaced every meter for their given DEM's providing me with a gradient profile for the stream in each year. In addition to the stream centerline, cross-sectional profiles were taken along the reach at specific areas in the study area to compare rates of change laterally along the reach. Also using a onemeter interval all transects were given elevation attributes. Using the Stack Profile tool on each transect, comparison of all years' lidar runs were made to show overall change in channel morphology at each cross section.

The combination of change detection, longitudinal profile, and latitudinal profile methods allows us to get a more detailed look at the morphological adjustments taking place in the reach and inferences can be made from the data involving changes to bedform based on changes in water surface elevations. Understanding the rates of change in correlation to the geology and discharge of the river, can allow inferences to be made along the river and be a welcome addition to planning development along a given reach giving credence to geotechnical realities within a given run; this process emulates the traditional field survey techniques done in previous studies.

Observing changes in downstream gradient and comparing that to the geology of the region allows the inference of rates of change in bedform and sediment changes in the reach, showing areas of both aggradation and degradation across subsequent years of lidar to monitor stream health and morphology over time. Cross section comparison allows us 
to look at the migratory progress within a reach, again showing areas of aggradation and degradation in the floodplain and allows for monitoring of areas prone to avulsion. This also shows areas within the reach of resistant geology that would provide a natural barrier to channel migration.

This process can also elucidate areas of reverse migration from one year to the next. Once full channel migration equilibrium is reached in either lateral direction, more force will be exerted in down cutting within the gradient, given local geologic constraints. Understanding of the active channel properties within a given area allows for proper planning and active mitigation within a watershed environment. 


\subsection{Results}

\subsection{Lidar Comparison}

All data were created and compared using ESRI ${ }^{\circledR}$ ArcMap 10 with the 3D-Analyst Extension. Active channel polygons were compared against each year of lidar acquisition for overall change in the channel features, lateral migration, and channel incision over time. The active channel was clipped from the previous year's active channel to get total areas of change along and area was calculated; areas less than or equal to $20 \mathrm{~m}^{2}$ were excluded from the output polygons. Each polygon was given a unique identifier using a letter of the alphabet; note that if all the letters were used the identifier would start over putting a Z before the letters and cycling back through from the beginning of the alphabet.

To get the difference in change for each polygon it was necessary to extract values from the rasters being compared to each other. The polygons were then used to extract elevation values just within the areas of change for each year's lidar. Each year's subsequent change raster was then subtracted from the previous year's change raster and a difference raster was created. I compared the total amount of positive change and negative change in the reach to come up with the total difference in active channel margin change over the fiveyear period. Table 4 shows the outputs of this process with a total aggradation amounts for each acquisition year. Overall change in active channel margins from Transect A through

Transect H, from 2006 to 2012 showed a net change of $-139,417 \mathrm{~m}^{3}$. The majority of channel change happens over year one, with approximately half that same rate of change occurring over the next four years; the final year of the study shows the first net gain. 
Table 4. Total lidar derived active channel margin change from 2006 - 2012. Note: Areas of degradation and aggradation are only for changes in the active channel margins. Note that in the 2011 to 2012 returns the data shows the reach to be in quasi-equilibrium.

\begin{tabular}{cccccccc}
\hline Lidar \\
Year & $\begin{array}{c}\text { Area of } \\
(+) \\
\text { Change } \\
\left(\mathrm{m}^{2}\right)\end{array}$ & $\begin{array}{c}\text { Area of } \\
(-) \\
\text { Change } \\
\left(\mathrm{m}^{2}\right)\end{array}$ & $\begin{array}{c}\text { Surface } \\
\text { Change } \\
(\mathrm{m})(+)\end{array}$ & $\begin{array}{c}\text { Surface } \\
\text { Change } \\
(\mathrm{m})(-)\end{array}$ & $\begin{array}{c}\text { Sum } \\
\text { Degradation } \\
\left(\mathrm{m}^{3}\right)\end{array}$ & $\begin{array}{c}\text { Sum } \\
\text { Aggradation } \\
\left(\mathrm{m}^{3}\right)\end{array}$ & $\begin{array}{c}\text { Net } \\
\text { Change } \\
\left(\mathrm{m}^{3}\right)\end{array}$ \\
\hline 06 to 08 & 4,070 & 33,301 & 9.7 & -21.9 & $-89,338$ & 11,433 & $-77,905$ \\
08 to 11 & 8,135 & 28,820 & 2.2 & -33.6 & $-34,546$ & 767 & $-33,779$ \\
11 to 12 & 2,538 & 3,907 & 7.2 & -2.0 & -874 & 939 & 65 \\
06 to 12 & 3,106 & 36,895 & 2.5 & -46.1 & $-145,649$ & 6,232 & $-139,417$ \\
\hline
\end{tabular}

To look at the overall change in net area and morphology for the reservoir impoundment I created a series of polygons based on the elevation at the dam head shown in the 2006 lidar. This polygon encompassed the entirety of the canyon for the study reach using the margins of channel transects created for the lateral profile comparisons. The bare earth DEM for each lidar year was clipped by that shape and the rasters were subtracted from each other to show overall change in the reach between 2006 and 2012. The same process was used to extract the values of change for each year and a comparison of the total change across all returns.

To find the average change over the reach, the mean was extracted for each lidar acquisition year change raster and the area of difference was found by taking the area of the change raster and subtracting that from the area of the polygon demarcating the floodplain/impoundment extent. This area of difference was then multiplied by the mean of the depth change giving the average net change of the study reach over the five-year period. Again, the most significant year of net change occurs in year one; Table 5 shows the details for each year's comparison. Of note again is the overall adjustment shown between 2006 and 2012. The difference in channel depth and changes in the terraces and 
gravel bars from the initial reservoir level to the 2012 lidar flight shows how quickly the reach was able to establish quasi-equilibrium.

To further show how effective lidar is for examining channel evolution morphology a longitudinal profile was created to examine the effects from the knickpoint of the impoundment to just downstream of the spillway location. To accomplish this a stream centerline was digitized for each year of lidar at a 1:1,000 scale to ensure the best accuracy of the reach conditions. Each centerline then had the values extracted from their corresponding lidar acquisition DEM resulting in a channel water surface elevation longitudinal profile line.

Table 5. Total impoundment area change 2006-2012. Note: Average overall net change of the study area over the course of six years.

\begin{tabular}{cccccc}
\hline Lidar Year & $\begin{array}{c}\text { Area of Change } \\
\left(\mathrm{m}^{2}\right)\end{array}$ & $\begin{array}{c}\text { Area of } \\
\text { Reservoir }\left(\mathrm{m}^{2}\right)\end{array}$ & $\begin{array}{c}\text { Area Difference } \\
\left(\mathrm{m}^{2}\right)\end{array}$ & $\begin{array}{c}\text { Surface } \\
\text { Change }(\mathrm{m})\end{array}$ & $\begin{array}{c}\text { Net Change } \\
\left(\mathrm{m}^{3}\right)\end{array}$ \\
\hline 06 to 08 & $3,720,115$ & $3,729,626$ & 9,511 & -1.1 & $-10,462$ \\
08 to 11 & $3,719,308$ & $3,729,626$ & 10,318 & -0.8 & $-8,254$ \\
11 to 12 & $3,713,011$ & $3,729,626$ & 16,615 & -0.1 & -997 \\
06 to 12 & $3,714,625$ & $3,729,626$ & 15,001 & -1.9 & $-28,502$ \\
\hline
\end{tabular}

Transect points were marked on the profile to correlate what was being seen longitudinally with the areas that were chosen for the lateral profile examination of the lidar acquisitions. Comparison of the longitudinal profiles correlates to the findings shown in the earlier discussion on extracting the difference between rasters. The gradient profile graph shown in Figure 21 shows the feature changes in bedform and areas of aggradation and degradation that are consistent with the data discussed from previous studies on dam removal.

Rates of change along the stream profile are variable. Transects $\mathrm{A}-\mathrm{H}$, are used to highlight changes from below the dam site (A) to the upstream extent of the former 
reservoir influence and knickpoint migration $(\mathrm{H})$. All transects are drawn from the leftward bank to the rightward bank downstream. Flux between 2006 and 2008; 2011 and 2012 being minimal and would suggest equilibrium at the knickpoint. Point $\mathrm{F}$ and $\mathrm{G}$ mark the margins of the wooden crib structure to the north and the south; almost five meters of change can be seen between 2006 and 2008 at this point.

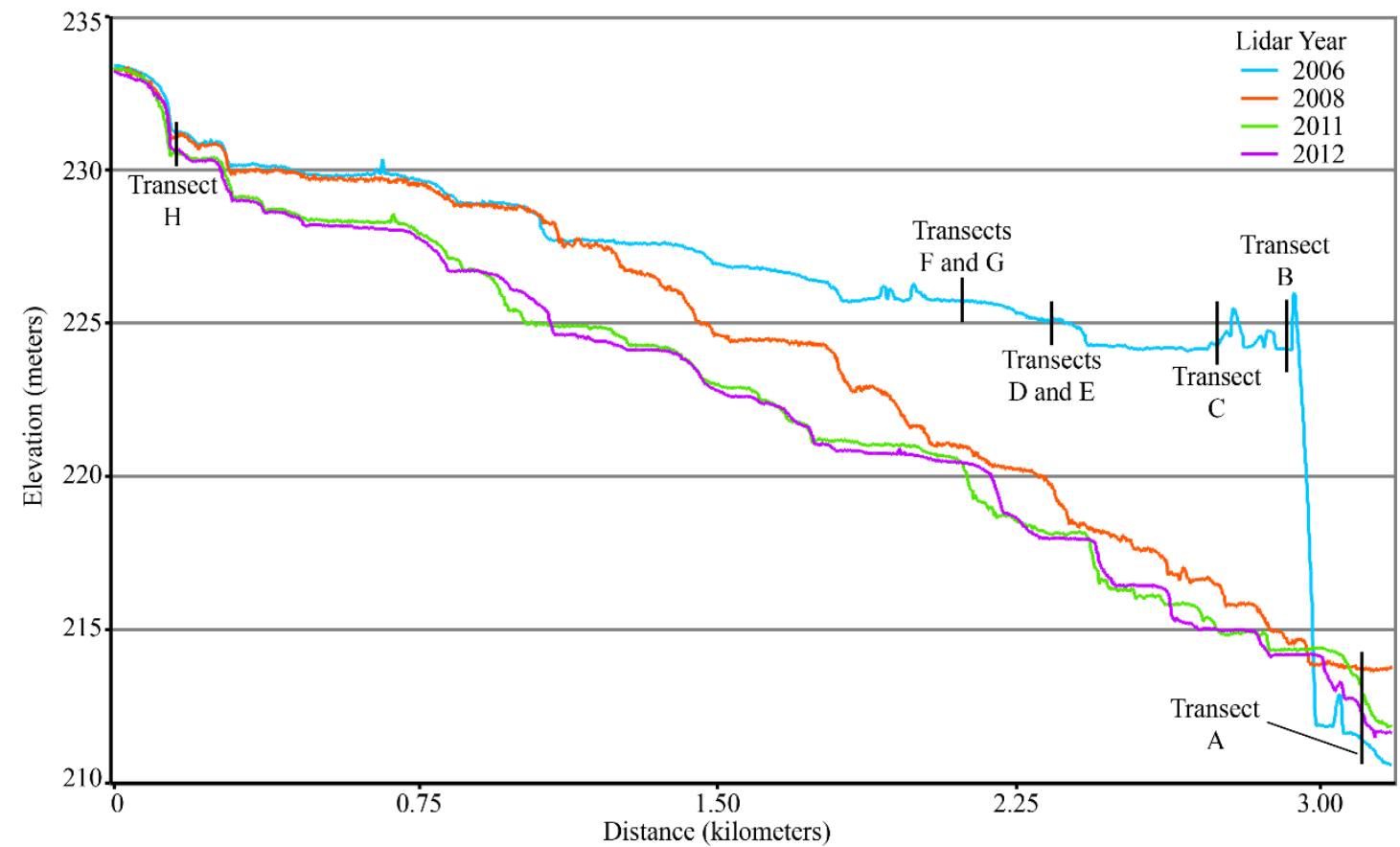

Figure 21. Water surface elevation longitudinal profile gradient. While bedform is not something that can be mapped at this point using lidar, inferences in bedform activity can be made by comparing the downstream difference between profile years. The highpoint shown in the 2006 line just downstream from Transect B is the Marmot Dam prior to removal.

Note that the channels are not running in the same course through the part of the reach and thus the lateral position of each channel is not identical and will be explored further in the lateral profile discussion. Change between 2008 and 2011 at this point is approximately one meter, and the change between 2011 and 2012 show the bed aggrading slightly and advancing the head of the rapid downstream close to 50 meters. 
Transect D marks the end of the rapid at the gravel bar and Transect E cross cuts the lahar dike that impedes the progress of the Sandy River and creates the pool conditions found at the end of the lahar dike before the second rapid/riffle. Figure 8 and Figure 20 show photos of the current conditions compared with the field collection photos from 2014; while the changes are not large there have been continued adjustments to the channel profile and depositional features on the reach.

Transect $\mathrm{C}$ bisects the gravel bar between GPS Point B and GPS Point C. At this cross section there is close to an 8-meter difference between the 2006 lidar acquisition and the 2008 flight. Close to 1 meter of continued degradation occurred between 2008 and 2011 with nearly no change noted between 2011 and 2012 at this point on the reach. Transect B marks the approximate location of the dam head and has 10 meters of change between the 2006 flight reservoir height and the first year of channel incision with some minor degradation occurring between 2008 and 2011, and again between 2011 and 2012.

There is a steady stair step down the profile gradient suggesting the aggradation adjustments moving downstream as discussed in the earlier portions of this paper. Transect A marks the approximate location of the spillway for the original dam, the area is well scoured and armored, so the channel continues to stay connected to this portion of the reach, there is noticeable aggradation between 2006 and 2008 of approximately three meters, with continued degradation occurring between 2008 and 2011, and slowing between 2011 and 2012. Appendix C: Appendix C: Stream Gradient Profiles shows the elevation point tables used from each year's lidar flight to create the stream gradient profile. Appendix B: Appendix B: Transect Tables shows the detailed elevation points applied to each profile using the Stack Profile tool. Each of these transect profiles is discussed in 
detail in the following section. I will begin at the upstream point which correlates to the visible knickpoint in all four lidar returns. Figure 22 shows the changes over the course of five years to the reservoirs knickpoint. This point is visible in the lidar and corresponds to the calculations given by Major and others, 2012 for the knickpoint retreat.

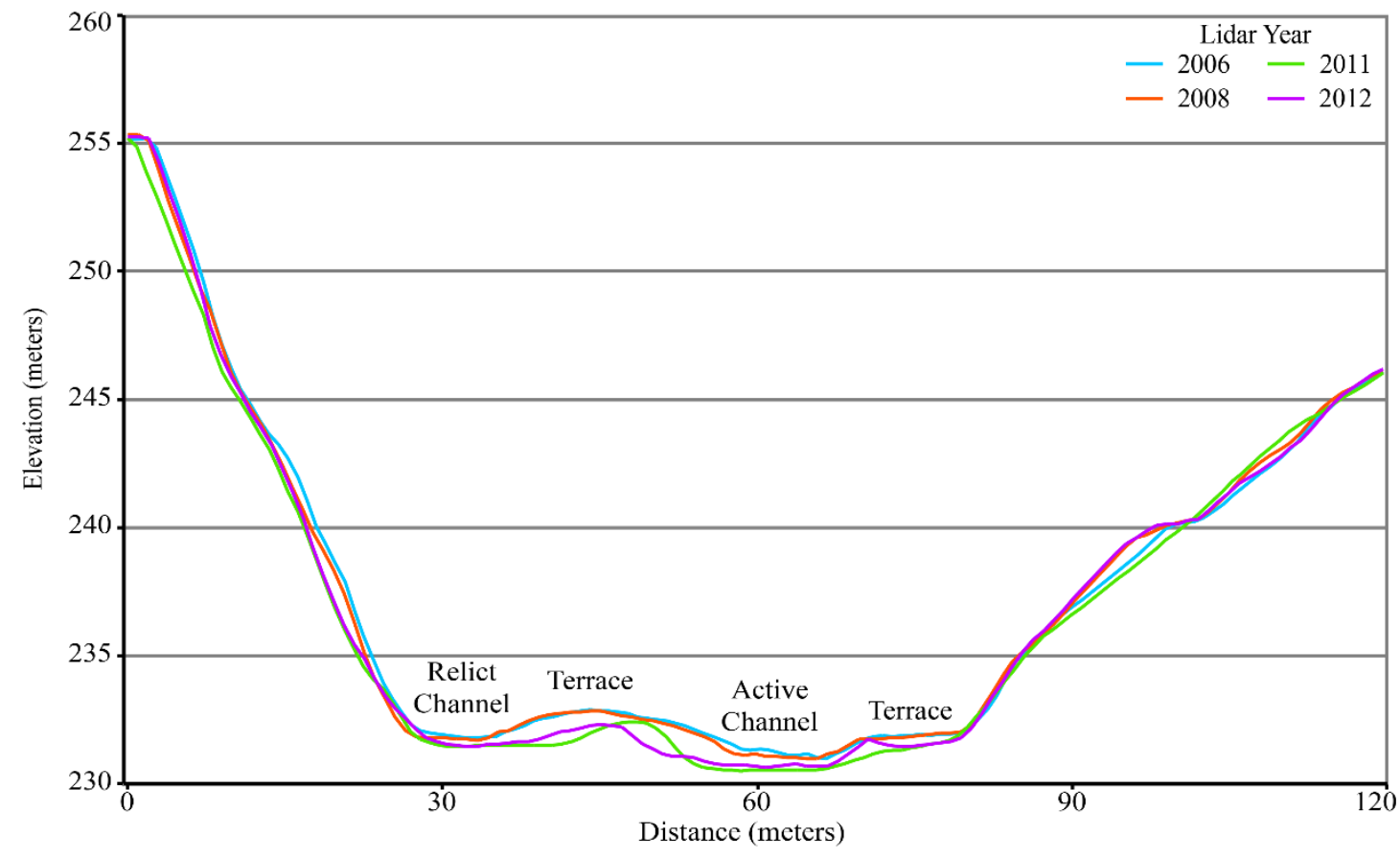

Figure 22. Transect $\mathrm{H}$ is located at the uppermost limit of the reservoirs influence.

Transect $\mathrm{H}$ trends south to north in the downstream direction. Terraces are visible in the profile, one to the south with approximately a 1-meter offset between 2006 and 2012, and a lesser terrace to the north that has some channel characteristics and would most likely be engaged in peak winter flow conditions. The overall change to the channel has approximately 1 meter of degradation and close to one meter of channel migration to the south by the 2012 lidar acquisition; note, this could be attributed to the increased accuracy between the original flight and the 2012 flight. The profile again shows that at this location on the river the channel has reached equilibrium and continues to adjust the margins of the 
channel laterally and the downstream gradient consistent with what was shown by Doyle et al., (2003b).

Transect $\mathrm{G}$ bisects the floodplain at the southernmost margin of the wooden crib structure (Figure 23) and trends northwest to southeast. Channel characteristics and the overall change are noticeable in this profile. Transect $\mathrm{G}$ is located 40-meters downstream of the riffle/rapid head.

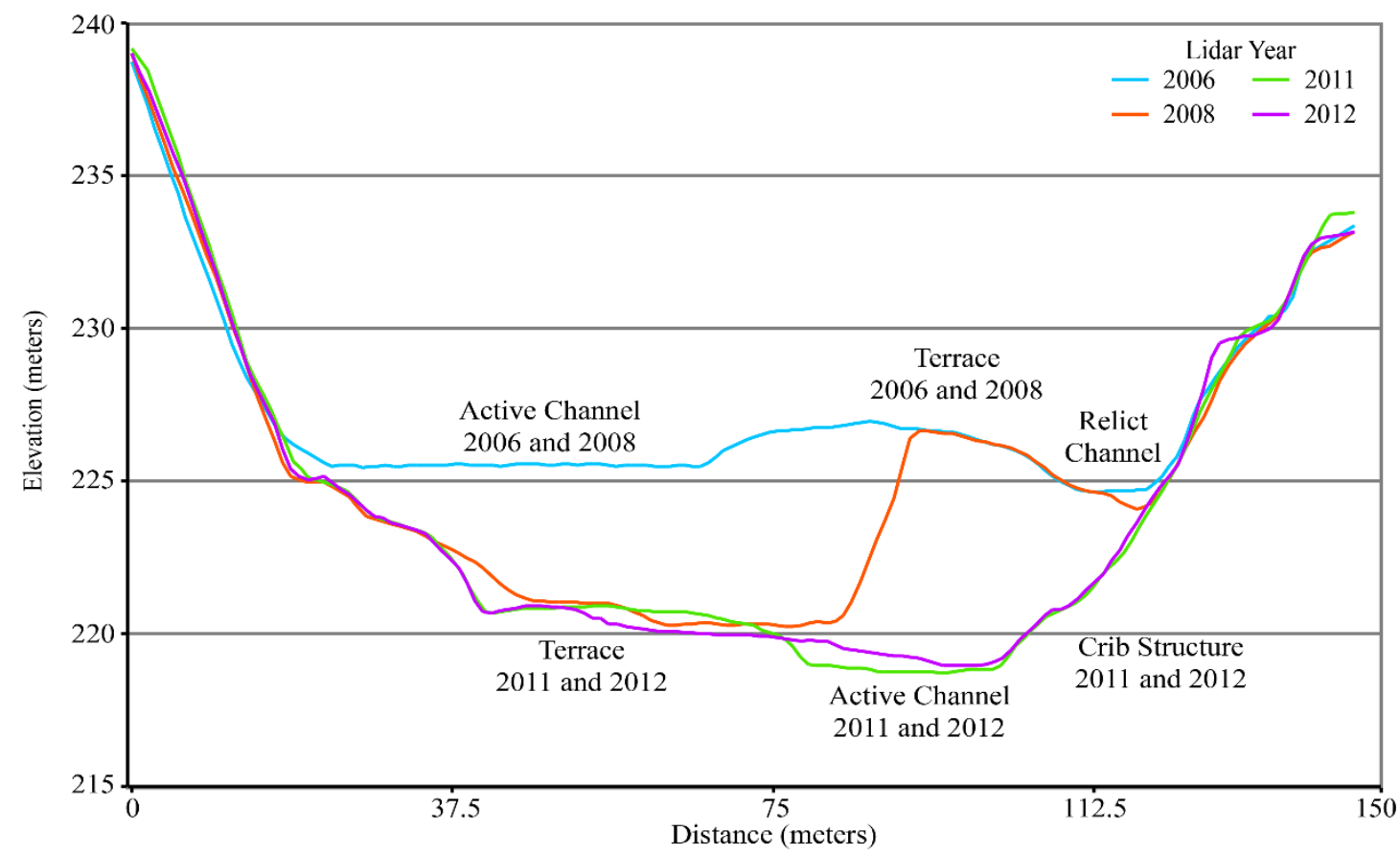

Figure 23. Transect $\mathrm{G}$ follows the southern edge of the crib structure. 2011 is the first indication of it on the lidar profile.

The 2006 water surface elevation and channel position show an 40 -meter channel to the west and an $~ 12$-meter wide relict channel on the east bank separated by a $\sim 30$-meter wide by $\sim 1$-meter high terrace. 2008 shows a decrease of $\sim 5$-meters in water surface elevation there is a $\sim 12$-meter wide by $\sim 1$-meter high terrace to the west. The channel is still migrating east and a thick sediment wedge $\sim 35$-meters wide by $\sim 5$-meters high is still being mobilized by the river in 2008 . The relict channel on the east side of the sediment 
wedge is just beginning the downward cutting of the sediment wedge. The river continues to change significantly between 2008 and 2011; the 30,000 cfs flood event in 2009 would have had an impact on the mobilization between year 1 and year 4 after the dam removal. The 2011 channel is $\sim 30$-meters wide and has engaged the western edge of the wooden crib structure.

The boulder/cobble terrace to the west has aggraded eastward from 2008 and is $\sim 30$ meters wide from the western bank to the streambank. In 2012 the profile of the gravel bar is dipping east, and the active channel has narrowed significantly between 2011 and 2012. The streambank on the eastern slope is well established between the two years; again the 39,000 cfs 2011 flood event is evident in the 2012 profile in the realignment of the cobble bar and channel position, with aggradation and degradation occurring in the channel and on the bar.

Transect F is similar to Transect G, as it bisects the floodplain at the northernmost margin of the wooden crib structure (Figure 24) and trends northwest to southeast. We see almost identical channel characteristics in this profile to those of Transect $\mathrm{G}$. Transect $\mathrm{F}$ is located 47-meters downstream of the riffle/rapid head, the 2006 water surface elevation and channel position show an $\sim 40$-meter channel to the west and an $\sim 15$-meter wide relict channel on the east bank separated by a $\sim 30$-meter wide by $\sim 1$-meter high terrace. 2008 shows a decrease of $\sim 5$-meters in water surface elevation, and there is a $\sim 12$-meter wide by $\sim 1$-meter high terrace to the west that than slopes up at $\sim 45^{\circ}$ angle. Again, we see the channel is still migrating east and a thick sediment wedge is still being mobilized by the river in 2008. The relict channel on the east side of the sediment wedge is a bit more 
pronounced in the downward cutting of the sediment wedge, and we see a slight dip to the west towards the active channel.

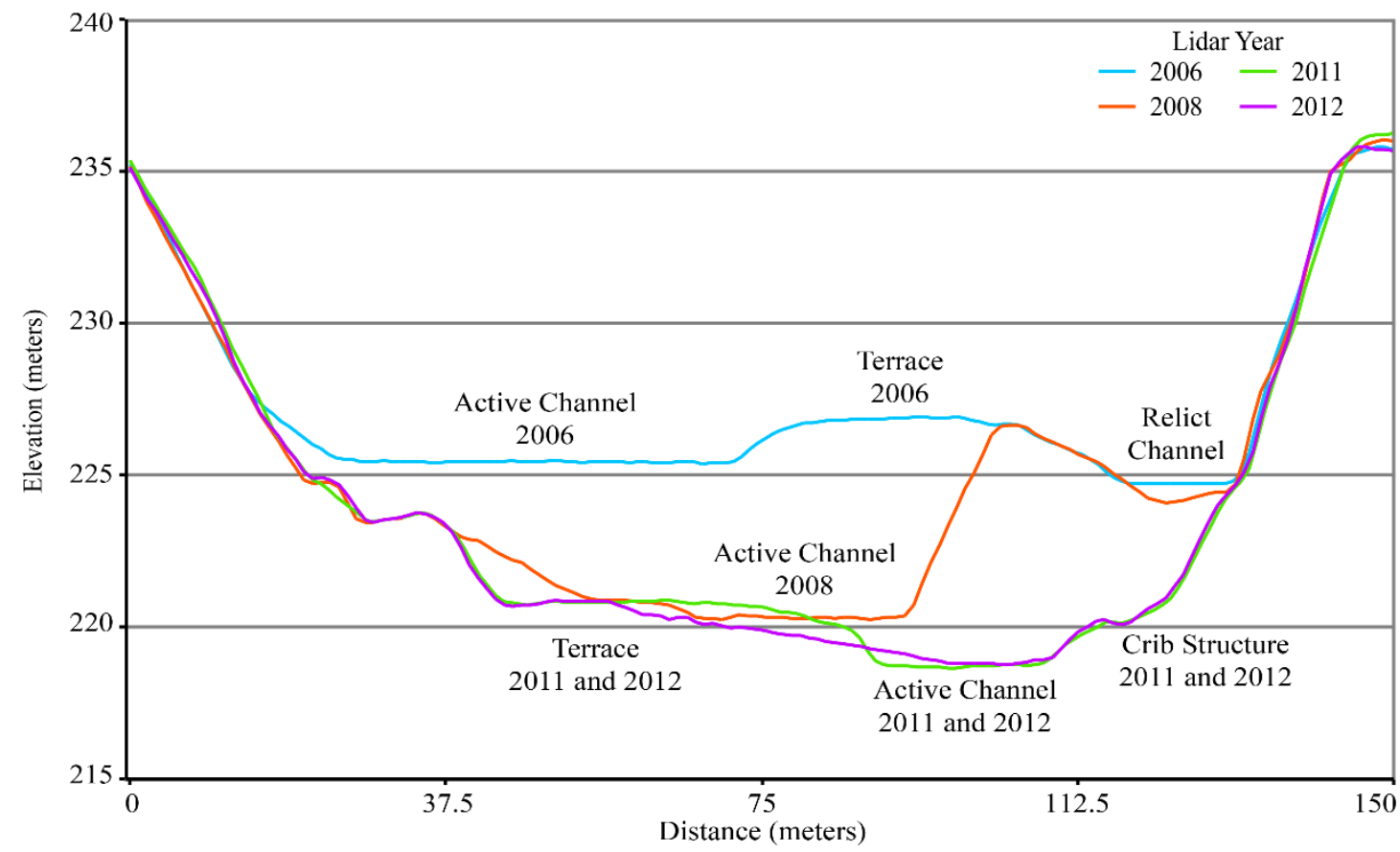

Figure 24. Transect $\mathrm{F}$ follows the northern edge of the crib structure. 2011 is the first indication of it on the lidar profile.

The 2009 flood event continues to change the profile significantly between 2008 and 2011 and would have had an impact on the mobilization between year 1 and year 4 after the dam removal. The 2011 channel is $~ 30$-meters wide and has engaged the western edge of the wooden crib structure. The boulder/cobble terrace to the west has aggraded eastward from 2008 and is $~ 30$-meters wide from the western bank to the streambank. We see the same eastward dip to the bar and narrowing of the active channel between 2011 and 2012 . The streambank on the eastern slope is well established between the two years, and we see here again the 2011 flood event evidence in the 2012 profile in the realignment of the cobble bar and channel position with aggradation and degradation occurring in the channel and on the bar. 
Transect E trends west to east and was drawn to cover the end of the gravel bar to the west of the active channel in 2014. Figure 25 compares the lidar returns and allows us to see the overall change in the channel at this prominent point. The gravel bar is to the west of the wooden crib structure and marks the southern edge of the pool and riffle that have formed as of 2018. The bar extends $\sim 170$-meters from the head of the rapids to the northwesterly bend the river takes when it reaches the lahar dike at the end of the run. The 2006 profile line shows a prominent terrace on the west bank $\sim 30$-meters wide which slopes down to the east $\sim 1.5$-meters before entering the main channel.

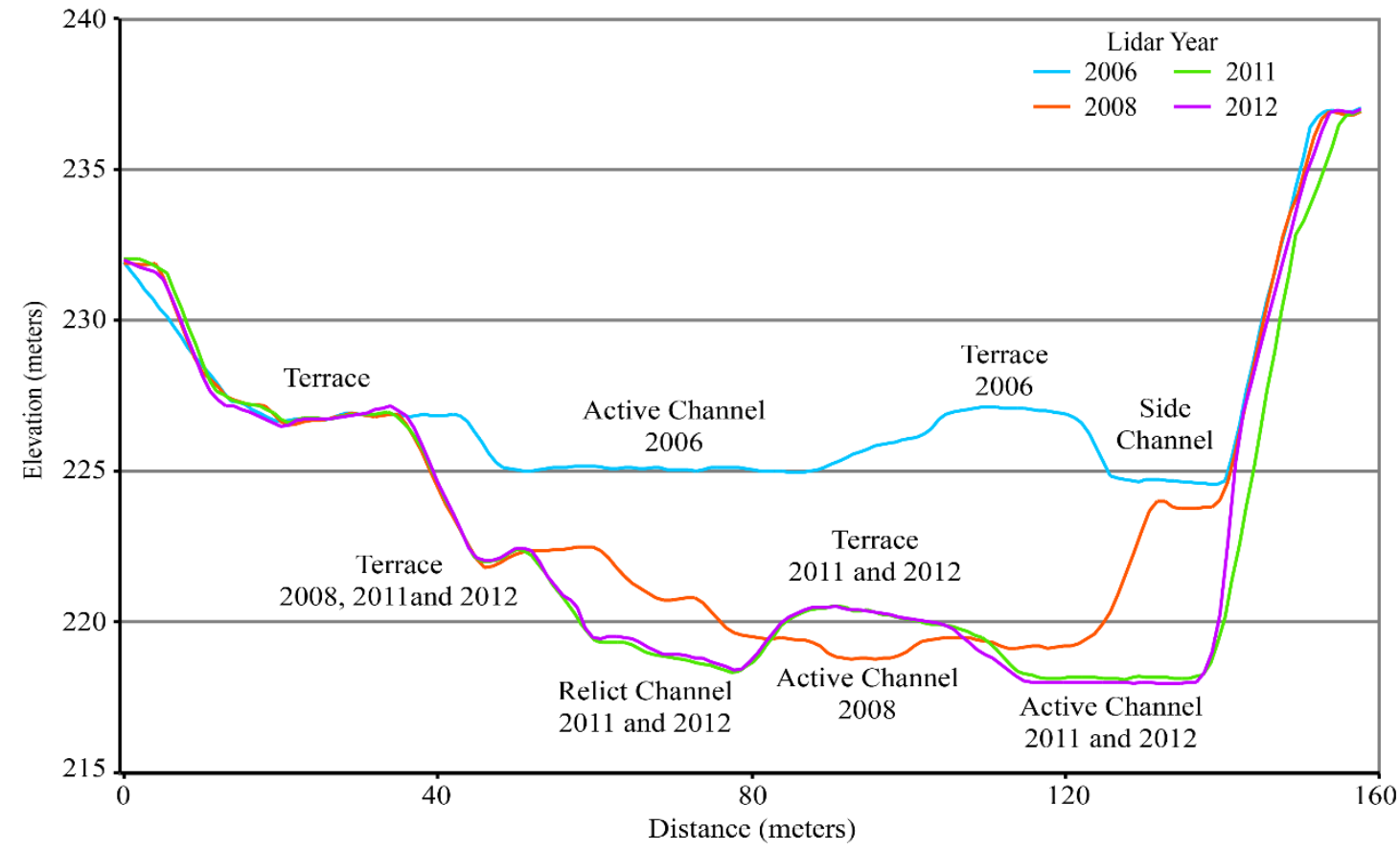

Figure 25. Transect E crosses the end of the active channel in 2011/2012 and traverses the northern portion of the gravel terrace head.

We can see the channel is $\sim 45$-meters wide at this point and slopes up to the east $\sim 2.5$ meters to another prominent terrace $~ 20$-meters wide, this dips sharply eastward into the 15-meter wide side channel before hitting the near shear bank on the eastern side of the 
bar. The immediate difference in this portion of the reach from 2006 to 2008 is striking and further shows the amount of sediment that was removed from the former impoundment.

We notice the terrace has retreated to the west $\sim 5$-meters and dropped $\sim 5$-meters to create another terrace $\sim 20$-meters wide. The bank dips again towards the east two more times forming smaller terraces that indicate the current floodplain margins at the time of acquisition. The active channel at this point of the river is $\sim 15$-meters wide and slopes up to the east to form another $~ 15$-meter wide terrace on the eastern bank of the channel. We can still see a portion of the side channel and part of the sediment wedge that is a continuation from the previous two transects immediately upstream from this point.

The 2011 and 2012 lidar profiles are nearly identical at this point of the river and correspond to what was shown in the photos from 2014 and 2018. We see that the terrace created in 2008 is a remnant of itself and the bank dips to the east $\sim 80^{\circ}$ before entering the new overflow channel that dips east $\sim 45^{\circ}$ before rising to the east $\sim 2.5$-meters to the head of the gravel bar. This well armored boulder/cobble terrace is $\sim 30$-meters wide and dips slightly towards the active channel. The terrace then dips down $\sim 2$-meters to the active channel which is $\sim 30$-meters wide at the end of the run before entering the pool/riffle portion of the reach.

Transect $\mathrm{D}$ is a west to east trending line that marks the end of the rapid/run and creates conditions that lead to the $\sim 60$-meter pool/riffle complex in the northwest bend of the river. An interesting feature in this profile is how it shows the exposure of the resistant lahar dike and the change in channel morphology that came from that exposure. Figure 26 compares the lidar returns and shows the geologic feature emerging from the reservoir sediment. As we can see in the image, the 2006 profile shows a broad pool with a couple of small terraces 
flanking east and west. The channel width is $\sim 45$-meters at this point with a sand and gravel terrace to the west and a portion of the lahar head at the edge of the channel to the east. The eastern bank is shear and well-formed based on the lidar returns.

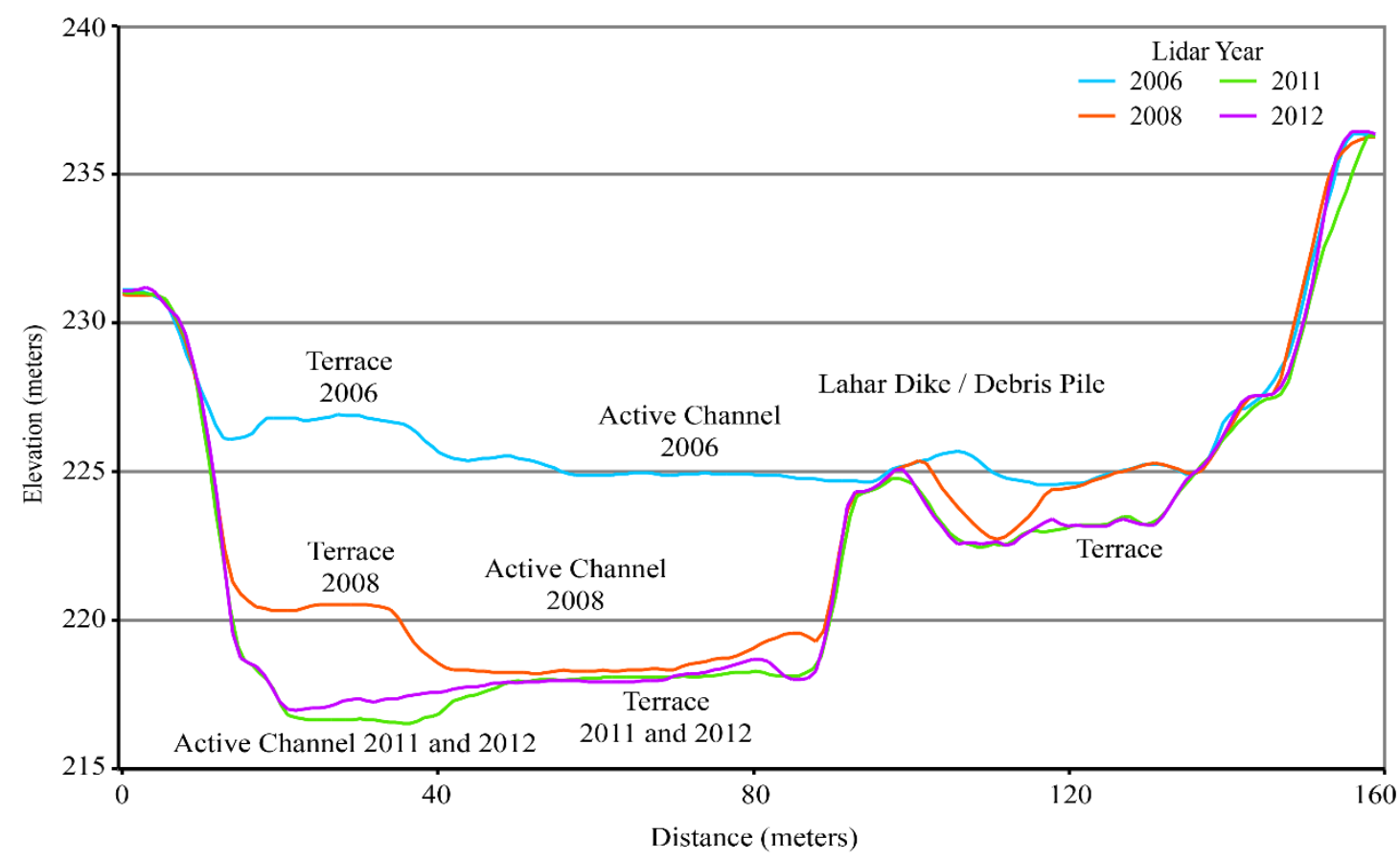

Figure 26. Transect $\mathrm{D}$ traverses the exposed lahar dike that influences the current stream pattern in the reach.

The most immediate effect we see in the 2008 profile is the $\sim 8$-meter drop in water surface for the channel creating $~ 20$-meter wide terrace on the western bank with $\sim 45$ meter wide channel that abuts the lahar dike, which rises sharply out of the channel to the east. Some scour can be seen atop the lahar dike in 2008 and would correspond to the end of the sediment wedge that was most likely the effect of presence of the lahar dike under the reservoir surface, acting as a dam. This sediment wedge appears as a beach in the 2005 NAIP imagery and has since been washed downstream.

The only real change we see from 2008 to 2012 is the further erosion and downcutting of the western terrace by another 4 -meters. Also, note the angled gradient of the channel 
that is running north at this point of the river as it rounds the lahar dike and heads into another short rapid set before hitting the smaller lahar dike 225 -meters downstream. Between that point there are two more pool/riffle complexes that are well established. Further erosion can be seen on top of the lahar dike exposing a knickpoint in the top of the structure that currently accumulates significant amounts of LWD.

Transect $\mathrm{C}$ is a southwest to northeast trending line that bisects the center of the gravel bar between GPS Point B and GPS Point C (Table 3). The western bank is a shear slope that is well constrained given that the lidar profile return shows insignificant change (within noise tolerances discussed in Faux et al., 2009). The main channel is shown as a broad gently sloping pool that then rises at a gentle angle when reaching the eastern bank in the 2006 profile (Figure 27).

The main channel is $\sim 55$-meters wide at this point and $\sim 10$-meters deep based on the lidar profile. Again, 2008 shows immediate change to the channel profile with $\sim 8$-meter drop in the water surface elevation and shifting to a 20 -meter wide channel with increase velocity based on the constraining $\sim 25$-meter wide terrace to the west and the steep slope of the bank to the east. The change between 2011 and 2012 is relatively minor, some aggradation of the gravel bar on the eastern bank with some slope degradation on the eastern bank forming what appears to be a low-relief slump. The main channel has migrated laterally west $\sim 15$-meters incising $\sim 1.5$-meters into the 2008 terrace and forming the $\sim 20$ meter wide channel shown in the profile. 


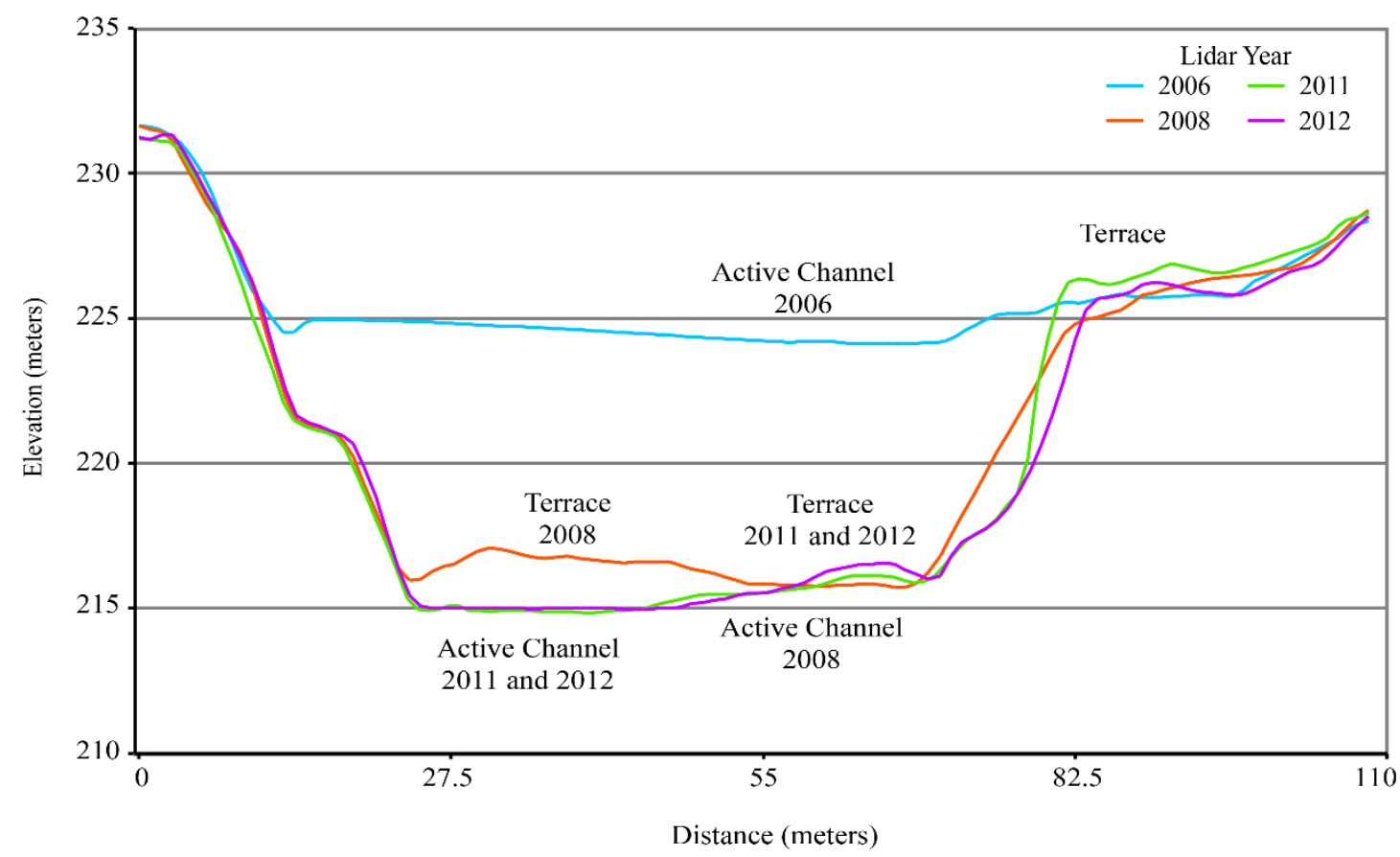

Figure 27. Transect $\mathrm{C}$ traverses the gravel bar and sandy beach terrace that has formed downstream of one of the lahar dikes.

From 2012 to 2018 this section has continued to aggrade with the gravel bar showing 1-meter offset from the current bank full conditions in the October 2014 photos to a well aggraded sandy beach in the July 2018 photos. This suggests that the river continues to redistribute sediment from the Upper Sandy River and given the quasi-equilibrium state of the river currently, the gravel bars will continue to aggrade and form stable beach fronts. This spot will continue to see aggradation of the beach until the lahar dikes erode enough for the rivers energy to adjust the channel features; the current meandering course the river takes in the $\sim 300$-meters from the end of the first rapid run to this bar is an ideal setting for the continued aggradation of this bar.

Transect B is a south to north trending line that bisects the approximate location of the Marmot Dam spillway head. Figure 28 shows the profile comparison with the most notable feature being the initial drop in the water surface elevation. Between the 2006 and 2008 lidar flights there is $~ 10$-meter drop in the water surface elevation of the main channel 
which is $\sim 75$-meters wide pool at point of the river. In 2008 we see the channel is $\sim 25$ meters wide and slopes sharply up on the north bank; given the lidar profile for the 2008 to 2012 shows a sharp angle of repose but since this is the old outer wall of the dam the slope is stable.

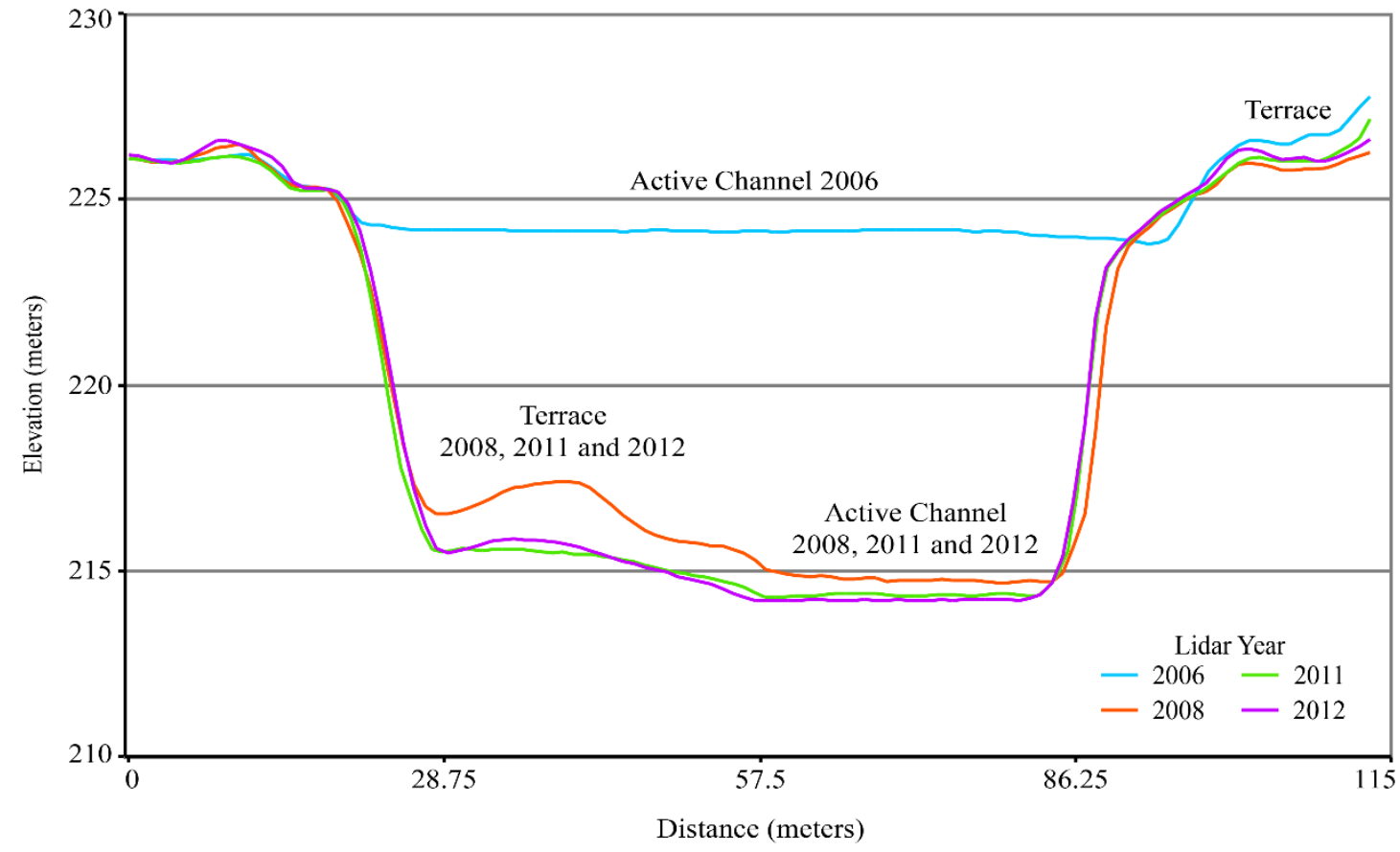

Figure 28. Transect B is located just above the location for the head of the Marmot Dam.

In both the 2011 and 2012 profile lines we see that the main channel is now $~ 30$-meters wide and slopes gently to the south forming $\sim 25$-meter bar gently dipping to the north $\sim 10^{\circ}$. The channel profile for this point of the study area shows a stable river system in equilibrium for the reach characteristics. The channel is highly constrained at his point, which is why there is little lateral movement. This is also why it made for an effective dam location.

The approximate foot of the spillway was chosen for the location of Transect A (Figure 29). The transect is a south to north trending line bisecting the width of the spillway chute and the portion of the diversion channel that was used to divert water to Roslyn Lake $\sim 5$ - 
meters west of the footbridge at the site location. When the dam was decommissioned there was no longer a need for the diversion channel, so it was closed, and Roslyn Lake ceased to exist. Return profiles show what has been consistent in the literature reviewed for this report.

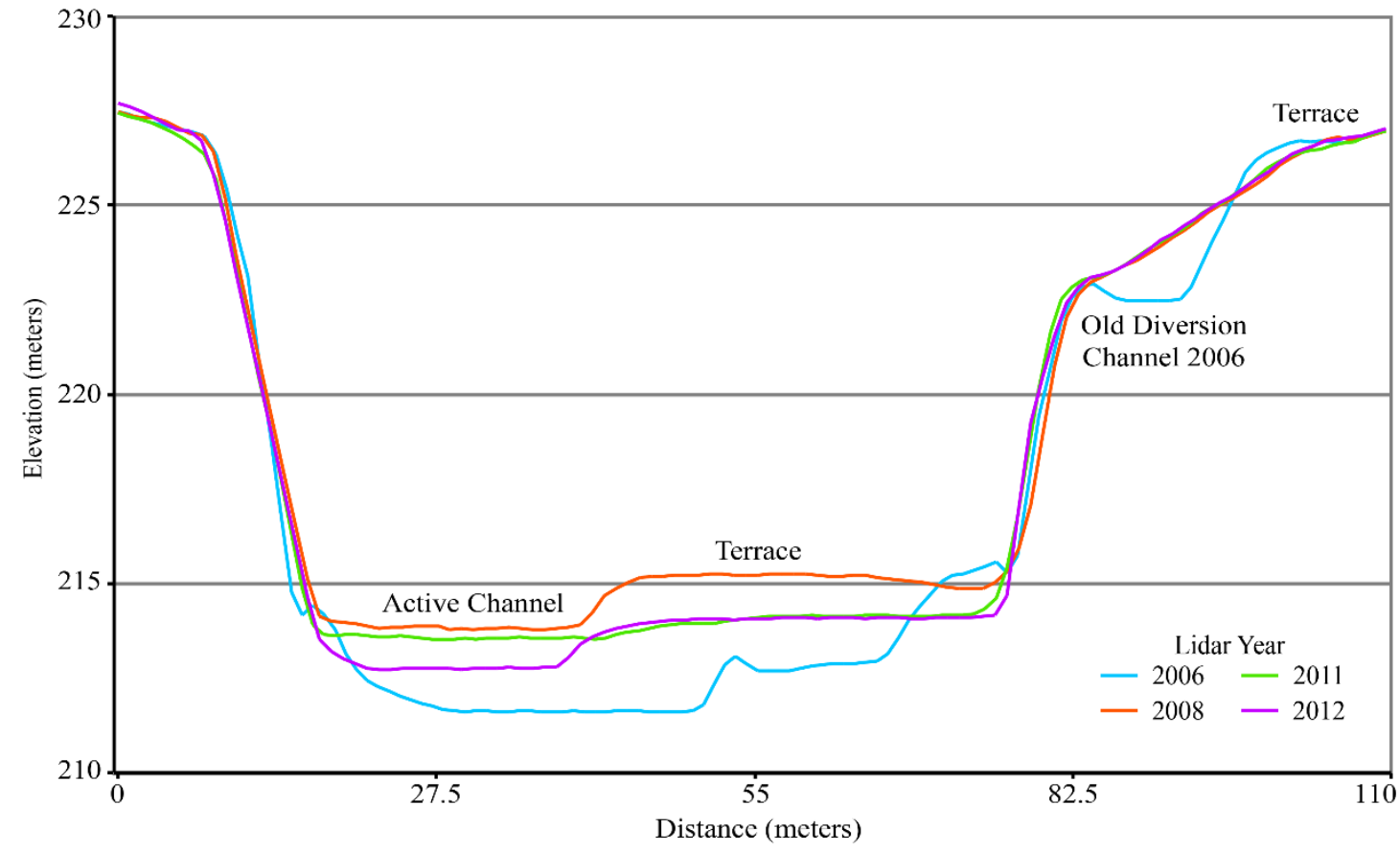

Figure 29. Transect A is just downstream of the location of the Marmot Dam spillway.

Removal of the dam saw an immediate downstream aggradation followed by continued degradation until the channel reached quasi-equilibrium. The 2006 profile line shows $\sim 25$ meter wide channel and $\sim 12$-meter wide side channel $\sim 1$-meter higher than the main channel to the north. The old diversion canal channel is shown and is $\sim 7$-meters wide.

The first thing to note is the initial aggradation from the removal of the Marmot Dam in 2006 to the profile line in 2008. As noted earlier in this paper, immediately following the removal of a dam the downstream region from the dam shows immediate aggradation, which based on the 2008 profile is $\sim 2.5$-meters in both the channel area and the gravel bar terrace that aggraded on the north bank of the river at this transect. Given that there were 
two significant flood events that occurred between the 2008 and 2012 lidar flights, we can infer what those events did to this portion of the reach.

The spillway was devoid of any significant sediment for the life of the Marmot Dam and was thus a well armored chute traveling west downstream of the dam before trending to the southwest $\sim 730$-meters downstream of Transect A. The aggradation accumulation from the 2008 profile was dispersed across the width of the transect canyon with less than a meter of degradation in the channel and $\sim 1$-meter of degradation to the gravel bar from 2008, creating $~ 55$-meter wide channel with a slight dip up to the north. The 2012 profile line comes in the immediate aftermath of the 2011 flood event that devastated parts of the Upper Sandy River in the Village at Mt. Hood segment of the river. We can see some continued degradation of the channel of $\sim 1$-meter but no real change to the profile of the gravel bar to the north; hence, we can assume by this profile line that the remaining sediment that had aggraded in the channel from the 2009 flood event was further pulsed out of the channel giving us the 2012 channel profile shown.

The cross-section profiles are another valuable tool for showing the usefulness of lidar for monitoring and identifying geomorphic features. With this study I have compared three different aspect of mapping with lidar that allows for the identification of in stream features and correlation to existing literature on the expectations involved in the removal of a dam and the amount of time needed for the system to reach stability.

\subsection{Discussion}

My continued research into channel morphology characteristics with lidar to supplement existing field techniques will add to the list of available resource management tools at our 
disposal. This study does have its limits given the accuracy of detecting the different geomorphic changes. Lidar accuracy itself will vary depending on the stream reach profile, angle of incidence and overall ground cover for the reach as well as the time of year the study was flown will also affect the data (English, 2008). Being able to use and test methods from other researchers will help me further refine my own methods. Evaluating the overall geomorphic change will provide a potential understanding of the reach dynamics. It is important to factor in flow, channel morphology and ground clutter when using lidar to detect water surface changes. At the time of the lidar acquisition field survey of the region was done to determine accuracy of the data for the flight tied to know ground control points. Comparison of sequential lidar provides a valuable tool for looking at channel morphology and should be considered as a necessary step in monitoring river channel change and morphology. It should also be used to make planning decisions in areas of active migration potential and to inform the public of the potential hazards associated with a given region. The technique has shown that the removal of the Marmot Dam the Sandy River has reached equilibrium with little potential for significant lateral migration in the reach due to geologic constraints.

Channel morphology present in the 2006 lidar (Figure 30) shows a long sandy bar dominating the upper reach in the same relative position as the lahar dike and crib structure and another sand bar to the northwest near another lahar intrusion(GPS points D, G, H, J, and K). This is corroborated by the 2005 NAIP imagery of the reservoir with a visible side channel to the east of the reservoir. The active channel has a low sinuosity and is noticeably wider than the channel in subsequent years. Prominent features visible in the transect profiles show the bar and terrace configuration of the reach. Figure 31 shows the rapid 


\section{Channel Characteristics \\ Pre-removal}

Plate 2

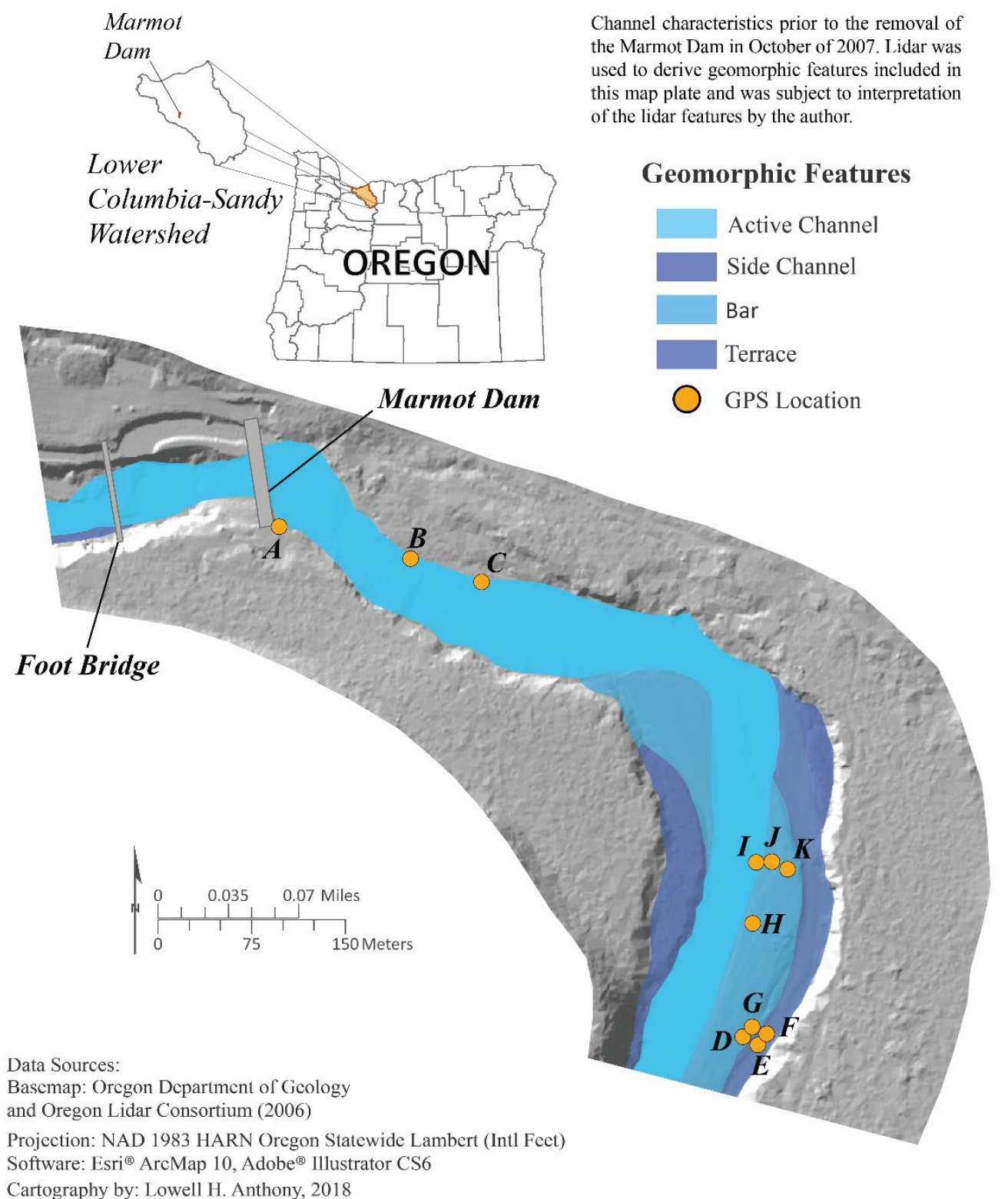

Software: E

Cartography by: Lowell H. Anthony, 2018

Figure 30. Geomorphic features present in the 2006 lidar acquisition. Note the long bar of sediment in line with the lahar intrusions visible now. 


\section{Channel Characteristics \\ Year 1 after removal}

Plate 3

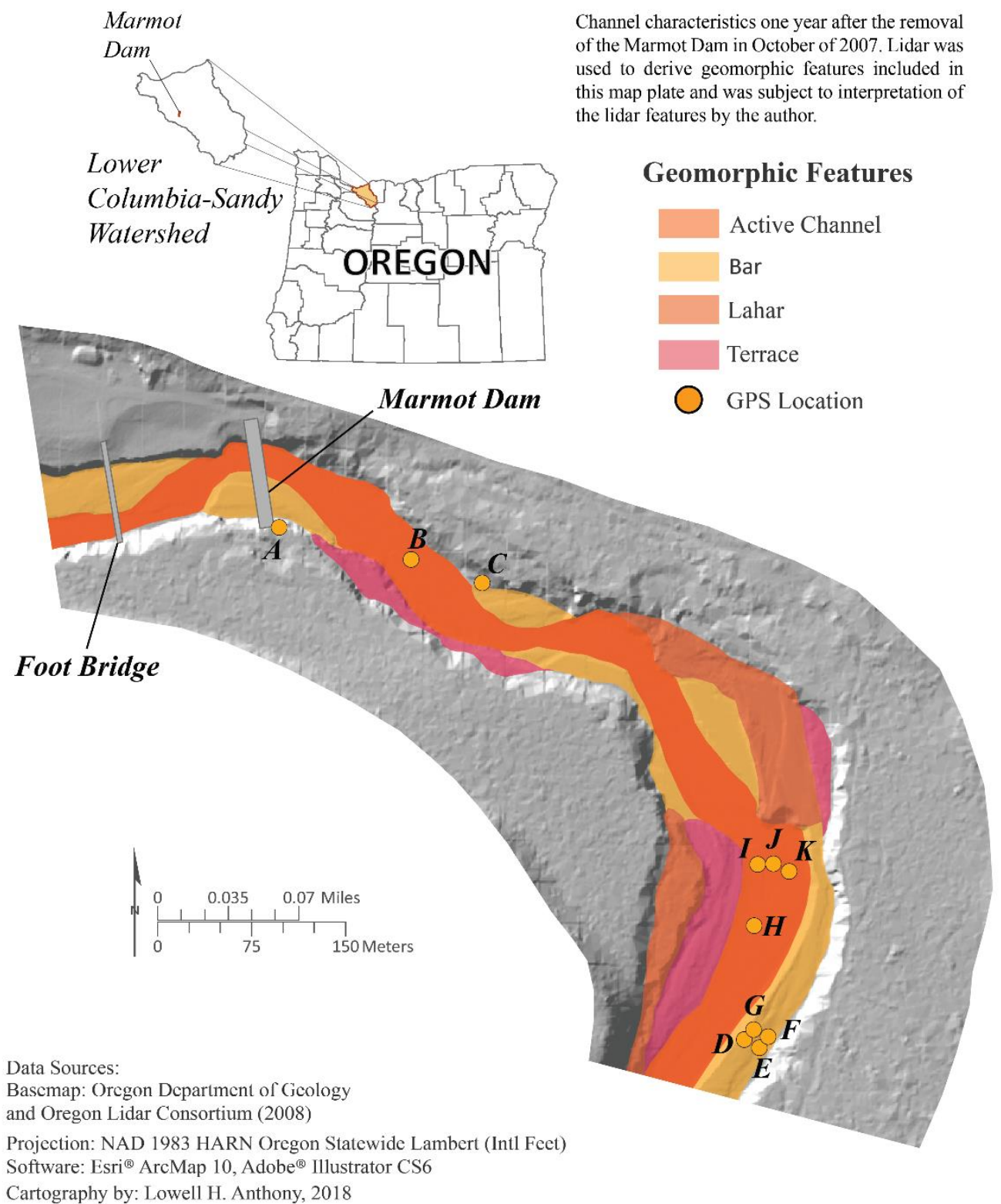

Software: Esti

Cartography by: Lowell H. Anthony, 2018

Figure 31. Geomorphic features present in the 2008 lidar acquisition. Note the lahar intrusions is just becoming visible now. 
change in channel morphology one year after the removal of the dam. There is a noticeable shift in the active channel margin and thalweg and the first signs of the lahar intrusion begins to appear.

The morphological characteristics shown in the transects for the 2008 lidar acquisition are prominent in this map. The bar visible in the 2006 lidar has been completely mobilized and a wide alluvium terrace dominates the eastern shore still covering the crib structure (GPS points $\mathrm{D}-\mathrm{G}$ ). A more permanent terrace has formed to the east of the lahar intrusion and on the southwest bank across from GPS point B. The active channel has a more sinuous pattern and the influence of the lahar intrusion is shown. Alluvium and gravel bars are shown on either flank of the active channel and continue to progress downstream as would be expected based the research discussed in this paper. The point density difference between the 2006 and 2008 acquisitions is noticeable in the detail of the features shown.

The current positioning of the active channel and bars adds testament to the competency of the Sandy River. A comparison of active channel margins (Figure 32) shows the amount of lateral offset between the two lidar flights, of note is the change in sinuosity between years also correlates to known research on the progression of meander bends in relation to bar and terrace formation what was mentioned in the various papers on CEM's. When comparing active channels, the first features noticeable is the influence of the lahar intrusion on the active channel position. The scour of the prominent bar that was visible in 2006 shows how much sediment had accumulated behind the lahar intrusion and the lack of cohesion in that portion of the reach due to its influence. In the 2011 lidar the morphology is more pronounced with the lahar intrusion still influencing the channel pattern and the first appearance of the crib structure showing on a lidar return. 


\section{Channel Change Year 1 after removal}

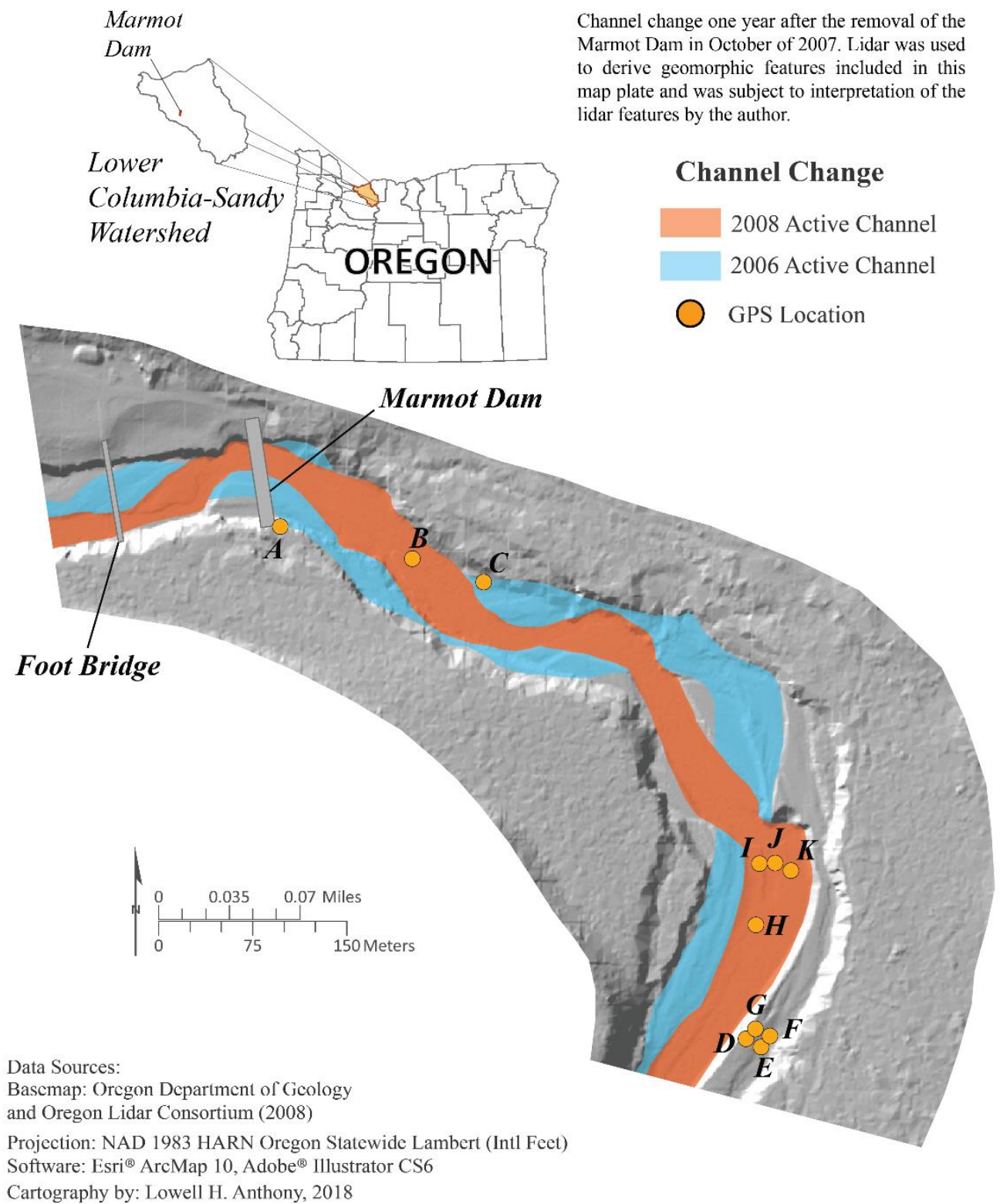

Figure 32. Active channel change between 2006 and 2008. The influence of the lahar in channel pattern is evident here.

As previously mentioned in this paper January of 2011 saw a significant flood event that undoubtedly helped shape the confines of the channel and the features, we see in the 
hillshade DEM. The active channel has migrated significantly to the east (Figure 33) and the large gravel bar is beginning to form with the margins of the head prominent already in the hillshade (GPS points I - K).

The alluvium terrace that was visible and made a prominent feature in the 2008 lidar has mostly eroded into the channel and is contributing to the aggradation of the sand bar downstream at GPS points B and C. Given the competence of the Sandy and the geologic constraints the lahar dike placed on the reservoir I hypothesis that the large reverser sorted cobbles that make up the gravel bar at GPS point $\mathrm{H}$ were deposited during past debris flows and the effect of the resistant dikes created these impediments in the reach, that are technically their own geomorphic formations now given the lack of sufficient flow to continue to mobilize them. The morphology here has created a narrow constriction point that creates a pool-riffle formation near GPS point I on the bar head.

Comparing the active channel change between the 2008 flight and 2011 flight we again see the continued shift in thalweg to the northwest with sinuosity curve continuing to progress downstream and the meander bends becoming more stable in their position (Figure 34). The bars continue to aggrade at GPS points B and C. The 39,000 cfs event from January would be a significant driver in the morphological changes shown in the active channel position between these two lidar flights. Figure 35 shows how the river has continued to incise over the years, and the adjustments to the overall geomorphology of the reach, with the gravel/boulder bars moving slightly downstream and aggradation of sediment occurring at the end of the last armored bar just upstream ( $\sim 150$ meters $)$ of the area the dam was in prior to removal. 


\section{Channel Characteristics \\ Year 4 after removal}

Plate 5

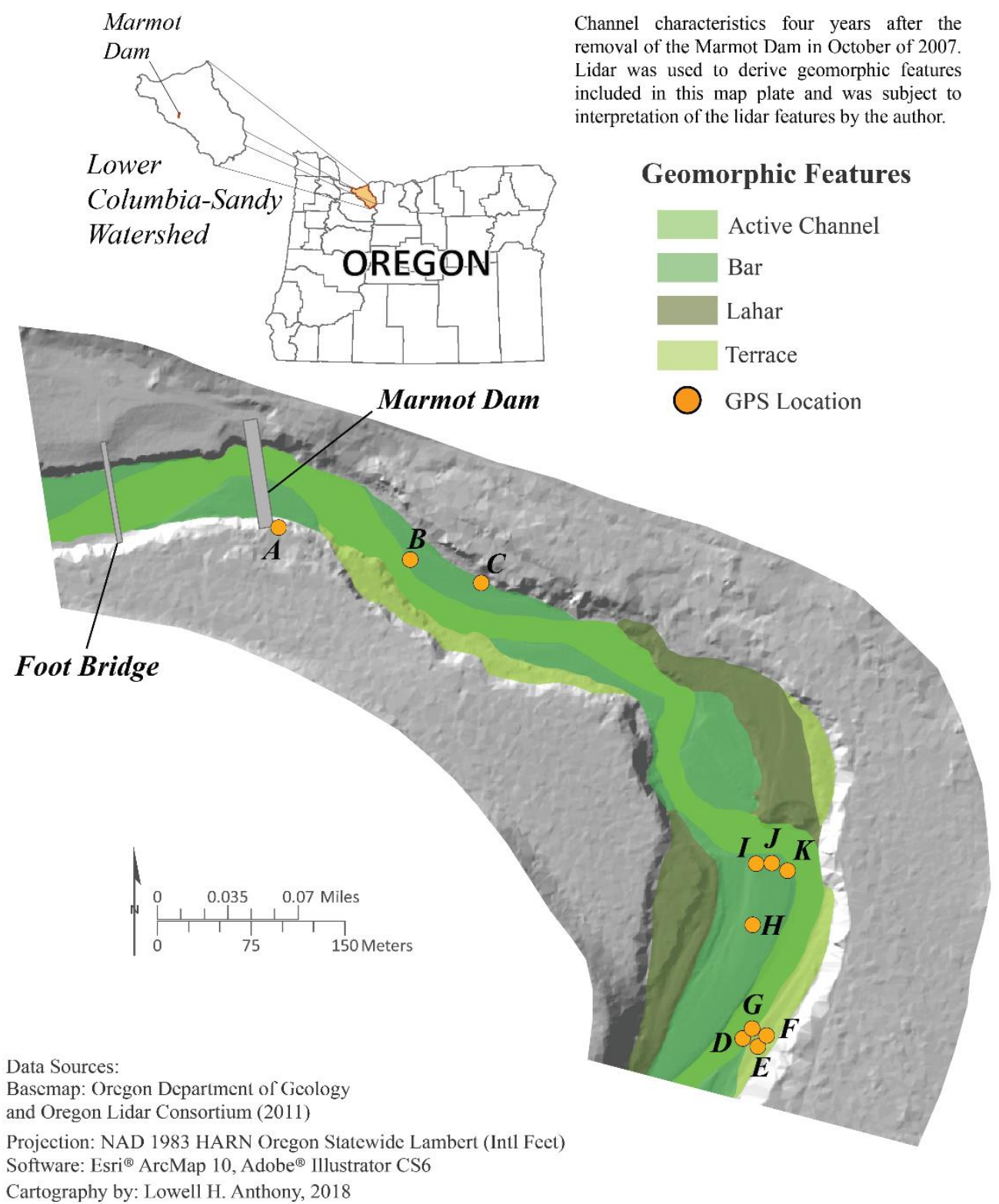

Software: Esri

Cartography by: Lowell H. Anthony, 2018

Figure 33. Channel morphology shown in the 2011 hillshade DEM, the lahar intrusion is a driving factor the shape of the reach and influence on the aggradation of the gravel bar marked by GPS point $\mathrm{H}$. 


\section{Channel Change \\ Year 4 after removal}

Plate 6

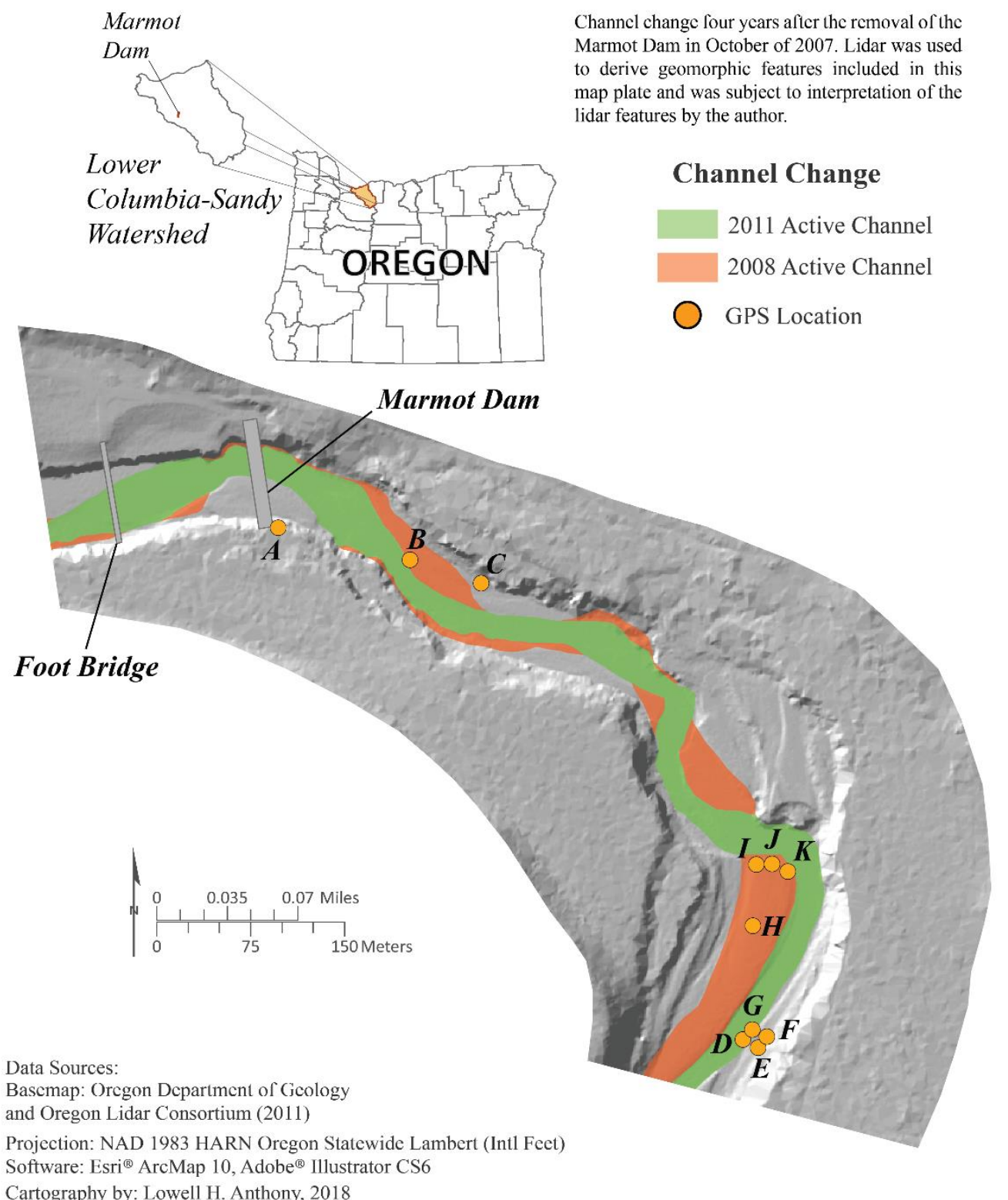

Software: Es

Cartography by: Lowell H. Anthony, 2018

Figure 34. Active channel change between 2008 and 2011. We see the bar being built back up at GPS point $\mathrm{H}$.

Sediment aggradation on the last bar significantly increased from the 2014 field study and photos taken in July of 2018 extending the end of the bar $\sim 50$ meters and creating a 
broad beach ( 12 meters) with intermixed boulders ( $>256 \mathrm{~mm})$ and cobbles $(64-256 \mathrm{~mm})$. Lack of significant vegetation on the bar and the beach show the continued power of the seasonal variability of the Sandy River and will lead to continued changes in the geomorphic characteristics of the bar. Further weathering and plucking of the lahar dikes have not made significant changes to their margins, nor in the channel position the cobble and boulder bars that extend to the north behind each of the two significant lahar intrusions on the east bank.

The lahar dike at the foreset bed of the bar has not had significant change to it structure; the sign and cross that had been atop the intrusion were removed when the area was made a park. The riffle to the south the lahar has extended $\sim 1$ meter downstream based from visual comparisons and a pool now encompasses the front two thirds of the lahar intrusion extending from front to back east to west before continuing into another riffle and deep main channel. The southernmost lahar dike still has a significant accumulation of large wooded debris on top of it, and some smoothing and removal of the fines on the south face as the river come around it to the northwest. The backside of the dike continues to exfoliate from cavitation forces during peak flows, but the difference is negligible between the 2014 and 2018 field excursions.

The wooden crib structure that emerged from the sediment between 2008 and 2011 has continued to come apart and the channel has migrated laterally to the east under the structure $\sim 1$ meter as shown in the July 2018 photos. The log ramp that had extended into the pool/rapid in the 2014 field photos no longer exists and a large boulder is $\sim 1$ meter to the west in the middle of the rapids. The bar on either side of this channel are $\sim 0.5$ meters high with a good angle of repose and well armored boulder structure. The remaining 


\section{Channel Characteristics \\ Year 5 after removal}

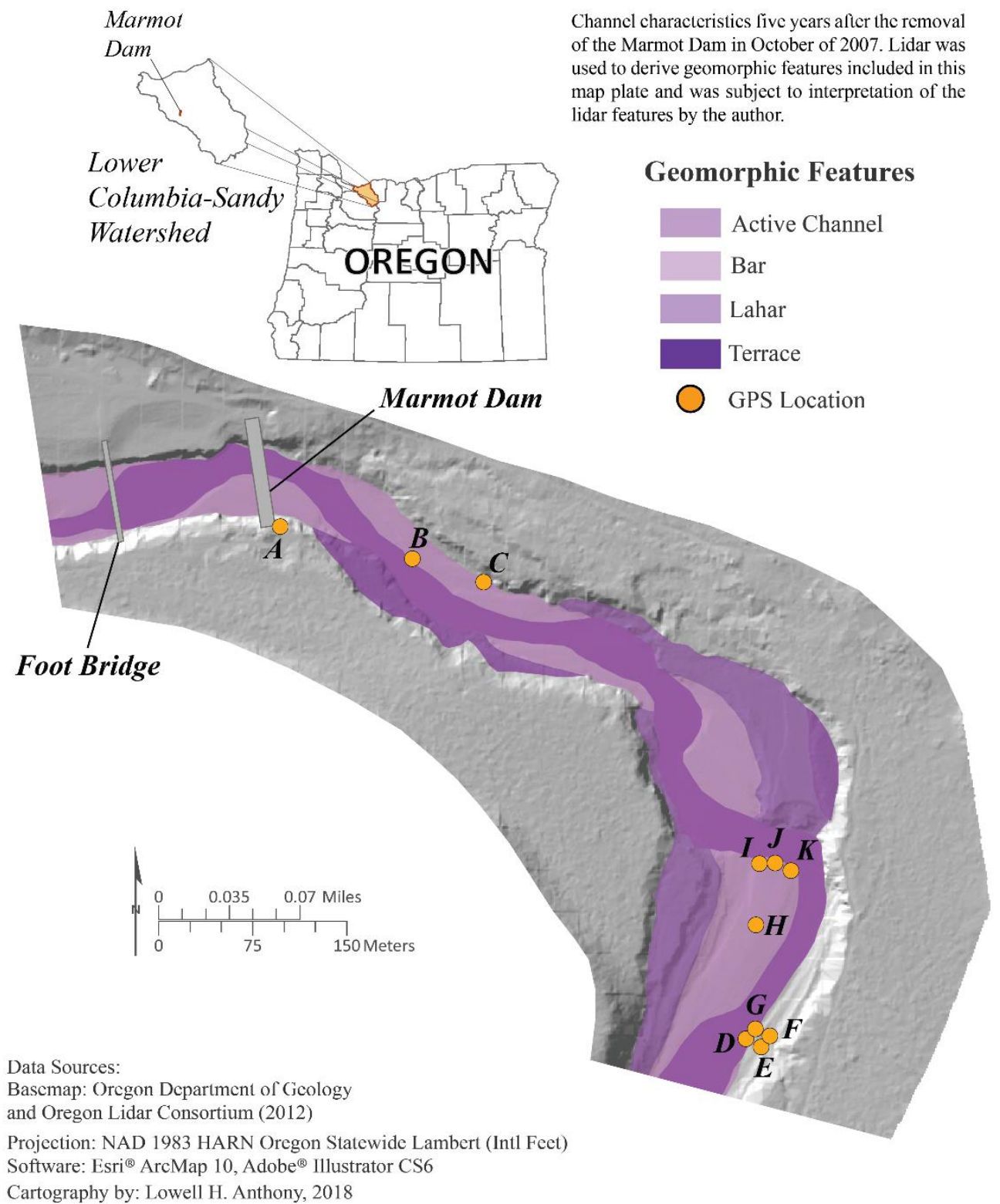

Figure 35. Channel morphology shown in the 2012 hillshade DEM, the minor adjustments to the morphology from 2011 shows a channel close to equilibrium.

wooden structure is mostly buried in the boulders and cobbles on the east bank and will still be a significant point on the river for some time. 
The cobble and boulder bar to the west of the wooden structure has continued to aggrade and is $\sim 1$ meter higher than in the 2014 field study based on current conditions. The boulders on all the bars are reverse sorted and intermixed with sand and gravel showing the competency of the river during winter flows. The pool at the end of this bar has migrated $\sim 0.5$ meters to the south and degraded the sediment that had accumulated at the end of that same bar in 2014, creating a 1-meter-deep pool in the area the beach had occupied in 2014. A low-grade riffle sets at the top of this pool and the original pool from 2014 has increased flow but is still $\sim 1$ meter deep. The rapid/riffle complex directly northwest of the pool going downstream remains unchanged in any measurable way visually, but the lahar intrusion on the west bank has continued to erode and now has an $\sim 0.5$-meter channel between its outer margin and the main intrusion embedded in the bank.

Subsequent slope failures on the west bank have allowed some minor lateral migration to the west and could account for some of the additional sediment found on the further downstream bar discussed above. The geomorphic characteristics noted in the lidar acquisitions and corroborated by time in the field, both in 2014 and 2018, show that lidar is a significant tool for helping identify and monitor geomorphic features and fluvial geomorphic conditions. The gradient profiles show aggradation and degradation upstream and downstream of the dam removal with the 2011 and 2012 comparisons showing the former impoundment coming into equilibrium and showing the importance of sequential lidar for looking for fluxes between lidar years (Figure 36). Change shown in each of the lateral profiles are significant between 2006 and 2008, and again between 2008 and 2011, with the 2011 and 2012 profiles showing the quasi-equilibrium state of the river at the time of those flights. Channel width and sinuosity showed significant change between the 


\section{Channel Change \\ Year 5 after removal}

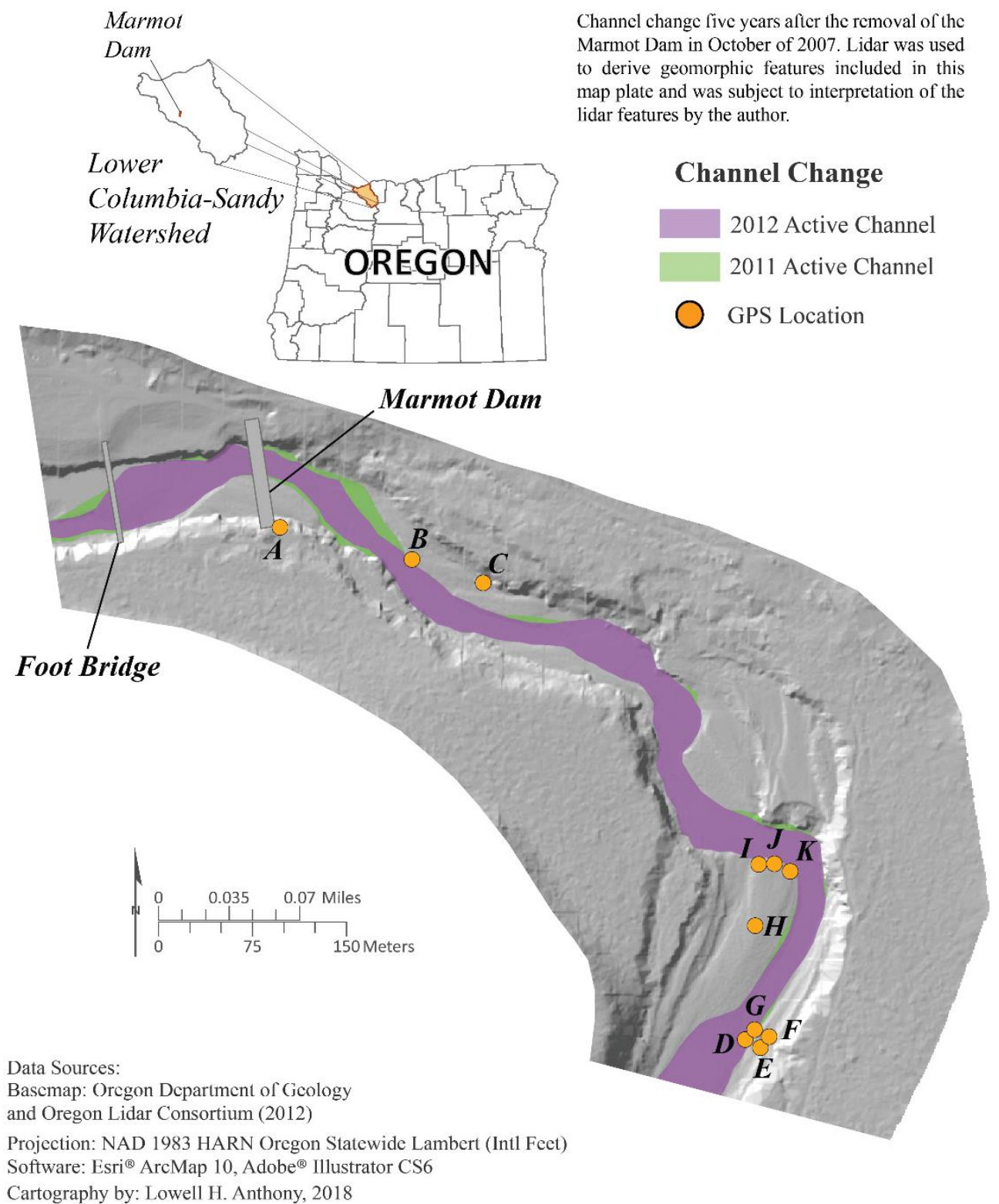

Software: Esri

Cartography by: Lowell H. Anthony, 2018

Figure 36. Active channel change between 2011 and 2012. Very little overall change in the channel thalweg, further degradation of the channel has occurred.

original 2006 lidar flight and the 2012 acquisition (Figure 37). There was an overall net elevation change of -1.9 meters across the entire impoundment from 2006 to 2012 and a 
total of $-28,502$ cubic meters of change in the overall morphological characteristics that we see in the 2012 bare earth hillshade DEM. The sand bar visible in the 2006 hillshade has been replaced by a large cobbled bar that is approximately 3 meters high at the head. Overall geomorphic change is a result of the underlying geology of the reach and overall competence of the Sandy River. High resolution map plates of the figures in this section are available at the end of this paper in.

The need for tools that allow researchers to efficiently, safely, and cost effectively monitor river morphology continues to grow. High resolution lidar provides fluvial geomorphologists the ability to monitor flow hydraulics in relation to channel morphology, flood prone and avulsion potential areas, aggradation and degradation of the channel margins and allows for more comprehensive analysis of the entire drainage system and the impacts to the overall watershed environment. When used in conjunction with existing CEMs the ability of lidar to monitor an array of processes at work simultaneously strengthens the already strong argument in its favor. I have shown that sequential lidar runs over a short temporal range provides a comprehensive tool for identifying and monitoring channel morphology.

Some things that I would do differently given what I found in this research would be to first work with the LAS data files and use the 3D visualization tools available in QT Modeler to better define the overall geomorphological response in the reach, and to more accurately classify the active channel bank margins. Another benefit to using the raw LAS data is that it also allows you to look at the individual point returns and evaluate the classification for each point, giving you even more control on the level of detail for the features you want to define and extract. 
Channel Comparison

Plate 9

\section{$2006-2012$}

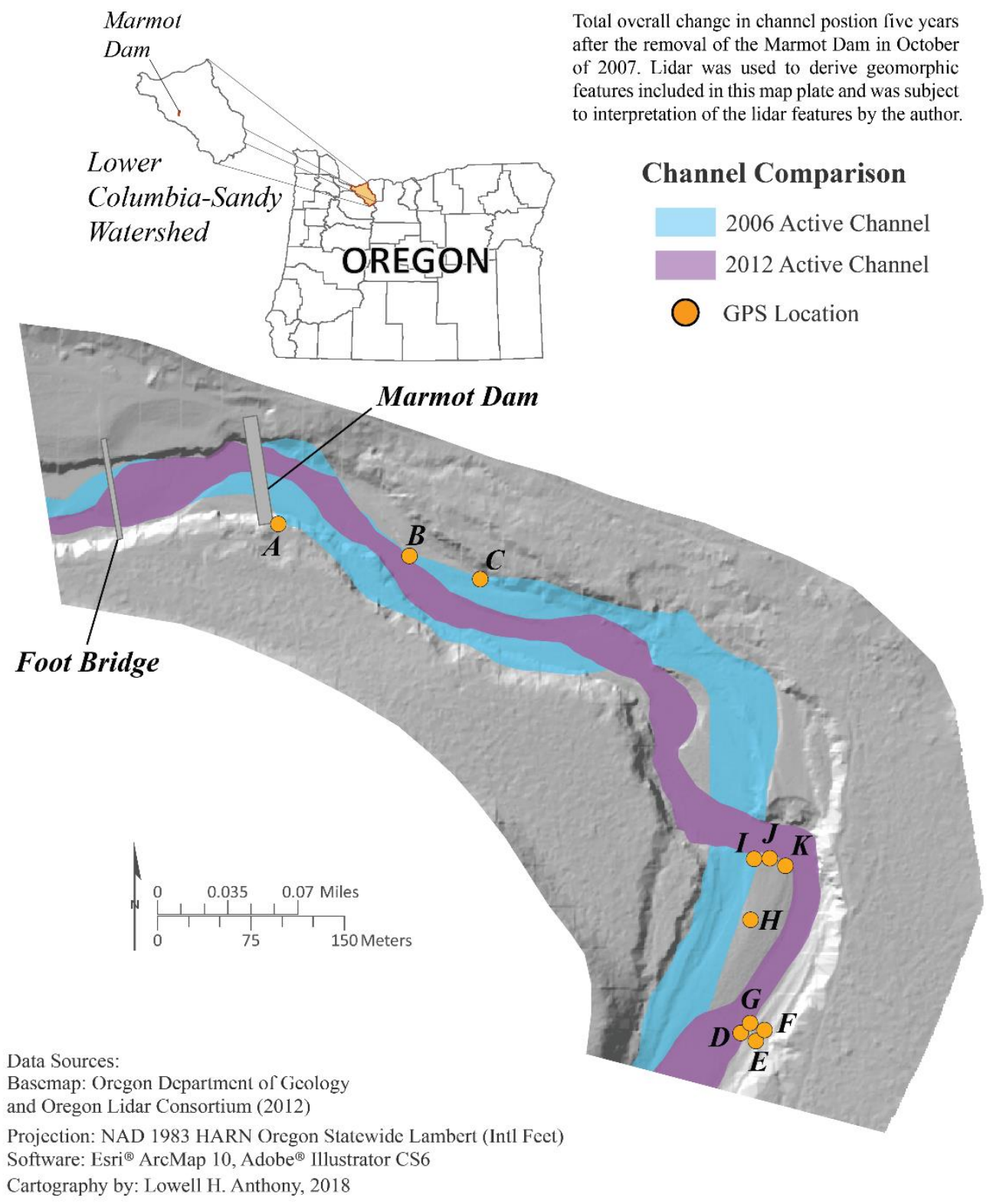

Figure 37. Overall active channel pattern changes between 2006 and 2012. Morphological differences in the overall configuration of the channel position and elevation is shown.

I would also explore more of the techniques for using the orthophoto color bands to derive depth features to extrapolate bathymetric feature in the 2006 - 2011 lidar runs so I 
could compare them to the 2012 bathymetric data that I did not look at in this study since there wasn't other data to compare it to. Adding the bathymetric component to the overall response of the Marmot Dam removal would have given more weight to my hypothesis that water surface elevations differences between lidar flights indicate changes in bedform that correlate to the continued downstream adjustment of the reach.

For future work on this concept and continued monitoring of the study area I would like to explore the use of unmanned aerial vehicles (UAV) and structure from motion (SFM) as an additional tool to provide more detailed analysis of geomorphic change in the area and as an additional tool to use with sequential lidar. Given the progression of the technology, the use of drone mounted lidar sensors aren't the far off and the overall technological advances in lidar continue to open avenues for more detailed studies of the overall bedform change using bathymetric lidar. I will continue to delve into the use of lidar for monitoring geohazards and hazard mitigation in our region and hope to influence decisions around the appropriate temporal schedule for sequential lidar acquisitions. The need for a defined schedule based on geomorphic and geologic principles is something that should be undertaken on a regional level with Oregon and the rest of the Pacific Northwest leading the way in the advancement of this valuable technology. 


\subsection{Conclusion}

The study furthers the case for sequential lidar on highly competent and marginally active river systems such as the Sandy River. Most rivers flowing out of the mountain ranges west of the Cascades have the potential for lateral migration, and lidar is an asset in the monitoring and planning that should be involved along the most vulnerable of areas. As climate change continues to adjust the storage capacity and intensity of winter storms in the Pacific Northwest, the need for proper mitigation and planning around the likelihood of channel migration occurring will become a reality.

Proper study of the potential for channel migration induced avulsions needs sequential lidar for monitoring. The practical uses of lidar are further enhanced by sequential acquisition flights from between three and five years depending on a reaches channel migration potential and vulnerability of local populations and infrastructure. Susceptibility should be measured for hazard potential, and sequential lidar is a necessary tool to accomplish this. Of all the findings in this study the one of most import is that of having sequential data to formulate best management practices and hazard mitigation/response efforts around natural hazards in areas prone to flood.

The impact of the seasonal variability for the Sandy River was shown in the quick overall transition of the reach to a state of equilibrium, more specifically the $30,000 \mathrm{cfs}$ flow that occurred on January $2^{\text {nd }}, 2009$ and the 39,000 cfs flow on January $14^{\text {th }}, 2011$ produced the most dramatic geomorphic change between the 2008 and 2011 lidar acquisitions. The river has continued to incise and migrate laterally to the east above the lahar intrusion and to west just beyond it, erosion of the bed materials (including cobbles, boulders and bedrock) are unlikely and sediment fines continue to scour off the boulder 
bar above the lahar intrusion and aggrade downstream at GPS points B and C, forming a coarse boulder and sand mixed beach at GPS point $\mathrm{C}$, and transitioning to a sand covered beach at GPS point B. The intrusive lahar dikes are highly resistant and will likely take many decades to erode, thus establishing equilibrium in the rivers current state.

The sequential DEMs permitted calculation of reach-scale volumetric erosion and aggradation rates, although it was necessary to set a threshold to account for noise in the lidar of \pm 2.5 feet and polygon area size $\leq 20 \mathrm{~m}^{2}$ to account for vegetation and low pulse return data near the margins and interpolation errors in digitizing. My study further shows the capability of lidar to measure rates of aggradation and degradation for an entire river system instead of reach specific extrapolations and that repeat lidar flights can more than adequately assess the changing nature of entire stream reaches more rapidly and more cost effectively than traditional field techniques. As such the more active a hazard in a given region the more often lidar should be acquired. Rivers in the western portion of the Pacific Northwest are highly competent and have greater avulsion potential then their counterparts on the eastern side of the Cascades and areas to the west of the Coast Range and coastal regions necessitate a more aggressive acquisition schedule. Having robust sequential lidar data in populated areas is an asset to hazard management and disaster recovery efforts after events. It also allows us to make informed decisions regarding infrastructure placement and urban planning in areas not as heavily developed. 
I have shown the effectiveness of sequential lidar as a tool to detect morphological changes, providing a safe, efficient and effective tool for fluvial geomorphologists and showing the necessity of both sequential lidar and up to date lidar. Some things to consider from this research:

- The utility of Lidar to do river management with repeat returns, having successive lidar acquisitions run on the watershed level will help us to gain insight into the correlation to precipitation events and geomorphological change in a given reach.

- Lidar can be used to assess the validity of channel evolution models. Sequential runs of lidar can be used to adjust the overall effectiveness of current CEM's and create new ones that consider reach specific geomorphology.

- Dam removal projects should incorporate initial lidar flights prior to removal and follow acquisitions based on known CEM's for the region and overall region-specific physiography.

- Sequential lidar should be used for hazard mitigation and geohazards analysis with an acquisition timeframe that is appropriate for the region's physiography, geology, geomorphology and the return interval of the hazard being monitored. 


\subsection{References}

Abbe, T., Reinhart, M. A., Higgins, S., French, D., and Wilson, J., (2015). Flood erosion hazard mitigation evaluation, Upper Sandy River, Clackamas County, Oregon, prepared for Clackamas County Emergency Management: Seattle, Wash., Natural Systems Design, 88 p.

American Rivers, (2018). Restoring damaged rivers Web. 8 December 2018. https://www.americanrivers.org/threats-solutions/restoring-damaged-rivers/.

Anthony, L. H., Williams, M. C., and Bauer, J. B., (2019) (expected). Natural Hazard risk report for the Lower Columbia-Sandy watershed, Oregon: Oregon Department of Geology and Mineral Industries Interpretive Map Series IMS-59, 57 p.

Babbitt, B. (2002). What goes up, may come down. BioScience. 52 (8): 656-658.

Biddulph, Matilda. (2015). Hyporheic zone: in situ sampling. In book: Geomorphological Techniques, Chapter: 3.11.1, Publisher: British Society for Geomorphology, Editors: Lucy Clarke, Jo Nield

Burns, W. J., Mickelson, K. A., and Madin, I. P., (2016). Landslide susceptibility overview map of Oregon: Oregon Department of Geology and Mineral Industries Open-File Report O-16-02, 48 p.

Burroughs, B. A., D. B. Hayes, K. D. Klomp, J. F. Hansen, and J. Mistak. (2009). Effects of Stronach Dam removal on fluvial geomorphology in the Pine River, Michigan, United States. Geomorphology. 110 (3-4): 96-107.

Cannatelli, K., \& Curran, J. (2012). Importance of hydrology on channel evolution following dam removal: case study and conceptual model. Journal of Hydraulic Engineering, (May). doi:10.1061/(ASCE)HY.1943-7900.0000526

Chang, H. H. (2008). Case study of fluvial modeling of river responses to dam removal. Journal of Hydraulic Engineering, 134(3), 295-303.

Charlton, R. (2008). Channel Form and Behavior. In Fundamentals of fluvial geomorphology. Pp.131-133. London: Routledge.

De Rose, R. C., and L. R. Basher. (2011). Measurement of river bank and cliff erosion from sequential LIDAR and historical aerial photography. Geomorphology. 126 (1-2): 132-147.

Doyle, M. W., E. H. Stanley, A. R. Selle, J. M. Stofleth, and J. M. Harbor. (2003a). Predicting the depth of erosion in reservoirs following dam removal using bank stability analysis. International journal of Sediment Research. 18: 115-121.

Doyle, M. W. (2003b). Channel adjustments following two dam removals in Wisconsin. Water Resources Research 39 (1):1-15.

Doyle, M. W., E. H. Stanley, C. H. Orr, A. R. Selle, S. A. Sethi, and J. M. Harbor. (2005). Stream ecosystem response to small dam removal: lessons from the heartland. Geomorphology 71 (1-2):227-244.

Doyle, M. W., E. H. Stanley, J. M. Harbor. (2002). Geomorphic analogies for assessing probable channel response to dam removal. JAWRA Journal of the American Water Resources Association, 38(6), 1567-1579.

Endreny, T. A., (2003). Fluvial Geomorphology Module, UCAR COMET Program and NOAA River Forecast Center, http://www.fgmorph.com, Syracuse, NY. 
English, J. T. (2008). Effectiveness of extracting water surface slopes from lidar data within the active channel: Sandy River, Oregon, USA (Master thesis). Retrieved from https://core.ac.uk/download/pdf/36684192.pdf

English, J. T., (2013). Change detection analysis using serial lidar data along a portion of the Upper Sandy River, Multnomah and Clackamas Counties, Oregon: Oregon Department of Geology and Mineral Industries Open-File Report O-13-01, 1 p.

Evans, J. S., A. T. Hudak, R. Faux, and A. M. S. Smith. (2009). Discrete return lidar in natural resources: recommendations for project planning, data processing, and deliverables. Remote Sensing. 1: 776-794.

Faux, R. N., J. M. Buffington, M. G. Whitley, S. H. Lanigan and B. B Roper. (2009). Chapter 6. - Use of airborne near-infrared lidar for determining channel cross-section characteristics and monitoring aquatic habitat in Pacific Northwest rivers: a preliminary analysis. $\quad$ Retrieved May 18, from http://www.fs.fed.us/rm/pubs_other/rmrs_2009_faux_r001.pdf

Higgs, S. and M. Bowman. (2002). The Ecology of Dam Removal. American Rivers. (p. $15)$.

Hilldale, R. C. and Raff, D. (2008), Assessing the ability of airborne lidar to map river bathymetry. Earth Surf. Process. Landforms, 33: 773-783.

Knighton, D. (1984). Fluvial forms and processes. London: E. Arnold

Leopold, L. B., T. Maddock Jr. (1953). The hydraulic geometry of stream channels and some physiographic implications. US Geological Survey Professional Paper. (252): 164.

Major, J. J., K. R. Spicer, A. Rhode, J. E. O’Connor, H. M. Bragg, D. Q. Tanner, C. W. Anderson, J. R. Wallick, and G. E. Grant. (2008). Initial fluvial response to the removal of Oregon's Marmot Dam. Eos, Transactions American Geophysical Union 89 (27):241.

Major J. J., O'Connor J.E., Keith M.K., Spicer K.R., Bragg H.M., Wallick J.R. et al., (2012). Geomorphic response of the Sandy River, Oregon, to removal of Marmot Dam. US Geological Survey Professional Paper. (1792): 1-76.

Neave, M., S. Rayburg, and A. Swan. (2009). River channel change following dam removal in an ephemeral stream. Australian Geographer. 40 (2): 235-246.

Pearson A. J., Snyder N.P., and Collins M.J. (2011). Rates and processes of channel response to dam removal with a sand-filled impoundment. Water Resources Research.47 (8).

Pierson, T. C., Scott, W.E., Vallance, J.W., and Pringle, P.T., (2009). Eruption-related lahars and sedimentation response downstream of Mount Hood: Field guide to volcaniclastic deposits along the Sandy River, Oregon, in O'Connor, J.E., Dorsey, R.J., and Madin, I.P., eds., Volcanoes to Vineyards: Geologic Field Trips through the Dynamic Landscape of the Pacific Northwest: Geological Society of America Field Guide 15, p. 221-236.

Sawyer, A. M., G. B. Pasternack, H. J. Moir, and A. A. Fulton. (2009). Riffle-pool maintenance and flow convergence routing observed on a large gravel-bed river. Geomorphology. 114 (3): 143-160.

Schumm, S. A. (1977). The fluvial system. New York: Wiley. 
Winter, T.C., J.W. Harvey, O.L. Franke, and W.M. Alley. (1998). Ground Water and Surface Water a Single Resource. Retrieved May 30 from https://pubs.usgs.gov/circ/circ1139/\#pdf 


\subsection{Appendix A: Differential Correction File}

Searching for base files...

File H:ISandyRiverDatalBaselUNAVCO, Boring, OR (p427) \p4272860.14o.Z downloaded. File H:ISandyRiverDatalBaselUNAVCO, Boring, OR (p427) \brdc2860.14n.Z downloaded. Successfully found or downloaded 2 of 2 files. Search complete.

--------Base Data Details:

Using reference position from base provider: Name: UNAVCO, Boring, OR (p427)

Position:

$122^{\circ} 20^{\prime} 26.26803 " \mathrm{~W}, 149.22 \mathrm{~m}$

Source: H:ISandyRiverDatalBaselUNAVCO, Boring, OR (p427) p4272860.14o.Z

Local time: 10/12/2014 4:59:45 PM to 10/13/2014 4:59:30 PM

Position: $45^{\circ} 25^{\prime} 48.64440 " \mathrm{~N}$, $122^{\circ} 20^{\prime} 26.27160^{\prime \prime} \mathrm{W}, 149.32 \mathrm{~m}, 0.01 \mathrm{~m}$ Antenna height

Distance from base provider: $0.08 \mathrm{~m}$

--------Coverage Details:

Rover file: R101313A.SSF

Local time: 10/13/2014 1:57:49 PM to

10/13/2014 2:23:15 PM

$100 \%$ total coverage

$100 \%$ coverage by $\mathrm{p} 4272860.14 \mathrm{o} . \mathrm{Z}$

Rover file: R101311A.SSF

Local time: 10/13/2014 11:41:50 AM to 10/13/2014 11:45:35 AM

$100 \%$ total coverage

$100 \%$ coverage by $\mathrm{p} 4272860.14 \mathrm{o} . \mathrm{Z}$

Rover file: R101312A.SSF

Local time: 10/13/2014 12:14:54 PM to

10/13/2014 12:17:55 PM

$100 \%$ total coverage

$100 \%$ coverage by $\mathrm{p} 4272860.14 \mathrm{o} . \mathrm{Z}$

Rover file: R101312B.SSF

Local time: 10/13/2014 12:28:17 PM to 10/13/2014 12:31:00 PM

$100 \%$ total coverage

$100 \%$ coverage by $\mathrm{p} 4272860.14 \mathrm{o} . \mathrm{Z}$

Rover file: R101314A.SSF

Local time: 10/13/2014 2:37:05 PM to 10/13/2014 2:41:10 PM
$100 \%$ total coverage

$100 \%$ coverage by $\mathrm{p} 4272860.14 \mathrm{o} . \mathrm{Z}$

Rover file: R101314B.SSF

Local time: 10/13/2014 2:44:27 PM to

10/13/2014 2:49:40 PM

$100 \%$ total coverage

$100 \%$ coverage by $\mathrm{p} 4272860.14 \mathrm{o} . \mathrm{Z}$

Differentially correcting...

Differential correction settings:

Use smart automatic filtering: On

Re-correct real-time positions: On

Output positions: Corrected only

Processing rover file, R101313A.SSF ...

...to output file,

H:ISandyRiverDatalR101313A.cor

Carrier processing...

No carrier processing performed as file has no carrier data

Corrected 0 positions

Code processing...

processing

Selected 160 positions for post-

Corrected 146 positions

Failed to correct 14 positions

Filtered out 14 uncorrected positions output)

(only "Corrected" positions selected for

Processing rover file, R101311A.SSF ...

...to output file,

H:ISandyRiverData\R101311A.cor

Carrier processing...

No carrier processing performed as file has no carrier data

Corrected 0 positions

Code processing...

Selected 30 positions for post-processing Corrected 30 positions

Processing rover file, R101312A.SSF ...

...to output file,

H:\SandyRiverData\R101312A.cor

Carrier processing...

No carrier processing performed as file has no carrier data 
Corrected 0 positions

Code processing...

Selected 32 positions for post-processing

Corrected 32 positions

Processing rover file, R101312B.SSF ...

...to output file,

H: SSandyRiverData\R101312B.cor

Carrier processing...

No carrier processing performed as file has no carrier data

Corrected 0 positions

Code processing...

Selected 30 positions for post-processing Corrected 29 positions

Failed to correct 1 positions

Filtered out 1 uncorrected positions output)

(only "Corrected" positions selected for

Processing rover file, R101314A.SSF ...

...to output file,

H:ISandyRiverData\R101314A.cor

Carrier processing...

No carrier processing performed as file has no carrier data

Corrected 0 positions

Code processing...

Selected 30 positions for post-processing

Corrected 30 positions

Processing rover file, R101314B.SSF ...

Differential correction complete. ...to

output

file,

H: SSandyRiverDatalR101314B.cor

Carrier processing...

No carrier processing performed as file has no carrier data

Corrected 0 positions

Code processing...

Selected 44 positions for post-processing

Corrected 43 positions

Failed to correct 1 positions

Filtered out 1 uncorrected positions output) (only "Corrected" positions selected for

Differential Correction Summary:

6 files processed. In these files:

$310(95.09 \%)$ of 326 selected positions

were code corrected by post-processing

$0(0.00 \%)$ of 0 selected positions were carrier corrected by post-processing

Estimated accuracies for 310 corrected positions are as follows:

Range Percentage

$\begin{array}{cc}0-5 \mathrm{~cm} & - \\ 5-15 \mathrm{~cm} & - \\ 15-30 \mathrm{~cm} & - \\ 30-50 \mathrm{~cm} & 63.55 \% \\ 0.5-1 \mathrm{~m} & 36.45 \% \\ 1-2 \mathrm{~m} & - \\ 2-5 \mathrm{~m} & - \\ >5 \mathrm{~m} & -\end{array}$


10.0 Appendix B: Transect Tables

10.1 Transect A - Spillway - All Returns:

\begin{tabular}{|c|c|c|c|c|c|c|c|}
\hline Distance & Z & DEM & Year & Distance & Z & DEM & Year \\
\hline 0.000 & 227.485 & Surface & 2006 & 0.000 & 227.428 & Surface & 2011 \\
\hline 2.983 & 227.414 & Surface & 2006 & 2.983 & 227.343 & Surface & 2011 \\
\hline 5.965 & 227.276 & Surface & 2006 & 5.965 & 227.265 & Surface & 2011 \\
\hline 8.948 & 227.183 & Surface & 2006 & 8.948 & 227.169 & Surface & 2011 \\
\hline 11.930 & 227.123 & Surface & 2006 & 11.930 & 227.047 & Surface & 2011 \\
\hline 14.913 & 227.058 & Surface & 2006 & 14.913 & 226.914 & Surface & 2011 \\
\hline 17.895 & 226.985 & Surface & 2006 & 17.895 & 226.777 & Surface & 2011 \\
\hline 20.878 & 226.917 & Surface & 2006 & 20.878 & 226.583 & Surface & 2011 \\
\hline 23.860 & 226.822 & Surface & 2006 & 23.860 & 226.367 & Surface & 2011 \\
\hline 26.843 & 226.355 & Surface & 2006 & 26.843 & 225.692 & Surface & 2011 \\
\hline 29.826 & 225.426 & Surface & 2006 & 29.826 & 224.476 & Surface & 2011 \\
\hline 32.808 & 224.213 & Surface & 2006 & 32.808 & 223.136 & Surface & 2011 \\
\hline 35.791 & 223.121 & Surface & 2006 & 35.791 & 221.798 & Surface & 2011 \\
\hline 38.774 & 220.981 & Surface & 2006 & 38.774 & 220.459 & Surface & 2011 \\
\hline 41.756 & 218.937 & Surface & 2006 & 41.756 & 219.109 & Surface & 2011 \\
\hline 44.739 & 216.808 & Surface & 2006 & 44.739 & 217.728 & Surface & 2011 \\
\hline 47.721 & 214.775 & Surface & 2006 & 47.721 & 216.311 & Surface & 2011 \\
\hline 50.704 & 214.174 & Surface & 2006 & 50.704 & 214.854 & Surface & 2011 \\
\hline 53.686 & 214.417 & Surface & 2006 & 53.686 & 213.889 & Surface & 2011 \\
\hline 56.669 & 214.194 & Surface & 2006 & 56.669 & 213.636 & Surface & 2011 \\
\hline 59.651 & 213.784 & Surface & 2006 & 59.651 & 213.629 & Surface & 2011 \\
\hline 62.634 & 213.140 & Surface & 2006 & 62.634 & 213.643 & Surface & 2011 \\
\hline 65.617 & 212.720 & Surface & 2006 & 65.617 & 213.644 & Surface & 2011 \\
\hline 68.599 & 212.437 & Surface & 2006 & 68.599 & 213.610 & Surface & 2011 \\
\hline 71.582 & 212.280 & Surface & 2006 & 71.582 & 213.580 & Surface & 2011 \\
\hline 74.564 & 212.151 & Surface & 2006 & 74.564 & 213.597 & Surface & 2011 \\
\hline 77.547 & 212.014 & Surface & 2006 & 77.547 & 213.608 & Surface & 2011 \\
\hline 80.529 & 211.906 & Surface & 2006 & 80.529 & 213.595 & Surface & 2011 \\
\hline 83.512 & 211.820 & Surface & 2006 & 83.512 & 213.567 & Surface & 2011 \\
\hline 86.494 & 211.748 & Surface & 2006 & 86.494 & 213.534 & Surface & 2011 \\
\hline 89.477 & 211.660 & Surface & 2006 & 89.477 & 213.512 & Surface & 2011 \\
\hline 92.460 & 211.629 & Surface & 2006 & 92.460 & 213.520 & Surface & 2011 \\
\hline 95.442 & 211.609 & Surface & 2006 & 95.442 & 213.541 & Surface & 2011 \\
\hline 98.425 & 211.619 & Surface & 2006 & 98.425 & 213.536 & Surface & 2011 \\
\hline 101.407 & 211.630 & Surface & 2006 & 101.407 & 213.537 & Surface & 2011 \\
\hline 104.390 & 211.615 & Surface & 2006 & 104.390 & 213.549 & Surface & 2011 \\
\hline 107.373 & 211.609 & Surface & 2006 & 107.373 & 213.561 & Surface & 2011 \\
\hline 110.355 & 211.627 & Surface & 2006 & 110.355 & 213.576 & Surface & 2011 \\
\hline 113.338 & 211.622 & Surface & 2006 & 113.338 & 213.561 & Surface & 2011 \\
\hline 116.320 & 211.596 & Surface & 2006 & 116.320 & 213.546 & Surface & 2011 \\
\hline 119.303 & 211.595 & Surface & 2006 & 119.303 & 213.550 & Surface & 2011 \\
\hline 122.285 & 211.608 & Surface & 2006 & 122.285 & 213.567 & Surface & 2011 \\
\hline 125.268 & 211.619 & Surface & 2006 & 125.268 & 213.571 & Surface & 2011 \\
\hline 128.250 & 211.613 & Surface & 2006 & 128.250 & 213.537 & Surface & 2011 \\
\hline
\end{tabular}




\begin{tabular}{|c|c|c|c|c|c|c|}
\hline 131.233 & 211.606 & Surface & 2006 & 131.233 & 213.514 & Surface \\
\hline 134.216 & 211.611 & Surface & 2006 & 134.216 & 213.546 & Surface \\
\hline 137.198 & 211.626 & Surface & 2006 & 137.198 & 213.636 & Surface \\
\hline 140.181 & 211.634 & Surface & 2006 & 140.181 & 213.698 & Surface \\
\hline 143.163 & 211.622 & Surface & 2006 & 143.163 & 213.755 & Surface \\
\hline 146.146 & 211.613 & Surface & 2006 & 146.146 & 213.818 & Surface \\
\hline 149.128 & 211.609 & Surface & 2006 & 149.128 & 213.862 & Surface \\
\hline 152.111 & 211.598 & Surface & 2006 & 152.111 & 213.906 & Surface \\
\hline 155.094 & 211.604 & Surface & 2006 & 155.094 & 213.928 & Surface \\
\hline 158.076 & 211.622 & Surface & 2006 & 158.076 & 213.940 & Surface \\
\hline 161.059 & 211.806 & Surface & 2006 & 161.059 & 213.931 & Surface \\
\hline 164.041 & 212.325 & Surface & 2006 & 164.041 & 213.946 & Surface \\
\hline 167.024 & 212.863 & Surface & 2006 & 167.024 & 213.998 & Surface \\
\hline 170.006 & 213.073 & Surface & 2006 & 170.006 & 214.045 & Surface \\
\hline 172.989 & 212.870 & Surface & 2006 & 172.989 & 214.058 & Surface \\
\hline 175.971 & 212.686 & Surface & 2006 & 175.971 & 214.081 & Surface \\
\hline 178.954 & 212.692 & Surface & 2006 & 178.954 & 214.120 & Surface \\
\hline 181.937 & 212.684 & Surface & 2006 & 181.937 & 214.133 & Surface \\
\hline 184.919 & 212.699 & Surface & 2006 & 184.919 & 214.125 & Surface \\
\hline 187.902 & 212.754 & Surface & 2006 & 187.902 & 214.130 & Surface \\
\hline 190.884 & 212.811 & Surface & 2006 & 190.884 & 214.155 & Surface \\
\hline 193.867 & 212.838 & Surface & 2006 & 193.867 & 214.143 & Surface \\
\hline 196.850 & 212.880 & Surface & 2006 & 196.850 & 214.124 & Surface \\
\hline 199.832 & 212.889 & Surface & 2006 & 199.832 & 214.115 & Surface \\
\hline 202.815 & 212.894 & Surface & 2006 & 202.815 & 214.129 & Surface \\
\hline 205.797 & 212.925 & Surface & 2006 & 205.797 & 214.156 & Surface \\
\hline 208.780 & 212.956 & Surface & 2006 & 208.780 & 214.166 & Surface \\
\hline 211.762 & 213.131 & Surface & 2006 & 211.762 & 214.155 & Surface \\
\hline 214.745 & 213.552 & Surface & 2006 & 214.745 & 214.137 & Surface \\
\hline 217.727 & 214.017 & Surface & 2006 & 217.727 & 214.130 & Surface \\
\hline 220.710 & 214.379 & Surface & 2006 & 220.710 & 214.137 & Surface \\
\hline 223.693 & 214.746 & Surface & 2006 & 223.693 & 214.138 & Surface \\
\hline 226.675 & 215.063 & Surface & 2006 & 226.675 & 214.153 & Surface \\
\hline 229.658 & 215.202 & Surface & 2006 & 229.658 & 214.170 & Surface \\
\hline 232.640 & 215.240 & Surface & 2006 & 232.640 & 214.171 & Surface \\
\hline 235.623 & 215.339 & Surface & 2006 & 235.623 & 214.204 & Surface \\
\hline 238.605 & 215.453 & Surface & 2006 & 238.605 & 214.318 & Surface \\
\hline 241.588 & 215.583 & Surface & 2006 & 241.588 & 214.622 & Surface \\
\hline 244.570 & 215.290 & Surface & 2006 & 244.570 & 215.422 & Surface \\
\hline 247.553 & 215.743 & Surface & 2006 & 247.553 & 216.765 & Surface \\
\hline 250.536 & 217.553 & Surface & 2006 & 250.536 & 218.441 & Surface \\
\hline 253.518 & 219.411 & Surface & 2006 & 253.518 & 220.152 & Surface \\
\hline 256.501 & 220.719 & Surface & 2006 & 256.501 & 221.656 & Surface \\
\hline 259.484 & 221.949 & Surface & 2006 & 259.484 & 222.511 & Surface \\
\hline 262.466 & 222.542 & Surface & 2006 & 262.466 & 222.835 & Surface \\
\hline 265.449 & 222.848 & Surface & 2006 & 265.449 & 223.025 & Surface \\
\hline 268.431 & 222.923 & Surface & 2006 & 268.431 & 223.087 & Surface \\
\hline 271.414 & 222.752 & Surface & 2006 & 271.414 & 223.146 & Surface \\
\hline 274.396 & 222.555 & Surface & 2006 & 274.396 & 223.285 & Surface \\
\hline
\end{tabular}




\begin{tabular}{|c|c|c|c|c|c|c|c|}
\hline 277.379 & 222.488 & Surface & 2006 & 277.379 & 223.448 & Surface & 2011 \\
\hline 280.361 & 222.469 & Surface & 2006 & 280.361 & 223.622 & Surface & 2011 \\
\hline 283.344 & 222.486 & Surface & 2006 & 283.344 & 223.808 & Surface & 2011 \\
\hline 286.327 & 222.480 & Surface & 2006 & 286.327 & 223.999 & Surface & 2011 \\
\hline 289.309 & 222.491 & Surface & 2006 & 289.309 & 224.180 & Surface & 2011 \\
\hline 292.292 & 222.505 & Surface & 2006 & 292.292 & 224.343 & Surface & 2011 \\
\hline 295.274 & 222.836 & Surface & 2006 & 295.274 & 224.537 & Surface & 2011 \\
\hline 298.257 & 223.453 & Surface & 2006 & 298.257 & 224.783 & Surface & 2011 \\
\hline 301.239 & 224.033 & Surface & 2006 & 301.239 & 224.955 & Surface & 2011 \\
\hline 304.222 & 224.601 & Surface & 2006 & 304.222 & 225.086 & Surface & 2011 \\
\hline 307.204 & 225.268 & Surface & 2006 & 307.204 & 225.280 & Surface & 2011 \\
\hline 310.187 & 225.860 & Surface & 2006 & 310.187 & 225.479 & Surface & 2011 \\
\hline 313.170 & 226.198 & Surface & 2006 & 313.170 & 225.717 & Surface & 2011 \\
\hline 316.152 & 226.375 & Surface & 2006 & 316.152 & 225.955 & Surface & 2011 \\
\hline 319.135 & 226.511 & Surface & 2006 & 319.135 & 226.126 & Surface & 2011 \\
\hline 322.118 & 226.643 & Surface & 2006 & 322.118 & 226.288 & Surface & 2011 \\
\hline 325.100 & 226.692 & Surface & 2006 & 325.100 & 226.379 & Surface & 2011 \\
\hline 328.083 & 226.681 & Surface & 2006 & 328.083 & 226.438 & Surface & 2011 \\
\hline 331.065 & 226.690 & Surface & 2006 & 331.065 & 226.490 & Surface & 2011 \\
\hline 334.048 & 226.666 & Surface & 2006 & 334.048 & 226.583 & Surface & 2011 \\
\hline 337.030 & 226.627 & Surface & 2006 & 337.030 & 226.626 & Surface & 2011 \\
\hline 340.013 & 226.695 & Surface & 2006 & 340.013 & 226.686 & Surface & 2011 \\
\hline 342.995 & 226.788 & Surface & 2006 & 342.995 & 226.797 & Surface & 2011 \\
\hline 345.978 & 226.917 & Surface & 2006 & 345.978 & 226.899 & Surface & 2011 \\
\hline 348.961 & 227.005 & Surface & 2006 & 348.961 & 226.956 & Surface & 2011 \\
\hline 0.000 & 227.482 & Surface & 2008 & 0.000 & 227.706 & Surface & 2012 \\
\hline 3.261 & 227.360 & Surface & 2008 & 3.261 & 227.616 & Surface & 2012 \\
\hline 6.523 & 227.323 & Surface & 2008 & 6.523 & 227.464 & Surface & 2012 \\
\hline 9.784 & 227.314 & Surface & 2008 & 9.784 & 227.328 & Surface & 2012 \\
\hline 13.045 & 227.220 & Surface & 2008 & 13.045 & 227.163 & Surface & 2012 \\
\hline 16.307 & 227.047 & Surface & 2008 & 16.307 & 226.987 & Surface & 2012 \\
\hline 19.568 & 226.902 & Surface & 2008 & 19.568 & 226.962 & Surface & 2012 \\
\hline 22.829 & 226.867 & Surface & 2008 & 22.829 & 226.696 & Surface & 2012 \\
\hline 26.090 & 226.420 & Surface & 2008 & 26.090 & 225.808 & Surface & 2012 \\
\hline 29.352 & 225.158 & Surface & 2008 & 29.352 & 224.585 & Surface & 2012 \\
\hline 32.613 & 223.704 & Surface & 2008 & 32.613 & 223.201 & Surface & 2012 \\
\hline 35.875 & 222.259 & Surface & 2008 & 35.875 & 221.781 & Surface & 2012 \\
\hline 39.136 & 220.825 & Surface & 2008 & 39.136 & 220.354 & Surface & 2012 \\
\hline 42.397 & 219.388 & Surface & 2008 & 42.397 & 218.926 & Surface & 2012 \\
\hline 45.658 & 217.947 & Surface & 2008 & 45.658 & 217.499 & Surface & 2012 \\
\hline 48.920 & 216.514 & Surface & 2008 & 48.920 & 216.071 & Surface & 2012 \\
\hline 52.181 & 215.121 & Surface & 2008 & 52.181 & 214.643 & Surface & 2012 \\
\hline 55.442 & 214.137 & Surface & 2008 & 55.442 & 213.506 & Surface & 2012 \\
\hline 58.703 & 213.999 & Surface & 2008 & 58.703 & 213.215 & Surface & 2012 \\
\hline 61.965 & 213.961 & Surface & 2008 & 61.965 & 213.021 & Surface & 2012 \\
\hline 65.226 & 213.923 & Surface & 2008 & 65.226 & 212.883 & Surface & 2012 \\
\hline 68.488 & 213.874 & Surface & 2008 & 68.488 & 212.760 & Surface & 2012 \\
\hline 71.749 & 213.812 & Surface & 2008 & 71.749 & 212.719 & Surface & 2012 \\
\hline 75.010 & 213.825 & Surface & 2008 & 75.010 & 212.717 & Surface & 2012 \\
\hline
\end{tabular}




\begin{tabular}{|c|c|c|c|c|c|c|c|}
\hline 78.271 & 213.847 & Surface & 2008 & 78.271 & 212.737 & Surface & 2012 \\
\hline 81.533 & 213.860 & Surface & 2008 & 81.533 & 212.746 & Surface & 2012 \\
\hline 84.794 & 213.868 & Surface & 2008 & 84.794 & 212.750 & Surface & 2012 \\
\hline 88.056 & 213.875 & Surface & 2008 & 88.056 & 212.750 & Surface & 2012 \\
\hline 91.317 & 213.791 & Surface & 2008 & 91.317 & 212.739 & Surface & 2012 \\
\hline 94.578 & 213.800 & Surface & 2008 & 94.578 & 212.727 & Surface & 2012 \\
\hline 97.839 & 213.787 & Surface & 2008 & 97.839 & 212.739 & Surface & 2012 \\
\hline 101.101 & 213.799 & Surface & 2008 & 101.101 & 212.750 & Surface & 2012 \\
\hline 104.362 & 213.816 & Surface & 2008 & 104.362 & 212.752 & Surface & 2012 \\
\hline 107.623 & 213.838 & Surface & 2008 & 107.623 & 212.785 & Surface & 2012 \\
\hline 110.885 & 213.820 & Surface & 2008 & 110.885 & 212.754 & Surface & 2012 \\
\hline 114.146 & 213.784 & Surface & 2008 & 114.146 & 212.767 & Surface & 2012 \\
\hline 117.407 & 213.789 & Surface & 2008 & 117.407 & 212.782 & Surface & 2012 \\
\hline 120.669 & 213.807 & Surface & 2008 & 120.669 & 212.781 & Surface & 2012 \\
\hline 123.930 & 213.847 & Surface & 2008 & 123.930 & 213.035 & Surface & 2012 \\
\hline 127.191 & 213.901 & Surface & 2008 & 127.191 & 213.376 & Surface & 2012 \\
\hline 130.453 & 214.216 & Surface & 2008 & 130.453 & 213.590 & Surface & 2012 \\
\hline 133.714 & 214.666 & Surface & 2008 & 133.714 & 213.719 & Surface & 2012 \\
\hline 136.975 & 214.878 & Surface & 2008 & 136.975 & 213.795 & Surface & 2012 \\
\hline 140.236 & 215.017 & Surface & 2008 & 140.236 & 213.871 & Surface & 2012 \\
\hline 143.498 & 215.146 & Surface & 2008 & 143.498 & 213.926 & Surface & 2012 \\
\hline 146.759 & 215.174 & Surface & 2008 & 146.759 & 213.952 & Surface & 2012 \\
\hline 150.020 & 215.192 & Surface & 2008 & 150.020 & 213.988 & Surface & 2012 \\
\hline 153.282 & 215.225 & Surface & 2008 & 153.282 & 214.024 & Surface & 2012 \\
\hline 156.543 & 215.227 & Surface & 2008 & 156.543 & 214.037 & Surface & 2012 \\
\hline 159.804 & 215.219 & Surface & 2008 & 159.804 & 214.051 & Surface & 2012 \\
\hline 163.066 & 215.245 & Surface & 2008 & 163.066 & 214.059 & Surface & 2012 \\
\hline 166.327 & 215.257 & Surface & 2008 & 166.327 & 214.050 & Surface & 2012 \\
\hline 169.588 & 215.229 & Surface & 2008 & 169.588 & 214.047 & Surface & 2012 \\
\hline 172.849 & 215.204 & Surface & 2008 & 172.849 & 214.057 & Surface & 2012 \\
\hline 176.111 & 215.219 & Surface & 2008 & 176.111 & 214.065 & Surface & 2012 \\
\hline 179.372 & 215.254 & Surface & 2008 & 179.372 & 214.079 & Surface & 2012 \\
\hline 182.634 & 215.261 & Surface & 2008 & 182.634 & 214.087 & Surface & 2012 \\
\hline 185.895 & 215.260 & Surface & 2008 & 185.895 & 214.090 & Surface & 2012 \\
\hline 189.156 & 215.247 & Surface & 2008 & 189.156 & 214.082 & Surface & 2012 \\
\hline 192.417 & 215.226 & Surface & 2008 & 192.417 & 214.079 & Surface & 2012 \\
\hline 195.679 & 215.193 & Surface & 2008 & 195.679 & 214.087 & Surface & 2012 \\
\hline 198.940 & 215.191 & Surface & 2008 & 198.940 & 214.083 & Surface & 2012 \\
\hline 202.202 & 215.223 & Surface & 2008 & 202.202 & 214.087 & Surface & 2012 \\
\hline 205.463 & 215.205 & Surface & 2008 & 205.463 & 214.080 & Surface & 2012 \\
\hline 208.724 & 215.144 & Surface & 2008 & 208.724 & 214.094 & Surface & 2012 \\
\hline 211.985 & 215.105 & Surface & 2008 & 211.985 & 214.087 & Surface & 2012 \\
\hline 215.247 & 215.097 & Surface & 2008 & 215.247 & 214.081 & Surface & 2012 \\
\hline 218.508 & 215.062 & Surface & 2008 & 218.508 & 214.071 & Surface & 2012 \\
\hline 221.769 & 215.021 & Surface & 2008 & 221.769 & 214.076 & Surface & 2012 \\
\hline 225.031 & 214.962 & Surface & 2008 & 225.031 & 214.083 & Surface & 2012 \\
\hline 228.292 & 214.908 & Surface & 2008 & 228.292 & 214.089 & Surface & 2012 \\
\hline 231.553 & 214.872 & Surface & 2008 & 231.553 & 214.095 & Surface & 2012 \\
\hline 234.815 & 214.861 & Surface & 2008 & 234.815 & 214.110 & Surface & 2012 \\
\hline
\end{tabular}




\begin{tabular}{|c|c|c|c|c|c|c|}
\hline 238.076 & 214.863 & Surface & 2008 & 238.076 & 214.139 & Surface \\
\hline 241.337 & 215.027 & Surface & 2008 & 241.337 & 214.144 & Surface \\
\hline 244.598 & 215.298 & Surface & 2008 & 244.598 & 214.688 & Surface \\
\hline 247.860 & 215.936 & Surface & 2008 & 247.860 & 217.016 & Surface \\
\hline 251.121 & 217.077 & Surface & 2008 & 251.121 & 219.250 & Surface \\
\hline 254.382 & 218.916 & Surface & 2008 & 254.382 & 220.454 & Surface \\
\hline 257.644 & 220.745 & Surface & 2008 & 257.644 & 221.562 & Surface \\
\hline 260.905 & 222.042 & Surface & 2008 & 260.905 & 222.432 & Surface \\
\hline 264.166 & 222.630 & Surface & 2008 & 264.166 & 222.834 & Surface \\
\hline 267.428 & 222.948 & Surface & 2008 & 267.428 & 223.078 & Surface \\
\hline 270.689 & 223.097 & Surface & 2008 & 270.689 & 223.144 & Surface \\
\hline 273.950 & 223.233 & Surface & 2008 & 273.950 & 223.254 & Surface \\
\hline 277.212 & 223.398 & Surface & 2008 & 277.212 & 223.417 & Surface \\
\hline 280.473 & 223.546 & Surface & 2008 & 280.473 & 223.596 & Surface \\
\hline 283.734 & 223.732 & Surface & 2008 & 283.734 & 223.814 & Surface \\
\hline 286.995 & 223.926 & Surface & 2008 & 286.995 & 224.066 & Surface \\
\hline 290.257 & 224.146 & Surface & 2008 & 290.257 & 224.256 & Surface \\
\hline 293.518 & 224.326 & Surface & 2008 & 293.518 & 224.450 & Surface \\
\hline 296.780 & 224.546 & Surface & 2008 & 296.780 & 224.662 & Surface \\
\hline 300.041 & 224.817 & Surface & 2008 & 300.041 & 224.888 & Surface \\
\hline 303.302 & 225.006 & Surface & 2008 & 303.302 & 225.077 & Surface \\
\hline 306.563 & 225.182 & Surface & 2008 & 306.563 & 225.233 & Surface \\
\hline 309.825 & 225.373 & Surface & 2008 & 309.825 & 225.443 & Surface \\
\hline 313.086 & 225.552 & Surface & 2008 & 313.086 & 225.665 & Surface \\
\hline 316.348 & 225.774 & Surface & 2008 & 316.348 & 225.885 & Surface \\
\hline 319.609 & 226.052 & Surface & 2008 & 319.609 & 226.135 & Surface \\
\hline 322.870 & 226.257 & Surface & 2008 & 322.870 & 226.338 & Surface \\
\hline 326.131 & 226.405 & Surface & 2008 & 326.131 & 226.474 & Surface \\
\hline 329.393 & 226.581 & Surface & 2008 & 329.393 & 226.578 & Surface \\
\hline 332.654 & 226.750 & Surface & 2008 & 332.654 & 226.697 & Surface \\
\hline 335.915 & 226.790 & Surface & 2008 & 335.915 & 226.746 & Surface \\
\hline 339.177 & 226.747 & Surface & 2008 & 339.177 & 226.789 & Surface \\
\hline 342.438 & 226.762 & Surface & 2008 & 342.438 & 226.840 & Surface \\
\hline 345.699 & 226.857 & Surface & 2008 & 345.699 & 226.920 & Surface \\
\hline 348.961 & 226.951 & Surface & 2008 & 348.961 & 227.008 & Surface \\
\hline
\end{tabular}


10.2 Transect B - Dam Head - All Returns:

\begin{tabular}{|c|c|c|c|c|c|c|c|}
\hline Distance & $\mathrm{Z}$ & DEM & Year & Distance & $\mathrm{Z}$ & DEM & Year \\
\hline 0.000 & 226.088 & Surface & 2006 & 0.000 & 226.090 & Surface & 2011 \\
\hline 2.998 & 226.066 & Surface & 2006 & 2.998 & 226.056 & Surface & 2011 \\
\hline 5.997 & 226.041 & Surface & 2006 & 5.997 & 226.027 & Surface & 2011 \\
\hline 8.996 & 226.051 & Surface & 2006 & 8.996 & 226.003 & Surface & 2011 \\
\hline 11.994 & 226.055 & Surface & 2006 & 11.994 & 225.987 & Surface & 2011 \\
\hline 14.993 & 226.034 & Surface & 2006 & 14.993 & 225.976 & Surface & 2011 \\
\hline 17.992 & 226.050 & Surface & 2006 & 17.992 & 225.991 & Surface & 2011 \\
\hline 20.990 & 226.077 & Surface & 2006 & 20.990 & 226.046 & Surface & 2011 \\
\hline 23.989 & 226.110 & Surface & 2006 & 23.989 & 226.097 & Surface & 2011 \\
\hline 26.987 & 226.134 & Surface & 2006 & 26.987 & 226.142 & Surface & 2011 \\
\hline 29.986 & 226.161 & Surface & 2006 & 29.986 & 226.158 & Surface & 2011 \\
\hline 32.985 & 226.187 & Surface & 2006 & 32.985 & 226.133 & Surface & 2011 \\
\hline 35.983 & 226.190 & Surface & 2006 & 35.983 & 226.071 & Surface & 2011 \\
\hline 38.982 & 226.080 & Surface & 2006 & 38.982 & 225.959 & Surface & 2011 \\
\hline 41.981 & 225.888 & Surface & 2006 & 41.981 & 225.786 & Surface & 2011 \\
\hline 44.979 & 225.682 & Surface & 2006 & 44.979 & 225.545 & Surface & 2011 \\
\hline 47.978 & 225.475 & Surface & 2006 & 47.978 & 225.294 & Surface & 2011 \\
\hline 50.976 & 225.359 & Surface & 2006 & 50.976 & 225.234 & Surface & 2011 \\
\hline 53.975 & 225.316 & Surface & 2006 & 53.975 & 225.230 & Surface & 2011 \\
\hline 56.973 & 225.297 & Surface & 2006 & 56.973 & 225.245 & Surface & 2011 \\
\hline 59.972 & 225.264 & Surface & 2006 & 59.972 & 225.262 & Surface & 2011 \\
\hline 62.971 & 225.004 & Surface & 2006 & 62.971 & 225.084 & Surface & 2011 \\
\hline 65.969 & 224.663 & Surface & 2006 & 65.969 & 224.482 & Surface & 2011 \\
\hline 68.968 & 224.382 & Surface & 2006 & 68.968 & 223.610 & Surface & 2011 \\
\hline 71.966 & 224.304 & Surface & 2006 & 71.966 & 222.370 & Surface & 2011 \\
\hline 74.965 & 224.295 & Surface & 2006 & 74.965 & 220.833 & Surface & 2011 \\
\hline 77.964 & 224.249 & Surface & 2006 & 77.964 & 219.150 & Surface & 2011 \\
\hline 80.962 & 224.194 & Surface & 2006 & 80.962 & 217.773 & Surface & 2011 \\
\hline 83.961 & 224.182 & Surface & 2006 & 83.961 & 216.909 & Surface & 2011 \\
\hline 86.960 & 224.182 & Surface & 2006 & 86.960 & 216.122 & Surface & 2011 \\
\hline 89.958 & 224.184 & Surface & 2006 & 89.958 & 215.559 & Surface & 2011 \\
\hline 92.957 & 224.187 & Surface & 2006 & 92.957 & 215.508 & Surface & 2011 \\
\hline 95.955 & 224.189 & Surface & 2006 & 95.955 & 215.544 & Surface & 2011 \\
\hline 98.954 & 224.189 & Surface & 2006 & 98.954 & 215.584 & Surface & 2011 \\
\hline 101.952 & 224.188 & Surface & 2006 & 101.952 & 215.573 & Surface & 2011 \\
\hline 104.951 & 224.189 & Surface & 2006 & 104.951 & 215.534 & Surface & 2011 \\
\hline 107.950 & 224.180 & Surface & 2006 & 107.950 & 215.554 & Surface & 2011 \\
\hline 110.948 & 224.160 & Surface & 2006 & 110.948 & 215.557 & Surface & 2011 \\
\hline 113.947 & 224.145 & Surface & 2006 & 113.947 & 215.559 & Surface & 2011 \\
\hline 116.945 & 224.142 & Surface & 2006 & 116.945 & 215.554 & Surface & 2011 \\
\hline 119.944 & 224.143 & Surface & 2006 & 119.944 & 215.524 & Surface & 2011 \\
\hline 122.943 & 224.145 & Surface & 2006 & 122.943 & 215.494 & Surface & 2011 \\
\hline 125.941 & 224.150 & Surface & 2006 & 125.941 & 215.485 & Surface & 2011 \\
\hline 128.940 & 224.154 & Surface & 2006 & 128.940 & 215.488 & Surface & 2011 \\
\hline 131.939 & 224.158 & Surface & 2006 & 131.939 & 215.444 & Surface & 2011 \\
\hline
\end{tabular}




\begin{tabular}{|c|c|c|c|c|c|c|}
\hline 134.937 & 224.159 & Surface & 2006 & 134.937 & 215.450 & Surface \\
\hline 137.936 & 224.159 & Surface & 2006 & 137.936 & 215.427 & Surface \\
\hline 140.934 & 224.155 & Surface & 2006 & 140.934 & 215.373 & Surface \\
\hline 143.933 & 224.145 & Surface & 2006 & 143.933 & 215.330 & Surface \\
\hline 146.931 & 224.126 & Surface & 2006 & 146.931 & 215.286 & Surface \\
\hline 149.930 & 224.134 & Surface & 2006 & 149.930 & 215.233 & Surface \\
\hline 152.929 & 224.157 & Surface & 2006 & 152.929 & 215.161 & Surface \\
\hline 155.927 & 224.168 & Surface & 2006 & 155.927 & 215.086 & Surface \\
\hline 158.926 & 224.167 & Surface & 2006 & 158.926 & 215.008 & Surface \\
\hline 161.925 & 224.154 & Surface & 2006 & 161.925 & 214.951 & Surface \\
\hline 164.923 & 224.153 & Surface & 2006 & 164.923 & 214.939 & Surface \\
\hline 167.922 & 224.148 & Surface & 2006 & 167.922 & 214.866 & Surface \\
\hline 170.920 & 224.133 & Surface & 2006 & 170.920 & 214.823 & Surface \\
\hline 173.919 & 224.123 & Surface & 2006 & 173.919 & 214.761 & Surface \\
\hline 176.918 & 224.123 & Surface & 2006 & 176.918 & 214.693 & Surface \\
\hline 179.916 & 224.126 & Surface & 2006 & 179.916 & 214.624 & Surface \\
\hline 182.915 & 224.133 & Surface & 2006 & 182.915 & 214.539 & Surface \\
\hline 185.913 & 224.139 & Surface & 2006 & 185.913 & 214.427 & Surface \\
\hline 188.912 & 224.142 & Surface & 2006 & 188.912 & 214.299 & Surface \\
\hline 191.910 & 224.124 & Surface & 2006 & 191.910 & 214.300 & Surface \\
\hline 194.909 & 224.118 & Surface & 2006 & 194.909 & 214.303 & Surface \\
\hline 197.908 & 224.133 & Surface & 2006 & 197.908 & 214.306 & Surface \\
\hline 200.906 & 224.133 & Surface & 2006 & 200.906 & 214.309 & Surface \\
\hline 203.905 & 224.138 & Surface & 2006 & 203.905 & 214.323 & Surface \\
\hline 206.904 & 224.148 & Surface & 2006 & 206.904 & 214.349 & Surface \\
\hline 209.902 & 224.144 & Surface & 2006 & 209.902 & 214.374 & Surface \\
\hline 212.901 & 224.140 & Surface & 2006 & 212.901 & 214.381 & Surface \\
\hline 215.899 & 224.159 & Surface & 2006 & 215.899 & 214.380 & Surface \\
\hline 218.898 & 224.173 & Surface & 2006 & 218.898 & 214.373 & Surface \\
\hline 221.897 & 224.168 & Surface & 2006 & 221.897 & 214.370 & Surface \\
\hline 224.895 & 224.167 & Surface & 2006 & 224.895 & 214.342 & Surface \\
\hline 227.894 & 224.164 & Surface & 2006 & 227.894 & 214.328 & Surface \\
\hline 230.892 & 224.162 & Surface & 2006 & 230.892 & 214.335 & Surface \\
\hline 233.891 & 224.161 & Surface & 2006 & 233.891 & 214.322 & Surface \\
\hline 236.889 & 224.162 & Surface & 2006 & 236.889 & 214.331 & Surface \\
\hline 239.888 & 224.163 & Surface & 2006 & 239.888 & 214.348 & Surface \\
\hline 242.887 & 224.166 & Surface & 2006 & 242.887 & 214.359 & Surface \\
\hline 245.885 & 224.162 & Surface & 2006 & 245.885 & 214.342 & Surface \\
\hline 248.884 & 224.144 & Surface & 2006 & 248.884 & 214.325 & Surface \\
\hline 251.883 & 224.127 & Surface & 2006 & 251.883 & 214.326 & Surface \\
\hline 254.881 & 224.132 & Surface & 2006 & 254.881 & 214.354 & Surface \\
\hline 257.880 & 224.128 & Surface & 2006 & 257.880 & 214.368 & Surface \\
\hline 260.878 & 224.125 & Surface & 2006 & 260.878 & 214.372 & Surface \\
\hline 263.877 & 224.107 & Surface & 2006 & 263.877 & 214.351 & Surface \\
\hline 266.876 & 224.064 & Surface & 2006 & 266.876 & 214.314 & Surface \\
\hline 269.874 & 224.023 & Surface & 2006 & 269.874 & 214.332 & Surface \\
\hline 272.873 & 224.007 & Surface & 2006 & 272.873 & 214.527 & Surface \\
\hline 275.871 & 223.987 & Surface & 2006 & 275.871 & 214.883 & Surface \\
\hline 278.870 & 223.973 & Surface & 2006 & 278.870 & 215.538 & Surface \\
\hline
\end{tabular}




\begin{tabular}{|c|c|c|c|c|c|c|c|}
\hline 281.868 & 223.971 & Surface & 2006 & 281.868 & 217.089 & Surface & 2011 \\
\hline 284.867 & 223.962 & Surface & 2006 & 284.867 & 219.574 & Surface & 2011 \\
\hline 287.866 & 223.962 & Surface & 2006 & 287.866 & 222.011 & Surface & 2011 \\
\hline 290.864 & 223.951 & Surface & 2006 & 290.864 & 223.139 & Surface & 2011 \\
\hline 293.863 & 223.925 & Surface & 2006 & 293.863 & 223.578 & Surface & 2011 \\
\hline 296.862 & 223.901 & Surface & 2006 & 296.862 & 223.861 & Surface & 2011 \\
\hline 299.860 & 223.846 & Surface & 2006 & 299.860 & 224.102 & Surface & 2011 \\
\hline 302.859 & 223.795 & Surface & 2006 & 302.859 & 224.327 & Surface & 2011 \\
\hline 305.857 & 223.816 & Surface & 2006 & 305.857 & 224.539 & Surface & 2011 \\
\hline 308.856 & 223.918 & Surface & 2006 & 308.856 & 224.726 & Surface & 2011 \\
\hline 311.855 & 224.302 & Surface & 2006 & 311.855 & 224.884 & Surface & 2011 \\
\hline 314.853 & 224.820 & Surface & 2006 & 314.853 & 225.047 & Surface & 2011 \\
\hline 317.852 & 225.312 & Surface & 2006 & 317.852 & 225.179 & Surface & 2011 \\
\hline 320.850 & 225.731 & Surface & 2006 & 320.850 & 225.337 & Surface & 2011 \\
\hline 323.849 & 226.024 & Surface & 2006 & 323.849 & 225.563 & Surface & 2011 \\
\hline 326.847 & 226.258 & Surface & 2006 & 326.847 & 225.791 & Surface & 2011 \\
\hline 329.846 & 226.441 & Surface & 2006 & 329.846 & 225.978 & Surface & 2011 \\
\hline 332.845 & 226.562 & Surface & 2006 & 332.845 & 226.104 & Surface & 2011 \\
\hline 335.843 & 226.583 & Surface & 2006 & 335.843 & 226.112 & Surface & 2011 \\
\hline 338.842 & 226.531 & Surface & 2006 & 338.842 & 226.072 & Surface & 2011 \\
\hline 341.841 & 226.482 & Surface & 2006 & 341.841 & 226.041 & Surface & 2011 \\
\hline 344.839 & 226.478 & Surface & 2006 & 344.839 & 226.044 & Surface & 2011 \\
\hline 347.838 & 226.609 & Surface & 2006 & 347.838 & 226.048 & Surface & 2011 \\
\hline 350.836 & 226.744 & Surface & 2006 & 350.836 & 226.032 & Surface & 2011 \\
\hline 353.835 & 226.734 & Surface & 2006 & 353.835 & 226.023 & Surface & 2011 \\
\hline 356.834 & 226.749 & Surface & 2006 & 356.834 & 226.134 & Surface & 2011 \\
\hline 359.832 & 226.863 & Surface & 2006 & 359.832 & 226.292 & Surface & 2011 \\
\hline 362.831 & 227.190 & Surface & 2006 & 362.831 & 226.446 & Surface & 2011 \\
\hline 365.830 & 227.471 & Surface & 2006 & 365.830 & 226.653 & Surface & 2011 \\
\hline 368.828 & 227.754 & Surface & 2006 & 368.828 & 227.140 & Surface & 2011 \\
\hline 0.000 & 226.090 & Surface & 2008 & 0.000 & 226.179 & Surface & 2012 \\
\hline 3.264 & 226.049 & Surface & 2008 & 3.264 & 226.158 & Surface & 2012 \\
\hline 6.528 & 226.005 & Surface & 2008 & 6.528 & 226.077 & Surface & 2012 \\
\hline 9.792 & 226.010 & Surface & 2008 & 9.792 & 225.996 & Surface & 2012 \\
\hline 13.056 & 226.006 & Surface & 2008 & 13.056 & 225.979 & Surface & 2012 \\
\hline 16.320 & 226.041 & Surface & 2008 & 16.320 & 226.056 & Surface & 2012 \\
\hline 19.584 & 226.145 & Surface & 2008 & 19.584 & 226.225 & Surface & 2012 \\
\hline 22.848 & 226.271 & Surface & 2008 & 22.848 & 226.422 & Surface & 2012 \\
\hline 26.112 & 226.388 & Surface & 2008 & 26.112 & 226.570 & Surface & 2012 \\
\hline 29.376 & 226.418 & Surface & 2008 & 29.376 & 226.570 & Surface & 2012 \\
\hline 32.640 & 226.465 & Surface & 2008 & 32.640 & 226.472 & Surface & 2012 \\
\hline 35.904 & 226.329 & Surface & 2008 & 35.904 & 226.374 & Surface & 2012 \\
\hline 39.167 & 226.045 & Surface & 2008 & 39.167 & 226.276 & Surface & 2012 \\
\hline 42.432 & 225.795 & Surface & 2008 & 42.432 & 226.126 & Surface & 2012 \\
\hline 45.696 & 225.511 & Surface & 2008 & 45.696 & 225.860 & Surface & 2012 \\
\hline 48.959 & 225.349 & Surface & 2008 & 48.959 & 225.441 & Surface & 2012 \\
\hline 52.223 & 225.335 & Surface & 2008 & 52.223 & 225.312 & Surface & 2012 \\
\hline 55.487 & 225.319 & Surface & 2008 & 55.487 & 225.285 & Surface & 2012 \\
\hline 58.751 & 225.272 & Surface & 2008 & 58.751 & 225.268 & Surface & 2012 \\
\hline
\end{tabular}




\begin{tabular}{|c|c|c|c|c|c|c|c|}
\hline 62.015 & 224.933 & Surface & 2008 & 62.015 & 225.186 & Surface & 2012 \\
\hline 65.279 & 224.291 & Surface & 2008 & 65.279 & 224.864 & Surface & 2012 \\
\hline 68.543 & 223.543 & Surface & 2008 & 68.543 & 224.142 & Surface & 2012 \\
\hline 71.807 & 222.624 & Surface & 2008 & 71.807 & 223.083 & Surface & 2012 \\
\hline 75.071 & 221.326 & Surface & 2008 & 75.071 & 221.786 & Surface & 2012 \\
\hline 78.335 & 219.760 & Surface & 2008 & 78.335 & 220.027 & Surface & 2012 \\
\hline 81.599 & 218.384 & Surface & 2008 & 81.599 & 218.446 & Surface & 2012 \\
\hline 84.863 & 217.329 & Surface & 2008 & 84.863 & 217.202 & Surface & 2012 \\
\hline 88.127 & 216.723 & Surface & 2008 & 88.127 & 216.202 & Surface & 2012 \\
\hline 91.391 & 216.533 & Surface & 2008 & 91.391 & 215.595 & Surface & 2012 \\
\hline 94.655 & 216.537 & Surface & 2008 & 94.655 & 215.481 & Surface & 2012 \\
\hline 97.919 & 216.591 & Surface & 2008 & 97.919 & 215.526 & Surface & 2012 \\
\hline 101.183 & 216.689 & Surface & 2008 & 101.183 & 215.601 & Surface & 2012 \\
\hline 104.447 & 216.816 & Surface & 2008 & 104.447 & 215.697 & Surface & 2012 \\
\hline 107.711 & 216.950 & Surface & 2008 & 107.711 & 215.782 & Surface & 2012 \\
\hline 110.975 & 217.095 & Surface & 2008 & 110.975 & 215.819 & Surface & 2012 \\
\hline 114.239 & 217.216 & Surface & 2008 & 114.239 & 215.842 & Surface & 2012 \\
\hline 117.503 & 217.273 & Surface & 2008 & 117.503 & 215.839 & Surface & 2012 \\
\hline 120.767 & 217.318 & Surface & 2008 & 120.767 & 215.815 & Surface & 2012 \\
\hline 124.031 & 217.366 & Surface & 2008 & 124.031 & 215.783 & Surface & 2012 \\
\hline 127.295 & 217.382 & Surface & 2008 & 127.295 & 215.752 & Surface & 2012 \\
\hline 130.558 & 217.380 & Surface & 2008 & 130.558 & 215.711 & Surface & 2012 \\
\hline 133.823 & 217.347 & Surface & 2008 & 133.823 & 215.643 & Surface & 2012 \\
\hline 137.087 & 217.222 & Surface & 2008 & 137.087 & 215.550 & Surface & 2012 \\
\hline 140.350 & 216.984 & Surface & 2008 & 140.350 & 215.440 & Surface & 2012 \\
\hline 143.614 & 216.741 & Surface & 2008 & 143.614 & 215.344 & Surface & 2012 \\
\hline 146.878 & 216.505 & Surface & 2008 & 146.878 & 215.259 & Surface & 2012 \\
\hline 150.142 & 216.279 & Surface & 2008 & 150.142 & 215.177 & Surface & 2012 \\
\hline 153.406 & 216.069 & Surface & 2008 & 153.406 & 215.087 & Surface & 2012 \\
\hline 156.670 & 215.942 & Surface & 2008 & 156.670 & 215.029 & Surface & 2012 \\
\hline 159.934 & 215.841 & Surface & 2008 & 159.934 & 214.965 & Surface & 2012 \\
\hline 163.198 & 215.785 & Surface & 2008 & 163.198 & 214.841 & Surface & 2012 \\
\hline 166.462 & 215.747 & Surface & 2008 & 166.462 & 214.755 & Surface & 2012 \\
\hline 169.726 & 215.723 & Surface & 2008 & 169.726 & 214.718 & Surface & 2012 \\
\hline 172.990 & 215.672 & Surface & 2008 & 172.990 & 214.628 & Surface & 2012 \\
\hline 176.254 & 215.648 & Surface & 2008 & 176.254 & 214.504 & Surface & 2012 \\
\hline 179.518 & 215.576 & Surface & 2008 & 179.518 & 214.384 & Surface & 2012 \\
\hline 182.782 & 215.471 & Surface & 2008 & 182.782 & 214.273 & Surface & 2012 \\
\hline 186.046 & 215.278 & Surface & 2008 & 186.046 & 214.194 & Surface & 2012 \\
\hline 189.310 & 215.021 & Surface & 2008 & 189.310 & 214.189 & Surface & 2012 \\
\hline 192.574 & 214.952 & Surface & 2008 & 192.574 & 214.181 & Surface & 2012 \\
\hline 195.838 & 214.903 & Surface & 2008 & 195.838 & 214.190 & Surface & 2012 \\
\hline 199.102 & 214.866 & Surface & 2008 & 199.102 & 214.202 & Surface & 2012 \\
\hline 202.366 & 214.847 & Surface & 2008 & 202.366 & 214.215 & Surface & 2012 \\
\hline 205.630 & 214.852 & Surface & 2008 & 205.630 & 214.209 & Surface & 2012 \\
\hline 208.894 & 214.840 & Surface & 2008 & 208.894 & 214.207 & Surface & 2012 \\
\hline 212.158 & 214.773 & Surface & 2008 & 212.158 & 214.206 & Surface & 2012 \\
\hline 215.422 & 214.755 & Surface & 2008 & 215.422 & 214.201 & Surface & 2012 \\
\hline 218.686 & 214.811 & Surface & 2008 & 218.686 & 214.211 & Surface & 2012 \\
\hline
\end{tabular}




\begin{tabular}{|c|c|c|c|c|c|c|c|}
\hline 221.949 & 214.792 & Surface & 2008 & 221.949 & 214.205 & Surface & 2012 \\
\hline 225.214 & 214.708 & Surface & 2008 & 225.214 & 214.204 & Surface & 2012 \\
\hline 28.478 & 214.745 & Surface & 2008 & 228.478 & 214.215 & Surface & 2012 \\
\hline 231.741 & 214.734 & Surface & 2008 & 231.741 & 214.203 & Surface & 2012 \\
\hline 235.005 & 214.720 & Surface & 2008 & 235.005 & 214.208 & Surface & 2012 \\
\hline 238.270 & 214.746 & Surface & 2008 & 238.270 & 214.193 & Surface & 2012 \\
\hline 241.533 & 214.767 & Surface & 2008 & 241.533 & 214.210 & Surface & 2012 \\
\hline 244.797 & 214.747 & Surface & 2008 & 244.797 & 214.178 & Surface & 2012 \\
\hline 248.061 & 214.749 & Surface & 2008 & 248.061 & 214.219 & Surface & 2012 \\
\hline 251.325 & 214.738 & Surface & 2008 & 251.325 & 214.225 & Surface & 2012 \\
\hline 254.589 & 214.694 & Surface & 2008 & 254.589 & 214.228 & Surface & 2012 \\
\hline 257.853 & 214.659 & Surface & 2008 & 257.853 & 214.212 & Surface & 2012 \\
\hline 261.117 & 214.671 & Surface & 2008 & 261.117 & 214.215 & Surface & 2012 \\
\hline 264.381 & 214.705 & Surface & 2008 & 264.381 & 214.203 & Surface & 2012 \\
\hline 267.645 & 214.726 & Surface & 2008 & 267.645 & 214.256 & Surface & 2012 \\
\hline 270.909 & 214.714 & Surface & 2008 & 270.909 & 214.361 & Surface & 2012 \\
\hline 274.173 & 214.712 & Surface & 2008 & 274.173 & 214.672 & Surface & 2012 \\
\hline 277.437 & 214.928 & Surface & 2008 & 277.437 & 215.366 & Surface & 2012 \\
\hline 280.701 & 215.626 & Surface & 2008 & 280.701 & 216.844 & Surface & 2012 \\
\hline 283.965 & 216.533 & Surface & 2008 & 283.965 & 218.974 & Surface & 2012 \\
\hline 287.229 & 218.729 & Surface & 2008 & 287.229 & 221.816 & Surface & 2012 \\
\hline 290.493 & 221.550 & Surface & 2008 & 290.493 & 223.167 & Surface & 2012 \\
\hline 293.757 & 223.110 & Surface & 2008 & 293.757 & 223.611 & Surface & 2012 \\
\hline 297.021 & 223.735 & Surface & 2008 & 297.021 & 223.918 & Surface & 2012 \\
\hline 300.285 & 224.050 & Surface & 2008 & 300.285 & 224.186 & Surface & 2012 \\
\hline 303.549 & 224.287 & Surface & 2008 & 303.549 & 224.437 & Surface & 2012 \\
\hline 306.813 & 224.545 & Surface & 2008 & 306.813 & 224.678 & Surface & 2012 \\
\hline 310.077 & 224.744 & Surface & 2008 & 310.077 & 224.878 & Surface & 2012 \\
\hline 313.341 & 224.936 & Surface & 2008 & 313.341 & 225.074 & Surface & 2012 \\
\hline 316.605 & 225.098 & Surface & 2008 & 316.605 & 225.217 & Surface & 2012 \\
\hline 319.869 & 225.207 & Surface & 2008 & 319.869 & 225.409 & Surface & 2012 \\
\hline 323.132 & 225.401 & Surface & 2008 & 323.132 & 225.740 & Surface & 2012 \\
\hline 326.396 & 225.725 & Surface & 2008 & 326.396 & 226.107 & Surface & 2012 \\
\hline 329.661 & 225.928 & Surface & 2008 & 329.661 & 226.315 & Surface & 2012 \\
\hline 332.924 & 225.955 & Surface & 2008 & 332.924 & 226.344 & Surface & 2012 \\
\hline 336.188 & 225.925 & Surface & 2008 & 336.188 & 226.284 & Surface & 2012 \\
\hline 339.452 & 225.860 & Surface & 2008 & 339.452 & 226.165 & Surface & 2012 \\
\hline 342.716 & 225.787 & Surface & 2008 & 342.716 & 226.069 & Surface & 2012 \\
\hline 345.980 & 225.764 & Surface & 2008 & 345.980 & 226.109 & Surface & 2012 \\
\hline 349.244 & 225.794 & Surface & 2008 & 349.244 & 226.127 & Surface & 2012 \\
\hline 352.508 & 225.800 & Surface & 2008 & 352.508 & 226.046 & Surface & 2012 \\
\hline 355.772 & 225.827 & Surface & 2008 & 355.772 & 226.019 & Surface & 2012 \\
\hline 359.036 & 225.934 & Surface & 2008 & 359.036 & 226.127 & Surface & 2012 \\
\hline 362.300 & 226.056 & Surface & 2008 & 362.300 & 226.244 & Surface & 2012 \\
\hline 365.564 & 226.156 & Surface & 2008 & 365.564 & 226.402 & Surface & 2012 \\
\hline 368.828 & 226.267 & Surface & 2008 & 368.828 & 226.594 & Surface & 2012 \\
\hline
\end{tabular}


10.3 Transect C - Gravel Bar - All Returns:

\begin{tabular}{|c|c|c|c|c|c|c|c|}
\hline Distance & $\mathrm{Z}$ & DEM & Year & Distance & $\mathrm{Z}$ & DEM & Year \\
\hline 0.000 & 231.647 & Surface & 2006 & 0.000 & 231.190 & Surface & 2011 \\
\hline 2.993 & 231.593 & Surface & 2006 & 2.993 & 231.152 & Surface & 2011 \\
\hline 5.987 & 231.525 & Surface & 2006 & 5.987 & 231.121 & Surface & 2011 \\
\hline 8.980 & 231.323 & Surface & 2006 & 8.980 & 231.060 & Surface & 2011 \\
\hline 11.973 & 231.024 & Surface & 2006 & 11.973 & 230.775 & Surface & 2011 \\
\hline 14.966 & 230.641 & Surface & 2006 & 14.966 & 230.162 & Surface & 2011 \\
\hline 17.960 & 230.096 & Surface & 2006 & 17.960 & 229.548 & Surface & 2011 \\
\hline 20.953 & 229.308 & Surface & 2006 & 20.953 & 228.858 & Surface & 2011 \\
\hline 23.946 & 228.484 & Surface & 2006 & 23.946 & 228.003 & Surface & 2011 \\
\hline 26.939 & 227.639 & Surface & 2006 & 26.939 & 227.070 & Surface & 2011 \\
\hline 29.933 & 226.678 & Surface & 2006 & 29.933 & 226.095 & Surface & 2011 \\
\hline 32.926 & 225.949 & Surface & 2006 & 32.926 & 225.076 & Surface & 2011 \\
\hline 35.919 & 225.422 & Surface & 2006 & 35.919 & 224.102 & Surface & 2011 \\
\hline 38.913 & 224.934 & Surface & 2006 & 38.913 & 223.122 & Surface & 2011 \\
\hline 41.906 & 224.533 & Surface & 2006 & 41.906 & 222.130 & Surface & 2011 \\
\hline 44.899 & 224.510 & Surface & 2006 & 44.899 & 221.493 & Surface & 2011 \\
\hline 47.893 & 224.847 & Surface & 2006 & 47.893 & 221.284 & Surface & 2011 \\
\hline 50.886 & 224.976 & Surface & 2006 & 50.886 & 221.175 & Surface & 2011 \\
\hline 53.879 & 224.978 & Surface & 2006 & 53.879 & 221.066 & Surface & 2011 \\
\hline 56.872 & 224.968 & Surface & 2006 & 56.872 & 220.915 & Surface & 2011 \\
\hline 59.866 & 224.957 & Surface & 2006 & 59.866 & 220.538 & Surface & 2011 \\
\hline 62.859 & 224.947 & Surface & 2006 & 62.859 & 219.695 & Surface & 2011 \\
\hline 65.852 & 224.936 & Surface & 2006 & 65.852 & 218.848 & Surface & 2011 \\
\hline 68.846 & 224.925 & Surface & 2006 & 68.846 & 218.029 & Surface & 2011 \\
\hline 71.839 & 224.915 & Surface & 2006 & 71.839 & 217.188 & Surface & 2011 \\
\hline 74.832 & 224.906 & Surface & 2006 & 74.832 & 216.246 & Surface & 2011 \\
\hline 77.825 & 224.894 & Surface & 2006 & 77.825 & 215.346 & Surface & 2011 \\
\hline 80.819 & 224.881 & Surface & 2006 & 80.819 & 214.980 & Surface & 2011 \\
\hline 83.812 & 224.866 & Surface & 2006 & 83.812 & 214.929 & Surface & 2011 \\
\hline 86.805 & 224.849 & Surface & 2006 & 86.805 & 214.972 & Surface & 2011 \\
\hline 89.798 & 224.833 & Surface & 2006 & 89.798 & 215.063 & Surface & 2011 \\
\hline 92.792 & 224.813 & Surface & 2006 & 92.792 & 215.098 & Surface & 2011 \\
\hline 95.785 & 224.795 & Surface & 2006 & 95.785 & 214.928 & Surface & 2011 \\
\hline 98.778 & 224.777 & Surface & 2006 & 98.778 & 214.905 & Surface & 2011 \\
\hline 101.772 & 224.757 & Surface & 2006 & 101.772 & 214.899 & Surface & 2011 \\
\hline 104.765 & 224.739 & Surface & 2006 & 104.765 & 214.910 & Surface & 2011 \\
\hline 107.758 & 224.721 & Surface & 2006 & 107.758 & 214.917 & Surface & 2011 \\
\hline 110.751 & 224.702 & Surface & 2006 & 110.751 & 214.908 & Surface & 2011 \\
\hline 113.745 & 224.683 & Surface & 2006 & 113.745 & 214.902 & Surface & 2011 \\
\hline 116.738 & 224.664 & Surface & 2006 & 116.738 & 214.883 & Surface & 2011 \\
\hline 119.731 & 224.647 & Surface & 2006 & 119.731 & 214.867 & Surface & 2011 \\
\hline 122.724 & 224.628 & Surface & 2006 & 122.724 & 214.878 & Surface & 2011 \\
\hline 125.718 & 224.606 & Surface & 2006 & 125.718 & 214.869 & Surface & 2011 \\
\hline 128.711 & 224.591 & Surface & 2006 & 128.711 & 214.846 & Surface & 2011 \\
\hline 131.704 & 224.568 & Surface & 2006 & 131.704 & 214.834 & Surface & 2011 \\
\hline
\end{tabular}




\begin{tabular}{|c|c|c|c|c|c|c|}
\hline 134.698 & 224.549 & Surface & 2006 & 134.698 & 214.880 & Surface \\
\hline 137.691 & 224.528 & Surface & 2006 & 137.691 & 214.930 & Surface \\
\hline 140.684 & 224.505 & Surface & 2006 & 140.684 & 214.936 & Surface \\
\hline 143.678 & 224.486 & Surface & 2006 & 143.678 & 214.966 & Surface \\
\hline 146.671 & 224.465 & Surface & 2006 & 146.671 & 215.008 & Surface \\
\hline 149.664 & 224.444 & Surface & 2006 & 149.664 & 215.108 & Surface \\
\hline 152.657 & 224.423 & Surface & 2006 & 152.657 & 215.210 & Surface \\
\hline 155.651 & 224.400 & Surface & 2006 & 155.651 & 215.288 & Surface \\
\hline 158.644 & 224.379 & Surface & 2006 & 158.644 & 215.358 & Surface \\
\hline 161.637 & 224.359 & Surface & 2006 & 161.637 & 215.422 & Surface \\
\hline 164.631 & 224.338 & Surface & 2006 & 164.631 & 215.463 & Surface \\
\hline 167.624 & 224.318 & Surface & 2006 & 167.624 & 215.470 & Surface \\
\hline 170.617 & 224.295 & Surface & 2006 & 170.617 & 215.464 & Surface \\
\hline 173.610 & 224.273 & Surface & 2006 & 173.610 & 215.479 & Surface \\
\hline 176.604 & 224.253 & Surface & 2006 & 176.604 & 215.493 & Surface \\
\hline 179.597 & 224.232 & Surface & 2006 & 179.597 & 215.521 & Surface \\
\hline 182.590 & 224.211 & Surface & 2006 & 182.590 & 215.564 & Surface \\
\hline 185.583 & 224.191 & Surface & 2006 & 185.583 & 215.608 & Surface \\
\hline 188.577 & 224.171 & Surface & 2006 & 188.577 & 215.651 & Surface \\
\hline 191.570 & 224.181 & Surface & 2006 & 191.570 & 215.693 & Surface \\
\hline 194.563 & 224.193 & Surface & 2006 & 194.563 & 215.738 & Surface \\
\hline 197.557 & 224.205 & Surface & 2006 & 197.557 & 215.812 & Surface \\
\hline 200.550 & 224.208 & Surface & 2006 & 200.550 & 215.911 & Surface \\
\hline 203.543 & 224.172 & Surface & 2006 & 203.543 & 216.047 & Surface \\
\hline 206.536 & 224.111 & Surface & 2006 & 206.536 & 216.113 & Surface \\
\hline 209.530 & 224.132 & Surface & 2006 & 209.530 & 216.121 & Surface \\
\hline 212.523 & 224.137 & Surface & 2006 & 212.523 & 216.139 & Surface \\
\hline 215.516 & 224.121 & Surface & 2006 & 215.516 & 216.122 & Surface \\
\hline 218.510 & 224.123 & Surface & 2006 & 218.510 & 216.074 & Surface \\
\hline 221.503 & 224.119 & Surface & 2006 & 221.503 & 215.958 & Surface \\
\hline 224.496 & 224.136 & Surface & 2006 & 224.496 & 215.889 & Surface \\
\hline 227.489 & 224.166 & Surface & 2006 & 227.489 & 215.913 & Surface \\
\hline 230.483 & 224.162 & Surface & 2006 & 230.483 & 216.139 & Surface \\
\hline 233.476 & 224.180 & Surface & 2006 & 233.476 & 216.464 & Surface \\
\hline 236.469 & 224.369 & Surface & 2006 & 236.469 & 216.901 & Surface \\
\hline 239.462 & 224.590 & Surface & 2006 & 239.462 & 217.300 & Surface \\
\hline 242.456 & 224.770 & Surface & 2006 & 242.456 & 217.557 & Surface \\
\hline 245.449 & 224.942 & Surface & 2006 & 245.449 & 217.772 & Surface \\
\hline 248.442 & 225.103 & Surface & 2006 & 248.442 & 218.080 & Surface \\
\hline 251.436 & 225.179 & Surface & 2006 & 251.436 & 218.531 & Surface \\
\hline 254.429 & 225.178 & Surface & 2006 & 254.429 & 218.927 & Surface \\
\hline 257.422 & 225.165 & Surface & 2006 & 257.422 & 220.170 & Surface \\
\hline 260.416 & 225.212 & Surface & 2006 & 260.416 & 222.639 & Surface \\
\hline 263.409 & 225.349 & Surface & 2006 & 263.409 & 224.315 & Surface \\
\hline 266.402 & 225.530 & Surface & 2006 & 266.402 & 225.658 & Surface \\
\hline 269.395 & 225.568 & Surface & 2006 & 269.395 & 226.243 & Surface \\
\hline 272.388 & 225.529 & Surface & 2006 & 272.388 & 226.355 & Surface \\
\hline 275.382 & 225.586 & Surface & 2006 & 275.382 & 226.336 & Surface \\
\hline 278.375 & 225.680 & Surface & 2006 & 278.375 & 226.187 & Surface \\
\hline
\end{tabular}




\begin{tabular}{|c|c|c|c|c|c|c|c|}
\hline 281.369 & 225.779 & Surface & 2006 & 281.369 & 226.141 & Surface & 2011 \\
\hline 284.362 & 225.835 & Surface & 2006 & 284.362 & 226.241 & Surface & 2011 \\
\hline 287.355 & 225.778 & Surface & 2006 & 287.355 & 226.360 & Surface & 2011 \\
\hline 290.348 & 225.701 & Surface & 2006 & 290.348 & 226.479 & Surface & 2011 \\
\hline 293.342 & 225.715 & Surface & 2006 & 293.342 & 226.580 & Surface & 2011 \\
\hline 296.335 & 225.736 & Surface & 2006 & 296.335 & 226.762 & Surface & 2011 \\
\hline 299.328 & 225.760 & Surface & 2006 & 299.328 & 226.861 & Surface & 2011 \\
\hline 302.321 & 225.776 & Surface & 2006 & 302.321 & 226.802 & Surface & 2011 \\
\hline 305.315 & 225.791 & Surface & 2006 & 305.315 & 226.724 & Surface & 2011 \\
\hline 308.308 & 225.804 & Surface & 2006 & 308.308 & 226.641 & Surface & 2011 \\
\hline 311.301 & 225.804 & Surface & 2006 & 311.301 & 226.579 & Surface & 2011 \\
\hline 314.295 & 225.765 & Surface & 2006 & 314.295 & 226.570 & Surface & 2011 \\
\hline 317.288 & 225.763 & Surface & 2006 & 317.288 & 226.646 & Surface & 2011 \\
\hline 320.281 & 226.014 & Surface & 2006 & 320.281 & 226.747 & Surface & 2011 \\
\hline 323.275 & 226.284 & Surface & 2006 & 323.275 & 226.854 & Surface & 2011 \\
\hline 326.268 & 226.456 & Surface & 2006 & 326.268 & 226.969 & Surface & 2011 \\
\hline 329.261 & 226.625 & Surface & 2006 & 329.261 & 227.085 & Surface & 2011 \\
\hline 332.254 & 226.813 & Surface & 2006 & 332.254 & 227.201 & Surface & 2011 \\
\hline 335.247 & 226.999 & Surface & 2006 & 335.247 & 227.317 & Surface & 2011 \\
\hline 338.241 & 227.186 & Surface & 2006 & 338.241 & 227.432 & Surface & 2011 \\
\hline 341.234 & 227.373 & Surface & 2006 & 341.234 & 227.547 & Surface & 2011 \\
\hline 344.227 & 227.556 & Surface & 2006 & 344.227 & 227.777 & Surface & 2011 \\
\hline 347.221 & 227.746 & Surface & 2006 & 347.221 & 228.147 & Surface & 2011 \\
\hline 350.214 & 228.036 & Surface & 2006 & 350.214 & 228.413 & Surface & 2011 \\
\hline 353.207 & 228.231 & Surface & 2006 & 353.207 & 228.491 & Surface & 2011 \\
\hline 356.201 & 228.359 & Surface & 2006 & 356.201 & 228.643 & Surface & 2011 \\
\hline 0.000 & 231.627 & Surface & 2008 & 0.000 & 231.250 & Surface & 2012 \\
\hline 3.268 & 231.532 & Surface & 2008 & 3.268 & 231.171 & Surface & 2012 \\
\hline 6.536 & 231.435 & Surface & 2008 & 6.536 & 231.313 & Surface & 2012 \\
\hline 9.804 & 231.116 & Surface & 2008 & 9.804 & 231.336 & Surface & 2012 \\
\hline 13.072 & 230.392 & Surface & 2008 & 13.072 & 230.750 & Surface & 2012 \\
\hline 16.340 & 229.642 & Surface & 2008 & 16.340 & 230.051 & Surface & 2012 \\
\hline 19.607 & 228.958 & Surface & 2008 & 19.607 & 229.333 & Surface & 2012 \\
\hline 22.875 & 228.378 & Surface & 2008 & 22.875 & 228.610 & Surface & 2012 \\
\hline 26.143 & 227.867 & Surface & 2008 & 26.143 & 227.895 & Surface & 2012 \\
\hline 29.411 & 227.269 & Surface & 2008 & 29.411 & 227.147 & Surface & 2012 \\
\hline 32.679 & 226.169 & Surface & 2008 & 32.679 & 226.289 & Surface & 2012 \\
\hline 35.947 & 224.883 & Surface & 2008 & 35.947 & 225.228 & Surface & 2012 \\
\hline 39.215 & 223.597 & Surface & 2008 & 39.215 & 223.878 & Surface & 2012 \\
\hline 42.483 & 222.242 & Surface & 2008 & 42.483 & 222.515 & Surface & 2012 \\
\hline 45.751 & 221.483 & Surface & 2008 & 45.751 & 221.647 & Surface & 2012 \\
\hline 49.019 & 221.318 & Surface & 2008 & 49.019 & 221.386 & Surface & 2012 \\
\hline 52.286 & 221.246 & Surface & 2008 & 52.286 & 221.287 & Surface & 2012 \\
\hline 55.554 & 221.105 & Surface & 2008 & 55.554 & 221.109 & Surface & 2012 \\
\hline 58.822 & 220.789 & Surface & 2008 & 58.822 & 220.946 & Surface & 2012 \\
\hline 62.090 & 220.220 & Surface & 2008 & 62.090 & 220.670 & Surface & 2012 \\
\hline 65.358 & 219.277 & Surface & 2008 & 65.358 & 219.881 & Surface & 2012 \\
\hline 68.626 & 218.321 & Surface & 2008 & 68.626 & 218.848 & Surface & 2012 \\
\hline 71.894 & 217.357 & Surface & 2008 & 71.894 & 217.596 & Surface & 2012 \\
\hline
\end{tabular}




\begin{tabular}{|c|c|c|c|c|c|c|c|}
\hline 75.162 & 216.367 & Surface & 2008 & 75.162 & 216.322 & Surface & 2012 \\
\hline 78.429 & 215.954 & Surface & 2008 & 78.429 & 215.450 & Surface & 2012 \\
\hline 81.697 & 216.010 & Surface & 2008 & 81.697 & 215.083 & Surface & 2012 \\
\hline 84.965 & 216.283 & Surface & 2008 & 84.965 & 215.010 & Surface & 2012 \\
\hline 88.233 & 216.438 & Surface & 2008 & 88.233 & 215.007 & Surface & 2012 \\
\hline 91.501 & 216.535 & Surface & 2008 & 91.501 & 215.012 & Surface & 2012 \\
\hline 94.769 & 216.754 & Surface & 2008 & 94.769 & 215.008 & Surface & 2012 \\
\hline 98.037 & 216.957 & Surface & 2008 & 98.037 & 215.001 & Surface & 2012 \\
\hline 101.305 & 217.069 & Surface & 2008 & 101.305 & 215.009 & Surface & 2012 \\
\hline 104.573 & 217.051 & Surface & 2008 & 104.573 & 214.992 & Surface & 2012 \\
\hline 107.840 & 216.946 & Surface & 2008 & 107.840 & 215.013 & Surface & 2012 \\
\hline 111.109 & 216.844 & Surface & 2008 & 111.109 & 215.003 & Surface & 2012 \\
\hline 114.376 & 216.779 & Surface & 2008 & 114.376 & 214.977 & Surface & 2012 \\
\hline 117.644 & 216.728 & Surface & 2008 & 117.644 & 214.985 & Surface & 2012 \\
\hline 120.912 & 216.744 & Surface & 2008 & 120.912 & 214.995 & Surface & 2012 \\
\hline 124.180 & 216.796 & Surface & 2008 & 124.180 & 214.983 & Surface & 2012 \\
\hline 127.448 & 216.724 & Surface & 2008 & 127.448 & 214.985 & Surface & 2012 \\
\hline 130.716 & 216.673 & Surface & 2008 & 130.716 & 214.990 & Surface & 2012 \\
\hline 133.984 & 216.658 & Surface & 2008 & 133.984 & 214.990 & Surface & 2012 \\
\hline 137.252 & 216.602 & Surface & 2008 & 137.252 & 214.999 & Surface & 2012 \\
\hline 140.519 & 216.577 & Surface & 2008 & 140.519 & 214.979 & Surface & 2012 \\
\hline 143.787 & 216.597 & Surface & 2008 & 143.787 & 214.971 & Surface & 2012 \\
\hline 147.055 & 216.613 & Surface & 2008 & 147.055 & 214.977 & Surface & 2012 \\
\hline 150.323 & 216.613 & Surface & 2008 & 150.323 & 214.988 & Surface & 2012 \\
\hline 153.591 & 216.582 & Surface & 2008 & 153.591 & 214.993 & Surface & 2012 \\
\hline 156.859 & 216.486 & Surface & 2008 & 156.859 & 215.026 & Surface & 2012 \\
\hline 160.127 & 216.358 & Surface & 2008 & 160.127 & 215.144 & Surface & 2012 \\
\hline 163.395 & 216.272 & Surface & 2008 & 163.395 & 215.201 & Surface & 2012 \\
\hline 166.663 & 216.182 & Surface & 2008 & 166.663 & 215.278 & Surface & 2012 \\
\hline 169.930 & 216.088 & Surface & 2008 & 169.930 & 215.320 & Surface & 2012 \\
\hline 173.198 & 215.971 & Surface & 2008 & 173.198 & 215.436 & Surface & 2012 \\
\hline 176.466 & 215.839 & Surface & 2008 & 176.466 & 215.528 & Surface & 2012 \\
\hline 179.734 & 215.836 & Surface & 2008 & 179.734 & 215.519 & Surface & 2012 \\
\hline 183.002 & 215.823 & Surface & 2008 & 183.002 & 215.558 & Surface & 2012 \\
\hline 186.270 & 215.819 & Surface & 2008 & 186.270 & 215.667 & Surface & 2012 \\
\hline 189.538 & 215.816 & Surface & 2008 & 189.538 & 215.760 & Surface & 2012 \\
\hline 192.806 & 215.803 & Surface & 2008 & 192.806 & 215.881 & Surface & 2012 \\
\hline 196.074 & 215.769 & Surface & 2008 & 196.074 & 216.093 & Surface & 2012 \\
\hline 199.341 & 215.752 & Surface & 2008 & 199.341 & 216.279 & Surface & 2012 \\
\hline 202.609 & 215.796 & Surface & 2008 & 202.609 & 216.352 & Surface & 2012 \\
\hline 205.877 & 215.813 & Surface & 2008 & 205.877 & 216.436 & Surface & 2012 \\
\hline 209.145 & 215.832 & Surface & 2008 & 209.145 & 216.502 & Surface & 2012 \\
\hline 212.413 & 215.825 & Surface & 2008 & 212.413 & 216.527 & Surface & 2012 \\
\hline 215.681 & 215.793 & Surface & 2008 & 215.681 & 216.550 & Surface & 2012 \\
\hline 218.949 & 215.737 & Surface & 2008 & 218.949 & 216.501 & Surface & 2012 \\
\hline 222.217 & 215.720 & Surface & 2008 & 222.217 & 216.332 & Surface & 2012 \\
\hline 225.485 & 215.887 & Surface & 2008 & 225.485 & 216.167 & Surface & 2012 \\
\hline 228.753 & 216.251 & Surface & 2008 & 228.753 & 216.014 & Surface & 2012 \\
\hline 232.020 & 216.819 & Surface & 2008 & 232.020 & 216.122 & Surface & 2012 \\
\hline
\end{tabular}




\begin{tabular}{|c|c|c|c|c|c|c|}
\hline 235.288 & 217.506 & Surface & 2008 & 235.288 & 216.807 & Surface \\
\hline 238.556 & 218.213 & Surface & 2008 & 238.556 & 217.279 & Surface \\
\hline 241.824 & 218.924 & Surface & 2008 & 241.824 & 217.522 & Surface \\
\hline 245.092 & 219.595 & Surface & 2008 & 245.092 & 217.716 & Surface \\
\hline 248.360 & 220.300 & Surface & 2008 & 248.360 & 217.993 & Surface \\
\hline 251.628 & 220.996 & Surface & 2008 & 251.628 & 218.439 & Surface \\
\hline 254.896 & 221.659 & Surface & 2008 & 254.896 & 219.017 & Surface \\
\hline 258.164 & 222.353 & Surface & 2008 & 258.164 & 219.753 & Surface \\
\hline 261.432 & 223.039 & Surface & 2008 & 261.432 & 220.621 & Surface \\
\hline 264.700 & 223.768 & Surface & 2008 & 264.700 & 221.649 & Surface \\
\hline 267.967 & 224.489 & Surface & 2008 & 267.967 & 222.920 & Surface \\
\hline 271.235 & 224.814 & Surface & 2008 & 271.235 & 224.231 & Surface \\
\hline 274.503 & 224.956 & Surface & 2008 & 274.503 & 225.282 & Surface \\
\hline 277.771 & 225.042 & Surface & 2008 & 277.771 & 225.683 & Surface \\
\hline 281.039 & 225.148 & Surface & 2008 & 281.039 & 225.737 & Surface \\
\hline 284.307 & 225.292 & Surface & 2008 & 284.307 & 225.801 & Surface \\
\hline 287.575 & 225.538 & Surface & 2008 & 287.575 & 225.933 & Surface \\
\hline 290.843 & 225.792 & Surface & 2008 & 290.843 & 226.178 & Surface \\
\hline 294.111 & 225.898 & Surface & 2008 & 294.111 & 226.232 & Surface \\
\hline 297.378 & 225.982 & Surface & 2008 & 297.378 & 226.209 & Surface \\
\hline 300.646 & 226.081 & Surface & 2008 & 300.646 & 226.117 & Surface \\
\hline 303.914 & 226.181 & Surface & 2008 & 303.914 & 225.990 & Surface \\
\hline 307.182 & 226.274 & Surface & 2008 & 307.182 & 225.912 & Surface \\
\hline 310.450 & 226.352 & Surface & 2008 & 310.450 & 225.863 & Surface \\
\hline 313.718 & 226.414 & Surface & 2008 & 313.718 & 225.820 & Surface \\
\hline 316.986 & 226.457 & Surface & 2008 & 316.986 & 225.787 & Surface \\
\hline 320.254 & 226.485 & Surface & 2008 & 320.254 & 225.846 & Surface \\
\hline 323.522 & 226.515 & Surface & 2008 & 323.522 & 226.004 & Surface \\
\hline 326.789 & 226.588 & Surface & 2008 & 326.789 & 226.204 & Surface \\
\hline 330.057 & 226.661 & Surface & 2008 & 330.057 & 226.399 & Surface \\
\hline 333.325 & 226.726 & Surface & 2008 & 333.325 & 226.587 & Surface \\
\hline 336.593 & 226.868 & Surface & 2008 & 336.593 & 226.719 & Surface \\
\hline 339.861 & 227.122 & Surface & 2008 & 339.861 & 226.801 & Surface \\
\hline 343.129 & 227.408 & Surface & 2008 & 343.129 & 226.984 & Surface \\
\hline 346.397 & 227.716 & Surface & 2008 & 346.397 & 227.387 & Surface \\
\hline 349.665 & 228.076 & Surface & 2008 & 349.665 & 227.819 & Surface \\
\hline 352.933 & 228.457 & Surface & 2008 & 352.933 & 228.169 & Surface \\
\hline 356.201 & 228.713 & Surface & 2008 & 356.201 & 228.535 & Surface \\
\hline
\end{tabular}


10.4 Transect D - Lahar Dike - All Returns:

\begin{tabular}{|c|c|c|c|c|c|c|c|}
\hline Distance & Z & DEM & Year & Distance & $\mathrm{Z}$ & DEM & Year \\
\hline 0.000 & 231.131 & Surface & 2006 & 0.000 & 231.034 & Surface & 2011 \\
\hline 2.994 & 231.140 & Surface & 2006 & 2.994 & 231.007 & Surface & 2011 \\
\hline 5.988 & 231.111 & Surface & 2006 & 5.988 & 230.992 & Surface & 2011 \\
\hline 8.982 & 231.047 & Surface & 2006 & 8.982 & 230.992 & Surface & 2011 \\
\hline 11.976 & 230.962 & Surface & 2006 & 11.976 & 230.954 & Surface & 2011 \\
\hline 14.970 & 230.847 & Surface & 2006 & 14.970 & 230.902 & Surface & 2011 \\
\hline 17.964 & 230.721 & Surface & 2006 & 17.964 & 230.795 & Surface & 2011 \\
\hline 20.958 & 230.253 & Surface & 2006 & 20.958 & 230.431 & Surface & 2011 \\
\hline 23.952 & 229.620 & Surface & 2006 & 23.952 & 229.937 & Surface & 2011 \\
\hline 26.945 & 228.936 & Surface & 2006 & 26.945 & 229.398 & Surface & 2011 \\
\hline 29.940 & 228.316 & Surface & 2006 & 29.940 & 228.455 & Surface & 2011 \\
\hline 32.933 & 227.718 & Surface & 2006 & 32.933 & 226.979 & Surface & 2011 \\
\hline 35.927 & 227.119 & Surface & 2006 & 35.927 & 225.343 & Surface & 2011 \\
\hline 38.921 & 226.566 & Surface & 2006 & 38.921 & 223.699 & Surface & 2011 \\
\hline 41.915 & 226.121 & Surface & 2006 & 41.915 & 222.046 & Surface & 2011 \\
\hline 44.909 & 226.081 & Surface & 2006 & 44.909 & 220.398 & Surface & 2011 \\
\hline 47.903 & 226.125 & Surface & 2006 & 47.903 & 219.136 & Surface & 2011 \\
\hline 50.897 & 226.176 & Surface & 2006 & 50.897 & 218.651 & Surface & 2011 \\
\hline 53.891 & 226.296 & Surface & 2006 & 53.891 & 218.468 & Surface & 2011 \\
\hline 56.885 & 226.557 & Surface & 2006 & 56.885 & 218.252 & Surface & 2011 \\
\hline 59.879 & 226.809 & Surface & 2006 & 59.879 & 217.998 & Surface & 2011 \\
\hline 62.873 & 226.814 & Surface & 2006 & 62.873 & 217.666 & Surface & 2011 \\
\hline 65.867 & 226.787 & Surface & 2006 & 65.867 & 217.121 & Surface & 2011 \\
\hline 68.861 & 226.812 & Surface & 2006 & 68.861 & 216.808 & Surface & 2011 \\
\hline 71.855 & 226.789 & Surface & 2006 & 71.855 & 216.711 & Surface & 2011 \\
\hline 74.849 & 226.739 & Surface & 2006 & 74.849 & 216.667 & Surface & 2011 \\
\hline 77.843 & 226.710 & Surface & 2006 & 77.843 & 216.648 & Surface & 2011 \\
\hline 80.836 & 226.747 & Surface & 2006 & 80.836 & 216.647 & Surface & 2011 \\
\hline 83.831 & 226.799 & Surface & 2006 & 83.831 & 216.639 & Surface & 2011 \\
\hline 86.824 & 226.855 & Surface & 2006 & 86.824 & 216.633 & Surface & 2011 \\
\hline 89.818 & 226.910 & Surface & 2006 & 89.818 & 216.633 & Surface & 2011 \\
\hline 92.812 & 226.900 & Surface & 2006 & 92.812 & 216.647 & Surface & 2011 \\
\hline 95.806 & 226.876 & Surface & 2006 & 95.806 & 216.657 & Surface & 2011 \\
\hline 98.800 & 226.866 & Surface & 2006 & 98.800 & 216.667 & Surface & 2011 \\
\hline 101.794 & 226.800 & Surface & 2006 & 101.794 & 216.656 & Surface & 2011 \\
\hline 104.788 & 226.755 & Surface & 2006 & 104.788 & 216.648 & Surface & 2011 \\
\hline 107.782 & 226.707 & Surface & 2006 & 107.782 & 216.604 & Surface & 2011 \\
\hline 110.776 & 226.691 & Surface & 2006 & 110.776 & 216.557 & Surface & 2011 \\
\hline 113.770 & 226.649 & Surface & 2006 & 113.770 & 216.570 & Surface & 2011 \\
\hline 116.764 & 226.581 & Surface & 2006 & 116.764 & 216.511 & Surface & 2011 \\
\hline 119.758 & 226.489 & Surface & 2006 & 119.758 & 216.510 & Surface & 2011 \\
\hline 122.752 & 226.271 & Surface & 2006 & 122.752 & 216.600 & Surface & 2011 \\
\hline 125.746 & 226.045 & Surface & 2006 & 125.746 & 216.701 & Surface & 2011 \\
\hline 128.740 & 225.851 & Surface & 2006 & 128.740 & 216.765 & Surface & 2011 \\
\hline 131.734 & 225.650 & Surface & 2006 & 131.734 & 216.836 & Surface & 2011 \\
\hline
\end{tabular}




\begin{tabular}{|c|c|c|c|c|c|c|}
\hline 134.727 & 225.508 & Surface & 2006 & 134.727 & 217.072 & Surface \\
\hline 137.721 & 225.430 & Surface & 2006 & 137.721 & 217.292 & Surface \\
\hline 140.715 & 225.392 & Surface & 2006 & 140.715 & 217.374 & Surface \\
\hline 143.709 & 225.362 & Surface & 2006 & 143.709 & 217.427 & Surface \\
\hline 146.703 & 225.403 & Surface & 2006 & 146.703 & 217.485 & Surface \\
\hline 149.697 & 225.424 & Surface & 2006 & 149.697 & 217.569 & Surface \\
\hline 152.691 & 225.453 & Surface & 2006 & 152.691 & 217.648 & Surface \\
\hline 155.685 & 225.492 & Surface & 2006 & 155.685 & 217.734 & Surface \\
\hline 158.679 & 225.508 & Surface & 2006 & 158.679 & 217.829 & Surface \\
\hline 161.673 & 225.516 & Surface & 2006 & 161.673 & 217.909 & Surface \\
\hline 164.667 & 225.460 & Surface & 2006 & 164.667 & 217.947 & Surface \\
\hline 167.661 & 225.392 & Surface & 2006 & 167.661 & 217.928 & Surface \\
\hline 170.655 & 225.341 & Surface & 2006 & 170.655 & 217.951 & Surface \\
\hline 173.649 & 225.287 & Surface & 2006 & 173.649 & 217.990 & Surface \\
\hline 176.643 & 225.208 & Surface & 2006 & 176.643 & 217.996 & Surface \\
\hline 179.637 & 225.102 & Surface & 2006 & 179.637 & 217.989 & Surface \\
\hline 182.630 & 225.008 & Surface & 2006 & 182.630 & 217.969 & Surface \\
\hline 185.625 & 224.912 & Surface & 2006 & 185.625 & 217.981 & Surface \\
\hline 188.618 & 224.894 & Surface & 2006 & 188.618 & 218.017 & Surface \\
\hline 191.612 & 224.895 & Surface & 2006 & 191.612 & 218.037 & Surface \\
\hline 194.607 & 224.878 & Surface & 2006 & 194.607 & 218.042 & Surface \\
\hline 197.600 & 224.861 & Surface & 2006 & 197.600 & 218.054 & Surface \\
\hline 200.594 & 224.867 & Surface & 2006 & 200.594 & 218.072 & Surface \\
\hline 203.588 & 224.884 & Surface & 2006 & 203.588 & 218.080 & Surface \\
\hline 206.582 & 224.906 & Surface & 2006 & 206.582 & 218.069 & Surface \\
\hline 209.576 & 224.928 & Surface & 2006 & 209.576 & 218.062 & Surface \\
\hline 212.570 & 224.954 & Surface & 2006 & 212.570 & 218.073 & Surface \\
\hline 215.564 & 224.970 & Surface & 2006 & 215.564 & 218.077 & Surface \\
\hline 218.558 & 224.952 & Surface & 2006 & 218.558 & 218.075 & Surface \\
\hline 221.552 & 224.924 & Surface & 2006 & 221.552 & 218.072 & Surface \\
\hline 224.546 & 224.897 & Surface & 2006 & 224.546 & 218.067 & Surface \\
\hline 227.540 & 224.895 & Surface & 2006 & 227.540 & 218.078 & Surface \\
\hline 230.534 & 224.899 & Surface & 2006 & 230.534 & 218.099 & Surface \\
\hline 233.528 & 224.919 & Surface & 2006 & 233.528 & 218.104 & Surface \\
\hline 236.521 & 224.937 & Surface & 2006 & 236.521 & 218.090 & Surface \\
\hline 239.516 & 224.938 & Surface & 2006 & 239.516 & 218.087 & Surface \\
\hline 242.510 & 224.946 & Surface & 2006 & 242.510 & 218.105 & Surface \\
\hline 245.503 & 224.930 & Surface & 2006 & 245.503 & 218.136 & Surface \\
\hline 248.497 & 224.907 & Surface & 2006 & 248.497 & 218.157 & Surface \\
\hline 251.491 & 224.905 & Surface & 2006 & 251.491 & 218.190 & Surface \\
\hline 254.485 & 224.907 & Surface & 2006 & 254.485 & 218.223 & Surface \\
\hline 257.479 & 224.909 & Surface & 2006 & 257.479 & 218.236 & Surface \\
\hline 260.473 & 224.910 & Surface & 2006 & 260.473 & 218.258 & Surface \\
\hline 263.467 & 224.887 & Surface & 2006 & 263.467 & 218.273 & Surface \\
\hline 266.461 & 224.860 & Surface & 2006 & 266.461 & 218.242 & Surface \\
\hline 269.455 & 224.861 & Surface & 2006 & 269.455 & 218.175 & Surface \\
\hline 272.449 & 224.860 & Surface & 2006 & 272.449 & 218.137 & Surface \\
\hline 275.443 & 224.834 & Surface & 2006 & 275.443 & 218.132 & Surface \\
\hline 278.437 & 224.800 & Surface & 2006 & 278.437 & 218.110 & Surface \\
\hline
\end{tabular}




\begin{tabular}{|c|c|c|c|c|c|c|}
\hline 281.431 & 224.771 & Surface & 2006 & 281.431 & 218.136 & Surface \\
\hline 284.425 & 224.770 & Surface & 2006 & 284.425 & 218.234 & Surface \\
\hline 287.419 & 224.769 & Surface & 2006 & 287.419 & 218.348 & Surface \\
\hline 290.413 & 224.727 & Surface & 2006 & 290.413 & 218.774 & Surface \\
\hline 293.406 & 224.699 & Surface & 2006 & 293.406 & 219.687 & Surface \\
\hline 296.401 & 224.681 & Surface & 2006 & 296.401 & 220.809 & Surface \\
\hline 299.394 & 224.670 & Surface & 2006 & 299.394 & 222.125 & Surface \\
\hline 302.388 & 224.671 & Surface & 2006 & 302.388 & 223.461 & Surface \\
\hline 305.382 & 224.662 & Surface & 2006 & 305.382 & 224.152 & Surface \\
\hline 308.376 & 224.643 & Surface & 2006 & 308.376 & 224.277 & Surface \\
\hline 311.370 & 224.651 & Surface & 2006 & 311.370 & 224.342 & Surface \\
\hline 314.364 & 224.735 & Surface & 2006 & 314.364 & 224.424 & Surface \\
\hline 317.358 & 224.914 & Surface & 2006 & 317.358 & 224.601 & Surface \\
\hline 320.352 & 225.072 & Surface & 2006 & 320.352 & 224.768 & Surface \\
\hline 323.346 & 225.146 & Surface & 2006 & 323.346 & 224.752 & Surface \\
\hline 326.340 & 225.220 & Surface & 2006 & 326.340 & 224.666 & Surface \\
\hline 329.334 & 225.285 & Surface & 2006 & 329.334 & 224.546 & Surface \\
\hline 332.328 & 225.353 & Surface & 2006 & 332.328 & 224.285 & Surface \\
\hline 335.322 & 225.417 & Surface & 2006 & 335.322 & 223.900 & Surface \\
\hline 338.316 & 225.503 & Surface & 2006 & 338.316 & 223.510 & Surface \\
\hline 341.310 & 225.587 & Surface & 2006 & 341.310 & 223.182 & Surface \\
\hline 344.304 & 225.640 & Surface & 2006 & 344.304 & 222.908 & Surface \\
\hline 347.297 & 225.675 & Surface & 2006 & 347.297 & 222.700 & Surface \\
\hline 350.292 & 225.653 & Surface & 2006 & 350.292 & 222.589 & Surface \\
\hline 353.285 & 225.531 & Surface & 2006 & 353.285 & 222.506 & Surface \\
\hline 356.279 & 225.348 & Surface & 2006 & 356.279 & 222.454 & Surface \\
\hline 359.273 & 225.139 & Surface & 2006 & 359.273 & 222.489 & Surface \\
\hline 362.267 & 224.969 & Surface & 2006 & 362.267 & 222.547 & Surface \\
\hline 365.261 & 224.834 & Surface & 2006 & 365.261 & 222.533 & Surface \\
\hline 368.255 & 224.745 & Surface & 2006 & 368.255 & 222.562 & Surface \\
\hline 371.249 & 224.715 & Surface & 2006 & 371.249 & 222.719 & Surface \\
\hline 374.243 & 224.673 & Surface & 2006 & 374.243 & 222.873 & Surface \\
\hline 377.237 & 224.626 & Surface & 2006 & 377.237 & 222.983 & Surface \\
\hline 380.231 & 224.566 & Surface & 2006 & 380.231 & 223.005 & Surface \\
\hline 383.225 & 224.546 & Surface & 2006 & 383.225 & 222.976 & Surface \\
\hline 386.219 & 224.561 & Surface & 2006 & 386.219 & 222.995 & Surface \\
\hline 389.213 & 224.578 & Surface & 2006 & 389.213 & 223.037 & Surface \\
\hline 392.207 & 224.595 & Surface & 2006 & 392.207 & 223.093 & Surface \\
\hline 395.201 & 224.607 & Surface & 2006 & 395.201 & 223.163 & Surface \\
\hline 398.195 & 224.582 & Surface & 2006 & 398.195 & 223.206 & Surface \\
\hline 401.188 & 224.636 & Surface & 2006 & 401.188 & 223.207 & Surface \\
\hline 404.183 & 224.754 & Surface & 2006 & 404.183 & 223.181 & Surface \\
\hline 407.177 & 224.841 & Surface & 2006 & 407.177 & 223.192 & Surface \\
\hline 410.170 & 224.909 & Surface & 2006 & 410.170 & 223.256 & Surface \\
\hline 413.164 & 224.980 & Surface & 2006 & 413.164 & 223.353 & Surface \\
\hline 416.158 & 225.043 & Surface & 2006 & 416.158 & 223.475 & Surface \\
\hline 419.152 & 225.094 & Surface & 2006 & 419.152 & 223.475 & Surface \\
\hline 422.146 & 225.174 & Surface & 2006 & 422.146 & 223.336 & Surface \\
\hline 425.140 & 225.237 & Surface & 2006 & 425.140 & 223.218 & Surface \\
\hline
\end{tabular}




\begin{tabular}{|c|c|c|c|c|c|c|c|}
\hline 428.134 & 225.260 & Surface & 2006 & 428.134 & 223.266 & Surface & 2011 \\
\hline 431.128 & 225.248 & Surface & 2006 & 431.128 & 223.409 & Surface & 2011 \\
\hline 434.122 & 225.203 & Surface & 2006 & 434.122 & 223.663 & Surface & 2011 \\
\hline 437.116 & 225.117 & Surface & 2006 & 437.116 & 224.059 & Surface & 2011 \\
\hline 440.110 & 225.006 & Surface & 2006 & 440.110 & 224.392 & Surface & 2011 \\
\hline 443.104 & 224.887 & Surface & 2006 & 443.104 & 224.635 & Surface & 2011 \\
\hline 446.098 & 224.909 & Surface & 2006 & 446.098 & 224.944 & Surface & 2011 \\
\hline 449.092 & 225.132 & Surface & 2006 & 449.092 & 225.223 & Surface & 2011 \\
\hline 452.086 & 225.517 & Surface & 2006 & 452.086 & 225.485 & Surface & 2011 \\
\hline 455.080 & 226.049 & Surface & 2006 & 455.080 & 225.773 & Surface & 2011 \\
\hline 458.073 & 226.647 & Surface & 2006 & 458.073 & 226.089 & Surface & 2011 \\
\hline 461.068 & 226.973 & Surface & 2006 & 461.068 & 226.376 & Surface & 2011 \\
\hline 464.061 & 227.062 & Surface & 2006 & 464.061 & 226.627 & Surface & 2011 \\
\hline 467.055 & 227.138 & Surface & 2006 & 467.055 & 226.878 & Surface & 2011 \\
\hline 470.049 & 227.273 & Surface & 2006 & 470.049 & 227.128 & Surface & 2011 \\
\hline 473.043 & 227.467 & Surface & 2006 & 473.043 & 227.334 & Surface & 2011 \\
\hline 476.037 & 227.722 & Surface & 2006 & 476.037 & 227.437 & Surface & 2011 \\
\hline 479.031 & 228.076 & Surface & 2006 & 479.031 & 227.488 & Surface & 2011 \\
\hline 482.025 & 228.481 & Surface & 2006 & 482.025 & 227.599 & Surface & 2011 \\
\hline 485.019 & 228.904 & Surface & 2006 & 485.019 & 228.044 & Surface & 2011 \\
\hline 488.013 & 229.657 & Surface & 2006 & 488.013 & 228.898 & Surface & 2011 \\
\hline 491.007 & 230.702 & Surface & 2006 & 491.007 & 229.793 & Surface & 2011 \\
\hline 494.001 & 231.759 & Surface & 2006 & 494.001 & 230.765 & Surface & 2011 \\
\hline 496.995 & 232.754 & Surface & 2006 & 496.995 & 231.760 & Surface & 2011 \\
\hline 499.989 & 233.675 & Surface & 2006 & 499.989 & 232.546 & Surface & 2011 \\
\hline 502.983 & 234.569 & Surface & 2006 & 502.983 & 233.160 & Surface & 2011 \\
\hline 505.977 & 235.454 & Surface & 2006 & 505.977 & 233.791 & Surface & 2011 \\
\hline 508.971 & 236.124 & Surface & 2006 & 508.971 & 234.446 & Surface & 2011 \\
\hline 511.964 & 236.359 & Surface & 2006 & 511.964 & 235.125 & Surface & 2011 \\
\hline 514.959 & 236.344 & Surface & 2006 & 514.959 & 235.806 & Surface & 2011 \\
\hline 517.952 & 236.311 & Surface & 2006 & 517.952 & 236.266 & Surface & 2011 \\
\hline 520.946 & 236.284 & Surface & 2006 & 520.946 & 236.270 & Surface & 2011 \\
\hline 0.000 & 230.949 & Surface & 2008 & 0.000 & 231.074 & Surface & 2012 \\
\hline 3.276 & 230.932 & Surface & 2008 & 3.276 & 231.076 & Surface & 2012 \\
\hline 6.553 & 230.918 & Surface & 2008 & 6.553 & 231.129 & Surface & 2012 \\
\hline 9.829 & 230.938 & Surface & 2008 & 9.829 & 231.187 & Surface & 2012 \\
\hline 13.106 & 230.948 & Surface & 2008 & 13.106 & 231.075 & Surface & 2012 \\
\hline 16.382 & 230.832 & Surface & 2008 & 16.382 & 230.745 & Surface & 2012 \\
\hline 19.658 & 230.557 & Surface & 2008 & 19.658 & 230.448 & Surface & 2012 \\
\hline 22.935 & 230.059 & Surface & 2008 & 22.935 & 230.210 & Surface & 2012 \\
\hline 26.211 & 229.383 & Surface & 2008 & 26.211 & 229.642 & Surface & 2012 \\
\hline 29.488 & 228.491 & Surface & 2008 & 29.488 & 228.627 & Surface & 2012 \\
\hline 32.764 & 227.207 & Surface & 2008 & 32.764 & 227.478 & Surface & 2012 \\
\hline 36.040 & 225.714 & Surface & 2008 & 36.040 & 225.958 & Surface & 2012 \\
\hline 39.317 & 224.097 & Surface & 2008 & 39.317 & 224.176 & Surface & 2012 \\
\hline 42.593 & 222.377 & Surface & 2008 & 42.593 & 221.743 & Surface & 2012 \\
\hline 45.870 & 221.291 & Surface & 2008 & 45.870 & 219.556 & Surface & 2012 \\
\hline 49.146 & 220.894 & Surface & 2008 & 49.146 & 218.729 & Surface & 2012 \\
\hline 52.422 & 220.633 & Surface & 2008 & 52.422 & 218.572 & Surface & 2012 \\
\hline
\end{tabular}




\begin{tabular}{|c|c|c|c|c|c|c|c|}
\hline 55.699 & 220.459 & Surface & 2008 & 55.699 & 218.429 & Surface & 2012 \\
\hline 58.975 & 220.389 & Surface & 2008 & 58.975 & 218.148 & Surface & 2012 \\
\hline 62.251 & 220.334 & Surface & 2008 & 62.251 & 217.711 & Surface & 2012 \\
\hline 65.528 & 220.328 & Surface & 2008 & 65.528 & 217.246 & Surface & 2012 \\
\hline 68.804 & 220.322 & Surface & 2008 & 68.804 & 217.013 & Surface & 2012 \\
\hline 72.081 & 220.324 & Surface & 2008 & 72.081 & 216.971 & Surface & 2012 \\
\hline 75.357 & 220.388 & Surface & 2008 & 75.357 & 216.997 & Surface & 2012 \\
\hline 78.634 & 220.463 & Surface & 2008 & 78.634 & 217.025 & Surface & 2012 \\
\hline 81.910 & 220.501 & Surface & 2008 & 81.910 & 217.050 & Surface & 2012 \\
\hline 85.186 & 220.518 & Surface & 2008 & 85.186 & 217.081 & Surface & 2012 \\
\hline 88.463 & 220.527 & Surface & 2008 & 88.463 & 217.145 & Surface & 2012 \\
\hline 91.739 & 220.533 & Surface & 2008 & 91.739 & 217.265 & Surface & 2012 \\
\hline 95.015 & 220.524 & Surface & 2008 & 95.015 & 217.337 & Surface & 2012 \\
\hline 98.292 & 220.528 & Surface & 2008 & 98.292 & 217.355 & Surface & 2012 \\
\hline 101.568 & 220.512 & Surface & 2008 & 101.568 & 217.293 & Surface & 2012 \\
\hline 104.845 & 220.484 & Surface & 2008 & 104.845 & 217.258 & Surface & 2012 \\
\hline 108.121 & 220.459 & Surface & 2008 & 108.121 & 217.322 & Surface & 2012 \\
\hline 111.397 & 220.352 & Surface & 2008 & 111.397 & 217.350 & Surface & 2012 \\
\hline 114.674 & 220.083 & Surface & 2008 & 114.674 & 217.364 & Surface & 2012 \\
\hline 117.950 & 219.647 & Surface & 2008 & 117.950 & 217.428 & Surface & 2012 \\
\hline 121.226 & 219.247 & Surface & 2008 & 121.226 & 217.493 & Surface & 2012 \\
\hline 124.503 & 218.975 & Surface & 2008 & 124.503 & 217.534 & Surface & 2012 \\
\hline 127.779 & 218.763 & Surface & 2008 & 127.779 & 217.541 & Surface & 2012 \\
\hline 131.056 & 218.560 & Surface & 2008 & 131.056 & 217.545 & Surface & 2012 \\
\hline 134.332 & 218.395 & Surface & 2008 & 134.332 & 217.591 & Surface & 2012 \\
\hline 137.609 & 218.322 & Surface & 2008 & 137.609 & 217.660 & Surface & 2012 \\
\hline 140.885 & 218.313 & Surface & 2008 & 140.885 & 217.738 & Surface & 2012 \\
\hline 144.161 & 218.315 & Surface & 2008 & 144.161 & 217.767 & Surface & 2012 \\
\hline 147.437 & 218.298 & Surface & 2008 & 147.437 & 217.766 & Surface & 2012 \\
\hline 150.714 & 218.274 & Surface & 2008 & 150.714 & 217.809 & Surface & 2012 \\
\hline 153.990 & 218.250 & Surface & 2008 & 153.990 & 217.867 & Surface & 2012 \\
\hline 157.267 & 218.228 & Surface & 2008 & 157.267 & 217.898 & Surface & 2012 \\
\hline 160.543 & 218.221 & Surface & 2008 & 160.543 & 217.902 & Surface & 2012 \\
\hline 163.820 & 218.227 & Surface & 2008 & 163.820 & 217.900 & Surface & 2012 \\
\hline 167.096 & 218.221 & Surface & 2008 & 167.096 & 217.904 & Surface & 2012 \\
\hline 170.372 & 218.206 & Surface & 2008 & 170.372 & 217.918 & Surface & 2012 \\
\hline 173.649 & 218.203 & Surface & 2008 & 173.649 & 217.940 & Surface & 2012 \\
\hline 176.925 & 218.238 & Surface & 2008 & 176.925 & 217.950 & Surface & 2012 \\
\hline 180.201 & 218.287 & Surface & 2008 & 180.201 & 217.955 & Surface & 2012 \\
\hline 183.478 & 218.308 & Surface & 2008 & 183.478 & 217.963 & Surface & 2012 \\
\hline 186.754 & 218.280 & Surface & 2008 & 186.754 & 217.962 & Surface & 2012 \\
\hline 190.031 & 218.269 & Surface & 2008 & 190.031 & 217.954 & Surface & 2012 \\
\hline 193.307 & 218.274 & Surface & 2008 & 193.307 & 217.934 & Surface & 2012 \\
\hline 196.584 & 218.283 & Surface & 2008 & 196.584 & 217.929 & Surface & 2012 \\
\hline 199.860 & 218.304 & Surface & 2008 & 199.860 & 217.932 & Surface & 2012 \\
\hline 203.136 & 218.284 & Surface & 2008 & 203.136 & 217.923 & Surface & 2012 \\
\hline 206.413 & 218.284 & Surface & 2008 & 206.413 & 217.920 & Surface & 2012 \\
\hline 209.689 & 218.313 & Surface & 2008 & 209.689 & 217.918 & Surface & 2012 \\
\hline 212.965 & 218.325 & Surface & 2008 & 212.965 & 217.937 & Surface & 2012 \\
\hline
\end{tabular}




\begin{tabular}{|c|c|c|c|c|c|c|c|}
\hline 216.242 & 218.332 & Surface & 2008 & 216.242 & 217.960 & Surface & 2012 \\
\hline 219.518 & 218.353 & Surface & 2008 & 219.518 & 217.965 & Surface & 2012 \\
\hline 222.795 & 218.360 & Surface & 2008 & 222.795 & 217.954 & Surface & 2012 \\
\hline 226.071 & 218.304 & Surface & 2008 & 226.071 & 218.002 & Surface & 2012 \\
\hline 229.347 & 218.327 & Surface & 2008 & 229.347 & 218.103 & Surface & 2012 \\
\hline 232.624 & 218.441 & Surface & 2008 & 232.624 & 218.177 & Surface & 2012 \\
\hline 235.900 & 218.508 & Surface & 2008 & 235.900 & 218.201 & Surface & 2012 \\
\hline 239.177 & 218.553 & Surface & 2008 & 239.177 & 218.195 & Surface & 2012 \\
\hline 242.453 & 218.603 & Surface & 2008 & 242.453 & 218.244 & Surface & 2012 \\
\hline 245.729 & 218.672 & Surface & 2008 & 245.729 & 218.306 & Surface & 2012 \\
\hline 249.006 & 218.716 & Surface & 2008 & 249.006 & 218.357 & Surface & 2012 \\
\hline 252.282 & 218.720 & Surface & 2008 & 252.282 & 218.435 & Surface & 2012 \\
\hline 255.559 & 218.785 & Surface & 2008 & 255.559 & 218.514 & Surface & 2012 \\
\hline 258.835 & 218.919 & Surface & 2008 & 258.835 & 218.601 & Surface & 2012 \\
\hline 262.111 & 219.058 & Surface & 2008 & 262.111 & 218.682 & Surface & 2012 \\
\hline 265.388 & 219.188 & Surface & 2008 & 265.388 & 218.700 & Surface & 2012 \\
\hline 268.664 & 219.312 & Surface & 2008 & 268.664 & 218.587 & Surface & 2012 \\
\hline 271.941 & 219.416 & Surface & 2008 & 271.941 & 218.360 & Surface & 2012 \\
\hline 275.217 & 219.508 & Surface & 2008 & 275.217 & 218.117 & Surface & 2012 \\
\hline 278.493 & 219.578 & Surface & 2008 & 278.493 & 218.007 & Surface & 2012 \\
\hline 281.770 & 219.549 & Surface & 2008 & 281.770 & 218.006 & Surface & 2012 \\
\hline 285.046 & 219.426 & Surface & 2008 & 285.046 & 218.028 & Surface & 2012 \\
\hline 288.322 & 219.289 & Surface & 2008 & 288.322 & 218.263 & Surface & 2012 \\
\hline 291.599 & 219.633 & Surface & 2008 & 291.599 & 219.187 & Surface & 2012 \\
\hline 294.875 & 220.809 & Surface & 2008 & 294.875 & 220.485 & Surface & 2012 \\
\hline 298.152 & 222.318 & Surface & 2008 & 298.152 & 222.183 & Surface & 2012 \\
\hline 301.428 & 223.663 & Surface & 2008 & 301.428 & 223.836 & Surface & 2012 \\
\hline 304.705 & 224.296 & Surface & 2008 & 304.705 & 224.308 & Surface & 2012 \\
\hline 307.981 & 224.275 & Surface & 2008 & 307.981 & 224.320 & Surface & 2012 \\
\hline 311.257 & 224.364 & Surface & 2008 & 311.257 & 224.395 & Surface & 2012 \\
\hline 314.534 & 224.507 & Surface & 2008 & 314.534 & 224.545 & Surface & 2012 \\
\hline 317.810 & 224.745 & Surface & 2008 & 317.810 & 224.783 & Surface & 2012 \\
\hline 321.086 & 225.042 & Surface & 2008 & 321.086 & 225.057 & Surface & 2012 \\
\hline 324.363 & 225.157 & Surface & 2008 & 324.363 & 225.067 & Surface & 2012 \\
\hline 327.639 & 225.233 & Surface & 2008 & 327.639 & 224.743 & Surface & 2012 \\
\hline 330.916 & 225.351 & Surface & 2008 & 330.916 & 224.317 & Surface & 2012 \\
\hline 334.192 & 225.287 & Surface & 2008 & 334.192 & 223.895 & Surface & 2012 \\
\hline 337.469 & 224.872 & Surface & 2008 & 337.469 & 223.480 & Surface & 2012 \\
\hline 340.745 & 224.425 & Surface & 2008 & 340.745 & 223.151 & Surface & 2012 \\
\hline 344.021 & 224.102 & Surface & 2008 & 344.021 & 222.814 & Surface & 2012 \\
\hline 347.297 & 223.791 & Surface & 2008 & 347.297 & 222.579 & Surface & 2012 \\
\hline 350.574 & 223.512 & Surface & 2008 & 350.574 & 222.582 & Surface & 2012 \\
\hline 353.850 & 223.240 & Surface & 2008 & 353.850 & 222.598 & Surface & 2012 \\
\hline 357.127 & 222.982 & Surface & 2008 & 357.127 & 222.557 & Surface & 2012 \\
\hline 360.403 & 222.806 & Surface & 2008 & 360.403 & 222.611 & Surface & 2012 \\
\hline 363.680 & 222.733 & Surface & 2008 & 363.680 & 222.620 & Surface & 2012 \\
\hline 366.956 & 222.808 & Surface & 2008 & 366.956 & 222.522 & Surface & 2012 \\
\hline 370.232 & 222.996 & Surface & 2008 & 370.232 & 222.573 & Surface & 2012 \\
\hline 373.509 & 223.236 & Surface & 2008 & 373.509 & 222.804 & Surface & 2012 \\
\hline
\end{tabular}




\begin{tabular}{|c|c|c|c|c|c|c|c|}
\hline 376.785 & 223.495 & Surface & 2008 & 376.785 & 222.966 & Surface & 2012 \\
\hline 380.061 & 223.809 & Surface & 2008 & 380.061 & 223.111 & Surface & 2012 \\
\hline 383.338 & 224.202 & Surface & 2008 & 383.338 & 223.277 & Surface & 2012 \\
\hline 386.614 & 224.411 & Surface & 2008 & 386.614 & 223.382 & Surface & 2012 \\
\hline 389.891 & 224.416 & Surface & 2008 & 389.891 & 223.258 & Surface & 2012 \\
\hline 393.167 & 224.436 & Surface & 2008 & 393.167 & 223.178 & Surface & 2012 \\
\hline 396.444 & 224.486 & Surface & 2008 & 396.444 & 223.197 & Surface & 2012 \\
\hline 399.720 & 224.564 & Surface & 2008 & 399.720 & 223.177 & Surface & 2012 \\
\hline 402.996 & 224.671 & Surface & 2008 & 402.996 & 223.178 & Surface & 2012 \\
\hline 406.273 & 224.767 & Surface & 2008 & 406.273 & 223.168 & Surface & 2012 \\
\hline 409.549 & 224.858 & Surface & 2008 & 409.549 & 223.158 & Surface & 2012 \\
\hline 412.825 & 224.947 & Surface & 2008 & 412.825 & 223.303 & Surface & 2012 \\
\hline 416.102 & 225.019 & Surface & 2008 & 416.102 & 223.399 & Surface & 2012 \\
\hline 419.378 & 225.083 & Surface & 2008 & 419.378 & 223.315 & Surface & 2012 \\
\hline 422.655 & 225.153 & Surface & 2008 & 422.655 & 223.221 & Surface & 2012 \\
\hline 425.931 & 225.241 & Surface & 2008 & 425.931 & 223.212 & Surface & 2012 \\
\hline 429.207 & 225.292 & Surface & 2008 & 429.207 & 223.218 & Surface & 2012 \\
\hline 432.484 & 225.255 & Surface & 2008 & 432.484 & 223.465 & Surface & 2012 \\
\hline 435.760 & 225.164 & Surface & 2008 & 435.760 & 223.892 & Surface & 2012 \\
\hline 439.037 & 225.064 & Surface & 2008 & 439.037 & 224.316 & Surface & 2012 \\
\hline 442.313 & 224.963 & Surface & 2008 & 442.313 & 224.710 & Surface & 2012 \\
\hline 445.589 & 224.907 & Surface & 2008 & 445.589 & 224.962 & Surface & 2012 \\
\hline 448.866 & 225.042 & Surface & 2008 & 448.866 & 225.152 & Surface & 2012 \\
\hline 452.142 & 225.386 & Surface & 2008 & 452.142 & 225.491 & Surface & 2012 \\
\hline 455.418 & 225.816 & Surface & 2008 & 455.418 & 225.897 & Surface & 2012 \\
\hline 458.695 & 226.269 & Surface & 2008 & 458.695 & 226.363 & Surface & 2012 \\
\hline 461.971 & 226.696 & Surface & 2008 & 461.971 & 226.899 & Surface & 2012 \\
\hline 465.248 & 227.143 & Surface & 2008 & 465.248 & 227.305 & Surface & 2012 \\
\hline 468.524 & 227.471 & Surface & 2008 & 468.524 & 227.512 & Surface & 2012 \\
\hline 471.801 & 227.570 & Surface & 2008 & 471.801 & 227.566 & Surface & 2012 \\
\hline 475.077 & 227.561 & Surface & 2008 & 475.077 & 227.565 & Surface & 2012 \\
\hline 478.353 & 227.598 & Surface & 2008 & 478.353 & 227.596 & Surface & 2012 \\
\hline 481.630 & 228.116 & Surface & 2008 & 481.630 & 227.833 & Surface & 2012 \\
\hline 484.906 & 229.226 & Surface & 2008 & 484.906 & 228.377 & Surface & 2012 \\
\hline 488.182 & 230.334 & Surface & 2008 & 488.182 & 229.197 & Surface & 2012 \\
\hline 491.459 & 231.438 & Surface & 2008 & 491.459 & 230.172 & Surface & 2012 \\
\hline 494.735 & 232.553 & Surface & 2008 & 494.735 & 231.328 & Surface & 2012 \\
\hline 498.012 & 233.745 & Surface & 2008 & 498.012 & 232.823 & Surface & 2012 \\
\hline 501.288 & 234.855 & Surface & 2008 & 501.288 & 234.394 & Surface & 2012 \\
\hline 504.565 & 235.489 & Surface & 2008 & 504.565 & 235.562 & Surface & 2012 \\
\hline 507.841 & 235.804 & Surface & 2008 & 507.841 & 236.127 & Surface & 2012 \\
\hline 511.117 & 236.034 & Surface & 2008 & 511.117 & 236.431 & Surface & 2012 \\
\hline 514.393 & 236.146 & Surface & 2008 & 514.393 & 236.454 & Surface & 2012 \\
\hline 517.670 & 236.258 & Surface & 2008 & 517.670 & 236.430 & Surface & 2012 \\
\hline 520.946 & 236.234 & Surface & 2008 & 520.946 & 236.359 & Surface & 2012 \\
\hline
\end{tabular}


10.5 Transect E - End of Rapids/Pool Bank - All Returns:

\begin{tabular}{|c|c|c|c|c|c|c|c|}
\hline Distance & $\mathrm{Z}$ & DEM & Year & Distance & $\mathrm{Z}$ & DEM & Year \\
\hline 0.000 & 231.918 & Surface & 2006 & 0.000 & 232.047 & Surface & 2011 \\
\hline 2.989 & 231.636 & Surface & 2006 & 2.989 & 232.039 & Surface & 2011 \\
\hline 5.979 & 231.335 & Surface & 2006 & 5.979 & 232.022 & Surface & 2011 \\
\hline 8.968 & 231.015 & Surface & 2006 & 8.968 & 231.966 & Surface & 2011 \\
\hline 11.957 & 230.714 & Surface & 2006 & 11.957 & 231.846 & Surface & 2011 \\
\hline 14.946 & 230.413 & Surface & 2006 & 14.946 & 231.728 & Surface & 2011 \\
\hline 17.936 & 230.114 & Surface & 2006 & 17.936 & 231.543 & Surface & 2011 \\
\hline 20.925 & 229.804 & Surface & 2006 & 20.925 & 231.014 & Surface & 2011 \\
\hline 23.915 & 229.443 & Surface & 2006 & 23.915 & 230.394 & Surface & 2011 \\
\hline 26.904 & 229.073 & Surface & 2006 & 26.904 & 229.798 & Surface & 2011 \\
\hline 29.893 & 228.752 & Surface & 2006 & 29.893 & 229.193 & Surface & 2011 \\
\hline 32.882 & 228.474 & Surface & 2006 & 32.882 & 228.583 & Surface & 2011 \\
\hline 35.872 & 228.205 & Surface & 2006 & 35.872 & 227.974 & Surface & 2011 \\
\hline 38.861 & 227.904 & Surface & 2006 & 38.861 & 227.659 & Surface & 2011 \\
\hline 41.850 & 227.559 & Surface & 2006 & 41.850 & 227.504 & Surface & 2011 \\
\hline 44.839 & 227.327 & Surface & 2006 & 44.839 & 227.364 & Surface & 2011 \\
\hline 47.829 & 227.283 & Surface & 2006 & 47.829 & 227.277 & Surface & 2011 \\
\hline 50.818 & 227.187 & Surface & 2006 & 50.818 & 227.245 & Surface & 2011 \\
\hline 53.807 & 227.058 & Surface & 2006 & 53.807 & 227.212 & Surface & 2011 \\
\hline 56.797 & 226.924 & Surface & 2006 & 56.797 & 227.144 & Surface & 2011 \\
\hline 59.786 & 226.791 & Surface & 2006 & 59.786 & 227.048 & Surface & 2011 \\
\hline 62.775 & 226.663 & Surface & 2006 & 62.775 & 226.911 & Surface & 2011 \\
\hline 65.765 & 226.637 & Surface & 2006 & 65.765 & 226.728 & Surface & 2011 \\
\hline 68.754 & 226.675 & Surface & 2006 & 68.754 & 226.594 & Surface & 2011 \\
\hline 71.743 & 226.717 & Surface & 2006 & 71.743 & 226.670 & Surface & 2011 \\
\hline 74.733 & 226.738 & Surface & 2006 & 74.733 & 226.775 & Surface & 2011 \\
\hline 77.722 & 226.753 & Surface & 2006 & 77.722 & 226.778 & Surface & 2011 \\
\hline 80.711 & 226.701 & Surface & 2006 & 80.711 & 226.748 & Surface & 2011 \\
\hline 83.700 & 226.692 & Surface & 2006 & 83.700 & 226.715 & Surface & 2011 \\
\hline 86.690 & 226.745 & Surface & 2006 & 86.690 & 226.732 & Surface & 2011 \\
\hline 89.679 & 226.825 & Surface & 2006 & 89.679 & 226.788 & Surface & 2011 \\
\hline 92.668 & 226.906 & Surface & 2006 & 92.668 & 226.840 & Surface & 2011 \\
\hline 95.658 & 226.933 & Surface & 2006 & 95.658 & 226.879 & Surface & 2011 \\
\hline 98.647 & 226.893 & Surface & 2006 & 98.647 & 226.888 & Surface & 2011 \\
\hline 101.636 & 226.844 & Surface & 2006 & 101.636 & 226.884 & Surface & 2011 \\
\hline 104.626 & 226.824 & Surface & 2006 & 104.626 & 226.896 & Surface & 2011 \\
\hline 107.615 & 226.854 & Surface & 2006 & 107.615 & 226.921 & Surface & 2011 \\
\hline 110.604 & 226.894 & Surface & 2006 & 110.604 & 226.946 & Surface & 2011 \\
\hline 113.594 & 226.891 & Surface & 2006 & 113.594 & 226.856 & Surface & 2011 \\
\hline 116.583 & 226.854 & Surface & 2006 & 116.583 & 226.632 & Surface & 2011 \\
\hline 119.572 & 226.819 & Surface & 2006 & 119.572 & 226.364 & Surface & 2011 \\
\hline 122.561 & 226.830 & Surface & 2006 & 122.561 & 226.021 & Surface & 2011 \\
\hline 125.551 & 226.861 & Surface & 2006 & 125.551 & 225.596 & Surface & 2011 \\
\hline 128.540 & 226.860 & Surface & 2006 & 128.540 & 225.099 & Surface & 2011 \\
\hline 131.529 & 226.848 & Surface & 2006 & 131.529 & 224.564 & Surface & 2011 \\
\hline
\end{tabular}




\begin{tabular}{|c|c|c|c|c|c|c|}
\hline 134.518 & 226.853 & Surface & 2006 & 134.518 & 224.030 & Surface \\
\hline 137.508 & 226.868 & Surface & 2006 & 137.508 & 223.580 & Surface \\
\hline 140.497 & 226.843 & Surface & 2006 & 140.497 & 223.118 & Surface \\
\hline 143.486 & 226.629 & Surface & 2006 & 143.486 & 222.553 & Surface \\
\hline 146.476 & 226.309 & Surface & 2006 & 146.476 & 222.147 & Surface \\
\hline 149.465 & 225.966 & Surface & 2006 & 149.465 & 222.001 & Surface \\
\hline 152.454 & 225.597 & Surface & 2006 & 152.454 & 221.987 & Surface \\
\hline 155.444 & 225.268 & Surface & 2006 & 155.444 & 222.021 & Surface \\
\hline 158.433 & 225.107 & Surface & 2006 & 158.433 & 222.133 & Surface \\
\hline 161.422 & 225.067 & Surface & 2006 & 161.422 & 222.299 & Surface \\
\hline 164.412 & 225.037 & Surface & 2006 & 164.412 & 222.386 & Surface \\
\hline 167.401 & 224.994 & Surface & 2006 & 167.401 & 222.356 & Surface \\
\hline 170.390 & 224.986 & Surface & 2006 & 170.390 & 222.198 & Surface \\
\hline 173.379 & 225.027 & Surface & 2006 & 173.379 & 221.931 & Surface \\
\hline 176.369 & 225.087 & Surface & 2006 & 176.369 & 221.606 & Surface \\
\hline 179.358 & 225.116 & Surface & 2006 & 179.358 & 221.232 & Surface \\
\hline 182.347 & 225.124 & Surface & 2006 & 182.347 & 220.934 & Surface \\
\hline 185.337 & 225.132 & Surface & 2006 & 185.337 & 220.640 & Surface \\
\hline 188.326 & 225.140 & Surface & 2006 & 188.326 & 220.294 & Surface \\
\hline 191.315 & 225.147 & Surface & 2006 & 191.315 & 219.939 & Surface \\
\hline 194.305 & 225.154 & Surface & 2006 & 194.305 & 219.597 & Surface \\
\hline 197.294 & 225.145 & Surface & 2006 & 197.294 & 219.366 & Surface \\
\hline 200.283 & 225.114 & Surface & 2006 & 200.283 & 219.308 & Surface \\
\hline 203.273 & 225.092 & Surface & 2006 & 203.273 & 219.309 & Surface \\
\hline 206.262 & 225.089 & Surface & 2006 & 206.262 & 219.321 & Surface \\
\hline 209.251 & 225.107 & Surface & 2006 & 209.251 & 219.302 & Surface \\
\hline 212.240 & 225.110 & Surface & 2006 & 212.240 & 219.222 & Surface \\
\hline 215.230 & 225.089 & Surface & 2006 & 215.230 & 219.107 & Surface \\
\hline 218.219 & 225.083 & Surface & 2006 & 218.219 & 219.011 & Surface \\
\hline 221.208 & 225.118 & Surface & 2006 & 221.208 & 218.922 & Surface \\
\hline 224.197 & 225.109 & Surface & 2006 & 224.197 & 218.888 & Surface \\
\hline 227.187 & 225.048 & Surface & 2006 & 227.187 & 218.852 & Surface \\
\hline 230.176 & 225.047 & Surface & 2006 & 230.176 & 218.790 & Surface \\
\hline 233.166 & 225.057 & Surface & 2006 & 233.166 & 218.750 & Surface \\
\hline 236.155 & 225.020 & Surface & 2006 & 236.155 & 218.703 & Surface \\
\hline 239.144 & 225.011 & Surface & 2006 & 239.144 & 218.658 & Surface \\
\hline 242.133 & 225.052 & Surface & 2006 & 242.133 & 218.614 & Surface \\
\hline 245.123 & 225.104 & Surface & 2006 & 245.123 & 218.559 & Surface \\
\hline 248.112 & 225.128 & Surface & 2006 & 248.112 & 218.495 & Surface \\
\hline 251.101 & 225.132 & Surface & 2006 & 251.101 & 218.410 & Surface \\
\hline 254.091 & 225.127 & Surface & 2006 & 254.091 & 218.336 & Surface \\
\hline 257.080 & 225.110 & Surface & 2006 & 257.080 & 218.352 & Surface \\
\hline 260.069 & 225.083 & Surface & 2006 & 260.069 & 218.508 & Surface \\
\hline 263.058 & 225.038 & Surface & 2006 & 263.058 & 218.692 & Surface \\
\hline 266.048 & 225.011 & Surface & 2006 & 266.048 & 218.921 & Surface \\
\hline 269.037 & 225.007 & Surface & 2006 & 269.037 & 219.227 & Surface \\
\hline 272.027 & 225.009 & Surface & 2006 & 272.027 & 219.568 & Surface \\
\hline 275.016 & 225.001 & Surface & 2006 & 275.016 & 219.863 & Surface \\
\hline 278.005 & 224.974 & Surface & 2006 & 278.005 & 220.075 & Surface \\
\hline
\end{tabular}




\begin{tabular}{|c|c|c|c|c|c|c|}
\hline 280.994 & 224.956 & Surface & 2006 & 280.994 & 220.191 & Surface \\
\hline 283.984 & 224.965 & Surface & 2006 & 283.984 & 220.310 & Surface \\
\hline 286.973 & 224.981 & Surface & 2006 & 286.973 & 220.404 & Surface \\
\hline 289.962 & 225.029 & Surface & 2006 & 289.962 & 220.438 & Surface \\
\hline 292.952 & 225.121 & Surface & 2006 & 292.952 & 220.456 & Surface \\
\hline 295.941 & 225.227 & Surface & 2006 & 295.941 & 220.516 & Surface \\
\hline 298.930 & 225.323 & Surface & 2006 & 298.930 & 220.534 & Surface \\
\hline 301.919 & 225.440 & Surface & 2006 & 301.919 & 220.468 & Surface \\
\hline 304.909 & 225.555 & Surface & 2006 & 304.909 & 220.377 & Surface \\
\hline 307.898 & 225.637 & Surface & 2006 & 307.898 & 220.369 & Surface \\
\hline 310.888 & 225.729 & Surface & 2006 & 310.888 & 220.385 & Surface \\
\hline 313.877 & 225.825 & Surface & 2006 & 313.877 & 220.327 & Surface \\
\hline 316.866 & 225.884 & Surface & 2006 & 316.866 & 220.248 & Surface \\
\hline 319.855 & 225.905 & Surface & 2006 & 319.855 & 220.222 & Surface \\
\hline 322.845 & 225.958 & Surface & 2006 & 322.845 & 220.178 & Surface \\
\hline 325.834 & 226.045 & Surface & 2006 & 325.834 & 220.127 & Surface \\
\hline 328.823 & 226.079 & Surface & 2006 & 328.823 & 220.097 & Surface \\
\hline 331.812 & 226.127 & Surface & 2006 & 331.812 & 220.041 & Surface \\
\hline 334.802 & 226.220 & Surface & 2006 & 334.802 & 219.970 & Surface \\
\hline 337.791 & 226.413 & Surface & 2006 & 337.791 & 219.937 & Surface \\
\hline 340.780 & 226.713 & Surface & 2006 & 340.780 & 219.918 & Surface \\
\hline 343.770 & 226.892 & Surface & 2006 & 343.770 & 219.895 & Surface \\
\hline 346.759 & 226.957 & Surface & 2006 & 346.759 & 219.832 & Surface \\
\hline 349.748 & 226.987 & Surface & 2006 & 349.748 & 219.742 & Surface \\
\hline 352.738 & 227.037 & Surface & 2006 & 352.738 & 219.662 & Surface \\
\hline 355.727 & 227.088 & Surface & 2006 & 355.727 & 219.579 & Surface \\
\hline 358.716 & 227.117 & Surface & 2006 & 358.716 & 219.487 & Surface \\
\hline 361.706 & 227.127 & Surface & 2006 & 361.706 & 219.324 & Surface \\
\hline 364.695 & 227.107 & Surface & 2006 & 364.695 & 219.089 & Surface \\
\hline 367.684 & 227.091 & Surface & 2006 & 367.684 & 218.824 & Surface \\
\hline 370.673 & 227.086 & Surface & 2006 & 370.673 & 218.565 & Surface \\
\hline 373.663 & 227.085 & Surface & 2006 & 373.663 & 218.380 & Surface \\
\hline 376.652 & 227.078 & Surface & 2006 & 376.652 & 218.295 & Surface \\
\hline 379.641 & 227.043 & Surface & 2006 & 379.641 & 218.224 & Surface \\
\hline 382.631 & 227.014 & Surface & 2006 & 382.631 & 218.170 & Surface \\
\hline 385.620 & 226.999 & Surface & 2006 & 385.620 & 218.128 & Surface \\
\hline 388.609 & 226.971 & Surface & 2006 & 388.609 & 218.101 & Surface \\
\hline 391.599 & 226.923 & Surface & 2006 & 391.599 & 218.138 & Surface \\
\hline 394.588 & 226.864 & Surface & 2006 & 394.588 & 218.143 & Surface \\
\hline 397.577 & 226.772 & Surface & 2006 & 397.577 & 218.151 & Surface \\
\hline 400.567 & 226.592 & Surface & 2006 & 400.567 & 218.159 & Surface \\
\hline 403.556 & 226.253 & Surface & 2006 & 403.556 & 218.154 & Surface \\
\hline 406.545 & 225.782 & Surface & 2006 & 406.545 & 218.151 & Surface \\
\hline 409.534 & 225.276 & Surface & 2006 & 409.534 & 218.124 & Surface \\
\hline 412.524 & 224.829 & Surface & 2006 & 412.524 & 218.126 & Surface \\
\hline 415.513 & 224.746 & Surface & 2006 & 415.513 & 218.121 & Surface \\
\hline 418.502 & 224.731 & Surface & 2006 & 418.502 & 218.098 & Surface \\
\hline 421.491 & 224.683 & Surface & 2006 & 421.491 & 218.161 & Surface \\
\hline 424.481 & 224.630 & Surface & 2006 & 424.481 & 218.194 & Surface \\
\hline
\end{tabular}




\begin{tabular}{|c|c|c|c|c|c|c|c|}
\hline 427.470 & 224.710 & Surface & 2006 & 427.470 & 218.141 & Surface & 2011 \\
\hline 430.459 & 224.724 & Surface & 2006 & 430.459 & 218.156 & Surface & 2011 \\
\hline 433.449 & 224.702 & Surface & 2006 & 433.449 & 218.170 & Surface & 2011 \\
\hline 436.438 & 224.680 & Surface & 2006 & 436.438 & 218.160 & Surface & 2011 \\
\hline 439.427 & 224.660 & Surface & 2006 & 439.427 & 218.139 & Surface & 2011 \\
\hline 442.417 & 224.640 & Surface & 2006 & 442.417 & 218.122 & Surface & 2011 \\
\hline 445.406 & 224.622 & Surface & 2006 & 445.406 & 218.123 & Surface & 2011 \\
\hline 448.395 & 224.604 & Surface & 2006 & 448.395 & 218.197 & Surface & 2011 \\
\hline 451.385 & 224.585 & Surface & 2006 & 451.385 & 218.241 & Surface & 2011 \\
\hline 454.374 & 224.565 & Surface & 2006 & 454.374 & 218.652 & Surface & 2011 \\
\hline 457.363 & 224.558 & Surface & 2006 & 457.363 & 219.299 & Surface & 2011 \\
\hline 460.352 & 224.674 & Surface & 2006 & 460.352 & 220.165 & Surface & 2011 \\
\hline 463.342 & 225.429 & Surface & 2006 & 463.342 & 221.377 & Surface & 2011 \\
\hline 466.331 & 226.527 & Surface & 2006 & 466.331 & 222.565 & Surface & 2011 \\
\hline 469.320 & 227.621 & Surface & 2006 & 469.320 & 223.790 & Surface & 2011 \\
\hline 472.310 & 228.711 & Surface & 2006 & 472.310 & 225.082 & Surface & 2011 \\
\hline 475.299 & 229.756 & Surface & 2006 & 475.299 & 226.378 & Surface & 2011 \\
\hline 478.288 & 230.677 & Surface & 2006 & 478.288 & 227.674 & Surface & 2011 \\
\hline 481.278 & 231.599 & Surface & 2006 & 481.278 & 228.970 & Surface & 2011 \\
\hline 484.267 & 232.522 & Surface & 2006 & 484.267 & 230.266 & Surface & 2011 \\
\hline 487.256 & 233.445 & Surface & 2006 & 487.256 & 231.563 & Surface & 2011 \\
\hline 490.246 & 234.406 & Surface & 2006 & 490.246 & 232.820 & Surface & 2011 \\
\hline 493.235 & 235.401 & Surface & 2006 & 493.235 & 233.264 & Surface & 2011 \\
\hline 496.224 & 236.382 & Surface & 2006 & 496.224 & 233.815 & Surface & 2011 \\
\hline 499.213 & 236.762 & Surface & 2006 & 499.213 & 234.423 & Surface & 2011 \\
\hline 502.203 & 236.913 & Surface & 2006 & 502.203 & 235.060 & Surface & 2011 \\
\hline 505.192 & 236.961 & Surface & 2006 & 505.192 & 235.773 & Surface & 2011 \\
\hline 508.181 & 236.936 & Surface & 2006 & 508.181 & 236.497 & Surface & 2011 \\
\hline 511.170 & 236.921 & Surface & 2006 & 511.170 & 236.806 & Surface & 2011 \\
\hline 514.160 & 236.928 & Surface & 2006 & 514.160 & 236.843 & Surface & 2011 \\
\hline 517.149 & 237.042 & Surface & 2006 & 517.149 & 236.944 & Surface & 2011 \\
\hline 0.000 & 231.891 & Surface & 2008 & 0.000 & 232.002 & Surface & 2012 \\
\hline 3.273 & 231.873 & Surface & 2008 & 3.273 & 231.887 & Surface & 2012 \\
\hline 6.546 & 231.843 & Surface & 2008 & 6.546 & 231.777 & Surface & 2012 \\
\hline 9.819 & 231.824 & Surface & 2008 & 9.819 & 231.681 & Surface & 2012 \\
\hline 13.092 & 231.877 & Surface & 2008 & 13.092 & 231.597 & Surface & 2012 \\
\hline 16.366 & 231.430 & Surface & 2008 & 16.366 & 231.342 & Surface & 2012 \\
\hline 19.638 & 230.764 & Surface & 2008 & 19.638 & 230.793 & Surface & 2012 \\
\hline 22.912 & 230.079 & Surface & 2008 & 22.912 & 230.144 & Surface & 2012 \\
\hline 26.185 & 229.408 & Surface & 2008 & 26.185 & 229.495 & Surface & 2012 \\
\hline 29.458 & 228.746 & Surface & 2008 & 29.458 & 228.839 & Surface & 2012 \\
\hline 32.731 & 228.285 & Surface & 2008 & 32.731 & 228.161 & Surface & 2012 \\
\hline 36.004 & 228.055 & Surface & 2008 & 36.004 & 227.637 & Surface & 2012 \\
\hline 39.277 & 227.804 & Surface & 2008 & 39.277 & 227.357 & Surface & 2012 \\
\hline 42.550 & 227.478 & Surface & 2008 & 42.550 & 227.146 & Surface & 2012 \\
\hline 45.823 & 227.363 & Surface & 2008 & 45.823 & 227.152 & Surface & 2012 \\
\hline 49.096 & 227.284 & Surface & 2008 & 49.096 & 227.059 & Surface & 2012 \\
\hline 52.370 & 227.213 & Surface & 2008 & 52.370 & 226.945 & Surface & 2012 \\
\hline 55.643 & 227.196 & Surface & 2008 & 55.643 & 226.829 & Surface & 2012 \\
\hline
\end{tabular}




\begin{tabular}{|c|c|c|c|c|c|c|c|}
\hline 58.916 & 227.168 & Surface & 2008 & 58.916 & 226.740 & Surface & 2012 \\
\hline 62.189 & 226.932 & Surface & 2008 & 62.189 & 226.617 & Surface & 2012 \\
\hline 65.462 & 226.623 & Surface & 2008 & 65.462 & 226.481 & Surface & 2012 \\
\hline 68.735 & 226.535 & Surface & 2008 & 68.735 & 226.527 & Surface & 2012 \\
\hline 72.008 & 226.555 & Surface & 2008 & 72.008 & 226.675 & Surface & 2012 \\
\hline 75.281 & 226.645 & Surface & 2008 & 75.281 & 226.734 & Surface & 2012 \\
\hline 78.554 & 226.695 & Surface & 2008 & 78.554 & 226.731 & Surface & 2012 \\
\hline 81.828 & 226.680 & Surface & 2008 & 81.828 & 226.739 & Surface & 2012 \\
\hline 85.100 & 226.726 & Surface & 2008 & 85.100 & 226.732 & Surface & 2012 \\
\hline 88.374 & 226.807 & Surface & 2008 & 88.374 & 226.747 & Surface & 2012 \\
\hline 91.647 & 226.854 & Surface & 2008 & 91.647 & 226.814 & Surface & 2012 \\
\hline 94.920 & 226.901 & Surface & 2008 & 94.920 & 226.845 & Surface & 2012 \\
\hline 98.193 & 226.886 & Surface & 2008 & 98.193 & 226.871 & Surface & 2012 \\
\hline 101.466 & 226.853 & Surface & 2008 & 101.466 & 226.916 & Surface & 2012 \\
\hline 104.739 & 226.813 & Surface & 2008 & 104.739 & 227.024 & Surface & 2012 \\
\hline 108.012 & 226.836 & Surface & 2008 & 108.012 & 227.097 & Surface & 2012 \\
\hline 111.285 & 226.887 & Surface & 2008 & 111.285 & 227.141 & Surface & 2012 \\
\hline 114.558 & 226.871 & Surface & 2008 & 114.558 & 227.019 & Surface & 2012 \\
\hline 117.832 & 226.545 & Surface & 2008 & 117.832 & 226.832 & Surface & 2012 \\
\hline 121.104 & 226.123 & Surface & 2008 & 121.104 & 226.430 & Surface & 2012 \\
\hline 124.378 & 225.646 & Surface & 2008 & 124.378 & 225.898 & Surface & 2012 \\
\hline 127.651 & 225.042 & Surface & 2008 & 127.651 & 225.279 & Surface & 2012 \\
\hline 130.924 & 224.461 & Surface & 2008 & 130.924 & 224.635 & Surface & 2012 \\
\hline 134.197 & 223.929 & Surface & 2008 & 134.197 & 224.111 & Surface & 2012 \\
\hline 137.470 & 223.473 & Surface & 2008 & 137.470 & 223.599 & Surface & 2012 \\
\hline 140.743 & 222.980 & Surface & 2008 & 140.743 & 223.041 & Surface & 2012 \\
\hline 144.016 & 222.505 & Surface & 2008 & 144.016 & 222.436 & Surface & 2012 \\
\hline 147.289 & 222.083 & Surface & 2008 & 147.289 & 222.109 & Surface & 2012 \\
\hline 150.562 & 221.808 & Surface & 2008 & 150.562 & 222.027 & Surface & 2012 \\
\hline 153.836 & 221.834 & Surface & 2008 & 153.836 & 222.028 & Surface & 2012 \\
\hline 157.109 & 221.969 & Surface & 2008 & 157.109 & 222.118 & Surface & 2012 \\
\hline 160.382 & 222.111 & Surface & 2008 & 160.382 & 222.300 & Surface & 2012 \\
\hline 163.655 & 222.237 & Surface & 2008 & 163.655 & 222.431 & Surface & 2012 \\
\hline 166.928 & 222.330 & Surface & 2008 & 166.928 & 222.437 & Surface & 2012 \\
\hline 170.201 & 222.366 & Surface & 2008 & 170.201 & 222.352 & Surface & 2012 \\
\hline 173.474 & 222.343 & Surface & 2008 & 173.474 & 221.999 & Surface & 2012 \\
\hline 176.747 & 222.358 & Surface & 2008 & 176.747 & 221.533 & Surface & 2012 \\
\hline 180.020 & 222.383 & Surface & 2008 & 180.020 & 221.181 & Surface & 2012 \\
\hline 183.294 & 222.417 & Surface & 2008 & 183.294 & 220.866 & Surface & 2012 \\
\hline 186.566 & 222.453 & Surface & 2008 & 186.566 & 220.708 & Surface & 2012 \\
\hline 189.840 & 222.493 & Surface & 2008 & 189.840 & 220.468 & Surface & 2012 \\
\hline 193.113 & 222.492 & Surface & 2008 & 193.113 & 219.805 & Surface & 2012 \\
\hline 196.386 & 222.490 & Surface & 2008 & 196.386 & 219.466 & Surface & 2012 \\
\hline 199.659 & 222.368 & Surface & 2008 & 199.659 & 219.457 & Surface & 2012 \\
\hline 202.932 & 222.102 & Surface & 2008 & 202.932 & 219.506 & Surface & 2012 \\
\hline 206.205 & 221.816 & Surface & 2008 & 206.205 & 219.501 & Surface & 2012 \\
\hline 209.478 & 221.528 & Surface & 2008 & 209.478 & 219.485 & Surface & 2012 \\
\hline 212.751 & 221.290 & Surface & 2008 & 212.751 & 219.423 & Surface & 2012 \\
\hline 216.024 & 221.131 & Surface & 2008 & 216.024 & 219.326 & Surface & 2012 \\
\hline
\end{tabular}




\begin{tabular}{|c|c|c|c|c|c|c|c|}
\hline 219.298 & 220.974 & Surface & 2008 & 219.298 & 219.177 & Surface & 2012 \\
\hline 222.570 & 220.805 & Surface & 2008 & 222.570 & 219.032 & Surface & 2012 \\
\hline 225.844 & 220.730 & Surface & 2008 & 225.844 & 218.919 & Surface & 2012 \\
\hline 229.117 & 220.734 & Surface & 2008 & 229.117 & 218.930 & Surface & 2012 \\
\hline 232.390 & 220.747 & Surface & 2008 & 232.390 & 218.913 & Surface & 2012 \\
\hline 235.663 & 220.792 & Surface & 2008 & 235.663 & 218.871 & Surface & 2012 \\
\hline 238.936 & 220.817 & Surface & 2008 & 238.936 & 218.811 & Surface & 2012 \\
\hline 242.209 & 220.647 & Surface & 2008 & 242.209 & 218.783 & Surface & 2012 \\
\hline 245.482 & 220.376 & Surface & 2008 & 245.482 & 218.696 & Surface & 2012 \\
\hline 248.755 & 220.060 & Surface & 2008 & 248.755 & 218.606 & Surface & 2012 \\
\hline 252.028 & 219.810 & Surface & 2008 & 252.028 & 218.512 & Surface & 2012 \\
\hline 255.302 & 219.633 & Surface & 2008 & 255.302 & 218.420 & Surface & 2012 \\
\hline 258.575 & 219.544 & Surface & 2008 & 258.575 & 218.456 & Surface & 2012 \\
\hline 261.848 & 219.501 & Surface & 2008 & 261.848 & 218.676 & Surface & 2012 \\
\hline 265.121 & 219.461 & Surface & 2008 & 265.121 & 218.951 & Surface & 2012 \\
\hline 268.394 & 219.426 & Surface & 2008 & 268.394 & 219.304 & Surface & 2012 \\
\hline 271.667 & 219.446 & Surface & 2008 & 271.667 & 219.659 & Surface & 2012 \\
\hline 274.940 & 219.470 & Surface & 2008 & 274.940 & 219.970 & Surface & 2012 \\
\hline 278.213 & 219.447 & Surface & 2008 & 278.213 & 220.180 & Surface & 2012 \\
\hline 281.486 & 219.406 & Surface & 2008 & 281.486 & 220.293 & Surface & 2012 \\
\hline 284.759 & 219.400 & Surface & 2008 & 284.759 & 220.414 & Surface & 2012 \\
\hline 288.032 & 219.333 & Surface & 2008 & 288.032 & 220.490 & Surface & 2012 \\
\hline 291.306 & 219.185 & Surface & 2008 & 291.306 & 220.489 & Surface & 2012 \\
\hline 294.579 & 218.961 & Surface & 2008 & 294.579 & 220.491 & Surface & 2012 \\
\hline 297.852 & 218.857 & Surface & 2008 & 297.852 & 220.516 & Surface & 2012 \\
\hline 301.125 & 218.799 & Surface & 2008 & 301.125 & 220.453 & Surface & 2012 \\
\hline 304.398 & 218.766 & Surface & 2008 & 304.398 & 220.414 & Surface & 2012 \\
\hline 307.671 & 218.783 & Surface & 2008 & 307.671 & 220.402 & Surface & 2012 \\
\hline 310.944 & 218.785 & Surface & 2008 & 310.944 & 220.374 & Surface & 2012 \\
\hline 314.217 & 218.776 & Surface & 2008 & 314.217 & 220.325 & Surface & 2012 \\
\hline 317.490 & 218.784 & Surface & 2008 & 317.490 & 220.269 & Surface & 2012 \\
\hline 320.764 & 218.812 & Surface & 2008 & 320.764 & 220.237 & Surface & 2012 \\
\hline 324.036 & 218.897 & Surface & 2008 & 324.036 & 220.174 & Surface & 2012 \\
\hline 327.310 & 219.015 & Surface & 2008 & 327.310 & 220.124 & Surface & 2012 \\
\hline 330.583 & 219.181 & Surface & 2008 & 330.583 & 220.086 & Surface & 2012 \\
\hline 333.856 & 219.344 & Surface & 2008 & 333.856 & 220.034 & Surface & 2012 \\
\hline 337.129 & 219.415 & Surface & 2008 & 337.129 & 219.998 & Surface & 2012 \\
\hline 340.402 & 219.444 & Surface & 2008 & 340.402 & 219.954 & Surface & 2012 \\
\hline 343.675 & 219.478 & Surface & 2008 & 343.675 & 219.892 & Surface & 2012 \\
\hline 346.948 & 219.485 & Surface & 2008 & 346.948 & 219.749 & Surface & 2012 \\
\hline 350.221 & 219.490 & Surface & 2008 & 350.221 & 219.530 & Surface & 2012 \\
\hline 353.494 & 219.473 & Surface & 2008 & 353.494 & 219.305 & Surface & 2012 \\
\hline 356.768 & 219.417 & Surface & 2008 & 356.768 & 219.096 & Surface & 2012 \\
\hline 360.041 & 219.372 & Surface & 2008 & 360.041 & 218.940 & Surface & 2012 \\
\hline 363.314 & 219.323 & Surface & 2008 & 363.314 & 218.790 & Surface & 2012 \\
\hline 366.587 & 219.216 & Surface & 2008 & 366.587 & 218.599 & Surface & 2012 \\
\hline 369.860 & 219.136 & Surface & 2008 & 369.860 & 218.394 & Surface & 2012 \\
\hline 373.133 & 219.133 & Surface & 2008 & 373.133 & 218.182 & Surface & 2012 \\
\hline 376.406 & 219.149 & Surface & 2008 & 376.406 & 218.035 & Surface & 2012 \\
\hline
\end{tabular}




\begin{tabular}{|c|c|c|c|c|c|c|c|}
\hline 379.679 & 219.181 & Surface & 2008 & 379.679 & 217.988 & Surface & 2012 \\
\hline 382.952 & 219.192 & Surface & 2008 & 382.952 & 218.001 & Surface & 2012 \\
\hline 386.225 & 219.135 & Surface & 2008 & 386.225 & 217.990 & Surface & 2012 \\
\hline 389.498 & 219.179 & Surface & 2008 & 389.498 & 217.989 & Surface & 2012 \\
\hline 392.772 & 219.212 & Surface & 2008 & 392.772 & 218.005 & Surface & 2012 \\
\hline 396.045 & 219.191 & Surface & 2008 & 396.045 & 217.999 & Surface & 2012 \\
\hline 399.318 & 219.280 & Surface & 2008 & 399.318 & 217.996 & Surface & 2012 \\
\hline 402.591 & 219.423 & Surface & 2008 & 402.591 & 218.000 & Surface & 2012 \\
\hline 405.864 & 219.613 & Surface & 2008 & 405.864 & 218.000 & Surface & 2012 \\
\hline 409.137 & 219.901 & Surface & 2008 & 409.137 & 218.005 & Surface & 2012 \\
\hline 412.410 & 220.325 & Surface & 2008 & 412.410 & 218.010 & Surface & 2012 \\
\hline 415.683 & 220.936 & Surface & 2008 & 415.683 & 218.005 & Surface & 2012 \\
\hline 418.956 & 221.601 & Surface & 2008 & 418.956 & 217.987 & Surface & 2012 \\
\hline 422.230 & 222.269 & Surface & 2008 & 422.230 & 217.979 & Surface & 2012 \\
\hline 425.502 & 223.006 & Surface & 2008 & 425.502 & 217.991 & Surface & 2012 \\
\hline 428.776 & 223.670 & Surface & 2008 & 428.776 & 217.990 & Surface & 2012 \\
\hline 432.049 & 224.011 & Surface & 2008 & 432.049 & 217.980 & Surface & 2012 \\
\hline 435.322 & 223.985 & Surface & 2008 & 435.322 & 217.974 & Surface & 2012 \\
\hline 438.595 & 223.816 & Surface & 2008 & 438.595 & 217.968 & Surface & 2012 \\
\hline 441.868 & 223.741 & Surface & 2008 & 441.868 & 217.978 & Surface & 2012 \\
\hline 445.141 & 223.746 & Surface & 2008 & 445.141 & 217.996 & Surface & 2012 \\
\hline 448.414 & 223.771 & Surface & 2008 & 448.414 & 218.013 & Surface & 2012 \\
\hline 451.687 & 223.792 & Surface & 2008 & 451.687 & 218.338 & Surface & 2012 \\
\hline 454.960 & 223.810 & Surface & 2008 & 454.960 & 219.015 & Surface & 2012 \\
\hline 458.234 & 224.022 & Surface & 2008 & 458.234 & 220.237 & Surface & 2012 \\
\hline 461.506 & 224.632 & Surface & 2008 & 461.506 & 222.604 & Surface & 2012 \\
\hline 464.780 & 225.661 & Surface & 2008 & 464.780 & 225.296 & Surface & 2012 \\
\hline 468.053 & 226.891 & Surface & 2008 & 468.053 & 226.950 & Surface & 2012 \\
\hline 471.326 & 228.121 & Surface & 2008 & 471.326 & 227.930 & Surface & 2012 \\
\hline 474.599 & 229.340 & Surface & 2008 & 474.599 & 228.965 & Surface & 2012 \\
\hline 477.872 & 230.534 & Surface & 2008 & 477.872 & 229.958 & Surface & 2012 \\
\hline 481.145 & 231.647 & Surface & 2008 & 481.145 & 230.908 & Surface & 2012 \\
\hline 484.418 & 232.744 & Surface & 2008 & 484.418 & 231.901 & Surface & 2012 \\
\hline 487.691 & 233.586 & Surface & 2008 & 487.691 & 232.880 & Surface & 2012 \\
\hline 490.964 & 234.220 & Surface & 2008 & 490.964 & 233.912 & Surface & 2012 \\
\hline 494.238 & 235.159 & Surface & 2008 & 494.238 & 234.826 & Surface & 2012 \\
\hline 497.511 & 236.095 & Surface & 2008 & 497.511 & 235.509 & Surface & 2012 \\
\hline 500.784 & 236.673 & Surface & 2008 & 500.784 & 236.286 & Surface & 2012 \\
\hline 504.057 & 236.908 & Surface & 2008 & 504.057 & 236.883 & Surface & 2012 \\
\hline 507.330 & 236.889 & Surface & 2008 & 507.330 & 236.973 & Surface & 2012 \\
\hline 510.603 & 236.801 & Surface & 2008 & 510.603 & 236.904 & Surface & 2012 \\
\hline 513.876 & 236.792 & Surface & 2008 & 513.876 & 236.894 & Surface & 2012 \\
\hline 517.149 & 236.917 & Surface & 2008 & 517.149 & 237.000 & Surface & 2012 \\
\hline
\end{tabular}


10.6 Transect F - Crib Structure North Points - All Returns:

\begin{tabular}{|c|c|c|c|c|c|c|c|}
\hline Distance & Z & DEM & Year & Distance & $\mathrm{Z}$ & DEM & Year \\
\hline 0.000 & 235.255 & Surface & 2006 & 0.000 & 235.362 & Surface & 2011 \\
\hline 2.983 & 234.858 & Surface & 2006 & 2.983 & 234.888 & Surface & 2011 \\
\hline 5.967 & 234.358 & Surface & 2006 & 5.967 & 234.417 & Surface & 2011 \\
\hline 8.950 & 233.824 & Surface & 2006 & 8.950 & 233.984 & Surface & 2011 \\
\hline 11.933 & 233.288 & Surface & 2006 & 11.933 & 233.562 & Surface & 2011 \\
\hline 14.917 & 232.752 & Surface & 2006 & 14.917 & 233.161 & Surface & 2011 \\
\hline 17.900 & 232.215 & Surface & 2006 & 17.900 & 232.715 & Surface & 2011 \\
\hline 20.883 & 231.677 & Surface & 2006 & 20.883 & 232.292 & Surface & 2011 \\
\hline 23.866 & 231.167 & Surface & 2006 & 23.866 & 231.908 & Surface & 2011 \\
\hline 26.850 & 230.663 & Surface & 2006 & 26.850 & 231.424 & Surface & 2011 \\
\hline 29.833 & 230.157 & Surface & 2006 & 29.833 & 230.851 & Surface & 2011 \\
\hline 32.816 & 229.643 & Surface & 2006 & 32.816 & 230.300 & Surface & 2011 \\
\hline 35.800 & 229.129 & Surface & 2006 & 35.800 & 229.741 & Surface & 2011 \\
\hline 38.783 & 228.615 & Surface & 2006 & 38.783 & 229.177 & Surface & 2011 \\
\hline 41.766 & 228.130 & Surface & 2006 & 41.766 & 228.617 & Surface & 2011 \\
\hline 44.750 & 227.732 & Surface & 2006 & 44.750 & 228.083 & Surface & 2011 \\
\hline 47.733 & 227.427 & Surface & 2006 & 47.733 & 227.608 & Surface & 2011 \\
\hline 50.716 & 227.181 & Surface & 2006 & 50.716 & 227.054 & Surface & 2011 \\
\hline 53.700 & 226.983 & Surface & 2006 & 53.700 & 226.466 & Surface & 2011 \\
\hline 56.683 & 226.780 & Surface & 2006 & 56.683 & 226.132 & Surface & 2011 \\
\hline 59.666 & 226.585 & Surface & 2006 & 59.666 & 225.797 & Surface & 2011 \\
\hline 62.650 & 226.388 & Surface & 2006 & 62.650 & 225.462 & Surface & 2011 \\
\hline 65.633 & 226.194 & Surface & 2006 & 65.633 & 225.127 & Surface & 2011 \\
\hline 68.616 & 225.991 & Surface & 2006 & 68.616 & 224.844 & Surface & 2011 \\
\hline 71.599 & 225.866 & Surface & 2006 & 71.599 & 224.706 & Surface & 2011 \\
\hline 74.583 & 225.696 & Surface & 2006 & 74.583 & 224.505 & Surface & 2011 \\
\hline 77.566 & 225.576 & Surface & 2006 & 77.566 & 224.287 & Surface & 2011 \\
\hline 80.549 & 225.523 & Surface & 2006 & 80.549 & 224.090 & Surface & 2011 \\
\hline 83.533 & 225.514 & Surface & 2006 & 83.533 & 223.898 & Surface & 2011 \\
\hline 86.516 & 225.459 & Surface & 2006 & 86.516 & 223.685 & Surface & 2011 \\
\hline 89.499 & 225.443 & Surface & 2006 & 89.499 & 223.497 & Surface & 2011 \\
\hline 92.483 & 225.444 & Surface & 2006 & 92.483 & 223.463 & Surface & 2011 \\
\hline 95.466 & 225.468 & Surface & 2006 & 95.466 & 223.519 & Surface & 2011 \\
\hline 98.449 & 225.458 & Surface & 2006 & 98.449 & 223.555 & Surface & 2011 \\
\hline 101.432 & 225.436 & Surface & 2006 & 101.432 & 223.586 & Surface & 2011 \\
\hline 104.416 & 225.433 & Surface & 2006 & 104.416 & 223.640 & Surface & 2011 \\
\hline 107.399 & 225.423 & Surface & 2006 & 107.399 & 223.708 & Surface & 2011 \\
\hline 110.382 & 225.436 & Surface & 2006 & 110.382 & 223.747 & Surface & 2011 \\
\hline 113.366 & 225.410 & Surface & 2006 & 113.366 & 223.676 & Surface & 2011 \\
\hline 116.349 & 225.386 & Surface & 2006 & 116.349 & 223.516 & Surface & 2011 \\
\hline 119.333 & 225.426 & Surface & 2006 & 119.333 & 223.338 & Surface & 2011 \\
\hline 122.316 & 225.447 & Surface & 2006 & 122.316 & 223.093 & Surface & 2011 \\
\hline 125.299 & 225.459 & Surface & 2006 & 125.299 & 222.620 & Surface & 2011 \\
\hline 128.282 & 225.442 & Surface & 2006 & 128.282 & 222.104 & Surface & 2011 \\
\hline 131.266 & 225.443 & Surface & 2006 & 131.266 & 221.660 & Surface & 2011 \\
\hline
\end{tabular}




\begin{tabular}{|c|c|c|c|c|c|c|}
\hline 134.249 & 225.445 & Surface & 2006 & 134.249 & 221.341 & Surface \\
\hline 137.232 & 225.439 & Surface & 2006 & 137.232 & 221.041 & Surface \\
\hline 140.216 & 225.453 & Surface & 2006 & 140.216 & 220.844 & Surface \\
\hline 143.199 & 225.465 & Surface & 2006 & 143.199 & 220.801 & Surface \\
\hline 146.182 & 225.445 & Surface & 2006 & 146.182 & 220.760 & Surface \\
\hline 149.166 & 225.454 & Surface & 2006 & 149.166 & 220.738 & Surface \\
\hline 152.149 & 225.459 & Surface & 2006 & 152.149 & 220.753 & Surface \\
\hline 155.132 & 225.460 & Surface & 2006 & 155.132 & 220.795 & Surface \\
\hline 158.115 & 225.473 & Surface & 2006 & 158.115 & 220.836 & Surface \\
\hline 161.099 & 225.476 & Surface & 2006 & 161.099 & 220.832 & Surface \\
\hline 164.082 & 225.450 & Surface & 2006 & 164.082 & 220.809 & Surface \\
\hline 167.065 & 225.424 & Surface & 2006 & 167.065 & 220.800 & Surface \\
\hline 170.049 & 225.405 & Surface & 2006 & 170.049 & 220.794 & Surface \\
\hline 173.032 & 225.438 & Surface & 2006 & 173.032 & 220.793 & Surface \\
\hline 176.015 & 225.443 & Surface & 2006 & 176.015 & 220.795 & Surface \\
\hline 178.999 & 225.440 & Surface & 2006 & 178.999 & 220.789 & Surface \\
\hline 181.982 & 225.451 & Surface & 2006 & 181.982 & 220.780 & Surface \\
\hline 184.965 & 225.424 & Surface & 2006 & 184.965 & 220.791 & Surface \\
\hline 187.948 & 225.409 & Surface & 2006 & 187.948 & 220.827 & Surface \\
\hline 190.932 & 225.423 & Surface & 2006 & 190.932 & 220.844 & Surface \\
\hline 193.915 & 225.421 & Surface & 2006 & 193.915 & 220.838 & Surface \\
\hline 196.899 & 225.414 & Surface & 2006 & 196.899 & 220.845 & Surface \\
\hline 199.882 & 225.411 & Surface & 2006 & 199.882 & 220.875 & Surface \\
\hline 202.865 & 225.415 & Surface & 2006 & 202.865 & 220.863 & Surface \\
\hline 205.849 & 225.420 & Surface & 2006 & 205.849 & 220.837 & Surface \\
\hline 208.832 & 225.429 & Surface & 2006 & 208.832 & 220.804 & Surface \\
\hline 211.815 & 225.433 & Surface & 2006 & 211.815 & 220.780 & Surface \\
\hline 214.798 & 225.376 & Surface & 2006 & 214.798 & 220.791 & Surface \\
\hline 217.782 & 225.404 & Surface & 2006 & 217.782 & 220.794 & Surface \\
\hline 220.765 & 225.405 & Surface & 2006 & 220.765 & 220.768 & Surface \\
\hline 223.748 & 225.411 & Surface & 2006 & 223.748 & 220.742 & Surface \\
\hline 226.732 & 225.433 & Surface & 2006 & 226.732 & 220.727 & Surface \\
\hline 229.715 & 225.600 & Surface & 2006 & 229.715 & 220.706 & Surface \\
\hline 232.698 & 225.849 & Surface & 2006 & 232.698 & 220.674 & Surface \\
\hline 235.682 & 226.046 & Surface & 2006 & 235.682 & 220.672 & Surface \\
\hline 238.665 & 226.202 & Surface & 2006 & 238.665 & 220.628 & Surface \\
\hline 241.648 & 226.359 & Surface & 2006 & 241.648 & 220.557 & Surface \\
\hline 244.631 & 226.496 & Surface & 2006 & 244.631 & 220.499 & Surface \\
\hline 247.615 & 226.580 & Surface & 2006 & 247.615 & 220.463 & Surface \\
\hline 250.598 & 226.655 & Surface & 2006 & 250.598 & 220.425 & Surface \\
\hline 253.581 & 226.707 & Surface & 2006 & 253.581 & 220.356 & Surface \\
\hline 256.565 & 226.735 & Surface & 2006 & 256.565 & 220.239 & Surface \\
\hline 259.548 & 226.758 & Surface & 2006 & 259.548 & 220.145 & Surface \\
\hline 262.531 & 226.785 & Surface & 2006 & 262.531 & 220.110 & Surface \\
\hline 265.515 & 226.801 & Surface & 2006 & 265.515 & 220.067 & Surface \\
\hline 268.498 & 226.812 & Surface & 2006 & 268.498 & 220.016 & Surface \\
\hline 271.481 & 226.831 & Surface & 2006 & 271.481 & 219.890 & Surface \\
\hline 274.465 & 226.842 & Surface & 2006 & 274.465 & 219.637 & Surface \\
\hline 277.448 & 226.835 & Surface & 2006 & 277.448 & 219.202 & Surface \\
\hline
\end{tabular}




\begin{tabular}{|c|c|c|c|c|c|c|}
\hline 280.431 & 226.825 & Surface & 2006 & 280.431 & 218.897 & Surface \\
\hline 283.415 & 226.843 & Surface & 2006 & 283.415 & 218.740 & Surface \\
\hline 286.398 & 226.874 & Surface & 2006 & 286.398 & 218.722 & Surface \\
\hline 289.381 & 226.868 & Surface & 2006 & 289.381 & 218.719 & Surface \\
\hline 292.365 & 226.876 & Surface & 2006 & 292.365 & 218.710 & Surface \\
\hline 295.348 & 226.906 & Surface & 2006 & 295.348 & 218.686 & Surface \\
\hline 298.331 & 226.902 & Surface & 2006 & 298.331 & 218.687 & Surface \\
\hline 301.314 & 226.894 & Surface & 2006 & 301.314 & 218.686 & Surface \\
\hline 304.298 & 226.881 & Surface & 2006 & 304.298 & 218.672 & Surface \\
\hline 307.281 & 226.881 & Surface & 2006 & 307.281 & 218.649 & Surface \\
\hline 310.264 & 226.924 & Surface & 2006 & 310.264 & 218.640 & Surface \\
\hline 313.248 & 226.876 & Surface & 2006 & 313.248 & 218.671 & Surface \\
\hline 316.231 & 226.812 & Surface & 2006 & 316.231 & 218.713 & Surface \\
\hline 319.214 & 226.757 & Surface & 2006 & 319.214 & 218.736 & Surface \\
\hline 322.198 & 226.672 & Surface & 2006 & 322.198 & 218.738 & Surface \\
\hline 325.181 & 226.655 & Surface & 2006 & 325.181 & 218.736 & Surface \\
\hline 328.164 & 226.667 & Surface & 2006 & 328.164 & 218.740 & Surface \\
\hline 331.147 & 226.683 & Surface & 2006 & 331.147 & 218.745 & Surface \\
\hline 334.131 & 226.629 & Surface & 2006 & 334.131 & 218.741 & Surface \\
\hline 337.114 & 226.496 & Surface & 2006 & 337.114 & 218.734 & Surface \\
\hline 340.097 & 226.340 & Surface & 2006 & 340.097 & 218.744 & Surface \\
\hline 343.081 & 226.194 & Surface & 2006 & 343.081 & 218.802 & Surface \\
\hline 346.064 & 226.085 & Surface & 2006 & 346.064 & 218.977 & Surface \\
\hline 349.047 & 225.984 & Surface & 2006 & 349.047 & 219.219 & Surface \\
\hline 352.031 & 225.863 & Surface & 2006 & 352.031 & 219.428 & Surface \\
\hline 355.014 & 225.755 & Surface & 2006 & 355.014 & 219.613 & Surface \\
\hline 357.997 & 225.677 & Surface & 2006 & 357.997 & 219.742 & Surface \\
\hline 360.981 & 225.503 & Surface & 2006 & 360.981 & 219.885 & Surface \\
\hline 363.964 & 225.346 & Surface & 2006 & 363.964 & 220.005 & Surface \\
\hline 366.947 & 225.121 & Surface & 2006 & 366.947 & 220.144 & Surface \\
\hline 369.931 & 224.934 & Surface & 2006 & 369.931 & 220.147 & Surface \\
\hline 372.914 & 224.784 & Surface & 2006 & 372.914 & 220.109 & Surface \\
\hline 375.897 & 224.727 & Surface & 2006 & 375.897 & 220.213 & Surface \\
\hline 378.880 & 224.722 & Surface & 2006 & 378.880 & 220.339 & Surface \\
\hline 381.864 & 224.724 & Surface & 2006 & 381.864 & 220.427 & Surface \\
\hline 384.847 & 224.721 & Surface & 2006 & 384.847 & 220.549 & Surface \\
\hline 387.830 & 224.718 & Surface & 2006 & 387.830 & 220.701 & Surface \\
\hline 390.814 & 224.713 & Surface & 2006 & 390.814 & 220.914 & Surface \\
\hline 393.797 & 224.710 & Surface & 2006 & 393.797 & 221.277 & Surface \\
\hline 396.780 & 224.712 & Surface & 2006 & 396.780 & 221.738 & Surface \\
\hline 399.764 & 224.722 & Surface & 2006 & 399.764 & 222.241 & Surface \\
\hline 402.747 & 224.727 & Surface & 2006 & 402.747 & 222.740 & Surface \\
\hline 405.730 & 224.726 & Surface & 2006 & 405.730 & 223.241 & Surface \\
\hline 408.714 & 224.725 & Surface & 2006 & 408.714 & 223.756 & Surface \\
\hline 411.697 & 224.726 & Surface & 2006 & 411.697 & 224.212 & Surface \\
\hline 414.680 & 224.787 & Surface & 2006 & 414.680 & 224.523 & Surface \\
\hline 417.663 & 225.040 & Surface & 2006 & 417.663 & 224.785 & Surface \\
\hline 420.647 & 225.720 & Surface & 2006 & 420.647 & 225.215 & Surface \\
\hline 423.630 & 226.726 & Surface & 2006 & 423.630 & 226.112 & Surface \\
\hline
\end{tabular}




\begin{tabular}{|c|c|c|c|c|c|c|c|}
\hline 426.613 & 227.724 & Surface & 2006 & 426.613 & 227.107 & Surface & 2011 \\
\hline 429.597 & 228.658 & Surface & 2006 & 429.597 & 227.976 & Surface & 2011 \\
\hline 432.580 & 229.404 & Surface & 2006 & 432.580 & 228.746 & Surface & 2011 \\
\hline 435.564 & 230.079 & Surface & 2006 & 435.564 & 229.412 & Surface & 2011 \\
\hline 438.547 & 230.783 & Surface & 2006 & 438.547 & 230.085 & Surface & 2011 \\
\hline 441.530 & 231.617 & Surface & 2006 & 441.530 & 231.089 & Surface & 2011 \\
\hline 444.513 & 232.469 & Surface & 2006 & 444.513 & 231.940 & Surface & 2011 \\
\hline 447.497 & 233.236 & Surface & 2006 & 447.497 & 232.729 & Surface & 2011 \\
\hline 450.480 & 233.874 & Surface & 2006 & 450.480 & 233.518 & Surface & 2011 \\
\hline 453.463 & 234.489 & Surface & 2006 & 453.463 & 234.306 & Surface & 2011 \\
\hline 456.447 & 235.104 & Surface & 2006 & 456.447 & 235.129 & Surface & 2011 \\
\hline 459.430 & 235.569 & Surface & 2006 & 459.430 & 235.723 & Surface & 2011 \\
\hline 462.413 & 235.658 & Surface & 2006 & 462.413 & 236.048 & Surface & 2011 \\
\hline 465.397 & 235.705 & Surface & 2006 & 465.397 & 236.173 & Surface & 2011 \\
\hline 468.380 & 235.810 & Surface & 2006 & 468.380 & 236.206 & Surface & 2011 \\
\hline 471.363 & 235.807 & Surface & 2006 & 471.363 & 236.219 & Surface & 2011 \\
\hline 474.346 & 235.731 & Surface & 2006 & 474.346 & 236.245 & Surface & 2011 \\
\hline 0.000 & 235.150 & Surface & 2008 & 0.000 & 235.104 & Surface & 2012 \\
\hline 3.271 & 234.561 & Surface & 2008 & 3.271 & 234.561 & Surface & 2012 \\
\hline 6.543 & 233.947 & Surface & 2008 & 6.543 & 234.091 & Surface & 2012 \\
\hline 9.814 & 233.454 & Surface & 2008 & 9.814 & 233.666 & Surface & 2012 \\
\hline 13.085 & 232.908 & Surface & 2008 & 13.085 & 233.228 & Surface & 2012 \\
\hline 16.357 & 232.366 & Surface & 2008 & 16.357 & 232.733 & Surface & 2012 \\
\hline 19.628 & 231.830 & Surface & 2008 & 19.628 & 232.235 & Surface & 2012 \\
\hline 22.900 & 231.301 & Surface & 2008 & 22.900 & 231.758 & Surface & 2012 \\
\hline 26.171 & 230.763 & Surface & 2008 & 26.171 & 231.269 & Surface & 2012 \\
\hline 29.442 & 230.214 & Surface & 2008 & 29.442 & 230.731 & Surface & 2012 \\
\hline 32.714 & 229.677 & Surface & 2008 & 32.714 & 230.163 & Surface & 2012 \\
\hline 35.985 & 229.143 & Surface & 2008 & 35.985 & 229.447 & Surface & 2012 \\
\hline 39.256 & 228.604 & Surface & 2008 & 39.256 & 228.710 & Surface & 2012 \\
\hline 42.527 & 228.034 & Surface & 2008 & 42.527 & 228.093 & Surface & 2012 \\
\hline 45.799 & 227.470 & Surface & 2008 & 45.799 & 227.564 & Surface & 2012 \\
\hline 49.070 & 226.991 & Surface & 2008 & 49.070 & 227.045 & Surface & 2012 \\
\hline 52.342 & 226.542 & Surface & 2008 & 52.342 & 226.630 & Surface & 2012 \\
\hline 55.613 & 226.103 & Surface & 2008 & 55.613 & 226.270 & Surface & 2012 \\
\hline 58.884 & 225.666 & Surface & 2008 & 58.884 & 225.885 & Surface & 2012 \\
\hline 62.156 & 225.226 & Surface & 2008 & 62.156 & 225.503 & Surface & 2012 \\
\hline 65.427 & 224.840 & Surface & 2008 & 65.427 & 225.120 & Surface & 2012 \\
\hline 68.698 & 224.713 & Surface & 2008 & 68.698 & 224.888 & Surface & 2012 \\
\hline 71.970 & 224.762 & Surface & 2008 & 71.970 & 224.910 & Surface & 2012 \\
\hline 75.241 & 224.753 & Surface & 2008 & 75.241 & 224.854 & Surface & 2012 \\
\hline 78.513 & 224.602 & Surface & 2008 & 78.513 & 224.683 & Surface & 2012 \\
\hline 81.784 & 224.118 & Surface & 2008 & 81.784 & 224.344 & Surface & 2012 \\
\hline 85.055 & 223.545 & Surface & 2008 & 85.055 & 223.905 & Surface & 2012 \\
\hline 88.327 & 223.426 & Surface & 2008 & 88.327 & 223.538 & Surface & 2012 \\
\hline 91.598 & 223.433 & Surface & 2008 & 91.598 & 223.445 & Surface & 2012 \\
\hline 94.869 & 223.511 & Surface & 2008 & 94.869 & 223.528 & Surface & 2012 \\
\hline 98.141 & 223.551 & Surface & 2008 & 98.141 & 223.577 & Surface & 2012 \\
\hline 101.412 & 223.573 & Surface & 2008 & 101.412 & 223.602 & Surface & 2012 \\
\hline
\end{tabular}




\begin{tabular}{|c|c|c|c|c|c|c|c|}
\hline 104.683 & 223.665 & Surface & 2008 & 104.683 & 223.691 & Surface & 2012 \\
\hline 107.955 & 223.768 & Surface & 2008 & 107.955 & 223.751 & Surface & 2012 \\
\hline 111.226 & 223.726 & Surface & 2008 & 111.226 & 223.715 & Surface & 2012 \\
\hline 114.498 & 223.559 & Surface & 2008 & 114.498 & 223.605 & Surface & 2012 \\
\hline 117.769 & 223.359 & Surface & 2008 & 117.769 & 223.421 & Surface & 2012 \\
\hline 121.040 & 223.174 & Surface & 2008 & 121.040 & 223.135 & Surface & 2012 \\
\hline 124.311 & 222.963 & Surface & 2008 & 124.311 & 222.609 & Surface & 2012 \\
\hline 127.583 & 222.878 & Surface & 2008 & 127.583 & 222.035 & Surface & 2012 \\
\hline 130.854 & 222.824 & Surface & 2008 & 130.854 & 221.609 & Surface & 2012 \\
\hline 134.125 & 222.670 & Surface & 2008 & 134.125 & 221.292 & Surface & 2012 \\
\hline 137.397 & 222.477 & Surface & 2008 & 137.397 & 220.922 & Surface & 2012 \\
\hline 140.668 & 222.323 & Surface & 2008 & 140.668 & 220.708 & Surface & 2012 \\
\hline 143.940 & 222.211 & Surface & 2008 & 143.940 & 220.682 & Surface & 2012 \\
\hline 147.211 & 222.100 & Surface & 2008 & 147.211 & 220.716 & Surface & 2012 \\
\hline 150.482 & 221.925 & Surface & 2008 & 150.482 & 220.727 & Surface & 2012 \\
\hline 153.754 & 221.712 & Surface & 2008 & 153.754 & 220.772 & Surface & 2012 \\
\hline 157.025 & 221.512 & Surface & 2008 & 157.025 & 220.841 & Surface & 2012 \\
\hline 160.296 & 221.363 & Surface & 2008 & 160.296 & 220.860 & Surface & 2012 \\
\hline 163.568 & 221.232 & Surface & 2008 & 163.568 & 220.853 & Surface & 2012 \\
\hline 166.839 & 221.102 & Surface & 2008 & 166.839 & 220.846 & Surface & 2012 \\
\hline 170.110 & 220.999 & Surface & 2008 & 170.110 & 220.849 & Surface & 2012 \\
\hline 173.382 & 220.940 & Surface & 2008 & 173.382 & 220.835 & Surface & 2012 \\
\hline 176.653 & 220.892 & Surface & 2008 & 176.653 & 220.833 & Surface & 2012 \\
\hline 179.925 & 220.881 & Surface & 2008 & 179.925 & 220.829 & Surface & 2012 \\
\hline 183.196 & 220.894 & Surface & 2008 & 183.196 & 220.735 & Surface & 2012 \\
\hline 186.467 & 220.874 & Surface & 2008 & 186.467 & 220.625 & Surface & 2012 \\
\hline 189.739 & 220.844 & Surface & 2008 & 189.739 & 220.505 & Surface & 2012 \\
\hline 193.010 & 220.818 & Surface & 2008 & 193.010 & 220.399 & Surface & 2012 \\
\hline 196.281 & 220.804 & Surface & 2008 & 196.281 & 220.406 & Surface & 2012 \\
\hline 199.553 & 220.770 & Surface & 2008 & 199.553 & 220.357 & Surface & 2012 \\
\hline 202.824 & 220.738 & Surface & 2008 & 202.824 & 220.246 & Surface & 2012 \\
\hline 206.095 & 220.615 & Surface & 2008 & 206.095 & 220.332 & Surface & 2012 \\
\hline 209.367 & 220.474 & Surface & 2008 & 209.367 & 220.338 & Surface & 2012 \\
\hline 212.638 & 220.329 & Surface & 2008 & 212.638 & 220.159 & Surface & 2012 \\
\hline 215.909 & 220.279 & Surface & 2008 & 215.909 & 220.083 & Surface & 2012 \\
\hline 219.181 & 220.297 & Surface & 2008 & 219.181 & 220.116 & Surface & 2012 \\
\hline 222.452 & 220.240 & Surface & 2008 & 222.452 & 220.021 & Surface & 2012 \\
\hline 225.723 & 220.315 & Surface & 2008 & 225.723 & 219.980 & Surface & 2012 \\
\hline 228.995 & 220.404 & Surface & 2008 & 228.995 & 220.005 & Surface & 2012 \\
\hline 232.266 & 220.362 & Surface & 2008 & 232.266 & 219.978 & Surface & 2012 \\
\hline 235.538 & 220.344 & Surface & 2008 & 235.538 & 219.918 & Surface & 2012 \\
\hline 238.809 & 220.336 & Surface & 2008 & 238.809 & 219.863 & Surface & 2012 \\
\hline 242.080 & 220.336 & Surface & 2008 & 242.080 & 219.789 & Surface & 2012 \\
\hline 245.352 & 220.305 & Surface & 2008 & 245.352 & 219.763 & Surface & 2012 \\
\hline 248.623 & 220.296 & Surface & 2008 & 248.623 & 219.727 & Surface & 2012 \\
\hline 251.894 & 220.294 & Surface & 2008 & 251.894 & 219.717 & Surface & 2012 \\
\hline 255.166 & 220.280 & Surface & 2008 & 255.166 & 219.660 & Surface & 2012 \\
\hline 258.437 & 220.311 & Surface & 2008 & 258.437 & 219.582 & Surface & 2012 \\
\hline 261.708 & 220.315 & Surface & 2008 & 261.708 & 219.536 & Surface & 2012 \\
\hline
\end{tabular}




\begin{tabular}{|c|c|c|c|c|c|c|c|}
\hline 264.980 & 220.294 & Surface & 2008 & 264.980 & 219.496 & Surface & 2012 \\
\hline 268.251 & 220.310 & Surface & 2008 & 268.251 & 219.440 & Surface & 2012 \\
\hline 271.523 & 220.316 & Surface & 2008 & 271.523 & 219.420 & Surface & 2012 \\
\hline 274.794 & 220.270 & Surface & 2008 & 274.794 & 219.369 & Surface & 2012 \\
\hline 278.065 & 220.253 & Surface & 2008 & 278.065 & 219.280 & Surface & 2012 \\
\hline 281.337 & 220.293 & Surface & 2008 & 281.337 & 219.220 & Surface & 2012 \\
\hline 284.608 & 220.320 & Surface & 2008 & 284.608 & 219.192 & Surface & 2012 \\
\hline 287.879 & 220.329 & Surface & 2008 & 287.879 & 219.170 & Surface & 2012 \\
\hline 291.150 & 220.366 & Surface & 2008 & 291.150 & 219.117 & Surface & 2012 \\
\hline 294.422 & 220.712 & Surface & 2008 & 294.422 & 219.041 & Surface & 2012 \\
\hline 297.693 & 221.407 & Surface & 2008 & 297.693 & 218.942 & Surface & 2012 \\
\hline 300.965 & 222.126 & Surface & 2008 & 300.965 & 218.909 & Surface & 2012 \\
\hline 304.236 & 222.709 & Surface & 2008 & 304.236 & 218.874 & Surface & 2012 \\
\hline 307.507 & 223.325 & Surface & 2008 & 307.507 & 218.788 & Surface & 2012 \\
\hline 310.779 & 223.974 & Surface & 2008 & 310.779 & 218.798 & Surface & 2012 \\
\hline 314.050 & 224.550 & Surface & 2008 & 314.050 & 218.801 & Surface & 2012 \\
\hline 317.321 & 225.085 & Surface & 2008 & 317.321 & 218.807 & Surface & 2012 \\
\hline 320.593 & 225.719 & Surface & 2008 & 320.593 & 218.800 & Surface & 2012 \\
\hline 323.864 & 226.329 & Surface & 2008 & 323.864 & 218.785 & Surface & 2012 \\
\hline 327.136 & 226.619 & Surface & 2008 & 327.136 & 218.754 & Surface & 2012 \\
\hline 330.407 & 226.628 & Surface & 2008 & 330.407 & 218.751 & Surface & 2012 \\
\hline 333.678 & 226.626 & Surface & 2008 & 333.678 & 218.803 & Surface & 2012 \\
\hline 336.950 & 226.559 & Surface & 2008 & 336.950 & 218.853 & Surface & 2012 \\
\hline 340.221 & 226.365 & Surface & 2008 & 340.221 & 218.904 & Surface & 2012 \\
\hline 343.492 & 226.196 & Surface & 2008 & 343.492 & 218.919 & Surface & 2012 \\
\hline 346.764 & 226.061 & Surface & 2008 & 346.764 & 218.984 & Surface & 2012 \\
\hline 350.035 & 225.944 & Surface & 2008 & 350.035 & 219.270 & Surface & 2012 \\
\hline 353.306 & 225.829 & Surface & 2008 & 353.306 & 219.582 & Surface & 2012 \\
\hline 356.578 & 225.681 & Surface & 2008 & 356.578 & 219.821 & Surface & 2012 \\
\hline 359.849 & 225.557 & Surface & 2008 & 359.849 & 219.986 & Surface & 2012 \\
\hline 363.120 & 225.439 & Surface & 2008 & 363.120 & 220.200 & Surface & 2012 \\
\hline 366.392 & 225.293 & Surface & 2008 & 366.392 & 220.226 & Surface & 2012 \\
\hline 369.663 & 225.056 & Surface & 2008 & 369.663 & 220.130 & Surface & 2012 \\
\hline 372.934 & 224.830 & Surface & 2008 & 372.934 & 220.066 & Surface & 2012 \\
\hline 376.206 & 224.649 & Surface & 2008 & 376.206 & 220.176 & Surface & 2012 \\
\hline 379.477 & 224.424 & Surface & 2008 & 379.477 & 220.400 & Surface & 2012 \\
\hline 382.748 & 224.252 & Surface & 2008 & 382.748 & 220.600 & Surface & 2012 \\
\hline 386.020 & 224.178 & Surface & 2008 & 386.020 & 220.756 & Surface & 2012 \\
\hline 389.291 & 224.098 & Surface & 2008 & 389.291 & 220.962 & Surface & 2012 \\
\hline 392.563 & 224.126 & Surface & 2008 & 392.563 & 221.320 & Surface & 2012 \\
\hline 395.834 & 224.175 & Surface & 2008 & 395.834 & 221.716 & Surface & 2012 \\
\hline 399.105 & 224.245 & Surface & 2008 & 399.105 & 222.262 & Surface & 2012 \\
\hline 402.377 & 224.340 & Surface & 2008 & 402.377 & 222.937 & Surface & 2012 \\
\hline 405.648 & 224.401 & Surface & 2008 & 405.648 & 223.529 & Surface & 2012 \\
\hline 408.919 & 224.437 & Surface & 2008 & 408.919 & 224.012 & Surface & 2012 \\
\hline 412.191 & 224.451 & Surface & 2008 & 412.191 & 224.354 & Surface & 2012 \\
\hline 415.462 & 224.701 & Surface & 2008 & 415.462 & 224.666 & Surface & 2012 \\
\hline 418.733 & 225.504 & Surface & 2008 & 418.733 & 225.061 & Surface & 2012 \\
\hline 422.005 & 226.834 & Surface & 2008 & 422.005 & 225.778 & Surface & 2012 \\
\hline
\end{tabular}




$\begin{array}{llllllll}425.276 & 227.743 & \text { Surface } & 2008 & 425.276 & 226.832 & \text { Surface } & 2012 \\ 428.548 & 228.368 & \text { Surface } & 2008 & 428.548 & 227.919 & \text { Surface } & 2012 \\ 431.819 & 228.959 & \text { Surface } & 2008 & 431.819 & 228.691 & \text { Surface } & 2012 \\ 435.090 & 229.699 & \text { Surface } & 2008 & 435.090 & 229.405 & \text { Surface } & 2012 \\ 438.361 & 230.560 & \text { Surface } & 2008 & 438.361 & 230.502 & \text { Surface } & 2012 \\ 441.633 & 231.513 & \text { Surface } & 2008 & 441.633 & 231.689 & \text { Surface } & 2012 \\ 444.904 & 232.783 & \text { Surface } & 2008 & 444.904 & 232.798 & \text { Surface } & 2012 \\ 448.175 & 234.133 & \text { Surface } & 2008 & 448.175 & 233.949 & \text { Surface } & 2012 \\ 451.447 & 234.989 & \text { Surface } & 2008 & 451.447 & 234.937 & \text { Surface } & 2012 \\ 454.718 & 235.216 & \text { Surface } & 2008 & 454.718 & 235.327 & \text { Surface } & 2012 \\ 457.990 & 235.373 & \text { Surface } & 2008 & 457.990 & 235.609 & \text { Surface } & 2012 \\ 461.261 & 235.651 & \text { Surface } & 2008 & 461.261 & 235.811 & \text { Surface } & 2012 \\ 464.532 & 235.875 & \text { Surface } & 2008 & 464.532 & 235.801 & \text { Surface } & 2012 \\ 467.804 & 235.975 & \text { Surface } & 2008 & 467.804 & 235.728 & \text { Surface } & 2012 \\ 471.075 & 236.020 & \text { Surface } & 2008 & 471.075 & 235.711 & \text { Surface } & 2012 \\ 474.346 & 236.013 & \text { Surface } & 2008 & 474.346 & 235.673 & \text { Surface } & 2012\end{array}$


10.7 Transect G - Crib Structure South Points - All Returns:

\begin{tabular}{|c|c|c|c|c|c|c|c|}
\hline Distance & Z & DEM & Year & Distance & $\mathrm{Z}$ & DEM & Year \\
\hline 0.000 & 238.709 & Surface & 2006 & 0.000 & 239.177 & Surface & 2011 \\
\hline 2.996 & 237.991 & Surface & 2006 & 2.996 & 238.806 & Surface & 2011 \\
\hline 5.993 & 237.267 & Surface & 2006 & 5.993 & 238.439 & Surface & 2011 \\
\hline 8.989 & 236.543 & Surface & 2006 & 8.989 & 237.793 & Surface & 2011 \\
\hline 11.985 & 235.823 & Surface & 2006 & 11.985 & 237.068 & Surface & 2011 \\
\hline 14.981 & 235.098 & Surface & 2006 & 14.981 & 236.343 & Surface & 2011 \\
\hline 17.978 & 234.391 & Surface & 2006 & 17.978 & 235.621 & Surface & 2011 \\
\hline 20.974 & 233.650 & Surface & 2006 & 20.974 & 234.898 & Surface & 2011 \\
\hline 23.970 & 232.977 & Surface & 2006 & 23.970 & 234.176 & Surface & 2011 \\
\hline 26.966 & 232.291 & Surface & 2006 & 26.966 & 233.454 & Surface & 2011 \\
\hline 29.963 & 231.594 & Surface & 2006 & 29.963 & 232.733 & Surface & 2011 \\
\hline 32.959 & 230.985 & Surface & 2006 & 32.959 & 232.012 & Surface & 2011 \\
\hline 35.955 & 230.285 & Surface & 2006 & 35.955 & 231.274 & Surface & 2011 \\
\hline 38.952 & 229.498 & Surface & 2006 & 38.952 & 230.471 & Surface & 2011 \\
\hline 41.948 & 228.873 & Surface & 2006 & 41.948 & 229.654 & Surface & 2011 \\
\hline 44.944 & 228.393 & Surface & 2006 & 44.944 & 228.906 & Surface & 2011 \\
\hline 47.940 & 227.957 & Surface & 2006 & 47.940 & 228.352 & Surface & 2011 \\
\hline 50.937 & 227.506 & Surface & 2006 & 50.937 & 227.871 & Surface & 2011 \\
\hline 53.933 & 227.093 & Surface & 2006 & 53.933 & 227.344 & Surface & 2011 \\
\hline 56.929 & 226.692 & Surface & 2006 & 56.929 & 226.822 & Surface & 2011 \\
\hline 59.926 & 226.383 & Surface & 2006 & 59.926 & 226.296 & Surface & 2011 \\
\hline 62.922 & 226.211 & Surface & 2006 & 62.922 & 225.654 & Surface & 2011 \\
\hline 65.918 & 226.054 & Surface & 2006 & 65.918 & 225.395 & Surface & 2011 \\
\hline 68.914 & 225.911 & Surface & 2006 & 68.914 & 225.132 & Surface & 2011 \\
\hline 71.911 & 225.764 & Surface & 2006 & 71.911 & 225.026 & Surface & 2011 \\
\hline 74.907 & 225.604 & Surface & 2006 & 74.907 & 224.944 & Surface & 2011 \\
\hline 77.903 & 225.481 & Surface & 2006 & 77.903 & 224.863 & Surface & 2011 \\
\hline 80.900 & 225.504 & Surface & 2006 & 80.900 & 224.789 & Surface & 2011 \\
\hline 83.896 & 225.512 & Surface & 2006 & 83.896 & 224.663 & Surface & 2011 \\
\hline 86.892 & 225.509 & Surface & 2006 & 86.892 & 224.441 & Surface & 2011 \\
\hline 89.888 & 225.425 & Surface & 2006 & 89.888 & 224.158 & Surface & 2011 \\
\hline 92.885 & 225.462 & Surface & 2006 & 92.885 & 223.911 & Surface & 2011 \\
\hline 95.881 & 225.488 & Surface & 2006 & 95.881 & 223.789 & Surface & 2011 \\
\hline 98.877 & 225.513 & Surface & 2006 & 98.877 & 223.722 & Surface & 2011 \\
\hline 101.873 & 225.500 & Surface & 2006 & 101.873 & 223.655 & Surface & 2011 \\
\hline 104.870 & 225.492 & Surface & 2006 & 104.870 & 223.578 & Surface & 2011 \\
\hline 107.866 & 225.508 & Surface & 2006 & 107.866 & 223.492 & Surface & 2011 \\
\hline 110.862 & 225.536 & Surface & 2006 & 110.862 & 223.409 & Surface & 2011 \\
\hline 113.859 & 225.534 & Surface & 2006 & 113.859 & 223.319 & Surface & 2011 \\
\hline 116.855 & 225.534 & Surface & 2006 & 116.855 & 223.146 & Surface & 2011 \\
\hline 119.851 & 225.534 & Surface & 2006 & 119.851 & 222.872 & Surface & 2011 \\
\hline 122.847 & 225.537 & Surface & 2006 & 122.847 & 222.621 & Surface & 2011 \\
\hline 125.844 & 225.550 & Surface & 2006 & 125.844 & 222.334 & Surface & 2011 \\
\hline 128.840 & 225.545 & Surface & 2006 & 128.840 & 221.940 & Surface & 2011 \\
\hline 131.836 & 225.534 & Surface & 2006 & 131.836 & 221.507 & Surface & 2011 \\
\hline
\end{tabular}




\begin{tabular}{|c|c|c|c|c|c|c|}
\hline 134.833 & 225.535 & Surface & 2006 & 134.833 & 221.069 & Surface \\
\hline 137.829 & 225.539 & Surface & 2006 & 137.829 & 220.709 & Surface \\
\hline 140.825 & 225.525 & Surface & 2006 & 140.825 & 220.687 & Surface \\
\hline 143.821 & 225.498 & Surface & 2006 & 143.821 & 220.730 & Surface \\
\hline 146.818 & 225.509 & Surface & 2006 & 146.818 & 220.758 & Surface \\
\hline 149.814 & 225.546 & Surface & 2006 & 149.814 & 220.803 & Surface \\
\hline 152.810 & 225.565 & Surface & 2006 & 152.810 & 220.837 & Surface \\
\hline 155.807 & 225.555 & Surface & 2006 & 155.807 & 220.857 & Surface \\
\hline 158.803 & 225.541 & Surface & 2006 & 158.803 & 220.850 & Surface \\
\hline 161.799 & 225.538 & Surface & 2006 & 161.799 & 220.838 & Surface \\
\hline 164.795 & 225.539 & Surface & 2006 & 164.795 & 220.849 & Surface \\
\hline 167.792 & 225.561 & Surface & 2006 & 167.792 & 220.871 & Surface \\
\hline 170.788 & 225.576 & Surface & 2006 & 170.788 & 220.894 & Surface \\
\hline 173.784 & 225.536 & Surface & 2006 & 173.784 & 220.893 & Surface \\
\hline 176.780 & 225.545 & Surface & 2006 & 176.780 & 220.895 & Surface \\
\hline 179.777 & 225.558 & Surface & 2006 & 179.777 & 220.915 & Surface \\
\hline 182.773 & 225.553 & Surface & 2006 & 182.773 & 220.919 & Surface \\
\hline 185.769 & 225.531 & Surface & 2006 & 185.769 & 220.913 & Surface \\
\hline 188.766 & 225.499 & Surface & 2006 & 188.766 & 220.880 & Surface \\
\hline 191.762 & 225.515 & Surface & 2006 & 191.762 & 220.845 & Surface \\
\hline 194.758 & 225.511 & Surface & 2006 & 194.758 & 220.808 & Surface \\
\hline 197.754 & 225.510 & Surface & 2006 & 197.754 & 220.778 & Surface \\
\hline 200.751 & 225.505 & Surface & 2006 & 200.751 & 220.759 & Surface \\
\hline 203.747 & 225.492 & Surface & 2006 & 203.747 & 220.737 & Surface \\
\hline 206.743 & 225.472 & Surface & 2006 & 206.743 & 220.731 & Surface \\
\hline 209.740 & 225.486 & Surface & 2006 & 209.740 & 220.726 & Surface \\
\hline 212.736 & 225.515 & Surface & 2006 & 212.736 & 220.718 & Surface \\
\hline 215.732 & 225.506 & Surface & 2006 & 215.732 & 220.709 & Surface \\
\hline 218.728 & 225.481 & Surface & 2006 & 218.728 & 220.686 & Surface \\
\hline 221.725 & 225.488 & Surface & 2006 & 221.725 & 220.643 & Surface \\
\hline 224.721 & 225.614 & Surface & 2006 & 224.721 & 220.596 & Surface \\
\hline 227.717 & 225.804 & Surface & 2006 & 227.717 & 220.534 & Surface \\
\hline 230.713 & 225.994 & Surface & 2006 & 230.713 & 220.488 & Surface \\
\hline 233.710 & 226.140 & Surface & 2006 & 233.710 & 220.407 & Surface \\
\hline 236.706 & 226.257 & Surface & 2006 & 236.706 & 220.362 & Surface \\
\hline 239.702 & 226.363 & Surface & 2006 & 239.702 & 220.326 & Surface \\
\hline 242.699 & 226.452 & Surface & 2006 & 242.699 & 220.260 & Surface \\
\hline 245.695 & 226.522 & Surface & 2006 & 245.695 & 220.130 & Surface \\
\hline 248.691 & 226.582 & Surface & 2006 & 248.691 & 220.040 & Surface \\
\hline 251.687 & 226.636 & Surface & 2006 & 251.687 & 219.944 & Surface \\
\hline 254.683 & 226.655 & Surface & 2006 & 254.683 & 219.803 & Surface \\
\hline 257.680 & 226.666 & Surface & 2006 & 257.680 & 219.587 & Surface \\
\hline 260.676 & 226.665 & Surface & 2006 & 260.676 & 219.246 & Surface \\
\hline 263.673 & 226.701 & Surface & 2006 & 263.673 & 218.995 & Surface \\
\hline 266.669 & 226.750 & Surface & 2006 & 266.669 & 218.970 & Surface \\
\hline 269.665 & 226.756 & Surface & 2006 & 269.665 & 218.976 & Surface \\
\hline 272.661 & 226.755 & Surface & 2006 & 272.661 & 218.945 & Surface \\
\hline 275.658 & 226.783 & Surface & 2006 & 275.658 & 218.911 & Surface \\
\hline 278.654 & 226.831 & Surface & 2006 & 278.654 & 218.895 & Surface \\
\hline
\end{tabular}




\begin{tabular}{|c|c|c|c|c|c|c|}
\hline 281.650 & 226.883 & Surface & 2006 & 281.650 & 218.886 & Surface \\
\hline 284.647 & 226.924 & Surface & 2006 & 284.647 & 218.873 & Surface \\
\hline 287.643 & 226.951 & Surface & 2006 & 287.643 & 218.831 & Surface \\
\hline 290.639 & 226.934 & Surface & 2006 & 290.639 & 218.760 & Surface \\
\hline 293.635 & 226.871 & Surface & 2006 & 293.635 & 218.745 & Surface \\
\hline 296.632 & 226.782 & Surface & 2006 & 296.632 & 218.773 & Surface \\
\hline 299.628 & 226.726 & Surface & 2006 & 299.628 & 218.762 & Surface \\
\hline 302.624 & 226.719 & Surface & 2006 & 302.624 & 218.767 & Surface \\
\hline 305.620 & 226.717 & Surface & 2006 & 305.620 & 218.768 & Surface \\
\hline 308.617 & 226.692 & Surface & 2006 & 308.617 & 218.770 & Surface \\
\hline 311.613 & 226.648 & Surface & 2006 & 311.613 & 218.749 & Surface \\
\hline 314.609 & 226.628 & Surface & 2006 & 314.609 & 218.731 & Surface \\
\hline 317.606 & 226.615 & Surface & 2006 & 317.606 & 218.721 & Surface \\
\hline 320.602 & 226.589 & Surface & 2006 & 320.602 & 218.742 & Surface \\
\hline 323.598 & 226.509 & Surface & 2006 & 323.598 & 218.790 & Surface \\
\hline 326.594 & 226.427 & Surface & 2006 & 326.594 & 218.830 & Surface \\
\hline 329.590 & 226.348 & Surface & 2006 & 329.590 & 218.826 & Surface \\
\hline 332.587 & 226.279 & Surface & 2006 & 332.587 & 218.837 & Surface \\
\hline 335.583 & 226.213 & Surface & 2006 & 335.583 & 218.832 & Surface \\
\hline 338.580 & 226.149 & Surface & 2006 & 338.580 & 218.977 & Surface \\
\hline 341.576 & 226.075 & Surface & 2006 & 341.576 & 219.279 & Surface \\
\hline 344.572 & 225.991 & Surface & 2006 & 344.572 & 219.590 & Surface \\
\hline 347.568 & 225.897 & Surface & 2006 & 347.568 & 219.858 & Surface \\
\hline 350.565 & 225.703 & Surface & 2006 & 350.565 & 220.114 & Surface \\
\hline 353.561 & 225.501 & Surface & 2006 & 353.561 & 220.357 & Surface \\
\hline 356.557 & 225.324 & Surface & 2006 & 356.557 & 220.555 & Surface \\
\hline 359.553 & 225.102 & Surface & 2006 & 359.553 & 220.694 & Surface \\
\hline 362.550 & 224.954 & Surface & 2006 & 362.550 & 220.799 & Surface \\
\hline 365.546 & 224.829 & Surface & 2006 & 365.546 & 220.903 & Surface \\
\hline 368.542 & 224.738 & Surface & 2006 & 368.542 & 221.031 & Surface \\
\hline 371.539 & 224.675 & Surface & 2006 & 371.539 & 221.223 & Surface \\
\hline 374.535 & 224.635 & Surface & 2006 & 374.535 & 221.530 & Surface \\
\hline 377.531 & 224.647 & Surface & 2006 & 377.531 & 221.864 & Surface \\
\hline 380.527 & 224.686 & Surface & 2006 & 380.527 & 222.138 & Surface \\
\hline 383.524 & 224.694 & Surface & 2006 & 383.524 & 222.369 & Surface \\
\hline 386.520 & 224.694 & Surface & 2006 & 386.520 & 222.632 & Surface \\
\hline 389.516 & 224.696 & Surface & 2006 & 389.516 & 223.037 & Surface \\
\hline 392.513 & 224.701 & Surface & 2006 & 392.513 & 223.442 & Surface \\
\hline 395.509 & 224.738 & Surface & 2006 & 395.509 & 223.878 & Surface \\
\hline 398.505 & 224.905 & Surface & 2006 & 398.505 & 224.278 & Surface \\
\hline 401.501 & 225.179 & Surface & 2006 & 401.501 & 224.677 & Surface \\
\hline 404.497 & 225.489 & Surface & 2006 & 404.497 & 225.075 & Surface \\
\hline 407.494 & 225.816 & Surface & 2006 & 407.494 & 225.483 & Surface \\
\hline 410.490 & 226.375 & Surface & 2006 & 410.490 & 226.055 & Surface \\
\hline 413.486 & 227.113 & Surface & 2006 & 413.486 & 226.668 & Surface \\
\hline 416.483 & 227.640 & Surface & 2006 & 416.483 & 227.259 & Surface \\
\hline 419.479 & 228.016 & Surface & 2006 & 419.479 & 227.769 & Surface \\
\hline 422.475 & 228.394 & Surface & 2006 & 422.475 & 228.254 & Surface \\
\hline 425.472 & 228.770 & Surface & 2006 & 425.472 & 228.735 & Surface \\
\hline
\end{tabular}




\begin{tabular}{|c|c|c|c|c|c|c|c|}
\hline 428.468 & 229.133 & Surface & 2006 & 428.468 & 229.216 & Surface & 2011 \\
\hline 431.464 & 229.411 & Surface & 2006 & 431.464 & 229.684 & Surface & 2011 \\
\hline 434.460 & 229.661 & Surface & 2006 & 434.460 & 229.933 & Surface & 2011 \\
\hline 437.457 & 229.905 & Surface & 2006 & 437.457 & 230.048 & Surface & 2011 \\
\hline 440.453 & 230.136 & Surface & 2006 & 440.453 & 230.172 & Surface & 2011 \\
\hline 443.449 & 230.385 & Surface & 2006 & 443.449 & 230.253 & Surface & 2011 \\
\hline 446.446 & 230.391 & Surface & 2006 & 446.446 & 230.480 & Surface & 2011 \\
\hline 449.442 & 230.595 & Surface & 2006 & 449.442 & 230.836 & Surface & 2011 \\
\hline 452.438 & 231.028 & Surface & 2006 & 452.438 & 231.273 & Surface & 2011 \\
\hline 455.434 & 231.812 & Surface & 2006 & 455.434 & 231.786 & Surface & 2011 \\
\hline 458.430 & 232.464 & Surface & 2006 & 458.430 & 232.325 & Surface & 2011 \\
\hline 461.427 & 232.614 & Surface & 2006 & 461.427 & 232.865 & Surface & 2011 \\
\hline 464.423 & 232.747 & Surface & 2006 & 464.423 & 233.384 & Surface & 2011 \\
\hline 467.420 & 232.888 & Surface & 2006 & 467.420 & 233.722 & Surface & 2011 \\
\hline 470.416 & 233.041 & Surface & 2006 & 470.416 & 233.744 & Surface & 2011 \\
\hline 473.412 & 233.196 & Surface & 2006 & 473.412 & 233.766 & Surface & 2011 \\
\hline 476.408 & 233.364 & Surface & 2006 & 476.408 & 233.789 & Surface & 2011 \\
\hline 0.000 & 238.985 & Surface & 2008 & 0.000 & 238.989 & Surface & 2012 \\
\hline 3.263 & 238.188 & Surface & 2008 & 3.263 & 238.361 & Surface & 2012 \\
\hline 6.526 & 237.433 & Surface & 2008 & 6.526 & 237.742 & Surface & 2012 \\
\hline 9.789 & 236.705 & Surface & 2008 & 9.789 & 237.080 & Surface & 2012 \\
\hline 13.052 & 235.984 & Surface & 2008 & 13.052 & 236.407 & Surface & 2012 \\
\hline 16.315 & 235.250 & Surface & 2008 & 16.315 & 235.729 & Surface & 2012 \\
\hline 19.578 & 234.535 & Surface & 2008 & 19.578 & 235.018 & Surface & 2012 \\
\hline 22.841 & 233.827 & Surface & 2008 & 22.841 & 234.223 & Surface & 2012 \\
\hline 26.105 & 233.106 & Surface & 2008 & 26.105 & 233.423 & Surface & 2012 \\
\hline 29.368 & 232.382 & Surface & 2008 & 29.368 & 232.692 & Surface & 2012 \\
\hline 32.631 & 231.670 & Surface & 2008 & 32.631 & 231.844 & Surface & 2012 \\
\hline 35.894 & 230.933 & Surface & 2008 & 35.894 & 230.991 & Surface & 2012 \\
\hline 39.157 & 230.137 & Surface & 2008 & 39.157 & 230.202 & Surface & 2012 \\
\hline 42.420 & 229.323 & Surface & 2008 & 42.420 & 229.319 & Surface & 2012 \\
\hline 45.683 & 228.512 & Surface & 2008 & 45.683 & 228.560 & Surface & 2012 \\
\hline 48.946 & 227.771 & Surface & 2008 & 48.946 & 228.001 & Surface & 2012 \\
\hline 52.209 & 227.063 & Surface & 2008 & 52.209 & 227.498 & Surface & 2012 \\
\hline 55.472 & 226.315 & Surface & 2008 & 55.472 & 226.879 & Surface & 2012 \\
\hline 58.735 & 225.612 & Surface & 2008 & 58.735 & 226.021 & Surface & 2012 \\
\hline 61.998 & 225.171 & Surface & 2008 & 61.998 & 225.383 & Surface & 2012 \\
\hline 65.262 & 225.024 & Surface & 2008 & 65.262 & 225.154 & Surface & 2012 \\
\hline 68.524 & 224.976 & Surface & 2008 & 68.524 & 225.041 & Surface & 2012 \\
\hline 71.788 & 224.976 & Surface & 2008 & 71.788 & 225.083 & Surface & 2012 \\
\hline 75.051 & 224.958 & Surface & 2008 & 75.051 & 225.147 & Surface & 2012 \\
\hline 78.314 & 224.804 & Surface & 2008 & 78.314 & 224.911 & Surface & 2012 \\
\hline 81.577 & 224.649 & Surface & 2008 & 81.577 & 224.738 & Surface & 2012 \\
\hline 84.840 & 224.493 & Surface & 2008 & 84.840 & 224.580 & Surface & 2012 \\
\hline 88.103 & 224.156 & Surface & 2008 & 88.103 & 224.309 & Surface & 2012 \\
\hline 91.366 & 223.824 & Surface & 2008 & 91.366 & 224.022 & Surface & 2012 \\
\hline 94.629 & 223.760 & Surface & 2008 & 94.629 & 223.856 & Surface & 2012 \\
\hline 97.892 & 223.694 & Surface & 2008 & 97.892 & 223.784 & Surface & 2012 \\
\hline 101.155 & 223.598 & Surface & 2008 & 101.155 & 223.659 & Surface & 2012 \\
\hline
\end{tabular}




\begin{tabular}{|c|c|c|c|c|c|c|c|}
\hline 104.418 & 223.510 & Surface & 2008 & 104.418 & 223.568 & Surface & 2012 \\
\hline 107.681 & 223.427 & Surface & 2008 & 107.681 & 223.487 & Surface & 2012 \\
\hline 110.944 & 223.341 & Surface & 2008 & 110.944 & 223.405 & Surface & 2012 \\
\hline 114.208 & 223.212 & Surface & 2008 & 114.208 & 223.271 & Surface & 2012 \\
\hline 117.470 & 223.059 & Surface & 2008 & 117.470 & 223.011 & Surface & 2012 \\
\hline 120.734 & 222.937 & Surface & 2008 & 120.734 & 222.719 & Surface & 2012 \\
\hline 123.997 & 222.793 & Surface & 2008 & 123.997 & 222.489 & Surface & 2012 \\
\hline 127.260 & 222.622 & Surface & 2008 & 127.260 & 222.173 & Surface & 2012 \\
\hline 130.523 & 222.492 & Surface & 2008 & 130.523 & 221.676 & Surface & 2012 \\
\hline 133.786 & 222.369 & Surface & 2008 & 133.786 & 221.098 & Surface & 2012 \\
\hline 137.049 & 222.168 & Surface & 2008 & 137.049 & 220.727 & Surface & 2012 \\
\hline 140.312 & 221.897 & Surface & 2008 & 140.312 & 220.683 & Surface & 2012 \\
\hline 143.575 & 221.635 & Surface & 2008 & 143.575 & 220.745 & Surface & 2012 \\
\hline 146.838 & 221.455 & Surface & 2008 & 146.838 & 220.793 & Surface & 2012 \\
\hline 150.101 & 221.283 & Surface & 2008 & 150.101 & 220.842 & Surface & 2012 \\
\hline 153.364 & 221.155 & Surface & 2008 & 153.364 & 220.902 & Surface & 2012 \\
\hline 156.627 & 221.093 & Surface & 2008 & 156.627 & 220.905 & Surface & 2012 \\
\hline 159.890 & 221.084 & Surface & 2008 & 159.890 & 220.902 & Surface & 2012 \\
\hline 163.154 & 221.037 & Surface & 2008 & 163.154 & 220.870 & Surface & 2012 \\
\hline 166.417 & 221.036 & Surface & 2008 & 166.417 & 220.861 & Surface & 2012 \\
\hline 169.680 & 221.056 & Surface & 2008 & 169.680 & 220.849 & Surface & 2012 \\
\hline 172.943 & 221.057 & Surface & 2008 & 172.943 & 220.817 & Surface & 2012 \\
\hline 176.206 & 221.008 & Surface & 2008 & 176.206 & 220.674 & Surface & 2012 \\
\hline 179.469 & 220.993 & Surface & 2008 & 179.469 & 220.540 & Surface & 2012 \\
\hline 182.732 & 220.991 & Surface & 2008 & 182.732 & 220.509 & Surface & 2012 \\
\hline 185.995 & 220.982 & Surface & 2008 & 185.995 & 220.321 & Surface & 2012 \\
\hline 189.258 & 220.911 & Surface & 2008 & 189.258 & 220.312 & Surface & 2012 \\
\hline 192.521 & 220.833 & Surface & 2008 & 192.521 & 220.257 & Surface & 2012 \\
\hline 195.784 & 220.712 & Surface & 2008 & 195.784 & 220.182 & Surface & 2012 \\
\hline 199.047 & 220.586 & Surface & 2008 & 199.047 & 220.158 & Surface & 2012 \\
\hline 202.311 & 220.442 & Surface & 2008 & 202.311 & 220.125 & Surface & 2012 \\
\hline 205.573 & 220.343 & Surface & 2008 & 205.573 & 220.091 & Surface & 2012 \\
\hline 208.837 & 220.274 & Surface & 2008 & 208.837 & 220.072 & Surface & 2012 \\
\hline 212.100 & 220.283 & Surface & 2008 & 212.100 & 220.091 & Surface & 2012 \\
\hline 215.363 & 220.316 & Surface & 2008 & 215.363 & 220.057 & Surface & 2012 \\
\hline 218.626 & 220.315 & Surface & 2008 & 218.626 & 220.033 & Surface & 2012 \\
\hline 221.889 & 220.356 & Surface & 2008 & 221.889 & 220.017 & Surface & 2012 \\
\hline 225.152 & 220.373 & Surface & 2008 & 225.152 & 219.989 & Surface & 2012 \\
\hline 228.415 & 220.321 & Surface & 2008 & 228.415 & 219.955 & Surface & 2012 \\
\hline 231.678 & 220.263 & Surface & 2008 & 231.678 & 219.946 & Surface & 2012 \\
\hline 234.941 & 220.278 & Surface & 2008 & 234.941 & 219.952 & Surface & 2012 \\
\hline 238.204 & 220.305 & Surface & 2008 & 238.204 & 219.955 & Surface & 2012 \\
\hline 241.467 & 220.312 & Surface & 2008 & 241.467 & 219.952 & Surface & 2012 \\
\hline 244.730 & 220.310 & Surface & 2008 & 244.730 & 219.940 & Surface & 2012 \\
\hline 247.993 & 220.300 & Surface & 2008 & 247.993 & 219.905 & Surface & 2012 \\
\hline 251.257 & 220.285 & Surface & 2008 & 251.257 & 219.869 & Surface & 2012 \\
\hline 254.519 & 220.243 & Surface & 2008 & 254.519 & 219.823 & Surface & 2012 \\
\hline 257.783 & 220.240 & Surface & 2008 & 257.783 & 219.801 & Surface & 2012 \\
\hline 261.046 & 220.277 & Surface & 2008 & 261.046 & 219.764 & Surface & 2012 \\
\hline
\end{tabular}




\begin{tabular}{|c|c|c|c|c|c|c|c|}
\hline 264.309 & 220.352 & Surface & 2008 & 264.309 & 219.781 & Surface & 2012 \\
\hline 267.572 & 220.380 & Surface & 2008 & 267.572 & 219.767 & Surface & 2012 \\
\hline 270.835 & 220.367 & Surface & 2008 & 270.835 & 219.746 & Surface & 2012 \\
\hline 274.098 & 220.389 & Surface & 2008 & 274.098 & 219.659 & Surface & 2012 \\
\hline 277.361 & 220.606 & Surface & 2008 & 277.361 & 219.524 & Surface & 2012 \\
\hline 280.624 & 221.039 & Surface & 2008 & 280.624 & 219.488 & Surface & 2012 \\
\hline 283.887 & 221.715 & Surface & 2008 & 283.887 & 219.433 & Surface & 2012 \\
\hline 287.150 & 222.418 & Surface & 2008 & 287.150 & 219.402 & Surface & 2012 \\
\hline 290.413 & 223.060 & Surface & 2008 & 290.413 & 219.360 & Surface & 2012 \\
\hline 293.676 & 223.662 & Surface & 2008 & 293.676 & 219.322 & Surface & 2012 \\
\hline 296.940 & 224.439 & Surface & 2008 & 296.940 & 219.285 & Surface & 2012 \\
\hline 300.202 & 225.441 & Surface & 2008 & 300.202 & 219.261 & Surface & 2012 \\
\hline 303.466 & 226.388 & Surface & 2008 & 303.466 & 219.226 & Surface & 2012 \\
\hline 306.729 & 226.637 & Surface & 2008 & 306.729 & 219.187 & Surface & 2012 \\
\hline 309.992 & 226.636 & Surface & 2008 & 309.992 & 219.125 & Surface & 2012 \\
\hline 313.255 & 226.591 & Surface & 2008 & 313.255 & 219.051 & Surface & 2012 \\
\hline 316.518 & 226.542 & Surface & 2008 & 316.518 & 218.975 & Surface & 2012 \\
\hline 319.781 & 226.542 & Surface & 2008 & 319.781 & 218.955 & Surface & 2012 \\
\hline 323.044 & 226.499 & Surface & 2008 & 323.044 & 218.971 & Surface & 2012 \\
\hline 326.307 & 226.409 & Surface & 2008 & 326.307 & 218.965 & Surface & 2012 \\
\hline 329.570 & 226.324 & Surface & 2008 & 329.570 & 218.968 & Surface & 2012 \\
\hline 332.833 & 226.262 & Surface & 2008 & 332.833 & 219.011 & Surface & 2012 \\
\hline 336.096 & 226.218 & Surface & 2008 & 336.096 & 219.065 & Surface & 2012 \\
\hline 339.359 & 226.155 & Surface & 2008 & 339.359 & 219.182 & Surface & 2012 \\
\hline 342.622 & 226.065 & Surface & 2008 & 342.622 & 219.468 & Surface & 2012 \\
\hline 345.886 & 225.975 & Surface & 2008 & 345.886 & 219.793 & Surface & 2012 \\
\hline 349.148 & 225.839 & Surface & 2008 & 349.148 & 220.022 & Surface & 2012 \\
\hline 352.412 & 225.642 & Surface & 2008 & 352.412 & 220.299 & Surface & 2012 \\
\hline 355.675 & 225.425 & Surface & 2008 & 355.675 & 220.585 & Surface & 2012 \\
\hline 358.938 & 225.208 & Surface & 2008 & 358.938 & 220.782 & Surface & 2012 \\
\hline 362.201 & 225.037 & Surface & 2008 & 362.201 & 220.815 & Surface & 2012 \\
\hline 365.464 & 224.880 & Surface & 2008 & 365.464 & 220.936 & Surface & 2012 \\
\hline 368.727 & 224.766 & Surface & 2008 & 368.727 & 221.142 & Surface & 2012 \\
\hline 371.990 & 224.675 & Surface & 2008 & 371.990 & 221.455 & Surface & 2012 \\
\hline 375.253 & 224.640 & Surface & 2008 & 375.253 & 221.672 & Surface & 2012 \\
\hline 378.516 & 224.612 & Surface & 2008 & 378.516 & 221.961 & Surface & 2012 \\
\hline 381.779 & 224.523 & Surface & 2008 & 381.779 & 222.396 & Surface & 2012 \\
\hline 385.042 & 224.339 & Surface & 2008 & 385.042 & 222.730 & Surface & 2012 \\
\hline 388.305 & 224.181 & Surface & 2008 & 388.305 & 223.218 & Surface & 2012 \\
\hline 391.568 & 224.097 & Surface & 2008 & 391.568 & 223.659 & Surface & 2012 \\
\hline 394.832 & 224.159 & Surface & 2008 & 394.832 & 224.081 & Surface & 2012 \\
\hline 398.094 & 224.321 & Surface & 2008 & 398.094 & 224.469 & Surface & 2012 \\
\hline 401.358 & 224.625 & Surface & 2008 & 401.358 & 224.859 & Surface & 2012 \\
\hline 404.621 & 225.126 & Surface & 2008 & 404.621 & 225.186 & Surface & 2012 \\
\hline 407.884 & 225.616 & Surface & 2008 & 407.884 & 225.577 & Surface & 2012 \\
\hline 411.147 & 226.101 & Surface & 2008 & 411.147 & 226.239 & Surface & 2012 \\
\hline 414.410 & 226.580 & Surface & 2008 & 414.410 & 227.040 & Surface & 2012 \\
\hline 417.673 & 227.065 & Surface & 2008 & 417.673 & 228.024 & Surface & 2012 \\
\hline 420.936 & 227.624 & Surface & 2008 & 420.936 & 229.035 & Surface & 2012 \\
\hline
\end{tabular}




$\begin{array}{llllllll}424.199 & 228.271 & \text { Surface } & 2008 & 424.199 & 229.528 & \text { Surface } & 2012 \\ 427.462 & 228.792 & \text { Surface } & 2008 & 427.462 & 229.621 & \text { Surface } & 2012 \\ 430.725 & 229.179 & \text { Surface } & 2008 & 430.725 & 229.679 & \text { Surface } & 2012 \\ 433.988 & 229.472 & \text { Surface } & 2008 & 433.988 & 229.749 & \text { Surface } & 2012 \\ 437.251 & 229.717 & \text { Surface } & 2008 & 437.251 & 229.758 & \text { Surface } & 2012 \\ 440.515 & 229.982 & \text { Surface } & 2008 & 440.515 & 229.862 & \text { Surface } & 2012 \\ 443.778 & 230.187 & \text { Surface } & 2008 & 443.778 & 230.019 & \text { Surface } & 2012 \\ 447.040 & 230.457 & \text { Surface } & 2008 & 447.040 & 230.282 & \text { Surface } & 2012 \\ 450.304 & 230.933 & \text { Surface } & 2008 & 450.304 & 230.899 & \text { Surface } & 2012 \\ 453.567 & 231.581 & \text { Surface } & 2008 & 453.567 & 231.695 & \text { Surface } & 2012 \\ 456.830 & 232.161 & \text { Surface } & 2008 & 456.830 & 232.351 & \text { Surface } & 2012 \\ 460.093 & 232.476 & \text { Surface } & 2008 & 460.093 & 232.765 & \text { Surface } & 2012 \\ 463.356 & 232.620 & \text { Surface } & 2008 & 463.356 & 232.954 & \text { Surface } & 2012 \\ 466.619 & 232.698 & \text { Surface } & 2008 & 466.619 & 233.015 & \text { Surface } & 2012 \\ 469.882 & 232.839 & \text { Surface } & 2008 & 469.882 & 233.052 & \text { Surface } & 2012 \\ 473.145 & 232.987 & \text { Surface } & 2008 & 473.145 & 233.092 & \text { Surface } & 2012 \\ 476.408 & 233.155 & \text { Surface } & 2008 & 476.408 & 233.160 & \text { Surface } & 2012\end{array}$


10.8 Transect H - Knickpoint - All Returns:

\begin{tabular}{|c|c|c|c|c|c|c|c|}
\hline Distance & $\mathrm{Z}$ & DEM & Year & Distance & $\mathrm{Z}$ & DEM & Year \\
\hline 0.000 & 255.134 & Surface & 2006 & 0.000 & 255.179 & Surface & 2011 \\
\hline 2.998 & 255.158 & Surface & 2006 & 2.998 & 254.859 & Surface & 2011 \\
\hline 5.997 & 255.194 & Surface & 2006 & 5.997 & 253.906 & Surface & 2011 \\
\hline 8.995 & 254.814 & Surface & 2006 & 8.995 & 252.939 & Surface & 2011 \\
\hline 11.994 & 253.873 & Surface & 2006 & 11.994 & 252.009 & Surface & 2011 \\
\hline 14.992 & 252.880 & Surface & 2006 & 14.992 & 251.090 & Surface & 2011 \\
\hline 17.991 & 251.895 & Surface & 2006 & 17.991 & 250.171 & Surface & 2011 \\
\hline 20.989 & 250.856 & Surface & 2006 & 20.989 & 249.254 & Surface & 2011 \\
\hline 23.988 & 249.606 & Surface & 2006 & 23.988 & 248.307 & Surface & 2011 \\
\hline 26.986 & 248.250 & Surface & 2006 & 26.986 & 247.019 & Surface & 2011 \\
\hline 29.984 & 247.145 & Surface & 2006 & 29.984 & 246.082 & Surface & 2011 \\
\hline 32.983 & 246.202 & Surface & 2006 & 32.983 & 245.479 & Surface & 2011 \\
\hline 35.981 & 245.411 & Surface & 2006 & 35.981 & 244.904 & Surface & 2011 \\
\hline 38.980 & 244.772 & Surface & 2006 & 38.980 & 244.254 & Surface & 2011 \\
\hline 41.978 & 244.163 & Surface & 2006 & 41.978 & 243.629 & Surface & 2011 \\
\hline 44.977 & 243.654 & Surface & 2006 & 44.977 & 243.076 & Surface & 2011 \\
\hline 47.975 & 243.264 & Surface & 2006 & 47.975 & 242.276 & Surface & 2011 \\
\hline 50.973 & 242.709 & Surface & 2006 & 50.973 & 241.409 & Surface & 2011 \\
\hline 53.972 & 241.929 & Surface & 2006 & 53.972 & 240.585 & Surface & 2011 \\
\hline 56.970 & 241.028 & Surface & 2006 & 56.970 & 239.674 & Surface & 2011 \\
\hline 59.969 & 240.020 & Surface & 2006 & 59.969 & 238.737 & Surface & 2011 \\
\hline 62.967 & 239.354 & Surface & 2006 & 62.967 & 237.796 & Surface & 2011 \\
\hline 65.965 & 238.637 & Surface & 2006 & 65.965 & 236.857 & Surface & 2011 \\
\hline 68.964 & 237.849 & Surface & 2006 & 68.964 & 235.956 & Surface & 2011 \\
\hline 71.963 & 236.787 & Surface & 2006 & 71.963 & 235.211 & Surface & 2011 \\
\hline 74.961 & 235.771 & Surface & 2006 & 74.961 & 234.540 & Surface & 2011 \\
\hline 77.959 & 234.874 & Surface & 2006 & 77.959 & 234.061 & Surface & 2011 \\
\hline 80.958 & 233.943 & Surface & 2006 & 80.958 & 233.678 & Surface & 2011 \\
\hline 83.956 & 233.309 & Surface & 2006 & 83.956 & 233.173 & Surface & 2011 \\
\hline 86.955 & 232.788 & Surface & 2006 & 86.955 & 232.600 & Surface & 2011 \\
\hline 89.953 & 232.289 & Surface & 2006 & 89.953 & 232.034 & Surface & 2011 \\
\hline 92.951 & 232.043 & Surface & 2006 & 92.951 & 231.705 & Surface & 2011 \\
\hline 95.950 & 231.969 & Surface & 2006 & 95.950 & 231.574 & Surface & 2011 \\
\hline 98.948 & 231.903 & Surface & 2006 & 98.948 & 231.483 & Surface & 2011 \\
\hline 101.947 & 231.849 & Surface & 2006 & 101.947 & 231.442 & Surface & 2011 \\
\hline 104.945 & 231.803 & Surface & 2006 & 104.945 & 231.431 & Surface & 2011 \\
\hline 107.944 & 231.792 & Surface & 2006 & 107.944 & 231.452 & Surface & 2011 \\
\hline 110.942 & 231.779 & Surface & 2006 & 110.942 & 231.470 & Surface & 2011 \\
\hline 113.941 & 231.808 & Surface & 2006 & 113.941 & 231.479 & Surface & 2011 \\
\hline 116.939 & 231.848 & Surface & 2006 & 116.939 & 231.479 & Surface & 2011 \\
\hline 119.938 & 232.000 & Surface & 2006 & 119.938 & 231.477 & Surface & 2011 \\
\hline 122.936 & 232.116 & Surface & 2006 & 122.936 & 231.481 & Surface & 2011 \\
\hline 125.934 & 232.272 & Surface & 2006 & 125.934 & 231.493 & Surface & 2011 \\
\hline 128.933 & 232.457 & Surface & 2006 & 128.933 & 231.480 & Surface & 2011 \\
\hline 131.931 & 232.546 & Surface & 2006 & 131.931 & 231.468 & Surface & 2011 \\
\hline
\end{tabular}




\begin{tabular}{|c|c|c|c|c|c|c|}
\hline 134.930 & 232.611 & Surface & 2006 & 134.930 & 231.485 & Surface \\
\hline 137.928 & 232.709 & Surface & 2006 & 137.928 & 231.546 & Surface \\
\hline 140.927 & 232.769 & Surface & 2006 & 140.927 & 231.636 & Surface \\
\hline 143.925 & 232.835 & Surface & 2006 & 143.925 & 231.781 & Surface \\
\hline 146.923 & 232.857 & Surface & 2006 & 146.923 & 231.952 & Surface \\
\hline 149.922 & 232.834 & Surface & 2006 & 149.922 & 232.117 & Surface \\
\hline 152.920 & 232.816 & Surface & 2006 & 152.920 & 232.251 & Surface \\
\hline 155.919 & 232.795 & Surface & 2006 & 155.919 & 232.350 & Surface \\
\hline 158.917 & 232.724 & Surface & 2006 & 158.917 & 232.418 & Surface \\
\hline 161.916 & 232.615 & Surface & 2006 & 161.916 & 232.421 & Surface \\
\hline 164.914 & 232.549 & Surface & 2006 & 164.914 & 232.349 & Surface \\
\hline 167.913 & 232.500 & Surface & 2006 & 167.913 & 232.174 & Surface \\
\hline 170.911 & 232.451 & Surface & 2006 & 170.911 & 231.836 & Surface \\
\hline 173.909 & 232.370 & Surface & 2006 & 173.909 & 231.397 & Surface \\
\hline 176.908 & 232.239 & Surface & 2006 & 176.908 & 231.002 & Surface \\
\hline 179.906 & 232.101 & Surface & 2006 & 179.906 & 230.770 & Surface \\
\hline 182.905 & 231.978 & Surface & 2006 & 182.905 & 230.618 & Surface \\
\hline 185.903 & 231.841 & Surface & 2006 & 185.903 & 230.574 & Surface \\
\hline 188.901 & 231.693 & Surface & 2006 & 188.901 & 230.536 & Surface \\
\hline 191.900 & 231.518 & Surface & 2006 & 191.900 & 230.509 & Surface \\
\hline 194.898 & 231.365 & Surface & 2006 & 194.898 & 230.503 & Surface \\
\hline 197.897 & 231.317 & Surface & 2006 & 197.897 & 230.525 & Surface \\
\hline 200.895 & 231.320 & Surface & 2006 & 200.895 & 230.527 & Surface \\
\hline 203.894 & 231.295 & Surface & 2006 & 203.894 & 230.532 & Surface \\
\hline 206.892 & 231.190 & Surface & 2006 & 206.892 & 230.538 & Surface \\
\hline 209.891 & 231.125 & Surface & 2006 & 209.891 & 230.543 & Surface \\
\hline 212.889 & 231.112 & Surface & 2006 & 212.889 & 230.517 & Surface \\
\hline 215.887 & 231.148 & Surface & 2006 & 215.887 & 230.511 & Surface \\
\hline 218.886 & 231.007 & Surface & 2006 & 218.886 & 230.530 & Surface \\
\hline 221.884 & 230.977 & Surface & 2006 & 221.884 & 230.571 & Surface \\
\hline 224.883 & 231.159 & Surface & 2006 & 224.883 & 230.660 & Surface \\
\hline 227.881 & 231.307 & Surface & 2006 & 227.881 & 230.787 & Surface \\
\hline 230.880 & 231.540 & Surface & 2006 & 230.880 & 230.913 & Surface \\
\hline 233.878 & 231.743 & Surface & 2006 & 233.878 & 231.028 & Surface \\
\hline 236.876 & 231.836 & Surface & 2006 & 236.876 & 231.139 & Surface \\
\hline 239.875 & 231.855 & Surface & 2006 & 239.875 & 231.244 & Surface \\
\hline 242.873 & 231.842 & Surface & 2006 & 242.873 & 231.290 & Surface \\
\hline 245.872 & 231.868 & Surface & 2006 & 245.872 & 231.312 & Surface \\
\hline 248.870 & 231.881 & Surface & 2006 & 248.870 & 231.371 & Surface \\
\hline 251.869 & 231.910 & Surface & 2006 & 251.869 & 231.439 & Surface \\
\hline 254.867 & 231.862 & Surface & 2006 & 254.867 & 231.515 & Surface \\
\hline 257.866 & 231.928 & Surface & 2006 & 257.866 & 231.590 & Surface \\
\hline 260.864 & 231.923 & Surface & 2006 & 260.864 & 231.686 & Surface \\
\hline 263.863 & 231.957 & Surface & 2006 & 263.863 & 231.880 & Surface \\
\hline 266.861 & 232.110 & Surface & 2006 & 266.861 & 232.212 & Surface \\
\hline 269.859 & 232.434 & Surface & 2006 & 269.859 & 232.647 & Surface \\
\hline 272.858 & 232.876 & Surface & 2006 & 272.858 & 233.093 & Surface \\
\hline 275.856 & 233.387 & Surface & 2006 & 275.856 & 233.548 & Surface \\
\hline 278.855 & 234.065 & Surface & 2006 & 278.855 & 234.009 & Surface \\
\hline
\end{tabular}




\begin{tabular}{|c|c|c|c|c|c|c|c|}
\hline 281.853 & 234.699 & Surface & 2006 & 281.853 & 234.470 & Surface & 2011 \\
\hline 284.851 & 235.124 & Surface & 2006 & 284.851 & 234.932 & Surface & 2011 \\
\hline 287.850 & 235.489 & Surface & 2006 & 287.850 & 235.380 & Surface & 2011 \\
\hline 290.849 & 235.851 & Surface & 2006 & 290.849 & 235.750 & Surface & 2011 \\
\hline 293.847 & 236.209 & Surface & 2006 & 293.847 & 236.015 & Surface & 2011 \\
\hline 296.845 & 236.561 & Surface & 2006 & 296.845 & 236.286 & Surface & 2011 \\
\hline 299.844 & 236.879 & Surface & 2006 & 299.844 & 236.574 & Surface & 2011 \\
\hline 302.842 & 237.174 & Surface & 2006 & 302.842 & 236.864 & Surface & 2011 \\
\hline 305.841 & 237.462 & Surface & 2006 & 305.841 & 237.153 & Surface & 2011 \\
\hline 308.839 & 237.749 & Surface & 2006 & 308.839 & 237.443 & Surface & 2011 \\
\hline 311.837 & 238.038 & Surface & 2006 & 311.837 & 237.733 & Surface & 2011 \\
\hline 314.836 & 238.328 & Surface & 2006 & 314.836 & 238.022 & Surface & 2011 \\
\hline 317.834 & 238.627 & Surface & 2006 & 317.834 & 238.312 & Surface & 2011 \\
\hline 320.833 & 238.944 & Surface & 2006 & 320.833 & 238.601 & Surface & 2011 \\
\hline 323.831 & 239.276 & Surface & 2006 & 323.831 & 238.891 & Surface & 2011 \\
\hline 326.830 & 239.612 & Surface & 2006 & 326.830 & 239.181 & Surface & 2011 \\
\hline 329.828 & 239.936 & Surface & 2006 & 329.828 & 239.481 & Surface & 2011 \\
\hline 332.826 & 240.146 & Surface & 2006 & 332.826 & 239.780 & Surface & 2011 \\
\hline 335.825 & 240.172 & Surface & 2006 & 335.825 & 240.062 & Surface & 2011 \\
\hline 338.823 & 240.208 & Surface & 2006 & 338.823 & 240.370 & Surface & 2011 \\
\hline 341.822 & 240.369 & Surface & 2006 & 341.822 & 240.680 & Surface & 2011 \\
\hline 344.820 & 240.597 & Surface & 2006 & 344.820 & 241.054 & Surface & 2011 \\
\hline 347.819 & 240.918 & Surface & 2006 & 347.819 & 241.447 & Surface & 2011 \\
\hline 350.817 & 241.226 & Surface & 2006 & 350.817 & 241.806 & Surface & 2011 \\
\hline 353.816 & 241.515 & Surface & 2006 & 353.816 & 242.108 & Surface & 2011 \\
\hline 356.814 & 241.797 & Surface & 2006 & 356.814 & 242.437 & Surface & 2011 \\
\hline 359.812 & 242.075 & Surface & 2006 & 359.812 & 242.762 & Surface & 2011 \\
\hline 362.811 & 242.367 & Surface & 2006 & 362.811 & 243.082 & Surface & 2011 \\
\hline 365.809 & 242.673 & Surface & 2006 & 365.809 & 243.401 & Surface & 2011 \\
\hline 368.808 & 242.991 & Surface & 2006 & 368.808 & 243.719 & Surface & 2011 \\
\hline 371.806 & 243.328 & Surface & 2006 & 371.806 & 243.980 & Surface & 2011 \\
\hline 374.805 & 243.867 & Surface & 2006 & 374.805 & 244.207 & Surface & 2011 \\
\hline 377.803 & 244.240 & Surface & 2006 & 377.803 & 244.422 & Surface & 2011 \\
\hline 380.802 & 244.530 & Surface & 2006 & 380.802 & 244.647 & Surface & 2011 \\
\hline 383.800 & 244.874 & Surface & 2006 & 383.800 & 244.875 & Surface & 2011 \\
\hline 386.798 & 245.184 & Surface & 2006 & 386.798 & 245.105 & Surface & 2011 \\
\hline 389.797 & 245.416 & Surface & 2006 & 389.797 & 245.334 & Surface & 2011 \\
\hline 392.795 & 245.621 & Surface & 2006 & 392.795 & 245.564 & Surface & 2011 \\
\hline 395.794 & 245.827 & Surface & 2006 & 395.794 & 245.804 & Surface & 2011 \\
\hline 398.792 & 246.073 & Surface & 2006 & 398.792 & 246.031 & Surface & 2011 \\
\hline 0.000 & 255.321 & Surface & 2008 & 0.000 & 255.239 & Surface & 2012 \\
\hline 3.269 & 255.341 & Surface & 2008 & 3.269 & 255.232 & Surface & 2012 \\
\hline 6.537 & 255.092 & Surface & 2008 & 6.537 & 255.179 & Surface & 2012 \\
\hline 9.806 & 253.902 & Surface & 2008 & 9.806 & 254.245 & Surface & 2012 \\
\hline 13.075 & 252.762 & Surface & 2008 & 13.075 & 253.193 & Surface & 2012 \\
\hline 16.344 & 251.666 & Surface & 2008 & 16.344 & 252.079 & Surface & 2012 \\
\hline 19.613 & 250.554 & Surface & 2008 & 19.613 & 250.825 & Surface & 2012 \\
\hline 22.882 & 249.391 & Surface & 2008 & 22.882 & 249.422 & Surface & 2012 \\
\hline 26.150 & 248.459 & Surface & 2008 & 26.150 & 247.853 & Surface & 2012 \\
\hline
\end{tabular}




\begin{tabular}{|c|c|c|c|c|c|c|c|}
\hline 29.419 & 247.239 & Surface & 2008 & 29.419 & 246.762 & Surface & 2012 \\
\hline 32.688 & 246.036 & Surface & 2008 & 32.688 & 245.897 & Surface & 2012 \\
\hline 35.957 & 245.305 & Surface & 2008 & 35.957 & 245.285 & Surface & 2012 \\
\hline 39.226 & 244.636 & Surface & 2008 & 39.226 & 244.508 & Surface & 2012 \\
\hline 42.494 & 243.993 & Surface & 2008 & 42.494 & 243.858 & Surface & 2012 \\
\hline 45.763 & 243.236 & Surface & 2008 & 45.763 & 243.195 & Surface & 2012 \\
\hline 49.032 & 242.432 & Surface & 2008 & 49.032 & 242.281 & Surface & 2012 \\
\hline 52.301 & 241.540 & Surface & 2008 & 52.301 & 241.359 & Surface & 2012 \\
\hline 55.569 & 240.637 & Surface & 2008 & 55.569 & 240.383 & Surface & 2012 \\
\hline 58.838 & 239.845 & Surface & 2008 & 58.838 & 239.260 & Surface & 2012 \\
\hline 62.107 & 239.115 & Surface & 2008 & 62.107 & 238.165 & Surface & 2012 \\
\hline 65.376 & 238.365 & Surface & 2008 & 65.376 & 237.134 & Surface & 2012 \\
\hline 68.645 & 237.458 & Surface & 2008 & 68.645 & 236.207 & Surface & 2012 \\
\hline 71.913 & 236.264 & Surface & 2008 & 71.913 & 235.448 & Surface & 2012 \\
\hline 75.182 & 235.051 & Surface & 2008 & 75.182 & 234.834 & Surface & 2012 \\
\hline 78.451 & 234.126 & Surface & 2008 & 78.451 & 234.138 & Surface & 2012 \\
\hline 81.720 & 233.348 & Surface & 2008 & 81.720 & 233.454 & Surface & 2012 \\
\hline 84.989 & 232.596 & Surface & 2008 & 84.989 & 232.942 & Surface & 2012 \\
\hline 88.257 & 232.045 & Surface & 2008 & 88.257 & 232.544 & Surface & 2012 \\
\hline 91.526 & 231.845 & Surface & 2008 & 91.526 & 232.104 & Surface & 2012 \\
\hline 94.795 & 231.780 & Surface & 2008 & 94.795 & 231.754 & Surface & 2012 \\
\hline 98.064 & 231.763 & Surface & 2008 & 98.064 & 231.608 & Surface & 2012 \\
\hline 101.332 & 231.761 & Surface & 2008 & 101.332 & 231.536 & Surface & 2012 \\
\hline 104.601 & 231.752 & Surface & 2008 & 104.601 & 231.489 & Surface & 2012 \\
\hline 107.870 & 231.718 & Surface & 2008 & 107.870 & 231.439 & Surface & 2012 \\
\hline 111.139 & 231.697 & Surface & 2008 & 111.139 & 231.455 & Surface & 2012 \\
\hline 114.408 & 231.791 & Surface & 2008 & 114.408 & 231.517 & Surface & 2012 \\
\hline 117.676 & 231.994 & Surface & 2008 & 117.676 & 231.543 & Surface & 2012 \\
\hline 120.945 & 232.069 & Surface & 2008 & 120.945 & 231.600 & Surface & 2012 \\
\hline 124.214 & 232.267 & Surface & 2008 & 124.214 & 231.612 & Surface & 2012 \\
\hline 127.483 & 232.454 & Surface & 2008 & 127.483 & 231.650 & Surface & 2012 \\
\hline 130.752 & 232.576 & Surface & 2008 & 130.752 & 231.747 & Surface & 2012 \\
\hline 134.020 & 232.690 & Surface & 2008 & 134.020 & 231.886 & Surface & 2012 \\
\hline 137.289 & 232.754 & Surface & 2008 & 137.289 & 231.998 & Surface & 2012 \\
\hline 140.558 & 232.779 & Surface & 2008 & 140.558 & 232.083 & Surface & 2012 \\
\hline 143.827 & 232.781 & Surface & 2008 & 143.827 & 232.143 & Surface & 2012 \\
\hline 147.096 & 232.811 & Surface & 2008 & 147.096 & 232.251 & Surface & 2012 \\
\hline 150.364 & 232.824 & Surface & 2008 & 150.364 & 232.285 & Surface & 2012 \\
\hline 153.633 & 232.723 & Surface & 2008 & 153.633 & 232.240 & Surface & 2012 \\
\hline 156.902 & 232.632 & Surface & 2008 & 156.902 & 232.193 & Surface & 2012 \\
\hline 160.171 & 232.571 & Surface & 2008 & 160.171 & 231.871 & Surface & 2012 \\
\hline 163.439 & 232.489 & Surface & 2008 & 163.439 & 231.551 & Surface & 2012 \\
\hline 166.708 & 232.427 & Surface & 2008 & 166.708 & 231.350 & Surface & 2012 \\
\hline 169.977 & 232.350 & Surface & 2008 & 169.977 & 231.170 & Surface & 2012 \\
\hline 173.246 & 232.258 & Surface & 2008 & 173.246 & 231.062 & Surface & 2012 \\
\hline 176.515 & 232.130 & Surface & 2008 & 176.515 & 231.078 & Surface & 2012 \\
\hline 179.783 & 231.969 & Surface & 2008 & 179.783 & 231.025 & Surface & 2012 \\
\hline 183.052 & 231.813 & Surface & 2008 & 183.052 & 230.869 & Surface & 2012 \\
\hline 186.321 & 231.636 & Surface & 2008 & 186.321 & 230.775 & Surface & 2012 \\
\hline
\end{tabular}




\begin{tabular}{|c|c|c|c|c|c|c|c|}
\hline 189.590 & 231.366 & Surface & 2008 & 189.590 & 230.737 & Surface & 2012 \\
\hline 192.858 & 231.147 & Surface & 2008 & 192.858 & 230.712 & Surface & 2012 \\
\hline 196.127 & 231.121 & Surface & 2008 & 196.127 & 230.709 & Surface & 2012 \\
\hline 199.396 & 231.132 & Surface & 2008 & 199.396 & 230.674 & Surface & 2012 \\
\hline 202.665 & 231.075 & Surface & 2008 & 202.665 & 230.630 & Surface & 2012 \\
\hline 205.934 & 231.034 & Surface & 2008 & 205.934 & 230.661 & Surface & 2012 \\
\hline 209.202 & 231.012 & Surface & 2008 & 209.202 & 230.712 & Surface & 2012 \\
\hline 212.471 & 231.002 & Surface & 2008 & 212.471 & 230.768 & Surface & 2012 \\
\hline 215.740 & 230.977 & Surface & 2008 & 215.740 & 230.672 & Surface & 2012 \\
\hline 219.009 & 230.970 & Surface & 2008 & 219.009 & 230.666 & Surface & 2012 \\
\hline 222.278 & 231.136 & Surface & 2008 & 222.278 & 230.693 & Surface & 2012 \\
\hline 225.546 & 231.260 & Surface & 2008 & 225.546 & 230.877 & Surface & 2012 \\
\hline 228.815 & 231.497 & Surface & 2008 & 228.815 & 231.130 & Surface & 2012 \\
\hline 232.084 & 231.724 & Surface & 2008 & 232.084 & 231.435 & Surface & 2012 \\
\hline 235.353 & 231.716 & Surface & 2008 & 235.353 & 231.733 & Surface & 2012 \\
\hline 238.621 & 231.716 & Surface & 2008 & 238.621 & 231.591 & Surface & 2012 \\
\hline 241.890 & 231.754 & Surface & 2008 & 241.890 & 231.494 & Surface & 2012 \\
\hline 245.159 & 231.772 & Surface & 2008 & 245.159 & 231.426 & Surface & 2012 \\
\hline 248.428 & 231.813 & Surface & 2008 & 248.428 & 231.440 & Surface & 2012 \\
\hline 251.697 & 231.860 & Surface & 2008 & 251.697 & 231.490 & Surface & 2012 \\
\hline 254.965 & 231.901 & Surface & 2008 & 254.965 & 231.530 & Surface & 2012 \\
\hline 258.234 & 231.950 & Surface & 2008 & 258.234 & 231.583 & Surface & 2012 \\
\hline 261.503 & 231.978 & Surface & 2008 & 261.503 & 231.645 & Surface & 2012 \\
\hline 264.772 & 232.029 & Surface & 2008 & 264.772 & 231.796 & Surface & 2012 \\
\hline 268.041 & 232.327 & Surface & 2008 & 268.041 & 232.137 & Surface & 2012 \\
\hline 271.309 & 232.908 & Surface & 2008 & 271.309 & 232.735 & Surface & 2012 \\
\hline 274.578 & 233.564 & Surface & 2008 & 274.578 & 233.404 & Surface & 2012 \\
\hline 277.847 & 234.223 & Surface & 2008 & 277.847 & 233.998 & Surface & 2012 \\
\hline 281.116 & 234.770 & Surface & 2008 & 281.116 & 234.553 & Surface & 2012 \\
\hline 284.384 & 235.151 & Surface & 2008 & 284.384 & 235.112 & Surface & 2012 \\
\hline 287.653 & 235.489 & Surface & 2008 & 287.653 & 235.598 & Surface & 2012 \\
\hline 290.922 & 235.841 & Surface & 2008 & 290.922 & 235.971 & Surface & 2012 \\
\hline 294.191 & 236.237 & Surface & 2008 & 294.191 & 236.378 & Surface & 2012 \\
\hline 297.460 & 236.664 & Surface & 2008 & 297.460 & 236.818 & Surface & 2012 \\
\hline 300.728 & 237.090 & Surface & 2008 & 300.728 & 237.258 & Surface & 2012 \\
\hline 303.997 & 237.520 & Surface & 2008 & 303.997 & 237.695 & Surface & 2012 \\
\hline 307.266 & 237.950 & Surface & 2008 & 307.266 & 238.129 & Surface & 2012 \\
\hline 310.535 & 238.386 & Surface & 2008 & 310.535 & 238.566 & Surface & 2012 \\
\hline 313.804 & 238.822 & Surface & 2008 & 313.804 & 238.982 & Surface & 2012 \\
\hline 317.072 & 239.255 & Surface & 2008 & 317.072 & 239.338 & Surface & 2012 \\
\hline 320.341 & 239.596 & Surface & 2008 & 320.341 & 239.619 & Surface & 2012 \\
\hline 323.610 & 239.707 & Surface & 2008 & 323.610 & 239.857 & Surface & 2012 \\
\hline 326.879 & 239.909 & Surface & 2008 & 326.879 & 240.056 & Surface & 2012 \\
\hline 330.147 & 240.065 & Surface & 2008 & 330.147 & 240.109 & Surface & 2012 \\
\hline 333.417 & 240.179 & Surface & 2008 & 333.417 & 240.117 & Surface & 2012 \\
\hline 336.685 & 240.251 & Surface & 2008 & 336.685 & 240.262 & Surface & 2012 \\
\hline 339.954 & 240.313 & Surface & 2008 & 339.954 & 240.314 & Surface & 2012 \\
\hline 343.223 & 240.617 & Surface & 2008 & 343.223 & 240.641 & Surface & 2012 \\
\hline 346.491 & 240.958 & Surface & 2008 & 346.491 & 240.977 & Surface & 2012 \\
\hline
\end{tabular}




$\begin{array}{llllllll}349.760 & 241.333 & \text { Surface } & 2008 & 349.760 & 241.319 & \text { Surface } & 2012 \\ 353.029 & 241.790 & \text { Surface } & 2008 & 353.029 & 241.698 & \text { Surface } & 2012 \\ 356.298 & 242.202 & \text { Surface } & 2008 & 356.298 & 241.940 & \text { Surface } & 2012 \\ 359.567 & 242.520 & \text { Surface } & 2008 & 359.567 & 242.202 & \text { Surface } & 2012 \\ 362.836 & 242.807 & \text { Surface } & 2008 & 362.836 & 242.484 & \text { Surface } & 2012 \\ 366.104 & 243.070 & \text { Surface } & 2008 & 366.104 & 242.779 & \text { Surface } & 2012 \\ 369.373 & 243.316 & \text { Surface } & 2008 & 369.373 & 243.081 & \text { Surface } & 2012 \\ 372.642 & 243.688 & \text { Surface } & 2008 & 372.642 & 243.403 & \text { Surface } & 2012 \\ 375.911 & 244.173 & \text { Surface } & 2008 & 375.911 & 243.824 & \text { Surface } & 2012 \\ 379.180 & 244.645 & \text { Surface } & 2008 & 379.180 & 244.311 & \text { Surface } & 2012 \\ 382.448 & 244.999 & \text { Surface } & 2008 & 382.448 & 244.796 & \text { Surface } & 2012 \\ 385.717 & 245.248 & \text { Surface } & 2008 & 385.717 & 245.173 & \text { Surface } & 2012 \\ 388.986 & 245.476 & \text { Surface } & 2008 & 388.986 & 245.452 & \text { Surface } & 2012 \\ 392.255 & 245.700 & \text { Surface } & 2008 & 392.255 & 245.716 & \text { Surface } & 2012 \\ 395.523 & 245.920 & \text { Surface } & 2008 & 395.523 & 245.993 & \text { Surface } & 2012 \\ 398.792 & 246.064 & \text { Surface } & 2008 & 398.792 & 246.194 & \text { Surface } & 2012\end{array}$




\subsection{Appendix C: Stream Gradient Profiles}

11.1 Stream Gradient - 2006 Lidar:

\begin{tabular}{ccll}
\hline Distance & $\mathrm{Z}$ & DEM & Year \\
\hline 0.000 & 233.418 & Surface & 2006 \\
2.980 & 233.419 & Surface & 2006 \\
5.959 & 233.413 & Surface & 2006 \\
8.939 & 233.407 & Surface & 2006 \\
11.919 & 233.406 & Surface & 2006 \\
14.899 & 233.402 & Surface & 2006 \\
17.878 & 233.403 & Surface & 2006 \\
20.858 & 233.417 & Surface & 2006 \\
23.838 & 233.412 & Surface & 2006 \\
26.818 & 233.405 & Surface & 2006 \\
29.797 & 233.417 & Surface & 2006 \\
32.777 & 233.399 & Surface & 2006 \\
35.757 & 233.390 & Surface & 2006 \\
38.737 & 233.406 & Surface & 2006 \\
41.716 & 233.399 & Surface & 2006 \\
44.696 & 233.394 & Surface & 2006 \\
47.676 & 233.390 & Surface & 2006 \\
50.656 & 233.370 & Surface & 2006 \\
53.635 & 233.391 & Surface & 2006 \\
56.615 & 233.376 & Surface & 2006 \\
59.595 & 233.366 & Surface & 2006 \\
62.575 & 233.356 & Surface & 2006 \\
65.555 & 233.352 & Surface & 2006 \\
68.534 & 233.350 & Surface & 2006 \\
71.514 & 233.357 & Surface & 2006 \\
74.494 & 233.356 & Surface & 2006 \\
77.444 & 233.343 & Surface & 2006 \\
80.394 & 233.348 & Surface & 2006 \\
83.345 & 233.351 & Surface & 2006 \\
86.295 & 233.347 & Surface & 2006 \\
89.245 & 233.325 & Surface & 2006 \\
92.196 & 233.327 & Surface & 2006 \\
95.146 & 233.330 & Surface & 2006 \\
98.096 & 233.341 & Surface & 2006 \\
101.047 & 233.331 & Surface & 2006 \\
103.997 & 233.325 & Surface & 2006 \\
106.947 & 233.313 & Surface & 2006 \\
109.898 & 233.315 & Surface & 2006 \\
112.848 & 233.311 & Surface & 2006 \\
115.798 & 233.310 & Surface & 2006 \\
118.749 & 233.309 & Surface & 2006 \\
121.699 & 233.302 & Surface & 2006 \\
124.649 & 233.297 & Surface & 2006 \\
& & &
\end{tabular}

\begin{tabular}{ccll}
\hline Distance & Z & DEM & Year \\
\hline 5284.769 & 226.781 & Surface & 2006 \\
5287.708 & 226.777 & Surface & 2006 \\
5290.647 & 226.784 & Surface & 2006 \\
5293.586 & 226.786 & Surface & 2006 \\
5296.524 & 226.762 & Surface & 2006 \\
5299.463 & 226.781 & Surface & 2006 \\
5302.402 & 226.786 & Surface & 2006 \\
5305.341 & 226.789 & Surface & 2006 \\
5308.280 & 226.781 & Surface & 2006 \\
5311.218 & 226.791 & Surface & 2006 \\
5314.157 & 226.792 & Surface & 2006 \\
5317.096 & 226.790 & Surface & 2006 \\
5320.035 & 226.787 & Surface & 2006 \\
5322.973 & 226.808 & Surface & 2006 \\
5325.912 & 226.831 & Surface & 2006 \\
5328.851 & 226.829 & Surface & 2006 \\
5331.790 & 226.822 & Surface & 2006 \\
5334.728 & 226.793 & Surface & 2006 \\
5337.667 & 226.782 & Surface & 2006 \\
5340.606 & 226.783 & Surface & 2006 \\
5343.544 & 226.779 & Surface & 2006 \\
5346.483 & 226.766 & Surface & 2006 \\
5349.422 & 226.770 & Surface & 2006 \\
5352.408 & 226.773 & Surface & 2006 \\
5355.394 & 226.752 & Surface & 2006 \\
5358.380 & 226.770 & Surface & 2006 \\
5361.366 & 226.777 & Surface & 2006 \\
5364.351 & 226.802 & Surface & 2006 \\
5367.337 & 226.826 & Surface & 2006 \\
5370.323 & 226.787 & Surface & 2006 \\
5373.309 & 226.784 & Surface & 2006 \\
5376.295 & 226.781 & Surface & 2006 \\
5379.281 & 226.777 & Surface & 2006 \\
5382.267 & 226.761 & Surface & 2006 \\
5385.253 & 226.767 & Surface & 2006 \\
5388.238 & 226.784 & Surface & 2006 \\
5391.224 & 226.792 & Surface & 2006 \\
5394.210 & 226.786 & Surface & 2006 \\
5397.196 & 226.783 & Surface & 2006 \\
5400.182 & 226.751 & Surface & 2006 \\
5403.168 & 226.753 & Surface & 2006 \\
5406.154 & 226.775 & Surface & 2006 \\
5409.140 & 226.779 & Surface & 2006
\end{tabular}




\begin{tabular}{|c|c|c|c|c|c|c|c|}
\hline 127.600 & 233.278 & Surface & 2006 & 5412.126 & 226.768 & Surface & 2006 \\
\hline 130.550 & 233.285 & Surface & 2006 & 5415.111 & 226.759 & Surface & 2006 \\
\hline 133.500 & 233.284 & Surface & 2006 & 5418.097 & 226.761 & Surface & 2006 \\
\hline 136.451 & 233.278 & Surface & 2006 & 5421.083 & 226.760 & Surface & 2006 \\
\hline 139.401 & 233.274 & Surface & 2006 & 5424.069 & 226.753 & Surface & 2006 \\
\hline 142.351 & 233.282 & Surface & 2006 & 5427.055 & 226.755 & Surface & 2006 \\
\hline 145.302 & 233.295 & Surface & 2006 & 5430.041 & 226.759 & Surface & 2006 \\
\hline 148.252 & 233.287 & Surface & 2006 & 5433.026 & 226.757 & Surface & 2006 \\
\hline 151.202 & 233.279 & Surface & 2006 & 5436.013 & 226.751 & Surface & 2006 \\
\hline 154.153 & 233.272 & Surface & 2006 & 5438.998 & 226.741 & Surface & 2006 \\
\hline 157.103 & 233.270 & Surface & 2006 & 5441.984 & 226.748 & Surface & 2006 \\
\hline 160.053 & 233.245 & Surface & 2006 & 5444.970 & 226.715 & Surface & 2006 \\
\hline 163.003 & 233.236 & Surface & 2006 & 5447.956 & 226.695 & Surface & 2006 \\
\hline 165.954 & 233.247 & Surface & 2006 & 5450.942 & 226.697 & Surface & 2006 \\
\hline 168.904 & 233.276 & Surface & 2006 & 5453.928 & 226.712 & Surface & 2006 \\
\hline 171.854 & 233.272 & Surface & 2006 & 5456.913 & 226.719 & Surface & 2006 \\
\hline 174.805 & 233.249 & Surface & 2006 & 5459.899 & 226.695 & Surface & 2006 \\
\hline 177.755 & 233.253 & Surface & 2006 & 5462.885 & 226.695 & Surface & 2006 \\
\hline 180.705 & 233.240 & Surface & 2006 & 5465.871 & 226.707 & Surface & 2006 \\
\hline 183.656 & 233.230 & Surface & 2006 & 5468.857 & 226.716 & Surface & 2006 \\
\hline 186.606 & 233.224 & Surface & 2006 & 5471.843 & 226.726 & Surface & 2006 \\
\hline 189.556 & 233.213 & Surface & 2006 & 5474.829 & 226.748 & Surface & 2006 \\
\hline 192.507 & 233.219 & Surface & 2006 & 5477.815 & 226.760 & Surface & 2006 \\
\hline 195.457 & 233.220 & Surface & 2006 & 5480.801 & 226.762 & Surface & 2006 \\
\hline 198.407 & 233.205 & Surface & 2006 & 5483.792 & 226.722 & Surface & 2006 \\
\hline 201.358 & 233.189 & Surface & 2006 & 5486.783 & 226.694 & Surface & 2006 \\
\hline 204.308 & 233.183 & Surface & 2006 & 5489.774 & 226.685 & Surface & 2006 \\
\hline 207.258 & 233.200 & Surface & 2006 & 5492.765 & 226.694 & Surface & 2006 \\
\hline 210.209 & 233.183 & Surface & 2006 & 5495.756 & 226.689 & Surface & 2006 \\
\hline 213.159 & 233.178 & Surface & 2006 & 5498.747 & 226.686 & Surface & 2006 \\
\hline 216.109 & 233.141 & Surface & 2006 & 5501.738 & 226.701 & Surface & 2006 \\
\hline 219.059 & 233.141 & Surface & 2006 & 5504.729 & 226.697 & Surface & 2006 \\
\hline 222.010 & 233.158 & Surface & 2006 & 5507.720 & 226.681 & Surface & 2006 \\
\hline 224.960 & 233.170 & Surface & 2006 & 5510.711 & 226.681 & Surface & 2006 \\
\hline 227.910 & 233.163 & Surface & 2006 & 5513.702 & 226.677 & Surface & 2006 \\
\hline 230.861 & 233.174 & Surface & 2006 & 5516.693 & 226.675 & Surface & 2006 \\
\hline 233.811 & 233.169 & Surface & 2006 & 5519.684 & 226.669 & Surface & 2006 \\
\hline 236.761 & 233.138 & Surface & 2006 & 5522.675 & 226.672 & Surface & 2006 \\
\hline 239.712 & 233.104 & Surface & 2006 & 5525.666 & 226.670 & Surface & 2006 \\
\hline 242.662 & 233.115 & Surface & 2006 & 5528.658 & 226.658 & Surface & 2006 \\
\hline 245.584 & 233.115 & Surface & 2006 & 5531.648 & 226.658 & Surface & 2006 \\
\hline 248.507 & 233.124 & Surface & 2006 & 5534.639 & 226.666 & Surface & 2006 \\
\hline 251.429 & 233.127 & Surface & 2006 & 5537.631 & 226.664 & Surface & 2006 \\
\hline 254.351 & 233.132 & Surface & 2006 & 5540.622 & 226.656 & Surface & 2006 \\
\hline 257.274 & 233.114 & Surface & 2006 & 5543.613 & 226.654 & Surface & 2006 \\
\hline 260.196 & 233.106 & Surface & 2006 & 5546.604 & 226.653 & Surface & 2006 \\
\hline 263.118 & 233.065 & Surface & 2006 & 5549.595 & 226.641 & Surface & 2006 \\
\hline 266.041 & 233.052 & Surface & 2006 & 5552.586 & 226.635 & Surface & 2006 \\
\hline 268.963 & 233.055 & Surface & 2006 & 5555.577 & 226.640 & Surface & 2006 \\
\hline
\end{tabular}




\begin{tabular}{|c|c|c|c|c|c|c|c|}
\hline 271.885 & 233.062 & Surface & 2006 & 5558.568 & 226.646 & Surface & 2006 \\
\hline 274.807 & 233.047 & Surface & 2006 & 5561.559 & 226.641 & Surface & 2006 \\
\hline 277.730 & 233.022 & Surface & 2006 & 5564.550 & 226.650 & Surface & 2006 \\
\hline 280.652 & 233.028 & Surface & 2006 & 5567.541 & 226.643 & Surface & 2006 \\
\hline 283.574 & 233.036 & Surface & 2006 & 5570.532 & 226.633 & Surface & 2006 \\
\hline 286.497 & 233.022 & Surface & 2006 & 5573.523 & 226.645 & Surface & 2006 \\
\hline 289.419 & 233.012 & Surface & 2006 & 5576.514 & 226.637 & Surface & 2006 \\
\hline 292.341 & 233.012 & Surface & 2006 & 5579.506 & 226.637 & Surface & 2006 \\
\hline 295.264 & 233.009 & Surface & 2006 & 5582.497 & 226.635 & Surface & 2006 \\
\hline 298.186 & 232.985 & Surface & 2006 & 5585.496 & 226.632 & Surface & 2006 \\
\hline 301.108 & 232.985 & Surface & 2006 & 5588.495 & 226.629 & Surface & 2006 \\
\hline 304.030 & 232.959 & Surface & 2006 & 5591.494 & 226.628 & Surface & 2006 \\
\hline 306.953 & 232.960 & Surface & 2006 & 5594.493 & 226.621 & Surface & 2006 \\
\hline 309.875 & 232.966 & Surface & 2006 & 5597.493 & 226.641 & Surface & 2006 \\
\hline 312.797 & 232.954 & Surface & 2006 & 5600.492 & 226.638 & Surface & 2006 \\
\hline 315.719 & 232.942 & Surface & 2006 & 5603.491 & 226.634 & Surface & 2006 \\
\hline 318.642 & 232.943 & Surface & 2006 & 5606.490 & 226.625 & Surface & 2006 \\
\hline 321.564 & 232.920 & Surface & 2006 & 5609.489 & 226.614 & Surface & 2006 \\
\hline 324.487 & 232.897 & Surface & 2006 & 5612.489 & 226.598 & Surface & 2006 \\
\hline 327.409 & 232.868 & Surface & 2006 & 5615.488 & 226.588 & Surface & 2006 \\
\hline 330.345 & 232.868 & Surface & 2006 & 5618.487 & 226.600 & Surface & 2006 \\
\hline 333.282 & 232.873 & Surface & 2006 & 5621.486 & 226.617 & Surface & 2006 \\
\hline 336.218 & 232.859 & Surface & 2006 & 5624.485 & 226.617 & Surface & 2006 \\
\hline 339.155 & 232.842 & Surface & 2006 & 5627.485 & 226.579 & Surface & 2006 \\
\hline 342.091 & 232.842 & Surface & 2006 & 5630.484 & 226.582 & Surface & 2006 \\
\hline 345.028 & 232.828 & Surface & 2006 & 5633.483 & 226.601 & Surface & 2006 \\
\hline 347.965 & 232.810 & Surface & 2006 & 5636.482 & 226.602 & Surface & 2006 \\
\hline 350.901 & 232.803 & Surface & 2006 & 5639.481 & 226.580 & Surface & 2006 \\
\hline 353.838 & 232.792 & Surface & 2006 & 5642.480 & 226.581 & Surface & 2006 \\
\hline 356.774 & 232.783 & Surface & 2006 & 5645.480 & 226.582 & Surface & 2006 \\
\hline 359.710 & 232.787 & Surface & 2006 & 5648.479 & 226.555 & Surface & 2006 \\
\hline 362.647 & 232.768 & Surface & 2006 & 5651.478 & 226.570 & Surface & 2006 \\
\hline 365.584 & 232.777 & Surface & 2006 & 5654.477 & 226.568 & Surface & 2006 \\
\hline 368.520 & 232.744 & Surface & 2006 & 5657.477 & 226.570 & Surface & 2006 \\
\hline 371.457 & 232.726 & Surface & 2006 & 5660.476 & 226.582 & Surface & 2006 \\
\hline 374.393 & 232.711 & Surface & 2006 & 5663.475 & 226.558 & Surface & 2006 \\
\hline 377.330 & 232.705 & Surface & 2006 & 5666.474 & 226.556 & Surface & 2006 \\
\hline 380.266 & 232.703 & Surface & 2006 & 5669.473 & 226.539 & Surface & 2006 \\
\hline 383.203 & 232.700 & Surface & 2006 & 5672.472 & 226.568 & Surface & 2006 \\
\hline 386.139 & 232.639 & Surface & 2006 & 5675.472 & 226.567 & Surface & 2006 \\
\hline 389.076 & 232.598 & Surface & 2006 & 5678.471 & 226.559 & Surface & 2006 \\
\hline 392.013 & 232.552 & Surface & 2006 & 5681.470 & 226.553 & Surface & 2006 \\
\hline 394.949 & 232.527 & Surface & 2006 & 5684.437 & 226.550 & Surface & 2006 \\
\hline 397.885 & 232.526 & Surface & 2006 & 5687.403 & 226.521 & Surface & 2006 \\
\hline 400.822 & 232.505 & Surface & 2006 & 5690.370 & 226.518 & Surface & 2006 \\
\hline 403.758 & 232.474 & Surface & 2006 & 5693.336 & 226.519 & Surface & 2006 \\
\hline 406.695 & 232.450 & Surface & 2006 & 5696.303 & 226.514 & Surface & 2006 \\
\hline 409.632 & 232.432 & Surface & 2006 & 5699.269 & 226.512 & Surface & 2006 \\
\hline 412.568 & 232.430 & Surface & 2006 & 5702.236 & 226.508 & Surface & 2006 \\
\hline
\end{tabular}




\begin{tabular}{|c|c|c|c|c|c|c|c|}
\hline 415.505 & 232.443 & Surface & 2006 & 5705.202 & 226.497 & Surface & 2006 \\
\hline 418.441 & 232.407 & Surface & 2006 & 5708.169 & 226.487 & Surface & 2006 \\
\hline 421.378 & 232.404 & Surface & 2006 & 5711.135 & 226.493 & Surface & 2006 \\
\hline 424.314 & 232.413 & Surface & 2006 & 5714.102 & 226.508 & Surface & 2006 \\
\hline 427.251 & 232.356 & Surface & 2006 & 5717.068 & 226.494 & Surface & 2006 \\
\hline 430.187 & 232.333 & Surface & 2006 & 5720.035 & 226.502 & Surface & 2006 \\
\hline 433.124 & 232.331 & Surface & 2006 & 5723.001 & 226.494 & Surface & 2006 \\
\hline 436.060 & 232.236 & Surface & 2006 & 5725.968 & 226.481 & Surface & 2006 \\
\hline 438.997 & 232.168 & Surface & 2006 & 5728.935 & 226.464 & Surface & 2006 \\
\hline 441.956 & 232.144 & Surface & 2006 & 5731.901 & 226.444 & Surface & 2006 \\
\hline 444.915 & 232.080 & Surface & 2006 & 5734.868 & 226.454 & Surface & 2006 \\
\hline 447.874 & 232.006 & Surface & 2006 & 5737.834 & 226.433 & Surface & 2006 \\
\hline 450.834 & 231.947 & Surface & 2006 & 5740.801 & 226.426 & Surface & 2006 \\
\hline 453.793 & 231.863 & Surface & 2006 & 5743.767 & 226.426 & Surface & 2006 \\
\hline 456.752 & 231.788 & Surface & 2006 & 5746.734 & 226.423 & Surface & 2006 \\
\hline 459.711 & 231.714 & Surface & 2006 & 5749.700 & 226.407 & Surface & 2006 \\
\hline 462.671 & 231.639 & Surface & 2006 & 5752.667 & 226.424 & Surface & 2006 \\
\hline 465.630 & 231.566 & Surface & 2006 & 5755.633 & 226.412 & Surface & 2006 \\
\hline 468.589 & 231.448 & Surface & 2006 & 5758.600 & 226.408 & Surface & 2006 \\
\hline 471.548 & 231.347 & Surface & 2006 & 5761.566 & 226.419 & Surface & 2006 \\
\hline 474.508 & 231.306 & Surface & 2006 & 5764.533 & 226.401 & Surface & 2006 \\
\hline 477.467 & 231.321 & Surface & 2006 & 5767.495 & 226.434 & Surface & 2006 \\
\hline 480.426 & 231.326 & Surface & 2006 & 5770.457 & 226.423 & Surface & 2006 \\
\hline 483.385 & 231.309 & Surface & 2006 & 5773.419 & 226.419 & Surface & 2006 \\
\hline 486.344 & 231.285 & Surface & 2006 & 5776.381 & 226.416 & Surface & 2006 \\
\hline 489.304 & 231.260 & Surface & 2006 & 5779.343 & 226.409 & Surface & 2006 \\
\hline 492.263 & 231.251 & Surface & 2006 & 5782.304 & 226.405 & Surface & 2006 \\
\hline 495.222 & 231.230 & Surface & 2006 & 5785.266 & 226.411 & Surface & 2006 \\
\hline 498.181 & 231.239 & Surface & 2006 & 5788.228 & 226.422 & Surface & 2006 \\
\hline 501.140 & 231.260 & Surface & 2006 & 5791.190 & 226.409 & Surface & 2006 \\
\hline 504.093 & 231.257 & Surface & 2006 & 5794.152 & 226.395 & Surface & 2006 \\
\hline 507.046 & 231.249 & Surface & 2006 & 5797.114 & 226.384 & Surface & 2006 \\
\hline 509.998 & 231.262 & Surface & 2006 & 5800.076 & 226.378 & Surface & 2006 \\
\hline 512.951 & 231.248 & Surface & 2006 & 5803.038 & 226.386 & Surface & 2006 \\
\hline 515.904 & 231.269 & Surface & 2006 & 5806.000 & 226.396 & Surface & 2006 \\
\hline 518.856 & 231.263 & Surface & 2006 & 5808.962 & 226.393 & Surface & 2006 \\
\hline 521.809 & 231.257 & Surface & 2006 & 5811.924 & 226.386 & Surface & 2006 \\
\hline 524.761 & 231.260 & Surface & 2006 & 5814.886 & 226.401 & Surface & 2006 \\
\hline 527.714 & 231.284 & Surface & 2006 & 5817.848 & 226.408 & Surface & 2006 \\
\hline 530.667 & 231.275 & Surface & 2006 & 5820.810 & 226.402 & Surface & 2006 \\
\hline 533.620 & 231.252 & Surface & 2006 & 5823.772 & 226.394 & Surface & 2006 \\
\hline 536.572 & 231.249 & Surface & 2006 & 5826.734 & 226.384 & Surface & 2006 \\
\hline 539.525 & 231.233 & Surface & 2006 & 5829.695 & 226.367 & Surface & 2006 \\
\hline 542.477 & 231.244 & Surface & 2006 & 5832.657 & 226.361 & Surface & 2006 \\
\hline 545.430 & 231.254 & Surface & 2006 & 5835.619 & 226.383 & Surface & 2006 \\
\hline 548.383 & 231.258 & Surface & 2006 & 5838.534 & 226.366 & Surface & 2006 \\
\hline 551.336 & 231.229 & Surface & 2006 & 5841.448 & 226.357 & Surface & 2006 \\
\hline 554.288 & 231.224 & Surface & 2006 & 5844.363 & 226.353 & Surface & 2006 \\
\hline 557.241 & 231.247 & Surface & 2006 & 5847.278 & 226.361 & Surface & 2006 \\
\hline
\end{tabular}




\begin{tabular}{|c|c|c|c|c|c|c|c|}
\hline 560.193 & 231.247 & Surface & 2006 & 5850.192 & 226.364 & Surface & 2006 \\
\hline 563.146 & 231.201 & Surface & 2006 & 5853.107 & 226.338 & Surface & 2006 \\
\hline 566.099 & 231.202 & Surface & 2006 & 5856.022 & 226.322 & Surface & 2006 \\
\hline 569.051 & 231.233 & Surface & 2006 & 5858.936 & 226.356 & Surface & 2006 \\
\hline 572.004 & 231.244 & Surface & 2006 & 5861.851 & 226.368 & Surface & 2006 \\
\hline 574.957 & 231.215 & Surface & 2006 & 5864.765 & 226.312 & Surface & 2006 \\
\hline 577.854 & 231.214 & Surface & 2006 & 5867.680 & 226.342 & Surface & 2006 \\
\hline 580.751 & 231.225 & Surface & 2006 & 5870.595 & 226.334 & Surface & 2006 \\
\hline 583.648 & 231.214 & Surface & 2006 & 5873.509 & 226.325 & Surface & 2006 \\
\hline 586.545 & 231.190 & Surface & 2006 & 5876.424 & 226.338 & Surface & 2006 \\
\hline 589.442 & 231.179 & Surface & 2006 & 5879.339 & 226.354 & Surface & 2006 \\
\hline 592.340 & 231.183 & Surface & 2006 & 5882.253 & 226.365 & Surface & 2006 \\
\hline 595.237 & 231.184 & Surface & 2006 & 5885.168 & 226.339 & Surface & 2006 \\
\hline 598.134 & 231.179 & Surface & 2006 & 5888.083 & 226.316 & Surface & 2006 \\
\hline 601.031 & 231.168 & Surface & 2006 & 5890.997 & 226.316 & Surface & 2006 \\
\hline 603.928 & 231.163 & Surface & 2006 & 5893.912 & 226.318 & Surface & 2006 \\
\hline 606.825 & 231.166 & Surface & 2006 & 5896.826 & 226.325 & Surface & 2006 \\
\hline 609.723 & 231.156 & Surface & 2006 & 5899.741 & 226.307 & Surface & 2006 \\
\hline 612.620 & 231.158 & Surface & 2006 & 5902.656 & 226.312 & Surface & 2006 \\
\hline 615.517 & 231.148 & Surface & 2006 & 5905.570 & 226.327 & Surface & 2006 \\
\hline 618.414 & 231.121 & Surface & 2006 & 5908.485 & 226.329 & Surface & 2006 \\
\hline 621.396 & 231.124 & Surface & 2006 & 5911.400 & 226.317 & Surface & 2006 \\
\hline 624.377 & 231.117 & Surface & 2006 & 5914.314 & 226.317 & Surface & 2006 \\
\hline 627.359 & 231.100 & Surface & 2006 & 5917.229 & 226.304 & Surface & 2006 \\
\hline 630.341 & 231.036 & Surface & 2006 & 5920.143 & 226.306 & Surface & 2006 \\
\hline 633.322 & 231.082 & Surface & 2006 & 5923.096 & 226.317 & Surface & 2006 \\
\hline 636.304 & 231.087 & Surface & 2006 & 5926.048 & 226.238 & Surface & 2006 \\
\hline 639.285 & 231.037 & Surface & 2006 & 5929.000 & 226.208 & Surface & 2006 \\
\hline 642.267 & 231.050 & Surface & 2006 & 5931.952 & 226.208 & Surface & 2006 \\
\hline 645.249 & 231.033 & Surface & 2006 & 5934.905 & 226.193 & Surface & 2006 \\
\hline 648.230 & 231.048 & Surface & 2006 & 5937.857 & 226.180 & Surface & 2006 \\
\hline 651.212 & 231.007 & Surface & 2006 & 5940.809 & 226.172 & Surface & 2006 \\
\hline 654.194 & 231.019 & Surface & 2006 & 5943.762 & 226.179 & Surface & 2006 \\
\hline 657.176 & 230.999 & Surface & 2006 & 5946.714 & 226.167 & Surface & 2006 \\
\hline 660.157 & 230.968 & Surface & 2006 & 5949.666 & 226.155 & Surface & 2006 \\
\hline 662.902 & 230.947 & Surface & 2006 & 5952.618 & 226.165 & Surface & 2006 \\
\hline 665.647 & 230.945 & Surface & 2006 & 5955.570 & 226.183 & Surface & 2006 \\
\hline 668.392 & 230.945 & Surface & 2006 & 5958.523 & 226.185 & Surface & 2006 \\
\hline 671.137 & 230.923 & Surface & 2006 & 5961.475 & 226.164 & Surface & 2006 \\
\hline 673.882 & 230.909 & Surface & 2006 & 5964.427 & 226.140 & Surface & 2006 \\
\hline 676.627 & 230.937 & Surface & 2006 & 5967.380 & 226.149 & Surface & 2006 \\
\hline 679.372 & 230.937 & Surface & 2006 & 5970.332 & 226.128 & Surface & 2006 \\
\hline 682.117 & 230.896 & Surface & 2006 & 5973.284 & 226.102 & Surface & 2006 \\
\hline 684.862 & 230.874 & Surface & 2006 & 5976.236 & 226.101 & Surface & 2006 \\
\hline 687.608 & 230.867 & Surface & 2006 & 5979.188 & 226.095 & Surface & 2006 \\
\hline 690.491 & 230.858 & Surface & 2006 & 5982.141 & 226.092 & Surface & 2006 \\
\hline 693.375 & 230.851 & Surface & 2006 & 5985.093 & 226.081 & Surface & 2006 \\
\hline 696.258 & 230.862 & Surface & 2006 & 5988.045 & 226.062 & Surface & 2006 \\
\hline 699.142 & 230.851 & Surface & 2006 & 5990.998 & 226.043 & Surface & 2006 \\
\hline
\end{tabular}




\begin{tabular}{|c|c|c|c|c|c|c|c|}
\hline 702.026 & 230.847 & Surface & 2006 & 5993.960 & 226.020 & Surface & 2006 \\
\hline 704.909 & 230.850 & Surface & 2006 & 5996.922 & 225.990 & Surface & 2006 \\
\hline 707.793 & 230.843 & Surface & 2006 & 5999.885 & 225.956 & Surface & 2006 \\
\hline 710.677 & 230.840 & Surface & 2006 & 6002.847 & 225.942 & Surface & 2006 \\
\hline 713.609 & 230.845 & Surface & 2006 & 6005.809 & 225.925 & Surface & 2006 \\
\hline 716.542 & 230.839 & Surface & 2006 & 6008.772 & 225.904 & Surface & 2006 \\
\hline 719.475 & 230.839 & Surface & 2006 & 6011.734 & 225.880 & Surface & 2006 \\
\hline 722.408 & 230.855 & Surface & 2006 & 6014.696 & 225.861 & Surface & 2006 \\
\hline 725.341 & 230.863 & Surface & 2006 & 6017.658 & 225.857 & Surface & 2006 \\
\hline 728.274 & 230.869 & Surface & 2006 & 6020.621 & 225.841 & Surface & 2006 \\
\hline 731.207 & 230.858 & Surface & 2006 & 6023.583 & 225.809 & Surface & 2006 \\
\hline 734.139 & 230.884 & Surface & 2006 & 6026.545 & 225.764 & Surface & 2006 \\
\hline 737.072 & 230.902 & Surface & 2006 & 6029.507 & 225.727 & Surface & 2006 \\
\hline 740.005 & 230.866 & Surface & 2006 & 6032.470 & 225.701 & Surface & 2006 \\
\hline 742.938 & 230.867 & Surface & 2006 & 6035.432 & 225.703 & Surface & 2006 \\
\hline 745.890 & 230.890 & Surface & 2006 & 6038.394 & 225.717 & Surface & 2006 \\
\hline 748.841 & 230.894 & Surface & 2006 & 6041.328 & 225.729 & Surface & 2006 \\
\hline 751.793 & 230.916 & Surface & 2006 & 6044.261 & 225.729 & Surface & 2006 \\
\hline 754.745 & 230.976 & Surface & 2006 & 6047.194 & 225.735 & Surface & 2006 \\
\hline 757.696 & 230.861 & Surface & 2006 & 6050.128 & 225.720 & Surface & 2006 \\
\hline 760.648 & 230.824 & Surface & 2006 & 6053.061 & 225.714 & Surface & 2006 \\
\hline 763.600 & 230.866 & Surface & 2006 & 6055.995 & 225.717 & Surface & 2006 \\
\hline 766.552 & 230.868 & Surface & 2006 & 6058.928 & 225.706 & Surface & 2006 \\
\hline 769.503 & 230.827 & Surface & 2006 & 6061.862 & 225.727 & Surface & 2006 \\
\hline 772.455 & 230.831 & Surface & 2006 & 6064.795 & 225.748 & Surface & 2006 \\
\hline 775.417 & 230.833 & Surface & 2006 & 6067.728 & 225.706 & Surface & 2006 \\
\hline 778.380 & 230.852 & Surface & 2006 & 6070.662 & 225.724 & Surface & 2006 \\
\hline 781.342 & 230.866 & Surface & 2006 & 6073.595 & 225.722 & Surface & 2006 \\
\hline 784.304 & 230.882 & Surface & 2006 & 6076.529 & 225.735 & Surface & 2006 \\
\hline 787.266 & 230.890 & Surface & 2006 & 6079.462 & 225.700 & Surface & 2006 \\
\hline 790.229 & 230.855 & Surface & 2006 & 6082.396 & 225.692 & Surface & 2006 \\
\hline 793.191 & 230.825 & Surface & 2006 & 6085.329 & 225.685 & Surface & 2006 \\
\hline 796.153 & 230.881 & Surface & 2006 & 6088.263 & 225.695 & Surface & 2006 \\
\hline 799.116 & 230.942 & Surface & 2006 & 6091.196 & 225.724 & Surface & 2006 \\
\hline 802.078 & 230.984 & Surface & 2006 & 6094.048 & 225.729 & Surface & 2006 \\
\hline 805.040 & 230.951 & Surface & 2006 & 6096.899 & 225.692 & Surface & 2006 \\
\hline 808.002 & 230.908 & Surface & 2006 & 6099.751 & 225.667 & Surface & 2006 \\
\hline 810.965 & 230.874 & Surface & 2006 & 6102.602 & 225.687 & Surface & 2006 \\
\hline 813.927 & 230.850 & Surface & 2006 & 6105.454 & 225.690 & Surface & 2006 \\
\hline 816.889 & 230.855 & Surface & 2006 & 6108.305 & 225.687 & Surface & 2006 \\
\hline 819.852 & 230.878 & Surface & 2006 & 6111.157 & 225.712 & Surface & 2006 \\
\hline 822.765 & 230.896 & Surface & 2006 & 6114.008 & 225.734 & Surface & 2006 \\
\hline 825.679 & 230.896 & Surface & 2006 & 6116.860 & 225.755 & Surface & 2006 \\
\hline 828.592 & 230.880 & Surface & 2006 & 6119.711 & 225.770 & Surface & 2006 \\
\hline 831.506 & 230.888 & Surface & 2006 & 6122.563 & 225.748 & Surface & 2006 \\
\hline 834.419 & 230.893 & Surface & 2006 & 6125.414 & 225.737 & Surface & 2006 \\
\hline 837.333 & 230.890 & Surface & 2006 & 6128.266 & 225.713 & Surface & 2006 \\
\hline 840.246 & 230.916 & Surface & 2006 & 6131.118 & 225.722 & Surface & 2006 \\
\hline 843.160 & 230.932 & Surface & 2006 & 6133.969 & 225.722 & Surface & 2006 \\
\hline
\end{tabular}




\begin{tabular}{|c|c|c|c|c|c|c|c|}
\hline 846.073 & 230.913 & Surface & 2006 & 6136.821 & 225.719 & Surface & 2006 \\
\hline 848.987 & 230.914 & Surface & 2006 & 6139.817 & 225.693 & Surface & 2006 \\
\hline 851.900 & 230.918 & Surface & 2006 & 6142.813 & 225.717 & Surface & 2006 \\
\hline 854.814 & 230.909 & Surface & 2006 & 6145.809 & 225.744 & Surface & 2006 \\
\hline 857.727 & 230.917 & Surface & 2006 & 6148.805 & 225.707 & Surface & 2006 \\
\hline 860.641 & 230.907 & Surface & 2006 & 6151.802 & 225.714 & Surface & 2006 \\
\hline 863.555 & 230.927 & Surface & 2006 & 6154.798 & 225.708 & Surface & 2006 \\
\hline 866.468 & 230.912 & Surface & 2006 & 6157.794 & 225.707 & Surface & 2006 \\
\hline 869.382 & 230.908 & Surface & 2006 & 6160.790 & 225.707 & Surface & 2006 \\
\hline 872.295 & 230.892 & Surface & 2006 & 6163.787 & 225.707 & Surface & 2006 \\
\hline 875.228 & 230.884 & Surface & 2006 & 6166.783 & 225.716 & Surface & 2006 \\
\hline 878.161 & 230.885 & Surface & 2006 & 6169.779 & 225.712 & Surface & 2006 \\
\hline 881.094 & 230.888 & Surface & 2006 & 6172.776 & 225.709 & Surface & 2006 \\
\hline 884.027 & 230.887 & Surface & 2006 & 6175.772 & 225.732 & Surface & 2006 \\
\hline 886.960 & 230.878 & Surface & 2006 & 6178.768 & 225.727 & Surface & 2006 \\
\hline 889.892 & 230.870 & Surface & 2006 & 6181.764 & 225.725 & Surface & 2006 \\
\hline 892.825 & 230.851 & Surface & 2006 & 6184.761 & 225.737 & Surface & 2006 \\
\hline 895.758 & 230.808 & Surface & 2006 & 6187.656 & 225.735 & Surface & 2006 \\
\hline 898.691 & 230.800 & Surface & 2006 & 6190.553 & 225.742 & Surface & 2006 \\
\hline 901.624 & 230.772 & Surface & 2006 & 6193.448 & 225.753 & Surface & 2006 \\
\hline 904.557 & 230.724 & Surface & 2006 & 6196.345 & 225.762 & Surface & 2006 \\
\hline 907.347 & 230.714 & Surface & 2006 & 6199.241 & 225.762 & Surface & 2006 \\
\hline 910.137 & 230.729 & Surface & 2006 & 6202.137 & 225.757 & Surface & 2006 \\
\hline 912.927 & 230.704 & Surface & 2006 & 6205.033 & 225.764 & Surface & 2006 \\
\hline 915.717 & 230.638 & Surface & 2006 & 6207.929 & 225.772 & Surface & 2006 \\
\hline 918.507 & 230.594 & Surface & 2006 & 6210.825 & 225.774 & Surface & 2006 \\
\hline 921.297 & 230.537 & Surface & 2006 & 6213.721 & 225.787 & Surface & 2006 \\
\hline 924.087 & 230.513 & Surface & 2006 & 6216.617 & 225.795 & Surface & 2006 \\
\hline 926.877 & 230.514 & Surface & 2006 & 6219.513 & 225.785 & Surface & 2006 \\
\hline 929.667 & 230.452 & Surface & 2006 & 6222.409 & 225.776 & Surface & 2006 \\
\hline 932.457 & 230.355 & Surface & 2006 & 6225.305 & 225.772 & Surface & 2006 \\
\hline 935.247 & 230.299 & Surface & 2006 & 6228.201 & 225.770 & Surface & 2006 \\
\hline 938.096 & 230.273 & Surface & 2006 & 6231.097 & 225.750 & Surface & 2006 \\
\hline 940.944 & 230.264 & Surface & 2006 & 6233.993 & 225.769 & Surface & 2006 \\
\hline 943.792 & 230.226 & Surface & 2006 & 6236.889 & 225.793 & Surface & 2006 \\
\hline 946.640 & 230.178 & Surface & 2006 & 6239.781 & 225.817 & Surface & 2006 \\
\hline 949.489 & 230.140 & Surface & 2006 & 6242.673 & 225.813 & Surface & 2006 \\
\hline 952.337 & 230.114 & Surface & 2006 & 6245.564 & 225.816 & Surface & 2006 \\
\hline 955.185 & 230.106 & Surface & 2006 & 6248.456 & 225.810 & Surface & 2006 \\
\hline 958.033 & 230.096 & Surface & 2006 & 6251.348 & 225.803 & Surface & 2006 \\
\hline 960.882 & 230.082 & Surface & 2006 & 6254.240 & 225.799 & Surface & 2006 \\
\hline 963.730 & 230.105 & Surface & 2006 & 6257.131 & 225.793 & Surface & 2006 \\
\hline 966.579 & 230.150 & Surface & 2006 & 6260.023 & 225.790 & Surface & 2006 \\
\hline 969.427 & 230.169 & Surface & 2006 & 6262.915 & 225.792 & Surface & 2006 \\
\hline 972.275 & 230.181 & Surface & 2006 & 6265.807 & 225.795 & Surface & 2006 \\
\hline 975.124 & 230.193 & Surface & 2006 & 6268.699 & 225.798 & Surface & 2006 \\
\hline 977.972 & 230.169 & Surface & 2006 & 6271.590 & 225.799 & Surface & 2006 \\
\hline 980.820 & 230.139 & Surface & 2006 & 6274.482 & 225.795 & Surface & 2006 \\
\hline 983.746 & 230.139 & Surface & 2006 & 6277.374 & 225.792 & Surface & 2006 \\
\hline
\end{tabular}




\begin{tabular}{|c|c|c|c|c|c|c|c|}
\hline 986.672 & 230.121 & Surface & 2006 & 6280.265 & 225.795 & Surface & 2006 \\
\hline 989.598 & 230.109 & Surface & 2006 & 6283.157 & 225.793 & Surface & 2006 \\
\hline 992.524 & 230.125 & Surface & 2006 & 6286.049 & 225.792 & Surface & 2006 \\
\hline 995.450 & 230.152 & Surface & 2006 & 6288.941 & 225.789 & Surface & 2006 \\
\hline 998.375 & 230.154 & Surface & 2006 & 6291.833 & 225.785 & Surface & 2006 \\
\hline 1001.301 & 230.148 & Surface & 2006 & 6294.726 & 225.788 & Surface & 2006 \\
\hline 1004.227 & 230.140 & Surface & 2006 & 6297.620 & 225.788 & Surface & 2006 \\
\hline 1007.153 & 230.128 & Surface & 2006 & 6300.513 & 225.798 & Surface & 2006 \\
\hline 1010.079 & 230.128 & Surface & 2006 & 6303.407 & 225.810 & Surface & 2006 \\
\hline 1013.005 & 230.125 & Surface & 2006 & 6306.300 & 225.820 & Surface & 2006 \\
\hline 1015.931 & 230.131 & Surface & 2006 & 6309.194 & 225.832 & Surface & 2006 \\
\hline 1018.857 & 230.160 & Surface & 2006 & 6312.087 & 225.841 & Surface & 2006 \\
\hline 1021.783 & 230.166 & Surface & 2006 & 6314.981 & 225.840 & Surface & 2006 \\
\hline 1024.708 & 230.152 & Surface & 2006 & 6317.874 & 225.840 & Surface & 2006 \\
\hline 1027.669 & 230.141 & Surface & 2006 & 6320.768 & 225.841 & Surface & 2006 \\
\hline 1030.630 & 230.146 & Surface & 2006 & 6323.661 & 225.843 & Surface & 2006 \\
\hline 1033.591 & 230.154 & Surface & 2006 & 6326.555 & 225.845 & Surface & 2006 \\
\hline 1036.552 & 230.155 & Surface & 2006 & 6329.448 & 225.848 & Surface & 2006 \\
\hline 1039.513 & 230.155 & Surface & 2006 & 6332.342 & 225.849 & Surface & 2006 \\
\hline 1042.474 & 230.140 & Surface & 2006 & 6335.236 & 225.852 & Surface & 2006 \\
\hline 1045.435 & 230.138 & Surface & 2006 & 6338.129 & 225.854 & Surface & 2006 \\
\hline 1048.396 & 230.158 & Surface & 2006 & 6341.023 & 225.856 & Surface & 2006 \\
\hline 1051.357 & 230.151 & Surface & 2006 & 6343.916 & 225.859 & Surface & 2006 \\
\hline 1054.318 & 230.150 & Surface & 2006 & 6346.782 & 225.868 & Surface & 2006 \\
\hline 1057.279 & 230.151 & Surface & 2006 & 6349.648 & 225.915 & Surface & 2006 \\
\hline 1060.240 & 230.146 & Surface & 2006 & 6352.513 & 226.014 & Surface & 2006 \\
\hline 1063.201 & 230.163 & Surface & 2006 & 6355.379 & 226.102 & Surface & 2006 \\
\hline 1066.161 & 230.137 & Surface & 2006 & 6358.245 & 226.150 & Surface & 2006 \\
\hline 1069.123 & 230.135 & Surface & 2006 & 6361.111 & 226.158 & Surface & 2006 \\
\hline 1072.084 & 230.143 & Surface & 2006 & 6363.976 & 226.169 & Surface & 2006 \\
\hline 1075.044 & 230.143 & Surface & 2006 & 6366.842 & 226.177 & Surface & 2006 \\
\hline 1077.894 & 230.140 & Surface & 2006 & 6369.708 & 226.185 & Surface & 2006 \\
\hline 1080.744 & 230.129 & Surface & 2006 & 6372.573 & 226.193 & Surface & 2006 \\
\hline 1083.594 & 230.138 & Surface & 2006 & 6375.439 & 226.203 & Surface & 2006 \\
\hline 1086.444 & 230.131 & Surface & 2006 & 6378.305 & 226.210 & Surface & 2006 \\
\hline 1089.294 & 230.123 & Surface & 2006 & 6381.171 & 226.212 & Surface & 2006 \\
\hline 1092.143 & 230.136 & Surface & 2006 & 6384.036 & 226.212 & Surface & 2006 \\
\hline 1094.993 & 230.146 & Surface & 2006 & 6386.902 & 226.212 & Surface & 2006 \\
\hline 1097.843 & 230.170 & Surface & 2006 & 6389.768 & 226.195 & Surface & 2006 \\
\hline 1100.693 & 230.169 & Surface & 2006 & 6392.634 & 226.109 & Surface & 2006 \\
\hline 1103.543 & 230.159 & Surface & 2006 & 6395.499 & 225.992 & Surface & 2006 \\
\hline 1106.393 & 230.154 & Surface & 2006 & 6398.466 & 225.881 & Surface & 2006 \\
\hline 1109.242 & 230.164 & Surface & 2006 & 6401.432 & 225.796 & Surface & 2006 \\
\hline 1112.092 & 230.166 & Surface & 2006 & 6404.397 & 225.763 & Surface & 2006 \\
\hline 1114.942 & 230.165 & Surface & 2006 & 6407.364 & 225.761 & Surface & 2006 \\
\hline 1117.855 & 230.167 & Surface & 2006 & 6410.330 & 225.780 & Surface & 2006 \\
\hline 1120.768 & 230.139 & Surface & 2006 & 6413.296 & 225.859 & Surface & 2006 \\
\hline 1123.681 & 230.130 & Surface & 2006 & 6416.262 & 225.939 & Surface & 2006 \\
\hline 1126.594 & 230.144 & Surface & 2006 & 6419.228 & 226.017 & Surface & 2006 \\
\hline
\end{tabular}




\begin{tabular}{|c|c|c|c|c|c|c|c|}
\hline 1129.506 & 230.145 & Surface & 2006 & 6422.194 & 226.090 & Surface & 2006 \\
\hline 1132.419 & 230.167 & Surface & 2006 & 6425.160 & 226.122 & Surface & 2006 \\
\hline 1135.332 & 230.180 & Surface & 2006 & 6428.126 & 226.114 & Surface & 2006 \\
\hline 1138.245 & 230.186 & Surface & 2006 & 6431.093 & 226.106 & Surface & 2006 \\
\hline 1141.158 & 230.179 & Surface & 2006 & 6434.084 & 226.099 & Surface & 2006 \\
\hline 1144.071 & 230.190 & Surface & 2006 & 6437.077 & 226.087 & Surface & 2006 \\
\hline 1146.984 & 230.196 & Surface & 2006 & 6440.069 & 226.072 & Surface & 2006 \\
\hline 1149.896 & 230.187 & Surface & 2006 & 6443.061 & 226.062 & Surface & 2006 \\
\hline 1152.810 & 230.175 & Surface & 2006 & 6446.053 & 226.055 & Surface & 2006 \\
\hline 1155.722 & 230.184 & Surface & 2006 & 6449.045 & 226.024 & Surface & 2006 \\
\hline 1158.635 & 230.190 & Surface & 2006 & 6452.037 & 225.993 & Surface & 2006 \\
\hline 1161.548 & 230.163 & Surface & 2006 & 6455.029 & 225.962 & Surface & 2006 \\
\hline 1164.461 & 230.166 & Surface & 2006 & 6458.021 & 225.929 & Surface & 2006 \\
\hline 1167.374 & 230.178 & Surface & 2006 & 6461.013 & 225.898 & Surface & 2006 \\
\hline 1170.287 & 230.191 & Surface & 2006 & 6464.005 & 225.861 & Surface & 2006 \\
\hline 1173.200 & 230.186 & Surface & 2006 & 6466.997 & 225.806 & Surface & 2006 \\
\hline 1176.113 & 230.196 & Surface & 2006 & 6469.989 & 225.779 & Surface & 2006 \\
\hline 1179.026 & 230.202 & Surface & 2006 & 6472.981 & 225.786 & Surface & 2006 \\
\hline 1181.847 & 230.186 & Surface & 2006 & 6475.973 & 225.792 & Surface & 2006 \\
\hline 1184.668 & 230.165 & Surface & 2006 & 6478.965 & 225.796 & Surface & 2006 \\
\hline 1187.489 & 230.140 & Surface & 2006 & 6481.957 & 225.797 & Surface & 2006 \\
\hline 1190.310 & 230.124 & Surface & 2006 & 6484.949 & 225.796 & Surface & 2006 \\
\hline 1193.131 & 230.146 & Surface & 2006 & 6487.941 & 225.792 & Surface & 2006 \\
\hline 1195.953 & 230.159 & Surface & 2006 & 6490.933 & 225.791 & Surface & 2006 \\
\hline 1198.774 & 230.159 & Surface & 2006 & 6493.925 & 225.789 & Surface & 2006 \\
\hline 1201.595 & 230.133 & Surface & 2006 & 6496.917 & 225.788 & Surface & 2006 \\
\hline 1204.416 & 230.113 & Surface & 2006 & 6499.909 & 225.787 & Surface & 2006 \\
\hline 1207.237 & 230.114 & Surface & 2006 & 6502.901 & 225.782 & Surface & 2006 \\
\hline 1210.097 & 230.133 & Surface & 2006 & 6505.893 & 225.775 & Surface & 2006 \\
\hline 1212.956 & 230.134 & Surface & 2006 & 6508.885 & 225.769 & Surface & 2006 \\
\hline 1215.816 & 230.118 & Surface & 2006 & 6511.871 & 225.772 & Surface & 2006 \\
\hline 1218.675 & 230.117 & Surface & 2006 & 6514.858 & 225.764 & Surface & 2006 \\
\hline 1221.534 & 230.118 & Surface & 2006 & 6517.844 & 225.775 & Surface & 2006 \\
\hline 1224.394 & 230.118 & Surface & 2006 & 6520.830 & 225.775 & Surface & 2006 \\
\hline 1227.253 & 230.129 & Surface & 2006 & 6523.816 & 225.758 & Surface & 2006 \\
\hline 1230.113 & 230.140 & Surface & 2006 & 6526.802 & 225.732 & Surface & 2006 \\
\hline 1232.972 & 230.141 & Surface & 2006 & 6529.789 & 225.731 & Surface & 2006 \\
\hline 1235.831 & 230.126 & Surface & 2006 & 6532.775 & 225.734 & Surface & 2006 \\
\hline 1238.691 & 230.125 & Surface & 2006 & 6535.761 & 225.736 & Surface & 2006 \\
\hline 1241.550 & 230.116 & Surface & 2006 & 6538.747 & 225.737 & Surface & 2006 \\
\hline 1244.533 & 230.105 & Surface & 2006 & 6541.733 & 225.738 & Surface & 2006 \\
\hline 1247.515 & 230.105 & Surface & 2006 & 6544.719 & 225.737 & Surface & 2006 \\
\hline 1250.498 & 230.100 & Surface & 2006 & 6547.706 & 225.738 & Surface & 2006 \\
\hline 1253.481 & 230.089 & Surface & 2006 & 6550.617 & 225.739 & Surface & 2006 \\
\hline 1256.463 & 230.085 & Surface & 2006 & 6553.529 & 225.741 & Surface & 2006 \\
\hline 1259.445 & 230.097 & Surface & 2006 & 6556.440 & 225.742 & Surface & 2006 \\
\hline 1262.428 & 230.104 & Surface & 2006 & 6559.352 & 225.743 & Surface & 2006 \\
\hline 1265.411 & 230.101 & Surface & 2006 & 6562.264 & 225.744 & Surface & 2006 \\
\hline 1268.393 & 230.109 & Surface & 2006 & 6565.175 & 225.743 & Surface & 2006 \\
\hline
\end{tabular}




\begin{tabular}{|c|c|c|c|c|c|c|c|}
\hline 1271.376 & 230.111 & Surface & 2006 & 6568.087 & 225.744 & Surface & 2006 \\
\hline 1274.359 & 230.096 & Surface & 2006 & 6570.998 & 225.746 & Surface & 2006 \\
\hline 1277.341 & 230.096 & Surface & 2006 & 6573.910 & 225.747 & Surface & 2006 \\
\hline 1280.323 & 230.099 & Surface & 2006 & 6576.821 & 225.748 & Surface & 2006 \\
\hline 1283.306 & 230.098 & Surface & 2006 & 6579.733 & 225.750 & Surface & 2006 \\
\hline 1286.289 & 230.104 & Surface & 2006 & 6582.644 & 225.750 & Surface & 2006 \\
\hline 1289.271 & 230.088 & Surface & 2006 & 6585.556 & 225.749 & Surface & 2006 \\
\hline 1292.254 & 230.089 & Surface & 2006 & 6588.467 & 225.750 & Surface & 2006 \\
\hline 1295.236 & 230.102 & Surface & 2006 & 6591.379 & 225.752 & Surface & 2006 \\
\hline 1298.219 & 230.106 & Surface & 2006 & 6594.365 & 225.754 & Surface & 2006 \\
\hline 1301.201 & 230.117 & Surface & 2006 & 6597.350 & 225.784 & Surface & 2006 \\
\hline 1304.184 & 230.135 & Surface & 2006 & 6600.335 & 225.833 & Surface & 2006 \\
\hline 1307.167 & 230.130 & Surface & 2006 & 6603.321 & 225.911 & Surface & 2006 \\
\hline 1310.149 & 230.121 & Surface & 2006 & 6606.306 & 226.038 & Surface & 2006 \\
\hline 1313.132 & 230.113 & Surface & 2006 & 6609.292 & 226.169 & Surface & 2006 \\
\hline 1316.047 & 230.110 & Surface & 2006 & 6612.277 & 226.219 & Surface & 2006 \\
\hline 1318.963 & 230.104 & Surface & 2006 & 6615.262 & 226.220 & Surface & 2006 \\
\hline 1321.878 & 230.106 & Surface & 2006 & 6618.248 & 226.229 & Surface & 2006 \\
\hline 1324.793 & 230.117 & Surface & 2006 & 6621.233 & 226.238 & Surface & 2006 \\
\hline 1327.709 & 230.116 & Surface & 2006 & 6624.218 & 226.248 & Surface & 2006 \\
\hline 1330.624 & 230.107 & Surface & 2006 & 6627.204 & 226.265 & Surface & 2006 \\
\hline 1333.539 & 230.112 & Surface & 2006 & 6630.189 & 226.283 & Surface & 2006 \\
\hline 1336.455 & 230.129 & Surface & 2006 & 6633.175 & 226.257 & Surface & 2006 \\
\hline 1339.370 & 230.130 & Surface & 2006 & 6636.160 & 226.223 & Surface & 2006 \\
\hline 1342.285 & 230.123 & Surface & 2006 & 6639.146 & 226.190 & Surface & 2006 \\
\hline 1345.201 & 230.111 & Surface & 2006 & 6642.131 & 226.157 & Surface & 2006 \\
\hline 1348.116 & 230.106 & Surface & 2006 & 6645.117 & 226.121 & Surface & 2006 \\
\hline 1351.032 & 230.099 & Surface & 2006 & 6648.102 & 226.096 & Surface & 2006 \\
\hline 1353.953 & 230.088 & Surface & 2006 & 6651.087 & 226.051 & Surface & 2006 \\
\hline 1356.875 & 230.081 & Surface & 2006 & 6653.952 & 226.025 & Surface & 2006 \\
\hline 1359.797 & 230.078 & Surface & 2006 & 6656.816 & 226.020 & Surface & 2006 \\
\hline 1362.718 & 230.079 & Surface & 2006 & 6659.681 & 226.020 & Surface & 2006 \\
\hline 1365.640 & 230.081 & Surface & 2006 & 6662.545 & 226.019 & Surface & 2006 \\
\hline 1368.562 & 230.081 & Surface & 2006 & 6665.410 & 226.019 & Surface & 2006 \\
\hline 1371.483 & 230.088 & Surface & 2006 & 6668.275 & 226.017 & Surface & 2006 \\
\hline 1374.405 & 230.097 & Surface & 2006 & 6671.139 & 226.018 & Surface & 2006 \\
\hline 1377.326 & 230.105 & Surface & 2006 & 6674.004 & 226.016 & Surface & 2006 \\
\hline 1380.248 & 230.115 & Surface & 2006 & 6676.868 & 226.017 & Surface & 2006 \\
\hline 1383.170 & 230.125 & Surface & 2006 & 6679.733 & 226.024 & Surface & 2006 \\
\hline 1386.091 & 230.131 & Surface & 2006 & 6682.598 & 226.012 & Surface & 2006 \\
\hline 1389.013 & 230.125 & Surface & 2006 & 6685.462 & 225.975 & Surface & 2006 \\
\hline 1391.934 & 230.111 & Surface & 2006 & 6688.327 & 225.941 & Surface & 2006 \\
\hline 1394.856 & 230.097 & Surface & 2006 & 6691.191 & 225.894 & Surface & 2006 \\
\hline 1397.778 & 230.088 & Surface & 2006 & 6694.056 & 225.873 & Surface & 2006 \\
\hline 1400.699 & 230.087 & Surface & 2006 & 6696.921 & 225.873 & Surface & 2006 \\
\hline 1403.621 & 230.088 & Surface & 2006 & 6699.785 & 225.874 & Surface & 2006 \\
\hline 1406.543 & 230.085 & Surface & 2006 & 6702.650 & 225.875 & Surface & 2006 \\
\hline 1409.464 & 230.097 & Surface & 2006 & 6705.514 & 225.876 & Surface & 2006 \\
\hline 1412.407 & 230.116 & Surface & 2006 & 6708.479 & 225.884 & Surface & 2006 \\
\hline
\end{tabular}




\begin{tabular}{|c|c|c|c|c|c|c|c|}
\hline 1415.349 & 230.107 & Surface & 2006 & 6711.445 & 225.900 & Surface & 2006 \\
\hline 1418.291 & 230.092 & Surface & 2006 & 6714.410 & 225.916 & Surface & 2006 \\
\hline 1421.234 & 230.076 & Surface & 2006 & 6717.376 & 225.932 & Surface & 2006 \\
\hline 1424.176 & 230.069 & Surface & 2006 & 6720.341 & 225.899 & Surface & 2006 \\
\hline 1427.118 & 230.069 & Surface & 2006 & 6723.307 & 225.817 & Surface & 2006 \\
\hline 1430.060 & 230.056 & Surface & 2006 & 6726.272 & 225.790 & Surface & 2006 \\
\hline 1433.003 & 230.049 & Surface & 2006 & 6729.238 & 225.794 & Surface & 2006 \\
\hline 1435.945 & 230.052 & Surface & 2006 & 6732.203 & 225.798 & Surface & 2006 \\
\hline 1438.887 & 230.045 & Surface & 2006 & 6735.169 & 225.802 & Surface & 2006 \\
\hline 1441.830 & 230.029 & Surface & 2006 & 6738.134 & 225.803 & Surface & 2006 \\
\hline 1444.772 & 230.027 & Surface & 2006 & 6741.100 & 225.790 & Surface & 2006 \\
\hline 1447.714 & 230.035 & Surface & 2006 & 6744.065 & 225.784 & Surface & 2006 \\
\hline 1450.657 & 230.036 & Surface & 2006 & 6747.031 & 225.778 & Surface & 2006 \\
\hline 1453.599 & 230.038 & Surface & 2006 & 6749.996 & 225.773 & Surface & 2006 \\
\hline 1456.541 & 230.027 & Surface & 2006 & 6752.962 & 225.768 & Surface & 2006 \\
\hline 1459.484 & 230.010 & Surface & 2006 & 6755.927 & 225.761 & Surface & 2006 \\
\hline 1462.426 & 230.004 & Surface & 2006 & 6758.892 & 225.755 & Surface & 2006 \\
\hline 1465.368 & 230.019 & Surface & 2006 & 6761.887 & 225.750 & Surface & 2006 \\
\hline 1468.311 & 230.009 & Surface & 2006 & 6764.882 & 225.744 & Surface & 2006 \\
\hline 1471.253 & 229.988 & Surface & 2006 & 6767.877 & 225.748 & Surface & 2006 \\
\hline 1474.195 & 229.991 & Surface & 2006 & 6770.872 & 225.751 & Surface & 2006 \\
\hline 1477.138 & 229.993 & Surface & 2006 & 6773.867 & 225.748 & Surface & 2006 \\
\hline 1480.080 & 229.991 & Surface & 2006 & 6776.862 & 225.749 & Surface & 2006 \\
\hline 1483.022 & 229.985 & Surface & 2006 & 6779.856 & 225.738 & Surface & 2006 \\
\hline 1485.964 & 229.977 & Surface & 2006 & 6782.852 & 225.727 & Surface & 2006 \\
\hline 1488.907 & 229.976 & Surface & 2006 & 6785.847 & 225.745 & Surface & 2006 \\
\hline 1491.849 & 229.967 & Surface & 2006 & 6788.841 & 225.756 & Surface & 2006 \\
\hline 1494.792 & 229.969 & Surface & 2006 & 6791.836 & 225.759 & Surface & 2006 \\
\hline 1497.734 & 229.951 & Surface & 2006 & 6794.831 & 225.762 & Surface & 2006 \\
\hline 1500.676 & 229.955 & Surface & 2006 & 6797.826 & 225.766 & Surface & 2006 \\
\hline 1503.618 & 229.969 & Surface & 2006 & 6800.821 & 225.770 & Surface & 2006 \\
\hline 1506.561 & 229.975 & Surface & 2006 & 6803.816 & 225.773 & Surface & 2006 \\
\hline 1509.503 & 229.960 & Surface & 2006 & 6806.811 & 225.775 & Surface & 2006 \\
\hline 1512.439 & 229.959 & Surface & 2006 & 6809.806 & 225.779 & Surface & 2006 \\
\hline 1515.374 & 229.966 & Surface & 2006 & 6812.800 & 225.778 & Surface & 2006 \\
\hline 1518.310 & 229.960 & Surface & 2006 & 6815.795 & 225.774 & Surface & 2006 \\
\hline 1521.245 & 229.945 & Surface & 2006 & 6818.790 & 225.777 & Surface & 2006 \\
\hline 1524.181 & 229.943 & Surface & 2006 & 6821.785 & 225.793 & Surface & 2006 \\
\hline 1527.117 & 229.951 & Surface & 2006 & 6824.780 & 225.801 & Surface & 2006 \\
\hline 1530.052 & 229.959 & Surface & 2006 & 6827.775 & 225.780 & Surface & 2006 \\
\hline 1532.988 & 229.958 & Surface & 2006 & 6830.770 & 225.769 & Surface & 2006 \\
\hline 1535.923 & 229.952 & Surface & 2006 & 6833.714 & 225.766 & Surface & 2006 \\
\hline 1538.859 & 229.938 & Surface & 2006 & 6836.659 & 225.767 & Surface & 2006 \\
\hline 1541.795 & 229.938 & Surface & 2006 & 6839.603 & 225.769 & Surface & 2006 \\
\hline 1544.730 & 229.922 & Surface & 2006 & 6842.547 & 225.760 & Surface & 2006 \\
\hline 1547.666 & 229.917 & Surface & 2006 & 6845.492 & 225.758 & Surface & 2006 \\
\hline 1550.601 & 229.916 & Surface & 2006 & 6848.436 & 225.761 & Surface & 2006 \\
\hline 1553.537 & 229.924 & Surface & 2006 & 6851.380 & 225.754 & Surface & 2006 \\
\hline 1556.473 & 229.921 & Surface & 2006 & 6854.324 & 225.713 & Surface & 2006 \\
\hline
\end{tabular}




\begin{tabular}{|c|c|c|c|c|c|c|c|}
\hline 1559.408 & 229.919 & Surface & 2006 & 6857.269 & 225.688 & Surface & 2006 \\
\hline 1562.344 & 229.909 & Surface & 2006 & 6860.213 & 225.709 & Surface & 2006 \\
\hline 1565.279 & 229.905 & Surface & 2006 & 6863.158 & 225.730 & Surface & 2006 \\
\hline 1568.215 & 229.901 & Surface & 2006 & 6866.102 & 225.745 & Surface & 2006 \\
\hline 1571.151 & 229.896 & Surface & 2006 & 6869.047 & 225.752 & Surface & 2006 \\
\hline 1574.086 & 229.887 & Surface & 2006 & 6871.991 & 225.753 & Surface & 2006 \\
\hline 1577.022 & 229.884 & Surface & 2006 & 6874.935 & 225.750 & Surface & 2006 \\
\hline 1579.958 & 229.882 & Surface & 2006 & 6877.880 & 225.750 & Surface & 2006 \\
\hline 1582.893 & 229.863 & Surface & 2006 & 6880.824 & 225.746 & Surface & 2006 \\
\hline 1585.829 & 229.864 & Surface & 2006 & 6883.769 & 225.742 & Surface & 2006 \\
\hline 1588.724 & 229.875 & Surface & 2006 & 6886.713 & 225.731 & Surface & 2006 \\
\hline 1591.621 & 229.856 & Surface & 2006 & 6889.657 & 225.709 & Surface & 2006 \\
\hline 1594.516 & 229.864 & Surface & 2006 & 6892.602 & 225.711 & Surface & 2006 \\
\hline 1597.412 & 229.860 & Surface & 2006 & 6895.546 & 225.714 & Surface & 2006 \\
\hline 1600.308 & 229.839 & Surface & 2006 & 6898.490 & 225.734 & Surface & 2006 \\
\hline 1603.204 & 229.861 & Surface & 2006 & 6901.434 & 225.748 & Surface & 2006 \\
\hline 1606.099 & 229.867 & Surface & 2006 & 6904.379 & 225.734 & Surface & 2006 \\
\hline 1608.996 & 229.856 & Surface & 2006 & 6907.323 & 225.716 & Surface & 2006 \\
\hline 1611.891 & 229.838 & Surface & 2006 & 6910.268 & 225.713 & Surface & 2006 \\
\hline 1614.787 & 229.827 & Surface & 2006 & 6913.212 & 225.714 & Surface & 2006 \\
\hline 1617.683 & 229.831 & Surface & 2006 & 6916.157 & 225.712 & Surface & 2006 \\
\hline 1620.579 & 229.832 & Surface & 2006 & 6919.101 & 225.714 & Surface & 2006 \\
\hline 1623.475 & 229.850 & Surface & 2006 & 6922.045 & 225.708 & Surface & 2006 \\
\hline 1626.371 & 229.857 & Surface & 2006 & 6924.990 & 225.702 & Surface & 2006 \\
\hline 1629.333 & 229.839 & Surface & 2006 & 6927.968 & 225.703 & Surface & 2006 \\
\hline 1632.295 & 229.833 & Surface & 2006 & 6930.946 & 225.712 & Surface & 2006 \\
\hline 1635.257 & 229.869 & Surface & 2006 & 6933.925 & 225.717 & Surface & 2006 \\
\hline 1638.219 & 229.841 & Surface & 2006 & 6936.903 & 225.729 & Surface & 2006 \\
\hline 1641.182 & 229.828 & Surface & 2006 & 6939.881 & 225.747 & Surface & 2006 \\
\hline 1644.144 & 229.833 & Surface & 2006 & 6942.859 & 225.755 & Surface & 2006 \\
\hline 1647.106 & 229.845 & Surface & 2006 & 6945.838 & 225.764 & Surface & 2006 \\
\hline 1650.069 & 229.848 & Surface & 2006 & 6948.816 & 225.755 & Surface & 2006 \\
\hline 1653.031 & 229.848 & Surface & 2006 & 6951.794 & 225.747 & Surface & 2006 \\
\hline 1655.993 & 229.853 & Surface & 2006 & 6954.773 & 225.748 & Surface & 2006 \\
\hline 1658.956 & 229.852 & Surface & 2006 & 6957.751 & 225.725 & Surface & 2006 \\
\hline 1661.918 & 229.855 & Surface & 2006 & 6960.729 & 225.710 & Surface & 2006 \\
\hline 1664.880 & 229.864 & Surface & 2006 & 6963.707 & 225.707 & Surface & 2006 \\
\hline 1667.842 & 229.864 & Surface & 2006 & 6966.686 & 225.713 & Surface & 2006 \\
\hline 1670.805 & 229.858 & Surface & 2006 & 6969.664 & 225.726 & Surface & 2006 \\
\hline 1673.767 & 229.854 & Surface & 2006 & 6972.642 & 225.731 & Surface & 2006 \\
\hline 1676.722 & 229.839 & Surface & 2006 & 6975.620 & 225.736 & Surface & 2006 \\
\hline 1679.678 & 229.822 & Surface & 2006 & 6978.598 & 225.737 & Surface & 2006 \\
\hline 1682.634 & 229.816 & Surface & 2006 & 6981.577 & 225.714 & Surface & 2006 \\
\hline 1685.590 & 229.824 & Surface & 2006 & 6984.555 & 225.716 & Surface & 2006 \\
\hline 1688.545 & 229.839 & Surface & 2006 & 6987.533 & 225.746 & Surface & 2006 \\
\hline 1691.501 & 229.828 & Surface & 2006 & 6990.512 & 225.738 & Surface & 2006 \\
\hline 1694.456 & 229.829 & Surface & 2006 & 6993.490 & 225.750 & Surface & 2006 \\
\hline 1697.412 & 229.831 & Surface & 2006 & 6996.468 & 225.750 & Surface & 2006 \\
\hline 1700.368 & 229.835 & Surface & 2006 & 6999.447 & 225.736 & Surface & 2006 \\
\hline
\end{tabular}




\begin{tabular}{|c|c|c|c|c|c|c|c|}
\hline 1703.323 & 229.790 & Surface & 2006 & 7002.425 & 225.729 & Surface & 2006 \\
\hline 1706.279 & 229.801 & Surface & 2006 & 7005.403 & 225.726 & Surface & 2006 \\
\hline 1709.235 & 229.822 & Surface & 2006 & 7008.381 & 225.725 & Surface & 2006 \\
\hline 1712.190 & 229.821 & Surface & 2006 & 7011.360 & 225.734 & Surface & 2006 \\
\hline 1715.146 & 229.819 & Surface & 2006 & 7014.338 & 225.746 & Surface & 2006 \\
\hline 1718.102 & 229.802 & Surface & 2006 & 7017.316 & 225.744 & Surface & 2006 \\
\hline 1721.057 & 229.808 & Surface & 2006 & 7020.256 & 225.735 & Surface & 2006 \\
\hline 1724.013 & 229.799 & Surface & 2006 & 7023.196 & 225.729 & Surface & 2006 \\
\hline 1726.969 & 229.789 & Surface & 2006 & 7026.136 & 225.722 & Surface & 2006 \\
\hline 1729.866 & 229.780 & Surface & 2006 & 7029.076 & 225.715 & Surface & 2006 \\
\hline 1732.763 & 229.787 & Surface & 2006 & 7032.016 & 225.707 & Surface & 2006 \\
\hline 1735.660 & 229.801 & Surface & 2006 & 7034.956 & 225.701 & Surface & 2006 \\
\hline 1738.558 & 229.818 & Surface & 2006 & 7037.895 & 225.699 & Surface & 2006 \\
\hline 1741.455 & 229.800 & Surface & 2006 & 7040.835 & 225.700 & Surface & 2006 \\
\hline 1744.352 & 229.789 & Surface & 2006 & 7043.775 & 225.700 & Surface & 2006 \\
\hline 1747.250 & 229.811 & Surface & 2006 & 7046.715 & 225.701 & Surface & 2006 \\
\hline 1750.147 & 229.815 & Surface & 2006 & 7049.655 & 225.702 & Surface & 2006 \\
\hline 1753.044 & 229.819 & Surface & 2006 & 7052.595 & 225.704 & Surface & 2006 \\
\hline 1755.941 & 229.822 & Surface & 2006 & 7055.535 & 225.705 & Surface & 2006 \\
\hline 1758.839 & 229.799 & Surface & 2006 & 7058.474 & 225.707 & Surface & 2006 \\
\hline 1761.736 & 229.797 & Surface & 2006 & 7061.414 & 225.708 & Surface & 2006 \\
\hline 1764.633 & 229.807 & Surface & 2006 & 7064.354 & 225.710 & Surface & 2006 \\
\hline 1767.530 & 229.814 & Surface & 2006 & 7067.294 & 225.712 & Surface & 2006 \\
\hline 1770.428 & 229.822 & Surface & 2006 & 7070.234 & 225.718 & Surface & 2006 \\
\hline 1773.325 & 229.791 & Surface & 2006 & 7073.174 & 225.723 & Surface & 2006 \\
\hline 1776.222 & 229.792 & Surface & 2006 & 7076.114 & 225.728 & Surface & 2006 \\
\hline 1779.119 & 229.809 & Surface & 2006 & 7079.053 & 225.733 & Surface & 2006 \\
\hline 1782.017 & 229.801 & Surface & 2006 & 7081.993 & 225.741 & Surface & 2006 \\
\hline 1784.914 & 229.801 & Surface & 2006 & 7084.933 & 225.719 & Surface & 2006 \\
\hline 1787.811 & 229.784 & Surface & 2006 & 7087.873 & 225.689 & Surface & 2006 \\
\hline 1790.709 & 229.799 & Surface & 2006 & 7090.813 & 225.691 & Surface & 2006 \\
\hline 1793.606 & 229.811 & Surface & 2006 & 7093.753 & 225.699 & Surface & 2006 \\
\hline 1796.503 & 229.812 & Surface & 2006 & 7096.693 & 225.708 & Surface & 2006 \\
\hline 1799.400 & 229.802 & Surface & 2006 & 7099.633 & 225.724 & Surface & 2006 \\
\hline 1802.370 & 229.773 & Surface & 2006 & 7102.572 & 225.729 & Surface & 2006 \\
\hline 1805.340 & 229.785 & Surface & 2006 & 7105.512 & 225.717 & Surface & 2006 \\
\hline 1808.310 & 229.807 & Surface & 2006 & 7108.452 & 225.715 & Surface & 2006 \\
\hline 1811.280 & 229.794 & Surface & 2006 & 7111.392 & 225.720 & Surface & 2006 \\
\hline 1814.250 & 229.772 & Surface & 2006 & 7114.332 & 225.716 & Surface & 2006 \\
\hline 1817.220 & 229.774 & Surface & 2006 & 7117.272 & 225.717 & Surface & 2006 \\
\hline 1820.190 & 229.783 & Surface & 2006 & 7120.212 & 225.726 & Surface & 2006 \\
\hline 1823.159 & 229.785 & Surface & 2006 & 7123.152 & 225.713 & Surface & 2006 \\
\hline 1826.129 & 229.816 & Surface & 2006 & 7126.091 & 225.686 & Surface & 2006 \\
\hline 1829.099 & 229.826 & Surface & 2006 & 7129.031 & 225.679 & Surface & 2006 \\
\hline 1832.069 & 229.800 & Surface & 2006 & 7131.946 & 225.687 & Surface & 2006 \\
\hline 1835.039 & 229.798 & Surface & 2006 & 7134.860 & 225.710 & Surface & 2006 \\
\hline 1838.009 & 229.835 & Surface & 2006 & 7137.775 & 225.714 & Surface & 2006 \\
\hline 1840.979 & 229.835 & Surface & 2006 & 7140.689 & 225.717 & Surface & 2006 \\
\hline 1843.948 & 229.839 & Surface & 2006 & 7143.604 & 225.710 & Surface & 2006 \\
\hline
\end{tabular}




\begin{tabular}{|c|c|c|c|c|c|c|c|}
\hline 1846.918 & 229.853 & Surface & 2006 & 7146.518 & 225.685 & Surface & 2006 \\
\hline 1849.888 & 229.829 & Surface & 2006 & 7149.433 & 225.674 & Surface & 2006 \\
\hline 1852.858 & 229.809 & Surface & 2006 & 7152.348 & 225.674 & Surface & 2006 \\
\hline 1855.828 & 229.825 & Surface & 2006 & 7155.262 & 225.681 & Surface & 2006 \\
\hline 1858.798 & 229.809 & Surface & 2006 & 7158.177 & 225.675 & Surface & 2006 \\
\hline 1861.768 & 229.807 & Surface & 2006 & 7161.091 & 225.658 & Surface & 2006 \\
\hline 1864.738 & 229.816 & Surface & 2006 & 7164.006 & 225.665 & Surface & 2006 \\
\hline 1867.707 & 229.801 & Surface & 2006 & 7166.920 & 225.671 & Surface & 2006 \\
\hline 1870.678 & 229.780 & Surface & 2006 & 7169.835 & 225.670 & Surface & 2006 \\
\hline 1873.647 & 229.813 & Surface & 2006 & 7172.749 & 225.663 & Surface & 2006 \\
\hline 1876.617 & 229.803 & Surface & 2006 & 7175.664 & 225.659 & Surface & 2006 \\
\hline 1879.587 & 229.794 & Surface & 2006 & 7178.578 & 225.677 & Surface & 2006 \\
\hline 1882.557 & 229.792 & Surface & 2006 & 7181.493 & 225.683 & Surface & 2006 \\
\hline 1885.527 & 229.792 & Surface & 2006 & 7184.407 & 225.668 & Surface & 2006 \\
\hline 1888.496 & 229.796 & Surface & 2006 & 7187.322 & 225.686 & Surface & 2006 \\
\hline 1891.467 & 229.778 & Surface & 2006 & 7190.237 & 225.703 & Surface & 2006 \\
\hline 1894.436 & 229.787 & Surface & 2006 & 7193.151 & 225.687 & Surface & 2006 \\
\hline 1897.406 & 229.826 & Surface & 2006 & 7196.070 & 225.665 & Surface & 2006 \\
\hline 1900.376 & 229.810 & Surface & 2006 & 7198.988 & 225.652 & Surface & 2006 \\
\hline 1903.308 & 229.795 & Surface & 2006 & 7201.907 & 225.647 & Surface & 2006 \\
\hline 1906.239 & 229.794 & Surface & 2006 & 7204.825 & 225.663 & Surface & 2006 \\
\hline 1909.171 & 229.792 & Surface & 2006 & 7207.743 & 225.665 & Surface & 2006 \\
\hline 1912.103 & 229.797 & Surface & 2006 & 7210.662 & 225.672 & Surface & 2006 \\
\hline 1915.034 & 229.818 & Surface & 2006 & 7213.580 & 225.669 & Surface & 2006 \\
\hline 1917.966 & 229.846 & Surface & 2006 & 7216.499 & 225.663 & Surface & 2006 \\
\hline 1920.898 & 229.851 & Surface & 2006 & 7219.417 & 225.673 & Surface & 2006 \\
\hline 1923.830 & 229.822 & Surface & 2006 & 7222.336 & 225.660 & Surface & 2006 \\
\hline 1926.761 & 229.813 & Surface & 2006 & 7225.254 & 225.647 & Surface & 2006 \\
\hline 1929.693 & 229.802 & Surface & 2006 & 7228.173 & 225.656 & Surface & 2006 \\
\hline 1932.625 & 229.803 & Surface & 2006 & 7231.091 & 225.679 & Surface & 2006 \\
\hline 1935.556 & 229.812 & Surface & 2006 & 7234.010 & 225.680 & Surface & 2006 \\
\hline 1938.488 & 229.818 & Surface & 2006 & 7236.928 & 225.653 & Surface & 2006 \\
\hline 1941.420 & 229.828 & Surface & 2006 & 7239.847 & 225.662 & Surface & 2006 \\
\hline 1944.351 & 229.833 & Surface & 2006 & 7242.765 & 225.647 & Surface & 2006 \\
\hline 1947.283 & 229.813 & Surface & 2006 & 7245.684 & 225.647 & Surface & 2006 \\
\hline 1950.215 & 229.793 & Surface & 2006 & 7248.602 & 225.641 & Surface & 2006 \\
\hline 1953.147 & 229.804 & Surface & 2006 & 7251.520 & 225.663 & Surface & 2006 \\
\hline 1956.078 & 229.816 & Surface & 2006 & 7254.439 & 225.675 & Surface & 2006 \\
\hline 1959.010 & 229.810 & Surface & 2006 & 7257.358 & 225.629 & Surface & 2006 \\
\hline 1961.864 & 229.808 & Surface & 2006 & 7260.276 & 225.611 & Surface & 2006 \\
\hline 1964.719 & 229.819 & Surface & 2006 & 7263.194 & 225.597 & Surface & 2006 \\
\hline 1967.573 & 229.813 & Surface & 2006 & 7266.113 & 225.623 & Surface & 2006 \\
\hline 1970.428 & 229.810 & Surface & 2006 & 7269.031 & 225.629 & Surface & 2006 \\
\hline 1973.282 & 229.818 & Surface & 2006 & 7271.950 & 225.625 & Surface & 2006 \\
\hline 1976.136 & 229.827 & Surface & 2006 & 7274.868 & 225.648 & Surface & 2006 \\
\hline 1978.991 & 229.818 & Surface & 2006 & 7277.787 & 225.647 & Surface & 2006 \\
\hline 1981.845 & 229.808 & Surface & 2006 & 7280.649 & 225.633 & Surface & 2006 \\
\hline 1984.700 & 229.811 & Surface & 2006 & 7283.511 & 225.621 & Surface & 2006 \\
\hline 1987.554 & 229.826 & Surface & 2006 & 7286.373 & 225.595 & Surface & 2006 \\
\hline
\end{tabular}




\begin{tabular}{|c|c|c|c|c|c|c|c|}
\hline 1990.408 & 229.825 & Surface & 2006 & 7289.236 & 225.593 & Surface & 2006 \\
\hline 1993.262 & 229.832 & Surface & 2006 & 7292.098 & 225.598 & Surface & 2006 \\
\hline 1996.117 & 229.836 & Surface & 2006 & 7294.960 & 225.616 & Surface & 2006 \\
\hline 1998.971 & 229.820 & Surface & 2006 & 7297.823 & 225.625 & Surface & 2006 \\
\hline 2001.826 & 229.794 & Surface & 2006 & 7300.685 & 225.617 & Surface & 2006 \\
\hline 2004.680 & 229.800 & Surface & 2006 & 7303.547 & 225.615 & Surface & 2006 \\
\hline 2007.534 & 229.827 & Surface & 2006 & 7306.409 & 225.596 & Surface & 2006 \\
\hline 2010.389 & 229.837 & Surface & 2006 & 7309.272 & 225.596 & Surface & 2006 \\
\hline 2013.243 & 229.829 & Surface & 2006 & 7312.134 & 225.606 & Surface & 2006 \\
\hline 2016.097 & 229.835 & Surface & 2006 & 7314.996 & 225.614 & Surface & 2006 \\
\hline 2019.058 & 229.841 & Surface & 2006 & 7317.859 & 225.630 & Surface & 2006 \\
\hline 2022.019 & 229.844 & Surface & 2006 & 7320.721 & 225.631 & Surface & 2006 \\
\hline 2024.980 & 229.844 & Surface & 2006 & 7323.583 & 225.604 & Surface & 2006 \\
\hline 2027.941 & 229.841 & Surface & 2006 & 7326.446 & 225.616 & Surface & 2006 \\
\hline 2030.902 & 229.828 & Surface & 2006 & 7329.308 & 225.599 & Surface & 2006 \\
\hline 2033.863 & 229.822 & Surface & 2006 & 7332.170 & 225.576 & Surface & 2006 \\
\hline 2036.824 & 229.816 & Surface & 2006 & 7335.092 & 225.579 & Surface & 2006 \\
\hline 2039.785 & 229.813 & Surface & 2006 & 7338.013 & 225.603 & Surface & 2006 \\
\hline 2042.746 & 229.811 & Surface & 2006 & 7340.935 & 225.629 & Surface & 2006 \\
\hline 2045.707 & 229.828 & Surface & 2006 & 7343.857 & 225.583 & Surface & 2006 \\
\hline 2048.668 & 229.828 & Surface & 2006 & 7346.778 & 225.575 & Surface & 2006 \\
\hline 2051.629 & 229.833 & Surface & 2006 & 7349.700 & 225.561 & Surface & 2006 \\
\hline 2054.590 & 229.848 & Surface & 2006 & 7352.622 & 225.564 & Surface & 2006 \\
\hline 2057.551 & 229.847 & Surface & 2006 & 7355.543 & 225.595 & Surface & 2006 \\
\hline 2060.512 & 229.830 & Surface & 2006 & 7358.465 & 225.587 & Surface & 2006 \\
\hline 2063.473 & 229.846 & Surface & 2006 & 7361.386 & 225.568 & Surface & 2006 \\
\hline 2066.434 & 229.848 & Surface & 2006 & 7364.308 & 225.559 & Surface & 2006 \\
\hline 2069.327 & 229.842 & Surface & 2006 & 7367.230 & 225.557 & Surface & 2006 \\
\hline 2072.221 & 229.836 & Surface & 2006 & 7370.152 & 225.570 & Surface & 2006 \\
\hline 2075.114 & 229.821 & Surface & 2006 & 7373.073 & 225.579 & Surface & 2006 \\
\hline 2078.008 & 229.807 & Surface & 2006 & 7375.995 & 225.560 & Surface & 2006 \\
\hline 2080.901 & 229.826 & Surface & 2006 & 7378.917 & 225.545 & Surface & 2006 \\
\hline 2083.795 & 229.850 & Surface & 2006 & 7381.838 & 225.548 & Surface & 2006 \\
\hline 2086.688 & 229.860 & Surface & 2006 & 7384.760 & 225.534 & Surface & 2006 \\
\hline 2089.582 & 229.867 & Surface & 2006 & 7387.682 & 225.516 & Surface & 2006 \\
\hline 2092.475 & 229.876 & Surface & 2006 & 7390.603 & 225.512 & Surface & 2006 \\
\hline 2095.369 & 229.885 & Surface & 2006 & 7393.596 & 225.520 & Surface & 2006 \\
\hline 2098.262 & 229.892 & Surface & 2006 & 7396.590 & 225.516 & Surface & 2006 \\
\hline 2101.156 & 229.886 & Surface & 2006 & 7399.584 & 225.503 & Surface & 2006 \\
\hline 2104.049 & 229.864 & Surface & 2006 & 7402.577 & 225.492 & Surface & 2006 \\
\hline 2106.943 & 229.849 & Surface & 2006 & 7405.571 & 225.497 & Surface & 2006 \\
\hline 2109.836 & 229.852 & Surface & 2006 & 7408.564 & 225.515 & Surface & 2006 \\
\hline 2112.823 & 229.853 & Surface & 2006 & 7411.557 & 225.544 & Surface & 2006 \\
\hline 2115.810 & 229.859 & Surface & 2006 & 7414.551 & 225.526 & Surface & 2006 \\
\hline 2118.797 & 229.853 & Surface & 2006 & 7417.545 & 225.511 & Surface & 2006 \\
\hline 2121.784 & 229.836 & Surface & 2006 & 7420.538 & 225.500 & Surface & 2006 \\
\hline 2124.771 & 229.825 & Surface & 2006 & 7423.532 & 225.497 & Surface & 2006 \\
\hline 2127.758 & 229.828 & Surface & 2006 & 7426.525 & 225.489 & Surface & 2006 \\
\hline 2130.745 & 229.831 & Surface & 2006 & 7429.519 & 225.475 & Surface & 2006 \\
\hline
\end{tabular}




\begin{tabular}{|c|c|c|c|c|c|c|c|}
\hline 2133.732 & 229.830 & Surface & 2006 & 7432.512 & 225.463 & Surface & 2006 \\
\hline 2136.720 & 229.833 & Surface & 2006 & 7435.506 & 225.446 & Surface & 2006 \\
\hline 2139.707 & 229.832 & Surface & 2006 & 7438.499 & 225.461 & Surface & 2006 \\
\hline 2142.694 & 229.827 & Surface & 2006 & 7441.493 & 225.463 & Surface & 2006 \\
\hline 2145.681 & 229.838 & Surface & 2006 & 7444.486 & 225.452 & Surface & 2006 \\
\hline 2148.668 & 229.858 & Surface & 2006 & 7447.480 & 225.439 & Surface & 2006 \\
\hline 2151.655 & 229.861 & Surface & 2006 & 7450.473 & 225.447 & Surface & 2006 \\
\hline 2154.642 & 229.858 & Surface & 2006 & 7453.467 & 225.437 & Surface & 2006 \\
\hline 2157.629 & 229.882 & Surface & 2006 & 7456.460 & 225.403 & Surface & 2006 \\
\hline 2160.598 & 229.930 & Surface & 2006 & 7459.454 & 225.394 & Surface & 2006 \\
\hline 2163.568 & 229.984 & Surface & 2006 & 7462.447 & 225.404 & Surface & 2006 \\
\hline 2166.538 & 230.027 & Surface & 2006 & 7465.358 & 225.389 & Surface & 2006 \\
\hline 2169.507 & 230.025 & Surface & 2006 & 7468.269 & 225.353 & Surface & 2006 \\
\hline 2172.477 & 230.008 & Surface & 2006 & 7471.180 & 225.326 & Surface & 2006 \\
\hline 2175.447 & 229.991 & Surface & 2006 & 7474.091 & 225.318 & Surface & 2006 \\
\hline 2178.416 & 229.973 & Surface & 2006 & 7477.002 & 225.322 & Surface & 2006 \\
\hline 2181.386 & 229.955 & Surface & 2006 & 7479.913 & 225.326 & Surface & 2006 \\
\hline 2184.356 & 229.937 & Surface & 2006 & 7482.824 & 225.332 & Surface & 2006 \\
\hline 2187.325 & 229.919 & Surface & 2006 & 7485.735 & 225.303 & Surface & 2006 \\
\hline 2190.295 & 229.902 & Surface & 2006 & 7488.646 & 225.325 & Surface & 2006 \\
\hline 2193.264 & 229.892 & Surface & 2006 & 7491.557 & 225.335 & Surface & 2006 \\
\hline 2196.234 & 229.885 & Surface & 2006 & 7494.468 & 225.342 & Surface & 2006 \\
\hline 2199.204 & 229.879 & Surface & 2006 & 7497.379 & 225.350 & Surface & 2006 \\
\hline 2202.174 & 229.872 & Surface & 2006 & 7500.290 & 225.334 & Surface & 2006 \\
\hline 2205.143 & 229.868 & Surface & 2006 & 7503.201 & 225.320 & Surface & 2006 \\
\hline 2208.113 & 229.862 & Surface & 2006 & 7506.112 & 225.302 & Surface & 2006 \\
\hline 2211.082 & 229.862 & Surface & 2006 & 7509.023 & 225.271 & Surface & 2006 \\
\hline 2214.052 & 229.870 & Surface & 2006 & 7511.934 & 225.310 & Surface & 2006 \\
\hline 2216.975 & 230.105 & Surface & 2006 & 7514.801 & 225.311 & Surface & 2006 \\
\hline 2219.897 & 230.263 & Surface & 2006 & 7517.667 & 225.306 & Surface & 2006 \\
\hline 2222.820 & 230.348 & Surface & 2006 & 7520.534 & 225.309 & Surface & 2006 \\
\hline 2225.742 & 230.361 & Surface & 2006 & 7523.401 & 225.304 & Surface & 2006 \\
\hline 2228.665 & 230.299 & Surface & 2006 & 7526.268 & 225.286 & Surface & 2006 \\
\hline 2231.588 & 230.165 & Surface & 2006 & 7529.134 & 225.295 & Surface & 2006 \\
\hline 2234.510 & 229.957 & Surface & 2006 & 7532.001 & 225.302 & Surface & 2006 \\
\hline 2237.432 & 229.882 & Surface & 2006 & 7534.868 & 225.295 & Surface & 2006 \\
\hline 2240.355 & 229.868 & Surface & 2006 & 7537.735 & 225.272 & Surface & 2006 \\
\hline 2243.278 & 229.864 & Surface & 2006 & 7540.601 & 225.237 & Surface & 2006 \\
\hline 2246.200 & 229.862 & Surface & 2006 & 7543.468 & 225.263 & Surface & 2006 \\
\hline 2249.123 & 229.861 & Surface & 2006 & 7546.335 & 225.265 & Surface & 2006 \\
\hline 2252.045 & 229.861 & Surface & 2006 & 7549.201 & 225.262 & Surface & 2006 \\
\hline 2254.968 & 229.863 & Surface & 2006 & 7552.068 & 225.263 & Surface & 2006 \\
\hline 2257.890 & 229.865 & Surface & 2006 & 7554.935 & 225.238 & Surface & 2006 \\
\hline 2260.813 & 229.866 & Surface & 2006 & 7557.802 & 225.232 & Surface & 2006 \\
\hline 2263.736 & 229.867 & Surface & 2006 & 7560.668 & 225.212 & Surface & 2006 \\
\hline 2266.658 & 229.866 & Surface & 2006 & 7563.535 & 225.164 & Surface & 2006 \\
\hline 2269.580 & 229.865 & Surface & 2006 & 7566.402 & 225.217 & Surface & 2006 \\
\hline 2272.503 & 229.865 & Surface & 2006 & 7569.268 & 225.253 & Surface & 2006 \\
\hline 2275.426 & 229.864 & Surface & 2006 & 7572.253 & 225.238 & Surface & 2006 \\
\hline
\end{tabular}




\begin{tabular}{|c|c|c|c|c|c|c|c|}
\hline 2278.348 & 229.862 & Surface & 2006 & 7575.238 & 225.236 & Surface & 2006 \\
\hline 2281.271 & 229.862 & Surface & 2006 & 7578.223 & 225.204 & Surface & 2006 \\
\hline 2284.193 & 229.861 & Surface & 2006 & 7581.207 & 225.158 & Surface & 2006 \\
\hline 2287.116 & 229.859 & Surface & 2006 & 7584.192 & 225.158 & Surface & 2006 \\
\hline 2290.038 & 229.859 & Surface & 2006 & 7587.177 & 225.191 & Surface & 2006 \\
\hline 2292.959 & 229.859 & Surface & 2006 & 7590.162 & 225.205 & Surface & 2006 \\
\hline 2295.881 & 229.858 & Surface & 2006 & 7593.146 & 225.214 & Surface & 2006 \\
\hline 2298.802 & 229.856 & Surface & 2006 & 7596.131 & 225.201 & Surface & 2006 \\
\hline 2301.724 & 229.856 & Surface & 2006 & 7599.115 & 225.182 & Surface & 2006 \\
\hline 2304.645 & 229.855 & Surface & 2006 & 7602.100 & 225.132 & Surface & 2006 \\
\hline 2307.566 & 229.853 & Surface & 2006 & 7605.085 & 225.141 & Surface & 2006 \\
\hline 2310.488 & 229.852 & Surface & 2006 & 7608.070 & 225.171 & Surface & 2006 \\
\hline 2313.409 & 229.852 & Surface & 2006 & 7611.054 & 225.174 & Surface & 2006 \\
\hline 2316.330 & 229.850 & Surface & 2006 & 7614.039 & 225.185 & Surface & 2006 \\
\hline 2319.251 & 229.850 & Surface & 2006 & 7617.024 & 225.190 & Surface & 2006 \\
\hline 2322.173 & 229.855 & Surface & 2006 & 7620.009 & 225.184 & Surface & 2006 \\
\hline 2325.094 & 229.862 & Surface & 2006 & 7622.993 & 225.177 & Surface & 2006 \\
\hline 2328.016 & 229.859 & Surface & 2006 & 7625.978 & 225.176 & Surface & 2006 \\
\hline 2330.937 & 229.856 & Surface & 2006 & 7628.963 & 225.178 & Surface & 2006 \\
\hline 2333.858 & 229.845 & Surface & 2006 & 7631.947 & 225.179 & Surface & 2006 \\
\hline 2336.780 & 229.839 & Surface & 2006 & 7634.932 & 225.134 & Surface & 2006 \\
\hline 2339.701 & 229.846 & Surface & 2006 & 7637.917 & 225.142 & Surface & 2006 \\
\hline 2342.622 & 229.841 & Surface & 2006 & 7640.901 & 225.145 & Surface & 2006 \\
\hline 2345.543 & 229.838 & Surface & 2006 & 7643.886 & 225.163 & Surface & 2006 \\
\hline 2348.465 & 229.833 & Surface & 2006 & 7646.871 & 225.169 & Surface & 2006 \\
\hline 2351.386 & 229.829 & Surface & 2006 & 7649.856 & 225.159 & Surface & 2006 \\
\hline 2354.308 & 229.836 & Surface & 2006 & 7652.840 & 225.125 & Surface & 2006 \\
\hline 2357.229 & 229.831 & Surface & 2006 & 7655.825 & 225.095 & Surface & 2006 \\
\hline 2360.150 & 229.824 & Surface & 2006 & 7658.810 & 225.075 & Surface & 2006 \\
\hline 2363.072 & 229.816 & Surface & 2006 & 7661.794 & 225.061 & Surface & 2006 \\
\hline 2365.993 & 229.809 & Surface & 2006 & 7664.779 & 225.085 & Surface & 2006 \\
\hline 2368.912 & 229.805 & Surface & 2006 & 7667.768 & 225.125 & Surface & 2006 \\
\hline 2371.832 & 229.802 & Surface & 2006 & 7670.756 & 225.127 & Surface & 2006 \\
\hline 2374.751 & 229.799 & Surface & 2006 & 7673.744 & 225.093 & Surface & 2006 \\
\hline 2377.671 & 229.796 & Surface & 2006 & 7676.732 & 225.086 & Surface & 2006 \\
\hline 2380.590 & 229.793 & Surface & 2006 & 7679.721 & 225.118 & Surface & 2006 \\
\hline 2383.510 & 229.790 & Surface & 2006 & 7682.709 & 225.139 & Surface & 2006 \\
\hline 2386.429 & 229.787 & Surface & 2006 & 7685.697 & 225.161 & Surface & 2006 \\
\hline 2389.348 & 229.784 & Surface & 2006 & 7688.686 & 225.164 & Surface & 2006 \\
\hline 2392.268 & 229.781 & Surface & 2006 & 7691.674 & 225.153 & Surface & 2006 \\
\hline 2395.187 & 229.778 & Surface & 2006 & 7694.663 & 225.088 & Surface & 2006 \\
\hline 2398.107 & 229.775 & Surface & 2006 & 7697.651 & 225.053 & Surface & 2006 \\
\hline 2401.026 & 229.775 & Surface & 2006 & 7700.639 & 225.090 & Surface & 2006 \\
\hline 2403.945 & 229.783 & Surface & 2006 & 7703.628 & 225.115 & Surface & 2006 \\
\hline 2406.865 & 229.788 & Surface & 2006 & 7706.616 & 225.124 & Surface & 2006 \\
\hline 2409.784 & 229.785 & Surface & 2006 & 7709.604 & 225.119 & Surface & 2006 \\
\hline 2412.704 & 229.782 & Surface & 2006 & 7712.593 & 225.092 & Surface & 2006 \\
\hline 2415.623 & 229.777 & Surface & 2006 & 7715.581 & 225.038 & Surface & 2006 \\
\hline 2418.543 & 229.771 & Surface & 2006 & 7718.496 & 225.043 & Surface & 2006 \\
\hline
\end{tabular}




\begin{tabular}{|c|c|c|c|c|c|c|c|}
\hline 2421.462 & 229.757 & Surface & 2006 & 7721.412 & 225.042 & Surface & 2006 \\
\hline 2424.382 & 229.734 & Surface & 2006 & 7724.327 & 225.054 & Surface & 2006 \\
\hline 2427.301 & 229.732 & Surface & 2006 & 7727.242 & 225.095 & Surface & 2006 \\
\hline 2430.220 & 229.728 & Surface & 2006 & 7730.158 & 225.124 & Surface & 2006 \\
\hline 2433.140 & 229.719 & Surface & 2006 & 7733.073 & 225.093 & Surface & 2006 \\
\hline 2436.059 & 229.710 & Surface & 2006 & 7735.989 & 225.097 & Surface & 2006 \\
\hline 2438.979 & 229.704 & Surface & 2006 & 7738.904 & 225.123 & Surface & 2006 \\
\hline 2441.898 & 229.695 & Surface & 2006 & 7741.819 & 225.128 & Surface & 2006 \\
\hline 2444.817 & 229.686 & Surface & 2006 & 7744.734 & 225.127 & Surface & 2006 \\
\hline 2447.737 & 229.685 & Surface & 2006 & 7747.650 & 225.093 & Surface & 2006 \\
\hline 2450.656 & 229.704 & Surface & 2006 & 7750.565 & 225.046 & Surface & 2006 \\
\hline 2453.597 & 229.729 & Surface & 2006 & 7753.481 & 225.066 & Surface & 2006 \\
\hline 2456.537 & 229.738 & Surface & 2006 & 7756.392 & 225.099 & Surface & 2006 \\
\hline 2459.477 & 229.713 & Surface & 2006 & 7759.303 & 225.068 & Surface & 2006 \\
\hline 2462.418 & 229.701 & Surface & 2006 & 7762.214 & 225.024 & Surface & 2006 \\
\hline 2465.358 & 229.706 & Surface & 2006 & 7765.125 & 225.010 & Surface & 2006 \\
\hline 2468.298 & 229.708 & Surface & 2006 & 7768.036 & 225.050 & Surface & 2006 \\
\hline 2471.239 & 229.715 & Surface & 2006 & 7770.947 & 225.110 & Surface & 2006 \\
\hline 2474.179 & 229.715 & Surface & 2006 & 7773.858 & 225.080 & Surface & 2006 \\
\hline 2477.119 & 229.721 & Surface & 2006 & 7776.769 & 225.070 & Surface & 2006 \\
\hline 2480.060 & 229.720 & Surface & 2006 & 7779.680 & 225.109 & Surface & 2006 \\
\hline 2483.000 & 229.713 & Surface & 2006 & 7782.591 & 225.106 & Surface & 2006 \\
\hline 2485.941 & 229.716 & Surface & 2006 & 7785.502 & 225.125 & Surface & 2006 \\
\hline 2488.881 & 229.708 & Surface & 2006 & 7788.413 & 225.147 & Surface & 2006 \\
\hline 2491.821 & 229.700 & Surface & 2006 & 7791.324 & 225.152 & Surface & 2006 \\
\hline 2494.761 & 229.693 & Surface & 2006 & 7794.235 & 225.132 & Surface & 2006 \\
\hline 2497.702 & 229.691 & Surface & 2006 & 7797.146 & 225.110 & Surface & 2006 \\
\hline 2500.642 & 229.689 & Surface & 2006 & 7800.057 & 225.103 & Surface & 2006 \\
\hline 2503.583 & 229.674 & Surface & 2006 & 7802.968 & 225.130 & Surface & 2006 \\
\hline 2506.523 & 229.686 & Surface & 2006 & 7805.937 & 225.134 & Surface & 2006 \\
\hline 2509.463 & 229.704 & Surface & 2006 & 7808.907 & 225.110 & Surface & 2006 \\
\hline 2512.404 & 229.690 & Surface & 2006 & 7811.877 & 225.089 & Surface & 2006 \\
\hline 2515.344 & 229.686 & Surface & 2006 & 7814.846 & 225.105 & Surface & 2006 \\
\hline 2518.284 & 229.672 & Surface & 2006 & 7817.816 & 225.106 & Surface & 2006 \\
\hline 2521.225 & 229.674 & Surface & 2006 & 7820.786 & 225.105 & Surface & 2006 \\
\hline 2524.165 & 229.670 & Surface & 2006 & 7823.755 & 225.089 & Surface & 2006 \\
\hline 2527.105 & 229.657 & Surface & 2006 & 7826.725 & 225.106 & Surface & 2006 \\
\hline 2530.089 & 229.633 & Surface & 2006 & 7829.694 & 225.077 & Surface & 2006 \\
\hline 2533.072 & 229.662 & Surface & 2006 & 7832.664 & 225.083 & Surface & 2006 \\
\hline 2536.055 & 229.646 & Surface & 2006 & 7835.634 & 225.119 & Surface & 2006 \\
\hline 2539.038 & 229.658 & Surface & 2006 & 7838.604 & 225.149 & Surface & 2006 \\
\hline 2542.021 & 229.634 & Surface & 2006 & 7841.573 & 225.080 & Surface & 2006 \\
\hline 2545.004 & 229.640 & Surface & 2006 & 7844.543 & 225.049 & Surface & 2006 \\
\hline 2547.987 & 229.632 & Surface & 2006 & 7847.513 & 225.071 & Surface & 2006 \\
\hline 2550.971 & 229.652 & Surface & 2006 & 7850.482 & 225.099 & Surface & 2006 \\
\hline 2553.954 & 229.642 & Surface & 2006 & 7853.452 & 225.128 & Surface & 2006 \\
\hline 2556.937 & 229.646 & Surface & 2006 & 7856.422 & 225.104 & Surface & 2006 \\
\hline 2559.920 & 229.642 & Surface & 2006 & 7859.391 & 225.083 & Surface & 2006 \\
\hline 2562.903 & 229.610 & Surface & 2006 & 7862.374 & 225.042 & Surface & 2006 \\
\hline
\end{tabular}




\begin{tabular}{|c|c|c|c|c|c|c|c|}
\hline 2565.886 & 229.645 & Surface & 2006 & 7865.356 & 225.089 & Surface & 2006 \\
\hline 2568.869 & 229.623 & Surface & 2006 & 7868.339 & 225.098 & Surface & 2006 \\
\hline 2571.853 & 229.643 & Surface & 2006 & 7871.322 & 225.062 & Surface & 2006 \\
\hline 2574.835 & 229.639 & Surface & 2006 & 7874.304 & 225.005 & Surface & 2006 \\
\hline 2577.819 & 229.639 & Surface & 2006 & 7877.287 & 224.978 & Surface & 2006 \\
\hline 2580.802 & 229.632 & Surface & 2006 & 7880.269 & 224.996 & Surface & 2006 \\
\hline 2583.785 & 229.613 & Surface & 2006 & 7883.252 & 224.994 & Surface & 2006 \\
\hline 2586.768 & 229.608 & Surface & 2006 & 7886.234 & 225.008 & Surface & 2006 \\
\hline 2589.751 & 229.628 & Surface & 2006 & 7889.217 & 225.028 & Surface & 2006 \\
\hline 2592.734 & 229.615 & Surface & 2006 & 7892.200 & 225.010 & Surface & 2006 \\
\hline 2595.717 & 229.616 & Surface & 2006 & 7895.182 & 225.000 & Surface & 2006 \\
\hline 2598.701 & 229.609 & Surface & 2006 & 7898.165 & 225.004 & Surface & 2006 \\
\hline 2601.684 & 229.625 & Surface & 2006 & 7901.148 & 224.999 & Surface & 2006 \\
\hline 2604.595 & 229.616 & Surface & 2006 & 7904.130 & 225.001 & Surface & 2006 \\
\hline 2607.507 & 229.604 & Surface & 2006 & 7906.999 & 225.000 & Surface & 2006 \\
\hline 2610.419 & 229.605 & Surface & 2006 & 7909.867 & 224.973 & Surface & 2006 \\
\hline 2613.330 & 229.603 & Surface & 2006 & 7912.736 & 224.962 & Surface & 2006 \\
\hline 2616.241 & 229.598 & Surface & 2006 & 7915.605 & 224.950 & Surface & 2006 \\
\hline 2619.153 & 229.601 & Surface & 2006 & 7918.474 & 224.944 & Surface & 2006 \\
\hline 2622.065 & 229.603 & Surface & 2006 & 7921.342 & 224.965 & Surface & 2006 \\
\hline 2624.976 & 229.595 & Surface & 2006 & 7924.211 & 224.993 & Surface & 2006 \\
\hline 2627.888 & 229.582 & Surface & 2006 & 7927.080 & 224.986 & Surface & 2006 \\
\hline 2630.799 & 229.571 & Surface & 2006 & 7929.948 & 224.973 & Surface & 2006 \\
\hline 2633.711 & 229.561 & Surface & 2006 & 7932.817 & 224.967 & Surface & 2006 \\
\hline 2636.622 & 229.569 & Surface & 2006 & 7935.685 & 224.957 & Surface & 2006 \\
\hline 2639.534 & 229.569 & Surface & 2006 & 7938.554 & 224.966 & Surface & 2006 \\
\hline 2642.445 & 229.550 & Surface & 2006 & 7941.423 & 224.989 & Surface & 2006 \\
\hline 2645.357 & 229.555 & Surface & 2006 & 7944.292 & 224.963 & Surface & 2006 \\
\hline 2648.269 & 229.557 & Surface & 2006 & 7947.160 & 224.933 & Surface & 2006 \\
\hline 2651.180 & 229.524 & Surface & 2006 & 7950.029 & 224.924 & Surface & 2006 \\
\hline 2654.091 & 229.544 & Surface & 2006 & 7952.898 & 224.907 & Surface & 2006 \\
\hline 2657.003 & 229.536 & Surface & 2006 & 7955.867 & 224.885 & Surface & 2006 \\
\hline 2659.915 & 229.542 & Surface & 2006 & 7958.836 & 224.864 & Surface & 2006 \\
\hline 2662.826 & 229.516 & Surface & 2006 & 7961.805 & 224.865 & Surface & 2006 \\
\hline 2665.738 & 229.503 & Surface & 2006 & 7964.774 & 224.894 & Surface & 2006 \\
\hline 2668.649 & 229.489 & Surface & 2006 & 7967.744 & 224.903 & Surface & 2006 \\
\hline 2671.561 & 229.461 & Surface & 2006 & 7970.713 & 224.904 & Surface & 2006 \\
\hline 2674.472 & 229.444 & Surface & 2006 & 7973.682 & 224.877 & Surface & 2006 \\
\hline 2677.388 & 229.450 & Surface & 2006 & 7976.651 & 224.853 & Surface & 2006 \\
\hline 2680.304 & 229.468 & Surface & 2006 & 7979.620 & 224.855 & Surface & 2006 \\
\hline 2683.220 & 229.469 & Surface & 2006 & 7982.590 & 224.828 & Surface & 2006 \\
\hline 2686.137 & 229.449 & Surface & 2006 & 7985.559 & 224.799 & Surface & 2006 \\
\hline 2689.052 & 229.429 & Surface & 2006 & 7988.528 & 224.812 & Surface & 2006 \\
\hline 2691.969 & 229.422 & Surface & 2006 & 7991.497 & 224.836 & Surface & 2006 \\
\hline 2694.885 & 229.424 & Surface & 2006 & 7994.466 & 224.879 & Surface & 2006 \\
\hline 2697.801 & 229.420 & Surface & 2006 & 7997.436 & 224.879 & Surface & 2006 \\
\hline 2700.717 & 229.402 & Surface & 2006 & 8000.405 & 224.860 & Surface & 2006 \\
\hline 2703.633 & 229.387 & Surface & 2006 & 8003.374 & 224.855 & Surface & 2006 \\
\hline 2706.549 & 229.403 & Surface & 2006 & 8006.343 & 224.857 & Surface & 2006 \\
\hline
\end{tabular}




\begin{tabular}{|c|c|c|c|c|c|c|c|}
\hline 2709.465 & 229.396 & Surface & 2006 & 8009.313 & 224.854 & Surface & 2006 \\
\hline 2712.381 & 229.357 & Surface & 2006 & 8012.282 & 224.804 & Surface & 2006 \\
\hline 2715.297 & 229.356 & Surface & 2006 & 8015.251 & 224.751 & Surface & 2006 \\
\hline 2718.213 & 229.360 & Surface & 2006 & 8018.220 & 224.732 & Surface & 2006 \\
\hline 2721.129 & 229.303 & Surface & 2006 & 8021.189 & 224.737 & Surface & 2006 \\
\hline 2724.045 & 229.283 & Surface & 2006 & 8024.159 & 224.722 & Surface & 2006 \\
\hline 2726.961 & 229.261 & Surface & 2006 & 8027.128 & 224.680 & Surface & 2006 \\
\hline 2729.768 & 229.278 & Surface & 2006 & 8030.080 & 224.681 & Surface & 2006 \\
\hline 2732.576 & 229.274 & Surface & 2006 & 8033.031 & 224.677 & Surface & 2006 \\
\hline 2735.383 & 229.249 & Surface & 2006 & 8035.983 & 224.652 & Surface & 2006 \\
\hline 2738.190 & 229.235 & Surface & 2006 & 8038.935 & 224.615 & Surface & 2006 \\
\hline 2740.998 & 229.219 & Surface & 2006 & 8041.886 & 224.550 & Surface & 2006 \\
\hline 2743.805 & 229.232 & Surface & 2006 & 8044.838 & 224.639 & Surface & 2006 \\
\hline 2746.612 & 229.244 & Surface & 2006 & 8047.790 & 224.550 & Surface & 2006 \\
\hline 2749.420 & 229.229 & Surface & 2006 & 8050.741 & 224.465 & Surface & 2006 \\
\hline 2752.227 & 229.208 & Surface & 2006 & 8053.693 & 224.390 & Surface & 2006 \\
\hline 2755.035 & 229.199 & Surface & 2006 & 8056.645 & 224.390 & Surface & 2006 \\
\hline 2757.842 & 229.192 & Surface & 2006 & 8059.598 & 224.354 & Surface & 2006 \\
\hline 2760.649 & 229.183 & Surface & 2006 & 8062.550 & 224.316 & Surface & 2006 \\
\hline 2763.457 & 229.175 & Surface & 2006 & 8065.503 & 224.302 & Surface & 2006 \\
\hline 2766.264 & 229.193 & Surface & 2006 & 8068.455 & 224.265 & Surface & 2006 \\
\hline 2769.223 & 229.186 & Surface & 2006 & 8071.408 & 224.255 & Surface & 2006 \\
\hline 2772.183 & 229.170 & Surface & 2006 & 8074.361 & 224.269 & Surface & 2006 \\
\hline 2775.142 & 229.122 & Surface & 2006 & 8077.314 & 224.321 & Surface & 2006 \\
\hline 2778.101 & 229.150 & Surface & 2006 & 8080.266 & 224.330 & Surface & 2006 \\
\hline 2781.061 & 229.158 & Surface & 2006 & 8083.219 & 224.298 & Surface & 2006 \\
\hline 2784.020 & 229.169 & Surface & 2006 & 8086.171 & 224.279 & Surface & 2006 \\
\hline 2786.979 & 229.160 & Surface & 2006 & 8089.124 & 224.302 & Surface & 2006 \\
\hline 2789.938 & 229.151 & Surface & 2006 & 8092.077 & 224.332 & Surface & 2006 \\
\hline 2792.898 & 229.147 & Surface & 2006 & 8095.030 & 224.307 & Surface & 2006 \\
\hline 2795.857 & 229.164 & Surface & 2006 & 8097.982 & 224.254 & Surface & 2006 \\
\hline 2798.816 & 229.179 & Surface & 2006 & 8100.935 & 224.274 & Surface & 2006 \\
\hline 2801.676 & 229.164 & Surface & 2006 & 8103.887 & 224.319 & Surface & 2006 \\
\hline 2804.535 & 229.167 & Surface & 2006 & 8106.840 & 224.313 & Surface & 2006 \\
\hline 2807.394 & 229.146 & Surface & 2006 & 8109.793 & 224.284 & Surface & 2006 \\
\hline 2810.254 & 229.119 & Surface & 2006 & 8112.746 & 224.262 & Surface & 2006 \\
\hline 2813.113 & 229.108 & Surface & 2006 & 8115.698 & 224.253 & Surface & 2006 \\
\hline 2815.973 & 229.111 & Surface & 2006 & 8118.651 & 224.270 & Surface & 2006 \\
\hline 2818.832 & 229.096 & Surface & 2006 & 8121.603 & 224.264 & Surface & 2006 \\
\hline 2821.691 & 229.111 & Surface & 2006 & 8124.556 & 224.237 & Surface & 2006 \\
\hline 2824.551 & 229.117 & Surface & 2006 & 8127.509 & 224.247 & Surface & 2006 \\
\hline 2827.410 & 229.074 & Surface & 2006 & 8130.462 & 224.272 & Surface & 2006 \\
\hline 2830.270 & 229.033 & Surface & 2006 & 8133.329 & 224.287 & Surface & 2006 \\
\hline 2833.129 & 229.053 & Surface & 2006 & 8136.196 & 224.295 & Surface & 2006 \\
\hline 2835.907 & 229.052 & Surface & 2006 & 8139.064 & 224.300 & Surface & 2006 \\
\hline 2838.686 & 229.014 & Surface & 2006 & 8141.931 & 224.303 & Surface & 2006 \\
\hline 2841.464 & 228.970 & Surface & 2006 & 8144.799 & 224.289 & Surface & 2006 \\
\hline 2844.242 & 228.940 & Surface & 2006 & 8147.666 & 224.284 & Surface & 2006 \\
\hline 2847.021 & 228.946 & Surface & 2006 & 8150.533 & 224.283 & Surface & 2006 \\
\hline
\end{tabular}




\begin{tabular}{|c|c|c|c|c|c|c|c|}
\hline 2849.799 & 228.941 & Surface & 2006 & 8153.401 & 224.273 & Surface & 2006 \\
\hline 2852.577 & 228.944 & Surface & 2006 & 8156.269 & 224.255 & Surface & 2006 \\
\hline 2855.355 & 228.958 & Surface & 2006 & 8159.136 & 224.251 & Surface & 2006 \\
\hline 2858.134 & 228.931 & Surface & 2006 & 8162.003 & 224.280 & Surface & 2006 \\
\hline 2860.912 & 228.937 & Surface & 2006 & 8164.871 & 224.292 & Surface & 2006 \\
\hline 2863.690 & 228.956 & Surface & 2006 & 8167.738 & 224.285 & Surface & 2006 \\
\hline 2866.469 & 228.959 & Surface & 2006 & 8170.606 & 224.275 & Surface & 2006 \\
\hline 2869.247 & 228.942 & Surface & 2006 & 8173.473 & 224.276 & Surface & 2006 \\
\hline 2872.198 & 228.957 & Surface & 2006 & 8176.340 & 224.291 & Surface & 2006 \\
\hline 2875.149 & 228.949 & Surface & 2006 & 8179.208 & 224.292 & Surface & 2006 \\
\hline 2878.099 & 228.926 & Surface & 2006 & 8182.075 & 224.291 & Surface & 2006 \\
\hline 2881.051 & 228.902 & Surface & 2006 & 8184.942 & 224.285 & Surface & 2006 \\
\hline 2884.001 & 228.904 & Surface & 2006 & 8187.810 & 224.274 & Surface & 2006 \\
\hline 2886.952 & 228.913 & Surface & 2006 & 8190.678 & 224.277 & Surface & 2006 \\
\hline 2889.903 & 228.924 & Surface & 2006 & 8193.545 & 224.293 & Surface & 2006 \\
\hline 2892.854 & 228.934 & Surface & 2006 & 8196.541 & 224.279 & Surface & 2006 \\
\hline 2895.805 & 228.936 & Surface & 2006 & 8199.537 & 224.268 & Surface & 2006 \\
\hline 2898.756 & 228.931 & Surface & 2006 & 8202.534 & 224.282 & Surface & 2006 \\
\hline 2901.706 & 228.933 & Surface & 2006 & 8205.530 & 224.261 & Surface & 2006 \\
\hline 2904.657 & 228.920 & Surface & 2006 & 8208.526 & 224.264 & Surface & 2006 \\
\hline 2907.608 & 228.900 & Surface & 2006 & 8211.522 & 224.284 & Surface & 2006 \\
\hline 2910.559 & 228.913 & Surface & 2006 & 8214.518 & 224.288 & Surface & 2006 \\
\hline 2913.510 & 228.912 & Surface & 2006 & 8217.515 & 224.272 & Surface & 2006 \\
\hline 2916.459 & 228.906 & Surface & 2006 & 8220.511 & 224.277 & Surface & 2006 \\
\hline 2919.408 & 228.917 & Surface & 2006 & 8223.507 & 224.284 & Surface & 2006 \\
\hline 2922.357 & 228.912 & Surface & 2006 & 8226.504 & 224.284 & Surface & 2006 \\
\hline 2925.307 & 228.901 & Surface & 2006 & 8229.500 & 224.284 & Surface & 2006 \\
\hline 2928.256 & 228.917 & Surface & 2006 & 8232.496 & 224.285 & Surface & 2006 \\
\hline 2931.205 & 228.965 & Surface & 2006 & 8235.492 & 224.272 & Surface & 2006 \\
\hline 2934.154 & 228.931 & Surface & 2006 & 8238.489 & 224.235 & Surface & 2006 \\
\hline 2937.104 & 228.889 & Surface & 2006 & 8241.485 & 224.233 & Surface & 2006 \\
\hline 2940.053 & 228.881 & Surface & 2006 & 8244.418 & 224.241 & Surface & 2006 \\
\hline 2943.002 & 228.919 & Surface & 2006 & 8247.351 & 224.247 & Surface & 2006 \\
\hline 2945.951 & 228.912 & Surface & 2006 & 8250.284 & 224.261 & Surface & 2006 \\
\hline 2948.901 & 228.931 & Surface & 2006 & 8253.216 & 224.259 & Surface & 2006 \\
\hline 2951.850 & 228.960 & Surface & 2006 & 8256.149 & 224.240 & Surface & 2006 \\
\hline 2954.799 & 228.936 & Surface & 2006 & 8259.082 & 224.251 & Surface & 2006 \\
\hline 2957.715 & 228.926 & Surface & 2006 & 8262.015 & 224.256 & Surface & 2006 \\
\hline 2960.630 & 228.905 & Surface & 2006 & 8264.947 & 224.232 & Surface & 2006 \\
\hline 2963.546 & 228.883 & Surface & 2006 & 8267.881 & 224.239 & Surface & 2006 \\
\hline 2966.461 & 228.892 & Surface & 2006 & 8270.813 & 224.238 & Surface & 2006 \\
\hline 2969.376 & 228.910 & Surface & 2006 & 8273.746 & 224.228 & Surface & 2006 \\
\hline 2972.292 & 228.890 & Surface & 2006 & 8276.725 & 224.228 & Surface & 2006 \\
\hline 2975.207 & 228.899 & Surface & 2006 & 8279.704 & 224.246 & Surface & 2006 \\
\hline 2978.122 & 228.867 & Surface & 2006 & 8282.683 & 224.235 & Surface & 2006 \\
\hline 2981.038 & 228.867 & Surface & 2006 & 8285.662 & 224.242 & Surface & 2006 \\
\hline 2983.953 & 228.864 & Surface & 2006 & 8288.642 & 224.232 & Surface & 2006 \\
\hline 2986.868 & 228.867 & Surface & 2006 & 8291.620 & 224.236 & Surface & 2006 \\
\hline 2989.784 & 228.891 & Surface & 2006 & 8294.600 & 224.257 & Surface & 2006 \\
\hline
\end{tabular}




\begin{tabular}{|c|c|c|c|c|c|c|c|}
\hline 2992.699 & 228.896 & Surface & 2006 & 8297.579 & 224.260 & Surface & 2006 \\
\hline 2995.632 & 228.898 & Surface & 2006 & 8300.558 & 224.238 & Surface & 2006 \\
\hline 2998.565 & 228.910 & Surface & 2006 & 8303.537 & 224.255 & Surface & 2006 \\
\hline 3001.498 & 228.935 & Surface & 2006 & 8306.516 & 224.278 & Surface & 2006 \\
\hline 3004.431 & 228.918 & Surface & 2006 & 8309.495 & 224.265 & Surface & 2006 \\
\hline 3007.364 & 228.903 & Surface & 2006 & 8312.474 & 224.270 & Surface & 2006 \\
\hline 3010.296 & 228.933 & Surface & 2006 & 8315.453 & 224.277 & Surface & 2006 \\
\hline 3013.229 & 228.921 & Surface & 2006 & 8318.432 & 224.273 & Surface & 2006 \\
\hline 3016.162 & 228.896 & Surface & 2006 & 8321.257 & 224.265 & Surface & 2006 \\
\hline 3019.095 & 228.901 & Surface & 2006 & 8324.082 & 224.261 & Surface & 2006 \\
\hline 3022.028 & 228.893 & Surface & 2006 & 8326.907 & 224.253 & Surface & 2006 \\
\hline 3024.961 & 228.889 & Surface & 2006 & 8329.732 & 224.264 & Surface & 2006 \\
\hline 3027.928 & 228.893 & Surface & 2006 & 8332.557 & 224.257 & Surface & 2006 \\
\hline 3030.895 & 228.893 & Surface & 2006 & 8335.381 & 224.257 & Surface & 2006 \\
\hline 3033.863 & 228.893 & Surface & 2006 & 8338.206 & 224.265 & Surface & 2006 \\
\hline 3036.831 & 228.905 & Surface & 2006 & 8341.031 & 224.270 & Surface & 2006 \\
\hline 3039.798 & 228.926 & Surface & 2006 & 8343.856 & 224.274 & Surface & 2006 \\
\hline 3042.765 & 228.917 & Surface & 2006 & 8346.681 & 224.264 & Surface & 2006 \\
\hline 3045.733 & 228.887 & Surface & 2006 & 8349.506 & 224.254 & Surface & 2006 \\
\hline 3048.701 & 228.871 & Surface & 2006 & 8352.331 & 224.244 & Surface & 2006 \\
\hline 3051.668 & 228.952 & Surface & 2006 & 8355.308 & 224.236 & Surface & 2006 \\
\hline 3054.635 & 228.971 & Surface & 2006 & 8358.286 & 224.229 & Surface & 2006 \\
\hline 3057.603 & 228.981 & Surface & 2006 & 8361.263 & 224.237 & Surface & 2006 \\
\hline 3060.570 & 228.967 & Surface & 2006 & 8364.241 & 224.245 & Surface & 2006 \\
\hline 3063.538 & 228.951 & Surface & 2006 & 8367.218 & 224.262 & Surface & 2006 \\
\hline 3066.505 & 228.935 & Surface & 2006 & 8370.195 & 224.272 & Surface & 2006 \\
\hline 3069.473 & 228.920 & Surface & 2006 & 8373.173 & 224.275 & Surface & 2006 \\
\hline 3072.440 & 228.912 & Surface & 2006 & 8376.150 & 224.277 & Surface & 2006 \\
\hline 3075.408 & 228.900 & Surface & 2006 & 8379.128 & 224.277 & Surface & 2006 \\
\hline 3078.375 & 228.894 & Surface & 2006 & 8382.105 & 224.273 & Surface & 2006 \\
\hline 3081.343 & 228.891 & Surface & 2006 & 8385.082 & 224.263 & Surface & 2006 \\
\hline 3084.310 & 228.886 & Surface & 2006 & 8388.060 & 224.252 & Surface & 2006 \\
\hline 3087.278 & 228.882 & Surface & 2006 & 8391.037 & 224.242 & Surface & 2006 \\
\hline 3090.245 & 228.879 & Surface & 2006 & 8394.015 & 224.230 & Surface & 2006 \\
\hline 3093.197 & 228.875 & Surface & 2006 & 8396.992 & 224.219 & Surface & 2006 \\
\hline 3096.149 & 228.870 & Surface & 2006 & 8399.969 & 224.216 & Surface & 2006 \\
\hline 3099.101 & 228.868 & Surface & 2006 & 8402.947 & 224.213 & Surface & 2006 \\
\hline 3102.053 & 228.865 & Surface & 2006 & 8405.820 & 224.209 & Surface & 2006 \\
\hline 3105.005 & 228.863 & Surface & 2006 & 8408.693 & 224.208 & Surface & 2006 \\
\hline 3107.957 & 228.892 & Surface & 2006 & 8411.566 & 224.215 & Surface & 2006 \\
\hline 3110.909 & 228.922 & Surface & 2006 & 8414.438 & 224.199 & Surface & 2006 \\
\hline 3113.861 & 228.943 & Surface & 2006 & 8417.311 & 224.172 & Surface & 2006 \\
\hline 3116.813 & 229.020 & Surface & 2006 & 8420.184 & 224.175 & Surface & 2006 \\
\hline 3119.764 & 229.009 & Surface & 2006 & 8423.057 & 224.189 & Surface & 2006 \\
\hline 3122.717 & 228.959 & Surface & 2006 & 8425.930 & 224.184 & Surface & 2006 \\
\hline 3125.668 & 228.923 & Surface & 2006 & 8428.803 & 224.183 & Surface & 2006 \\
\hline 3128.620 & 228.910 & Surface & 2006 & 8431.675 & 224.178 & Surface & 2006 \\
\hline 3131.572 & 228.928 & Surface & 2006 & 8434.548 & 224.189 & Surface & 2006 \\
\hline 3134.524 & 228.945 & Surface & 2006 & 8437.421 & 224.191 & Surface & 2006 \\
\hline
\end{tabular}




\begin{tabular}{|c|c|c|c|c|c|c|c|}
\hline 3137.476 & 228.942 & Surface & 2006 & 8440.294 & 224.182 & Surface & 2006 \\
\hline 3140.428 & 228.894 & Surface & 2006 & 8443.167 & 224.171 & Surface & 2006 \\
\hline 3143.380 & 228.921 & Surface & 2006 & 8446.040 & 224.167 & Surface & 2006 \\
\hline 3146.332 & 228.944 & Surface & 2006 & 8448.913 & 224.167 & Surface & 2006 \\
\hline 3149.283 & 228.940 & Surface & 2006 & 8451.786 & 224.156 & Surface & 2006 \\
\hline 3152.236 & 228.890 & Surface & 2006 & 8454.659 & 224.140 & Surface & 2006 \\
\hline 3155.187 & 228.897 & Surface & 2006 & 8457.531 & 224.156 & Surface & 2006 \\
\hline 3158.139 & 228.908 & Surface & 2006 & 8460.404 & 224.179 & Surface & 2006 \\
\hline 3161.091 & 228.913 & Surface & 2006 & 8463.229 & 224.191 & Surface & 2006 \\
\hline 3164.043 & 228.907 & Surface & 2006 & 8466.054 & 224.161 & Surface & 2006 \\
\hline 3166.995 & 228.914 & Surface & 2006 & 8468.879 & 224.157 & Surface & 2006 \\
\hline 3169.947 & 228.952 & Surface & 2006 & 8471.703 & 224.117 & Surface & 2006 \\
\hline 3172.899 & 228.945 & Surface & 2006 & 8474.528 & 224.140 & Surface & 2006 \\
\hline 3175.851 & 228.896 & Surface & 2006 & 8477.352 & 224.165 & Surface & 2006 \\
\hline 3178.803 & 228.881 & Surface & 2006 & 8480.177 & 224.166 & Surface & 2006 \\
\hline 3181.755 & 228.901 & Surface & 2006 & 8483.002 & 224.157 & Surface & 2006 \\
\hline 3184.707 & 228.933 & Surface & 2006 & 8485.827 & 224.134 & Surface & 2006 \\
\hline 3187.659 & 228.945 & Surface & 2006 & 8488.651 & 224.124 & Surface & 2006 \\
\hline 3190.610 & 228.929 & Surface & 2006 & 8491.476 & 224.138 & Surface & 2006 \\
\hline 3193.562 & 228.931 & Surface & 2006 & 8494.409 & 224.157 & Surface & 2006 \\
\hline 3196.514 & 228.934 & Surface & 2006 & 8497.343 & 224.151 & Surface & 2006 \\
\hline 3199.466 & 228.926 & Surface & 2006 & 8500.276 & 224.136 & Surface & 2006 \\
\hline 3202.428 & 228.922 & Surface & 2006 & 8503.210 & 224.139 & Surface & 2006 \\
\hline 3205.389 & 228.922 & Surface & 2006 & 8506.143 & 224.141 & Surface & 2006 \\
\hline 3208.350 & 228.912 & Surface & 2006 & 8509.077 & 224.141 & Surface & 2006 \\
\hline 3211.311 & 228.919 & Surface & 2006 & 8512.010 & 224.157 & Surface & 2006 \\
\hline 3214.273 & 228.935 & Surface & 2006 & 8514.944 & 224.139 & Surface & 2006 \\
\hline 3217.234 & 228.945 & Surface & 2006 & 8517.877 & 224.148 & Surface & 2006 \\
\hline 3220.195 & 228.944 & Surface & 2006 & 8520.810 & 224.143 & Surface & 2006 \\
\hline 3223.156 & 228.923 & Surface & 2006 & 8523.744 & 224.139 & Surface & 2006 \\
\hline 3226.118 & 228.912 & Surface & 2006 & 8526.677 & 224.131 & Surface & 2006 \\
\hline 3229.079 & 228.906 & Surface & 2006 & 8529.611 & 224.130 & Surface & 2006 \\
\hline 3232.040 & 228.906 & Surface & 2006 & 8532.544 & 224.130 & Surface & 2006 \\
\hline 3235.002 & 228.913 & Surface & 2006 & 8535.477 & 224.143 & Surface & 2006 \\
\hline 3237.963 & 228.900 & Surface & 2006 & 8538.411 & 224.151 & Surface & 2006 \\
\hline 3240.924 & 228.895 & Surface & 2006 & 8541.344 & 224.136 & Surface & 2006 \\
\hline 3243.885 & 228.910 & Surface & 2006 & 8544.278 & 224.144 & Surface & 2006 \\
\hline 3246.847 & 228.899 & Surface & 2006 & 8547.226 & 224.174 & Surface & 2006 \\
\hline 3249.808 & 228.887 & Surface & 2006 & 8550.173 & 224.189 & Surface & 2006 \\
\hline 3252.769 & 228.874 & Surface & 2006 & 8553.121 & 224.186 & Surface & 2006 \\
\hline 3255.730 & 228.859 & Surface & 2006 & 8556.069 & 224.187 & Surface & 2006 \\
\hline 3258.692 & 228.867 & Surface & 2006 & 8559.016 & 224.192 & Surface & 2006 \\
\hline 3261.653 & 228.887 & Surface & 2006 & 8561.964 & 224.194 & Surface & 2006 \\
\hline 3264.614 & 228.882 & Surface & 2006 & 8564.912 & 224.195 & Surface & 2006 \\
\hline 3267.575 & 228.868 & Surface & 2006 & 8567.860 & 224.192 & Surface & 2006 \\
\hline 3270.454 & 228.867 & Surface & 2006 & 8570.807 & 224.173 & Surface & 2006 \\
\hline 3273.333 & 228.865 & Surface & 2006 & 8573.755 & 224.154 & Surface & 2006 \\
\hline 3276.212 & 228.871 & Surface & 2006 & 8576.703 & 224.152 & Surface & 2006 \\
\hline 3279.090 & 228.869 & Surface & 2006 & 8579.650 & 224.141 & Surface & 2006 \\
\hline
\end{tabular}




\begin{tabular}{|c|c|c|c|c|c|c|c|}
\hline 3281.969 & 228.868 & Surface & 2006 & 8582.598 & 224.141 & Surface & 2006 \\
\hline 3284.847 & 228.866 & Surface & 2006 & 8585.546 & 224.146 & Surface & 2006 \\
\hline 3287.726 & 228.856 & Surface & 2006 & 8588.493 & 224.145 & Surface & 2006 \\
\hline 3290.605 & 228.821 & Surface & 2006 & 8591.441 & 224.180 & Surface & 2006 \\
\hline 3293.483 & 228.856 & Surface & 2006 & 8594.389 & 224.192 & Surface & 2006 \\
\hline 3296.362 & 228.883 & Surface & 2006 & 8597.337 & 224.171 & Surface & 2006 \\
\hline 3299.241 & 228.871 & Surface & 2006 & 8600.284 & 224.155 & Surface & 2006 \\
\hline 3302.120 & 228.845 & Surface & 2006 & 8603.232 & 224.144 & Surface & 2006 \\
\hline 3304.998 & 228.851 & Surface & 2006 & 8606.105 & 224.161 & Surface & 2006 \\
\hline 3307.877 & 228.852 & Surface & 2006 & 8608.977 & 224.181 & Surface & 2006 \\
\hline 3310.755 & 228.844 & Surface & 2006 & 8611.850 & 224.182 & Surface & 2006 \\
\hline 3313.634 & 228.847 & Surface & 2006 & 8614.722 & 224.181 & Surface & 2006 \\
\hline 3316.513 & 228.849 & Surface & 2006 & 8617.595 & 224.180 & Surface & 2006 \\
\hline 3319.391 & 228.846 & Surface & 2006 & 8620.467 & 224.177 & Surface & 2006 \\
\hline 3322.270 & 228.841 & Surface & 2006 & 8623.340 & 224.178 & Surface & 2006 \\
\hline 3325.149 & 228.842 & Surface & 2006 & 8626.212 & 224.181 & Surface & 2006 \\
\hline 3328.074 & 228.836 & Surface & 2006 & 8629.085 & 224.184 & Surface & 2006 \\
\hline 3331.001 & 228.826 & Surface & 2006 & 8631.957 & 224.190 & Surface & 2006 \\
\hline 3333.926 & 228.828 & Surface & 2006 & 8634.830 & 224.202 & Surface & 2006 \\
\hline 3336.852 & 228.839 & Surface & 2006 & 8637.584 & 224.205 & Surface & 2006 \\
\hline 3339.778 & 228.854 & Surface & 2006 & 8640.339 & 224.204 & Surface & 2006 \\
\hline 3342.704 & 228.861 & Surface & 2006 & 8643.093 & 224.202 & Surface & 2006 \\
\hline 3345.630 & 228.855 & Surface & 2006 & 8645.848 & 224.200 & Surface & 2006 \\
\hline 3348.556 & 228.846 & Surface & 2006 & 8648.603 & 224.197 & Surface & 2006 \\
\hline 3351.482 & 228.848 & Surface & 2006 & 8651.357 & 224.194 & Surface & 2006 \\
\hline 3354.408 & 228.845 & Surface & 2006 & 8654.112 & 224.192 & Surface & 2006 \\
\hline 3357.334 & 228.845 & Surface & 2006 & 8656.866 & 224.190 & Surface & 2006 \\
\hline 3360.260 & 228.829 & Surface & 2006 & 8659.621 & 224.190 & Surface & 2006 \\
\hline 3363.185 & 228.804 & Surface & 2006 & 8662.375 & 224.187 & Surface & 2006 \\
\hline 3366.111 & 228.812 & Surface & 2006 & 8665.130 & 224.182 & Surface & 2006 \\
\hline 3369.037 & 228.796 & Surface & 2006 & 8667.884 & 224.178 & Surface & 2006 \\
\hline 3371.976 & 228.801 & Surface & 2006 & 8670.707 & 224.176 & Surface & 2006 \\
\hline 3374.915 & 228.778 & Surface & 2006 & 8673.529 & 224.177 & Surface & 2006 \\
\hline 3377.853 & 228.771 & Surface & 2006 & 8676.351 & 224.177 & Surface & 2006 \\
\hline 3380.792 & 228.816 & Surface & 2006 & 8679.174 & 224.178 & Surface & 2006 \\
\hline 3383.731 & 228.803 & Surface & 2006 & 8681.996 & 224.177 & Surface & 2006 \\
\hline 3386.670 & 228.801 & Surface & 2006 & 8684.819 & 224.178 & Surface & 2006 \\
\hline 3389.609 & 228.788 & Surface & 2006 & 8687.641 & 224.180 & Surface & 2006 \\
\hline 3392.548 & 228.793 & Surface & 2006 & 8690.463 & 224.180 & Surface & 2006 \\
\hline 3395.486 & 228.801 & Surface & 2006 & 8693.285 & 224.180 & Surface & 2006 \\
\hline 3398.425 & 228.797 & Surface & 2006 & 8696.108 & 224.183 & Surface & 2006 \\
\hline 3401.364 & 228.798 & Surface & 2006 & 8698.930 & 224.189 & Surface & 2006 \\
\hline 3404.303 & 228.814 & Surface & 2006 & 8701.753 & 224.197 & Surface & 2006 \\
\hline 3407.241 & 228.794 & Surface & 2006 & 8704.575 & 224.204 & Surface & 2006 \\
\hline 3410.180 & 228.774 & Surface & 2006 & 8707.397 & 224.206 & Surface & 2006 \\
\hline 3413.119 & 228.740 & Surface & 2006 & 8710.220 & 224.199 & Surface & 2006 \\
\hline 3416.058 & 228.712 & Surface & 2006 & 8713.042 & 224.198 & Surface & 2006 \\
\hline 3418.997 & 228.702 & Surface & 2006 & 8715.958 & 224.197 & Surface & 2006 \\
\hline 3421.935 & 228.707 & Surface & 2006 & 8718.874 & 224.196 & Surface & 2006 \\
\hline
\end{tabular}




\begin{tabular}{|c|c|c|c|c|c|c|c|}
\hline 3424.874 & 228.705 & Surface & 2006 & 8721.790 & 224.195 & Surface & 2006 \\
\hline 3427.813 & 228.707 & Surface & 2006 & 8724.706 & 224.194 & Surface & 2006 \\
\hline 3430.752 & 228.709 & Surface & 2006 & 8727.622 & 224.193 & Surface & 2006 \\
\hline 3433.691 & 228.700 & Surface & 2006 & 8730.538 & 224.192 & Surface & 2006 \\
\hline 3436.629 & 228.678 & Surface & 2006 & 8733.454 & 224.191 & Surface & 2006 \\
\hline 3439.568 & 228.665 & Surface & 2006 & 8736.370 & 224.190 & Surface & 2006 \\
\hline 3442.507 & 228.652 & Surface & 2006 & 8739.286 & 224.189 & Surface & 2006 \\
\hline 3445.446 & 228.649 & Surface & 2006 & 8742.202 & 224.187 & Surface & 2006 \\
\hline 3448.384 & 228.643 & Surface & 2006 & 8745.118 & 224.186 & Surface & 2006 \\
\hline 3451.323 & 228.630 & Surface & 2006 & 8748.034 & 224.185 & Surface & 2006 \\
\hline 3454.262 & 228.637 & Surface & 2006 & 8750.954 & 224.185 & Surface & 2006 \\
\hline 3457.201 & 228.637 & Surface & 2006 & 8753.873 & 224.185 & Surface & 2006 \\
\hline 3460.140 & 228.628 & Surface & 2006 & 8756.793 & 224.185 & Surface & 2006 \\
\hline 3463.079 & 228.627 & Surface & 2006 & 8759.713 & 224.184 & Surface & 2006 \\
\hline 3466.017 & 228.610 & Surface & 2006 & 8762.633 & 224.184 & Surface & 2006 \\
\hline 3468.956 & 228.598 & Surface & 2006 & 8765.552 & 224.184 & Surface & 2006 \\
\hline 3471.895 & 228.601 & Surface & 2006 & 8768.472 & 224.184 & Surface & 2006 \\
\hline 3474.834 & 228.606 & Surface & 2006 & 8771.391 & 224.183 & Surface & 2006 \\
\hline 3477.773 & 228.608 & Surface & 2006 & 8774.311 & 224.183 & Surface & 2006 \\
\hline 3480.711 & 228.612 & Surface & 2006 & 8777.231 & 224.183 & Surface & 2006 \\
\hline 3483.650 & 228.605 & Surface & 2006 & 8779.985 & 224.182 & Surface & 2006 \\
\hline 3486.589 & 228.587 & Surface & 2006 & 8782.740 & 224.180 & Surface & 2006 \\
\hline 3489.528 & 228.569 & Surface & 2006 & 8785.494 & 224.179 & Surface & 2006 \\
\hline 3492.466 & 228.559 & Surface & 2006 & 8788.249 & 224.177 & Surface & 2006 \\
\hline 3495.405 & 228.581 & Surface & 2006 & 8791.003 & 224.176 & Surface & 2006 \\
\hline 3498.344 & 228.575 & Surface & 2006 & 8793.758 & 224.174 & Surface & 2006 \\
\hline 3501.283 & 228.584 & Surface & 2006 & 8796.512 & 224.173 & Surface & 2006 \\
\hline 3504.222 & 228.563 & Surface & 2006 & 8799.267 & 224.171 & Surface & 2006 \\
\hline 3507.160 & 228.539 & Surface & 2006 & 8802.021 & 224.170 & Surface & 2006 \\
\hline 3510.099 & 228.536 & Surface & 2006 & 8804.776 & 224.171 & Surface & 2006 \\
\hline 3513.038 & 228.527 & Surface & 2006 & 8807.531 & 224.173 & Surface & 2006 \\
\hline 3515.977 & 228.507 & Surface & 2006 & 8810.285 & 224.179 & Surface & 2006 \\
\hline 3518.916 & 228.512 & Surface & 2006 & 8812.981 & 224.183 & Surface & 2006 \\
\hline 3521.855 & 228.480 & Surface & 2006 & 8815.677 & 224.181 & Surface & 2006 \\
\hline 3524.801 & 228.434 & Surface & 2006 & 8818.373 & 224.180 & Surface & 2006 \\
\hline 3527.747 & 228.393 & Surface & 2006 & 8821.069 & 224.183 & Surface & 2006 \\
\hline 3530.694 & 228.322 & Surface & 2006 & 8823.765 & 224.184 & Surface & 2006 \\
\hline 3533.640 & 228.223 & Surface & 2006 & 8826.460 & 224.185 & Surface & 2006 \\
\hline 3536.586 & 228.126 & Surface & 2006 & 8829.156 & 224.187 & Surface & 2006 \\
\hline 3539.533 & 228.068 & Surface & 2006 & 8831.852 & 224.189 & Surface & 2006 \\
\hline 3542.479 & 228.075 & Surface & 2006 & 8834.548 & 224.190 & Surface & 2006 \\
\hline 3545.425 & 228.097 & Surface & 2006 & 8837.403 & 224.191 & Surface & 2006 \\
\hline 3548.372 & 228.063 & Surface & 2006 & 8840.257 & 224.193 & Surface & 2006 \\
\hline 3551.318 & 228.025 & Surface & 2006 & 8843.111 & 224.190 & Surface & 2006 \\
\hline 3554.264 & 228.003 & Surface & 2006 & 8845.966 & 224.188 & Surface & 2006 \\
\hline 3557.211 & 227.997 & Surface & 2006 & 8848.820 & 224.186 & Surface & 2006 \\
\hline 3560.157 & 227.967 & Surface & 2006 & 8851.674 & 224.184 & Surface & 2006 \\
\hline 3563.104 & 227.935 & Surface & 2006 & 8854.529 & 224.173 & Surface & 2006 \\
\hline 3566.050 & 227.885 & Surface & 2006 & 8857.383 & 224.165 & Surface & 2006 \\
\hline
\end{tabular}




\begin{tabular}{|c|c|c|c|c|c|c|c|}
\hline 3568.996 & 227.890 & Surface & 2006 & 8860.237 & 224.158 & Surface & 2006 \\
\hline 3571.943 & 227.898 & Surface & 2006 & 8863.092 & 224.153 & Surface & 2006 \\
\hline 3574.889 & 227.850 & Surface & 2006 & 8866.064 & 224.147 & Surface & 2006 \\
\hline 3577.835 & 227.832 & Surface & 2006 & 8869.035 & 224.141 & Surface & 2006 \\
\hline 3580.782 & 227.843 & Surface & 2006 & 8872.007 & 224.135 & Surface & 2006 \\
\hline 3583.728 & 227.829 & Surface & 2006 & 8874.979 & 224.129 & Surface & 2006 \\
\hline 3586.674 & 227.816 & Surface & 2006 & 8877.951 & 224.123 & Surface & 2006 \\
\hline 3589.657 & 227.791 & Surface & 2006 & 8880.923 & 224.117 & Surface & 2006 \\
\hline 3592.640 & 227.768 & Surface & 2006 & 8883.895 & 224.110 & Surface & 2006 \\
\hline 3595.622 & 227.755 & Surface & 2006 & 8886.866 & 224.104 & Surface & 2006 \\
\hline 3598.605 & 227.744 & Surface & 2006 & 8889.838 & 224.100 & Surface & 2006 \\
\hline 3601.587 & 227.743 & Surface & 2006 & 8892.810 & 224.095 & Surface & 2006 \\
\hline 3604.570 & 227.729 & Surface & 2006 & 8895.782 & 224.092 & Surface & 2006 \\
\hline 3607.552 & 227.729 & Surface & 2006 & 8898.754 & 224.093 & Surface & 2006 \\
\hline 3610.535 & 227.733 & Surface & 2006 & 8901.726 & 224.096 & Surface & 2006 \\
\hline 3613.518 & 227.755 & Surface & 2006 & 8904.698 & 224.101 & Surface & 2006 \\
\hline 3616.500 & 227.758 & Surface & 2006 & 8907.670 & 224.109 & Surface & 2006 \\
\hline 3619.483 & 227.737 & Surface & 2006 & 8910.641 & 224.107 & Surface & 2006 \\
\hline 3622.465 & 227.716 & Surface & 2006 & 8913.613 & 224.102 & Surface & 2006 \\
\hline 3625.378 & 227.711 & Surface & 2006 & 8916.585 & 224.106 & Surface & 2006 \\
\hline 3628.292 & 227.708 & Surface & 2006 & 8919.557 & 224.111 & Surface & 2006 \\
\hline 3631.205 & 227.708 & Surface & 2006 & 8922.452 & 224.114 & Surface & 2006 \\
\hline 3634.119 & 227.701 & Surface & 2006 & 8925.348 & 224.114 & Surface & 2006 \\
\hline 3637.032 & 227.695 & Surface & 2006 & 8928.243 & 224.113 & Surface & 2006 \\
\hline 3639.946 & 227.701 & Surface & 2006 & 8931.138 & 224.110 & Surface & 2006 \\
\hline 3642.859 & 227.704 & Surface & 2006 & 8934.034 & 224.109 & Surface & 2006 \\
\hline 3645.773 & 227.674 & Surface & 2006 & 8936.929 & 224.111 & Surface & 2006 \\
\hline 3648.686 & 227.674 & Surface & 2006 & 8939.824 & 224.162 & Surface & 2006 \\
\hline 3651.600 & 227.675 & Surface & 2006 & 8942.720 & 224.174 & Surface & 2006 \\
\hline 3654.513 & 227.690 & Surface & 2006 & 8945.615 & 224.173 & Surface & 2006 \\
\hline 3657.427 & 227.703 & Surface & 2006 & 8948.510 & 224.172 & Surface & 2006 \\
\hline 3660.340 & 227.701 & Surface & 2006 & 8951.406 & 224.174 & Surface & 2006 \\
\hline 3663.254 & 227.690 & Surface & 2006 & 8954.301 & 224.174 & Surface & 2006 \\
\hline 3666.167 & 227.705 & Surface & 2006 & 8957.196 & 224.174 & Surface & 2006 \\
\hline 3669.081 & 227.716 & Surface & 2006 & 8960.092 & 224.174 & Surface & 2006 \\
\hline 3671.994 & 227.707 & Surface & 2006 & 8962.987 & 224.174 & Surface & 2006 \\
\hline 3674.908 & 227.678 & Surface & 2006 & 8965.882 & 224.174 & Surface & 2006 \\
\hline 3677.821 & 227.722 & Surface & 2006 & 8968.778 & 224.174 & Surface & 2006 \\
\hline 3680.734 & 227.707 & Surface & 2006 & 8971.673 & 224.174 & Surface & 2006 \\
\hline 3683.648 & 227.694 & Surface & 2006 & 8974.568 & 224.175 & Surface & 2006 \\
\hline 3686.561 & 227.693 & Surface & 2006 & 8977.464 & 224.177 & Surface & 2006 \\
\hline 3689.475 & 227.676 & Surface & 2006 & 8980.357 & 224.177 & Surface & 2006 \\
\hline 3692.388 & 227.677 & Surface & 2006 & 8983.251 & 224.175 & Surface & 2006 \\
\hline 3695.302 & 227.686 & Surface & 2006 & 8986.144 & 224.175 & Surface & 2006 \\
\hline 3698.215 & 227.689 & Surface & 2006 & 8989.038 & 224.172 & Surface & 2006 \\
\hline 3701.129 & 227.668 & Surface & 2006 & 8991.931 & 224.172 & Surface & 2006 \\
\hline 3704.105 & 227.681 & Surface & 2006 & 8994.825 & 224.169 & Surface & 2006 \\
\hline 3707.082 & 227.692 & Surface & 2006 & 8997.718 & 224.169 & Surface & 2006 \\
\hline 3710.058 & 227.675 & Surface & 2006 & 9000.612 & 224.166 & Surface & 2006 \\
\hline
\end{tabular}




\begin{tabular}{|c|c|c|c|c|c|c|c|}
\hline 3713.035 & 227.668 & Surface & 2006 & 9003.505 & 224.165 & Surface & 2006 \\
\hline 3716.012 & 227.676 & Surface & 2006 & 9006.504 & 224.163 & Surface & 2006 \\
\hline 3718.988 & 227.694 & Surface & 2006 & 9009.503 & 224.162 & Surface & 2006 \\
\hline 3721.965 & 227.727 & Surface & 2006 & 9012.501 & 224.160 & Surface & 2006 \\
\hline 3724.941 & 227.709 & Surface & 2006 & 9015.500 & 224.159 & Surface & 2006 \\
\hline 3727.918 & 227.694 & Surface & 2006 & 9018.499 & 224.158 & Surface & 2006 \\
\hline 3730.895 & 227.682 & Surface & 2006 & 9021.497 & 224.156 & Surface & 2006 \\
\hline 3733.871 & 227.678 & Surface & 2006 & 9024.496 & 224.155 & Surface & 2006 \\
\hline 3736.848 & 227.689 & Surface & 2006 & 9027.494 & 224.153 & Surface & 2006 \\
\hline 3739.825 & 227.685 & Surface & 2006 & 9030.493 & 224.152 & Surface & 2006 \\
\hline 3742.801 & 227.676 & Surface & 2006 & 9033.492 & 224.150 & Surface & 2006 \\
\hline 3745.778 & 227.666 & Surface & 2006 & 9036.491 & 224.149 & Surface & 2006 \\
\hline 3748.754 & 227.620 & Surface & 2006 & 9039.489 & 224.147 & Surface & 2006 \\
\hline 3751.731 & 227.597 & Surface & 2006 & 9042.488 & 224.146 & Surface & 2006 \\
\hline 3754.708 & 227.577 & Surface & 2006 & 9045.486 & 224.143 & Surface & 2006 \\
\hline 3757.684 & 227.601 & Surface & 2006 & 9048.485 & 224.143 & Surface & 2006 \\
\hline 3760.661 & 227.636 & Surface & 2006 & 9051.484 & 224.141 & Surface & 2006 \\
\hline 3763.638 & 227.651 & Surface & 2006 & 9054.482 & 224.140 & Surface & 2006 \\
\hline 3766.614 & 227.674 & Surface & 2006 & 9057.481 & 224.138 & Surface & 2006 \\
\hline 3769.591 & 227.689 & Surface & 2006 & 9060.480 & 224.148 & Surface & 2006 \\
\hline 3772.544 & 227.688 & Surface & 2006 & 9063.478 & 224.167 & Surface & 2006 \\
\hline 3775.498 & 227.667 & Surface & 2006 & 9066.477 & 224.186 & Surface & 2006 \\
\hline 3778.451 & 227.663 & Surface & 2006 & 9069.429 & 224.213 & Surface & 2006 \\
\hline 3781.404 & 227.684 & Surface & 2006 & 9072.381 & 224.246 & Surface & 2006 \\
\hline 3784.358 & 227.690 & Surface & 2006 & 9075.333 & 224.276 & Surface & 2006 \\
\hline 3787.311 & 227.706 & Surface & 2006 & 9078.284 & 224.291 & Surface & 2006 \\
\hline 3790.264 & 227.705 & Surface & 2006 & 9081.236 & 224.301 & Surface & 2006 \\
\hline 3793.218 & 227.691 & Surface & 2006 & 9084.188 & 224.314 & Surface & 2006 \\
\hline 3796.171 & 227.674 & Surface & 2006 & 9087.140 & 224.337 & Surface & 2006 \\
\hline 3799.125 & 227.690 & Surface & 2006 & 9090.092 & 224.368 & Surface & 2006 \\
\hline 3802.078 & 227.702 & Surface & 2006 & 9093.044 & 224.365 & Surface & 2006 \\
\hline 3805.031 & 227.684 & Surface & 2006 & 9095.996 & 224.359 & Surface & 2006 \\
\hline 3807.985 & 227.693 & Surface & 2006 & 9098.948 & 224.353 & Surface & 2006 \\
\hline 3810.983 & 227.686 & Surface & 2006 & 9101.900 & 224.344 & Surface & 2006 \\
\hline 3813.981 & 227.697 & Surface & 2006 & 9104.852 & 224.338 & Surface & 2006 \\
\hline 3816.979 & 227.710 & Surface & 2006 & 9107.804 & 224.332 & Surface & 2006 \\
\hline 3819.977 & 227.711 & Surface & 2006 & 9110.755 & 224.325 & Surface & 2006 \\
\hline 3822.975 & 227.713 & Surface & 2006 & 9113.708 & 224.317 & Surface & 2006 \\
\hline 3825.973 & 227.719 & Surface & 2006 & 9116.659 & 224.311 & Surface & 2006 \\
\hline 3828.971 & 227.717 & Surface & 2006 & 9119.611 & 224.305 & Surface & 2006 \\
\hline 3831.969 & 227.708 & Surface & 2006 & 9122.563 & 224.298 & Surface & 2006 \\
\hline 3834.967 & 227.695 & Surface & 2006 & 9125.515 & 224.289 & Surface & 2006 \\
\hline 3837.965 & 227.702 & Surface & 2006 & 9128.467 & 224.284 & Surface & 2006 \\
\hline 3840.963 & 227.696 & Surface & 2006 & 9131.419 & 224.277 & Surface & 2006 \\
\hline 3843.962 & 227.706 & Surface & 2006 & 9134.371 & 224.272 & Surface & 2006 \\
\hline 3846.960 & 227.696 & Surface & 2006 & 9137.323 & 224.264 & Surface & 2006 \\
\hline 3849.958 & 227.713 & Surface & 2006 & 9140.274 & 224.279 & Surface & 2006 \\
\hline 3852.956 & 227.736 & Surface & 2006 & 9143.227 & 224.286 & Surface & 2006 \\
\hline 3855.954 & 227.732 & Surface & 2006 & 9146.178 & 224.289 & Surface & 2006 \\
\hline
\end{tabular}




\begin{tabular}{|c|c|c|c|c|c|c|c|}
\hline 3858.952 & 227.718 & Surface & 2006 & 9149.130 & 224.292 & Surface & 2006 \\
\hline 3861.950 & 227.719 & Surface & 2006 & 9152.082 & 224.297 & Surface & 2006 \\
\hline 3864.948 & 227.715 & Surface & 2006 & 9155.034 & 224.306 & Surface & 2006 \\
\hline 3867.943 & 227.714 & Surface & 2006 & 9157.986 & 224.333 & Surface & 2006 \\
\hline 3870.937 & 227.715 & Surface & 2006 & 9160.938 & 224.372 & Surface & 2006 \\
\hline 3873.932 & 227.725 & Surface & 2006 & 9163.890 & 224.412 & Surface & 2006 \\
\hline 3876.927 & 227.745 & Surface & 2006 & 9166.842 & 224.465 & Surface & 2006 \\
\hline 3879.922 & 227.762 & Surface & 2006 & 9169.794 & 224.473 & Surface & 2006 \\
\hline 3882.916 & 227.746 & Surface & 2006 & 9172.746 & 224.457 & Surface & 2006 \\
\hline 3885.911 & 227.730 & Surface & 2006 & 9175.698 & 224.477 & Surface & 2006 \\
\hline 3888.906 & 227.724 & Surface & 2006 & 9178.667 & 224.498 & Surface & 2006 \\
\hline 3891.901 & 227.721 & Surface & 2006 & 9181.636 & 224.517 & Surface & 2006 \\
\hline 3894.896 & 227.726 & Surface & 2006 & 9184.605 & 224.536 & Surface & 2006 \\
\hline 3897.890 & 227.715 & Surface & 2006 & 9187.574 & 224.557 & Surface & 2006 \\
\hline 3900.885 & 227.713 & Surface & 2006 & 9190.544 & 224.577 & Surface & 2006 \\
\hline 3903.880 & 227.719 & Surface & 2006 & 9193.513 & 224.598 & Surface & 2006 \\
\hline 3906.874 & 227.719 & Surface & 2006 & 9196.482 & 224.619 & Surface & 2006 \\
\hline 3909.869 & 227.721 & Surface & 2006 & 9199.451 & 224.639 & Surface & 2006 \\
\hline 3912.864 & 227.722 & Surface & 2006 & 9202.420 & 224.658 & Surface & 2006 \\
\hline 3915.859 & 227.722 & Surface & 2006 & 9205.389 & 224.677 & Surface & 2006 \\
\hline 3918.853 & 227.722 & Surface & 2006 & 9208.358 & 224.698 & Surface & 2006 \\
\hline 3921.786 & 227.719 & Surface & 2006 & 9211.328 & 224.710 & Surface & 2006 \\
\hline 3924.719 & 227.716 & Surface & 2006 & 9214.297 & 224.713 & Surface & 2006 \\
\hline 3927.652 & 227.708 & Surface & 2006 & 9217.266 & 224.715 & Surface & 2006 \\
\hline 3930.585 & 227.700 & Surface & 2006 & 9220.235 & 224.716 & Surface & 2006 \\
\hline 3933.518 & 227.696 & Surface & 2006 & 9223.204 & 224.714 & Surface & 2006 \\
\hline 3936.450 & 227.700 & Surface & 2006 & 9226.174 & 224.704 & Surface & 2006 \\
\hline 3939.383 & 227.696 & Surface & 2006 & 9229.050 & 224.685 & Surface & 2006 \\
\hline 3942.316 & 227.686 & Surface & 2006 & 9231.925 & 224.665 & Surface & 2006 \\
\hline 3945.249 & 227.685 & Surface & 2006 & 9234.801 & 224.646 & Surface & 2006 \\
\hline 3948.182 & 227.685 & Surface & 2006 & 9237.677 & 224.627 & Surface & 2006 \\
\hline 3951.115 & 227.695 & Surface & 2006 & 9240.553 & 224.608 & Surface & 2006 \\
\hline 3954.048 & 227.698 & Surface & 2006 & 9243.429 & 224.589 & Surface & 2006 \\
\hline 3956.980 & 227.708 & Surface & 2006 & 9246.305 & 224.570 & Surface & 2006 \\
\hline 3959.913 & 227.708 & Surface & 2006 & 9249.181 & 224.551 & Surface & 2006 \\
\hline 3962.846 & 227.696 & Surface & 2006 & 9252.057 & 224.569 & Surface & 2006 \\
\hline 3965.779 & 227.697 & Surface & 2006 & 9254.933 & 224.681 & Surface & 2006 \\
\hline 3968.712 & 227.690 & Surface & 2006 & 9257.809 & 224.846 & Surface & 2006 \\
\hline 3971.645 & 227.701 & Surface & 2006 & 9260.684 & 225.011 & Surface & 2006 \\
\hline 3974.577 & 227.701 & Surface & 2006 & 9263.560 & 225.176 & Surface & 2006 \\
\hline 3977.511 & 227.682 & Surface & 2006 & 9266.436 & 225.341 & Surface & 2006 \\
\hline 3980.443 & 227.685 & Surface & 2006 & 9269.312 & 225.473 & Surface & 2006 \\
\hline 3983.376 & 227.703 & Surface & 2006 & 9272.188 & 225.500 & Surface & 2006 \\
\hline 3986.171 & 227.691 & Surface & 2006 & 9275.064 & 225.486 & Surface & 2006 \\
\hline 3988.965 & 227.679 & Surface & 2006 & 9277.940 & 225.474 & Surface & 2006 \\
\hline 3991.759 & 227.687 & Surface & 2006 & 9280.816 & 225.465 & Surface & 2006 \\
\hline 3994.554 & 227.694 & Surface & 2006 & 9283.692 & 225.454 & Surface & 2006 \\
\hline 3997.348 & 227.697 & Surface & 2006 & 9286.568 & 225.432 & Surface & 2006 \\
\hline 4000.143 & 227.690 & Surface & 2006 & 9289.444 & 225.394 & Surface & 2006 \\
\hline
\end{tabular}




\begin{tabular}{|c|c|c|c|c|c|c|c|}
\hline 4002.937 & 227.689 & Surface & 2006 & 9292.397 & 225.342 & Surface & 2006 \\
\hline 4005.732 & 227.694 & Surface & 2006 & 9295.350 & 225.297 & Surface & 2006 \\
\hline 4008.526 & 227.692 & Surface & 2006 & 9298.304 & 225.269 & Surface & 2006 \\
\hline 4011.321 & 227.699 & Surface & 2006 & 9301.257 & 225.243 & Surface & 2006 \\
\hline 4014.116 & 227.702 & Surface & 2006 & 9304.210 & 225.202 & Surface & 2006 \\
\hline 4016.910 & 227.702 & Surface & 2006 & 9307.164 & 225.161 & Surface & 2006 \\
\hline 4019.704 & 227.701 & Surface & 2006 & 9310.117 & 225.120 & Surface & 2006 \\
\hline 4022.499 & 227.694 & Surface & 2006 & 9313.070 & 225.080 & Surface & 2006 \\
\hline 4025.382 & 227.684 & Surface & 2006 & 9316.024 & 225.039 & Surface & 2006 \\
\hline 4028.266 & 227.679 & Surface & 2006 & 9318.977 & 224.998 & Surface & 2006 \\
\hline 4031.149 & 227.687 & Surface & 2006 & 9321.930 & 224.958 & Surface & 2006 \\
\hline 4034.032 & 227.697 & Surface & 2006 & 9324.884 & 224.917 & Surface & 2006 \\
\hline 4036.916 & 227.706 & Surface & 2006 & 9327.837 & 224.876 & Surface & 2006 \\
\hline 4039.799 & 227.708 & Surface & 2006 & 9330.693 & 224.840 & Surface & 2006 \\
\hline 4042.682 & 227.706 & Surface & 2006 & 9333.548 & 224.804 & Surface & 2006 \\
\hline 4045.566 & 227.706 & Surface & 2006 & 9336.403 & 224.770 & Surface & 2006 \\
\hline 4048.449 & 227.702 & Surface & 2006 & 9339.259 & 224.734 & Surface & 2006 \\
\hline 4051.332 & 227.683 & Surface & 2006 & 9342.114 & 224.698 & Surface & 2006 \\
\hline 4054.216 & 227.666 & Surface & 2006 & 9344.970 & 224.663 & Surface & 2006 \\
\hline 4057.099 & 227.662 & Surface & 2006 & 9347.825 & 224.628 & Surface & 2006 \\
\hline 4059.982 & 227.667 & Surface & 2006 & 9350.681 & 224.591 & Surface & 2006 \\
\hline 4062.866 & 227.668 & Surface & 2006 & 9353.536 & 224.548 & Surface & 2006 \\
\hline 4065.863 & 227.663 & Surface & 2006 & 9356.392 & 224.489 & Surface & 2006 \\
\hline 4068.859 & 227.659 & Surface & 2006 & 9359.247 & 224.339 & Surface & 2006 \\
\hline 4071.856 & 227.659 & Surface & 2006 & 9362.103 & 224.233 & Surface & 2006 \\
\hline 4074.853 & 227.663 & Surface & 2006 & 9364.958 & 224.200 & Surface & 2006 \\
\hline 4077.850 & 227.671 & Surface & 2006 & 9367.813 & 224.208 & Surface & 2006 \\
\hline 4080.847 & 227.678 & Surface & 2006 & 9370.669 & 224.218 & Surface & 2006 \\
\hline 4083.844 & 227.680 & Surface & 2006 & 9373.524 & 224.227 & Surface & 2006 \\
\hline 4086.841 & 227.682 & Surface & 2006 & 9376.380 & 224.236 & Surface & 2006 \\
\hline 4089.838 & 227.684 & Surface & 2006 & 9379.235 & 224.242 & Surface & 2006 \\
\hline 4092.834 & 227.688 & Surface & 2006 & 9382.091 & 224.229 & Surface & 2006 \\
\hline 4095.831 & 227.686 & Surface & 2006 & 9385.085 & 224.212 & Surface & 2006 \\
\hline 4098.828 & 227.680 & Surface & 2006 & 9388.080 & 224.209 & Surface & 2006 \\
\hline 4101.825 & 227.675 & Surface & 2006 & 9391.075 & 224.213 & Surface & 2006 \\
\hline 4104.822 & 227.670 & Surface & 2006 & 9394.069 & 224.217 & Surface & 2006 \\
\hline 4107.819 & 227.664 & Surface & 2006 & 9397.064 & 224.221 & Surface & 2006 \\
\hline 4110.815 & 227.660 & Surface & 2006 & 9400.059 & 224.225 & Surface & 2006 \\
\hline 4113.813 & 227.667 & Surface & 2006 & 9403.054 & 224.229 & Surface & 2006 \\
\hline 4116.809 & 227.672 & Surface & 2006 & 9406.048 & 224.233 & Surface & 2006 \\
\hline 4119.806 & 227.676 & Surface & 2006 & 9409.043 & 224.233 & Surface & 2006 \\
\hline 4122.803 & 227.680 & Surface & 2006 & 9412.038 & 224.231 & Surface & 2006 \\
\hline 4125.800 & 227.687 & Surface & 2006 & 9415.033 & 224.229 & Surface & 2006 \\
\hline 4128.594 & 227.695 & Surface & 2006 & 9418.027 & 224.227 & Surface & 2006 \\
\hline 4131.389 & 227.706 & Surface & 2006 & 9421.022 & 224.224 & Surface & 2006 \\
\hline 4134.183 & 227.720 & Surface & 2006 & 9424.017 & 224.222 & Surface & 2006 \\
\hline 4136.978 & 227.731 & Surface & 2006 & 9427.011 & 224.220 & Surface & 2006 \\
\hline 4139.772 & 227.727 & Surface & 2006 & 9430.006 & 224.234 & Surface & 2006 \\
\hline 4142.567 & 227.704 & Surface & 2006 & 9433.001 & 224.276 & Surface & 2006 \\
\hline
\end{tabular}




\begin{tabular}{|c|c|c|c|c|c|c|c|}
\hline 4145.361 & 227.685 & Surface & 2006 & 9435.996 & 224.267 & Surface & 2006 \\
\hline 4148.156 & 227.663 & Surface & 2006 & 9438.996 & 224.272 & Surface & 2006 \\
\hline 4150.950 & 227.638 & Surface & 2006 & 9441.996 & 224.284 & Surface & 2006 \\
\hline 4153.745 & 227.633 & Surface & 2006 & 9444.996 & 224.300 & Surface & 2006 \\
\hline 4156.539 & 227.620 & Surface & 2006 & 9447.996 & 224.313 & Surface & 2006 \\
\hline 4159.334 & 227.609 & Surface & 2006 & 9450.996 & 224.326 & Surface & 2006 \\
\hline 4162.128 & 227.603 & Surface & 2006 & 9453.996 & 224.341 & Surface & 2006 \\
\hline 4164.923 & 227.598 & Surface & 2006 & 9456.996 & 224.355 & Surface & 2006 \\
\hline 4167.900 & 227.598 & Surface & 2006 & 9459.997 & 224.369 & Surface & 2006 \\
\hline 4170.878 & 227.604 & Surface & 2006 & 9462.997 & 224.386 & Surface & 2006 \\
\hline 4173.856 & 227.613 & Surface & 2006 & 9465.997 & 224.403 & Surface & 2006 \\
\hline 4176.833 & 227.622 & Surface & 2006 & 9468.997 & 224.420 & Surface & 2006 \\
\hline 4179.811 & 227.623 & Surface & 2006 & 9471.997 & 224.439 & Surface & 2006 \\
\hline 4182.788 & 227.613 & Surface & 2006 & 9474.997 & 224.457 & Surface & 2006 \\
\hline 4185.766 & 227.584 & Surface & 2006 & 9477.997 & 224.475 & Surface & 2006 \\
\hline 4188.744 & 227.603 & Surface & 2006 & 9480.997 & 224.494 & Surface & 2006 \\
\hline 4191.721 & 227.609 & Surface & 2006 & 9483.997 & 224.512 & Surface & 2006 \\
\hline 4194.699 & 227.628 & Surface & 2006 & 9486.997 & 224.530 & Surface & 2006 \\
\hline 4197.677 & 227.614 & Surface & 2006 & 9489.998 & 224.549 & Surface & 2006 \\
\hline 4200.654 & 227.595 & Surface & 2006 & 9492.998 & 224.566 & Surface & 2006 \\
\hline 4203.632 & 227.581 & Surface & 2006 & 9495.998 & 224.584 & Surface & 2006 \\
\hline 4206.609 & 227.584 & Surface & 2006 & 9498.998 & 224.603 & Surface & 2006 \\
\hline 4209.484 & 227.586 & Surface & 2006 & 9501.998 & 224.565 & Surface & 2006 \\
\hline 4212.358 & 227.590 & Surface & 2006 & 9504.874 & 224.514 & Surface & 2006 \\
\hline 4215.232 & 227.595 & Surface & 2006 & 9507.750 & 224.565 & Surface & 2006 \\
\hline 4218.107 & 227.619 & Surface & 2006 & 9510.626 & 224.535 & Surface & 2006 \\
\hline 4220.981 & 227.638 & Surface & 2006 & 9513.501 & 224.485 & Surface & 2006 \\
\hline 4223.855 & 227.646 & Surface & 2006 & 9516.377 & 224.436 & Surface & 2006 \\
\hline 4226.730 & 227.659 & Surface & 2006 & 9519.253 & 224.394 & Surface & 2006 \\
\hline 4229.604 & 227.624 & Surface & 2006 & 9522.129 & 224.363 & Surface & 2006 \\
\hline 4232.478 & 227.605 & Surface & 2006 & 9525.005 & 224.268 & Surface & 2006 \\
\hline 4235.353 & 227.611 & Surface & 2006 & 9527.881 & 224.189 & Surface & 2006 \\
\hline 4238.227 & 227.613 & Surface & 2006 & 9530.757 & 224.228 & Surface & 2006 \\
\hline 4241.101 & 227.608 & Surface & 2006 & 9533.633 & 224.294 & Surface & 2006 \\
\hline 4243.975 & 227.597 & Surface & 2006 & 9536.508 & 224.361 & Surface & 2006 \\
\hline 4246.850 & 227.576 & Surface & 2006 & 9539.384 & 224.427 & Surface & 2006 \\
\hline 4249.724 & 227.570 & Surface & 2006 & 9542.260 & 224.493 & Surface & 2006 \\
\hline 4252.598 & 227.573 & Surface & 2006 & 9545.136 & 224.560 & Surface & 2006 \\
\hline 4255.473 & 227.587 & Surface & 2006 & 9548.012 & 224.626 & Surface & 2006 \\
\hline 4258.347 & 227.605 & Surface & 2006 & 9550.887 & 224.693 & Surface & 2006 \\
\hline 4261.222 & 227.608 & Surface & 2006 & 9553.763 & 224.756 & Surface & 2006 \\
\hline 4264.096 & 227.587 & Surface & 2006 & 9556.639 & 224.757 & Surface & 2006 \\
\hline 4266.970 & 227.576 & Surface & 2006 & 9559.515 & 224.753 & Surface & 2006 \\
\hline 4269.844 & 227.589 & Surface & 2006 & 9562.391 & 224.750 & Surface & 2006 \\
\hline 4272.719 & 227.595 & Surface & 2006 & 9565.267 & 224.746 & Surface & 2006 \\
\hline 4275.637 & 227.593 & Surface & 2006 & 9568.143 & 224.743 & Surface & 2006 \\
\hline 4278.556 & 227.583 & Surface & 2006 & 9571.047 & 224.737 & Surface & 2006 \\
\hline 4281.474 & 227.536 & Surface & 2006 & 9573.950 & 224.733 & Surface & 2006 \\
\hline 4284.393 & 227.570 & Surface & 2006 & 9576.854 & 224.728 & Surface & 2006 \\
\hline
\end{tabular}




\begin{tabular}{|c|c|c|c|c|c|c|c|}
\hline 4287.311 & 227.580 & Surface & 2006 & 9579.757 & 224.724 & Surface & 2006 \\
\hline 4290.229 & 227.587 & Surface & 2006 & 9582.661 & 224.720 & Surface & 2006 \\
\hline 4293.148 & 227.588 & Surface & 2006 & 9585.564 & 224.716 & Surface & 2006 \\
\hline 4296.067 & 227.602 & Surface & 2006 & 9588.468 & 224.712 & Surface & 2006 \\
\hline 4298.985 & 227.603 & Surface & 2006 & 9591.371 & 224.708 & Surface & 2006 \\
\hline 4301.903 & 227.576 & Surface & 2006 & 9594.275 & 224.703 & Surface & 2006 \\
\hline 4304.822 & 227.550 & Surface & 2006 & 9597.178 & 224.698 & Surface & 2006 \\
\hline 4307.740 & 227.575 & Surface & 2006 & 9600.082 & 224.694 & Surface & 2006 \\
\hline 4310.659 & 227.585 & Surface & 2006 & 9602.985 & 224.690 & Surface & 2006 \\
\hline 4313.577 & 227.602 & Surface & 2006 & 9605.889 & 224.689 & Surface & 2006 \\
\hline 4316.496 & 227.598 & Surface & 2006 & 9608.792 & 224.701 & Surface & 2006 \\
\hline 4319.414 & 227.586 & Surface & 2006 & 9611.696 & 224.609 & Surface & 2006 \\
\hline 4322.333 & 227.594 & Surface & 2006 & 9614.600 & 224.440 & Surface & 2006 \\
\hline 4325.251 & 227.607 & Surface & 2006 & 9617.503 & 224.357 & Surface & 2006 \\
\hline 4328.170 & 227.607 & Surface & 2006 & 9620.407 & 224.304 & Surface & 2006 \\
\hline 4331.088 & 227.598 & Surface & 2006 & 9623.250 & 224.251 & Surface & 2006 \\
\hline 4334.006 & 227.604 & Surface & 2006 & 9626.092 & 224.200 & Surface & 2006 \\
\hline 4336.925 & 227.599 & Surface & 2006 & 9628.935 & 224.162 & Surface & 2006 \\
\hline 4339.844 & 227.580 & Surface & 2006 & 9631.778 & 224.153 & Surface & 2006 \\
\hline 4342.762 & 227.576 & Surface & 2006 & 9634.621 & 224.153 & Surface & 2006 \\
\hline 4345.680 & 227.583 & Surface & 2006 & 9637.464 & 224.153 & Surface & 2006 \\
\hline 4348.599 & 227.604 & Surface & 2006 & 9640.306 & 224.153 & Surface & 2006 \\
\hline 4351.517 & 227.612 & Surface & 2006 & 9643.149 & 224.155 & Surface & 2006 \\
\hline 4354.436 & 227.606 & Surface & 2006 & 9645.992 & 224.156 & Surface & 2006 \\
\hline 4357.354 & 227.603 & Surface & 2006 & 9648.834 & 224.156 & Surface & 2006 \\
\hline 4360.230 & 227.606 & Surface & 2006 & 9651.677 & 224.156 & Surface & 2006 \\
\hline 4363.106 & 227.605 & Surface & 2006 & 9654.520 & 224.156 & Surface & 2006 \\
\hline 4365.982 & 227.602 & Surface & 2006 & 9657.363 & 224.156 & Surface & 2006 \\
\hline 4368.858 & 227.596 & Surface & 2006 & 9660.187 & 224.158 & Surface & 2006 \\
\hline 4371.734 & 227.579 & Surface & 2006 & 9663.012 & 224.159 & Surface & 2006 \\
\hline 4374.609 & 227.557 & Surface & 2006 & 9665.837 & 224.159 & Surface & 2006 \\
\hline 4377.485 & 227.557 & Surface & 2006 & 9668.662 & 224.159 & Surface & 2006 \\
\hline 4380.361 & 227.586 & Surface & 2006 & 9671.486 & 224.162 & Surface & 2006 \\
\hline 4383.237 & 227.609 & Surface & 2006 & 9674.311 & 224.162 & Surface & 2006 \\
\hline 4386.113 & 227.604 & Surface & 2006 & 9677.136 & 224.162 & Surface & 2006 \\
\hline 4388.989 & 227.595 & Surface & 2006 & 9679.960 & 224.162 & Surface & 2006 \\
\hline 4391.864 & 227.607 & Surface & 2006 & 9682.785 & 224.163 & Surface & 2006 \\
\hline 4394.740 & 227.620 & Surface & 2006 & 9685.610 & 224.165 & Surface & 2006 \\
\hline 4397.616 & 227.614 & Surface & 2006 & 9688.435 & 224.165 & Surface & 2006 \\
\hline 4400.492 & 227.596 & Surface & 2006 & 9691.396 & 224.165 & Surface & 2006 \\
\hline 4403.368 & 227.587 & Surface & 2006 & 9694.356 & 224.165 & Surface & 2006 \\
\hline 4406.244 & 227.581 & Surface & 2006 & 9697.317 & 224.164 & Surface & 2006 \\
\hline 4409.119 & 227.575 & Surface & 2006 & 9700.278 & 224.162 & Surface & 2006 \\
\hline 4411.995 & 227.571 & Surface & 2006 & 9703.239 & 224.159 & Surface & 2006 \\
\hline 4414.871 & 227.572 & Surface & 2006 & 9706.200 & 224.159 & Surface & 2006 \\
\hline 4417.747 & 227.581 & Surface & 2006 & 9709.161 & 224.162 & Surface & 2006 \\
\hline 4420.623 & 227.563 & Surface & 2006 & 9712.122 & 224.162 & Surface & 2006 \\
\hline 4423.499 & 227.562 & Surface & 2006 & 9715.083 & 224.165 & Surface & 2006 \\
\hline 4426.363 & 227.567 & Surface & 2006 & 9718.044 & 224.162 & Surface & 2006 \\
\hline
\end{tabular}




\begin{tabular}{|c|c|c|c|c|c|c|c|}
\hline 4429.228 & 227.567 & Surface & 2006 & 9721.004 & 224.148 & Surface & 2006 \\
\hline 4432.093 & 227.577 & Surface & 2006 & 9723.965 & 224.137 & Surface & 2006 \\
\hline 4434.957 & 227.590 & Surface & 2006 & 9726.926 & 224.143 & Surface & 2006 \\
\hline 4437.822 & 227.597 & Surface & 2006 & 9729.887 & 224.145 & Surface & 2006 \\
\hline 4440.687 & 227.579 & Surface & 2006 & 9732.848 & 224.147 & Surface & 2006 \\
\hline 4443.551 & 227.567 & Surface & 2006 & 9735.809 & 224.135 & Surface & 2006 \\
\hline 4446.416 & 227.559 & Surface & 2006 & 9738.770 & 224.147 & Surface & 2006 \\
\hline 4449.281 & 227.579 & Surface & 2006 & 9741.731 & 224.150 & Surface & 2006 \\
\hline 4452.145 & 227.594 & Surface & 2006 & 9744.692 & 224.121 & Surface & 2006 \\
\hline 4455.010 & 227.586 & Surface & 2006 & 9747.653 & 224.125 & Surface & 2006 \\
\hline 4457.875 & 227.585 & Surface & 2006 & 9750.614 & 224.132 & Surface & 2006 \\
\hline 4460.739 & 227.592 & Surface & 2006 & 9753.575 & 224.122 & Surface & 2006 \\
\hline 4463.604 & 227.580 & Surface & 2006 & 9756.571 & 224.117 & Surface & 2006 \\
\hline 4466.469 & 227.589 & Surface & 2006 & 9759.567 & 224.246 & Surface & 2006 \\
\hline 4469.333 & 227.599 & Surface & 2006 & 9762.563 & 224.597 & Surface & 2006 \\
\hline 4472.198 & 227.591 & Surface & 2006 & 9765.560 & 225.025 & Surface & 2006 \\
\hline 4475.063 & 227.592 & Surface & 2006 & 9768.556 & 225.403 & Surface & 2006 \\
\hline 4477.927 & 227.597 & Surface & 2006 & 9771.552 & 225.561 & Surface & 2006 \\
\hline 4480.792 & 227.603 & Surface & 2006 & 9774.548 & 225.863 & Surface & 2006 \\
\hline 4483.744 & 227.586 & Surface & 2006 & 9777.545 & 225.988 & Surface & 2006 \\
\hline 4486.696 & 227.607 & Surface & 2006 & 9780.541 & 225.953 & Surface & 2006 \\
\hline 4489.647 & 227.596 & Surface & 2006 & 9783.537 & 225.950 & Surface & 2006 \\
\hline 4492.599 & 227.585 & Surface & 2006 & 9786.534 & 225.938 & Surface & 2006 \\
\hline 4495.551 & 227.598 & Surface & 2006 & 9789.530 & 225.901 & Surface & 2006 \\
\hline 4498.502 & 227.597 & Surface & 2006 & 9792.526 & 225.722 & Surface & 2006 \\
\hline 4501.454 & 227.572 & Surface & 2006 & 9795.522 & 225.438 & Surface & 2006 \\
\hline 4504.406 & 227.555 & Surface & 2006 & 9798.518 & 225.191 & Surface & 2006 \\
\hline 4507.358 & 227.571 & Surface & 2006 & 9801.515 & 225.014 & Surface & 2006 \\
\hline 4510.309 & 227.557 & Surface & 2006 & 9804.511 & 224.831 & Surface & 2006 \\
\hline 4513.261 & 227.546 & Surface & 2006 & 9807.507 & 224.684 & Surface & 2006 \\
\hline 4516.212 & 227.565 & Surface & 2006 & 9810.503 & 224.562 & Surface & 2006 \\
\hline 4519.164 & 227.577 & Surface & 2006 & 9813.500 & 224.371 & Surface & 2006 \\
\hline 4522.116 & 227.575 & Surface & 2006 & 9816.496 & 224.144 & Surface & 2006 \\
\hline 4525.068 & 227.577 & Surface & 2006 & 9819.492 & 223.868 & Surface & 2006 \\
\hline 4528.019 & 227.576 & Surface & 2006 & 9822.488 & 223.635 & Surface & 2006 \\
\hline 4530.971 & 227.572 & Surface & 2006 & 9825.485 & 223.509 & Surface & 2006 \\
\hline 4533.923 & 227.574 & Surface & 2006 & 9828.481 & 223.410 & Surface & 2006 \\
\hline 4536.874 & 227.587 & Surface & 2006 & 9831.477 & 223.288 & Surface & 2006 \\
\hline 4539.826 & 227.597 & Surface & 2006 & 9834.473 & 223.102 & Surface & 2006 \\
\hline 4542.733 & 227.606 & Surface & 2006 & 9837.469 & 222.888 & Surface & 2006 \\
\hline 4545.641 & 227.615 & Surface & 2006 & 9840.466 & 222.675 & Surface & 2006 \\
\hline 4548.549 & 227.615 & Surface & 2006 & 9843.462 & 222.442 & Surface & 2006 \\
\hline 4551.456 & 227.618 & Surface & 2006 & 9846.458 & 222.267 & Surface & 2006 \\
\hline 4554.364 & 227.620 & Surface & 2006 & 9849.455 & 222.070 & Surface & 2006 \\
\hline 4557.271 & 227.624 & Surface & 2006 & 9852.451 & 221.881 & Surface & 2006 \\
\hline 4560.179 & 227.626 & Surface & 2006 & 9855.447 & 221.708 & Surface & 2006 \\
\hline 4563.086 & 227.626 & Surface & 2006 & 9858.443 & 221.504 & Surface & 2006 \\
\hline 4565.994 & 227.622 & Surface & 2006 & 9861.440 & 221.275 & Surface & 2006 \\
\hline 4568.902 & 227.614 & Surface & 2006 & 9864.436 & 221.051 & Surface & 2006 \\
\hline
\end{tabular}




\begin{tabular}{|c|c|c|c|c|c|c|c|}
\hline 4571.809 & 227.581 & Surface & 2006 & 9867.432 & 220.863 & Surface & 2006 \\
\hline 4574.717 & 227.548 & Surface & 2006 & 9870.428 & 220.708 & Surface & 2006 \\
\hline 4577.624 & 227.564 & Surface & 2006 & 9873.425 & 220.524 & Surface & 2006 \\
\hline 4580.532 & 227.575 & Surface & 2006 & 9876.419 & 220.331 & Surface & 2006 \\
\hline 4583.439 & 227.573 & Surface & 2006 & 9879.413 & 220.125 & Surface & 2006 \\
\hline 4586.347 & 227.567 & Surface & 2006 & 9882.407 & 219.896 & Surface & 2006 \\
\hline 4589.254 & 227.585 & Surface & 2006 & 9885.401 & 219.637 & Surface & 2006 \\
\hline 4592.162 & 227.583 & Surface & 2006 & 9888.396 & 219.386 & Surface & 2006 \\
\hline 4595.069 & 227.576 & Surface & 2006 & 9891.390 & 219.187 & Surface & 2006 \\
\hline 4598.003 & 227.564 & Surface & 2006 & 9894.384 & 218.997 & Surface & 2006 \\
\hline 4600.938 & 227.553 & Surface & 2006 & 9897.378 & 218.738 & Surface & 2006 \\
\hline 4603.872 & 227.565 & Surface & 2006 & 9900.372 & 218.462 & Surface & 2006 \\
\hline 4606.806 & 227.579 & Surface & 2006 & 9903.367 & 218.196 & Surface & 2006 \\
\hline 4609.740 & 227.590 & Surface & 2006 & 9906.361 & 217.921 & Surface & 2006 \\
\hline 4612.674 & 227.588 & Surface & 2006 & 9909.355 & 217.619 & Surface & 2006 \\
\hline 4615.609 & 227.565 & Surface & 2006 & 9912.349 & 217.271 & Surface & 2006 \\
\hline 4618.543 & 227.550 & Surface & 2006 & 9915.343 & 216.922 & Surface & 2006 \\
\hline 4621.477 & 227.543 & Surface & 2006 & 9918.337 & 216.584 & Surface & 2006 \\
\hline 4624.411 & 227.541 & Surface & 2006 & 9921.332 & 216.243 & Surface & 2006 \\
\hline 4627.345 & 227.537 & Surface & 2006 & 9924.326 & 215.878 & Surface & 2006 \\
\hline 4630.279 & 227.535 & Surface & 2006 & 9927.320 & 215.493 & Surface & 2006 \\
\hline 4633.214 & 227.523 & Surface & 2006 & 9930.314 & 215.103 & Surface & 2006 \\
\hline 4636.148 & 227.520 & Surface & 2006 & 9933.308 & 214.732 & Surface & 2006 \\
\hline 4639.082 & 227.532 & Surface & 2006 & 9936.303 & 214.340 & Surface & 2006 \\
\hline 4642.016 & 227.559 & Surface & 2006 & 9939.297 & 213.948 & Surface & 2006 \\
\hline 4644.946 & 227.565 & Surface & 2006 & 9942.291 & 213.614 & Surface & 2006 \\
\hline 4647.875 & 227.573 & Surface & 2006 & 9945.285 & 213.259 & Surface & 2006 \\
\hline 4650.805 & 227.576 & Surface & 2006 & 9948.279 & 212.839 & Surface & 2006 \\
\hline 4653.734 & 227.580 & Surface & 2006 & 9951.274 & 212.396 & Surface & 2006 \\
\hline 4656.664 & 227.573 & Surface & 2006 & 9954.268 & 212.044 & Surface & 2006 \\
\hline 4659.593 & 227.569 & Surface & 2006 & 9957.262 & 211.920 & Surface & 2006 \\
\hline 4662.523 & 227.567 & Surface & 2006 & 9960.256 & 211.871 & Surface & 2006 \\
\hline 4665.452 & 227.570 & Surface & 2006 & 9963.250 & 211.877 & Surface & 2006 \\
\hline 4668.381 & 227.553 & Surface & 2006 & 9966.244 & 211.882 & Surface & 2006 \\
\hline 4671.311 & 227.531 & Surface & 2006 & 9969.239 & 211.888 & Surface & 2006 \\
\hline 4674.240 & 227.533 & Surface & 2006 & 9972.233 & 211.892 & Surface & 2006 \\
\hline 4677.170 & 227.524 & Surface & 2006 & 9975.227 & 211.898 & Surface & 2006 \\
\hline 4680.099 & 227.526 & Surface & 2006 & 9978.221 & 211.904 & Surface & 2006 \\
\hline 4683.029 & 227.521 & Surface & 2006 & 9981.215 & 211.905 & Surface & 2006 \\
\hline 4685.958 & 227.533 & Surface & 2006 & 9984.210 & 211.904 & Surface & 2006 \\
\hline 4688.874 & 227.544 & Surface & 2006 & 9987.204 & 211.901 & Surface & 2006 \\
\hline 4691.789 & 227.532 & Surface & 2006 & 9990.198 & 211.898 & Surface & 2006 \\
\hline 4694.704 & 227.518 & Surface & 2006 & 9993.192 & 211.894 & Surface & 2006 \\
\hline 4697.620 & 227.521 & Surface & 2006 & 9996.186 & 211.894 & Surface & 2006 \\
\hline 4700.535 & 227.526 & Surface & 2006 & 9999.160 & 211.890 & Surface & 2006 \\
\hline 4703.450 & 227.527 & Surface & 2006 & 10002.134 & 211.886 & Surface & 2006 \\
\hline 4706.366 & 227.515 & Surface & 2006 & 10005.108 & 211.882 & Surface & 2006 \\
\hline 4709.281 & 227.508 & Surface & 2006 & 10008.082 & 211.879 & Surface & 2006 \\
\hline 4712.196 & 227.482 & Surface & 2006 & 10011.056 & 211.877 & Surface & 2006 \\
\hline
\end{tabular}




\begin{tabular}{|c|c|c|c|c|c|c|c|}
\hline 4715.112 & 227.469 & Surface & 2006 & 10014.030 & 211.874 & Surface & 2006 \\
\hline 4718.027 & 227.506 & Surface & 2006 & 10017.003 & 211.870 & Surface & 2006 \\
\hline 4720.943 & 227.506 & Surface & 2006 & 10019.978 & 211.866 & Surface & 2006 \\
\hline 4723.858 & 227.479 & Surface & 2006 & 10022.951 & 211.864 & Surface & 2006 \\
\hline 4726.732 & 227.440 & Surface & 2006 & 10025.925 & 211.861 & Surface & 2006 \\
\hline 4729.606 & 227.457 & Surface & 2006 & 10028.899 & 211.856 & Surface & 2006 \\
\hline 4732.480 & 227.468 & Surface & 2006 & 10031.873 & 211.853 & Surface & 2006 \\
\hline 4735.354 & 227.464 & Surface & 2006 & 10034.847 & 211.853 & Surface & 2006 \\
\hline 4738.228 & 227.474 & Surface & 2006 & 10037.821 & 211.852 & Surface & 2006 \\
\hline 4741.102 & 227.475 & Surface & 2006 & 10040.795 & 211.853 & Surface & 2006 \\
\hline 4743.976 & 227.467 & Surface & 2006 & 10043.768 & 211.853 & Surface & 2006 \\
\hline 4746.850 & 227.450 & Surface & 2006 & 10046.743 & 211.852 & Surface & 2006 \\
\hline 4749.724 & 227.440 & Surface & 2006 & 10049.716 & 211.853 & Surface & 2006 \\
\hline 4752.598 & 227.457 & Surface & 2006 & 10052.690 & 211.853 & Surface & 2006 \\
\hline 4755.472 & 227.454 & Surface & 2006 & 10055.664 & 211.852 & Surface & 2006 \\
\hline 4758.346 & 227.457 & Surface & 2006 & 10058.638 & 211.852 & Surface & 2006 \\
\hline 4761.220 & 227.436 & Surface & 2006 & 10061.612 & 211.856 & Surface & 2006 \\
\hline 4764.094 & 227.428 & Surface & 2006 & 10064.586 & 211.856 & Surface & 2006 \\
\hline 4766.968 & 227.446 & Surface & 2006 & 10067.560 & 211.858 & Surface & 2006 \\
\hline 4769.842 & 227.443 & Surface & 2006 & 10070.533 & 211.860 & Surface & 2006 \\
\hline 4772.760 & 227.438 & Surface & 2006 & 10073.507 & 211.862 & Surface & 2006 \\
\hline 4775.677 & 227.441 & Surface & 2006 & 10076.481 & 211.864 & Surface & 2006 \\
\hline 4778.594 & 227.408 & Surface & 2006 & 10079.470 & 211.868 & Surface & 2006 \\
\hline 4781.512 & 227.458 & Surface & 2006 & 10082.459 & 211.870 & Surface & 2006 \\
\hline 4784.429 & 227.432 & Surface & 2006 & 10085.448 & 211.865 & Surface & 2006 \\
\hline 4787.347 & 227.444 & Surface & 2006 & 10088.437 & 211.849 & Surface & 2006 \\
\hline 4790.264 & 227.435 & Surface & 2006 & 10091.426 & 211.852 & Surface & 2006 \\
\hline 4793.182 & 227.423 & Surface & 2006 & 10094.415 & 211.862 & Surface & 2006 \\
\hline 4796.099 & 227.405 & Surface & 2006 & 10097.404 & 211.853 & Surface & 2006 \\
\hline 4799.017 & 227.401 & Surface & 2006 & 10100.393 & 211.837 & Surface & 2006 \\
\hline 4801.934 & 227.408 & Surface & 2006 & 10103.382 & 211.880 & Surface & 2006 \\
\hline 4804.851 & 227.390 & Surface & 2006 & 10106.371 & 211.927 & Surface & 2006 \\
\hline 4807.769 & 227.345 & Surface & 2006 & 10109.360 & 211.976 & Surface & 2006 \\
\hline 4810.686 & 227.367 & Surface & 2006 & 10112.349 & 212.029 & Surface & 2006 \\
\hline 4813.604 & 227.370 & Surface & 2006 & 10115.338 & 212.069 & Surface & 2006 \\
\hline 4816.521 & 227.351 & Surface & 2006 & 10118.327 & 212.176 & Surface & 2006 \\
\hline 4819.438 & 227.339 & Surface & 2006 & 10121.316 & 212.261 & Surface & 2006 \\
\hline 4822.356 & 227.341 & Surface & 2006 & 10124.305 & 212.349 & Surface & 2006 \\
\hline 4825.273 & 227.331 & Surface & 2006 & 10127.294 & 212.440 & Surface & 2006 \\
\hline 4828.191 & 227.324 & Surface & 2006 & 10130.283 & 212.505 & Surface & 2006 \\
\hline 4831.108 & 227.348 & Surface & 2006 & 10133.272 & 212.569 & Surface & 2006 \\
\hline 4834.094 & 227.349 & Surface & 2006 & 10136.261 & 212.651 & Surface & 2006 \\
\hline 4837.080 & 227.340 & Surface & 2006 & 10139.250 & 212.704 & Surface & 2006 \\
\hline 4840.067 & 227.328 & Surface & 2006 & 10142.239 & 212.770 & Surface & 2006 \\
\hline 4843.053 & 227.330 & Surface & 2006 & 10145.228 & 212.839 & Surface & 2006 \\
\hline 4846.039 & 227.325 & Surface & 2006 & 10148.217 & 212.875 & Surface & 2006 \\
\hline 4849.025 & 227.323 & Surface & 2006 & 10151.206 & 212.878 & Surface & 2006 \\
\hline 4852.012 & 227.314 & Surface & 2006 & 10154.194 & 212.880 & Surface & 2006 \\
\hline 4854.998 & 227.305 & Surface & 2006 & 10157.183 & 212.878 & Surface & 2006 \\
\hline
\end{tabular}




\begin{tabular}{|c|c|c|c|c|c|c|c|}
\hline 4857.984 & 227.303 & Surface & 2006 & 10160.172 & 212.882 & Surface & 2006 \\
\hline 4860.970 & 227.310 & Surface & 2006 & 10163.161 & 212.862 & Surface & 2006 \\
\hline 4863.956 & 227.328 & Surface & 2006 & 10166.150 & 212.770 & Surface & 2006 \\
\hline 4866.943 & 227.306 & Surface & 2006 & 10169.139 & 212.649 & Surface & 2006 \\
\hline 4869.929 & 227.288 & Surface & 2006 & 10172.128 & 212.508 & Surface & 2006 \\
\hline 4872.788 & 227.284 & Surface & 2006 & 10175.117 & 212.288 & Surface & 2006 \\
\hline 4875.647 & 227.287 & Surface & 2006 & 10178.106 & 212.044 & Surface & 2006 \\
\hline 4878.507 & 227.288 & Surface & 2006 & 10181.095 & 211.789 & Surface & 2006 \\
\hline 4881.366 & 227.283 & Surface & 2006 & 10184.084 & 211.652 & Surface & 2006 \\
\hline 4884.226 & 227.272 & Surface & 2006 & 10187.073 & 211.622 & Surface & 2006 \\
\hline 4887.085 & 227.269 & Surface & 2006 & 10190.062 & 211.613 & Surface & 2006 \\
\hline 4889.944 & 227.276 & Surface & 2006 & 10193.051 & 211.619 & Surface & 2006 \\
\hline 4892.804 & 227.252 & Surface & 2006 & 10196.040 & 211.602 & Surface & 2006 \\
\hline 4895.663 & 227.197 & Surface & 2006 & 10199.029 & 211.613 & Surface & 2006 \\
\hline 4898.523 & 227.182 & Surface & 2006 & 10202.018 & 211.633 & Surface & 2006 \\
\hline 4901.382 & 227.170 & Surface & 2006 & 10205.007 & 211.618 & Surface & 2006 \\
\hline 4904.241 & 227.178 & Surface & 2006 & 10207.996 & 211.639 & Surface & 2006 \\
\hline 4907.219 & 227.180 & Surface & 2006 & 10210.985 & 211.648 & Surface & 2006 \\
\hline 4910.197 & 227.179 & Surface & 2006 & 10213.974 & 211.647 & Surface & 2006 \\
\hline 4913.175 & 227.168 & Surface & 2006 & 10216.963 & 211.635 & Surface & 2006 \\
\hline 4916.152 & 227.139 & Surface & 2006 & 10219.952 & 211.641 & Surface & 2006 \\
\hline 4919.130 & 227.097 & Surface & 2006 & 10222.941 & 211.645 & Surface & 2006 \\
\hline 4922.108 & 227.124 & Surface & 2006 & 10225.930 & 211.608 & Surface & 2006 \\
\hline 4925.085 & 227.136 & Surface & 2006 & 10228.919 & 211.637 & Surface & 2006 \\
\hline 4928.063 & 227.115 & Surface & 2006 & 10231.908 & 211.677 & Surface & 2006 \\
\hline 4931.041 & 227.109 & Surface & 2006 & 10234.897 & 211.658 & Surface & 2006 \\
\hline 4934.018 & 227.095 & Surface & 2006 & 10237.886 & 211.642 & Surface & 2006 \\
\hline 4936.996 & 227.075 & Surface & 2006 & 10240.875 & 211.617 & Surface & 2006 \\
\hline 4939.974 & 227.072 & Surface & 2006 & 10243.864 & 211.609 & Surface & 2006 \\
\hline 4942.952 & 227.068 & Surface & 2006 & 10246.827 & 211.647 & Surface & 2006 \\
\hline 4945.929 & 227.060 & Surface & 2006 & 10249.790 & 211.660 & Surface & 2006 \\
\hline 4948.907 & 227.034 & Surface & 2006 & 10252.753 & 211.631 & Surface & 2006 \\
\hline 4951.885 & 227.014 & Surface & 2006 & 10255.716 & 211.615 & Surface & 2006 \\
\hline 4954.863 & 226.988 & Surface & 2006 & 10258.680 & 211.614 & Surface & 2006 \\
\hline 4957.840 & 226.993 & Surface & 2006 & 10261.643 & 211.615 & Surface & 2006 \\
\hline 4960.742 & 226.972 & Surface & 2006 & 10264.606 & 211.602 & Surface & 2006 \\
\hline 4963.642 & 226.931 & Surface & 2006 & 10267.569 & 211.587 & Surface & 2006 \\
\hline 4966.544 & 226.933 & Surface & 2006 & 10270.532 & 211.599 & Surface & 2006 \\
\hline 4969.445 & 226.928 & Surface & 2006 & 10273.495 & 211.613 & Surface & 2006 \\
\hline 4972.346 & 226.917 & Surface & 2006 & 10276.459 & 211.613 & Surface & 2006 \\
\hline 4975.247 & 226.942 & Surface & 2006 & 10279.422 & 211.596 & Surface & 2006 \\
\hline 4978.148 & 226.957 & Surface & 2006 & 10282.385 & 211.579 & Surface & 2006 \\
\hline 4981.049 & 226.975 & Surface & 2006 & 10285.348 & 211.588 & Surface & 2006 \\
\hline 4983.951 & 226.970 & Surface & 2006 & 10288.311 & 211.600 & Surface & 2006 \\
\hline 4986.852 & 226.955 & Surface & 2006 & 10291.274 & 211.605 & Surface & 2006 \\
\hline 4989.753 & 226.947 & Surface & 2006 & 10294.237 & 211.594 & Surface & 2006 \\
\hline 4992.654 & 226.936 & Surface & 2006 & 10297.201 & 211.576 & Surface & 2006 \\
\hline 4995.555 & 226.930 & Surface & 2006 & 10300.164 & 211.577 & Surface & 2006 \\
\hline 4998.457 & 226.929 & Surface & 2006 & 10303.127 & 211.566 & Surface & 2006 \\
\hline
\end{tabular}




\begin{tabular}{|c|c|c|c|c|c|c|c|}
\hline 5001.358 & 226.952 & Surface & 2006 & 10306.090 & 211.550 & Surface & 2006 \\
\hline 5004.259 & 226.927 & Surface & 2006 & 10309.053 & 211.539 & Surface & 2006 \\
\hline 5007.160 & 226.935 & Surface & 2006 & 10312.016 & 211.555 & Surface & 2006 \\
\hline 5010.061 & 226.937 & Surface & 2006 & 10314.980 & 211.543 & Surface & 2006 \\
\hline 5012.962 & 226.922 & Surface & 2006 & 10317.943 & 211.493 & Surface & 2006 \\
\hline 5015.863 & 226.926 & Surface & 2006 & 10320.906 & 211.468 & Surface & 2006 \\
\hline 5018.764 & 226.926 & Surface & 2006 & 10323.869 & 211.462 & Surface & 2006 \\
\hline 5021.666 & 226.920 & Surface & 2006 & 10326.832 & 211.455 & Surface & 2006 \\
\hline 5024.617 & 226.908 & Surface & 2006 & 10329.795 & 211.440 & Surface & 2006 \\
\hline 5027.568 & 226.906 & Surface & 2006 & 10332.759 & 211.442 & Surface & 2006 \\
\hline 5030.520 & 226.905 & Surface & 2006 & 10335.722 & 211.431 & Surface & 2006 \\
\hline 5033.471 & 226.892 & Surface & 2006 & 10338.685 & 211.426 & Surface & 2006 \\
\hline 5036.422 & 226.901 & Surface & 2006 & 10341.648 & 211.417 & Surface & 2006 \\
\hline 5039.373 & 226.898 & Surface & 2006 & 10344.611 & 211.387 & Surface & 2006 \\
\hline 5042.325 & 226.894 & Surface & 2006 & 10347.574 & 211.377 & Surface & 2006 \\
\hline 5045.276 & 226.881 & Surface & 2006 & 10350.538 & 211.398 & Surface & 2006 \\
\hline 5048.227 & 226.885 & Surface & 2006 & 10353.501 & 211.395 & Surface & 2006 \\
\hline 5051.179 & 226.872 & Surface & 2006 & 10356.464 & 211.373 & Surface & 2006 \\
\hline 5054.130 & 226.879 & Surface & 2006 & 10359.427 & 211.367 & Surface & 2006 \\
\hline 5057.081 & 226.874 & Surface & 2006 & 10362.390 & 211.368 & Surface & 2006 \\
\hline 5060.033 & 226.887 & Surface & 2006 & 10365.353 & 211.353 & Surface & 2006 \\
\hline 5062.984 & 226.885 & Surface & 2006 & 10368.316 & 211.325 & Surface & 2006 \\
\hline 5065.935 & 226.882 & Surface & 2006 & 10371.280 & 211.290 & Surface & 2006 \\
\hline 5068.886 & 226.876 & Surface & 2006 & 10374.243 & 211.289 & Surface & 2006 \\
\hline 5071.838 & 226.879 & Surface & 2006 & 10377.206 & 211.284 & Surface & 2006 \\
\hline 5074.789 & 226.857 & Surface & 2006 & 10380.169 & 211.271 & Surface & 2006 \\
\hline 5077.740 & 226.856 & Surface & 2006 & 10383.132 & 211.289 & Surface & 2006 \\
\hline 5080.692 & 226.841 & Surface & 2006 & 10386.096 & 211.286 & Surface & 2006 \\
\hline 5083.643 & 226.836 & Surface & 2006 & 10389.059 & 211.260 & Surface & 2006 \\
\hline 5086.594 & 226.828 & Surface & 2006 & 10392.022 & 211.254 & Surface & 2006 \\
\hline 5089.546 & 226.823 & Surface & 2006 & 10394.985 & 211.258 & Surface & 2006 \\
\hline 5092.497 & 226.850 & Surface & 2006 & 10397.948 & 211.265 & Surface & 2006 \\
\hline 5095.448 & 226.844 & Surface & 2006 & 10400.912 & 211.249 & Surface & 2006 \\
\hline 5098.399 & 226.849 & Surface & 2006 & 10403.875 & 211.203 & Surface & 2006 \\
\hline 5101.351 & 226.845 & Surface & 2006 & 10406.838 & 211.179 & Surface & 2006 \\
\hline 5104.302 & 226.836 & Surface & 2006 & 10409.801 & 211.174 & Surface & 2006 \\
\hline 5107.282 & 226.813 & Surface & 2006 & 10412.764 & 211.155 & Surface & 2006 \\
\hline 5110.262 & 226.827 & Surface & 2006 & 10415.727 & 211.160 & Surface & 2006 \\
\hline 5113.242 & 226.825 & Surface & 2006 & 10418.691 & 211.140 & Surface & 2006 \\
\hline 5116.223 & 226.822 & Surface & 2006 & 10421.654 & 211.132 & Surface & 2006 \\
\hline 5119.203 & 226.809 & Surface & 2006 & 10424.617 & 211.121 & Surface & 2006 \\
\hline 5122.183 & 226.806 & Surface & 2006 & 10427.580 & 211.130 & Surface & 2006 \\
\hline 5125.163 & 226.830 & Surface & 2006 & 10430.543 & 211.124 & Surface & 2006 \\
\hline 5128.143 & 226.841 & Surface & 2006 & 10433.506 & 211.106 & Surface & 2006 \\
\hline 5131.123 & 226.844 & Surface & 2006 & 10436.470 & 211.087 & Surface & 2006 \\
\hline 5134.104 & 226.818 & Surface & 2006 & 10439.433 & 211.083 & Surface & 2006 \\
\hline 5137.083 & 226.820 & Surface & 2006 & 10442.396 & 211.069 & Surface & 2006 \\
\hline 5140.064 & 226.828 & Surface & 2006 & 10445.359 & 211.069 & Surface & 2006 \\
\hline 5143.044 & 226.830 & Surface & 2006 & 10448.322 & 211.060 & Surface & 2006 \\
\hline
\end{tabular}




\begin{tabular}{|c|c|c|c|c|c|c|c|}
\hline 5146.024 & 226.837 & Surface & 2006 & 10451.285 & 211.056 & Surface & 2006 \\
\hline 5149.004 & 226.832 & Surface & 2006 & 10454.249 & 211.001 & Surface & 2006 \\
\hline 5151.984 & 226.830 & Surface & 2006 & 10457.212 & 210.930 & Surface & 2006 \\
\hline 5154.964 & 226.830 & Surface & 2006 & 10460.184 & 210.908 & Surface & 2006 \\
\hline 5157.926 & 226.847 & Surface & 2006 & 10463.157 & 210.919 & Surface & 2006 \\
\hline 5160.887 & 226.856 & Surface & 2006 & 10466.130 & 210.897 & Surface & 2006 \\
\hline 5163.849 & 226.846 & Surface & 2006 & 10469.102 & 210.887 & Surface & 2006 \\
\hline 5166.810 & 226.835 & Surface & 2006 & 10472.075 & 210.877 & Surface & 2006 \\
\hline 5169.772 & 226.849 & Surface & 2006 & 10475.047 & 210.859 & Surface & 2006 \\
\hline 5172.733 & 226.848 & Surface & 2006 & 10478.020 & 210.858 & Surface & 2006 \\
\hline 5175.695 & 226.849 & Surface & 2006 & 10480.993 & 210.863 & Surface & 2006 \\
\hline 5178.656 & 226.855 & Surface & 2006 & 10483.965 & 210.842 & Surface & 2006 \\
\hline 5181.618 & 226.850 & Surface & 2006 & 10486.938 & 210.817 & Surface & 2006 \\
\hline 5184.579 & 226.844 & Surface & 2006 & 10489.910 & 210.794 & Surface & 2006 \\
\hline 5187.541 & 226.848 & Surface & 2006 & 10492.883 & 210.784 & Surface & 2006 \\
\hline 5190.502 & 226.845 & Surface & 2006 & 10495.856 & 210.782 & Surface & 2006 \\
\hline 5193.463 & 226.838 & Surface & 2006 & 10498.828 & 210.785 & Surface & 2006 \\
\hline 5196.425 & 226.838 & Surface & 2006 & 10501.801 & 210.782 & Surface & 2006 \\
\hline 5199.386 & 226.845 & Surface & 2006 & 10504.773 & 210.754 & Surface & 2006 \\
\hline 5202.348 & 226.857 & Surface & 2006 & 10507.746 & 210.702 & Surface & 2006 \\
\hline 5205.309 & 226.860 & Surface & 2006 & 10510.719 & 210.685 & Surface & 2006 \\
\hline 5208.271 & 226.858 & Surface & 2006 & 10513.691 & 210.697 & Surface & 2006 \\
\hline 5211.232 & 226.861 & Surface & 2006 & 10516.664 & 210.697 & Surface & 2006 \\
\hline 5214.194 & 226.859 & Surface & 2006 & 10519.637 & 210.683 & Surface & 2006 \\
\hline 5217.155 & 226.852 & Surface & 2006 & 10522.609 & 210.672 & Surface & 2006 \\
\hline 5220.117 & 226.848 & Surface & 2006 & 10525.581 & 210.664 & Surface & 2006 \\
\hline 5223.078 & 226.839 & Surface & 2006 & 10528.554 & 210.659 & Surface & 2006 \\
\hline 5226.040 & 226.824 & Surface & 2006 & 10531.527 & 210.665 & Surface & 2006 \\
\hline 5229.001 & 226.822 & Surface & 2006 & 10534.499 & 210.661 & Surface & 2006 \\
\hline 5231.963 & 226.821 & Surface & 2006 & 10537.472 & 210.647 & Surface & 2006 \\
\hline 5234.924 & 226.830 & Surface & 2006 & 10540.445 & 210.666 & Surface & 2006 \\
\hline 5237.885 & 226.838 & Surface & 2006 & 10543.417 & 210.658 & Surface & 2006 \\
\hline 5240.797 & 226.847 & Surface & 2006 & 10546.390 & 210.635 & Surface & 2006 \\
\hline 5243.708 & 226.846 & Surface & 2006 & 10549.362 & 210.626 & Surface & 2006 \\
\hline 5246.620 & 226.840 & Surface & 2006 & 10552.335 & 210.632 & Surface & 2006 \\
\hline 5249.532 & 226.840 & Surface & 2006 & 10555.308 & 210.643 & Surface & 2006 \\
\hline 5252.443 & 226.880 & Surface & 2006 & 10558.280 & 210.634 & Surface & 2006 \\
\hline 5255.382 & 226.840 & Surface & 2006 & 10561.253 & 210.624 & Surface & 2006 \\
\hline 5258.321 & 226.806 & Surface & 2006 & 10564.225 & 210.627 & Surface & 2006 \\
\hline 5261.259 & 226.803 & Surface & 2006 & 10567.198 & 210.608 & Surface & 2006 \\
\hline 5264.198 & 226.816 & Surface & 2006 & 10570.171 & 210.602 & Surface & 2006 \\
\hline 5267.137 & 226.819 & Surface & 2006 & 10573.143 & 210.604 & Surface & 2006 \\
\hline 5270.076 & 226.818 & Surface & 2006 & 10576.116 & 210.598 & Surface & 2006 \\
\hline 5273.015 & 226.813 & Surface & 2006 & 10579.088 & 210.599 & Surface & 2006 \\
\hline 5275.953 & 226.813 & Surface & 2006 & 10582.061 & 210.602 & Surface & 2006 \\
\hline 5278.892 & 226.809 & Surface & 2006 & 10585.034 & 210.613 & Surface & 2006 \\
\hline 5281.831 & 226.796 & Surface & 2006 & 10588.006 & 210.608 & Surface & 2006 \\
\hline
\end{tabular}


11.2 Stream Gradient - 2008 Lidar:

\begin{tabular}{|c|c|c|c|c|c|c|c|}
\hline Distance & $\mathrm{Z}$ & DEM & Year & Distance & $\mathrm{Z}$ & DEM & Year \\
\hline 0.000 & 233.253 & Surface & 2008 & 5345.667 & 224.435 & Surface & 2008 \\
\hline 3.142 & 233.220 & Surface & 2008 & 5348.815 & 224.424 & Surface & 2008 \\
\hline 6.285 & 233.216 & Surface & 2008 & 5351.963 & 224.428 & Surface & 2008 \\
\hline 9.427 & 233.235 & Surface & 2008 & 5355.112 & 224.445 & Surface & 2008 \\
\hline 12.570 & 233.226 & Surface & 2008 & 5358.259 & 224.452 & Surface & 2008 \\
\hline 15.712 & 233.190 & Surface & 2008 & 5361.408 & 224.432 & Surface & 2008 \\
\hline 18.854 & 233.198 & Surface & 2008 & 5364.556 & 224.429 & Surface & 2008 \\
\hline 21.997 & 233.232 & Surface & 2008 & 5367.704 & 224.440 & Surface & 2008 \\
\hline 25.139 & 233.320 & Surface & 2008 & 5370.852 & 224.421 & Surface & 2008 \\
\hline 28.281 & 233.310 & Surface & 2008 & 5374.000 & 224.419 & Surface & 2008 \\
\hline 31.424 & 233.286 & Surface & 2008 & 5377.148 & 224.452 & Surface & 2008 \\
\hline 34.566 & 233.277 & Surface & 2008 & 5380.296 & 224.471 & Surface & 2008 \\
\hline 37.708 & 233.263 & Surface & 2008 & 5383.444 & 224.467 & Surface & 2008 \\
\hline 40.851 & 233.264 & Surface & 2008 & 5386.592 & 224.469 & Surface & 2008 \\
\hline 43.993 & 233.250 & Surface & 2008 & 5389.740 & 224.487 & Surface & 2008 \\
\hline 47.135 & 233.245 & Surface & 2008 & 5392.888 & 224.492 & Surface & 2008 \\
\hline 50.278 & 233.249 & Surface & 2008 & 5396.036 & 224.488 & Surface & 2008 \\
\hline 53.420 & 233.241 & Surface & 2008 & 5399.184 & 224.475 & Surface & 2008 \\
\hline 56.563 & 233.231 & Surface & 2008 & 5402.332 & 224.456 & Surface & 2008 \\
\hline 59.705 & 233.240 & Surface & 2008 & 5405.480 & 224.427 & Surface & 2008 \\
\hline 62.847 & 233.246 & Surface & 2008 & 5408.629 & 224.405 & Surface & 2008 \\
\hline 65.990 & 233.242 & Surface & 2008 & 5411.776 & 224.430 & Surface & 2008 \\
\hline 69.132 & 233.291 & Surface & 2008 & 5414.925 & 224.437 & Surface & 2008 \\
\hline 72.395 & 233.317 & Surface & 2008 & 5418.073 & 224.443 & Surface & 2008 \\
\hline 75.658 & 233.297 & Surface & 2008 & 5421.221 & 224.446 & Surface & 2008 \\
\hline 78.922 & 233.283 & Surface & 2008 & 5424.369 & 224.453 & Surface & 2008 \\
\hline 82.185 & 233.293 & Surface & 2008 & 5427.517 & 224.468 & Surface & 2008 \\
\hline 85.448 & 233.323 & Surface & 2008 & 5430.665 & 224.458 & Surface & 2008 \\
\hline 88.711 & 233.325 & Surface & 2008 & 5433.813 & 224.456 & Surface & 2008 \\
\hline 91.975 & 233.267 & Surface & 2008 & 5436.961 & 224.491 & Surface & 2008 \\
\hline 95.238 & 233.203 & Surface & 2008 & 5440.109 & 224.476 & Surface & 2008 \\
\hline 98.501 & 233.194 & Surface & 2008 & 5443.257 & 224.460 & Surface & 2008 \\
\hline 101.764 & 233.170 & Surface & 2008 & 5446.405 & 224.487 & Surface & 2008 \\
\hline 105.027 & 233.192 & Surface & 2008 & 5449.553 & 224.495 & Surface & 2008 \\
\hline 108.290 & 233.315 & Surface & 2008 & 5452.701 & 224.497 & Surface & 2008 \\
\hline 111.554 & 233.342 & Surface & 2008 & 5455.849 & 224.522 & Surface & 2008 \\
\hline 114.817 & 233.323 & Surface & 2008 & 5458.997 & 224.529 & Surface & 2008 \\
\hline 118.080 & 233.254 & Surface & 2008 & 5462.145 & 224.514 & Surface & 2008 \\
\hline 121.343 & 233.226 & Surface & 2008 & 5465.294 & 224.500 & Surface & 2008 \\
\hline 124.606 & 233.199 & Surface & 2008 & 5468.441 & 224.491 & Surface & 2008 \\
\hline 127.869 & 233.153 & Surface & 2008 & 5471.631 & 224.477 & Surface & 2008 \\
\hline 131.132 & 233.152 & Surface & 2008 & 5474.822 & 224.472 & Surface & 2008 \\
\hline 134.396 & 233.118 & Surface & 2008 & 5478.012 & 224.446 & Surface & 2008 \\
\hline 137.659 & 233.092 & Surface & 2008 & 5481.202 & 224.407 & Surface & 2008 \\
\hline 140.922 & 233.058 & Surface & 2008 & 5484.392 & 224.389 & Surface & 2008 \\
\hline
\end{tabular}




\begin{tabular}{|c|c|c|c|c|c|c|c|}
\hline 144.185 & 233.208 & Surface & 2008 & 5487.582 & 224.411 & Surface & 2008 \\
\hline 147.449 & 233.280 & Surface & 2008 & 5490.772 & 224.448 & Surface & 2008 \\
\hline 150.712 & 233.270 & Surface & 2008 & 5493.962 & 224.472 & Surface & 2008 \\
\hline 153.975 & 233.178 & Surface & 2008 & 5497.152 & 224.490 & Surface & 2008 \\
\hline 157.238 & 233.151 & Surface & 2008 & 5500.343 & 224.472 & Surface & 2008 \\
\hline 160.501 & 233.161 & Surface & 2008 & 5503.533 & 224.471 & Surface & 2008 \\
\hline 163.764 & 233.138 & Surface & 2008 & 5506.723 & 224.478 & Surface & 2008 \\
\hline 167.028 & 233.097 & Surface & 2008 & 5509.913 & 224.485 & Surface & 2008 \\
\hline 170.291 & 233.095 & Surface & 2008 & 5513.103 & 224.455 & Surface & 2008 \\
\hline 173.554 & 233.105 & Surface & 2008 & 5516.293 & 224.458 & Surface & 2008 \\
\hline 176.817 & 233.127 & Surface & 2008 & 5519.483 & 224.469 & Surface & 2008 \\
\hline 180.080 & 233.192 & Surface & 2008 & 5522.673 & 224.488 & Surface & 2008 \\
\hline 183.206 & 233.151 & Surface & 2008 & 5525.863 & 224.487 & Surface & 2008 \\
\hline 186.331 & 233.185 & Surface & 2008 & 5529.054 & 224.477 & Surface & 2008 \\
\hline 189.457 & 233.161 & Surface & 2008 & 5532.244 & 224.456 & Surface & 2008 \\
\hline 192.582 & 233.048 & Surface & 2008 & 5535.434 & 224.436 & Surface & 2008 \\
\hline 195.708 & 233.053 & Surface & 2008 & 5538.699 & 224.413 & Surface & 2008 \\
\hline 198.833 & 233.058 & Surface & 2008 & 5541.965 & 224.398 & Surface & 2008 \\
\hline 201.959 & 233.049 & Surface & 2008 & 5545.230 & 224.406 & Surface & 2008 \\
\hline 205.084 & 233.071 & Surface & 2008 & 5548.496 & 224.437 & Surface & 2008 \\
\hline 208.210 & 233.130 & Surface & 2008 & 5551.762 & 224.456 & Surface & 2008 \\
\hline 211.335 & 233.145 & Surface & 2008 & 5555.027 & 224.473 & Surface & 2008 \\
\hline 214.461 & 233.114 & Surface & 2008 & 5558.293 & 224.470 & Surface & 2008 \\
\hline 217.586 & 233.037 & Surface & 2008 & 5561.558 & 224.459 & Surface & 2008 \\
\hline 220.712 & 233.001 & Surface & 2008 & 5564.823 & 224.429 & Surface & 2008 \\
\hline 223.837 & 232.984 & Surface & 2008 & 5568.089 & 224.420 & Surface & 2008 \\
\hline 226.963 & 232.971 & Surface & 2008 & 5571.355 & 224.437 & Surface & 2008 \\
\hline 230.088 & 232.998 & Surface & 2008 & 5574.620 & 224.446 & Surface & 2008 \\
\hline 233.213 & 233.016 & Surface & 2008 & 5577.886 & 224.457 & Surface & 2008 \\
\hline 236.339 & 232.993 & Surface & 2008 & 5581.151 & 224.431 & Surface & 2008 \\
\hline 239.464 & 232.985 & Surface & 2008 & 5584.417 & 224.408 & Surface & 2008 \\
\hline 242.690 & 232.997 & Surface & 2008 & 5587.682 & 224.428 & Surface & 2008 \\
\hline 245.917 & 232.999 & Surface & 2008 & 5590.948 & 224.459 & Surface & 2008 \\
\hline 249.143 & 232.958 & Surface & 2008 & 5594.213 & 224.467 & Surface & 2008 \\
\hline 252.369 & 232.917 & Surface & 2008 & 5597.479 & 224.455 & Surface & 2008 \\
\hline 255.595 & 232.930 & Surface & 2008 & 5600.745 & 224.429 & Surface & 2008 \\
\hline 258.821 & 232.916 & Surface & 2008 & 5604.010 & 224.423 & Surface & 2008 \\
\hline 262.047 & 232.887 & Surface & 2008 & 5607.276 & 224.405 & Surface & 2008 \\
\hline 265.273 & 232.853 & Surface & 2008 & 5610.541 & 224.385 & Surface & 2008 \\
\hline 268.500 & 232.841 & Surface & 2008 & 5613.806 & 224.408 & Surface & 2008 \\
\hline 271.726 & 232.860 & Surface & 2008 & 5617.072 & 224.439 & Surface & 2008 \\
\hline 274.952 & 232.872 & Surface & 2008 & 5620.338 & 224.432 & Surface & 2008 \\
\hline 278.178 & 232.876 & Surface & 2008 & 5623.603 & 224.417 & Surface & 2008 \\
\hline 281.404 & 232.896 & Surface & 2008 & 5626.869 & 224.399 & Surface & 2008 \\
\hline 284.630 & 232.899 & Surface & 2008 & 5630.134 & 224.366 & Surface & 2008 \\
\hline 287.857 & 232.835 & Surface & 2008 & 5633.400 & 224.350 & Surface & 2008 \\
\hline 291.083 & 232.800 & Surface & 2008 & 5636.665 & 224.337 & Surface & 2008 \\
\hline 294.328 & 232.852 & Surface & 2008 & 5639.812 & 224.326 & Surface & 2008 \\
\hline 297.574 & 232.891 & Surface & 2008 & 5642.959 & 224.331 & Surface & 2008 \\
\hline
\end{tabular}




\begin{tabular}{|c|c|c|c|c|c|c|c|}
\hline 300.819 & 232.900 & Surface & 2008 & 5646.106 & 224.330 & Surface & 2008 \\
\hline 304.065 & 232.855 & Surface & 2008 & 5649.253 & 224.329 & Surface & 2008 \\
\hline 307.311 & 232.736 & Surface & 2008 & 5652.399 & 224.336 & Surface & 2008 \\
\hline 310.556 & 232.701 & Surface & 2008 & 5655.546 & 224.360 & Surface & 2008 \\
\hline 313.802 & 232.699 & Surface & 2008 & 5658.693 & 224.396 & Surface & 2008 \\
\hline 317.047 & 232.708 & Surface & 2008 & 5661.840 & 224.398 & Surface & 2008 \\
\hline 320.293 & 232.679 & Surface & 2008 & 5664.986 & 224.379 & Surface & 2008 \\
\hline 323.538 & 232.668 & Surface & 2008 & 5668.133 & 224.358 & Surface & 2008 \\
\hline 326.784 & 232.680 & Surface & 2008 & 5671.280 & 224.344 & Surface & 2008 \\
\hline 330.029 & 232.671 & Surface & 2008 & 5674.427 & 224.363 & Surface & 2008 \\
\hline 333.275 & 232.644 & Surface & 2008 & 5677.573 & 224.372 & Surface & 2008 \\
\hline 336.520 & 232.655 & Surface & 2008 & 5680.720 & 224.330 & Surface & 2008 \\
\hline 339.766 & 232.653 & Surface & 2008 & 5683.867 & 224.315 & Surface & 2008 \\
\hline 343.011 & 232.641 & Surface & 2008 & 5687.014 & 224.353 & Surface & 2008 \\
\hline 346.257 & 232.618 & Surface & 2008 & 5690.161 & 224.381 & Surface & 2008 \\
\hline 349.506 & 232.588 & Surface & 2008 & 5693.307 & 224.415 & Surface & 2008 \\
\hline 352.754 & 232.546 & Surface & 2008 & 5696.454 & 224.402 & Surface & 2008 \\
\hline 356.003 & 232.519 & Surface & 2008 & 5699.696 & 224.403 & Surface & 2008 \\
\hline 359.251 & 232.522 & Surface & 2008 & 5702.936 & 224.396 & Surface & 2008 \\
\hline 362.500 & 232.536 & Surface & 2008 & 5706.178 & 224.359 & Surface & 2008 \\
\hline 365.748 & 232.498 & Surface & 2008 & 5709.419 & 224.373 & Surface & 2008 \\
\hline 368.997 & 232.499 & Surface & 2008 & 5712.660 & 224.372 & Surface & 2008 \\
\hline 372.245 & 232.494 & Surface & 2008 & 5715.901 & 224.352 & Surface & 2008 \\
\hline 375.493 & 232.466 & Surface & 2008 & 5719.142 & 224.311 & Surface & 2008 \\
\hline 378.742 & 232.433 & Surface & 2008 & 5722.383 & 224.246 & Surface & 2008 \\
\hline 381.990 & 232.382 & Surface & 2008 & 5725.625 & 224.310 & Surface & 2008 \\
\hline 385.239 & 232.356 & Surface & 2008 & 5728.865 & 224.359 & Surface & 2008 \\
\hline 388.488 & 232.377 & Surface & 2008 & 5732.107 & 224.364 & Surface & 2008 \\
\hline 391.736 & 232.346 & Surface & 2008 & 5735.348 & 224.374 & Surface & 2008 \\
\hline 394.985 & 232.293 & Surface & 2008 & 5738.589 & 224.395 & Surface & 2008 \\
\hline 398.233 & 232.260 & Surface & 2008 & 5741.830 & 224.346 & Surface & 2008 \\
\hline 401.481 & 232.228 & Surface & 2008 & 5745.071 & 224.345 & Surface & 2008 \\
\hline 404.730 & 232.208 & Surface & 2008 & 5748.312 & 224.356 & Surface & 2008 \\
\hline 407.978 & 232.174 & Surface & 2008 & 5751.554 & 224.327 & Surface & 2008 \\
\hline 411.227 & 232.141 & Surface & 2008 & 5754.794 & 224.316 & Surface & 2008 \\
\hline 414.475 & 232.153 & Surface & 2008 & 5758.036 & 224.323 & Surface & 2008 \\
\hline 417.744 & 232.126 & Surface & 2008 & 5761.277 & 224.337 & Surface & 2008 \\
\hline 421.013 & 232.094 & Surface & 2008 & 5764.518 & 224.373 & Surface & 2008 \\
\hline 424.282 & 232.082 & Surface & 2008 & 5767.708 & 224.358 & Surface & 2008 \\
\hline 427.550 & 232.061 & Surface & 2008 & 5770.898 & 224.305 & Surface & 2008 \\
\hline 430.819 & 232.004 & Surface & 2008 & 5774.089 & 224.377 & Surface & 2008 \\
\hline 434.088 & 231.988 & Surface & 2008 & 5777.279 & 224.364 & Surface & 2008 \\
\hline 437.357 & 231.970 & Surface & 2008 & 5780.469 & 224.329 & Surface & 2008 \\
\hline 440.626 & 231.927 & Surface & 2008 & 5783.660 & 224.305 & Surface & 2008 \\
\hline 443.894 & 231.866 & Surface & 2008 & 5786.850 & 224.298 & Surface & 2008 \\
\hline 447.163 & 231.799 & Surface & 2008 & 5790.040 & 224.286 & Surface & 2008 \\
\hline 450.432 & 231.702 & Surface & 2008 & 5793.230 & 224.334 & Surface & 2008 \\
\hline 453.701 & 231.648 & Surface & 2008 & 5796.421 & 224.357 & Surface & 2008 \\
\hline 456.970 & 231.519 & Surface & 2008 & 5799.611 & 224.393 & Surface & 2008 \\
\hline
\end{tabular}




\begin{tabular}{|c|c|c|c|c|c|c|c|}
\hline 460.238 & 231.438 & Surface & 2008 & 5802.801 & 224.375 & Surface & 2008 \\
\hline 463.507 & 231.325 & Surface & 2008 & 5805.991 & 224.348 & Surface & 2008 \\
\hline 466.776 & 231.197 & Surface & 2008 & 5809.181 & 224.338 & Surface & 2008 \\
\hline 470.045 & 231.168 & Surface & 2008 & 5812.372 & 224.349 & Surface & 2008 \\
\hline 473.314 & 231.097 & Surface & 2008 & 5815.562 & 224.355 & Surface & 2008 \\
\hline 476.582 & 231.011 & Surface & 2008 & 5818.752 & 224.367 & Surface & 2008 \\
\hline 479.851 & 231.006 & Surface & 2008 & 5821.942 & 224.394 & Surface & 2008 \\
\hline 483.120 & 231.039 & Surface & 2008 & 5825.133 & 224.381 & Surface & 2008 \\
\hline 486.389 & 231.027 & Surface & 2008 & 5828.323 & 224.322 & Surface & 2008 \\
\hline 489.658 & 231.027 & Surface & 2008 & 5831.513 & 224.278 & Surface & 2008 \\
\hline 492.927 & 231.024 & Surface & 2008 & 5834.704 & 224.331 & Surface & 2008 \\
\hline 496.196 & 231.025 & Surface & 2008 & 5837.894 & 224.357 & Surface & 2008 \\
\hline 499.465 & 231.037 & Surface & 2008 & 5841.084 & 224.310 & Surface & 2008 \\
\hline 502.735 & 231.049 & Surface & 2008 & 5844.326 & 224.248 & Surface & 2008 \\
\hline 506.003 & 231.045 & Surface & 2008 & 5847.569 & 224.295 & Surface & 2008 \\
\hline 509.273 & 231.049 & Surface & 2008 & 5850.811 & 224.333 & Surface & 2008 \\
\hline 512.542 & 231.069 & Surface & 2008 & 5854.053 & 224.330 & Surface & 2008 \\
\hline 515.811 & 231.109 & Surface & 2008 & 5857.296 & 224.327 & Surface & 2008 \\
\hline 519.080 & 231.145 & Surface & 2008 & 5860.538 & 224.315 & Surface & 2008 \\
\hline 522.349 & 231.176 & Surface & 2008 & 5863.780 & 224.360 & Surface & 2008 \\
\hline 525.618 & 231.164 & Surface & 2008 & 5867.023 & 224.369 & Surface & 2008 \\
\hline 528.887 & 231.127 & Surface & 2008 & 5870.265 & 224.298 & Surface & 2008 \\
\hline 532.156 & 231.125 & Surface & 2008 & 5873.507 & 224.251 & Surface & 2008 \\
\hline 535.425 & 231.133 & Surface & 2008 & 5876.750 & 224.284 & Surface & 2008 \\
\hline 538.694 & 231.080 & Surface & 2008 & 5879.992 & 224.312 & Surface & 2008 \\
\hline 541.963 & 231.089 & Surface & 2008 & 5883.234 & 224.311 & Surface & 2008 \\
\hline 545.232 & 231.157 & Surface & 2008 & 5886.477 & 224.273 & Surface & 2008 \\
\hline 548.501 & 231.195 & Surface & 2008 & 5889.719 & 224.298 & Surface & 2008 \\
\hline 551.770 & 231.172 & Surface & 2008 & 5892.961 & 224.342 & Surface & 2008 \\
\hline 555.040 & 231.137 & Surface & 2008 & 5896.204 & 224.332 & Surface & 2008 \\
\hline 558.308 & 231.109 & Surface & 2008 & 5899.446 & 224.313 & Surface & 2008 \\
\hline 561.578 & 231.111 & Surface & 2008 & 5902.688 & 224.286 & Surface & 2008 \\
\hline 564.704 & 231.124 & Surface & 2008 & 5905.931 & 224.249 & Surface & 2008 \\
\hline 567.830 & 231.163 & Surface & 2008 & 5909.173 & 224.264 & Surface & 2008 \\
\hline 570.956 & 231.185 & Surface & 2008 & 5912.415 & 224.316 & Surface & 2008 \\
\hline 574.082 & 231.192 & Surface & 2008 & 5915.658 & 224.330 & Surface & 2008 \\
\hline 577.209 & 231.139 & Surface & 2008 & 5918.900 & 224.331 & Surface & 2008 \\
\hline 580.335 & 231.143 & Surface & 2008 & 5922.054 & 224.334 & Surface & 2008 \\
\hline 583.461 & 231.166 & Surface & 2008 & 5925.207 & 224.327 & Surface & 2008 \\
\hline 586.587 & 231.143 & Surface & 2008 & 5928.361 & 224.314 & Surface & 2008 \\
\hline 589.713 & 231.099 & Surface & 2008 & 5931.514 & 224.315 & Surface & 2008 \\
\hline 592.840 & 231.073 & Surface & 2008 & 5934.668 & 224.306 & Surface & 2008 \\
\hline 595.966 & 231.059 & Surface & 2008 & 5937.822 & 224.299 & Surface & 2008 \\
\hline 599.092 & 231.052 & Surface & 2008 & 5940.975 & 224.290 & Surface & 2008 \\
\hline 602.218 & 231.074 & Surface & 2008 & 5944.129 & 224.243 & Surface & 2008 \\
\hline 605.345 & 231.113 & Surface & 2008 & 5947.282 & 224.279 & Surface & 2008 \\
\hline 608.471 & 231.120 & Surface & 2008 & 5950.436 & 224.288 & Surface & 2008 \\
\hline 611.597 & 231.087 & Surface & 2008 & 5953.589 & 224.254 & Surface & 2008 \\
\hline 614.723 & 231.034 & Surface & 2008 & 5956.743 & 224.215 & Surface & 2008 \\
\hline
\end{tabular}




\begin{tabular}{|c|c|c|c|c|c|c|c|}
\hline 617.849 & 231.025 & Surface & 2008 & 5959.897 & 224.165 & Surface & 2008 \\
\hline 620.976 & 230.963 & Surface & 2008 & 5963.050 & 224.203 & Surface & 2008 \\
\hline 624.102 & 230.993 & Surface & 2008 & 5966.204 & 224.163 & Surface & 2008 \\
\hline 627.203 & 230.952 & Surface & 2008 & 5969.357 & 224.151 & Surface & 2008 \\
\hline 630.304 & 230.917 & Surface & 2008 & 5972.511 & 224.140 & Surface & 2008 \\
\hline 633.405 & 230.927 & Surface & 2008 & 5975.665 & 224.118 & Surface & 2008 \\
\hline 636.506 & 230.962 & Surface & 2008 & 5978.818 & 224.159 & Surface & 2008 \\
\hline 639.606 & 230.971 & Surface & 2008 & 5981.972 & 224.125 & Surface & 2008 \\
\hline 642.708 & 230.941 & Surface & 2008 & 5985.126 & 224.115 & Surface & 2008 \\
\hline 645.808 & 230.903 & Surface & 2008 & 5988.279 & 224.104 & Surface & 2008 \\
\hline 648.909 & 230.898 & Surface & 2008 & 5991.432 & 224.088 & Surface & 2008 \\
\hline 652.010 & 230.898 & Surface & 2008 & 5994.584 & 224.044 & Surface & 2008 \\
\hline 655.111 & 230.889 & Surface & 2008 & 5997.737 & 224.067 & Surface & 2008 \\
\hline 658.212 & 230.897 & Surface & 2008 & 6000.890 & 224.078 & Surface & 2008 \\
\hline 661.313 & 230.929 & Surface & 2008 & 6004.043 & 224.029 & Surface & 2008 \\
\hline 664.414 & 230.902 & Surface & 2008 & 6007.195 & 224.002 & Surface & 2008 \\
\hline 667.515 & 230.886 & Surface & 2008 & 6010.348 & 224.044 & Surface & 2008 \\
\hline 670.616 & 230.864 & Surface & 2008 & 6013.500 & 224.039 & Surface & 2008 \\
\hline 673.717 & 230.846 & Surface & 2008 & 6016.653 & 223.999 & Surface & 2008 \\
\hline 676.818 & 230.813 & Surface & 2008 & 6019.805 & 223.981 & Surface & 2008 \\
\hline 680.023 & 230.769 & Surface & 2008 & 6022.958 & 223.934 & Surface & 2008 \\
\hline 683.228 & 230.770 & Surface & 2008 & 6026.111 & 223.888 & Surface & 2008 \\
\hline 686.433 & 230.814 & Surface & 2008 & 6029.263 & 223.849 & Surface & 2008 \\
\hline 689.638 & 230.744 & Surface & 2008 & 6032.416 & 223.839 & Surface & 2008 \\
\hline 692.843 & 230.748 & Surface & 2008 & 6035.569 & 223.872 & Surface & 2008 \\
\hline 696.049 & 230.785 & Surface & 2008 & 6038.721 & 223.875 & Surface & 2008 \\
\hline 699.254 & 230.761 & Surface & 2008 & 6041.874 & 223.828 & Surface & 2008 \\
\hline 702.459 & 230.685 & Surface & 2008 & 6045.120 & 223.733 & Surface & 2008 \\
\hline 705.664 & 230.657 & Surface & 2008 & 6048.365 & 223.683 & Surface & 2008 \\
\hline 708.869 & 230.666 & Surface & 2008 & 6051.611 & 223.643 & Surface & 2008 \\
\hline 712.074 & 230.698 & Surface & 2008 & 6054.856 & 223.535 & Surface & 2008 \\
\hline 715.279 & 230.757 & Surface & 2008 & 6058.102 & 223.457 & Surface & 2008 \\
\hline 718.484 & 230.783 & Surface & 2008 & 6061.347 & 223.455 & Surface & 2008 \\
\hline 721.689 & 230.760 & Surface & 2008 & 6064.593 & 223.446 & Surface & 2008 \\
\hline 724.895 & 230.748 & Surface & 2008 & 6067.839 & 223.439 & Surface & 2008 \\
\hline 728.100 & 230.809 & Surface & 2008 & 6071.084 & 223.406 & Surface & 2008 \\
\hline 731.305 & 230.822 & Surface & 2008 & 6074.330 & 223.335 & Surface & 2008 \\
\hline 734.434 & 230.831 & Surface & 2008 & 6077.575 & 223.317 & Surface & 2008 \\
\hline 737.563 & 230.840 & Surface & 2008 & 6080.821 & 223.348 & Surface & 2008 \\
\hline 740.692 & 230.848 & Surface & 2008 & 6084.066 & 223.338 & Surface & 2008 \\
\hline 743.821 & 230.806 & Surface & 2008 & 6087.312 & 223.314 & Surface & 2008 \\
\hline 746.951 & 230.804 & Surface & 2008 & 6090.558 & 223.271 & Surface & 2008 \\
\hline 750.080 & 230.817 & Surface & 2008 & 6093.803 & 223.206 & Surface & 2008 \\
\hline 753.208 & 230.803 & Surface & 2008 & 6097.049 & 223.160 & Surface & 2008 \\
\hline 756.338 & 230.773 & Surface & 2008 & 6100.286 & 223.199 & Surface & 2008 \\
\hline 759.467 & 230.760 & Surface & 2008 & 6103.523 & 223.164 & Surface & 2008 \\
\hline 762.596 & 230.750 & Surface & 2008 & 6106.760 & 223.096 & Surface & 2008 \\
\hline 765.725 & 230.748 & Surface & 2008 & 6109.997 & 223.102 & Surface & 2008 \\
\hline 768.854 & 230.773 & Surface & 2008 & 6113.234 & 223.056 & Surface & 2008 \\
\hline
\end{tabular}




\begin{tabular}{|c|c|c|c|c|c|c|c|}
\hline 771.983 & 230.812 & Surface & 2008 & 6116.471 & 223.095 & Surface & 2008 \\
\hline 775.084 & 230.811 & Surface & 2008 & 6119.708 & 223.122 & Surface & 2008 \\
\hline 778.185 & 230.797 & Surface & 2008 & 6122.945 & 223.111 & Surface & 2008 \\
\hline 781.286 & 230.792 & Surface & 2008 & 6126.182 & 223.076 & Surface & 2008 \\
\hline 784.387 & 230.812 & Surface & 2008 & 6129.419 & 223.008 & Surface & 2008 \\
\hline 787.488 & 230.813 & Surface & 2008 & 6132.656 & 222.958 & Surface & 2008 \\
\hline 790.589 & 230.816 & Surface & 2008 & 6135.893 & 222.963 & Surface & 2008 \\
\hline 793.690 & 230.854 & Surface & 2008 & 6139.131 & 222.975 & Surface & 2008 \\
\hline 796.791 & 230.852 & Surface & 2008 & 6142.368 & 222.972 & Surface & 2008 \\
\hline 799.892 & 230.840 & Surface & 2008 & 6145.605 & 222.973 & Surface & 2008 \\
\hline 802.993 & 230.828 & Surface & 2008 & 6148.842 & 222.929 & Surface & 2008 \\
\hline 806.094 & 230.816 & Surface & 2008 & 6152.058 & 222.860 & Surface & 2008 \\
\hline 809.195 & 230.803 & Surface & 2008 & 6155.275 & 222.821 & Surface & 2008 \\
\hline 812.295 & 230.808 & Surface & 2008 & 6158.491 & 222.785 & Surface & 2008 \\
\hline 815.396 & 230.820 & Surface & 2008 & 6161.708 & 222.772 & Surface & 2008 \\
\hline 818.497 & 230.836 & Surface & 2008 & 6164.924 & 222.768 & Surface & 2008 \\
\hline 821.598 & 230.853 & Surface & 2008 & 6168.141 & 222.752 & Surface & 2008 \\
\hline 824.699 & 230.853 & Surface & 2008 & 6171.357 & 222.756 & Surface & 2008 \\
\hline 827.871 & 230.840 & Surface & 2008 & 6174.574 & 222.815 & Surface & 2008 \\
\hline 831.043 & 230.838 & Surface & 2008 & 6177.790 & 222.799 & Surface & 2008 \\
\hline 834.215 & 230.831 & Surface & 2008 & 6181.007 & 222.782 & Surface & 2008 \\
\hline 837.387 & 230.830 & Surface & 2008 & 6184.223 & 222.770 & Surface & 2008 \\
\hline 840.559 & 230.821 & Surface & 2008 & 6187.440 & 222.749 & Surface & 2008 \\
\hline 843.731 & 230.818 & Surface & 2008 & 6190.656 & 222.732 & Surface & 2008 \\
\hline 846.902 & 230.820 & Surface & 2008 & 6193.873 & 222.766 & Surface & 2008 \\
\hline 850.075 & 230.841 & Surface & 2008 & 6197.089 & 222.818 & Surface & 2008 \\
\hline 853.246 & 230.838 & Surface & 2008 & 6200.306 & 222.859 & Surface & 2008 \\
\hline 856.418 & 230.837 & Surface & 2008 & 6203.522 & 222.891 & Surface & 2008 \\
\hline 859.590 & 230.834 & Surface & 2008 & 6206.739 & 222.901 & Surface & 2008 \\
\hline 862.762 & 230.825 & Surface & 2008 & 6209.955 & 222.847 & Surface & 2008 \\
\hline 865.934 & 230.819 & Surface & 2008 & 6213.172 & 222.840 & Surface & 2008 \\
\hline 869.106 & 230.811 & Surface & 2008 & 6216.343 & 222.888 & Surface & 2008 \\
\hline 872.278 & 230.799 & Surface & 2008 & 6219.514 & 222.909 & Surface & 2008 \\
\hline 875.450 & 230.790 & Surface & 2008 & 6222.685 & 222.946 & Surface & 2008 \\
\hline 878.621 & 230.788 & Surface & 2008 & 6225.856 & 222.953 & Surface & 2008 \\
\hline 881.793 & 230.779 & Surface & 2008 & 6229.028 & 222.930 & Surface & 2008 \\
\hline 884.965 & 230.776 & Surface & 2008 & 6232.199 & 222.890 & Surface & 2008 \\
\hline 888.112 & 230.770 & Surface & 2008 & 6235.370 & 222.891 & Surface & 2008 \\
\hline 891.259 & 230.760 & Surface & 2008 & 6238.541 & 222.879 & Surface & 2008 \\
\hline 894.405 & 230.751 & Surface & 2008 & 6241.712 & 222.790 & Surface & 2008 \\
\hline 897.552 & 230.743 & Surface & 2008 & 6244.883 & 222.767 & Surface & 2008 \\
\hline 900.699 & 230.740 & Surface & 2008 & 6248.055 & 222.818 & Surface & 2008 \\
\hline 903.845 & 230.740 & Surface & 2008 & 6251.226 & 222.867 & Surface & 2008 \\
\hline 906.992 & 230.688 & Surface & 2008 & 6254.397 & 222.878 & Surface & 2008 \\
\hline 910.139 & 230.692 & Surface & 2008 & 6257.569 & 222.840 & Surface & 2008 \\
\hline 913.285 & 230.670 & Surface & 2008 & 6260.740 & 222.818 & Surface & 2008 \\
\hline 916.432 & 230.600 & Surface & 2008 & 6263.911 & 222.856 & Surface & 2008 \\
\hline 919.579 & 230.594 & Surface & 2008 & 6267.082 & 222.921 & Surface & 2008 \\
\hline 922.725 & 230.605 & Surface & 2008 & 6270.253 & 222.942 & Surface & 2008 \\
\hline
\end{tabular}




\begin{tabular}{|c|c|c|c|c|c|c|c|}
\hline 925.872 & 230.570 & Surface & 2008 & 6273.424 & 222.924 & Surface & 2008 \\
\hline 929.019 & 230.531 & Surface & 2008 & 6276.596 & 222.938 & Surface & 2008 \\
\hline 932.166 & 230.498 & Surface & 2008 & 6279.767 & 222.950 & Surface & 2008 \\
\hline 935.312 & 230.455 & Surface & 2008 & 6282.938 & 222.947 & Surface & 2008 \\
\hline 938.593 & 230.379 & Surface & 2008 & 6286.109 & 222.946 & Surface & 2008 \\
\hline 941.874 & 230.270 & Surface & 2008 & 6289.378 & 222.890 & Surface & 2008 \\
\hline 945.155 & 230.179 & Surface & 2008 & 6292.648 & 222.833 & Surface & 2008 \\
\hline 948.435 & 230.113 & Surface & 2008 & 6295.918 & 222.876 & Surface & 2008 \\
\hline 951.716 & 230.064 & Surface & 2008 & 6299.187 & 222.868 & Surface & 2008 \\
\hline 954.997 & 230.039 & Surface & 2008 & 6302.457 & 222.832 & Surface & 2008 \\
\hline 958.278 & 230.025 & Surface & 2008 & 6305.726 & 222.907 & Surface & 2008 \\
\hline 961.558 & 229.997 & Surface & 2008 & 6308.996 & 222.931 & Surface & 2008 \\
\hline 964.839 & 229.971 & Surface & 2008 & 6312.266 & 222.952 & Surface & 2008 \\
\hline 968.120 & 229.930 & Surface & 2008 & 6315.535 & 222.965 & Surface & 2008 \\
\hline 971.400 & 229.883 & Surface & 2008 & 6318.805 & 222.950 & Surface & 2008 \\
\hline 974.681 & 229.830 & Surface & 2008 & 6322.074 & 222.940 & Surface & 2008 \\
\hline 977.962 & 229.832 & Surface & 2008 & 6325.344 & 222.931 & Surface & 2008 \\
\hline 981.243 & 229.928 & Surface & 2008 & 6328.613 & 222.921 & Surface & 2008 \\
\hline 984.524 & 229.940 & Surface & 2008 & 6331.883 & 222.943 & Surface & 2008 \\
\hline 987.804 & 229.874 & Surface & 2008 & 6335.152 & 222.967 & Surface & 2008 \\
\hline 991.085 & 229.869 & Surface & 2008 & 6338.422 & 222.961 & Surface & 2008 \\
\hline 994.366 & 229.901 & Surface & 2008 & 6341.692 & 222.943 & Surface & 2008 \\
\hline 997.364 & 229.910 & Surface & 2008 & 6344.914 & 222.929 & Surface & 2008 \\
\hline 1000.363 & 229.875 & Surface & 2008 & 6348.137 & 222.906 & Surface & 2008 \\
\hline 1003.362 & 229.830 & Surface & 2008 & 6351.360 & 222.890 & Surface & 2008 \\
\hline 1006.361 & 229.851 & Surface & 2008 & 6354.583 & 222.881 & Surface & 2008 \\
\hline 1009.360 & 229.875 & Surface & 2008 & 6357.806 & 222.866 & Surface & 2008 \\
\hline 1012.358 & 229.877 & Surface & 2008 & 6361.029 & 222.854 & Surface & 2008 \\
\hline 1015.357 & 229.921 & Surface & 2008 & 6364.252 & 222.845 & Surface & 2008 \\
\hline 1018.356 & 229.980 & Surface & 2008 & 6367.475 & 222.837 & Surface & 2008 \\
\hline 1021.355 & 229.975 & Surface & 2008 & 6370.698 & 222.832 & Surface & 2008 \\
\hline 1024.353 & 229.976 & Surface & 2008 & 6373.921 & 222.833 & Surface & 2008 \\
\hline 1027.352 & 229.991 & Surface & 2008 & 6377.144 & 222.850 & Surface & 2008 \\
\hline 1030.526 & 229.978 & Surface & 2008 & 6380.366 & 222.860 & Surface & 2008 \\
\hline 1033.701 & 229.960 & Surface & 2008 & 6383.589 & 222.860 & Surface & 2008 \\
\hline 1036.875 & 229.959 & Surface & 2008 & 6386.812 & 222.838 & Surface & 2008 \\
\hline 1040.050 & 229.996 & Surface & 2008 & 6390.035 & 222.791 & Surface & 2008 \\
\hline 1043.224 & 229.981 & Surface & 2008 & 6393.258 & 222.776 & Surface & 2008 \\
\hline 1046.398 & 229.954 & Surface & 2008 & 6396.481 & 222.820 & Surface & 2008 \\
\hline 1049.573 & 229.943 & Surface & 2008 & 6399.704 & 222.853 & Surface & 2008 \\
\hline 1052.747 & 229.932 & Surface & 2008 & 6402.927 & 222.830 & Surface & 2008 \\
\hline 1055.921 & 229.914 & Surface & 2008 & 6406.150 & 222.757 & Surface & 2008 \\
\hline 1059.096 & 229.937 & Surface & 2008 & 6409.373 & 222.698 & Surface & 2008 \\
\hline 1062.270 & 229.934 & Surface & 2008 & 6412.595 & 222.684 & Surface & 2008 \\
\hline 1065.444 & 229.945 & Surface & 2008 & 6415.819 & 222.670 & Surface & 2008 \\
\hline 1068.619 & 229.960 & Surface & 2008 & 6419.041 & 222.671 & Surface & 2008 \\
\hline 1071.707 & 229.947 & Surface & 2008 & 6422.219 & 222.667 & Surface & 2008 \\
\hline 1074.795 & 229.930 & Surface & 2008 & 6425.396 & 222.716 & Surface & 2008 \\
\hline 1077.883 & 229.943 & Surface & 2008 & 6428.573 & 222.810 & Surface & 2008 \\
\hline
\end{tabular}




\begin{tabular}{|c|c|c|c|c|c|c|c|}
\hline 1080.971 & 229.968 & Surface & 2008 & 6431.750 & 222.735 & Surface & 2008 \\
\hline 1084.059 & 230.009 & Surface & 2008 & 6434.927 & 222.694 & Surface & 2008 \\
\hline 1087.148 & 229.989 & Surface & 2008 & 6438.105 & 222.667 & Surface & 2008 \\
\hline 1090.236 & 229.928 & Surface & 2008 & 6441.282 & 222.726 & Surface & 2008 \\
\hline 1093.324 & 229.900 & Surface & 2008 & 6444.459 & 222.754 & Surface & 2008 \\
\hline 1096.412 & 229.902 & Surface & 2008 & 6447.636 & 222.765 & Surface & 2008 \\
\hline 1099.500 & 229.938 & Surface & 2008 & 6450.813 & 222.725 & Surface & 2008 \\
\hline 1102.589 & 229.960 & Surface & 2008 & 6453.990 & 222.671 & Surface & 2008 \\
\hline 1105.677 & 229.946 & Surface & 2008 & 6457.168 & 222.641 & Surface & 2008 \\
\hline 1108.765 & 229.972 & Surface & 2008 & 6460.345 & 222.649 & Surface & 2008 \\
\hline 1111.853 & 229.963 & Surface & 2008 & 6463.522 & 222.669 & Surface & 2008 \\
\hline 1114.941 & 229.958 & Surface & 2008 & 6466.699 & 222.657 & Surface & 2008 \\
\hline 1118.029 & 229.954 & Surface & 2008 & 6469.877 & 222.632 & Surface & 2008 \\
\hline 1121.251 & 229.968 & Surface & 2008 & 6473.054 & 222.598 & Surface & 2008 \\
\hline 1124.472 & 229.958 & Surface & 2008 & 6476.231 & 222.596 & Surface & 2008 \\
\hline 1127.693 & 229.950 & Surface & 2008 & 6479.408 & 222.618 & Surface & 2008 \\
\hline 1130.914 & 229.979 & Surface & 2008 & 6482.586 & 222.622 & Surface & 2008 \\
\hline 1134.135 & 230.005 & Surface & 2008 & 6485.763 & 222.599 & Surface & 2008 \\
\hline 1137.356 & 230.006 & Surface & 2008 & 6488.940 & 222.565 & Surface & 2008 \\
\hline 1140.578 & 229.996 & Surface & 2008 & 6492.146 & 222.489 & Surface & 2008 \\
\hline 1143.799 & 229.980 & Surface & 2008 & 6495.353 & 222.508 & Surface & 2008 \\
\hline 1147.020 & 229.982 & Surface & 2008 & 6498.559 & 222.498 & Surface & 2008 \\
\hline 1150.241 & 230.011 & Surface & 2008 & 6501.766 & 222.497 & Surface & 2008 \\
\hline 1153.462 & 229.996 & Surface & 2008 & 6504.973 & 222.495 & Surface & 2008 \\
\hline 1156.683 & 229.970 & Surface & 2008 & 6508.179 & 222.498 & Surface & 2008 \\
\hline 1159.905 & 229.998 & Surface & 2008 & 6511.385 & 222.485 & Surface & 2008 \\
\hline 1163.126 & 230.031 & Surface & 2008 & 6514.592 & 222.457 & Surface & 2008 \\
\hline 1166.347 & 229.972 & Surface & 2008 & 6517.798 & 222.425 & Surface & 2008 \\
\hline 1169.568 & 229.941 & Surface & 2008 & 6521.005 & 222.399 & Surface & 2008 \\
\hline 1172.789 & 229.940 & Surface & 2008 & 6524.212 & 222.408 & Surface & 2008 \\
\hline 1176.010 & 229.951 & Surface & 2008 & 6527.418 & 222.404 & Surface & 2008 \\
\hline 1179.232 & 229.974 & Surface & 2008 & 6530.624 & 222.395 & Surface & 2008 \\
\hline 1182.453 & 230.018 & Surface & 2008 & 6533.831 & 222.350 & Surface & 2008 \\
\hline 1185.624 & 230.036 & Surface & 2008 & 6537.037 & 222.307 & Surface & 2008 \\
\hline 1188.794 & 230.029 & Surface & 2008 & 6540.244 & 222.302 & Surface & 2008 \\
\hline 1191.965 & 230.024 & Surface & 2008 & 6543.450 & 222.281 & Surface & 2008 \\
\hline 1195.136 & 230.013 & Surface & 2008 & 6546.657 & 222.274 & Surface & 2008 \\
\hline 1198.307 & 229.991 & Surface & 2008 & 6549.863 & 222.264 & Surface & 2008 \\
\hline 1201.477 & 229.947 & Surface & 2008 & 6553.070 & 222.235 & Surface & 2008 \\
\hline 1204.649 & 229.995 & Surface & 2008 & 6556.277 & 222.213 & Surface & 2008 \\
\hline 1207.819 & 230.046 & Surface & 2008 & 6559.483 & 222.183 & Surface & 2008 \\
\hline 1210.990 & 230.011 & Surface & 2008 & 6562.689 & 222.164 & Surface & 2008 \\
\hline 1214.161 & 230.019 & Surface & 2008 & 6565.896 & 222.153 & Surface & 2008 \\
\hline 1217.332 & 230.019 & Surface & 2008 & 6569.102 & 222.124 & Surface & 2008 \\
\hline 1220.502 & 229.994 & Surface & 2008 & 6572.371 & 222.068 & Surface & 2008 \\
\hline 1223.673 & 229.963 & Surface & 2008 & 6575.641 & 222.016 & Surface & 2008 \\
\hline 1226.844 & 229.932 & Surface & 2008 & 6578.910 & 221.988 & Surface & 2008 \\
\hline 1230.015 & 229.921 & Surface & 2008 & 6582.179 & 221.941 & Surface & 2008 \\
\hline 1233.186 & 229.910 & Surface & 2008 & 6585.448 & 221.944 & Surface & 2008 \\
\hline
\end{tabular}




\begin{tabular}{|c|c|c|c|c|c|c|c|}
\hline 1236.356 & 229.916 & Surface & 2008 & 6588.717 & 221.967 & Surface & 2008 \\
\hline 1239.504 & 229.954 & Surface & 2008 & 6591.986 & 221.993 & Surface & 2008 \\
\hline 1242.653 & 229.927 & Surface & 2008 & 6595.255 & 222.000 & Surface & 2008 \\
\hline 1245.800 & 229.922 & Surface & 2008 & 6598.524 & 221.997 & Surface & 2008 \\
\hline 1248.949 & 229.922 & Surface & 2008 & 6601.793 & 221.976 & Surface & 2008 \\
\hline 1252.097 & 229.924 & Surface & 2008 & 6605.062 & 221.918 & Surface & 2008 \\
\hline 1255.245 & 229.953 & Surface & 2008 & 6608.331 & 221.896 & Surface & 2008 \\
\hline 1258.393 & 229.944 & Surface & 2008 & 6611.600 & 221.837 & Surface & 2008 \\
\hline 1261.541 & 229.935 & Surface & 2008 & 6614.870 & 221.814 & Surface & 2008 \\
\hline 1264.689 & 229.947 & Surface & 2008 & 6618.139 & 221.816 & Surface & 2008 \\
\hline 1267.837 & 229.970 & Surface & 2008 & 6621.408 & 221.808 & Surface & 2008 \\
\hline 1270.985 & 229.946 & Surface & 2008 & 6624.677 & 221.776 & Surface & 2008 \\
\hline 1274.133 & 229.888 & Surface & 2008 & 6627.946 & 221.713 & Surface & 2008 \\
\hline 1277.281 & 229.889 & Surface & 2008 & 6631.215 & 221.679 & Surface & 2008 \\
\hline 1280.429 & 229.912 & Surface & 2008 & 6634.366 & 221.686 & Surface & 2008 \\
\hline 1283.577 & 229.903 & Surface & 2008 & 6637.516 & 221.689 & Surface & 2008 \\
\hline 1286.725 & 229.919 & Surface & 2008 & 6640.667 & 221.650 & Surface & 2008 \\
\hline 1289.873 & 229.948 & Surface & 2008 & 6643.818 & 221.630 & Surface & 2008 \\
\hline 1293.021 & 229.952 & Surface & 2008 & 6646.968 & 221.635 & Surface & 2008 \\
\hline 1296.169 & 229.964 & Surface & 2008 & 6650.119 & 221.583 & Surface & 2008 \\
\hline 1299.318 & 229.954 & Surface & 2008 & 6653.270 & 221.566 & Surface & 2008 \\
\hline 1302.466 & 229.951 & Surface & 2008 & 6656.420 & 221.586 & Surface & 2008 \\
\hline 1305.664 & 229.944 & Surface & 2008 & 6659.571 & 221.617 & Surface & 2008 \\
\hline 1308.863 & 229.949 & Surface & 2008 & 6662.722 & 221.672 & Surface & 2008 \\
\hline 1312.061 & 229.944 & Surface & 2008 & 6665.872 & 221.658 & Surface & 2008 \\
\hline 1315.260 & 229.919 & Surface & 2008 & 6669.023 & 221.627 & Surface & 2008 \\
\hline 1318.459 & 229.928 & Surface & 2008 & 6672.173 & 221.623 & Surface & 2008 \\
\hline 1321.657 & 229.947 & Surface & 2008 & 6675.324 & 221.641 & Surface & 2008 \\
\hline 1324.856 & 229.959 & Surface & 2008 & 6678.475 & 221.613 & Surface & 2008 \\
\hline 1328.055 & 229.930 & Surface & 2008 & 6681.625 & 221.647 & Surface & 2008 \\
\hline 1331.253 & 229.936 & Surface & 2008 & 6684.776 & 221.630 & Surface & 2008 \\
\hline 1334.452 & 229.985 & Surface & 2008 & 6687.927 & 221.636 & Surface & 2008 \\
\hline 1337.651 & 229.995 & Surface & 2008 & 6691.077 & 221.644 & Surface & 2008 \\
\hline 1340.849 & 230.017 & Surface & 2008 & 6694.228 & 221.641 & Surface & 2008 \\
\hline 1344.048 & 230.044 & Surface & 2008 & 6697.379 & 221.625 & Surface & 2008 \\
\hline 1347.246 & 230.050 & Surface & 2008 & 6700.529 & 221.616 & Surface & 2008 \\
\hline 1350.445 & 230.024 & Surface & 2008 & 6703.734 & 221.632 & Surface & 2008 \\
\hline 1353.644 & 230.012 & Surface & 2008 & 6706.938 & 221.642 & Surface & 2008 \\
\hline 1356.842 & 230.026 & Surface & 2008 & 6710.142 & 221.652 & Surface & 2008 \\
\hline 1360.020 & 230.039 & Surface & 2008 & 6713.346 & 221.670 & Surface & 2008 \\
\hline 1363.198 & 230.018 & Surface & 2008 & 6716.550 & 221.664 & Surface & 2008 \\
\hline 1366.376 & 229.992 & Surface & 2008 & 6719.754 & 221.653 & Surface & 2008 \\
\hline 1369.554 & 230.010 & Surface & 2008 & 6722.959 & 221.672 & Surface & 2008 \\
\hline 1372.732 & 230.000 & Surface & 2008 & 6726.163 & 221.683 & Surface & 2008 \\
\hline 1375.910 & 230.008 & Surface & 2008 & 6729.367 & 221.638 & Surface & 2008 \\
\hline 1379.088 & 230.010 & Surface & 2008 & 6732.571 & 221.594 & Surface & 2008 \\
\hline 1382.266 & 230.013 & Surface & 2008 & 6735.775 & 221.626 & Surface & 2008 \\
\hline 1385.444 & 229.965 & Surface & 2008 & 6738.979 & 221.664 & Surface & 2008 \\
\hline 1388.622 & 229.938 & Surface & 2008 & 6742.184 & 221.625 & Surface & 2008 \\
\hline
\end{tabular}




\begin{tabular}{|c|c|c|c|c|c|c|c|}
\hline 1391.799 & 229.981 & Surface & 2008 & 6745.388 & 221.554 & Surface & 2008 \\
\hline 1394.977 & 230.004 & Surface & 2008 & 6748.592 & 221.536 & Surface & 2008 \\
\hline 1398.155 & 230.004 & Surface & 2008 & 6751.796 & 221.536 & Surface & 2008 \\
\hline 1401.333 & 230.009 & Surface & 2008 & 6755.078 & 221.548 & Surface & 2008 \\
\hline 1404.511 & 229.997 & Surface & 2008 & 6758.360 & 221.570 & Surface & 2008 \\
\hline 1407.689 & 229.981 & Surface & 2008 & 6761.642 & 221.584 & Surface & 2008 \\
\hline 1410.867 & 229.943 & Surface & 2008 & 6764.923 & 221.587 & Surface & 2008 \\
\hline 1414.045 & 229.894 & Surface & 2008 & 6768.205 & 221.503 & Surface & 2008 \\
\hline 1417.223 & 229.927 & Surface & 2008 & 6771.487 & 221.417 & Surface & 2008 \\
\hline 1420.401 & 229.920 & Surface & 2008 & 6774.769 & 221.421 & Surface & 2008 \\
\hline 1423.578 & 229.928 & Surface & 2008 & 6778.051 & 221.428 & Surface & 2008 \\
\hline 1426.756 & 229.971 & Surface & 2008 & 6781.333 & 221.408 & Surface & 2008 \\
\hline 1429.934 & 229.980 & Surface & 2008 & 6784.615 & 221.412 & Surface & 2008 \\
\hline 1433.112 & 229.881 & Surface & 2008 & 6787.896 & 221.386 & Surface & 2008 \\
\hline 1436.290 & 229.894 & Surface & 2008 & 6791.178 & 221.341 & Surface & 2008 \\
\hline 1439.467 & 229.889 & Surface & 2008 & 6794.460 & 221.337 & Surface & 2008 \\
\hline 1442.644 & 229.885 & Surface & 2008 & 6797.742 & 221.338 & Surface & 2008 \\
\hline 1445.821 & 229.900 & Surface & 2008 & 6801.024 & 221.325 & Surface & 2008 \\
\hline 1448.998 & 229.905 & Surface & 2008 & 6804.305 & 221.314 & Surface & 2008 \\
\hline 1452.176 & 229.940 & Surface & 2008 & 6807.587 & 221.268 & Surface & 2008 \\
\hline 1455.353 & 229.941 & Surface & 2008 & 6810.869 & 221.184 & Surface & 2008 \\
\hline 1458.530 & 229.897 & Surface & 2008 & 6814.151 & 221.081 & Surface & 2008 \\
\hline 1461.707 & 229.911 & Surface & 2008 & 6817.433 & 221.065 & Surface & 2008 \\
\hline 1464.885 & 229.937 & Surface & 2008 & 6820.714 & 221.033 & Surface & 2008 \\
\hline 1468.062 & 229.970 & Surface & 2008 & 6823.996 & 221.081 & Surface & 2008 \\
\hline 1471.239 & 229.988 & Surface & 2008 & 6827.278 & 221.105 & Surface & 2008 \\
\hline 1474.416 & 229.931 & Surface & 2008 & 6830.560 & 221.099 & Surface & 2008 \\
\hline 1477.593 & 229.930 & Surface & 2008 & 6833.842 & 221.034 & Surface & 2008 \\
\hline 1480.770 & 229.931 & Surface & 2008 & 6837.124 & 220.995 & Surface & 2008 \\
\hline 1483.948 & 229.891 & Surface & 2008 & 6840.406 & 221.007 & Surface & 2008 \\
\hline 1487.125 & 229.903 & Surface & 2008 & 6843.687 & 221.035 & Surface & 2008 \\
\hline 1490.302 & 229.939 & Surface & 2008 & 6846.969 & 221.008 & Surface & 2008 \\
\hline 1493.479 & 229.950 & Surface & 2008 & 6850.251 & 221.000 & Surface & 2008 \\
\hline 1496.657 & 229.955 & Surface & 2008 & 6853.533 & 221.007 & Surface & 2008 \\
\hline 1499.834 & 229.939 & Surface & 2008 & 6856.798 & 221.040 & Surface & 2008 \\
\hline 1503.011 & 229.903 & Surface & 2008 & 6860.062 & 221.048 & Surface & 2008 \\
\hline 1506.292 & 229.879 & Surface & 2008 & 6863.327 & 221.010 & Surface & 2008 \\
\hline 1509.574 & 229.891 & Surface & 2008 & 6866.591 & 220.970 & Surface & 2008 \\
\hline 1512.855 & 229.903 & Surface & 2008 & 6869.856 & 220.974 & Surface & 2008 \\
\hline 1516.136 & 229.914 & Surface & 2008 & 6873.121 & 221.005 & Surface & 2008 \\
\hline 1519.418 & 229.912 & Surface & 2008 & 6876.385 & 221.064 & Surface & 2008 \\
\hline 1522.699 & 229.908 & Surface & 2008 & 6879.650 & 221.075 & Surface & 2008 \\
\hline 1525.980 & 229.867 & Surface & 2008 & 6882.915 & 221.128 & Surface & 2008 \\
\hline 1529.262 & 229.821 & Surface & 2008 & 6886.179 & 221.135 & Surface & 2008 \\
\hline 1532.543 & 229.867 & Surface & 2008 & 6889.444 & 221.072 & Surface & 2008 \\
\hline 1535.824 & 229.883 & Surface & 2008 & 6892.708 & 221.014 & Surface & 2008 \\
\hline 1539.106 & 229.903 & Surface & 2008 & 6895.973 & 221.019 & Surface & 2008 \\
\hline 1542.387 & 229.874 & Surface & 2008 & 6899.238 & 221.056 & Surface & 2008 \\
\hline 1545.669 & 229.864 & Surface & 2008 & 6902.502 & 221.090 & Surface & 2008 \\
\hline
\end{tabular}




\begin{tabular}{|c|c|c|c|c|c|c|c|}
\hline 1548.950 & 229.868 & Surface & 2008 & 6905.767 & 221.055 & Surface & 2008 \\
\hline 1552.231 & 229.872 & Surface & 2008 & 6909.031 & 221.015 & Surface & 2008 \\
\hline 1555.512 & 229.848 & Surface & 2008 & 6912.296 & 220.995 & Surface & 2008 \\
\hline 1558.794 & 229.820 & Surface & 2008 & 6915.561 & 221.013 & Surface & 2008 \\
\hline 1562.075 & 229.809 & Surface & 2008 & 6918.825 & 221.065 & Surface & 2008 \\
\hline 1565.357 & 229.765 & Surface & 2008 & 6922.090 & 221.053 & Surface & 2008 \\
\hline 1568.638 & 229.723 & Surface & 2008 & 6925.355 & 221.032 & Surface & 2008 \\
\hline 1571.919 & 229.759 & Surface & 2008 & 6928.619 & 221.025 & Surface & 2008 \\
\hline 1575.200 & 229.816 & Surface & 2008 & 6931.884 & 221.001 & Surface & 2008 \\
\hline 1578.482 & 229.865 & Surface & 2008 & 6935.148 & 221.002 & Surface & 2008 \\
\hline 1581.763 & 229.860 & Surface & 2008 & 6938.423 & 221.049 & Surface & 2008 \\
\hline 1585.045 & 229.755 & Surface & 2008 & 6941.696 & 221.077 & Surface & 2008 \\
\hline 1588.326 & 229.743 & Surface & 2008 & 6944.970 & 221.089 & Surface & 2008 \\
\hline 1591.607 & 229.755 & Surface & 2008 & 6948.244 & 221.096 & Surface & 2008 \\
\hline 1594.888 & 229.747 & Surface & 2008 & 6951.518 & 221.075 & Surface & 2008 \\
\hline 1598.170 & 229.770 & Surface & 2008 & 6954.792 & 221.092 & Surface & 2008 \\
\hline 1601.451 & 229.721 & Surface & 2008 & 6958.066 & 221.093 & Surface & 2008 \\
\hline 1604.733 & 229.713 & Surface & 2008 & 6961.340 & 221.105 & Surface & 2008 \\
\hline 1608.014 & 229.751 & Surface & 2008 & 6964.614 & 221.132 & Surface & 2008 \\
\hline 1611.295 & 229.769 & Surface & 2008 & 6967.887 & 221.118 & Surface & 2008 \\
\hline 1614.576 & 229.742 & Surface & 2008 & 6971.162 & 221.093 & Surface & 2008 \\
\hline 1617.858 & 229.742 & Surface & 2008 & 6974.435 & 221.056 & Surface & 2008 \\
\hline 1621.139 & 229.759 & Surface & 2008 & 6977.709 & 221.054 & Surface & 2008 \\
\hline 1624.421 & 229.744 & Surface & 2008 & 6980.983 & 221.041 & Surface & 2008 \\
\hline 1627.702 & 229.694 & Surface & 2008 & 6984.257 & 221.040 & Surface & 2008 \\
\hline 1630.983 & 229.641 & Surface & 2008 & 6987.531 & 221.053 & Surface & 2008 \\
\hline 1634.264 & 229.665 & Surface & 2008 & 6990.805 & 221.033 & Surface & 2008 \\
\hline 1637.546 & 229.709 & Surface & 2008 & 6994.079 & 221.041 & Surface & 2008 \\
\hline 1640.779 & 229.705 & Surface & 2008 & 6997.353 & 221.000 & Surface & 2008 \\
\hline 1644.011 & 229.693 & Surface & 2008 & 7000.627 & 220.960 & Surface & 2008 \\
\hline 1647.244 & 229.713 & Surface & 2008 & 7003.901 & 221.016 & Surface & 2008 \\
\hline 1650.477 & 229.705 & Surface & 2008 & 7007.174 & 221.039 & Surface & 2008 \\
\hline 1653.710 & 229.701 & Surface & 2008 & 7010.448 & 221.075 & Surface & 2008 \\
\hline 1656.942 & 229.679 & Surface & 2008 & 7013.722 & 221.056 & Surface & 2008 \\
\hline 1660.175 & 229.671 & Surface & 2008 & 7016.996 & 221.011 & Surface & 2008 \\
\hline 1663.408 & 229.716 & Surface & 2008 & 7020.270 & 221.010 & Surface & 2008 \\
\hline 1666.640 & 229.792 & Surface & 2008 & 7023.544 & 221.026 & Surface & 2008 \\
\hline 1669.873 & 229.824 & Surface & 2008 & 7026.818 & 221.037 & Surface & 2008 \\
\hline 1673.106 & 229.801 & Surface & 2008 & 7030.051 & 221.056 & Surface & 2008 \\
\hline 1676.339 & 229.768 & Surface & 2008 & 7033.285 & 221.043 & Surface & 2008 \\
\hline 1679.571 & 229.700 & Surface & 2008 & 7036.519 & 221.014 & Surface & 2008 \\
\hline 1682.804 & 229.668 & Surface & 2008 & 7039.753 & 220.990 & Surface & 2008 \\
\hline 1686.037 & 229.717 & Surface & 2008 & 7042.986 & 221.015 & Surface & 2008 \\
\hline 1689.269 & 229.736 & Surface & 2008 & 7046.220 & 221.015 & Surface & 2008 \\
\hline 1692.502 & 229.732 & Surface & 2008 & 7049.454 & 220.968 & Surface & 2008 \\
\hline 1695.735 & 229.698 & Surface & 2008 & 7052.688 & 220.932 & Surface & 2008 \\
\hline 1698.967 & 229.688 & Surface & 2008 & 7055.921 & 220.914 & Surface & 2008 \\
\hline 1702.200 & 229.716 & Surface & 2008 & 7059.155 & 220.913 & Surface & 2008 \\
\hline 1705.433 & 229.670 & Surface & 2008 & 7062.389 & 220.923 & Surface & 2008 \\
\hline
\end{tabular}




\begin{tabular}{|c|c|c|c|c|c|c|c|}
\hline 1708.666 & 229.700 & Surface & 2008 & 7065.623 & 220.950 & Surface & 2008 \\
\hline 1711.898 & 229.731 & Surface & 2008 & 7068.856 & 221.026 & Surface & 2008 \\
\hline 1715.131 & 229.750 & Surface & 2008 & 7072.090 & 221.045 & Surface & 2008 \\
\hline 1718.364 & 229.712 & Surface & 2008 & 7075.324 & 220.984 & Surface & 2008 \\
\hline 1721.596 & 229.658 & Surface & 2008 & 7078.558 & 220.955 & Surface & 2008 \\
\hline 1724.829 & 229.655 & Surface & 2008 & 7081.791 & 221.023 & Surface & 2008 \\
\hline 1728.062 & 229.682 & Surface & 2008 & 7085.025 & 220.960 & Surface & 2008 \\
\hline 1731.295 & 229.749 & Surface & 2008 & 7088.259 & 220.945 & Surface & 2008 \\
\hline 1734.527 & 229.748 & Surface & 2008 & 7091.493 & 220.974 & Surface & 2008 \\
\hline 1737.760 & 229.711 & Surface & 2008 & 7094.726 & 221.008 & Surface & 2008 \\
\hline 1741.001 & 229.689 & Surface & 2008 & 7097.960 & 220.978 & Surface & 2008 \\
\hline 1744.243 & 229.689 & Surface & 2008 & 7101.194 & 220.915 & Surface & 2008 \\
\hline 1747.484 & 229.693 & Surface & 2008 & 7104.428 & 220.927 & Surface & 2008 \\
\hline 1750.725 & 229.703 & Surface & 2008 & 7107.661 & 220.991 & Surface & 2008 \\
\hline 1753.967 & 229.728 & Surface & 2008 & 7110.895 & 220.975 & Surface & 2008 \\
\hline 1757.208 & 229.727 & Surface & 2008 & 7114.129 & 220.947 & Surface & 2008 \\
\hline 1760.449 & 229.720 & Surface & 2008 & 7117.362 & 220.924 & Surface & 2008 \\
\hline 1763.691 & 229.714 & Surface & 2008 & 7120.596 & 220.945 & Surface & 2008 \\
\hline 1766.932 & 229.743 & Surface & 2008 & 7123.830 & 220.926 & Surface & 2008 \\
\hline 1770.173 & 229.756 & Surface & 2008 & 7127.063 & 220.901 & Surface & 2008 \\
\hline 1773.414 & 229.741 & Surface & 2008 & 7130.297 & 221.008 & Surface & 2008 \\
\hline 1776.656 & 229.719 & Surface & 2008 & 7133.531 & 221.017 & Surface & 2008 \\
\hline 1779.897 & 229.685 & Surface & 2008 & 7136.765 & 220.950 & Surface & 2008 \\
\hline 1783.139 & 229.686 & Surface & 2008 & 7139.998 & 220.898 & Surface & 2008 \\
\hline 1786.380 & 229.693 & Surface & 2008 & 7143.278 & 220.896 & Surface & 2008 \\
\hline 1789.621 & 229.670 & Surface & 2008 & 7146.557 & 220.928 & Surface & 2008 \\
\hline 1792.862 & 229.637 & Surface & 2008 & 7149.837 & 220.933 & Surface & 2008 \\
\hline 1796.104 & 229.640 & Surface & 2008 & 7153.116 & 220.898 & Surface & 2008 \\
\hline 1799.345 & 229.687 & Surface & 2008 & 7156.396 & 220.872 & Surface & 2008 \\
\hline 1802.586 & 229.711 & Surface & 2008 & 7159.675 & 220.860 & Surface & 2008 \\
\hline 1805.828 & 229.697 & Surface & 2008 & 7162.955 & 220.889 & Surface & 2008 \\
\hline 1809.069 & 229.702 & Surface & 2008 & 7166.234 & 220.832 & Surface & 2008 \\
\hline 1812.310 & 229.729 & Surface & 2008 & 7169.514 & 220.793 & Surface & 2008 \\
\hline 1815.552 & 229.740 & Surface & 2008 & 7172.794 & 220.815 & Surface & 2008 \\
\hline 1818.793 & 229.734 & Surface & 2008 & 7176.073 & 220.802 & Surface & 2008 \\
\hline 1822.034 & 229.713 & Surface & 2008 & 7179.353 & 220.717 & Surface & 2008 \\
\hline 1825.276 & 229.697 & Surface & 2008 & 7182.632 & 220.636 & Surface & 2008 \\
\hline 1828.517 & 229.693 & Surface & 2008 & 7185.912 & 220.691 & Surface & 2008 \\
\hline 1831.758 & 229.694 & Surface & 2008 & 7189.191 & 220.744 & Surface & 2008 \\
\hline 1834.999 & 229.656 & Surface & 2008 & 7192.471 & 220.797 & Surface & 2008 \\
\hline 1838.241 & 229.715 & Surface & 2008 & 7195.750 & 220.799 & Surface & 2008 \\
\hline 1841.482 & 229.726 & Surface & 2008 & 7199.030 & 220.791 & Surface & 2008 \\
\hline 1844.723 & 229.710 & Surface & 2008 & 7202.309 & 220.736 & Surface & 2008 \\
\hline 1847.965 & 229.692 & Surface & 2008 & 7205.589 & 220.734 & Surface & 2008 \\
\hline 1851.206 & 229.685 & Surface & 2008 & 7208.868 & 220.729 & Surface & 2008 \\
\hline 1854.447 & 229.673 & Surface & 2008 & 7212.147 & 220.744 & Surface & 2008 \\
\hline 1857.689 & 229.659 & Surface & 2008 & 7215.427 & 220.781 & Surface & 2008 \\
\hline 1860.930 & 229.660 & Surface & 2008 & 7218.707 & 220.735 & Surface & 2008 \\
\hline 1864.171 & 229.690 & Surface & 2008 & 7221.986 & 220.649 & Surface & 2008 \\
\hline
\end{tabular}




\begin{tabular}{|c|c|c|c|c|c|c|c|}
\hline 1867.412 & 229.728 & Surface & 2008 & 7225.265 & 220.622 & Surface & 2008 \\
\hline 1870.654 & 229.746 & Surface & 2008 & 7228.545 & 220.629 & Surface & 2008 \\
\hline 1873.895 & 229.762 & Surface & 2008 & 7231.825 & 220.616 & Surface & 2008 \\
\hline 1877.136 & 229.725 & Surface & 2008 & 7235.104 & 220.632 & Surface & 2008 \\
\hline 1880.408 & 229.708 & Surface & 2008 & 7238.384 & 220.651 & Surface & 2008 \\
\hline 1883.679 & 229.712 & Surface & 2008 & 7241.663 & 220.612 & Surface & 2008 \\
\hline 1886.951 & 229.704 & Surface & 2008 & 7244.943 & 220.554 & Surface & 2008 \\
\hline 1890.222 & 229.740 & Surface & 2008 & 7248.222 & 220.540 & Surface & 2008 \\
\hline 1893.494 & 229.756 & Surface & 2008 & 7251.502 & 220.556 & Surface & 2008 \\
\hline 1896.765 & 229.721 & Surface & 2008 & 7254.781 & 220.552 & Surface & 2008 \\
\hline 1900.037 & 229.712 & Surface & 2008 & 7258.061 & 220.568 & Surface & 2008 \\
\hline 1903.308 & 229.706 & Surface & 2008 & 7261.340 & 220.582 & Surface & 2008 \\
\hline 1906.580 & 229.690 & Surface & 2008 & 7264.569 & 220.556 & Surface & 2008 \\
\hline 1909.851 & 229.682 & Surface & 2008 & 7267.798 & 220.554 & Surface & 2008 \\
\hline 1913.122 & 229.683 & Surface & 2008 & 7271.027 & 220.585 & Surface & 2008 \\
\hline 1916.394 & 229.685 & Surface & 2008 & 7274.257 & 220.584 & Surface & 2008 \\
\hline 1919.665 & 229.699 & Surface & 2008 & 7277.486 & 220.550 & Surface & 2008 \\
\hline 1922.937 & 229.716 & Surface & 2008 & 7280.715 & 220.502 & Surface & 2008 \\
\hline 1926.208 & 229.713 & Surface & 2008 & 7283.944 & 220.490 & Surface & 2008 \\
\hline 1929.480 & 229.673 & Surface & 2008 & 7287.173 & 220.485 & Surface & 2008 \\
\hline 1932.758 & 229.645 & Surface & 2008 & 7290.402 & 220.457 & Surface & 2008 \\
\hline 1936.036 & 229.661 & Surface & 2008 & 7293.631 & 220.478 & Surface & 2008 \\
\hline 1939.314 & 229.672 & Surface & 2008 & 7296.861 & 220.540 & Surface & 2008 \\
\hline 1942.592 & 229.660 & Surface & 2008 & 7300.090 & 220.527 & Surface & 2008 \\
\hline 1945.870 & 229.642 & Surface & 2008 & 7303.319 & 220.479 & Surface & 2008 \\
\hline 1949.148 & 229.631 & Surface & 2008 & 7306.548 & 220.417 & Surface & 2008 \\
\hline 1952.426 & 229.654 & Surface & 2008 & 7309.777 & 220.389 & Surface & 2008 \\
\hline 1955.704 & 229.696 & Surface & 2008 & 7313.006 & 220.421 & Surface & 2008 \\
\hline 1958.982 & 229.692 & Surface & 2008 & 7316.235 & 220.383 & Surface & 2008 \\
\hline 1962.260 & 229.679 & Surface & 2008 & 7319.465 & 220.371 & Surface & 2008 \\
\hline 1965.538 & 229.732 & Surface & 2008 & 7322.694 & 220.381 & Surface & 2008 \\
\hline 1968.816 & 229.740 & Surface & 2008 & 7325.923 & 220.415 & Surface & 2008 \\
\hline 1972.094 & 229.735 & Surface & 2008 & 7329.152 & 220.381 & Surface & 2008 \\
\hline 1975.372 & 229.704 & Surface & 2008 & 7332.381 & 220.364 & Surface & 2008 \\
\hline 1978.650 & 229.654 & Surface & 2008 & 7335.610 & 220.357 & Surface & 2008 \\
\hline 1981.928 & 229.658 & Surface & 2008 & 7338.839 & 220.359 & Surface & 2008 \\
\hline 1985.206 & 229.695 & Surface & 2008 & 7342.068 & 220.337 & Surface & 2008 \\
\hline 1988.484 & 229.683 & Surface & 2008 & 7345.298 & 220.284 & Surface & 2008 \\
\hline 1991.762 & 229.691 & Surface & 2008 & 7348.527 & 220.345 & Surface & 2008 \\
\hline 1995.040 & 229.704 & Surface & 2008 & 7351.693 & 220.378 & Surface & 2008 \\
\hline 1998.318 & 229.713 & Surface & 2008 & 7354.860 & 220.393 & Surface & 2008 \\
\hline 2001.596 & 229.710 & Surface & 2008 & 7358.027 & 220.407 & Surface & 2008 \\
\hline 2004.874 & 229.705 & Surface & 2008 & 7361.193 & 220.380 & Surface & 2008 \\
\hline 2008.152 & 229.672 & Surface & 2008 & 7364.360 & 220.366 & Surface & 2008 \\
\hline 2011.430 & 229.605 & Surface & 2008 & 7367.526 & 220.337 & Surface & 2008 \\
\hline 2014.708 & 229.607 & Surface & 2008 & 7370.693 & 220.342 & Surface & 2008 \\
\hline 2017.987 & 229.597 & Surface & 2008 & 7373.860 & 220.315 & Surface & 2008 \\
\hline 2021.264 & 229.642 & Surface & 2008 & 7377.026 & 220.307 & Surface & 2008 \\
\hline 2024.542 & 229.686 & Surface & 2008 & 7380.193 & 220.310 & Surface & 2008 \\
\hline
\end{tabular}




\begin{tabular}{|c|c|c|c|c|c|c|c|}
\hline 2027.821 & 229.704 & Surface & 2008 & 7383.360 & 220.314 & Surface & 2008 \\
\hline 2031.099 & 229.687 & Surface & 2008 & 7386.526 & 220.348 & Surface & 2008 \\
\hline 2034.376 & 229.679 & Surface & 2008 & 7389.693 & 220.354 & Surface & 2008 \\
\hline 2037.655 & 229.679 & Surface & 2008 & 7392.860 & 220.351 & Surface & 2008 \\
\hline 2040.933 & 229.700 & Surface & 2008 & 7396.026 & 220.323 & Surface & 2008 \\
\hline 2044.211 & 229.717 & Surface & 2008 & 7399.193 & 220.308 & Surface & 2008 \\
\hline 2047.489 & 229.716 & Surface & 2008 & 7402.360 & 220.319 & Surface & 2008 \\
\hline 2050.767 & 229.664 & Surface & 2008 & 7405.526 & 220.342 & Surface & 2008 \\
\hline 2054.045 & 229.681 & Surface & 2008 & 7408.693 & 220.295 & Surface & 2008 \\
\hline 2057.323 & 229.720 & Surface & 2008 & 7411.860 & 220.279 & Surface & 2008 \\
\hline 2060.601 & 229.738 & Surface & 2008 & 7415.026 & 220.282 & Surface & 2008 \\
\hline 2063.879 & 229.728 & Surface & 2008 & 7418.193 & 220.272 & Surface & 2008 \\
\hline 2067.157 & 229.709 & Surface & 2008 & 7421.359 & 220.286 & Surface & 2008 \\
\hline 2070.435 & 229.714 & Surface & 2008 & 7424.526 & 220.321 & Surface & 2008 \\
\hline 2073.713 & 229.723 & Surface & 2008 & 7427.693 & 220.328 & Surface & 2008 \\
\hline 2076.991 & 229.712 & Surface & 2008 & 7430.859 & 220.330 & Surface & 2008 \\
\hline 2080.269 & 229.674 & Surface & 2008 & 7434.098 & 220.313 & Surface & 2008 \\
\hline 2083.547 & 229.670 & Surface & 2008 & 7437.336 & 220.301 & Surface & 2008 \\
\hline 2086.825 & 229.692 & Surface & 2008 & 7440.574 & 220.279 & Surface & 2008 \\
\hline 2090.103 & 229.704 & Surface & 2008 & 7443.813 & 220.266 & Surface & 2008 \\
\hline 2093.381 & 229.672 & Surface & 2008 & 7447.051 & 220.250 & Surface & 2008 \\
\hline 2096.659 & 229.640 & Surface & 2008 & 7450.290 & 220.255 & Surface & 2008 \\
\hline 2099.937 & 229.618 & Surface & 2008 & 7453.528 & 220.291 & Surface & 2008 \\
\hline 2103.158 & 229.599 & Surface & 2008 & 7456.766 & 220.313 & Surface & 2008 \\
\hline 2106.379 & 229.667 & Surface & 2008 & 7460.005 & 220.279 & Surface & 2008 \\
\hline 2109.599 & 229.706 & Surface & 2008 & 7463.243 & 220.260 & Surface & 2008 \\
\hline 2112.820 & 229.667 & Surface & 2008 & 7466.481 & 220.280 & Surface & 2008 \\
\hline 2116.041 & 229.646 & Surface & 2008 & 7469.720 & 220.306 & Surface & 2008 \\
\hline 2119.262 & 229.655 & Surface & 2008 & 7472.958 & 220.293 & Surface & 2008 \\
\hline 2122.483 & 229.661 & Surface & 2008 & 7476.196 & 220.266 & Surface & 2008 \\
\hline 2125.703 & 229.625 & Surface & 2008 & 7479.435 & 220.278 & Surface & 2008 \\
\hline 2128.924 & 229.641 & Surface & 2008 & 7482.673 & 220.299 & Surface & 2008 \\
\hline 2132.145 & 229.666 & Surface & 2008 & 7485.911 & 220.296 & Surface & 2008 \\
\hline 2135.366 & 229.676 & Surface & 2008 & 7489.150 & 220.249 & Surface & 2008 \\
\hline 2138.587 & 229.682 & Surface & 2008 & 7492.388 & 220.247 & Surface & 2008 \\
\hline 2141.807 & 229.694 & Surface & 2008 & 7495.626 & 220.265 & Surface & 2008 \\
\hline 2145.028 & 229.714 & Surface & 2008 & 7498.865 & 220.308 & Surface & 2008 \\
\hline 2148.249 & 229.727 & Surface & 2008 & 7502.103 & 220.283 & Surface & 2008 \\
\hline 2151.470 & 229.723 & Surface & 2008 & 7505.333 & 220.250 & Surface & 2008 \\
\hline 2154.691 & 229.696 & Surface & 2008 & 7508.562 & 220.220 & Surface & 2008 \\
\hline 2157.911 & 229.653 & Surface & 2008 & 7511.791 & 220.211 & Surface & 2008 \\
\hline 2161.132 & 229.627 & Surface & 2008 & 7515.021 & 220.238 & Surface & 2008 \\
\hline 2164.353 & 229.626 & Surface & 2008 & 7518.250 & 220.242 & Surface & 2008 \\
\hline 2167.574 & 229.622 & Surface & 2008 & 7521.479 & 220.243 & Surface & 2008 \\
\hline 2170.795 & 229.682 & Surface & 2008 & 7524.709 & 220.241 & Surface & 2008 \\
\hline 2174.015 & 229.737 & Surface & 2008 & 7527.938 & 220.255 & Surface & 2008 \\
\hline 2177.236 & 229.746 & Surface & 2008 & 7531.168 & 220.256 & Surface & 2008 \\
\hline 2180.457 & 229.744 & Surface & 2008 & 7534.397 & 220.259 & Surface & 2008 \\
\hline 2183.678 & 229.726 & Surface & 2008 & 7537.626 & 220.247 & Surface & 2008 \\
\hline
\end{tabular}




\begin{tabular}{|c|c|c|c|c|c|c|c|}
\hline 2186.899 & 229.730 & Surface & 2008 & 7540.856 & 220.229 & Surface & 2008 \\
\hline 2190.084 & 229.733 & Surface & 2008 & 7544.085 & 220.233 & Surface & 2008 \\
\hline 2193.269 & 229.710 & Surface & 2008 & 7547.314 & 220.234 & Surface & 2008 \\
\hline 2196.454 & 229.710 & Surface & 2008 & 7550.544 & 220.241 & Surface & 2008 \\
\hline 2199.639 & 229.711 & Surface & 2008 & 7553.773 & 220.245 & Surface & 2008 \\
\hline 2202.824 & 229.715 & Surface & 2008 & 7557.002 & 220.264 & Surface & 2008 \\
\hline 2206.009 & 229.723 & Surface & 2008 & 7560.232 & 220.229 & Surface & 2008 \\
\hline 2209.194 & 229.723 & Surface & 2008 & 7563.461 & 220.183 & Surface & 2008 \\
\hline 2212.379 & 229.692 & Surface & 2008 & 7566.690 & 220.206 & Surface & 2008 \\
\hline 2215.563 & 229.653 & Surface & 2008 & 7569.920 & 220.241 & Surface & 2008 \\
\hline 2218.749 & 229.625 & Surface & 2008 & 7573.149 & 220.258 & Surface & 2008 \\
\hline 2221.933 & 229.675 & Surface & 2008 & 7576.379 & 220.266 & Surface & 2008 \\
\hline 2225.118 & 229.696 & Surface & 2008 & 7579.608 & 220.230 & Surface & 2008 \\
\hline 2228.303 & 229.741 & Surface & 2008 & 7582.837 & 220.210 & Surface & 2008 \\
\hline 2231.488 & 229.750 & Surface & 2008 & 7586.067 & 220.185 & Surface & 2008 \\
\hline 2234.673 & 229.750 & Surface & 2008 & 7589.296 & 220.145 & Surface & 2008 \\
\hline 2237.858 & 229.755 & Surface & 2008 & 7592.525 & 220.142 & Surface & 2008 \\
\hline 2241.043 & 229.751 & Surface & 2008 & 7595.755 & 220.170 & Surface & 2008 \\
\hline 2244.228 & 229.706 & Surface & 2008 & 7598.984 & 220.164 & Surface & 2008 \\
\hline 2247.413 & 229.698 & Surface & 2008 & 7602.204 & 220.204 & Surface & 2008 \\
\hline 2250.598 & 229.698 & Surface & 2008 & 7605.424 & 220.236 & Surface & 2008 \\
\hline 2253.783 & 229.698 & Surface & 2008 & 7608.644 & 220.258 & Surface & 2008 \\
\hline 2256.968 & 229.685 & Surface & 2008 & 7611.864 & 220.260 & Surface & 2008 \\
\hline 2260.153 & 229.673 & Surface & 2008 & 7615.084 & 220.217 & Surface & 2008 \\
\hline 2263.338 & 229.657 & Surface & 2008 & 7618.304 & 220.223 & Surface & 2008 \\
\hline 2266.523 & 229.664 & Surface & 2008 & 7621.524 & 220.226 & Surface & 2008 \\
\hline 2269.708 & 229.678 & Surface & 2008 & 7624.744 & 220.197 & Surface & 2008 \\
\hline 2272.893 & 229.693 & Surface & 2008 & 7627.964 & 220.178 & Surface & 2008 \\
\hline 2276.078 & 229.689 & Surface & 2008 & 7631.184 & 220.162 & Surface & 2008 \\
\hline 2279.263 & 229.678 & Surface & 2008 & 7634.404 & 220.178 & Surface & 2008 \\
\hline 2282.448 & 229.694 & Surface & 2008 & 7637.624 & 220.201 & Surface & 2008 \\
\hline 2285.720 & 229.715 & Surface & 2008 & 7640.843 & 220.201 & Surface & 2008 \\
\hline 2288.992 & 229.669 & Surface & 2008 & 7644.064 & 220.185 & Surface & 2008 \\
\hline 2292.263 & 229.588 & Surface & 2008 & 7647.284 & 220.182 & Surface & 2008 \\
\hline 2295.535 & 229.572 & Surface & 2008 & 7650.504 & 220.168 & Surface & 2008 \\
\hline 2298.807 & 229.600 & Surface & 2008 & 7653.723 & 220.152 & Surface & 2008 \\
\hline 2302.079 & 229.616 & Surface & 2008 & 7656.944 & 220.165 & Surface & 2008 \\
\hline 2305.351 & 229.618 & Surface & 2008 & 7660.163 & 220.232 & Surface & 2008 \\
\hline 2308.623 & 229.610 & Surface & 2008 & 7663.383 & 220.221 & Surface & 2008 \\
\hline 2311.895 & 229.607 & Surface & 2008 & 7666.603 & 220.166 & Surface & 2008 \\
\hline 2315.167 & 229.609 & Surface & 2008 & 7669.855 & 220.167 & Surface & 2008 \\
\hline 2318.439 & 229.614 & Surface & 2008 & 7673.106 & 220.168 & Surface & 2008 \\
\hline 2321.711 & 229.633 & Surface & 2008 & 7676.358 & 220.162 & Surface & 2008 \\
\hline 2324.983 & 229.653 & Surface & 2008 & 7679.609 & 220.140 & Surface & 2008 \\
\hline 2328.255 & 229.659 & Surface & 2008 & 7682.861 & 220.123 & Surface & 2008 \\
\hline 2331.527 & 229.653 & Surface & 2008 & 7686.112 & 220.132 & Surface & 2008 \\
\hline 2334.798 & 229.647 & Surface & 2008 & 7689.364 & 220.130 & Surface & 2008 \\
\hline 2338.070 & 229.654 & Surface & 2008 & 7692.615 & 220.102 & Surface & 2008 \\
\hline 2341.342 & 229.668 & Surface & 2008 & 7695.867 & 220.078 & Surface & 2008 \\
\hline
\end{tabular}




\begin{tabular}{|c|c|c|c|c|c|c|c|}
\hline 2344.614 & 229.663 & Surface & 2008 & 7699.118 & 220.024 & Surface & 2008 \\
\hline 2347.886 & 229.624 & Surface & 2008 & 7702.369 & 220.056 & Surface & 2008 \\
\hline 2351.158 & 229.577 & Surface & 2008 & 7705.621 & 220.098 & Surface & 2008 \\
\hline 2354.430 & 229.550 & Surface & 2008 & 7708.873 & 220.077 & Surface & 2008 \\
\hline 2357.702 & 229.615 & Surface & 2008 & 7712.124 & 220.044 & Surface & 2008 \\
\hline 2360.973 & 229.706 & Surface & 2008 & 7715.375 & 220.063 & Surface & 2008 \\
\hline 2364.245 & 229.719 & Surface & 2008 & 7718.627 & 220.041 & Surface & 2008 \\
\hline 2367.517 & 229.709 & Surface & 2008 & 7721.878 & 220.011 & Surface & 2008 \\
\hline 2370.714 & 229.701 & Surface & 2008 & 7725.130 & 220.008 & Surface & 2008 \\
\hline 2373.910 & 229.676 & Surface & 2008 & 7728.381 & 219.939 & Surface & 2008 \\
\hline 2377.106 & 229.665 & Surface & 2008 & 7731.633 & 219.981 & Surface & 2008 \\
\hline 2380.302 & 229.667 & Surface & 2008 & 7734.884 & 220.031 & Surface & 2008 \\
\hline 2383.498 & 229.678 & Surface & 2008 & 7738.136 & 220.013 & Surface & 2008 \\
\hline 2386.694 & 229.689 & Surface & 2008 & 7741.387 & 220.025 & Surface & 2008 \\
\hline 2389.891 & 229.676 & Surface & 2008 & 7744.639 & 220.026 & Surface & 2008 \\
\hline 2393.087 & 229.666 & Surface & 2008 & 7747.890 & 220.029 & Surface & 2008 \\
\hline 2396.283 & 229.689 & Surface & 2008 & 7751.142 & 220.046 & Surface & 2008 \\
\hline 2399.479 & 229.687 & Surface & 2008 & 7754.393 & 220.015 & Surface & 2008 \\
\hline 2402.675 & 229.686 & Surface & 2008 & 7757.599 & 219.996 & Surface & 2008 \\
\hline 2405.872 & 229.684 & Surface & 2008 & 7760.805 & 219.992 & Surface & 2008 \\
\hline 2409.068 & 229.682 & Surface & 2008 & 7764.011 & 220.014 & Surface & 2008 \\
\hline 2412.264 & 229.666 & Surface & 2008 & 7767.218 & 220.028 & Surface & 2008 \\
\hline 2415.460 & 229.598 & Surface & 2008 & 7770.424 & 219.979 & Surface & 2008 \\
\hline 2418.656 & 229.572 & Surface & 2008 & 7773.630 & 219.948 & Surface & 2008 \\
\hline 2421.853 & 229.601 & Surface & 2008 & 7776.836 & 219.841 & Surface & 2008 \\
\hline 2425.049 & 229.652 & Surface & 2008 & 7780.042 & 219.913 & Surface & 2008 \\
\hline 2428.245 & 229.717 & Surface & 2008 & 7783.248 & 219.946 & Surface & 2008 \\
\hline 2431.441 & 229.729 & Surface & 2008 & 7786.455 & 219.950 & Surface & 2008 \\
\hline 2434.638 & 229.709 & Surface & 2008 & 7789.661 & 219.909 & Surface & 2008 \\
\hline 2437.834 & 229.697 & Surface & 2008 & 7792.867 & 219.899 & Surface & 2008 \\
\hline 2441.030 & 229.681 & Surface & 2008 & 7796.073 & 219.867 & Surface & 2008 \\
\hline 2444.226 & 229.661 & Surface & 2008 & 7799.279 & 219.842 & Surface & 2008 \\
\hline 2447.422 & 229.626 & Surface & 2008 & 7802.485 & 219.848 & Surface & 2008 \\
\hline 2450.619 & 229.587 & Surface & 2008 & 7805.691 & 219.818 & Surface & 2008 \\
\hline 2453.815 & 229.551 & Surface & 2008 & 7808.898 & 219.761 & Surface & 2008 \\
\hline 2457.011 & 229.537 & Surface & 2008 & 7812.104 & 219.730 & Surface & 2008 \\
\hline 2460.207 & 229.508 & Surface & 2008 & 7815.243 & 219.725 & Surface & 2008 \\
\hline 2463.403 & 229.491 & Surface & 2008 & 7818.383 & 219.730 & Surface & 2008 \\
\hline 2466.600 & 229.497 & Surface & 2008 & 7821.523 & 219.763 & Surface & 2008 \\
\hline 2469.796 & 229.527 & Surface & 2008 & 7824.663 & 219.777 & Surface & 2008 \\
\hline 2472.992 & 229.548 & Surface & 2008 & 7827.802 & 219.761 & Surface & 2008 \\
\hline 2476.188 & 229.541 & Surface & 2008 & 7830.942 & 219.746 & Surface & 2008 \\
\hline 2479.385 & 229.570 & Surface & 2008 & 7834.081 & 219.735 & Surface & 2008 \\
\hline 2482.581 & 229.608 & Surface & 2008 & 7837.221 & 219.711 & Surface & 2008 \\
\hline 2485.777 & 229.567 & Surface & 2008 & 7840.361 & 219.620 & Surface & 2008 \\
\hline 2489.037 & 229.505 & Surface & 2008 & 7843.500 & 219.630 & Surface & 2008 \\
\hline 2492.297 & 229.480 & Surface & 2008 & 7846.640 & 219.617 & Surface & 2008 \\
\hline 2495.557 & 229.524 & Surface & 2008 & 7849.780 & 219.588 & Surface & 2008 \\
\hline 2498.818 & 229.577 & Surface & 2008 & 7852.919 & 219.559 & Surface & 2008 \\
\hline
\end{tabular}




\begin{tabular}{|c|c|c|c|c|c|c|c|}
\hline 2502.078 & 229.556 & Surface & 2008 & 7856.059 & 219.545 & Surface & 2008 \\
\hline 2505.337 & 229.550 & Surface & 2008 & 7859.285 & 219.492 & Surface & 2008 \\
\hline 2508.598 & 229.590 & Surface & 2008 & 7862.512 & 219.396 & Surface & 2008 \\
\hline 2511.858 & 229.556 & Surface & 2008 & 7865.739 & 219.364 & Surface & 2008 \\
\hline 2515.118 & 229.493 & Surface & 2008 & 7868.965 & 219.372 & Surface & 2008 \\
\hline 2518.378 & 229.508 & Surface & 2008 & 7872.192 & 219.338 & Surface & 2008 \\
\hline 2521.638 & 229.529 & Surface & 2008 & 7875.418 & 219.249 & Surface & 2008 \\
\hline 2524.898 & 229.533 & Surface & 2008 & 7878.645 & 219.178 & Surface & 2008 \\
\hline 2528.158 & 229.566 & Surface & 2008 & 7881.872 & 219.192 & Surface & 2008 \\
\hline 2531.418 & 229.596 & Surface & 2008 & 7885.098 & 219.254 & Surface & 2008 \\
\hline 2534.679 & 229.501 & Surface & 2008 & 7888.325 & 219.244 & Surface & 2008 \\
\hline 2537.939 & 229.446 & Surface & 2008 & 7891.551 & 219.181 & Surface & 2008 \\
\hline 2541.199 & 229.479 & Surface & 2008 & 7894.778 & 219.121 & Surface & 2008 \\
\hline 2544.459 & 229.531 & Surface & 2008 & 7898.005 & 219.079 & Surface & 2008 \\
\hline 2547.719 & 229.553 & Surface & 2008 & 7901.231 & 219.072 & Surface & 2008 \\
\hline 2550.979 & 229.555 & Surface & 2008 & 7904.295 & 219.077 & Surface & 2008 \\
\hline 2554.239 & 229.518 & Surface & 2008 & 7907.360 & 219.095 & Surface & 2008 \\
\hline 2557.500 & 229.544 & Surface & 2008 & 7910.424 & 219.062 & Surface & 2008 \\
\hline 2560.760 & 229.562 & Surface & 2008 & 7913.489 & 219.092 & Surface & 2008 \\
\hline 2564.020 & 229.550 & Surface & 2008 & 7916.553 & 219.108 & Surface & 2008 \\
\hline 2567.280 & 229.554 & Surface & 2008 & 7919.617 & 219.010 & Surface & 2008 \\
\hline 2570.540 & 229.578 & Surface & 2008 & 7922.682 & 218.999 & Surface & 2008 \\
\hline 2573.800 & 229.556 & Surface & 2008 & 7925.746 & 219.004 & Surface & 2008 \\
\hline 2577.060 & 229.523 & Surface & 2008 & 7928.811 & 219.033 & Surface & 2008 \\
\hline 2580.320 & 229.511 & Surface & 2008 & 7931.875 & 219.026 & Surface & 2008 \\
\hline 2583.581 & 229.496 & Surface & 2008 & 7934.939 & 218.973 & Surface & 2008 \\
\hline 2586.841 & 229.493 & Surface & 2008 & 7938.004 & 218.922 & Surface & 2008 \\
\hline 2590.101 & 229.449 & Surface & 2008 & 7941.068 & 218.930 & Surface & 2008 \\
\hline 2593.361 & 229.534 & Surface & 2008 & 7944.132 & 218.971 & Surface & 2008 \\
\hline 2596.621 & 229.497 & Surface & 2008 & 7947.197 & 218.924 & Surface & 2008 \\
\hline 2599.881 & 229.550 & Surface & 2008 & 7950.361 & 218.873 & Surface & 2008 \\
\hline 2603.141 & 229.570 & Surface & 2008 & 7953.524 & 218.872 & Surface & 2008 \\
\hline 2606.360 & 229.535 & Surface & 2008 & 7956.688 & 218.869 & Surface & 2008 \\
\hline 2609.579 & 229.473 & Surface & 2008 & 7959.852 & 218.831 & Surface & 2008 \\
\hline 2612.798 & 229.474 & Surface & 2008 & 7963.016 & 218.768 & Surface & 2008 \\
\hline 2616.017 & 229.441 & Surface & 2008 & 7966.180 & 218.705 & Surface & 2008 \\
\hline 2619.236 & 229.377 & Surface & 2008 & 7969.344 & 218.687 & Surface & 2008 \\
\hline 2622.455 & 229.362 & Surface & 2008 & 7972.507 & 218.673 & Surface & 2008 \\
\hline 2625.674 & 229.441 & Surface & 2008 & 7975.671 & 218.564 & Surface & 2008 \\
\hline 2628.893 & 229.443 & Surface & 2008 & 7978.789 & 218.539 & Surface & 2008 \\
\hline 2632.111 & 229.446 & Surface & 2008 & 7981.907 & 218.483 & Surface & 2008 \\
\hline 2635.330 & 229.463 & Surface & 2008 & 7985.024 & 218.459 & Surface & 2008 \\
\hline 2638.549 & 229.427 & Surface & 2008 & 7988.142 & 218.476 & Surface & 2008 \\
\hline 2641.768 & 229.369 & Surface & 2008 & 7991.260 & 218.493 & Surface & 2008 \\
\hline 2644.987 & 229.341 & Surface & 2008 & 7994.377 & 218.514 & Surface & 2008 \\
\hline 2648.206 & 229.346 & Surface & 2008 & 7997.495 & 218.550 & Surface & 2008 \\
\hline 2651.425 & 229.376 & Surface & 2008 & 8000.613 & 218.585 & Surface & 2008 \\
\hline 2654.644 & 229.410 & Surface & 2008 & 8003.730 & 218.562 & Surface & 2008 \\
\hline 2657.863 & 229.387 & Surface & 2008 & 8006.848 & 218.476 & Surface & 2008 \\
\hline
\end{tabular}




\begin{tabular}{|c|c|c|c|c|c|c|c|}
\hline 2661.082 & 229.364 & Surface & 2008 & 8009.966 & 218.438 & Surface & 2008 \\
\hline 2664.300 & 229.360 & Surface & 2008 & 8013.084 & 218.479 & Surface & 2008 \\
\hline 2667.520 & 229.374 & Surface & 2008 & 8016.201 & 218.519 & Surface & 2008 \\
\hline 2670.738 & 229.369 & Surface & 2008 & 8019.216 & 218.515 & Surface & 2008 \\
\hline 2673.957 & 229.340 & Surface & 2008 & 8022.232 & 218.506 & Surface & 2008 \\
\hline 2677.176 & 229.298 & Surface & 2008 & 8025.247 & 218.475 & Surface & 2008 \\
\hline 2680.395 & 229.266 & Surface & 2008 & 8028.262 & 218.446 & Surface & 2008 \\
\hline 2683.614 & 229.277 & Surface & 2008 & 8031.278 & 218.439 & Surface & 2008 \\
\hline 2686.833 & 229.294 & Surface & 2008 & 8034.293 & 218.463 & Surface & 2008 \\
\hline 2690.052 & 229.283 & Surface & 2008 & 8037.308 & 218.475 & Surface & 2008 \\
\hline 2693.271 & 229.271 & Surface & 2008 & 8040.324 & 218.443 & Surface & 2008 \\
\hline 2696.490 & 229.237 & Surface & 2008 & 8043.339 & 218.427 & Surface & 2008 \\
\hline 2699.709 & 229.205 & Surface & 2008 & 8046.354 & 218.422 & Surface & 2008 \\
\hline 2702.928 & 229.189 & Surface & 2008 & 8049.370 & 218.410 & Surface & 2008 \\
\hline 2706.147 & 229.237 & Surface & 2008 & 8052.483 & 218.371 & Surface & 2008 \\
\hline 2709.366 & 229.303 & Surface & 2008 & 8055.597 & 218.374 & Surface & 2008 \\
\hline 2712.585 & 229.291 & Surface & 2008 & 8058.710 & 218.325 & Surface & 2008 \\
\hline 2715.804 & 229.220 & Surface & 2008 & 8061.823 & 218.338 & Surface & 2008 \\
\hline 2719.022 & 229.153 & Surface & 2008 & 8064.937 & 218.372 & Surface & 2008 \\
\hline 2722.241 & 229.173 & Surface & 2008 & 8068.050 & 218.424 & Surface & 2008 \\
\hline 2725.460 & 229.202 & Surface & 2008 & 8071.164 & 218.470 & Surface & 2008 \\
\hline 2728.679 & 229.172 & Surface & 2008 & 8074.277 & 218.471 & Surface & 2008 \\
\hline 2731.898 & 229.179 & Surface & 2008 & 8077.390 & 218.441 & Surface & 2008 \\
\hline 2735.117 & 229.192 & Surface & 2008 & 8080.504 & 218.421 & Surface & 2008 \\
\hline 2738.336 & 229.137 & Surface & 2008 & 8083.617 & 218.411 & Surface & 2008 \\
\hline 2741.555 & 229.170 & Surface & 2008 & 8086.731 & 218.385 & Surface & 2008 \\
\hline 2744.774 & 229.148 & Surface & 2008 & 8089.844 & 218.401 & Surface & 2008 \\
\hline 2747.993 & 229.137 & Surface & 2008 & 8092.957 & 218.485 & Surface & 2008 \\
\hline 2751.211 & 229.088 & Surface & 2008 & 8096.071 & 218.527 & Surface & 2008 \\
\hline 2754.430 & 229.079 & Surface & 2008 & 8099.184 & 218.496 & Surface & 2008 \\
\hline 2757.649 & 229.099 & Surface & 2008 & 8102.298 & 218.437 & Surface & 2008 \\
\hline 2760.868 & 229.096 & Surface & 2008 & 8105.411 & 218.431 & Surface & 2008 \\
\hline 2764.087 & 229.085 & Surface & 2008 & 8108.675 & 218.431 & Surface & 2008 \\
\hline 2767.306 & 229.016 & Surface & 2008 & 8111.938 & 218.429 & Surface & 2008 \\
\hline 2770.525 & 229.033 & Surface & 2008 & 8115.202 & 218.453 & Surface & 2008 \\
\hline 2773.744 & 229.096 & Surface & 2008 & 8118.466 & 218.436 & Surface & 2008 \\
\hline 2776.963 & 229.118 & Surface & 2008 & 8121.729 & 218.415 & Surface & 2008 \\
\hline 2780.182 & 229.137 & Surface & 2008 & 8124.993 & 218.402 & Surface & 2008 \\
\hline 2783.401 & 229.152 & Surface & 2008 & 8128.257 & 218.407 & Surface & 2008 \\
\hline 2786.619 & 229.182 & Surface & 2008 & 8131.521 & 218.351 & Surface & 2008 \\
\hline 2789.742 & 229.161 & Surface & 2008 & 8134.784 & 218.309 & Surface & 2008 \\
\hline 2792.865 & 229.133 & Surface & 2008 & 8138.048 & 218.329 & Surface & 2008 \\
\hline 2795.988 & 229.095 & Surface & 2008 & 8141.312 & 218.317 & Surface & 2008 \\
\hline 2799.111 & 229.005 & Surface & 2008 & 8144.575 & 218.300 & Surface & 2008 \\
\hline 2802.233 & 228.980 & Surface & 2008 & 8147.839 & 218.322 & Surface & 2008 \\
\hline 2805.356 & 229.012 & Surface & 2008 & 8151.103 & 218.320 & Surface & 2008 \\
\hline 2808.479 & 229.020 & Surface & 2008 & 8154.366 & 218.257 & Surface & 2008 \\
\hline 2811.602 & 229.016 & Surface & 2008 & 8157.630 & 218.278 & Surface & 2008 \\
\hline 2814.725 & 229.003 & Surface & 2008 & 8160.894 & 218.318 & Surface & 2008 \\
\hline
\end{tabular}




\begin{tabular}{|c|c|c|c|c|c|c|c|}
\hline 2817.848 & 229.012 & Surface & 2008 & 8164.158 & 218.355 & Surface & 2008 \\
\hline 2820.970 & 228.989 & Surface & 2008 & 8167.414 & 218.333 & Surface & 2008 \\
\hline 2824.093 & 228.976 & Surface & 2008 & 8170.671 & 218.290 & Surface & 2008 \\
\hline 2827.216 & 228.970 & Surface & 2008 & 8173.928 & 218.286 & Surface & 2008 \\
\hline 2830.339 & 228.946 & Surface & 2008 & 8177.185 & 218.305 & Surface & 2008 \\
\hline 2833.462 & 228.917 & Surface & 2008 & 8180.442 & 218.259 & Surface & 2008 \\
\hline 2836.584 & 228.890 & Surface & 2008 & 8183.698 & 218.266 & Surface & 2008 \\
\hline 2839.707 & 228.865 & Surface & 2008 & 8186.955 & 218.270 & Surface & 2008 \\
\hline 2842.830 & 228.817 & Surface & 2008 & 8190.212 & 218.223 & Surface & 2008 \\
\hline 2845.953 & 228.809 & Surface & 2008 & 8193.469 & 218.160 & Surface & 2008 \\
\hline 2849.060 & 228.847 & Surface & 2008 & 8196.725 & 218.167 & Surface & 2008 \\
\hline 2852.167 & 228.888 & Surface & 2008 & 8199.982 & 218.223 & Surface & 2008 \\
\hline 2855.274 & 228.878 & Surface & 2008 & 8203.239 & 218.238 & Surface & 2008 \\
\hline 2858.381 & 228.835 & Surface & 2008 & 8206.496 & 218.235 & Surface & 2008 \\
\hline 2861.489 & 228.835 & Surface & 2008 & 8209.753 & 218.255 & Surface & 2008 \\
\hline 2864.596 & 228.841 & Surface & 2008 & 8213.009 & 218.265 & Surface & 2008 \\
\hline 2867.703 & 228.840 & Surface & 2008 & 8216.266 & 218.204 & Surface & 2008 \\
\hline 2870.810 & 228.867 & Surface & 2008 & 8219.523 & 218.216 & Surface & 2008 \\
\hline 2873.917 & 228.900 & Surface & 2008 & 8222.758 & 218.246 & Surface & 2008 \\
\hline 2877.024 & 228.900 & Surface & 2008 & 8225.993 & 218.273 & Surface & 2008 \\
\hline 2880.132 & 228.878 & Surface & 2008 & 8229.228 & 218.240 & Surface & 2008 \\
\hline 2883.239 & 228.841 & Surface & 2008 & 8232.463 & 218.184 & Surface & 2008 \\
\hline 2886.346 & 228.838 & Surface & 2008 & 8235.698 & 218.191 & Surface & 2008 \\
\hline 2889.453 & 228.841 & Surface & 2008 & 8238.933 & 218.172 & Surface & 2008 \\
\hline 2892.560 & 228.799 & Surface & 2008 & 8242.168 & 218.177 & Surface & 2008 \\
\hline 2895.668 & 228.780 & Surface & 2008 & 8245.403 & 218.196 & Surface & 2008 \\
\hline 2898.889 & 228.779 & Surface & 2008 & 8248.638 & 218.173 & Surface & 2008 \\
\hline 2902.110 & 228.787 & Surface & 2008 & 8251.873 & 218.105 & Surface & 2008 \\
\hline 2905.331 & 228.817 & Surface & 2008 & 8255.108 & 218.146 & Surface & 2008 \\
\hline 2908.552 & 228.842 & Surface & 2008 & 8258.343 & 218.257 & Surface & 2008 \\
\hline 2911.773 & 228.861 & Surface & 2008 & 8261.579 & 218.220 & Surface & 2008 \\
\hline 2914.995 & 228.863 & Surface & 2008 & 8264.814 & 218.174 & Surface & 2008 \\
\hline 2918.216 & 228.860 & Surface & 2008 & 8268.048 & 218.182 & Surface & 2008 \\
\hline 2921.437 & 228.864 & Surface & 2008 & 8271.284 & 218.215 & Surface & 2008 \\
\hline 2924.658 & 228.820 & Surface & 2008 & 8274.519 & 218.218 & Surface & 2008 \\
\hline 2927.879 & 228.809 & Surface & 2008 & 8277.754 & 218.192 & Surface & 2008 \\
\hline 2931.100 & 228.848 & Surface & 2008 & 8280.989 & 218.187 & Surface & 2008 \\
\hline 2934.322 & 228.884 & Surface & 2008 & 8284.224 & 218.177 & Surface & 2008 \\
\hline 2937.543 & 228.899 & Surface & 2008 & 8287.459 & 218.163 & Surface & 2008 \\
\hline 2940.764 & 228.884 & Surface & 2008 & 8290.694 & 218.147 & Surface & 2008 \\
\hline 2943.985 & 228.912 & Surface & 2008 & 8293.929 & 218.162 & Surface & 2008 \\
\hline 2947.206 & 228.875 & Surface & 2008 & 8297.164 & 218.149 & Surface & 2008 \\
\hline 2950.427 & 228.818 & Surface & 2008 & 8300.399 & 218.132 & Surface & 2008 \\
\hline 2953.649 & 228.802 & Surface & 2008 & 8303.634 & 218.189 & Surface & 2008 \\
\hline 2956.870 & 228.851 & Surface & 2008 & 8306.869 & 218.157 & Surface & 2008 \\
\hline 2960.091 & 228.873 & Surface & 2008 & 8310.104 & 218.125 & Surface & 2008 \\
\hline 2963.285 & 228.860 & Surface & 2008 & 8313.339 & 218.118 & Surface & 2008 \\
\hline 2966.478 & 228.869 & Surface & 2008 & 8316.574 & 218.137 & Surface & 2008 \\
\hline 2969.672 & 228.872 & Surface & 2008 & 8319.823 & 218.133 & Surface & 2008 \\
\hline
\end{tabular}




\begin{tabular}{|c|c|c|c|c|c|c|c|}
\hline 2972.865 & 228.854 & Surface & 2008 & 8323.071 & 218.123 & Surface & 2008 \\
\hline 2976.059 & 228.811 & Surface & 2008 & 8326.320 & 218.101 & Surface & 2008 \\
\hline 2979.252 & 228.763 & Surface & 2008 & 8329.569 & 218.026 & Surface & 2008 \\
\hline 2982.446 & 228.786 & Surface & 2008 & 8332.817 & 218.010 & Surface & 2008 \\
\hline 2985.639 & 228.812 & Surface & 2008 & 8336.066 & 218.049 & Surface & 2008 \\
\hline 2988.833 & 228.823 & Surface & 2008 & 8339.314 & 218.081 & Surface & 2008 \\
\hline 2992.026 & 228.850 & Surface & 2008 & 8342.563 & 218.103 & Surface & 2008 \\
\hline 2995.220 & 228.861 & Surface & 2008 & 8345.811 & 218.104 & Surface & 2008 \\
\hline 2998.414 & 228.852 & Surface & 2008 & 8349.060 & 218.104 & Surface & 2008 \\
\hline 3001.607 & 228.869 & Surface & 2008 & 8352.309 & 218.093 & Surface & 2008 \\
\hline 3004.801 & 228.893 & Surface & 2008 & 8355.557 & 218.045 & Surface & 2008 \\
\hline 3007.994 & 228.869 & Surface & 2008 & 8358.806 & 218.037 & Surface & 2008 \\
\hline 3011.188 & 228.847 & Surface & 2008 & 8362.054 & 218.035 & Surface & 2008 \\
\hline 3014.381 & 228.859 & Surface & 2008 & 8365.303 & 218.046 & Surface & 2008 \\
\hline 3017.575 & 228.852 & Surface & 2008 & 8368.551 & 218.050 & Surface & 2008 \\
\hline 3020.768 & 228.870 & Surface & 2008 & 8371.800 & 218.070 & Surface & 2008 \\
\hline 3023.962 & 228.870 & Surface & 2008 & 8375.048 & 218.026 & Surface & 2008 \\
\hline 3027.155 & 228.853 & Surface & 2008 & 8378.297 & 218.011 & Surface & 2008 \\
\hline 3030.349 & 228.850 & Surface & 2008 & 8381.546 & 218.001 & Surface & 2008 \\
\hline 3033.542 & 228.853 & Surface & 2008 & 8384.794 & 217.985 & Surface & 2008 \\
\hline 3036.736 & 228.860 & Surface & 2008 & 8388.042 & 217.976 & Surface & 2008 \\
\hline 3039.991 & 228.863 & Surface & 2008 & 8391.291 & 217.967 & Surface & 2008 \\
\hline 3043.246 & 228.881 & Surface & 2008 & 8394.540 & 217.954 & Surface & 2008 \\
\hline 3046.501 & 228.894 & Surface & 2008 & 8397.788 & 217.946 & Surface & 2008 \\
\hline 3049.756 & 228.895 & Surface & 2008 & 8401.037 & 217.921 & Surface & 2008 \\
\hline 3053.011 & 228.877 & Surface & 2008 & 8404.286 & 217.902 & Surface & 2008 \\
\hline 3056.266 & 228.863 & Surface & 2008 & 8407.534 & 217.883 & Surface & 2008 \\
\hline 3059.522 & 228.844 & Surface & 2008 & 8410.791 & 217.873 & Surface & 2008 \\
\hline 3062.776 & 228.801 & Surface & 2008 & 8414.049 & 217.880 & Surface & 2008 \\
\hline 3066.032 & 228.788 & Surface & 2008 & 8417.306 & 217.932 & Surface & 2008 \\
\hline 3069.287 & 228.839 & Surface & 2008 & 8420.563 & 218.043 & Surface & 2008 \\
\hline 3072.542 & 228.873 & Surface & 2008 & 8423.820 & 218.056 & Surface & 2008 \\
\hline 3075.797 & 228.864 & Surface & 2008 & 8427.078 & 218.082 & Surface & 2008 \\
\hline 3079.052 & 228.837 & Surface & 2008 & 8430.335 & 218.077 & Surface & 2008 \\
\hline 3082.307 & 228.826 & Surface & 2008 & 8433.592 & 218.051 & Surface & 2008 \\
\hline 3085.562 & 228.784 & Surface & 2008 & 8436.849 & 218.020 & Surface & 2008 \\
\hline 3088.817 & 228.752 & Surface & 2008 & 8440.106 & 217.985 & Surface & 2008 \\
\hline 3092.072 & 228.851 & Surface & 2008 & 8443.364 & 217.955 & Surface & 2008 \\
\hline 3095.327 & 228.863 & Surface & 2008 & 8446.621 & 217.956 & Surface & 2008 \\
\hline 3098.582 & 228.857 & Surface & 2008 & 8449.878 & 217.983 & Surface & 2008 \\
\hline 3101.837 & 228.841 & Surface & 2008 & 8453.136 & 218.029 & Surface & 2008 \\
\hline 3105.092 & 228.857 & Surface & 2008 & 8456.393 & 217.933 & Surface & 2008 \\
\hline 3108.347 & 228.889 & Surface & 2008 & 8459.650 & 217.880 & Surface & 2008 \\
\hline 3111.602 & 228.885 & Surface & 2008 & 8462.908 & 217.874 & Surface & 2008 \\
\hline 3114.857 & 228.839 & Surface & 2008 & 8466.165 & 217.905 & Surface & 2008 \\
\hline 3118.113 & 228.799 & Surface & 2008 & 8469.422 & 217.926 & Surface & 2008 \\
\hline 3121.367 & 228.794 & Surface & 2008 & 8472.679 & 217.921 & Surface & 2008 \\
\hline 3124.623 & 228.818 & Surface & 2008 & 8475.936 & 217.917 & Surface & 2008 \\
\hline 3127.878 & 228.857 & Surface & 2008 & 8479.194 & 217.966 & Surface & 2008 \\
\hline
\end{tabular}




\begin{tabular}{|c|c|c|c|c|c|c|c|}
\hline 3131.121 & 228.868 & Surface & 2008 & 8482.451 & 217.931 & Surface & 2008 \\
\hline 3134.365 & 228.849 & Surface & 2008 & 8485.708 & 217.933 & Surface & 2008 \\
\hline 3137.609 & 228.833 & Surface & 2008 & 8488.966 & 217.955 & Surface & 2008 \\
\hline 3140.853 & 228.822 & Surface & 2008 & 8492.223 & 217.901 & Surface & 2008 \\
\hline 3144.097 & 228.810 & Surface & 2008 & 8495.480 & 217.859 & Surface & 2008 \\
\hline 3147.340 & 228.811 & Surface & 2008 & 8498.737 & 217.856 & Surface & 2008 \\
\hline 3150.585 & 228.817 & Surface & 2008 & 8501.995 & 217.848 & Surface & 2008 \\
\hline 3153.828 & 228.843 & Surface & 2008 & 8505.252 & 217.819 & Surface & 2008 \\
\hline 3157.072 & 228.894 & Surface & 2008 & 8508.509 & 217.750 & Surface & 2008 \\
\hline 3160.316 & 228.880 & Surface & 2008 & 8511.766 & 217.701 & Surface & 2008 \\
\hline 3163.560 & 228.869 & Surface & 2008 & 8515.024 & 217.696 & Surface & 2008 \\
\hline 3166.804 & 228.847 & Surface & 2008 & 8518.281 & 217.693 & Surface & 2008 \\
\hline 3170.047 & 228.862 & Surface & 2008 & 8521.538 & 217.658 & Surface & 2008 \\
\hline 3173.291 & 228.805 & Surface & 2008 & 8524.795 & 217.622 & Surface & 2008 \\
\hline 3176.535 & 228.757 & Surface & 2008 & 8528.075 & 217.596 & Surface & 2008 \\
\hline 3179.779 & 228.719 & Surface & 2008 & 8531.354 & 217.621 & Surface & 2008 \\
\hline 3183.023 & 228.788 & Surface & 2008 & 8534.633 & 217.628 & Surface & 2008 \\
\hline 3186.266 & 228.845 & Surface & 2008 & 8537.913 & 217.613 & Surface & 2008 \\
\hline 3189.510 & 228.841 & Surface & 2008 & 8541.192 & 217.625 & Surface & 2008 \\
\hline 3192.754 & 228.831 & Surface & 2008 & 8544.471 & 217.656 & Surface & 2008 \\
\hline 3195.998 & 228.844 & Surface & 2008 & 8547.751 & 217.687 & Surface & 2008 \\
\hline 3199.242 & 228.841 & Surface & 2008 & 8551.030 & 217.684 & Surface & 2008 \\
\hline 3202.486 & 228.829 & Surface & 2008 & 8554.309 & 217.677 & Surface & 2008 \\
\hline 3205.730 & 228.809 & Surface & 2008 & 8557.589 & 217.687 & Surface & 2008 \\
\hline 3208.973 & 228.771 & Surface & 2008 & 8560.868 & 217.680 & Surface & 2008 \\
\hline 3212.217 & 228.746 & Surface & 2008 & 8564.147 & 217.668 & Surface & 2008 \\
\hline 3215.461 & 228.719 & Surface & 2008 & 8567.427 & 217.641 & Surface & 2008 \\
\hline 3218.737 & 228.739 & Surface & 2008 & 8570.706 & 217.622 & Surface & 2008 \\
\hline 3222.013 & 228.816 & Surface & 2008 & 8573.985 & 217.607 & Surface & 2008 \\
\hline 3225.288 & 228.859 & Surface & 2008 & 8577.265 & 217.579 & Surface & 2008 \\
\hline 3228.564 & 228.847 & Surface & 2008 & 8580.544 & 217.567 & Surface & 2008 \\
\hline 3231.840 & 228.787 & Surface & 2008 & 8583.823 & 217.558 & Surface & 2008 \\
\hline 3235.115 & 228.763 & Surface & 2008 & 8587.024 & 217.576 & Surface & 2008 \\
\hline 3238.391 & 228.770 & Surface & 2008 & 8590.224 & 217.608 & Surface & 2008 \\
\hline 3241.667 & 228.773 & Surface & 2008 & 8593.424 & 217.615 & Surface & 2008 \\
\hline 3244.942 & 228.792 & Surface & 2008 & 8596.624 & 217.597 & Surface & 2008 \\
\hline 3248.218 & 228.819 & Surface & 2008 & 8599.824 & 217.593 & Surface & 2008 \\
\hline 3251.494 & 228.808 & Surface & 2008 & 8603.025 & 217.613 & Surface & 2008 \\
\hline 3254.769 & 228.795 & Surface & 2008 & 8606.225 & 217.644 & Surface & 2008 \\
\hline 3258.045 & 228.777 & Surface & 2008 & 8609.425 & 217.681 & Surface & 2008 \\
\hline 3261.320 & 228.748 & Surface & 2008 & 8612.626 & 217.655 & Surface & 2008 \\
\hline 3264.596 & 228.727 & Surface & 2008 & 8615.826 & 217.595 & Surface & 2008 \\
\hline 3267.872 & 228.714 & Surface & 2008 & 8619.026 & 217.577 & Surface & 2008 \\
\hline 3271.147 & 228.697 & Surface & 2008 & 8622.227 & 217.637 & Surface & 2008 \\
\hline 3274.423 & 228.666 & Surface & 2008 & 8625.427 & 217.648 & Surface & 2008 \\
\hline 3277.699 & 228.669 & Surface & 2008 & 8628.627 & 217.644 & Surface & 2008 \\
\hline 3280.975 & 228.715 & Surface & 2008 & 8631.907 & 217.666 & Surface & 2008 \\
\hline 3284.251 & 228.773 & Surface & 2008 & 8635.187 & 217.680 & Surface & 2008 \\
\hline 3287.526 & 228.811 & Surface & 2008 & 8638.467 & 217.666 & Surface & 2008 \\
\hline
\end{tabular}




\begin{tabular}{|c|c|c|c|c|c|c|c|}
\hline 3290.802 & 228.798 & Surface & 2008 & 8641.747 & 217.639 & Surface & 2008 \\
\hline 3294.078 & 228.792 & Surface & 2008 & 8645.027 & 217.625 & Surface & 2008 \\
\hline 3297.353 & 228.772 & Surface & 2008 & 8648.307 & 217.639 & Surface & 2008 \\
\hline 3300.545 & 228.771 & Surface & 2008 & 8651.587 & 217.645 & Surface & 2008 \\
\hline 3303.738 & 228.791 & Surface & 2008 & 8654.867 & 217.646 & Surface & 2008 \\
\hline 3306.930 & 228.766 & Surface & 2008 & 8658.147 & 217.654 & Surface & 2008 \\
\hline 3310.123 & 228.739 & Surface & 2008 & 8661.427 & 217.634 & Surface & 2008 \\
\hline 3313.315 & 228.767 & Surface & 2008 & 8664.707 & 217.524 & Surface & 2008 \\
\hline 3316.508 & 228.785 & Surface & 2008 & 8667.987 & 217.553 & Surface & 2008 \\
\hline 3319.700 & 228.750 & Surface & 2008 & 8671.204 & 217.602 & Surface & 2008 \\
\hline 3322.893 & 228.700 & Surface & 2008 & 8674.420 & 217.650 & Surface & 2008 \\
\hline 3326.085 & 228.684 & Surface & 2008 & 8677.636 & 217.657 & Surface & 2008 \\
\hline 3329.277 & 228.714 & Surface & 2008 & 8680.852 & 217.626 & Surface & 2008 \\
\hline 3332.470 & 228.744 & Surface & 2008 & 8684.069 & 217.591 & Surface & 2008 \\
\hline 3335.662 & 228.752 & Surface & 2008 & 8687.285 & 217.594 & Surface & 2008 \\
\hline 3338.855 & 228.753 & Surface & 2008 & 8690.501 & 217.604 & Surface & 2008 \\
\hline 3342.047 & 228.748 & Surface & 2008 & 8693.717 & 217.611 & Surface & 2008 \\
\hline 3345.240 & 228.782 & Surface & 2008 & 8696.934 & 217.584 & Surface & 2008 \\
\hline 3348.432 & 228.773 & Surface & 2008 & 8700.150 & 217.545 & Surface & 2008 \\
\hline 3351.625 & 228.763 & Surface & 2008 & 8703.366 & 217.549 & Surface & 2008 \\
\hline 3354.817 & 228.725 & Surface & 2008 & 8706.582 & 217.560 & Surface & 2008 \\
\hline 3358.009 & 228.695 & Surface & 2008 & 8709.799 & 217.524 & Surface & 2008 \\
\hline 3361.202 & 228.723 & Surface & 2008 & 8713.023 & 217.507 & Surface & 2008 \\
\hline 3364.394 & 228.760 & Surface & 2008 & 8716.247 & 217.526 & Surface & 2008 \\
\hline 3367.587 & 228.740 & Surface & 2008 & 8719.471 & 217.525 & Surface & 2008 \\
\hline 3370.779 & 228.744 & Surface & 2008 & 8722.695 & 217.516 & Surface & 2008 \\
\hline 3373.972 & 228.769 & Surface & 2008 & 8725.919 & 217.502 & Surface & 2008 \\
\hline 3377.164 & 228.794 & Surface & 2008 & 8729.143 & 217.461 & Surface & 2008 \\
\hline 3380.357 & 228.796 & Surface & 2008 & 8732.367 & 217.421 & Surface & 2008 \\
\hline 3383.549 & 228.790 & Surface & 2008 & 8735.592 & 217.423 & Surface & 2008 \\
\hline 3386.779 & 228.811 & Surface & 2008 & 8738.816 & 217.405 & Surface & 2008 \\
\hline 3390.009 & 228.822 & Surface & 2008 & 8742.040 & 217.356 & Surface & 2008 \\
\hline 3393.240 & 228.826 & Surface & 2008 & 8745.264 & 217.379 & Surface & 2008 \\
\hline 3396.470 & 228.818 & Surface & 2008 & 8748.488 & 217.374 & Surface & 2008 \\
\hline 3399.700 & 228.804 & Surface & 2008 & 8751.713 & 217.409 & Surface & 2008 \\
\hline 3402.930 & 228.799 & Surface & 2008 & 8754.937 & 217.421 & Surface & 2008 \\
\hline 3406.160 & 228.790 & Surface & 2008 & 8758.161 & 217.405 & Surface & 2008 \\
\hline 3409.391 & 228.758 & Surface & 2008 & 8761.320 & 217.388 & Surface & 2008 \\
\hline 3412.621 & 228.730 & Surface & 2008 & 8764.479 & 217.378 & Surface & 2008 \\
\hline 3415.851 & 228.752 & Surface & 2008 & 8767.638 & 217.362 & Surface & 2008 \\
\hline 3419.082 & 228.753 & Surface & 2008 & 8770.797 & 217.344 & Surface & 2008 \\
\hline 3422.312 & 228.757 & Surface & 2008 & 8773.956 & 217.330 & Surface & 2008 \\
\hline 3425.542 & 228.733 & Surface & 2008 & 8777.115 & 217.317 & Surface & 2008 \\
\hline 3428.772 & 228.702 & Surface & 2008 & 8780.275 & 217.289 & Surface & 2008 \\
\hline 3432.002 & 228.684 & Surface & 2008 & 8783.434 & 217.254 & Surface & 2008 \\
\hline 3435.233 & 228.694 & Surface & 2008 & 8786.593 & 217.273 & Surface & 2008 \\
\hline 3438.463 & 228.700 & Surface & 2008 & 8789.752 & 217.295 & Surface & 2008 \\
\hline 3441.693 & 228.686 & Surface & 2008 & 8792.911 & 217.280 & Surface & 2008 \\
\hline 3444.924 & 228.671 & Surface & 2008 & 8796.070 & 217.186 & Surface & 2008 \\
\hline
\end{tabular}




\begin{tabular}{|c|c|c|c|c|c|c|c|}
\hline 3448.154 & 228.658 & Surface & 2008 & 8799.229 & 217.062 & Surface & 2008 \\
\hline 3451.384 & 228.635 & Surface & 2008 & 8802.388 & 216.963 & Surface & 2008 \\
\hline 3454.614 & 228.686 & Surface & 2008 & 8805.547 & 216.876 & Surface & 2008 \\
\hline 3457.844 & 228.744 & Surface & 2008 & 8808.753 & 216.814 & Surface & 2008 \\
\hline 3461.075 & 228.744 & Surface & 2008 & 8811.960 & 216.748 & Surface & 2008 \\
\hline 3464.305 & 228.641 & Surface & 2008 & 8815.166 & 216.799 & Surface & 2008 \\
\hline 3467.535 & 228.591 & Surface & 2008 & 8818.372 & 216.863 & Surface & 2008 \\
\hline 3470.765 & 228.623 & Surface & 2008 & 8821.578 & 216.857 & Surface & 2008 \\
\hline 3473.996 & 228.633 & Surface & 2008 & 8824.784 & 216.837 & Surface & 2008 \\
\hline 3477.226 & 228.655 & Surface & 2008 & 8827.991 & 216.868 & Surface & 2008 \\
\hline 3480.456 & 228.681 & Surface & 2008 & 8831.197 & 216.902 & Surface & 2008 \\
\hline 3483.686 & 228.652 & Surface & 2008 & 8834.403 & 216.882 & Surface & 2008 \\
\hline 3486.917 & 228.642 & Surface & 2008 & 8837.609 & 216.836 & Surface & 2008 \\
\hline 3490.147 & 228.664 & Surface & 2008 & 8840.815 & 216.807 & Surface & 2008 \\
\hline 3493.375 & 228.675 & Surface & 2008 & 8844.022 & 216.791 & Surface & 2008 \\
\hline 3496.604 & 228.679 & Surface & 2008 & 8847.228 & 216.787 & Surface & 2008 \\
\hline 3499.832 & 228.656 & Surface & 2008 & 8850.434 & 216.795 & Surface & 2008 \\
\hline 3503.061 & 228.640 & Surface & 2008 & 8853.640 & 216.803 & Surface & 2008 \\
\hline 3506.289 & 228.622 & Surface & 2008 & 8856.846 & 216.811 & Surface & 2008 \\
\hline 3509.517 & 228.628 & Surface & 2008 & 8860.052 & 216.811 & Surface & 2008 \\
\hline 3512.746 & 228.650 & Surface & 2008 & 8863.258 & 216.791 & Surface & 2008 \\
\hline 3515.974 & 228.629 & Surface & 2008 & 8866.454 & 216.776 & Surface & 2008 \\
\hline 3519.203 & 228.589 & Surface & 2008 & 8869.649 & 216.789 & Surface & 2008 \\
\hline 3522.431 & 228.549 & Surface & 2008 & 8872.844 & 216.817 & Surface & 2008 \\
\hline 3525.660 & 228.544 & Surface & 2008 & 8876.039 & 216.821 & Surface & 2008 \\
\hline 3528.888 & 228.591 & Surface & 2008 & 8879.234 & 216.832 & Surface & 2008 \\
\hline 3532.117 & 228.570 & Surface & 2008 & 8882.430 & 216.847 & Surface & 2008 \\
\hline 3535.345 & 228.557 & Surface & 2008 & 8885.625 & 216.830 & Surface & 2008 \\
\hline 3538.573 & 228.521 & Surface & 2008 & 8888.820 & 216.819 & Surface & 2008 \\
\hline 3541.802 & 228.493 & Surface & 2008 & 8892.015 & 216.753 & Surface & 2008 \\
\hline 3545.030 & 228.482 & Surface & 2008 & 8895.211 & 216.704 & Surface & 2008 \\
\hline 3548.259 & 228.498 & Surface & 2008 & 8898.406 & 216.674 & Surface & 2008 \\
\hline 3551.487 & 228.519 & Surface & 2008 & 8901.601 & 216.619 & Surface & 2008 \\
\hline 3554.716 & 228.533 & Surface & 2008 & 8904.796 & 216.610 & Surface & 2008 \\
\hline 3557.944 & 228.530 & Surface & 2008 & 8907.991 & 216.745 & Surface & 2008 \\
\hline 3561.044 & 228.507 & Surface & 2008 & 8911.186 & 216.825 & Surface & 2008 \\
\hline 3564.144 & 228.509 & Surface & 2008 & 8914.382 & 216.833 & Surface & 2008 \\
\hline 3567.244 & 228.529 & Surface & 2008 & 8917.577 & 216.856 & Surface & 2008 \\
\hline 3570.344 & 228.503 & Surface & 2008 & 8920.772 & 216.887 & Surface & 2008 \\
\hline 3573.444 & 228.485 & Surface & 2008 & 8923.967 & 216.912 & Surface & 2008 \\
\hline 3576.544 & 228.431 & Surface & 2008 & 8927.163 & 216.938 & Surface & 2008 \\
\hline 3579.644 & 228.377 & Surface & 2008 & 8930.358 & 216.969 & Surface & 2008 \\
\hline 3582.744 & 228.335 & Surface & 2008 & 8933.553 & 217.021 & Surface & 2008 \\
\hline 3585.843 & 228.265 & Surface & 2008 & 8936.748 & 217.045 & Surface & 2008 \\
\hline 3588.943 & 228.243 & Surface & 2008 & 8939.943 & 217.022 & Surface & 2008 \\
\hline 3592.043 & 228.312 & Surface & 2008 & 8943.139 & 216.999 & Surface & 2008 \\
\hline 3595.143 & 228.348 & Surface & 2008 & 8946.268 & 216.968 & Surface & 2008 \\
\hline 3598.243 & 228.355 & Surface & 2008 & 8949.398 & 216.938 & Surface & 2008 \\
\hline 3601.343 & 228.331 & Surface & 2008 & 8952.528 & 216.918 & Surface & 2008 \\
\hline
\end{tabular}




\begin{tabular}{|c|c|c|c|c|c|c|c|}
\hline 3604.443 & 228.312 & Surface & 2008 & 8955.658 & 216.891 & Surface & 2008 \\
\hline 3607.543 & 228.305 & Surface & 2008 & 8958.788 & 216.867 & Surface & 2008 \\
\hline 3610.643 & 228.313 & Surface & 2008 & 8961.917 & 216.821 & Surface & 2008 \\
\hline 3613.743 & 228.299 & Surface & 2008 & 8965.047 & 216.720 & Surface & 2008 \\
\hline 3616.875 & 228.310 & Surface & 2008 & 8968.177 & 216.692 & Surface & 2008 \\
\hline 3620.006 & 228.284 & Surface & 2008 & 8971.307 & 216.683 & Surface & 2008 \\
\hline 3623.138 & 228.257 & Surface & 2008 & 8974.437 & 216.680 & Surface & 2008 \\
\hline 3626.270 & 228.243 & Surface & 2008 & 8977.567 & 216.667 & Surface & 2008 \\
\hline 3629.402 & 228.263 & Surface & 2008 & 8980.696 & 216.684 & Surface & 2008 \\
\hline 3632.533 & 228.255 & Surface & 2008 & 8983.826 & 216.668 & Surface & 2008 \\
\hline 3635.665 & 228.245 & Surface & 2008 & 8986.956 & 216.600 & Surface & 2008 \\
\hline 3638.797 & 228.248 & Surface & 2008 & 8990.086 & 216.547 & Surface & 2008 \\
\hline 3641.928 & 228.239 & Surface & 2008 & 8993.216 & 216.534 & Surface & 2008 \\
\hline 3645.060 & 228.228 & Surface & 2008 & 8996.345 & 216.538 & Surface & 2008 \\
\hline 3648.192 & 228.208 & Surface & 2008 & 8999.475 & 216.566 & Surface & 2008 \\
\hline 3651.323 & 228.227 & Surface & 2008 & 9002.605 & 216.564 & Surface & 2008 \\
\hline 3654.455 & 228.265 & Surface & 2008 & 9005.735 & 216.561 & Surface & 2008 \\
\hline 3657.587 & 228.297 & Surface & 2008 & 9008.969 & 216.613 & Surface & 2008 \\
\hline 3660.719 & 228.288 & Surface & 2008 & 9012.202 & 216.627 & Surface & 2008 \\
\hline 3663.850 & 228.296 & Surface & 2008 & 9015.436 & 216.626 & Surface & 2008 \\
\hline 3667.120 & 228.332 & Surface & 2008 & 9018.670 & 216.642 & Surface & 2008 \\
\hline 3670.389 & 228.298 & Surface & 2008 & 9021.904 & 216.620 & Surface & 2008 \\
\hline 3673.659 & 228.259 & Surface & 2008 & 9025.137 & 216.606 & Surface & 2008 \\
\hline 3676.929 & 228.262 & Surface & 2008 & 9028.371 & 216.616 & Surface & 2008 \\
\hline 3680.198 & 228.222 & Surface & 2008 & 9031.605 & 216.633 & Surface & 2008 \\
\hline 3683.468 & 228.168 & Surface & 2008 & 9034.838 & 216.640 & Surface & 2008 \\
\hline 3686.737 & 228.136 & Surface & 2008 & 9038.072 & 216.637 & Surface & 2008 \\
\hline 3690.007 & 228.123 & Surface & 2008 & 9041.306 & 216.636 & Surface & 2008 \\
\hline 3693.277 & 228.101 & Surface & 2008 & 9044.540 & 216.626 & Surface & 2008 \\
\hline 3696.546 & 228.014 & Surface & 2008 & 9047.773 & 216.621 & Surface & 2008 \\
\hline 3699.816 & 227.953 & Surface & 2008 & 9051.007 & 216.626 & Surface & 2008 \\
\hline 3703.085 & 227.942 & Surface & 2008 & 9054.123 & 216.627 & Surface & 2008 \\
\hline 3706.355 & 227.900 & Surface & 2008 & 9057.240 & 216.643 & Surface & 2008 \\
\hline 3709.624 & 227.853 & Surface & 2008 & 9060.356 & 216.667 & Surface & 2008 \\
\hline 3712.894 & 227.741 & Surface & 2008 & 9063.473 & 216.639 & Surface & 2008 \\
\hline 3716.163 & 227.620 & Surface & 2008 & 9066.589 & 216.627 & Surface & 2008 \\
\hline 3719.433 & 227.595 & Surface & 2008 & 9069.705 & 216.661 & Surface & 2008 \\
\hline 3722.666 & 227.626 & Surface & 2008 & 9072.822 & 216.662 & Surface & 2008 \\
\hline 3725.899 & 227.633 & Surface & 2008 & 9075.938 & 216.650 & Surface & 2008 \\
\hline 3729.132 & 227.720 & Surface & 2008 & 9079.055 & 216.608 & Surface & 2008 \\
\hline 3732.364 & 227.762 & Surface & 2008 & 9082.171 & 216.627 & Surface & 2008 \\
\hline 3735.597 & 227.773 & Surface & 2008 & 9085.287 & 216.652 & Surface & 2008 \\
\hline 3738.830 & 227.776 & Surface & 2008 & 9088.404 & 216.651 & Surface & 2008 \\
\hline 3742.063 & 227.714 & Surface & 2008 & 9091.520 & 216.666 & Surface & 2008 \\
\hline 3745.296 & 227.685 & Surface & 2008 & 9094.637 & 216.674 & Surface & 2008 \\
\hline 3748.528 & 227.625 & Surface & 2008 & 9097.753 & 216.654 & Surface & 2008 \\
\hline 3751.761 & 227.573 & Surface & 2008 & 9100.899 & 216.660 & Surface & 2008 \\
\hline 3754.994 & 227.486 & Surface & 2008 & 9104.045 & 216.652 & Surface & 2008 \\
\hline 3758.227 & 227.500 & Surface & 2008 & 9107.191 & 216.636 & Surface & 2008 \\
\hline
\end{tabular}




\begin{tabular}{|c|c|c|c|c|c|c|c|}
\hline 3761.460 & 227.493 & Surface & 2008 & 9110.337 & 216.635 & Surface & 2008 \\
\hline 3764.544 & 227.490 & Surface & 2008 & 9113.483 & 216.638 & Surface & 2008 \\
\hline 3767.628 & 227.503 & Surface & 2008 & 9116.629 & 216.640 & Surface & 2008 \\
\hline 3770.713 & 227.522 & Surface & 2008 & 9119.775 & 216.643 & Surface & 2008 \\
\hline 3773.797 & 227.541 & Surface & 2008 & 9122.921 & 216.656 & Surface & 2008 \\
\hline 3776.882 & 227.560 & Surface & 2008 & 9126.067 & 216.694 & Surface & 2008 \\
\hline 3779.966 & 227.577 & Surface & 2008 & 9129.213 & 216.717 & Surface & 2008 \\
\hline 3783.050 & 227.580 & Surface & 2008 & 9132.360 & 216.712 & Surface & 2008 \\
\hline 3786.135 & 227.586 & Surface & 2008 & 9135.506 & 216.722 & Surface & 2008 \\
\hline 3789.219 & 227.590 & Surface & 2008 & 9138.651 & 216.721 & Surface & 2008 \\
\hline 3792.303 & 227.593 & Surface & 2008 & 9141.797 & 216.716 & Surface & 2008 \\
\hline 3795.388 & 227.600 & Surface & 2008 & 9144.944 & 216.711 & Surface & 2008 \\
\hline 3798.472 & 227.592 & Surface & 2008 & 9148.090 & 216.692 & Surface & 2008 \\
\hline 3801.556 & 227.573 & Surface & 2008 & 9151.236 & 216.655 & Surface & 2008 \\
\hline 3804.641 & 227.560 & Surface & 2008 & 9154.440 & 216.621 & Surface & 2008 \\
\hline 3807.725 & 227.542 & Surface & 2008 & 9157.645 & 216.620 & Surface & 2008 \\
\hline 3810.809 & 227.534 & Surface & 2008 & 9160.850 & 216.636 & Surface & 2008 \\
\hline 3813.939 & 227.534 & Surface & 2008 & 9164.055 & 216.641 & Surface & 2008 \\
\hline 3817.069 & 227.553 & Surface & 2008 & 9167.260 & 216.607 & Surface & 2008 \\
\hline 3820.199 & 227.559 & Surface & 2008 & 9170.465 & 216.561 & Surface & 2008 \\
\hline 3823.329 & 227.539 & Surface & 2008 & 9173.669 & 216.550 & Surface & 2008 \\
\hline 3826.459 & 227.523 & Surface & 2008 & 9176.874 & 216.543 & Surface & 2008 \\
\hline 3829.588 & 227.538 & Surface & 2008 & 9180.079 & 216.549 & Surface & 2008 \\
\hline 3832.718 & 227.538 & Surface & 2008 & 9183.284 & 216.556 & Surface & 2008 \\
\hline 3835.848 & 227.529 & Surface & 2008 & 9186.489 & 216.534 & Surface & 2008 \\
\hline 3838.978 & 227.512 & Surface & 2008 & 9189.693 & 216.501 & Surface & 2008 \\
\hline 3842.108 & 227.547 & Surface & 2008 & 9192.898 & 216.481 & Surface & 2008 \\
\hline 3845.237 & 227.588 & Surface & 2008 & 9196.103 & 216.484 & Surface & 2008 \\
\hline 3848.367 & 227.632 & Surface & 2008 & 9199.308 & 216.502 & Surface & 2008 \\
\hline 3851.497 & 227.669 & Surface & 2008 & 9202.513 & 216.486 & Surface & 2008 \\
\hline 3854.627 & 227.670 & Surface & 2008 & 9205.718 & 216.477 & Surface & 2008 \\
\hline 3857.757 & 227.690 & Surface & 2008 & 9208.922 & 216.472 & Surface & 2008 \\
\hline 3860.887 & 227.715 & Surface & 2008 & 9211.999 & 216.474 & Surface & 2008 \\
\hline 3864.016 & 227.742 & Surface & 2008 & 9215.074 & 216.485 & Surface & 2008 \\
\hline 3867.146 & 227.755 & Surface & 2008 & 9218.150 & 216.487 & Surface & 2008 \\
\hline 3870.407 & 227.737 & Surface & 2008 & 9221.226 & 216.490 & Surface & 2008 \\
\hline 3873.669 & 227.724 & Surface & 2008 & 9224.302 & 216.471 & Surface & 2008 \\
\hline 3876.930 & 227.703 & Surface & 2008 & 9227.378 & 216.462 & Surface & 2008 \\
\hline 3880.192 & 227.721 & Surface & 2008 & 9230.454 & 216.462 & Surface & 2008 \\
\hline 3883.453 & 227.762 & Surface & 2008 & 9233.529 & 216.446 & Surface & 2008 \\
\hline 3886.714 & 227.757 & Surface & 2008 & 9236.605 & 216.457 & Surface & 2008 \\
\hline 3889.976 & 227.715 & Surface & 2008 & 9239.681 & 216.469 & Surface & 2008 \\
\hline 3893.237 & 227.688 & Surface & 2008 & 9242.757 & 216.438 & Surface & 2008 \\
\hline 3896.498 & 227.685 & Surface & 2008 & 9245.833 & 216.392 & Surface & 2008 \\
\hline 3899.760 & 227.646 & Surface & 2008 & 9248.865 & 216.372 & Surface & 2008 \\
\hline 3903.021 & 227.593 & Surface & 2008 & 9251.897 & 216.330 & Surface & 2008 \\
\hline 3906.283 & 227.563 & Surface & 2008 & 9254.929 & 216.308 & Surface & 2008 \\
\hline 3909.544 & 227.555 & Surface & 2008 & 9257.961 & 216.277 & Surface & 2008 \\
\hline 3912.805 & 227.557 & Surface & 2008 & 9260.993 & 216.218 & Surface & 2008 \\
\hline
\end{tabular}




\begin{tabular}{|c|c|c|c|c|c|c|c|}
\hline 3916.066 & 227.555 & Surface & 2008 & 9264.025 & 216.160 & Surface & 2008 \\
\hline 3919.320 & 227.556 & Surface & 2008 & 9267.057 & 216.145 & Surface & 2008 \\
\hline 3922.574 & 227.554 & Surface & 2008 & 9270.089 & 216.132 & Surface & 2008 \\
\hline 3925.828 & 227.496 & Surface & 2008 & 9273.120 & 216.126 & Surface & 2008 \\
\hline 3929.082 & 227.492 & Surface & 2008 & 9276.153 & 216.059 & Surface & 2008 \\
\hline 3932.335 & 227.519 & Surface & 2008 & 9279.346 & 215.983 & Surface & 2008 \\
\hline 3935.589 & 227.540 & Surface & 2008 & 9282.539 & 215.900 & Surface & 2008 \\
\hline 3938.843 & 227.544 & Surface & 2008 & 9285.732 & 215.869 & Surface & 2008 \\
\hline 3942.096 & 227.545 & Surface & 2008 & 9288.925 & 215.839 & Surface & 2008 \\
\hline 3945.350 & 227.537 & Surface & 2008 & 9292.118 & 215.769 & Surface & 2008 \\
\hline 3948.604 & 227.525 & Surface & 2008 & 9295.311 & 215.776 & Surface & 2008 \\
\hline 3951.858 & 227.555 & Surface & 2008 & 9298.504 & 215.795 & Surface & 2008 \\
\hline 3955.112 & 227.584 & Surface & 2008 & 9301.697 & 215.826 & Surface & 2008 \\
\hline 3958.365 & 227.600 & Surface & 2008 & 9304.890 & 215.816 & Surface & 2008 \\
\hline 3961.619 & 227.600 & Surface & 2008 & 9308.000 & 215.798 & Surface & 2008 \\
\hline 3964.873 & 227.577 & Surface & 2008 & 9311.109 & 215.848 & Surface & 2008 \\
\hline 3968.126 & 227.503 & Surface & 2008 & 9314.219 & 215.857 & Surface & 2008 \\
\hline 3971.380 & 227.479 & Surface & 2008 & 9317.328 & 215.837 & Surface & 2008 \\
\hline 3974.634 & 227.449 & Surface & 2008 & 9320.438 & 215.797 & Surface & 2008 \\
\hline 3977.888 & 227.428 & Surface & 2008 & 9323.547 & 215.792 & Surface & 2008 \\
\hline 3981.142 & 227.437 & Surface & 2008 & 9326.657 & 215.798 & Surface & 2008 \\
\hline 3984.254 & 227.478 & Surface & 2008 & 9329.767 & 215.804 & Surface & 2008 \\
\hline 3987.367 & 227.491 & Surface & 2008 & 9332.876 & 215.842 & Surface & 2008 \\
\hline 3990.480 & 227.467 & Surface & 2008 & 9335.986 & 215.856 & Surface & 2008 \\
\hline 3993.592 & 227.535 & Surface & 2008 & 9339.095 & 215.861 & Surface & 2008 \\
\hline 3996.705 & 227.565 & Surface & 2008 & 9342.205 & 215.841 & Surface & 2008 \\
\hline 3999.818 & 227.568 & Surface & 2008 & 9345.314 & 215.803 & Surface & 2008 \\
\hline 4002.930 & 227.559 & Surface & 2008 & 9348.424 & 215.832 & Surface & 2008 \\
\hline 4006.043 & 227.526 & Surface & 2008 & 9351.534 & 215.842 & Surface & 2008 \\
\hline 4009.156 & 227.527 & Surface & 2008 & 9354.643 & 215.799 & Surface & 2008 \\
\hline 4012.269 & 227.533 & Surface & 2008 & 9357.824 & 215.826 & Surface & 2008 \\
\hline 4015.381 & 227.534 & Surface & 2008 & 9361.005 & 215.817 & Surface & 2008 \\
\hline 4018.494 & 227.535 & Surface & 2008 & 9364.186 & 215.798 & Surface & 2008 \\
\hline 4021.607 & 227.570 & Surface & 2008 & 9367.367 & 215.810 & Surface & 2008 \\
\hline 4024.719 & 227.621 & Surface & 2008 & 9370.549 & 215.817 & Surface & 2008 \\
\hline 4027.832 & 227.606 & Surface & 2008 & 9373.730 & 215.796 & Surface & 2008 \\
\hline 4030.945 & 227.566 & Surface & 2008 & 9376.911 & 215.782 & Surface & 2008 \\
\hline 4034.057 & 227.557 & Surface & 2008 & 9380.092 & 215.780 & Surface & 2008 \\
\hline 4037.303 & 227.549 & Surface & 2008 & 9383.273 & 215.774 & Surface & 2008 \\
\hline 4040.548 & 227.531 & Surface & 2008 & 9386.454 & 215.826 & Surface & 2008 \\
\hline 4043.794 & 227.531 & Surface & 2008 & 9389.635 & 215.837 & Surface & 2008 \\
\hline 4047.040 & 227.529 & Surface & 2008 & 9392.794 & 215.830 & Surface & 2008 \\
\hline 4050.285 & 227.527 & Surface & 2008 & 9395.952 & 215.848 & Surface & 2008 \\
\hline 4053.531 & 227.520 & Surface & 2008 & 9399.111 & 215.859 & Surface & 2008 \\
\hline 4056.776 & 227.526 & Surface & 2008 & 9402.269 & 215.827 & Surface & 2008 \\
\hline 4060.022 & 227.526 & Surface & 2008 & 9405.427 & 215.839 & Surface & 2008 \\
\hline 4063.268 & 227.519 & Surface & 2008 & 9408.586 & 215.865 & Surface & 2008 \\
\hline 4066.513 & 227.512 & Surface & 2008 & 9411.744 & 215.886 & Surface & 2008 \\
\hline 4069.759 & 227.512 & Surface & 2008 & 9414.903 & 215.893 & Surface & 2008 \\
\hline
\end{tabular}




\begin{tabular}{|c|c|c|c|c|c|c|c|}
\hline 4073.005 & 227.511 & Surface & 2008 & 9418.061 & 215.864 & Surface & 2008 \\
\hline 4076.250 & 227.511 & Surface & 2008 & 9421.220 & 215.831 & Surface & 2008 \\
\hline 4079.496 & 227.504 & Surface & 2008 & 9424.378 & 215.804 & Surface & 2008 \\
\hline 4082.676 & 227.480 & Surface & 2008 & 9427.536 & 215.796 & Surface & 2008 \\
\hline 4085.857 & 227.453 & Surface & 2008 & 9430.695 & 215.831 & Surface & 2008 \\
\hline 4089.038 & 227.429 & Surface & 2008 & 9433.853 & 215.862 & Surface & 2008 \\
\hline 4092.218 & 227.413 & Surface & 2008 & 9437.012 & 215.872 & Surface & 2008 \\
\hline 4095.399 & 227.395 & Surface & 2008 & 9440.170 & 215.878 & Surface & 2008 \\
\hline 4098.580 & 227.383 & Surface & 2008 & 9443.329 & 215.868 & Surface & 2008 \\
\hline 4101.760 & 227.363 & Surface & 2008 & 9446.440 & 215.833 & Surface & 2008 \\
\hline 4104.941 & 227.388 & Surface & 2008 & 9449.551 & 215.804 & Surface & 2008 \\
\hline 4108.122 & 227.421 & Surface & 2008 & 9452.662 & 215.810 & Surface & 2008 \\
\hline 4111.302 & 227.455 & Surface & 2008 & 9455.773 & 215.836 & Surface & 2008 \\
\hline 4114.483 & 227.471 & Surface & 2008 & 9458.884 & 215.859 & Surface & 2008 \\
\hline 4117.663 & 227.482 & Surface & 2008 & 9461.995 & 215.853 & Surface & 2008 \\
\hline 4120.844 & 227.496 & Surface & 2008 & 9465.106 & 215.832 & Surface & 2008 \\
\hline 4124.025 & 227.505 & Surface & 2008 & 9468.217 & 215.828 & Surface & 2008 \\
\hline 4127.205 & 227.484 & Surface & 2008 & 9471.328 & 215.836 & Surface & 2008 \\
\hline 4130.386 & 227.433 & Surface & 2008 & 9474.440 & 215.830 & Surface & 2008 \\
\hline 4133.567 & 227.402 & Surface & 2008 & 9477.551 & 215.858 & Surface & 2008 \\
\hline 4136.735 & 227.419 & Surface & 2008 & 9480.662 & 215.867 & Surface & 2008 \\
\hline 4139.902 & 227.435 & Surface & 2008 & 9483.773 & 215.860 & Surface & 2008 \\
\hline 4143.070 & 227.431 & Surface & 2008 & 9486.884 & 215.807 & Surface & 2008 \\
\hline 4146.239 & 227.404 & Surface & 2008 & 9489.995 & 215.853 & Surface & 2008 \\
\hline 4149.406 & 227.390 & Surface & 2008 & 9493.106 & 215.850 & Surface & 2008 \\
\hline 4152.574 & 227.429 & Surface & 2008 & 9496.217 & 215.800 & Surface & 2008 \\
\hline 4155.742 & 227.405 & Surface & 2008 & 9499.328 & 215.803 & Surface & 2008 \\
\hline 4158.910 & 227.350 & Surface & 2008 & 9502.439 & 215.839 & Surface & 2008 \\
\hline 4162.078 & 227.303 & Surface & 2008 & 9505.689 & 215.868 & Surface & 2008 \\
\hline 4165.246 & 227.282 & Surface & 2008 & 9508.938 & 215.841 & Surface & 2008 \\
\hline 4168.414 & 227.291 & Surface & 2008 & 9512.187 & 215.839 & Surface & 2008 \\
\hline 4171.582 & 227.303 & Surface & 2008 & 9515.436 & 215.857 & Surface & 2008 \\
\hline 4174.750 & 227.310 & Surface & 2008 & 9518.685 & 215.827 & Surface & 2008 \\
\hline 4177.918 & 227.292 & Surface & 2008 & 9521.934 & 215.802 & Surface & 2008 \\
\hline 4181.086 & 227.276 & Surface & 2008 & 9525.183 & 215.801 & Surface & 2008 \\
\hline 4184.254 & 227.260 & Surface & 2008 & 9528.433 & 215.794 & Surface & 2008 \\
\hline 4187.422 & 227.280 & Surface & 2008 & 9531.682 & 215.770 & Surface & 2008 \\
\hline 4190.589 & 227.287 & Surface & 2008 & 9534.931 & 215.795 & Surface & 2008 \\
\hline 4193.757 & 227.255 & Surface & 2008 & 9538.180 & 215.761 & Surface & 2008 \\
\hline 4196.858 & 227.192 & Surface & 2008 & 9541.429 & 215.751 & Surface & 2008 \\
\hline 4199.958 & 227.146 & Surface & 2008 & 9544.679 & 215.757 & Surface & 2008 \\
\hline 4203.058 & 227.093 & Surface & 2008 & 9547.928 & 215.748 & Surface & 2008 \\
\hline 4206.158 & 227.067 & Surface & 2008 & 9551.177 & 215.722 & Surface & 2008 \\
\hline 4209.258 & 227.066 & Surface & 2008 & 9554.426 & 215.647 & Surface & 2008 \\
\hline 4212.359 & 227.071 & Surface & 2008 & 9557.675 & 215.590 & Surface & 2008 \\
\hline 4215.459 & 227.073 & Surface & 2008 & 9560.912 & 215.567 & Surface & 2008 \\
\hline 4218.559 & 227.037 & Surface & 2008 & 9564.149 & 215.636 & Surface & 2008 \\
\hline 4221.659 & 226.990 & Surface & 2008 & 9567.387 & 215.677 & Surface & 2008 \\
\hline 4224.759 & 226.947 & Surface & 2008 & 9570.623 & 215.654 & Surface & 2008 \\
\hline
\end{tabular}




\begin{tabular}{|c|c|c|c|c|c|c|c|}
\hline 4227.860 & 226.881 & Surface & 2008 & 9573.861 & 215.612 & Surface & 2008 \\
\hline 4230.960 & 226.798 & Surface & 2008 & 9577.098 & 215.600 & Surface & 2008 \\
\hline 4234.060 & 226.820 & Surface & 2008 & 9580.335 & 215.551 & Surface & 2008 \\
\hline 4237.160 & 226.853 & Surface & 2008 & 9583.572 & 215.528 & Surface & 2008 \\
\hline 4240.328 & 226.860 & Surface & 2008 & 9586.809 & 215.520 & Surface & 2008 \\
\hline 4243.496 & 226.858 & Surface & 2008 & 9590.046 & 215.522 & Surface & 2008 \\
\hline 4246.664 & 226.851 & Surface & 2008 & 9593.283 & 215.517 & Surface & 2008 \\
\hline 4249.832 & 226.838 & Surface & 2008 & 9596.520 & 215.423 & Surface & 2008 \\
\hline 4253.000 & 226.794 & Surface & 2008 & 9599.757 & 215.395 & Surface & 2008 \\
\hline 4256.168 & 226.827 & Surface & 2008 & 9602.994 & 215.402 & Surface & 2008 \\
\hline 4259.336 & 226.832 & Surface & 2008 & 9606.231 & 215.414 & Surface & 2008 \\
\hline 4262.504 & 226.737 & Surface & 2008 & 9609.468 & 215.404 & Surface & 2008 \\
\hline 4265.672 & 226.685 & Surface & 2008 & 9612.706 & 215.386 & Surface & 2008 \\
\hline 4268.839 & 226.709 & Surface & 2008 & 9615.943 & 215.316 & Surface & 2008 \\
\hline 4272.008 & 226.724 & Surface & 2008 & 9619.180 & 215.276 & Surface & 2008 \\
\hline 4275.175 & 226.759 & Surface & 2008 & 9622.417 & 215.257 & Surface & 2008 \\
\hline 4278.343 & 226.769 & Surface & 2008 & 9625.654 & 215.246 & Surface & 2008 \\
\hline 4281.511 & 226.757 & Surface & 2008 & 9628.891 & 215.223 & Surface & 2008 \\
\hline 4284.679 & 226.699 & Surface & 2008 & 9632.128 & 215.172 & Surface & 2008 \\
\hline 4287.847 & 226.699 & Surface & 2008 & 9635.365 & 215.232 & Surface & 2008 \\
\hline 4291.015 & 226.722 & Surface & 2008 & 9638.474 & 215.232 & Surface & 2008 \\
\hline 4294.183 & 226.739 & Surface & 2008 & 9641.583 & 215.183 & Surface & 2008 \\
\hline 4297.351 & 226.748 & Surface & 2008 & 9644.692 & 215.147 & Surface & 2008 \\
\hline 4300.500 & 226.744 & Surface & 2008 & 9647.801 & 215.078 & Surface & 2008 \\
\hline 4303.649 & 226.719 & Surface & 2008 & 9650.910 & 215.057 & Surface & 2008 \\
\hline 4306.798 & 226.685 & Surface & 2008 & 9654.019 & 215.034 & Surface & 2008 \\
\hline 4309.947 & 226.698 & Surface & 2008 & 9657.128 & 215.005 & Surface & 2008 \\
\hline 4313.096 & 226.714 & Surface & 2008 & 9660.238 & 215.004 & Surface & 2008 \\
\hline 4316.245 & 226.730 & Surface & 2008 & 9663.347 & 215.031 & Surface & 2008 \\
\hline 4319.394 & 226.768 & Surface & 2008 & 9666.456 & 215.024 & Surface & 2008 \\
\hline 4322.544 & 226.743 & Surface & 2008 & 9669.565 & 215.041 & Surface & 2008 \\
\hline 4325.693 & 226.674 & Surface & 2008 & 9672.674 & 214.997 & Surface & 2008 \\
\hline 4328.842 & 226.638 & Surface & 2008 & 9675.783 & 214.944 & Surface & 2008 \\
\hline 4331.991 & 226.643 & Surface & 2008 & 9678.892 & 214.938 & Surface & 2008 \\
\hline 4335.140 & 226.667 & Surface & 2008 & 9682.001 & 214.954 & Surface & 2008 \\
\hline 4338.289 & 226.684 & Surface & 2008 & 9685.110 & 214.910 & Surface & 2008 \\
\hline 4341.438 & 226.703 & Surface & 2008 & 9688.360 & 214.916 & Surface & 2008 \\
\hline 4344.587 & 226.719 & Surface & 2008 & 9691.611 & 214.923 & Surface & 2008 \\
\hline 4347.736 & 226.686 & Surface & 2008 & 9694.861 & 214.942 & Surface & 2008 \\
\hline 4350.885 & 226.633 & Surface & 2008 & 9698.111 & 214.952 & Surface & 2008 \\
\hline 4354.034 & 226.689 & Surface & 2008 & 9701.361 & 214.961 & Surface & 2008 \\
\hline 4357.188 & 226.706 & Surface & 2008 & 9704.612 & 214.972 & Surface & 2008 \\
\hline 4360.341 & 226.659 & Surface & 2008 & 9707.862 & 215.006 & Surface & 2008 \\
\hline 4363.495 & 226.624 & Surface & 2008 & 9711.112 & 214.992 & Surface & 2008 \\
\hline 4366.649 & 226.625 & Surface & 2008 & 9714.363 & 214.950 & Surface & 2008 \\
\hline 4369.802 & 226.611 & Surface & 2008 & 9717.613 & 214.944 & Surface & 2008 \\
\hline 4372.956 & 226.616 & Surface & 2008 & 9720.773 & 214.930 & Surface & 2008 \\
\hline 4376.109 & 226.625 & Surface & 2008 & 9723.933 & 214.921 & Surface & 2008 \\
\hline 4379.263 & 226.634 & Surface & 2008 & 9727.092 & 214.938 & Surface & 2008 \\
\hline
\end{tabular}




\begin{tabular}{|c|c|c|c|c|c|c|c|}
\hline 4382.417 & 226.636 & Surface & 2008 & 9730.252 & 214.951 & Surface & 2008 \\
\hline 4385.570 & 226.618 & Surface & 2008 & 9733.412 & 214.915 & Surface & 2008 \\
\hline 4388.724 & 226.615 & Surface & 2008 & 9736.571 & 214.903 & Surface & 2008 \\
\hline 4391.878 & 226.624 & Surface & 2008 & 9739.731 & 214.910 & Surface & 2008 \\
\hline 4395.031 & 226.683 & Surface & 2008 & 9742.891 & 214.906 & Surface & 2008 \\
\hline 4398.185 & 226.718 & Surface & 2008 & 9746.051 & 214.935 & Surface & 2008 \\
\hline 4401.338 & 226.644 & Surface & 2008 & 9749.211 & 214.969 & Surface & 2008 \\
\hline 4404.492 & 226.622 & Surface & 2008 & 9752.432 & 214.967 & Surface & 2008 \\
\hline 4407.646 & 226.607 & Surface & 2008 & 9755.653 & 214.958 & Surface & 2008 \\
\hline 4410.799 & 226.579 & Surface & 2008 & 9758.874 & 214.927 & Surface & 2008 \\
\hline 4413.953 & 226.578 & Surface & 2008 & 9762.095 & 214.912 & Surface & 2008 \\
\hline 4417.107 & 226.585 & Surface & 2008 & 9765.316 & 214.904 & Surface & 2008 \\
\hline 4420.260 & 226.604 & Surface & 2008 & 9768.538 & 214.861 & Surface & 2008 \\
\hline 4423.414 & 226.599 & Surface & 2008 & 9771.759 & 214.860 & Surface & 2008 \\
\hline 4426.605 & 226.582 & Surface & 2008 & 9774.980 & 214.814 & Surface & 2008 \\
\hline 4429.797 & 226.552 & Surface & 2008 & 9778.201 & 214.769 & Surface & 2008 \\
\hline 4432.988 & 226.555 & Surface & 2008 & 9781.422 & 214.705 & Surface & 2008 \\
\hline 4436.180 & 226.561 & Surface & 2008 & 9784.667 & 214.702 & Surface & 2008 \\
\hline 4439.371 & 226.554 & Surface & 2008 & 9787.910 & 214.738 & Surface & 2008 \\
\hline 4442.563 & 226.542 & Surface & 2008 & 9791.155 & 214.766 & Surface & 2008 \\
\hline 4445.755 & 226.551 & Surface & 2008 & 9794.399 & 214.735 & Surface & 2008 \\
\hline 4448.946 & 226.583 & Surface & 2008 & 9797.643 & 214.704 & Surface & 2008 \\
\hline 4452.138 & 226.599 & Surface & 2008 & 9800.887 & 214.685 & Surface & 2008 \\
\hline 4455.329 & 226.584 & Surface & 2008 & 9804.131 & 214.647 & Surface & 2008 \\
\hline 4458.521 & 226.585 & Surface & 2008 & 9807.375 & 214.573 & Surface & 2008 \\
\hline 4461.712 & 226.627 & Surface & 2008 & 9810.620 & 214.555 & Surface & 2008 \\
\hline 4464.904 & 226.600 & Surface & 2008 & 9813.863 & 214.599 & Surface & 2008 \\
\hline 4468.096 & 226.551 & Surface & 2008 & 9817.108 & 214.578 & Surface & 2008 \\
\hline 4471.287 & 226.542 & Surface & 2008 & 9820.244 & 214.532 & Surface & 2008 \\
\hline 4474.479 & 226.556 & Surface & 2008 & 9823.379 & 214.508 & Surface & 2008 \\
\hline 4477.670 & 226.589 & Surface & 2008 & 9826.515 & 214.517 & Surface & 2008 \\
\hline 4480.862 & 226.568 & Surface & 2008 & 9829.651 & 214.557 & Surface & 2008 \\
\hline 4484.053 & 226.475 & Surface & 2008 & 9832.787 & 214.588 & Surface & 2008 \\
\hline 4487.201 & 226.510 & Surface & 2008 & 9835.923 & 214.584 & Surface & 2008 \\
\hline 4490.349 & 226.563 & Surface & 2008 & 9839.059 & 214.560 & Surface & 2008 \\
\hline 4493.497 & 226.601 & Surface & 2008 & 9842.194 & 214.576 & Surface & 2008 \\
\hline 4496.646 & 226.581 & Surface & 2008 & 9845.330 & 214.645 & Surface & 2008 \\
\hline 4499.793 & 226.533 & Surface & 2008 & 9848.466 & 214.678 & Surface & 2008 \\
\hline 4502.942 & 226.503 & Surface & 2008 & 9851.723 & 214.615 & Surface & 2008 \\
\hline 4506.090 & 226.504 & Surface & 2008 & 9854.980 & 214.554 & Surface & 2008 \\
\hline 4509.238 & 226.524 & Surface & 2008 & 9858.237 & 214.531 & Surface & 2008 \\
\hline 4512.386 & 226.533 & Surface & 2008 & 9861.494 & 214.519 & Surface & 2008 \\
\hline 4515.534 & 226.532 & Surface & 2008 & 9864.751 & 214.528 & Surface & 2008 \\
\hline 4518.682 & 226.502 & Surface & 2008 & 9868.008 & 214.579 & Surface & 2008 \\
\hline 4521.830 & 226.479 & Surface & 2008 & 9871.265 & 214.602 & Surface & 2008 \\
\hline 4524.978 & 226.459 & Surface & 2008 & 9874.522 & 214.602 & Surface & 2008 \\
\hline 4528.126 & 226.448 & Surface & 2008 & 9877.543 & 214.591 & Surface & 2008 \\
\hline 4531.274 & 226.464 & Surface & 2008 & 9880.563 & 214.671 & Surface & 2008 \\
\hline 4534.422 & 226.453 & Surface & 2008 & 9883.583 & 214.711 & Surface & 2008 \\
\hline
\end{tabular}




\begin{tabular}{|c|c|c|c|c|c|c|c|}
\hline 4537.570 & 226.448 & Surface & 2008 & 9886.604 & 214.691 & Surface & 2008 \\
\hline 4540.718 & 226.453 & Surface & 2008 & 9889.624 & 214.677 & Surface & 2008 \\
\hline 4543.866 & 226.457 & Surface & 2008 & 9892.645 & 214.670 & Surface & 2008 \\
\hline 4547.014 & 226.437 & Surface & 2008 & 9895.665 & 214.678 & Surface & 2008 \\
\hline 4550.162 & 226.406 & Surface & 2008 & 9898.686 & 214.669 & Surface & 2008 \\
\hline 4553.360 & 226.367 & Surface & 2008 & 9901.706 & 214.679 & Surface & 2008 \\
\hline 4556.557 & 226.332 & Surface & 2008 & 9904.727 & 214.681 & Surface & 2008 \\
\hline 4559.755 & 226.308 & Surface & 2008 & 9907.747 & 214.683 & Surface & 2008 \\
\hline 4562.952 & 226.258 & Surface & 2008 & 9910.907 & 214.683 & Surface & 2008 \\
\hline 4566.150 & 226.218 & Surface & 2008 & 9914.066 & 214.689 & Surface & 2008 \\
\hline 4569.347 & 226.220 & Surface & 2008 & 9917.226 & 214.703 & Surface & 2008 \\
\hline 4572.545 & 226.231 & Surface & 2008 & 9920.385 & 214.683 & Surface & 2008 \\
\hline 4575.742 & 226.213 & Surface & 2008 & 9923.544 & 214.668 & Surface & 2008 \\
\hline 4578.940 & 226.192 & Surface & 2008 & 9926.704 & 214.650 & Surface & 2008 \\
\hline 4582.137 & 226.185 & Surface & 2008 & 9929.863 & 214.647 & Surface & 2008 \\
\hline 4585.335 & 226.190 & Surface & 2008 & 9933.022 & 214.661 & Surface & 2008 \\
\hline 4588.532 & 226.189 & Surface & 2008 & 9936.182 & 214.660 & Surface & 2008 \\
\hline 4591.730 & 226.167 & Surface & 2008 & 9939.190 & 214.629 & Surface & 2008 \\
\hline 4594.927 & 226.153 & Surface & 2008 & 9942.199 & 214.628 & Surface & 2008 \\
\hline 4598.125 & 226.144 & Surface & 2008 & 9945.207 & 214.620 & Surface & 2008 \\
\hline 4601.322 & 226.152 & Surface & 2008 & 9948.215 & 214.568 & Surface & 2008 \\
\hline 4604.519 & 226.117 & Surface & 2008 & 9951.223 & 214.533 & Surface & 2008 \\
\hline 4607.717 & 226.071 & Surface & 2008 & 9954.232 & 214.554 & Surface & 2008 \\
\hline 4610.914 & 226.064 & Surface & 2008 & 9957.240 & 214.535 & Surface & 2008 \\
\hline 4614.112 & 226.076 & Surface & 2008 & 9960.248 & 214.504 & Surface & 2008 \\
\hline 4617.309 & 226.084 & Surface & 2008 & 9963.257 & 214.449 & Surface & 2008 \\
\hline 4620.507 & 226.131 & Surface & 2008 & 9966.265 & 214.338 & Surface & 2008 \\
\hline 4623.704 & 226.218 & Surface & 2008 & 9969.244 & 214.295 & Surface & 2008 \\
\hline 4626.902 & 226.168 & Surface & 2008 & 9972.223 & 214.244 & Surface & 2008 \\
\hline 4630.099 & 226.185 & Surface & 2008 & 9975.202 & 214.234 & Surface & 2008 \\
\hline 4633.297 & 226.159 & Surface & 2008 & 9978.181 & 214.123 & Surface & 2008 \\
\hline 4636.385 & 226.175 & Surface & 2008 & 9981.160 & 214.011 & Surface & 2008 \\
\hline 4639.473 & 226.214 & Surface & 2008 & 9984.139 & 213.938 & Surface & 2008 \\
\hline 4642.561 & 226.234 & Surface & 2008 & 9987.118 & 213.934 & Surface & 2008 \\
\hline 4645.649 & 226.227 & Surface & 2008 & 9990.097 & 213.944 & Surface & 2008 \\
\hline 4648.737 & 226.168 & Surface & 2008 & 9993.076 & 213.939 & Surface & 2008 \\
\hline 4651.826 & 226.149 & Surface & 2008 & 9996.227 & 213.900 & Surface & 2008 \\
\hline 4654.914 & 226.098 & Surface & 2008 & 9999.378 & 213.852 & Surface & 2008 \\
\hline 4658.002 & 226.049 & Surface & 2008 & 10002.528 & 213.871 & Surface & 2008 \\
\hline 4661.090 & 226.065 & Surface & 2008 & 10005.679 & 213.921 & Surface & 2008 \\
\hline 4664.178 & 226.074 & Surface & 2008 & 10008.830 & 213.930 & Surface & 2008 \\
\hline 4667.267 & 226.057 & Surface & 2008 & 10011.980 & 213.927 & Surface & 2008 \\
\hline 4670.355 & 226.068 & Surface & 2008 & 10015.131 & 213.908 & Surface & 2008 \\
\hline 4673.443 & 226.081 & Surface & 2008 & 10018.282 & 213.853 & Surface & 2008 \\
\hline 4676.531 & 226.088 & Surface & 2008 & 10021.432 & 213.828 & Surface & 2008 \\
\hline 4679.619 & 226.090 & Surface & 2008 & 10024.583 & 213.842 & Surface & 2008 \\
\hline 4682.707 & 226.101 & Surface & 2008 & 10027.733 & 213.853 & Surface & 2008 \\
\hline 4685.923 & 226.097 & Surface & 2008 & 10030.863 & 213.868 & Surface & 2008 \\
\hline 4689.139 & 226.065 & Surface & 2008 & 10033.993 & 213.859 & Surface & 2008 \\
\hline
\end{tabular}




\begin{tabular}{|c|c|c|c|c|c|c|c|}
\hline 4692.354 & 226.045 & Surface & 2008 & 10037.123 & 213.851 & Surface & 2008 \\
\hline 4695.570 & 226.044 & Surface & 2008 & 10040.253 & 213.873 & Surface & 2008 \\
\hline 4698.786 & 226.069 & Surface & 2008 & 10043.383 & 213.873 & Surface & 2008 \\
\hline 4702.002 & 226.069 & Surface & 2008 & 10046.513 & 213.872 & Surface & 2008 \\
\hline 4705.217 & 226.049 & Surface & 2008 & 10049.642 & 213.893 & Surface & 2008 \\
\hline 4708.433 & 226.062 & Surface & 2008 & 10052.772 & 213.893 & Surface & 2008 \\
\hline 4711.649 & 226.034 & Surface & 2008 & 10056.023 & 213.899 & Surface & 2008 \\
\hline 4714.864 & 225.969 & Surface & 2008 & 10059.274 & 213.870 & Surface & 2008 \\
\hline 4718.080 & 225.958 & Surface & 2008 & 10062.526 & 213.858 & Surface & 2008 \\
\hline 4721.296 & 225.950 & Surface & 2008 & 10065.777 & 213.845 & Surface & 2008 \\
\hline 4724.512 & 225.914 & Surface & 2008 & 10069.028 & 213.847 & Surface & 2008 \\
\hline 4727.727 & 225.970 & Surface & 2008 & 10072.279 & 213.876 & Surface & 2008 \\
\hline 4730.943 & 226.027 & Surface & 2008 & 10075.262 & 213.891 & Surface & 2008 \\
\hline 4734.159 & 226.005 & Surface & 2008 & 10078.245 & 213.895 & Surface & 2008 \\
\hline 4737.374 & 225.923 & Surface & 2008 & 10081.227 & 213.885 & Surface & 2008 \\
\hline 4740.590 & 225.951 & Surface & 2008 & 10084.209 & 213.866 & Surface & 2008 \\
\hline 4743.806 & 225.989 & Surface & 2008 & 10087.192 & 213.858 & Surface & 2008 \\
\hline 4747.022 & 225.970 & Surface & 2008 & 10090.175 & 213.907 & Surface & 2008 \\
\hline 4750.237 & 225.954 & Surface & 2008 & 10093.372 & 213.900 & Surface & 2008 \\
\hline 4753.450 & 225.935 & Surface & 2008 & 10096.570 & 213.909 & Surface & 2008 \\
\hline 4756.662 & 225.881 & Surface & 2008 & 10099.768 & 213.911 & Surface & 2008 \\
\hline 4759.874 & 225.836 & Surface & 2008 & 10102.965 & 213.911 & Surface & 2008 \\
\hline 4763.087 & 225.804 & Surface & 2008 & 10106.163 & 213.921 & Surface & 2008 \\
\hline 4766.299 & 225.772 & Surface & 2008 & 10109.361 & 213.914 & Surface & 2008 \\
\hline 4769.511 & 225.752 & Surface & 2008 & 10112.559 & 213.867 & Surface & 2008 \\
\hline 4772.723 & 225.749 & Surface & 2008 & 10115.757 & 213.861 & Surface & 2008 \\
\hline 4775.936 & 225.734 & Surface & 2008 & 10118.954 & 213.921 & Surface & 2008 \\
\hline 4779.148 & 225.681 & Surface & 2008 & 10122.152 & 213.934 & Surface & 2008 \\
\hline 4782.360 & 225.626 & Surface & 2008 & 10125.350 & 213.943 & Surface & 2008 \\
\hline 4785.573 & 225.626 & Surface & 2008 & 10128.400 & 213.907 & Surface & 2008 \\
\hline 4788.785 & 225.653 & Surface & 2008 & 10131.450 & 213.853 & Surface & 2008 \\
\hline 4791.944 & 225.664 & Surface & 2008 & 10134.500 & 213.873 & Surface & 2008 \\
\hline 4795.104 & 225.640 & Surface & 2008 & 10137.550 & 213.909 & Surface & 2008 \\
\hline 4798.263 & 225.569 & Surface & 2008 & 10140.600 & 213.953 & Surface & 2008 \\
\hline 4801.423 & 225.544 & Surface & 2008 & 10143.650 & 213.978 & Surface & 2008 \\
\hline 4804.582 & 225.556 & Surface & 2008 & 10146.700 & 213.986 & Surface & 2008 \\
\hline 4807.742 & 225.571 & Surface & 2008 & 10149.750 & 213.978 & Surface & 2008 \\
\hline 4810.901 & 225.515 & Surface & 2008 & 10152.800 & 213.986 & Surface & 2008 \\
\hline 4814.061 & 225.517 & Surface & 2008 & 10156.054 & 213.931 & Surface & 2008 \\
\hline 4817.220 & 225.507 & Surface & 2008 & 10159.307 & 213.904 & Surface & 2008 \\
\hline 4820.379 & 225.476 & Surface & 2008 & 10162.561 & 213.872 & Surface & 2008 \\
\hline 4823.539 & 225.517 & Surface & 2008 & 10165.815 & 213.887 & Surface & 2008 \\
\hline 4826.698 & 225.475 & Surface & 2008 & 10169.069 & 213.906 & Surface & 2008 \\
\hline 4829.858 & 225.452 & Surface & 2008 & 10172.323 & 213.871 & Surface & 2008 \\
\hline 4833.017 & 225.458 & Surface & 2008 & 10175.576 & 213.827 & Surface & 2008 \\
\hline 4836.176 & 225.454 & Surface & 2008 & 10178.830 & 213.878 & Surface & 2008 \\
\hline 4839.336 & 225.407 & Surface & 2008 & 10182.084 & 213.930 & Surface & 2008 \\
\hline 4842.495 & 225.341 & Surface & 2008 & 10185.338 & 213.901 & Surface & 2008 \\
\hline 4845.655 & 225.304 & Surface & 2008 & 10188.467 & 213.915 & Surface & 2008 \\
\hline
\end{tabular}




\begin{tabular}{|c|c|c|c|c|c|c|c|}
\hline 4848.816 & 225.303 & Surface & 2008 & 10191.597 & 213.888 & Surface & 2008 \\
\hline 4851.978 & 225.287 & Surface & 2008 & 10194.727 & 213.866 & Surface & 2008 \\
\hline 4855.139 & 225.210 & Surface & 2008 & 10197.857 & 213.883 & Surface & 2008 \\
\hline 4858.301 & 225.126 & Surface & 2008 & 10200.987 & 213.869 & Surface & 2008 \\
\hline 4861.462 & 225.068 & Surface & 2008 & 10204.117 & 213.835 & Surface & 2008 \\
\hline 4864.624 & 225.083 & Surface & 2008 & 10207.246 & 213.837 & Surface & 2008 \\
\hline 4867.786 & 225.080 & Surface & 2008 & 10210.376 & 213.848 & Surface & 2008 \\
\hline 4870.947 & 225.064 & Surface & 2008 & 10213.506 & 213.856 & Surface & 2008 \\
\hline 4874.109 & 225.043 & Surface & 2008 & 10216.636 & 213.898 & Surface & 2008 \\
\hline 4877.270 & 225.029 & Surface & 2008 & 10219.824 & 213.906 & Surface & 2008 \\
\hline 4880.432 & 225.040 & Surface & 2008 & 10223.013 & 213.853 & Surface & 2008 \\
\hline 4883.594 & 225.098 & Surface & 2008 & 10226.202 & 213.853 & Surface & 2008 \\
\hline 4886.755 & 225.081 & Surface & 2008 & 10229.390 & 213.816 & Surface & 2008 \\
\hline 4889.917 & 225.020 & Surface & 2008 & 10232.579 & 213.799 & Surface & 2008 \\
\hline 4893.111 & 224.990 & Surface & 2008 & 10235.768 & 213.766 & Surface & 2008 \\
\hline 4896.306 & 225.012 & Surface & 2008 & 10238.957 & 213.798 & Surface & 2008 \\
\hline 4899.500 & 225.002 & Surface & 2008 & 10242.145 & 213.825 & Surface & 2008 \\
\hline 4902.695 & 224.936 & Surface & 2008 & 10245.334 & 213.830 & Surface & 2008 \\
\hline 4905.889 & 224.940 & Surface & 2008 & 10248.383 & 213.767 & Surface & 2008 \\
\hline 4909.084 & 225.012 & Surface & 2008 & 10251.432 & 213.774 & Surface & 2008 \\
\hline 4912.279 & 224.990 & Surface & 2008 & 10254.482 & 213.846 & Surface & 2008 \\
\hline 4915.473 & 224.960 & Surface & 2008 & 10257.531 & 213.863 & Surface & 2008 \\
\hline 4918.668 & 224.957 & Surface & 2008 & 10260.580 & 213.865 & Surface & 2008 \\
\hline 4921.862 & 224.979 & Surface & 2008 & 10263.629 & 213.880 & Surface & 2008 \\
\hline 4925.057 & 225.001 & Surface & 2008 & 10266.678 & 213.850 & Surface & 2008 \\
\hline 4928.251 & 225.000 & Surface & 2008 & 10269.728 & 213.772 & Surface & 2008 \\
\hline 4931.446 & 224.973 & Surface & 2008 & 10272.777 & 213.765 & Surface & 2008 \\
\hline 4934.640 & 225.020 & Surface & 2008 & 10275.826 & 213.780 & Surface & 2008 \\
\hline 4937.835 & 225.021 & Surface & 2008 & 10278.875 & 213.805 & Surface & 2008 \\
\hline 4941.029 & 224.987 & Surface & 2008 & 10281.998 & 213.740 & Surface & 2008 \\
\hline 4944.224 & 224.937 & Surface & 2008 & 10285.120 & 213.810 & Surface & 2008 \\
\hline 4947.418 & 224.917 & Surface & 2008 & 10288.243 & 213.836 & Surface & 2008 \\
\hline 4950.528 & 224.973 & Surface & 2008 & 10291.365 & 213.809 & Surface & 2008 \\
\hline 4953.639 & 225.012 & Surface & 2008 & 10294.488 & 213.797 & Surface & 2008 \\
\hline 4956.748 & 224.978 & Surface & 2008 & 10297.610 & 213.788 & Surface & 2008 \\
\hline 4959.858 & 224.942 & Surface & 2008 & 10300.733 & 213.806 & Surface & 2008 \\
\hline 4962.968 & 224.926 & Surface & 2008 & 10303.855 & 213.827 & Surface & 2008 \\
\hline 4966.078 & 224.958 & Surface & 2008 & 10306.978 & 213.853 & Surface & 2008 \\
\hline 4969.188 & 224.980 & Surface & 2008 & 10310.100 & 213.849 & Surface & 2008 \\
\hline 4972.298 & 224.951 & Surface & 2008 & 10313.223 & 213.799 & Surface & 2008 \\
\hline 4975.408 & 224.921 & Surface & 2008 & 10316.345 & 213.780 & Surface & 2008 \\
\hline 4978.518 & 224.896 & Surface & 2008 & 10319.468 & 213.804 & Surface & 2008 \\
\hline 4981.628 & 224.879 & Surface & 2008 & 10322.590 & 213.800 & Surface & 2008 \\
\hline 4984.738 & 224.853 & Surface & 2008 & 10325.713 & 213.804 & Surface & 2008 \\
\hline 4987.848 & 224.827 & Surface & 2008 & 10328.835 & 213.813 & Surface & 2008 \\
\hline 4990.958 & 224.793 & Surface & 2008 & 10332.107 & 213.805 & Surface & 2008 \\
\hline 4994.068 & 224.796 & Surface & 2008 & 10335.378 & 213.775 & Surface & 2008 \\
\hline 4997.293 & 224.775 & Surface & 2008 & 10338.650 & 213.780 & Surface & 2008 \\
\hline 5000.517 & 224.708 & Surface & 2008 & 10341.921 & 213.797 & Surface & 2008 \\
\hline
\end{tabular}




\begin{tabular}{|c|c|c|c|c|c|c|c|}
\hline 5003.743 & 224.652 & Surface & 2008 & 10345.193 & 213.799 & Surface & 2008 \\
\hline 5006.967 & 224.660 & Surface & 2008 & 10348.464 & 213.796 & Surface & 2008 \\
\hline 5010.192 & 224.623 & Surface & 2008 & 10351.736 & 213.788 & Surface & 2008 \\
\hline 5013.417 & 224.582 & Surface & 2008 & 10355.007 & 213.803 & Surface & 2008 \\
\hline 5016.642 & 224.565 & Surface & 2008 & 10358.279 & 213.820 & Surface & 2008 \\
\hline 5019.866 & 224.620 & Surface & 2008 & 10361.550 & 213.821 & Surface & 2008 \\
\hline 5023.091 & 224.654 & Surface & 2008 & 10364.821 & 213.807 & Surface & 2008 \\
\hline 5026.316 & 224.669 & Surface & 2008 & 10368.093 & 213.791 & Surface & 2008 \\
\hline 5029.541 & 224.657 & Surface & 2008 & 10371.168 & 213.814 & Surface & 2008 \\
\hline 5032.765 & 224.588 & Surface & 2008 & 10374.243 & 213.835 & Surface & 2008 \\
\hline 5035.990 & 224.622 & Surface & 2008 & 10377.318 & 213.788 & Surface & 2008 \\
\hline 5039.215 & 224.610 & Surface & 2008 & 10380.392 & 213.744 & Surface & 2008 \\
\hline 5042.440 & 224.575 & Surface & 2008 & 10383.467 & 213.727 & Surface & 2008 \\
\hline 5045.665 & 224.607 & Surface & 2008 & 10386.542 & 213.709 & Surface & 2008 \\
\hline 5048.890 & 224.650 & Surface & 2008 & 10389.617 & 213.700 & Surface & 2008 \\
\hline 5052.114 & 224.622 & Surface & 2008 & 10392.692 & 213.712 & Surface & 2008 \\
\hline 5055.339 & 224.620 & Surface & 2008 & 10395.766 & 213.724 & Surface & 2008 \\
\hline 5058.564 & 224.623 & Surface & 2008 & 10398.841 & 213.724 & Surface & 2008 \\
\hline 5061.789 & 224.628 & Surface & 2008 & 10401.916 & 213.736 & Surface & 2008 \\
\hline 5065.013 & 224.618 & Surface & 2008 & 10404.991 & 213.764 & Surface & 2008 \\
\hline 5068.238 & 224.583 & Surface & 2008 & 10408.065 & 213.748 & Surface & 2008 \\
\hline 5071.463 & 224.572 & Surface & 2008 & 10411.140 & 213.737 & Surface & 2008 \\
\hline 5074.688 & 224.593 & Surface & 2008 & 10414.238 & 213.738 & Surface & 2008 \\
\hline 5077.913 & 224.570 & Surface & 2008 & 10417.336 & 213.759 & Surface & 2008 \\
\hline 5081.138 & 224.548 & Surface & 2008 & 10420.434 & 213.766 & Surface & 2008 \\
\hline 5084.362 & 224.575 & Surface & 2008 & 10423.532 & 213.768 & Surface & 2008 \\
\hline 5087.519 & 224.600 & Surface & 2008 & 10426.630 & 213.753 & Surface & 2008 \\
\hline 5090.675 & 224.557 & Surface & 2008 & 10429.728 & 213.710 & Surface & 2008 \\
\hline 5093.832 & 224.552 & Surface & 2008 & 10432.826 & 213.696 & Surface & 2008 \\
\hline 5096.989 & 224.586 & Surface & 2008 & 10435.924 & 213.714 & Surface & 2008 \\
\hline 5100.145 & 224.572 & Surface & 2008 & 10439.022 & 213.740 & Surface & 2008 \\
\hline 5103.302 & 224.550 & Surface & 2008 & 10442.120 & 213.746 & Surface & 2008 \\
\hline 5106.458 & 224.545 & Surface & 2008 & 10445.218 & 213.725 & Surface & 2008 \\
\hline 5109.615 & 224.518 & Surface & 2008 & 10448.316 & 213.697 & Surface & 2008 \\
\hline 5112.772 & 224.492 & Surface & 2008 & 10451.414 & 213.717 & Surface & 2008 \\
\hline 5115.928 & 224.505 & Surface & 2008 & 10454.512 & 213.692 & Surface & 2008 \\
\hline 5119.085 & 224.508 & Surface & 2008 & 10457.610 & 213.720 & Surface & 2008 \\
\hline 5122.241 & 224.555 & Surface & 2008 & 10460.708 & 213.777 & Surface & 2008 \\
\hline 5125.398 & 224.581 & Surface & 2008 & 10463.806 & 213.758 & Surface & 2008 \\
\hline 5128.554 & 224.524 & Surface & 2008 & 10467.039 & 213.682 & Surface & 2008 \\
\hline 5131.711 & 224.522 & Surface & 2008 & 10470.272 & 213.669 & Surface & 2008 \\
\hline 5134.868 & 224.495 & Surface & 2008 & 10473.504 & 213.677 & Surface & 2008 \\
\hline 5138.024 & 224.461 & Surface & 2008 & 10476.737 & 213.731 & Surface & 2008 \\
\hline 5141.181 & 224.435 & Surface & 2008 & 10479.969 & 213.740 & Surface & 2008 \\
\hline 5144.337 & 224.433 & Surface & 2008 & 10483.202 & 213.704 & Surface & 2008 \\
\hline 5147.494 & 224.408 & Surface & 2008 & 10486.434 & 213.713 & Surface & 2008 \\
\hline 5150.650 & 224.403 & Surface & 2008 & 10489.667 & 213.751 & Surface & 2008 \\
\hline 5153.807 & 224.394 & Surface & 2008 & 10492.900 & 213.738 & Surface & 2008 \\
\hline 5157.081 & 224.379 & Surface & 2008 & 10496.132 & 213.754 & Surface & 2008 \\
\hline
\end{tabular}




\begin{tabular}{|c|c|c|c|c|c|c|c|}
\hline 5160.354 & 224.358 & Surface & 2008 & 10499.365 & 213.714 & Surface & 2008 \\
\hline 5163.628 & 224.400 & Surface & 2008 & 10502.598 & 213.663 & Surface & 2008 \\
\hline 5166.901 & 224.480 & Surface & 2008 & 10505.830 & 213.656 & Surface & 2008 \\
\hline 5170.175 & 224.466 & Surface & 2008 & 10509.063 & 213.682 & Surface & 2008 \\
\hline 5173.449 & 224.417 & Surface & 2008 & 10512.295 & 213.703 & Surface & 2008 \\
\hline 5176.722 & 224.413 & Surface & 2008 & 10515.528 & 213.674 & Surface & 2008 \\
\hline 5179.996 & 224.420 & Surface & 2008 & 10518.760 & 213.647 & Surface & 2008 \\
\hline 5183.270 & 224.448 & Surface & 2008 & 10521.993 & 213.656 & Surface & 2008 \\
\hline 5186.543 & 224.435 & Surface & 2008 & 10525.226 & 213.659 & Surface & 2008 \\
\hline 5189.817 & 224.401 & Surface & 2008 & 10528.448 & 213.667 & Surface & 2008 \\
\hline 5193.091 & 224.410 & Surface & 2008 & 10531.670 & 213.672 & Surface & 2008 \\
\hline 5196.364 & 224.407 & Surface & 2008 & 10534.893 & 213.682 & Surface & 2008 \\
\hline 5199.638 & 224.358 & Surface & 2008 & 10538.115 & 213.727 & Surface & 2008 \\
\hline 5202.912 & 224.374 & Surface & 2008 & 10541.337 & 213.732 & Surface & 2008 \\
\hline 5206.185 & 224.436 & Surface & 2008 & 10544.560 & 213.711 & Surface & 2008 \\
\hline 5209.459 & 224.450 & Surface & 2008 & 10547.782 & 213.696 & Surface & 2008 \\
\hline 5212.732 & 224.450 & Surface & 2008 & 10551.004 & 213.700 & Surface & 2008 \\
\hline 5216.006 & 224.419 & Surface & 2008 & 10554.227 & 213.709 & Surface & 2008 \\
\hline 5219.279 & 224.397 & Surface & 2008 & 10557.449 & 213.673 & Surface & 2008 \\
\hline 5222.553 & 224.428 & Surface & 2008 & 10560.671 & 213.635 & Surface & 2008 \\
\hline 5225.726 & 224.465 & Surface & 2008 & 10563.894 & 213.708 & Surface & 2008 \\
\hline 5228.898 & 224.467 & Surface & 2008 & 10567.116 & 213.709 & Surface & 2008 \\
\hline 5232.071 & 224.466 & Surface & 2008 & 10570.338 & 213.699 & Surface & 2008 \\
\hline 5235.243 & 224.429 & Surface & 2008 & 10573.561 & 213.693 & Surface & 2008 \\
\hline 5238.415 & 224.431 & Surface & 2008 & 10576.735 & 213.681 & Surface & 2008 \\
\hline 5241.588 & 224.449 & Surface & 2008 & 10579.910 & 213.701 & Surface & 2008 \\
\hline 5244.760 & 224.438 & Surface & 2008 & 10583.084 & 213.722 & Surface & 2008 \\
\hline 5247.933 & 224.423 & Surface & 2008 & 10586.259 & 213.702 & Surface & 2008 \\
\hline 5251.105 & 224.441 & Surface & 2008 & 10589.433 & 213.687 & Surface & 2008 \\
\hline 5254.278 & 224.457 & Surface & 2008 & 10592.608 & 213.715 & Surface & 2008 \\
\hline 5257.450 & 224.473 & Surface & 2008 & 10595.782 & 213.745 & Surface & 2008 \\
\hline 5260.623 & 224.448 & Surface & 2008 & 10598.957 & 213.736 & Surface & 2008 \\
\hline 5263.795 & 224.416 & Surface & 2008 & 10602.131 & 213.721 & Surface & 2008 \\
\hline 5266.967 & 224.443 & Surface & 2008 & 10605.306 & 213.717 & Surface & 2008 \\
\hline 5270.140 & 224.426 & Surface & 2008 & 10608.480 & 213.709 & Surface & 2008 \\
\hline 5273.312 & 224.435 & Surface & 2008 & 10611.655 & 213.717 & Surface & 2008 \\
\hline 5276.485 & 224.455 & Surface & 2008 & 10614.829 & 213.728 & Surface & 2008 \\
\hline 5279.629 & 224.463 & Surface & 2008 & 10618.004 & 213.747 & Surface & 2008 \\
\hline 5282.773 & 224.472 & Surface & 2008 & 10621.204 & 213.744 & Surface & 2008 \\
\hline 5285.917 & 224.500 & Surface & 2008 & 10624.403 & 213.728 & Surface & 2008 \\
\hline 5289.061 & 224.481 & Surface & 2008 & 10627.603 & 213.718 & Surface & 2008 \\
\hline 5292.205 & 224.430 & Surface & 2008 & 10630.803 & 213.700 & Surface & 2008 \\
\hline 5295.349 & 224.423 & Surface & 2008 & 10634.003 & 213.700 & Surface & 2008 \\
\hline 5298.493 & 224.409 & Surface & 2008 & 10637.202 & 213.695 & Surface & 2008 \\
\hline 5301.638 & 224.458 & Surface & 2008 & 10640.402 & 213.712 & Surface & 2008 \\
\hline 5304.782 & 224.438 & Surface & 2008 & 10643.602 & 213.744 & Surface & 2008 \\
\hline 5307.926 & 224.445 & Surface & 2008 & 10646.802 & 213.724 & Surface & 2008 \\
\hline 5311.070 & 224.477 & Surface & 2008 & 10650.002 & 213.737 & Surface & 2008 \\
\hline 5314.214 & 224.500 & Surface & 2008 & 10653.201 & 213.756 & Surface & 2008 \\
\hline
\end{tabular}




$\begin{array}{llllllll}5317.358 & 224.485 & \text { Surface } & 2008 & 10656.401 & 213.770 & \text { Surface } & 2008 \\ 5320.502 & 224.462 & \text { Surface } & 2008 & 10659.601 & 213.774 & \text { Surface } & 2008 \\ 5323.647 & 224.464 & \text { Surface } & 2008 & 10662.801 & 213.749 & \text { Surface } & 2008 \\ 5326.791 & 224.465 & \text { Surface } & 2008 & 10666.001 & 213.722 & \text { Surface } & 2008 \\ 5329.935 & 224.489 & \text { Surface } & 2008 & 10669.200 & 213.780 & \text { Surface } & 2008 \\ 5333.079 & 224.482 & \text { Surface } & 2008 & 10671.777 & 213.789 & \text { Surface } & 2008 \\ 5336.223 & 224.455 & \text { Surface } & 2008 & 10674.354 & 213.789 & \text { Surface } & 2008 \\ 5339.371 & 224.439 & \text { Surface } & 2008 & 10676.931 & 213.784 & \text { Surface } & 2008\end{array}$


11.3 Stream Gradient - 2011 Lidar:

\begin{tabular}{|c|c|c|c|c|c|c|c|}
\hline Distance & $\mathrm{Z}$ & DEM & Year & Distance & $\mathrm{Z}$ & DEM & Year \\
\hline 0.000 & 233.306 & Surface & 2011 & 5355.962 & 222.779 & Surface & 2011 \\
\hline 2.980 & 233.295 & Surface & 2011 & 5358.877 & 222.798 & Surface & 2011 \\
\hline 5.959 & 233.280 & Surface & 2011 & 5361.792 & 222.815 & Surface & 2011 \\
\hline 8.939 & 233.255 & Surface & 2011 & 5364.706 & 222.808 & Surface & 2011 \\
\hline 11.919 & 233.258 & Surface & 2011 & 5367.621 & 222.787 & Surface & 2011 \\
\hline 14.899 & 233.280 & Surface & 2011 & 5370.536 & 222.766 & Surface & 2011 \\
\hline 17.878 & 233.291 & Surface & 2011 & 5373.450 & 222.766 & Surface & 2011 \\
\hline 20.858 & 233.282 & Surface & 2011 & 5376.365 & 222.764 & Surface & 2011 \\
\hline 23.838 & 233.256 & Surface & 2011 & 5379.280 & 222.752 & Surface & 2011 \\
\hline 26.818 & 233.252 & Surface & 2011 & 5382.194 & 222.750 & Surface & 2011 \\
\hline 29.797 & 233.277 & Surface & 2011 & 5385.109 & 222.749 & Surface & 2011 \\
\hline 32.777 & 233.295 & Surface & 2011 & 5388.024 & 222.779 & Surface & 2011 \\
\hline 35.757 & 233.302 & Surface & 2011 & 5390.938 & 222.767 & Surface & 2011 \\
\hline 38.737 & 233.287 & Surface & 2011 & 5393.853 & 222.737 & Surface & 2011 \\
\hline 41.716 & 233.287 & Surface & 2011 & 5396.767 & 222.705 & Surface & 2011 \\
\hline 44.696 & 233.278 & Surface & 2011 & 5399.682 & 222.684 & Surface & 2011 \\
\hline 47.676 & 233.283 & Surface & 2011 & 5402.597 & 222.663 & Surface & 2011 \\
\hline 50.656 & 233.273 & Surface & 2011 & 5405.511 & 222.645 & Surface & 2011 \\
\hline 53.635 & 233.248 & Surface & 2011 & 5408.426 & 222.612 & Surface & 2011 \\
\hline 56.615 & 233.248 & Surface & 2011 & 5411.341 & 222.580 & Surface & 2011 \\
\hline 59.595 & 233.275 & Surface & 2011 & 5414.255 & 222.556 & Surface & 2011 \\
\hline 62.575 & 233.283 & Surface & 2011 & 5417.170 & 222.541 & Surface & 2011 \\
\hline 65.555 & 233.285 & Surface & 2011 & 5420.085 & 222.537 & Surface & 2011 \\
\hline 68.534 & 233.284 & Surface & 2011 & 5422.999 & 222.531 & Surface & 2011 \\
\hline 71.514 & 233.277 & Surface & 2011 & 5425.914 & 222.523 & Surface & 2011 \\
\hline 74.494 & 233.274 & Surface & 2011 & 5428.872 & 222.514 & Surface & 2011 \\
\hline 77.444 & 233.269 & Surface & 2011 & 5431.831 & 222.488 & Surface & 2011 \\
\hline 80.394 & 233.258 & Surface & 2011 & 5434.789 & 222.477 & Surface & 2011 \\
\hline 83.345 & 233.258 & Surface & 2011 & 5437.748 & 222.485 & Surface & 2011 \\
\hline 86.295 & 233.259 & Surface & 2011 & 5440.706 & 222.489 & Surface & 2011 \\
\hline 89.245 & 233.252 & Surface & 2011 & 5443.664 & 222.485 & Surface & 2011 \\
\hline 92.196 & 233.244 & Surface & 2011 & 5446.623 & 222.485 & Surface & 2011 \\
\hline 95.146 & 233.264 & Surface & 2011 & 5449.581 & 222.476 & Surface & 2011 \\
\hline 98.096 & 233.248 & Surface & 2011 & 5452.540 & 222.485 & Surface & 2011 \\
\hline 101.047 & 233.242 & Surface & 2011 & 5455.498 & 222.499 & Surface & 2011 \\
\hline 103.997 & 233.241 & Surface & 2011 & 5458.457 & 222.502 & Surface & 2011 \\
\hline 106.947 & 233.232 & Surface & 2011 & 5461.415 & 222.478 & Surface & 2011 \\
\hline 109.898 & 233.225 & Surface & 2011 & 5464.373 & 222.468 & Surface & 2011 \\
\hline 112.848 & 233.234 & Surface & 2011 & 5467.332 & 222.473 & Surface & 2011 \\
\hline 115.798 & 233.240 & Surface & 2011 & 5470.290 & 222.475 & Surface & 2011 \\
\hline 118.749 & 233.235 & Surface & 2011 & 5473.248 & 222.462 & Surface & 2011 \\
\hline 121.699 & 233.241 & Surface & 2011 & 5476.207 & 222.468 & Surface & 2011 \\
\hline 124.649 & 233.257 & Surface & 2011 & 5479.165 & 222.451 & Surface & 2011 \\
\hline 127.600 & 233.264 & Surface & 2011 & 5482.124 & 222.438 & Surface & 2011 \\
\hline 130.550 & 233.261 & Surface & 2011 & 5485.082 & 222.430 & Surface & 2011 \\
\hline
\end{tabular}




\begin{tabular}{|c|c|c|c|c|c|c|c|}
\hline 133.500 & 233.230 & Surface & 2011 & 5488.041 & 222.434 & Surface & 2011 \\
\hline 136.451 & 233.211 & Surface & 2011 & 5490.999 & 222.448 & Surface & 2011 \\
\hline 139.401 & 233.213 & Surface & 2011 & 5493.958 & 222.447 & Surface & 2011 \\
\hline 142.351 & 233.213 & Surface & 2011 & 5496.916 & 222.420 & Surface & 2011 \\
\hline 145.302 & 233.206 & Surface & 2011 & 5499.874 & 222.388 & Surface & 2011 \\
\hline 148.252 & 233.174 & Surface & 2011 & 5502.833 & 222.391 & Surface & 2011 \\
\hline 151.202 & 233.154 & Surface & 2011 & 5505.791 & 222.393 & Surface & 2011 \\
\hline 154.153 & 233.157 & Surface & 2011 & 5508.749 & 222.392 & Surface & 2011 \\
\hline 157.103 & 233.168 & Surface & 2011 & 5511.708 & 222.392 & Surface & 2011 \\
\hline 160.053 & 233.166 & Surface & 2011 & 5514.674 & 222.390 & Surface & 2011 \\
\hline 163.003 & 233.160 & Surface & 2011 & 5517.639 & 222.388 & Surface & 2011 \\
\hline 165.954 & 233.156 & Surface & 2011 & 5520.605 & 222.391 & Surface & 2011 \\
\hline 168.904 & 233.145 & Surface & 2011 & 5523.570 & 222.391 & Surface & 2011 \\
\hline 171.854 & 233.141 & Surface & 2011 & 5526.536 & 222.373 & Surface & 2011 \\
\hline 174.805 & 233.139 & Surface & 2011 & 5529.502 & 222.367 & Surface & 2011 \\
\hline 177.755 & 233.140 & Surface & 2011 & 5532.468 & 222.366 & Surface & 2011 \\
\hline 180.705 & 233.134 & Surface & 2011 & 5535.433 & 222.349 & Surface & 2011 \\
\hline 183.656 & 233.097 & Surface & 2011 & 5538.399 & 222.329 & Surface & 2011 \\
\hline 186.606 & 233.076 & Surface & 2011 & 5541.364 & 222.346 & Surface & 2011 \\
\hline 189.556 & 233.088 & Surface & 2011 & 5544.330 & 222.360 & Surface & 2011 \\
\hline 192.507 & 233.105 & Surface & 2011 & 5547.296 & 222.346 & Surface & 2011 \\
\hline 195.457 & 233.093 & Surface & 2011 & 5550.261 & 222.309 & Surface & 2011 \\
\hline 198.407 & 233.080 & Surface & 2011 & 5553.227 & 222.263 & Surface & 2011 \\
\hline 201.358 & 233.082 & Surface & 2011 & 5556.193 & 222.255 & Surface & 2011 \\
\hline 204.308 & 233.087 & Surface & 2011 & 5559.158 & 222.283 & Surface & 2011 \\
\hline 207.258 & 233.076 & Surface & 2011 & 5562.124 & 222.284 & Surface & 2011 \\
\hline 210.209 & 233.056 & Surface & 2011 & 5565.089 & 222.265 & Surface & 2011 \\
\hline 213.159 & 233.056 & Surface & 2011 & 5568.055 & 222.239 & Surface & 2011 \\
\hline 216.109 & 233.048 & Surface & 2011 & 5571.021 & 222.229 & Surface & 2011 \\
\hline 219.059 & 233.041 & Surface & 2011 & 5573.986 & 222.248 & Surface & 2011 \\
\hline 222.010 & 233.048 & Surface & 2011 & 5576.952 & 222.254 & Surface & 2011 \\
\hline 224.960 & 233.060 & Surface & 2011 & 5579.918 & 222.252 & Surface & 2011 \\
\hline 227.910 & 233.057 & Surface & 2011 & 5582.883 & 222.256 & Surface & 2011 \\
\hline 230.861 & 233.039 & Surface & 2011 & 5585.849 & 222.262 & Surface & 2011 \\
\hline 233.811 & 233.020 & Surface & 2011 & 5588.815 & 222.232 & Surface & 2011 \\
\hline 236.761 & 233.007 & Surface & 2011 & 5591.705 & 222.164 & Surface & 2011 \\
\hline 239.712 & 233.015 & Surface & 2011 & 5594.595 & 222.165 & Surface & 2011 \\
\hline 242.662 & 233.027 & Surface & 2011 & 5597.485 & 222.161 & Surface & 2011 \\
\hline 245.584 & 233.004 & Surface & 2011 & 5600.375 & 222.157 & Surface & 2011 \\
\hline 248.507 & 232.980 & Surface & 2011 & 5603.265 & 222.156 & Surface & 2011 \\
\hline 251.429 & 232.964 & Surface & 2011 & 5606.155 & 222.165 & Surface & 2011 \\
\hline 254.351 & 232.966 & Surface & 2011 & 5609.045 & 222.159 & Surface & 2011 \\
\hline 257.274 & 232.957 & Surface & 2011 & 5611.935 & 222.137 & Surface & 2011 \\
\hline 260.196 & 232.951 & Surface & 2011 & 5614.825 & 222.115 & Surface & 2011 \\
\hline 263.118 & 232.932 & Surface & 2011 & 5617.715 & 222.080 & Surface & 2011 \\
\hline 266.041 & 232.904 & Surface & 2011 & 5620.605 & 222.047 & Surface & 2011 \\
\hline 268.963 & 232.886 & Surface & 2011 & 5623.495 & 222.035 & Surface & 2011 \\
\hline 271.885 & 232.853 & Surface & 2011 & 5626.385 & 222.039 & Surface & 2011 \\
\hline 274.807 & 232.874 & Surface & 2011 & 5629.275 & 222.032 & Surface & 2011 \\
\hline
\end{tabular}




\begin{tabular}{|c|c|c|c|c|c|c|c|}
\hline 277.730 & 232.876 & Surface & 2011 & 5632.165 & 222.020 & Surface & 2011 \\
\hline 280.652 & 232.867 & Surface & 2011 & 5635.055 & 221.999 & Surface & 2011 \\
\hline 283.574 & 232.849 & Surface & 2011 & 5637.945 & 221.964 & Surface & 2011 \\
\hline 286.497 & 232.816 & Surface & 2011 & 5640.835 & 221.922 & Surface & 2011 \\
\hline 289.419 & 232.782 & Surface & 2011 & 5643.833 & 221.876 & Surface & 2011 \\
\hline 292.341 & 232.778 & Surface & 2011 & 5646.832 & 221.851 & Surface & 2011 \\
\hline 295.264 & 232.772 & Surface & 2011 & 5649.830 & 221.876 & Surface & 2011 \\
\hline 298.186 & 232.750 & Surface & 2011 & 5652.828 & 221.861 & Surface & 2011 \\
\hline 301.108 & 232.731 & Surface & 2011 & 5655.826 & 221.861 & Surface & 2011 \\
\hline 304.030 & 232.728 & Surface & 2011 & 5658.825 & 221.862 & Surface & 2011 \\
\hline 306.953 & 232.736 & Surface & 2011 & 5661.823 & 221.827 & Surface & 2011 \\
\hline 309.875 & 232.729 & Surface & 2011 & 5664.822 & 221.783 & Surface & 2011 \\
\hline 312.797 & 232.709 & Surface & 2011 & 5667.820 & 221.788 & Surface & 2011 \\
\hline 315.719 & 232.626 & Surface & 2011 & 5670.818 & 221.795 & Surface & 2011 \\
\hline 318.642 & 232.553 & Surface & 2011 & 5673.816 & 221.792 & Surface & 2011 \\
\hline 321.564 & 232.592 & Surface & 2011 & 5676.815 & 221.787 & Surface & 2011 \\
\hline 324.487 & 232.608 & Surface & 2011 & 5679.813 & 221.795 & Surface & 2011 \\
\hline 327.409 & 232.588 & Surface & 2011 & 5682.811 & 221.811 & Surface & 2011 \\
\hline 330.345 & 232.549 & Surface & 2011 & 5685.810 & 221.803 & Surface & 2011 \\
\hline 333.282 & 232.531 & Surface & 2011 & 5688.808 & 221.794 & Surface & 2011 \\
\hline 336.218 & 232.523 & Surface & 2011 & 5691.806 & 221.777 & Surface & 2011 \\
\hline 339.155 & 232.486 & Surface & 2011 & 5694.805 & 221.780 & Surface & 2011 \\
\hline 342.091 & 232.458 & Surface & 2011 & 5697.803 & 221.807 & Surface & 2011 \\
\hline 345.028 & 232.454 & Surface & 2011 & 5700.801 & 221.826 & Surface & 2011 \\
\hline 347.965 & 232.428 & Surface & 2011 & 5703.799 & 221.820 & Surface & 2011 \\
\hline 350.901 & 232.377 & Surface & 2011 & 5706.752 & 221.806 & Surface & 2011 \\
\hline 353.838 & 232.343 & Surface & 2011 & 5709.705 & 221.792 & Surface & 2011 \\
\hline 356.774 & 232.320 & Surface & 2011 & 5712.658 & 221.791 & Surface & 2011 \\
\hline 359.710 & 232.286 & Surface & 2011 & 5715.611 & 221.781 & Surface & 2011 \\
\hline 362.647 & 232.255 & Surface & 2011 & 5718.564 & 221.776 & Surface & 2011 \\
\hline 365.584 & 232.242 & Surface & 2011 & 5721.517 & 221.763 & Surface & 2011 \\
\hline 368.520 & 232.237 & Surface & 2011 & 5724.470 & 221.736 & Surface & 2011 \\
\hline 371.457 & 232.196 & Surface & 2011 & 5727.423 & 221.724 & Surface & 2011 \\
\hline 374.393 & 232.186 & Surface & 2011 & 5730.376 & 221.767 & Surface & 2011 \\
\hline 377.330 & 232.169 & Surface & 2011 & 5733.329 & 221.801 & Surface & 2011 \\
\hline 380.266 & 232.110 & Surface & 2011 & 5736.282 & 221.809 & Surface & 2011 \\
\hline 383.203 & 232.039 & Surface & 2011 & 5739.235 & 221.807 & Surface & 2011 \\
\hline 386.139 & 232.041 & Surface & 2011 & 5742.187 & 221.804 & Surface & 2011 \\
\hline 389.076 & 232.063 & Surface & 2011 & 5745.140 & 221.776 & Surface & 2011 \\
\hline 392.013 & 232.046 & Surface & 2011 & 5748.093 & 221.766 & Surface & 2011 \\
\hline 394.949 & 231.991 & Surface & 2011 & 5751.046 & 221.752 & Surface & 2011 \\
\hline 397.885 & 231.978 & Surface & 2011 & 5753.999 & 221.740 & Surface & 2011 \\
\hline 400.822 & 231.955 & Surface & 2011 & 5756.952 & 221.745 & Surface & 2011 \\
\hline 403.758 & 231.917 & Surface & 2011 & 5759.905 & 221.750 & Surface & 2011 \\
\hline 406.695 & 231.895 & Surface & 2011 & 5762.858 & 221.732 & Surface & 2011 \\
\hline 409.632 & 231.863 & Surface & 2011 & 5765.811 & 221.708 & Surface & 2011 \\
\hline 412.568 & 231.791 & Surface & 2011 & 5768.704 & 221.721 & Surface & 2011 \\
\hline 415.505 & 231.681 & Surface & 2011 & 5771.597 & 221.740 & Surface & 2011 \\
\hline 418.441 & 231.615 & Surface & 2011 & 5774.490 & 221.743 & Surface & 2011 \\
\hline
\end{tabular}




\begin{tabular}{|c|c|c|c|c|c|c|c|}
\hline 421.378 & 231.608 & Surface & 2011 & 5777.383 & 221.741 & Surface & 2011 \\
\hline 424.314 & 231.583 & Surface & 2011 & 5780.277 & 221.734 & Surface & 2011 \\
\hline 427.251 & 231.542 & Surface & 2011 & 5783.170 & 221.724 & Surface & 2011 \\
\hline 430.187 & 231.499 & Surface & 2011 & 5786.063 & 221.719 & Surface & 2011 \\
\hline 433.124 & 231.453 & Surface & 2011 & 5788.956 & 221.716 & Surface & 2011 \\
\hline 436.060 & 231.357 & Surface & 2011 & 5791.849 & 221.718 & Surface & 2011 \\
\hline 438.997 & 231.293 & Surface & 2011 & 5794.742 & 221.716 & Surface & 2011 \\
\hline 441.792 & 231.201 & Surface & 2011 & 5797.636 & 221.716 & Surface & 2011 \\
\hline 444.586 & 231.118 & Surface & 2011 & 5800.529 & 221.687 & Surface & 2011 \\
\hline 447.381 & 231.073 & Surface & 2011 & 5803.422 & 221.661 & Surface & 2011 \\
\hline 450.176 & 230.997 & Surface & 2011 & 5806.315 & 221.678 & Surface & 2011 \\
\hline 452.970 & 230.908 & Surface & 2011 & 5809.208 & 221.701 & Surface & 2011 \\
\hline 455.765 & 230.816 & Surface & 2011 & 5812.101 & 221.662 & Surface & 2011 \\
\hline 458.560 & 230.754 & Surface & 2011 & 5814.994 & 221.528 & Surface & 2011 \\
\hline 461.465 & 230.711 & Surface & 2011 & 5817.888 & 221.519 & Surface & 2011 \\
\hline 464.370 & 230.630 & Surface & 2011 & 5820.781 & 221.532 & Surface & 2011 \\
\hline 467.275 & 230.538 & Surface & 2011 & 5823.674 & 221.537 & Surface & 2011 \\
\hline 470.180 & 230.459 & Surface & 2011 & 5826.567 & 221.503 & Surface & 2011 \\
\hline 473.085 & 230.451 & Surface & 2011 & 5829.460 & 221.450 & Surface & 2011 \\
\hline 475.989 & 230.492 & Surface & 2011 & 5832.353 & 221.435 & Surface & 2011 \\
\hline 478.894 & 230.514 & Surface & 2011 & 5835.247 & 221.432 & Surface & 2011 \\
\hline 481.799 & 230.531 & Surface & 2011 & 5838.140 & 221.414 & Surface & 2011 \\
\hline 484.704 & 230.532 & Surface & 2011 & 5841.033 & 221.405 & Surface & 2011 \\
\hline 487.609 & 230.542 & Surface & 2011 & 5843.926 & 221.381 & Surface & 2011 \\
\hline 490.514 & 230.554 & Surface & 2011 & 5846.819 & 221.298 & Surface & 2011 \\
\hline 493.419 & 230.563 & Surface & 2011 & 5849.681 & 221.226 & Surface & 2011 \\
\hline 496.324 & 230.560 & Surface & 2011 & 5852.542 & 221.150 & Surface & 2011 \\
\hline 499.229 & 230.548 & Surface & 2011 & 5855.403 & 221.110 & Surface & 2011 \\
\hline 502.134 & 230.553 & Surface & 2011 & 5858.265 & 221.091 & Surface & 2011 \\
\hline 505.039 & 230.563 & Surface & 2011 & 5861.126 & 221.082 & Surface & 2011 \\
\hline 507.944 & 230.547 & Surface & 2011 & 5863.987 & 221.091 & Surface & 2011 \\
\hline 510.849 & 230.538 & Surface & 2011 & 5866.849 & 221.106 & Surface & 2011 \\
\hline 513.754 & 230.542 & Surface & 2011 & 5869.710 & 221.125 & Surface & 2011 \\
\hline 516.659 & 230.558 & Surface & 2011 & 5872.571 & 221.134 & Surface & 2011 \\
\hline 519.564 & 230.565 & Surface & 2011 & 5875.433 & 221.107 & Surface & 2011 \\
\hline 522.469 & 230.564 & Surface & 2011 & 5878.294 & 221.112 & Surface & 2011 \\
\hline 525.374 & 230.562 & Surface & 2011 & 5881.156 & 221.145 & Surface & 2011 \\
\hline 528.279 & 230.557 & Surface & 2011 & 5884.017 & 221.166 & Surface & 2011 \\
\hline 531.184 & 230.558 & Surface & 2011 & 5886.878 & 221.169 & Surface & 2011 \\
\hline 534.163 & 230.542 & Surface & 2011 & 5889.720 & 221.164 & Surface & 2011 \\
\hline 537.143 & 230.496 & Surface & 2011 & 5892.562 & 221.157 & Surface & 2011 \\
\hline 540.122 & 230.526 & Surface & 2011 & 5895.404 & 221.162 & Surface & 2011 \\
\hline 543.101 & 230.590 & Surface & 2011 & 5898.247 & 221.175 & Surface & 2011 \\
\hline 546.081 & 230.599 & Surface & 2011 & 5901.089 & 221.193 & Surface & 2011 \\
\hline 549.060 & 230.581 & Surface & 2011 & 5903.931 & 221.201 & Surface & 2011 \\
\hline 552.039 & 230.540 & Surface & 2011 & 5906.772 & 221.193 & Surface & 2011 \\
\hline 555.018 & 230.500 & Surface & 2011 & 5909.614 & 221.183 & Surface & 2011 \\
\hline 557.998 & 230.501 & Surface & 2011 & 5912.456 & 221.221 & Surface & 2011 \\
\hline 560.977 & 230.520 & Surface & 2011 & 5915.298 & 221.220 & Surface & 2011 \\
\hline
\end{tabular}




\begin{tabular}{|c|c|c|c|c|c|c|c|}
\hline 563.719 & 230.525 & Surface & 2011 & 5918.141 & 221.222 & Surface & 2011 \\
\hline 566.461 & 230.521 & Surface & 2011 & 5920.983 & 221.230 & Surface & 2011 \\
\hline 569.202 & 230.512 & Surface & 2011 & 5923.825 & 221.239 & Surface & 2011 \\
\hline 571.944 & 230.481 & Surface & 2011 & 5926.667 & 221.220 & Surface & 2011 \\
\hline 574.686 & 230.478 & Surface & 2011 & 5929.560 & 221.180 & Surface & 2011 \\
\hline 577.428 & 230.504 & Surface & 2011 & 5932.453 & 221.193 & Surface & 2011 \\
\hline 580.169 & 230.518 & Surface & 2011 & 5935.346 & 221.210 & Surface & 2011 \\
\hline 582.911 & 230.507 & Surface & 2011 & 5938.239 & 221.204 & Surface & 2011 \\
\hline 585.653 & 230.493 & Surface & 2011 & 5941.133 & 221.187 & Surface & 2011 \\
\hline 588.482 & 230.492 & Surface & 2011 & 5944.026 & 221.197 & Surface & 2011 \\
\hline 591.312 & 230.489 & Surface & 2011 & 5946.919 & 221.203 & Surface & 2011 \\
\hline 594.142 & 230.470 & Surface & 2011 & 5949.812 & 221.188 & Surface & 2011 \\
\hline 596.972 & 230.466 & Surface & 2011 & 5952.705 & 221.170 & Surface & 2011 \\
\hline 599.802 & 230.494 & Surface & 2011 & 5955.598 & 221.170 & Surface & 2011 \\
\hline 602.632 & 230.503 & Surface & 2011 & 5958.491 & 221.191 & Surface & 2011 \\
\hline 605.461 & 230.489 & Surface & 2011 & 5961.384 & 221.199 & Surface & 2011 \\
\hline 608.291 & 230.485 & Surface & 2011 & 5964.278 & 221.198 & Surface & 2011 \\
\hline 611.121 & 230.498 & Surface & 2011 & 5967.171 & 221.189 & Surface & 2011 \\
\hline 613.951 & 230.482 & Surface & 2011 & 5970.064 & 221.179 & Surface & 2011 \\
\hline 616.780 & 230.469 & Surface & 2011 & 5972.957 & 221.160 & Surface & 2011 \\
\hline 619.610 & 230.437 & Surface & 2011 & 5975.850 & 221.156 & Surface & 2011 \\
\hline 622.440 & 230.402 & Surface & 2011 & 5978.744 & 221.165 & Surface & 2011 \\
\hline 625.270 & 230.383 & Surface & 2011 & 5981.637 & 221.183 & Surface & 2011 \\
\hline 628.214 & 230.385 & Surface & 2011 & 5984.530 & 221.190 & Surface & 2011 \\
\hline 631.158 & 230.386 & Surface & 2011 & 5987.423 & 221.176 & Surface & 2011 \\
\hline 634.102 & 230.370 & Surface & 2011 & 5990.316 & 221.167 & Surface & 2011 \\
\hline 637.046 & 230.357 & Surface & 2011 & 5993.209 & 221.166 & Surface & 2011 \\
\hline 639.990 & 230.355 & Surface & 2011 & 5996.103 & 221.158 & Surface & 2011 \\
\hline 642.934 & 230.355 & Surface & 2011 & 5998.996 & 221.154 & Surface & 2011 \\
\hline 645.878 & 230.353 & Surface & 2011 & 6001.889 & 221.162 & Surface & 2011 \\
\hline 648.822 & 230.357 & Surface & 2011 & 6004.782 & 221.171 & Surface & 2011 \\
\hline 651.766 & 230.360 & Surface & 2011 & 6007.675 & 221.178 & Surface & 2011 \\
\hline 654.709 & 230.363 & Surface & 2011 & 6010.660 & 221.179 & Surface & 2011 \\
\hline 657.653 & 230.380 & Surface & 2011 & 6013.645 & 221.175 & Surface & 2011 \\
\hline 660.597 & 230.394 & Surface & 2011 & 6016.630 & 221.169 & Surface & 2011 \\
\hline 663.541 & 230.366 & Surface & 2011 & 6019.614 & 221.165 & Surface & 2011 \\
\hline 666.486 & 230.355 & Surface & 2011 & 6022.599 & 221.168 & Surface & 2011 \\
\hline 669.429 & 230.356 & Surface & 2011 & 6025.584 & 221.169 & Surface & 2011 \\
\hline 672.373 & 230.363 & Surface & 2011 & 6028.568 & 221.157 & Surface & 2011 \\
\hline 675.317 & 230.364 & Surface & 2011 & 6031.553 & 221.169 & Surface & 2011 \\
\hline 678.261 & 230.372 & Surface & 2011 & 6034.538 & 221.179 & Surface & 2011 \\
\hline 681.205 & 230.383 & Surface & 2011 & 6037.523 & 221.186 & Surface & 2011 \\
\hline 684.149 & 230.353 & Surface & 2011 & 6040.508 & 221.164 & Surface & 2011 \\
\hline 687.093 & 230.366 & Surface & 2011 & 6043.492 & 221.139 & Surface & 2011 \\
\hline 690.037 & 230.372 & Surface & 2011 & 6046.477 & 221.149 & Surface & 2011 \\
\hline 692.981 & 230.360 & Surface & 2011 & 6049.462 & 221.175 & Surface & 2011 \\
\hline 695.925 & 230.366 & Surface & 2011 & 6052.447 & 221.189 & Surface & 2011 \\
\hline 698.869 & 230.361 & Surface & 2011 & 6055.432 & 221.182 & Surface & 2011 \\
\hline 701.813 & 230.355 & Surface & 2011 & 6058.416 & 221.182 & Surface & 2011 \\
\hline
\end{tabular}




\begin{tabular}{|c|c|c|c|c|c|c|c|}
\hline 704.675 & 230.315 & Surface & 2011 & 6061.401 & 221.181 & Surface & 2011 \\
\hline 707.538 & 230.299 & Surface & 2011 & 6064.386 & 221.163 & Surface & 2011 \\
\hline 710.400 & 230.322 & Surface & 2011 & 6067.370 & 221.167 & Surface & 2011 \\
\hline 713.263 & 230.344 & Surface & 2011 & 6070.355 & 221.180 & Surface & 2011 \\
\hline 716.125 & 230.347 & Surface & 2011 & 6073.340 & 221.185 & Surface & 2011 \\
\hline 718.988 & 230.335 & Surface & 2011 & 6076.325 & 221.173 & Surface & 2011 \\
\hline 721.902 & 230.327 & Surface & 2011 & 6079.310 & 221.170 & Surface & 2011 \\
\hline 724.817 & 230.337 & Surface & 2011 & 6082.294 & 221.181 & Surface & 2011 \\
\hline 727.731 & 230.352 & Surface & 2011 & 6085.279 & 221.191 & Surface & 2011 \\
\hline 730.646 & 230.372 & Surface & 2011 & 6088.258 & 221.173 & Surface & 2011 \\
\hline 733.560 & 230.372 & Surface & 2011 & 6091.237 & 221.192 & Surface & 2011 \\
\hline 736.474 & 230.355 & Surface & 2011 & 6094.215 & 221.178 & Surface & 2011 \\
\hline 739.389 & 230.346 & Surface & 2011 & 6097.194 & 221.180 & Surface & 2011 \\
\hline 742.303 & 230.345 & Surface & 2011 & 6100.173 & 221.181 & Surface & 2011 \\
\hline 745.218 & 230.342 & Surface & 2011 & 6103.152 & 221.181 & Surface & 2011 \\
\hline 748.132 & 230.358 & Surface & 2011 & 6106.131 & 221.180 & Surface & 2011 \\
\hline 751.047 & 230.367 & Surface & 2011 & 6109.109 & 221.172 & Surface & 2011 \\
\hline 753.962 & 230.347 & Surface & 2011 & 6112.088 & 221.170 & Surface & 2011 \\
\hline 756.865 & 230.341 & Surface & 2011 & 6115.067 & 221.166 & Surface & 2011 \\
\hline 759.769 & 230.352 & Surface & 2011 & 6118.046 & 221.158 & Surface & 2011 \\
\hline 762.673 & 230.361 & Surface & 2011 & 6121.024 & 221.156 & Surface & 2011 \\
\hline 765.576 & 230.362 & Surface & 2011 & 6124.003 & 221.177 & Surface & 2011 \\
\hline 768.480 & 230.369 & Surface & 2011 & 6126.982 & 221.194 & Surface & 2011 \\
\hline 771.384 & 230.364 & Surface & 2011 & 6129.899 & 221.204 & Surface & 2011 \\
\hline 774.287 & 230.356 & Surface & 2011 & 6132.817 & 221.186 & Surface & 2011 \\
\hline 777.191 & 230.344 & Surface & 2011 & 6135.734 & 221.169 & Surface & 2011 \\
\hline 780.095 & 230.347 & Surface & 2011 & 6138.652 & 221.157 & Surface & 2011 \\
\hline 782.998 & 230.347 & Surface & 2011 & 6141.569 & 221.157 & Surface & 2011 \\
\hline 785.902 & 230.360 & Surface & 2011 & 6144.487 & 221.157 & Surface & 2011 \\
\hline 788.891 & 230.373 & Surface & 2011 & 6147.404 & 221.154 & Surface & 2011 \\
\hline 791.880 & 230.377 & Surface & 2011 & 6150.322 & 221.147 & Surface & 2011 \\
\hline 794.868 & 230.378 & Surface & 2011 & 6153.239 & 221.147 & Surface & 2011 \\
\hline 797.857 & 230.372 & Surface & 2011 & 6156.157 & 221.149 & Surface & 2011 \\
\hline 800.846 & 230.362 & Surface & 2011 & 6159.074 & 221.148 & Surface & 2011 \\
\hline 803.834 & 230.380 & Surface & 2011 & 6161.992 & 221.148 & Surface & 2011 \\
\hline 806.823 & 230.399 & Surface & 2011 & 6164.909 & 221.133 & Surface & 2011 \\
\hline 809.812 & 230.397 & Surface & 2011 & 6167.827 & 221.139 & Surface & 2011 \\
\hline 812.801 & 230.380 & Surface & 2011 & 6170.744 & 221.151 & Surface & 2011 \\
\hline 815.789 & 230.370 & Surface & 2011 & 6173.662 & 221.151 & Surface & 2011 \\
\hline 818.778 & 230.377 & Surface & 2011 & 6176.579 & 221.140 & Surface & 2011 \\
\hline 821.767 & 230.380 & Surface & 2011 & 6179.497 & 221.123 & Surface & 2011 \\
\hline 824.755 & 230.370 & Surface & 2011 & 6182.414 & 221.119 & Surface & 2011 \\
\hline 827.744 & 230.389 & Surface & 2011 & 6185.332 & 221.132 & Surface & 2011 \\
\hline 830.733 & 230.390 & Surface & 2011 & 6188.249 & 221.151 & Surface & 2011 \\
\hline 833.722 & 230.393 & Surface & 2011 & 6191.167 & 221.158 & Surface & 2011 \\
\hline 836.652 & 230.389 & Surface & 2011 & 6194.084 & 221.145 & Surface & 2011 \\
\hline 839.582 & 230.386 & Surface & 2011 & 6197.002 & 221.132 & Surface & 2011 \\
\hline 842.512 & 230.391 & Surface & 2011 & 6199.919 & 221.127 & Surface & 2011 \\
\hline 845.443 & 230.387 & Surface & 2011 & 6202.836 & 221.122 & Surface & 2011 \\
\hline
\end{tabular}




\begin{tabular}{|c|c|c|c|c|c|c|c|}
\hline 848.372 & 230.377 & Surface & 2011 & 6205.753 & 221.111 & Surface & 2011 \\
\hline 851.303 & 230.366 & Surface & 2011 & 6208.669 & 221.116 & Surface & 2011 \\
\hline 854.233 & 230.352 & Surface & 2011 & 6211.586 & 221.125 & Surface & 2011 \\
\hline 857.163 & 230.381 & Surface & 2011 & 6214.503 & 221.125 & Surface & 2011 \\
\hline 860.093 & 230.399 & Surface & 2011 & 6217.419 & 221.115 & Surface & 2011 \\
\hline 863.024 & 230.379 & Surface & 2011 & 6220.336 & 221.120 & Surface & 2011 \\
\hline 865.954 & 230.349 & Surface & 2011 & 6223.253 & 221.142 & Surface & 2011 \\
\hline 868.884 & 230.318 & Surface & 2011 & 6226.169 & 221.156 & Surface & 2011 \\
\hline 871.814 & 230.290 & Surface & 2011 & 6229.086 & 221.142 & Surface & 2011 \\
\hline 874.744 & 230.264 & Surface & 2011 & 6232.003 & 221.126 & Surface & 2011 \\
\hline 877.675 & 230.250 & Surface & 2011 & 6234.919 & 221.103 & Surface & 2011 \\
\hline 880.605 & 230.252 & Surface & 2011 & 6237.836 & 221.093 & Surface & 2011 \\
\hline 883.535 & 230.255 & Surface & 2011 & 6240.753 & 221.100 & Surface & 2011 \\
\hline 886.465 & 230.259 & Surface & 2011 & 6243.669 & 221.101 & Surface & 2011 \\
\hline 889.459 & 230.268 & Surface & 2011 & 6246.586 & 221.088 & Surface & 2011 \\
\hline 892.452 & 230.274 & Surface & 2011 & 6249.503 & 221.081 & Surface & 2011 \\
\hline 895.445 & 230.273 & Surface & 2011 & 6252.419 & 221.079 & Surface & 2011 \\
\hline 898.439 & 230.267 & Surface & 2011 & 6255.336 & 221.073 & Surface & 2011 \\
\hline 901.432 & 230.253 & Surface & 2011 & 6258.253 & 221.069 & Surface & 2011 \\
\hline 904.426 & 230.240 & Surface & 2011 & 6261.169 & 221.065 & Surface & 2011 \\
\hline 907.419 & 230.218 & Surface & 2011 & 6264.086 & 221.060 & Surface & 2011 \\
\hline 910.413 & 230.128 & Surface & 2011 & 6267.003 & 221.049 & Surface & 2011 \\
\hline 913.406 & 230.121 & Surface & 2011 & 6269.919 & 221.058 & Surface & 2011 \\
\hline 916.399 & 230.092 & Surface & 2011 & 6272.836 & 221.056 & Surface & 2011 \\
\hline 919.393 & 230.054 & Surface & 2011 & 6275.772 & 221.037 & Surface & 2011 \\
\hline 922.386 & 230.041 & Surface & 2011 & 6278.707 & 221.042 & Surface & 2011 \\
\hline 925.379 & 230.040 & Surface & 2011 & 6281.643 & 221.044 & Surface & 2011 \\
\hline 928.373 & 230.000 & Surface & 2011 & 6284.579 & 221.021 & Surface & 2011 \\
\hline 931.366 & 229.845 & Surface & 2011 & 6287.515 & 221.005 & Surface & 2011 \\
\hline 934.276 & 229.845 & Surface & 2011 & 6290.450 & 221.021 & Surface & 2011 \\
\hline 937.185 & 229.803 & Surface & 2011 & 6293.386 & 221.038 & Surface & 2011 \\
\hline 940.095 & 229.746 & Surface & 2011 & 6296.322 & 221.034 & Surface & 2011 \\
\hline 943.004 & 229.758 & Surface & 2011 & 6299.258 & 221.034 & Surface & 2011 \\
\hline 945.913 & 229.756 & Surface & 2011 & 6302.193 & 221.030 & Surface & 2011 \\
\hline 948.823 & 229.719 & Surface & 2011 & 6305.129 & 221.026 & Surface & 2011 \\
\hline 951.732 & 229.680 & Surface & 2011 & 6308.065 & 221.004 & Surface & 2011 \\
\hline 954.642 & 229.645 & Surface & 2011 & 6311.001 & 220.996 & Surface & 2011 \\
\hline 957.551 & 229.607 & Surface & 2011 & 6313.936 & 220.999 & Surface & 2011 \\
\hline 960.461 & 229.574 & Surface & 2011 & 6316.872 & 220.997 & Surface & 2011 \\
\hline 963.370 & 229.537 & Surface & 2011 & 6319.808 & 221.005 & Surface & 2011 \\
\hline 966.279 & 229.521 & Surface & 2011 & 6322.743 & 221.032 & Surface & 2011 \\
\hline 969.189 & 229.509 & Surface & 2011 & 6325.679 & 220.994 & Surface & 2011 \\
\hline 972.098 & 229.481 & Surface & 2011 & 6328.615 & 220.995 & Surface & 2011 \\
\hline 975.008 & 229.423 & Surface & 2011 & 6331.551 & 221.009 & Surface & 2011 \\
\hline 977.917 & 229.362 & Surface & 2011 & 6334.486 & 221.026 & Surface & 2011 \\
\hline 980.827 & 229.327 & Surface & 2011 & 6337.479 & 221.041 & Surface & 2011 \\
\hline 983.620 & 229.307 & Surface & 2011 & 6340.472 & 221.008 & Surface & 2011 \\
\hline 986.413 & 229.252 & Surface & 2011 & 6343.466 & 220.984 & Surface & 2011 \\
\hline 989.206 & 229.168 & Surface & 2011 & 6346.459 & 221.000 & Surface & 2011 \\
\hline
\end{tabular}




\begin{tabular}{|c|c|c|c|c|c|c|c|}
\hline 991.999 & 229.099 & Surface & 2011 & 6349.452 & 221.018 & Surface & 2011 \\
\hline 994.792 & 229.051 & Surface & 2011 & 6352.445 & 221.023 & Surface & 2011 \\
\hline 997.585 & 229.057 & Surface & 2011 & 6355.439 & 221.021 & Surface & 2011 \\
\hline 1000.378 & 229.069 & Surface & 2011 & 6358.432 & 221.022 & Surface & 2011 \\
\hline 1003.171 & 229.083 & Surface & 2011 & 6361.425 & 221.044 & Surface & 2011 \\
\hline 1005.964 & 229.092 & Surface & 2011 & 6364.418 & 221.046 & Surface & 2011 \\
\hline 1008.757 & 229.101 & Surface & 2011 & 6367.412 & 221.042 & Surface & 2011 \\
\hline 1011.550 & 229.111 & Surface & 2011 & 6370.405 & 221.063 & Surface & 2011 \\
\hline 1014.343 & 229.115 & Surface & 2011 & 6373.398 & 221.061 & Surface & 2011 \\
\hline 1017.136 & 229.107 & Surface & 2011 & 6376.391 & 221.043 & Surface & 2011 \\
\hline 1019.929 & 229.099 & Surface & 2011 & 6379.384 & 221.041 & Surface & 2011 \\
\hline 1022.889 & 229.090 & Surface & 2011 & 6382.378 & 221.041 & Surface & 2011 \\
\hline 1025.849 & 229.084 & Surface & 2011 & 6385.371 & 221.041 & Surface & 2011 \\
\hline 1028.809 & 229.077 & Surface & 2011 & 6388.364 & 221.050 & Surface & 2011 \\
\hline 1031.770 & 229.101 & Surface & 2011 & 6391.357 & 221.048 & Surface & 2011 \\
\hline 1034.730 & 229.103 & Surface & 2011 & 6394.350 & 221.038 & Surface & 2011 \\
\hline 1037.690 & 229.119 & Surface & 2011 & 6397.239 & 221.016 & Surface & 2011 \\
\hline 1040.651 & 229.112 & Surface & 2011 & 6400.127 & 221.020 & Surface & 2011 \\
\hline 1043.611 & 229.115 & Surface & 2011 & 6403.016 & 221.030 & Surface & 2011 \\
\hline 1046.571 & 229.116 & Surface & 2011 & 6405.904 & 221.038 & Surface & 2011 \\
\hline 1049.531 & 229.115 & Surface & 2011 & 6408.792 & 221.045 & Surface & 2011 \\
\hline 1052.492 & 229.124 & Surface & 2011 & 6411.681 & 221.050 & Surface & 2011 \\
\hline 1055.452 & 229.126 & Surface & 2011 & 6414.570 & 221.053 & Surface & 2011 \\
\hline 1058.412 & 229.120 & Surface & 2011 & 6417.458 & 221.054 & Surface & 2011 \\
\hline 1061.372 & 229.113 & Surface & 2011 & 6420.346 & 221.039 & Surface & 2011 \\
\hline 1064.333 & 229.118 & Surface & 2011 & 6423.235 & 221.034 & Surface & 2011 \\
\hline 1067.293 & 229.118 & Surface & 2011 & 6426.123 & 221.031 & Surface & 2011 \\
\hline 1070.104 & 229.123 & Surface & 2011 & 6429.012 & 221.019 & Surface & 2011 \\
\hline 1072.915 & 229.124 & Surface & 2011 & 6431.900 & 221.017 & Surface & 2011 \\
\hline 1075.726 & 229.130 & Surface & 2011 & 6434.789 & 221.030 & Surface & 2011 \\
\hline 1078.537 & 229.132 & Surface & 2011 & 6437.677 & 221.036 & Surface & 2011 \\
\hline 1081.348 & 229.140 & Surface & 2011 & 6440.565 & 221.033 & Surface & 2011 \\
\hline 1084.159 & 229.137 & Surface & 2011 & 6443.454 & 221.020 & Surface & 2011 \\
\hline 1086.971 & 229.125 & Surface & 2011 & 6446.342 & 221.014 & Surface & 2011 \\
\hline 1089.781 & 229.115 & Surface & 2011 & 6449.231 & 221.014 & Surface & 2011 \\
\hline 1092.593 & 229.109 & Surface & 2011 & 6452.119 & 221.019 & Surface & 2011 \\
\hline 1095.403 & 229.133 & Surface & 2011 & 6455.007 & 221.004 & Surface & 2011 \\
\hline 1098.215 & 229.133 & Surface & 2011 & 6457.896 & 221.024 & Surface & 2011 \\
\hline 1101.026 & 229.112 & Surface & 2011 & 6460.784 & 221.031 & Surface & 2011 \\
\hline 1103.837 & 229.105 & Surface & 2011 & 6463.673 & 221.035 & Surface & 2011 \\
\hline 1106.648 & 229.104 & Surface & 2011 & 6466.561 & 221.047 & Surface & 2011 \\
\hline 1109.459 & 229.100 & Surface & 2011 & 6469.530 & 221.071 & Surface & 2011 \\
\hline 1112.394 & 229.097 & Surface & 2011 & 6472.499 & 221.065 & Surface & 2011 \\
\hline 1115.329 & 229.120 & Surface & 2011 & 6475.468 & 221.051 & Surface & 2011 \\
\hline 1118.264 & 229.124 & Surface & 2011 & 6478.437 & 221.049 & Surface & 2011 \\
\hline 1121.199 & 229.125 & Surface & 2011 & 6481.406 & 221.049 & Surface & 2011 \\
\hline 1124.134 & 229.128 & Surface & 2011 & 6484.376 & 221.058 & Surface & 2011 \\
\hline 1127.069 & 229.121 & Surface & 2011 & 6487.345 & 221.066 & Surface & 2011 \\
\hline 1130.004 & 229.107 & Surface & 2011 & 6490.314 & 221.057 & Surface & 2011 \\
\hline
\end{tabular}




\begin{tabular}{|c|c|c|c|c|c|c|c|}
\hline 1132.939 & 229.104 & Surface & 2011 & 6493.283 & 221.040 & Surface & 2011 \\
\hline 1135.875 & 229.111 & Surface & 2011 & 6496.252 & 221.021 & Surface & 2011 \\
\hline 1138.810 & 229.076 & Surface & 2011 & 6499.221 & 221.031 & Surface & 2011 \\
\hline 1141.745 & 229.058 & Surface & 2011 & 6502.190 & 221.042 & Surface & 2011 \\
\hline 1144.680 & 229.080 & Surface & 2011 & 6505.159 & 221.035 & Surface & 2011 \\
\hline 1147.615 & 229.104 & Surface & 2011 & 6508.128 & 221.042 & Surface & 2011 \\
\hline 1150.410 & 229.100 & Surface & 2011 & 6511.097 & 221.050 & Surface & 2011 \\
\hline 1153.206 & 229.087 & Surface & 2011 & 6514.066 & 221.053 & Surface & 2011 \\
\hline 1156.001 & 229.081 & Surface & 2011 & 6517.035 & 221.052 & Surface & 2011 \\
\hline 1158.797 & 229.076 & Surface & 2011 & 6520.005 & 221.056 & Surface & 2011 \\
\hline 1161.592 & 229.075 & Surface & 2011 & 6522.973 & 221.047 & Surface & 2011 \\
\hline 1164.387 & 229.085 & Surface & 2011 & 6525.943 & 221.027 & Surface & 2011 \\
\hline 1167.183 & 229.093 & Surface & 2011 & 6528.912 & 221.018 & Surface & 2011 \\
\hline 1169.978 & 229.090 & Surface & 2011 & 6531.881 & 221.014 & Surface & 2011 \\
\hline 1172.773 & 229.094 & Surface & 2011 & 6534.850 & 221.007 & Surface & 2011 \\
\hline 1175.569 & 229.090 & Surface & 2011 & 6537.819 & 220.998 & Surface & 2011 \\
\hline 1178.364 & 229.064 & Surface & 2011 & 6540.788 & 220.991 & Surface & 2011 \\
\hline 1181.160 & 229.038 & Surface & 2011 & 6543.757 & 220.994 & Surface & 2011 \\
\hline 1183.955 & 229.031 & Surface & 2011 & 6546.726 & 221.005 & Surface & 2011 \\
\hline 1186.765 & 229.061 & Surface & 2011 & 6549.695 & 221.015 & Surface & 2011 \\
\hline 1189.575 & 229.082 & Surface & 2011 & 6552.695 & 221.027 & Surface & 2011 \\
\hline 1192.384 & 229.076 & Surface & 2011 & 6555.694 & 221.040 & Surface & 2011 \\
\hline 1195.194 & 229.062 & Surface & 2011 & 6558.694 & 221.049 & Surface & 2011 \\
\hline 1198.003 & 229.067 & Surface & 2011 & 6561.693 & 221.048 & Surface & 2011 \\
\hline 1200.813 & 229.075 & Surface & 2011 & 6564.693 & 221.038 & Surface & 2011 \\
\hline 1203.623 & 229.065 & Surface & 2011 & 6567.692 & 221.032 & Surface & 2011 \\
\hline 1206.432 & 229.049 & Surface & 2011 & 6570.692 & 221.027 & Surface & 2011 \\
\hline 1209.242 & 229.039 & Surface & 2011 & 6573.691 & 221.020 & Surface & 2011 \\
\hline 1212.052 & 229.020 & Surface & 2011 & 6576.690 & 221.012 & Surface & 2011 \\
\hline 1214.861 & 228.993 & Surface & 2011 & 6579.690 & 221.003 & Surface & 2011 \\
\hline 1217.671 & 228.982 & Surface & 2011 & 6582.689 & 220.993 & Surface & 2011 \\
\hline 1220.481 & 228.992 & Surface & 2011 & 6585.689 & 220.987 & Surface & 2011 \\
\hline 1223.459 & 228.998 & Surface & 2011 & 6588.688 & 220.990 & Surface & 2011 \\
\hline 1226.437 & 228.997 & Surface & 2011 & 6591.688 & 220.994 & Surface & 2011 \\
\hline 1229.416 & 228.979 & Surface & 2011 & 6594.687 & 221.000 & Surface & 2011 \\
\hline 1232.394 & 228.988 & Surface & 2011 & 6597.687 & 221.006 & Surface & 2011 \\
\hline 1235.373 & 228.996 & Surface & 2011 & 6600.686 & 221.023 & Surface & 2011 \\
\hline 1238.351 & 228.929 & Surface & 2011 & 6603.686 & 221.052 & Surface & 2011 \\
\hline 1241.330 & 228.901 & Surface & 2011 & 6606.685 & 221.063 & Surface & 2011 \\
\hline 1244.308 & 228.881 & Surface & 2011 & 6609.684 & 221.055 & Surface & 2011 \\
\hline 1247.287 & 228.826 & Surface & 2011 & 6612.684 & 221.050 & Surface & 2011 \\
\hline 1250.265 & 228.770 & Surface & 2011 & 6615.683 & 221.046 & Surface & 2011 \\
\hline 1253.243 & 228.728 & Surface & 2011 & 6618.683 & 221.043 & Surface & 2011 \\
\hline 1256.222 & 228.712 & Surface & 2011 & 6621.682 & 221.040 & Surface & 2011 \\
\hline 1259.200 & 228.718 & Surface & 2011 & 6624.682 & 221.040 & Surface & 2011 \\
\hline 1261.958 & 228.720 & Surface & 2011 & 6627.681 & 221.037 & Surface & 2011 \\
\hline 1264.715 & 228.705 & Surface & 2011 & 6630.681 & 221.027 & Surface & 2011 \\
\hline 1267.473 & 228.685 & Surface & 2011 & 6633.667 & 221.016 & Surface & 2011 \\
\hline 1270.230 & 228.695 & Surface & 2011 & 6636.653 & 221.005 & Surface & 2011 \\
\hline
\end{tabular}




\begin{tabular}{|c|c|c|c|c|c|c|c|}
\hline 1272.988 & 228.701 & Surface & 2011 & 6639.639 & 221.003 & Surface & 2011 \\
\hline 1275.746 & 228.708 & Surface & 2011 & 6642.626 & 221.001 & Surface & 2011 \\
\hline 1278.503 & 228.701 & Surface & 2011 & 6645.612 & 220.990 & Surface & 2011 \\
\hline 1281.261 & 228.700 & Surface & 2011 & 6648.598 & 220.976 & Surface & 2011 \\
\hline 1284.018 & 228.706 & Surface & 2011 & 6651.584 & 220.969 & Surface & 2011 \\
\hline 1286.776 & 228.711 & Surface & 2011 & 6654.570 & 220.970 & Surface & 2011 \\
\hline 1289.533 & 228.717 & Surface & 2011 & 6657.557 & 220.986 & Surface & 2011 \\
\hline 1292.428 & 228.725 & Surface & 2011 & 6660.543 & 220.996 & Surface & 2011 \\
\hline 1295.324 & 228.717 & Surface & 2011 & 6663.529 & 221.011 & Surface & 2011 \\
\hline 1298.219 & 228.710 & Surface & 2011 & 6666.515 & 221.025 & Surface & 2011 \\
\hline 1301.115 & 228.710 & Surface & 2011 & 6669.501 & 221.015 & Surface & 2011 \\
\hline 1304.010 & 228.718 & Surface & 2011 & 6672.488 & 221.005 & Surface & 2011 \\
\hline 1306.905 & 228.744 & Surface & 2011 & 6675.474 & 220.973 & Surface & 2011 \\
\hline 1309.800 & 228.735 & Surface & 2011 & 6678.460 & 220.957 & Surface & 2011 \\
\hline 1312.696 & 228.714 & Surface & 2011 & 6681.446 & 220.979 & Surface & 2011 \\
\hline 1315.591 & 228.723 & Surface & 2011 & 6684.432 & 220.978 & Surface & 2011 \\
\hline 1318.487 & 228.730 & Surface & 2011 & 6687.419 & 220.965 & Surface & 2011 \\
\hline 1321.382 & 228.738 & Surface & 2011 & 6690.405 & 220.978 & Surface & 2011 \\
\hline 1324.277 & 228.738 & Surface & 2011 & 6693.391 & 220.990 & Surface & 2011 \\
\hline 1327.172 & 228.729 & Surface & 2011 & 6696.377 & 220.988 & Surface & 2011 \\
\hline 1330.068 & 228.727 & Surface & 2011 & 6699.363 & 220.985 & Surface & 2011 \\
\hline 1332.963 & 228.738 & Surface & 2011 & 6702.350 & 220.982 & Surface & 2011 \\
\hline 1335.789 & 228.741 & Surface & 2011 & 6705.336 & 220.981 & Surface & 2011 \\
\hline 1338.615 & 228.736 & Surface & 2011 & 6708.322 & 220.998 & Surface & 2011 \\
\hline 1341.441 & 228.730 & Surface & 2011 & 6711.212 & 221.003 & Surface & 2011 \\
\hline 1344.268 & 228.726 & Surface & 2011 & 6714.102 & 220.998 & Surface & 2011 \\
\hline 1347.094 & 228.726 & Surface & 2011 & 6716.992 & 220.995 & Surface & 2011 \\
\hline 1349.920 & 228.716 & Surface & 2011 & 6719.882 & 220.993 & Surface & 2011 \\
\hline 1352.746 & 228.727 & Surface & 2011 & 6722.772 & 220.986 & Surface & 2011 \\
\hline 1355.572 & 228.713 & Surface & 2011 & 6725.662 & 220.978 & Surface & 2011 \\
\hline 1358.398 & 228.711 & Surface & 2011 & 6728.552 & 220.969 & Surface & 2011 \\
\hline 1361.224 & 228.713 & Surface & 2011 & 6731.441 & 220.962 & Surface & 2011 \\
\hline 1364.050 & 228.708 & Surface & 2011 & 6734.331 & 220.955 & Surface & 2011 \\
\hline 1366.877 & 228.716 & Surface & 2011 & 6737.222 & 220.958 & Surface & 2011 \\
\hline 1369.696 & 228.729 & Surface & 2011 & 6740.111 & 220.977 & Surface & 2011 \\
\hline 1372.516 & 228.736 & Surface & 2011 & 6743.001 & 220.961 & Surface & 2011 \\
\hline 1375.335 & 228.731 & Surface & 2011 & 6745.891 & 220.939 & Surface & 2011 \\
\hline 1378.155 & 228.711 & Surface & 2011 & 6748.781 & 220.949 & Surface & 2011 \\
\hline 1380.975 & 228.708 & Surface & 2011 & 6751.671 & 220.941 & Surface & 2011 \\
\hline 1383.794 & 228.724 & Surface & 2011 & 6754.561 & 220.928 & Surface & 2011 \\
\hline 1386.614 & 228.731 & Surface & 2011 & 6757.451 & 220.920 & Surface & 2011 \\
\hline 1389.433 & 228.710 & Surface & 2011 & 6760.341 & 220.901 & Surface & 2011 \\
\hline 1392.253 & 228.708 & Surface & 2011 & 6763.231 & 220.879 & Surface & 2011 \\
\hline 1395.072 & 228.704 & Surface & 2011 & 6766.121 & 220.866 & Surface & 2011 \\
\hline 1397.892 & 228.701 & Surface & 2011 & 6769.011 & 220.876 & Surface & 2011 \\
\hline 1400.712 & 228.707 & Surface & 2011 & 6771.901 & 220.883 & Surface & 2011 \\
\hline 1403.531 & 228.717 & Surface & 2011 & 6774.791 & 220.885 & Surface & 2011 \\
\hline 1406.350 & 228.720 & Surface & 2011 & 6777.681 & 220.878 & Surface & 2011 \\
\hline 1409.170 & 228.722 & Surface & 2011 & 6780.571 & 220.870 & Surface & 2011 \\
\hline
\end{tabular}




\begin{tabular}{|c|c|c|c|c|c|c|c|}
\hline 1412.014 & 228.726 & Surface & 2011 & 6783.460 & 220.860 & Surface & 2011 \\
\hline 1414.857 & 228.704 & Surface & 2011 & 6786.351 & 220.840 & Surface & 2011 \\
\hline 1417.701 & 228.700 & Surface & 2011 & 6789.312 & 220.810 & Surface & 2011 \\
\hline 1420.545 & 228.709 & Surface & 2011 & 6792.274 & 220.846 & Surface & 2011 \\
\hline 1423.388 & 228.712 & Surface & 2011 & 6795.235 & 220.856 & Surface & 2011 \\
\hline 1426.232 & 228.711 & Surface & 2011 & 6798.197 & 220.843 & Surface & 2011 \\
\hline 1429.076 & 228.707 & Surface & 2011 & 6801.159 & 220.826 & Surface & 2011 \\
\hline 1431.920 & 228.695 & Surface & 2011 & 6804.121 & 220.812 & Surface & 2011 \\
\hline 1434.763 & 228.671 & Surface & 2011 & 6807.082 & 220.811 & Surface & 2011 \\
\hline 1437.607 & 228.651 & Surface & 2011 & 6810.044 & 220.787 & Surface & 2011 \\
\hline 1440.451 & 228.670 & Surface & 2011 & 6813.005 & 220.777 & Surface & 2011 \\
\hline 1443.294 & 228.671 & Surface & 2011 & 6815.967 & 220.775 & Surface & 2011 \\
\hline 1446.138 & 228.639 & Surface & 2011 & 6818.929 & 220.786 & Surface & 2011 \\
\hline 1449.074 & 228.645 & Surface & 2011 & 6821.890 & 220.800 & Surface & 2011 \\
\hline 1452.009 & 228.661 & Surface & 2011 & 6824.852 & 220.789 & Surface & 2011 \\
\hline 1454.945 & 228.654 & Surface & 2011 & 6827.814 & 220.777 & Surface & 2011 \\
\hline 1457.881 & 228.649 & Surface & 2011 & 6830.775 & 220.787 & Surface & 2011 \\
\hline 1460.817 & 228.636 & Surface & 2011 & 6833.737 & 220.751 & Surface & 2011 \\
\hline 1463.753 & 228.625 & Surface & 2011 & 6836.592 & 220.746 & Surface & 2011 \\
\hline 1466.689 & 228.624 & Surface & 2011 & 6839.447 & 220.757 & Surface & 2011 \\
\hline 1469.624 & 228.622 & Surface & 2011 & 6842.302 & 220.752 & Surface & 2011 \\
\hline 1472.560 & 228.613 & Surface & 2011 & 6845.157 & 220.761 & Surface & 2011 \\
\hline 1475.496 & 228.603 & Surface & 2011 & 6848.011 & 220.767 & Surface & 2011 \\
\hline 1478.432 & 228.595 & Surface & 2011 & 6850.866 & 220.759 & Surface & 2011 \\
\hline 1481.368 & 228.600 & Surface & 2011 & 6853.721 & 220.749 & Surface & 2011 \\
\hline 1484.304 & 228.606 & Surface & 2011 & 6856.576 & 220.750 & Surface & 2011 \\
\hline 1487.239 & 228.606 & Surface & 2011 & 6859.431 & 220.737 & Surface & 2011 \\
\hline 1490.175 & 228.592 & Surface & 2011 & 6862.286 & 220.714 & Surface & 2011 \\
\hline 1493.111 & 228.574 & Surface & 2011 & 6865.141 & 220.710 & Surface & 2011 \\
\hline 1496.047 & 228.570 & Surface & 2011 & 6867.996 & 220.725 & Surface & 2011 \\
\hline 1498.983 & 228.556 & Surface & 2011 & 6870.850 & 220.749 & Surface & 2011 \\
\hline 1501.918 & 228.536 & Surface & 2011 & 6873.705 & 220.739 & Surface & 2011 \\
\hline 1504.854 & 228.560 & Surface & 2011 & 6876.611 & 220.735 & Surface & 2011 \\
\hline 1507.799 & 228.588 & Surface & 2011 & 6879.516 & 220.724 & Surface & 2011 \\
\hline 1510.744 & 228.602 & Surface & 2011 & 6882.422 & 220.697 & Surface & 2011 \\
\hline 1513.689 & 228.597 & Surface & 2011 & 6885.328 & 220.726 & Surface & 2011 \\
\hline 1516.634 & 228.568 & Surface & 2011 & 6888.233 & 220.738 & Surface & 2011 \\
\hline 1519.579 & 228.563 & Surface & 2011 & 6891.139 & 220.740 & Surface & 2011 \\
\hline 1522.524 & 228.571 & Surface & 2011 & 6894.044 & 220.718 & Surface & 2011 \\
\hline 1525.469 & 228.565 & Surface & 2011 & 6896.950 & 220.700 & Surface & 2011 \\
\hline 1528.415 & 228.567 & Surface & 2011 & 6899.856 & 220.678 & Surface & 2011 \\
\hline 1531.360 & 228.535 & Surface & 2011 & 6902.761 & 220.658 & Surface & 2011 \\
\hline 1534.304 & 228.523 & Surface & 2011 & 6905.667 & 220.629 & Surface & 2011 \\
\hline 1537.249 & 228.536 & Surface & 2011 & 6908.573 & 220.646 & Surface & 2011 \\
\hline 1540.194 & 228.541 & Surface & 2011 & 6911.478 & 220.664 & Surface & 2011 \\
\hline 1543.140 & 228.537 & Surface & 2011 & 6914.384 & 220.672 & Surface & 2011 \\
\hline 1546.085 & 228.509 & Surface & 2011 & 6917.300 & 220.666 & Surface & 2011 \\
\hline 1549.030 & 228.464 & Surface & 2011 & 6920.215 & 220.690 & Surface & 2011 \\
\hline 1551.974 & 228.461 & Surface & 2011 & 6923.132 & 220.697 & Surface & 2011 \\
\hline
\end{tabular}




\begin{tabular}{|c|c|c|c|c|c|c|c|}
\hline 1554.919 & 228.476 & Surface & 2011 & 6926.047 & 220.690 & Surface & 2011 \\
\hline 1557.864 & 228.496 & Surface & 2011 & 6928.963 & 220.680 & Surface & 2011 \\
\hline 1560.810 & 228.469 & Surface & 2011 & 6931.879 & 220.666 & Surface & 2011 \\
\hline 1563.775 & 228.439 & Surface & 2011 & 6934.795 & 220.674 & Surface & 2011 \\
\hline 1566.741 & 228.456 & Surface & 2011 & 6937.711 & 220.683 & Surface & 2011 \\
\hline 1569.707 & 228.444 & Surface & 2011 & 6940.627 & 220.684 & Surface & 2011 \\
\hline 1572.672 & 228.422 & Surface & 2011 & 6943.543 & 220.673 & Surface & 2011 \\
\hline 1575.638 & 228.419 & Surface & 2011 & 6946.459 & 220.664 & Surface & 2011 \\
\hline 1578.603 & 228.423 & Surface & 2011 & 6949.375 & 220.678 & Surface & 2011 \\
\hline 1581.569 & 228.428 & Surface & 2011 & 6952.291 & 220.688 & Surface & 2011 \\
\hline 1584.534 & 228.440 & Surface & 2011 & 6955.207 & 220.673 & Surface & 2011 \\
\hline 1587.500 & 228.440 & Surface & 2011 & 6958.123 & 220.664 & Surface & 2011 \\
\hline 1590.466 & 228.429 & Surface & 2011 & 6961.039 & 220.668 & Surface & 2011 \\
\hline 1593.317 & 228.428 & Surface & 2011 & 6963.954 & 220.673 & Surface & 2011 \\
\hline 1596.169 & 228.430 & Surface & 2011 & 6966.871 & 220.671 & Surface & 2011 \\
\hline 1599.020 & 228.399 & Surface & 2011 & 6969.839 & 220.659 & Surface & 2011 \\
\hline 1601.871 & 228.385 & Surface & 2011 & 6972.808 & 220.658 & Surface & 2011 \\
\hline 1604.723 & 228.373 & Surface & 2011 & 6975.777 & 220.647 & Surface & 2011 \\
\hline 1607.574 & 228.385 & Surface & 2011 & 6978.745 & 220.639 & Surface & 2011 \\
\hline 1610.426 & 228.386 & Surface & 2011 & 6981.714 & 220.645 & Surface & 2011 \\
\hline 1613.277 & 228.372 & Surface & 2011 & 6984.682 & 220.649 & Surface & 2011 \\
\hline 1616.129 & 228.353 & Surface & 2011 & 6987.651 & 220.658 & Surface & 2011 \\
\hline 1618.980 & 228.347 & Surface & 2011 & 6990.620 & 220.669 & Surface & 2011 \\
\hline 1621.831 & 228.333 & Surface & 2011 & 6993.588 & 220.655 & Surface & 2011 \\
\hline 1624.683 & 228.313 & Surface & 2011 & 6996.557 & 220.622 & Surface & 2011 \\
\hline 1627.534 & 228.322 & Surface & 2011 & 6999.526 & 220.626 & Surface & 2011 \\
\hline 1630.385 & 228.344 & Surface & 2011 & 7002.494 & 220.652 & Surface & 2011 \\
\hline 1633.237 & 228.358 & Surface & 2011 & 7005.463 & 220.636 & Surface & 2011 \\
\hline 1636.085 & 228.356 & Surface & 2011 & 7008.432 & 220.642 & Surface & 2011 \\
\hline 1638.933 & 228.337 & Surface & 2011 & 7011.400 & 220.650 & Surface & 2011 \\
\hline 1641.781 & 228.338 & Surface & 2011 & 7014.369 & 220.647 & Surface & 2011 \\
\hline 1644.629 & 228.382 & Surface & 2011 & 7017.338 & 220.632 & Surface & 2011 \\
\hline 1647.477 & 228.382 & Surface & 2011 & 7020.212 & 220.640 & Surface & 2011 \\
\hline 1650.325 & 228.382 & Surface & 2011 & 7023.087 & 220.651 & Surface & 2011 \\
\hline 1653.173 & 228.383 & Surface & 2011 & 7025.962 & 220.631 & Surface & 2011 \\
\hline 1656.021 & 228.397 & Surface & 2011 & 7028.837 & 220.616 & Surface & 2011 \\
\hline 1658.869 & 228.400 & Surface & 2011 & 7031.712 & 220.624 & Surface & 2011 \\
\hline 1661.716 & 228.382 & Surface & 2011 & 7034.587 & 220.624 & Surface & 2011 \\
\hline 1664.564 & 228.365 & Surface & 2011 & 7037.462 & 220.616 & Surface & 2011 \\
\hline 1667.412 & 228.344 & Surface & 2011 & 7040.337 & 220.605 & Surface & 2011 \\
\hline 1670.260 & 228.345 & Surface & 2011 & 7043.212 & 220.594 & Surface & 2011 \\
\hline 1673.108 & 228.342 & Surface & 2011 & 7046.087 & 220.595 & Surface & 2011 \\
\hline 1675.956 & 228.353 & Surface & 2011 & 7048.962 & 220.610 & Surface & 2011 \\
\hline 1678.804 & 228.370 & Surface & 2011 & 7051.837 & 220.609 & Surface & 2011 \\
\hline 1681.652 & 228.374 & Surface & 2011 & 7054.712 & 220.583 & Surface & 2011 \\
\hline 1684.500 & 228.375 & Surface & 2011 & 7057.587 & 220.572 & Surface & 2011 \\
\hline 1687.348 & 228.380 & Surface & 2011 & 7060.462 & 220.560 & Surface & 2011 \\
\hline 1690.168 & 228.379 & Surface & 2011 & 7063.407 & 220.572 & Surface & 2011 \\
\hline 1692.987 & 228.387 & Surface & 2011 & 7066.353 & 220.597 & Surface & 2011 \\
\hline
\end{tabular}




\begin{tabular}{|c|c|c|c|c|c|c|c|}
\hline 1695.807 & 228.392 & Surface & 2011 & 7069.299 & 220.605 & Surface & 2011 \\
\hline 1698.627 & 228.388 & Surface & 2011 & 7072.244 & 220.602 & Surface & 2011 \\
\hline 1701.446 & 228.392 & Surface & 2011 & 7075.190 & 220.575 & Surface & 2011 \\
\hline 1704.265 & 228.394 & Surface & 2011 & 7078.136 & 220.548 & Surface & 2011 \\
\hline 1707.085 & 228.364 & Surface & 2011 & 7081.081 & 220.539 & Surface & 2011 \\
\hline 1709.905 & 228.392 & Surface & 2011 & 7084.027 & 220.518 & Surface & 2011 \\
\hline 1712.724 & 228.387 & Surface & 2011 & 7086.973 & 220.477 & Surface & 2011 \\
\hline 1715.544 & 228.361 & Surface & 2011 & 7089.919 & 220.498 & Surface & 2011 \\
\hline 1718.363 & 228.348 & Surface & 2011 & 7092.864 & 220.497 & Surface & 2011 \\
\hline 1721.183 & 228.347 & Surface & 2011 & 7095.810 & 220.490 & Surface & 2011 \\
\hline 1724.003 & 228.349 & Surface & 2011 & 7098.756 & 220.456 & Surface & 2011 \\
\hline 1726.822 & 228.366 & Surface & 2011 & 7101.701 & 220.437 & Surface & 2011 \\
\hline 1729.642 & 228.395 & Surface & 2011 & 7104.647 & 220.445 & Surface & 2011 \\
\hline 1732.489 & 228.404 & Surface & 2011 & 7107.593 & 220.450 & Surface & 2011 \\
\hline 1735.337 & 228.392 & Surface & 2011 & 7110.539 & 220.432 & Surface & 2011 \\
\hline 1738.185 & 228.375 & Surface & 2011 & 7113.517 & 220.414 & Surface & 2011 \\
\hline 1741.032 & 228.355 & Surface & 2011 & 7116.497 & 220.415 & Surface & 2011 \\
\hline 1743.880 & 228.380 & Surface & 2011 & 7119.476 & 220.431 & Surface & 2011 \\
\hline 1746.727 & 228.371 & Surface & 2011 & 7122.455 & 220.410 & Surface & 2011 \\
\hline 1749.575 & 228.349 & Surface & 2011 & 7125.434 & 220.293 & Surface & 2011 \\
\hline 1752.423 & 228.345 & Surface & 2011 & 7128.413 & 220.225 & Surface & 2011 \\
\hline 1755.271 & 228.361 & Surface & 2011 & 7131.392 & 220.179 & Surface & 2011 \\
\hline 1758.118 & 228.353 & Surface & 2011 & 7134.371 & 220.178 & Surface & 2011 \\
\hline 1760.966 & 228.351 & Surface & 2011 & 7137.350 & 220.195 & Surface & 2011 \\
\hline 1763.813 & 228.366 & Surface & 2011 & 7140.329 & 220.206 & Surface & 2011 \\
\hline 1766.661 & 228.357 & Surface & 2011 & 7143.308 & 220.194 & Surface & 2011 \\
\hline 1769.509 & 228.329 & Surface & 2011 & 7146.287 & 220.145 & Surface & 2011 \\
\hline 1772.356 & 228.315 & Surface & 2011 & 7149.142 & 220.120 & Surface & 2011 \\
\hline 1775.204 & 228.363 & Surface & 2011 & 7151.997 & 220.082 & Surface & 2011 \\
\hline 1778.052 & 228.346 & Surface & 2011 & 7154.852 & 220.019 & Surface & 2011 \\
\hline 1780.898 & 228.322 & Surface & 2011 & 7157.707 & 219.933 & Surface & 2011 \\
\hline 1783.744 & 228.339 & Surface & 2011 & 7160.562 & 219.883 & Surface & 2011 \\
\hline 1786.591 & 228.345 & Surface & 2011 & 7163.416 & 219.847 & Surface & 2011 \\
\hline 1789.437 & 228.335 & Surface & 2011 & 7166.271 & 219.821 & Surface & 2011 \\
\hline 1792.283 & 228.327 & Surface & 2011 & 7169.127 & 219.764 & Surface & 2011 \\
\hline 1795.130 & 228.309 & Surface & 2011 & 7171.981 & 219.700 & Surface & 2011 \\
\hline 1797.976 & 228.295 & Surface & 2011 & 7174.836 & 219.674 & Surface & 2011 \\
\hline 1800.823 & 228.300 & Surface & 2011 & 7177.691 & 219.649 & Surface & 2011 \\
\hline 1803.669 & 228.308 & Surface & 2011 & 7180.546 & 219.616 & Surface & 2011 \\
\hline 1806.515 & 228.307 & Surface & 2011 & 7183.401 & 219.582 & Surface & 2011 \\
\hline 1809.362 & 228.315 & Surface & 2011 & 7186.256 & 219.573 & Surface & 2011 \\
\hline 1812.208 & 228.348 & Surface & 2011 & 7189.183 & 219.548 & Surface & 2011 \\
\hline 1815.054 & 228.369 & Surface & 2011 & 7192.109 & 219.532 & Surface & 2011 \\
\hline 1817.901 & 228.354 & Surface & 2011 & 7195.036 & 219.538 & Surface & 2011 \\
\hline 1820.747 & 228.335 & Surface & 2011 & 7197.962 & 219.547 & Surface & 2011 \\
\hline 1823.593 & 228.332 & Surface & 2011 & 7200.889 & 219.517 & Surface & 2011 \\
\hline 1826.440 & 228.331 & Surface & 2011 & 7203.816 & 219.446 & Surface & 2011 \\
\hline 1829.286 & 228.338 & Surface & 2011 & 7206.742 & 219.353 & Surface & 2011 \\
\hline 1832.245 & 228.347 & Surface & 2011 & 7209.669 & 219.241 & Surface & 2011 \\
\hline
\end{tabular}




\begin{tabular}{|c|c|c|c|c|c|c|c|}
\hline 1835.203 & 228.356 & Surface & 2011 & 7212.596 & 219.281 & Surface & 2011 \\
\hline 1838.162 & 228.362 & Surface & 2011 & 7215.523 & 219.322 & Surface & 2011 \\
\hline 1841.121 & 228.326 & Surface & 2011 & 7218.449 & 219.374 & Surface & 2011 \\
\hline 1844.079 & 228.299 & Surface & 2011 & 7221.376 & 219.381 & Surface & 2011 \\
\hline 1847.038 & 228.327 & Surface & 2011 & 7224.303 & 219.385 & Surface & 2011 \\
\hline 1849.996 & 228.357 & Surface & 2011 & 7227.229 & 219.363 & Surface & 2011 \\
\hline 1852.955 & 228.348 & Surface & 2011 & 7230.156 & 219.335 & Surface & 2011 \\
\hline 1855.914 & 228.308 & Surface & 2011 & 7233.083 & 219.310 & Surface & 2011 \\
\hline 1858.872 & 228.335 & Surface & 2011 & 7236.010 & 219.254 & Surface & 2011 \\
\hline 1861.831 & 228.351 & Surface & 2011 & 7238.937 & 219.226 & Surface & 2011 \\
\hline 1864.790 & 228.355 & Surface & 2011 & 7241.863 & 219.218 & Surface & 2011 \\
\hline 1867.748 & 228.354 & Surface & 2011 & 7244.790 & 219.198 & Surface & 2011 \\
\hline 1870.707 & 228.351 & Surface & 2011 & 7247.717 & 219.200 & Surface & 2011 \\
\hline 1873.563 & 228.357 & Surface & 2011 & 7250.644 & 219.231 & Surface & 2011 \\
\hline 1876.420 & 228.364 & Surface & 2011 & 7253.571 & 219.238 & Surface & 2011 \\
\hline 1879.277 & 228.369 & Surface & 2011 & 7256.497 & 219.223 & Surface & 2011 \\
\hline 1882.133 & 228.357 & Surface & 2011 & 7259.424 & 219.180 & Surface & 2011 \\
\hline 1884.990 & 228.335 & Surface & 2011 & 7262.351 & 219.163 & Surface & 2011 \\
\hline 1887.847 & 228.323 & Surface & 2011 & 7265.278 & 219.169 & Surface & 2011 \\
\hline 1890.703 & 228.330 & Surface & 2011 & 7268.204 & 219.182 & Surface & 2011 \\
\hline 1893.560 & 228.346 & Surface & 2011 & 7271.131 & 219.194 & Surface & 2011 \\
\hline 1896.416 & 228.357 & Surface & 2011 & 7274.058 & 219.178 & Surface & 2011 \\
\hline 1899.273 & 228.348 & Surface & 2011 & 7276.985 & 219.149 & Surface & 2011 \\
\hline 1902.130 & 228.309 & Surface & 2011 & 7279.911 & 219.157 & Surface & 2011 \\
\hline 1904.986 & 228.276 & Surface & 2011 & 7282.838 & 219.172 & Surface & 2011 \\
\hline 1907.843 & 228.290 & Surface & 2011 & 7285.765 & 219.170 & Surface & 2011 \\
\hline 1910.700 & 228.321 & Surface & 2011 & 7288.692 & 219.158 & Surface & 2011 \\
\hline 1913.556 & 228.290 & Surface & 2011 & 7291.619 & 219.152 & Surface & 2011 \\
\hline 1916.413 & 228.283 & Surface & 2011 & 7294.545 & 219.146 & Surface & 2011 \\
\hline 1919.269 & 228.312 & Surface & 2011 & 7297.472 & 219.146 & Surface & 2011 \\
\hline 1922.126 & 228.330 & Surface & 2011 & 7300.366 & 219.141 & Surface & 2011 \\
\hline 1925.072 & 228.333 & Surface & 2011 & 7303.259 & 219.152 & Surface & 2011 \\
\hline 1928.018 & 228.325 & Surface & 2011 & 7306.153 & 219.149 & Surface & 2011 \\
\hline 1930.965 & 228.315 & Surface & 2011 & 7309.046 & 219.125 & Surface & 2011 \\
\hline 1933.911 & 228.326 & Surface & 2011 & 7311.940 & 219.096 & Surface & 2011 \\
\hline 1936.857 & 228.331 & Surface & 2011 & 7314.833 & 219.077 & Surface & 2011 \\
\hline 1939.804 & 228.318 & Surface & 2011 & 7317.727 & 219.083 & Surface & 2011 \\
\hline 1942.750 & 228.310 & Surface & 2011 & 7320.620 & 219.079 & Surface & 2011 \\
\hline 1945.697 & 228.316 & Surface & 2011 & 7323.514 & 219.063 & Surface & 2011 \\
\hline 1948.643 & 228.327 & Surface & 2011 & 7326.408 & 219.044 & Surface & 2011 \\
\hline 1951.590 & 228.318 & Surface & 2011 & 7329.301 & 218.973 & Surface & 2011 \\
\hline 1954.536 & 228.290 & Surface & 2011 & 7332.195 & 218.929 & Surface & 2011 \\
\hline 1957.482 & 228.267 & Surface & 2011 & 7335.088 & 218.932 & Surface & 2011 \\
\hline 1960.429 & 228.283 & Surface & 2011 & 7337.981 & 218.922 & Surface & 2011 \\
\hline 1963.375 & 228.332 & Surface & 2011 & 7340.875 & 218.933 & Surface & 2011 \\
\hline 1966.321 & 228.368 & Surface & 2011 & 7343.769 & 218.964 & Surface & 2011 \\
\hline 1969.268 & 228.369 & Surface & 2011 & 7346.662 & 219.007 & Surface & 2011 \\
\hline 1972.214 & 228.336 & Surface & 2011 & 7349.556 & 219.012 & Surface & 2011 \\
\hline 1975.161 & 228.291 & Surface & 2011 & 7352.411 & 219.008 & Surface & 2011 \\
\hline
\end{tabular}




\begin{tabular}{|c|c|c|c|c|c|c|c|}
\hline 1978.107 & 228.279 & Surface & 2011 & 7355.265 & 218.993 & Surface & 2011 \\
\hline 1981.053 & 228.304 & Surface & 2011 & 7358.120 & 218.981 & Surface & 2011 \\
\hline 1984.000 & 228.316 & Surface & 2011 & 7360.975 & 218.986 & Surface & 2011 \\
\hline 1986.946 & 228.319 & Surface & 2011 & 7363.830 & 218.995 & Surface & 2011 \\
\hline 1989.892 & 228.331 & Surface & 2011 & 7366.685 & 218.977 & Surface & 2011 \\
\hline 1992.839 & 228.347 & Surface & 2011 & 7369.540 & 218.956 & Surface & 2011 \\
\hline 1995.785 & 228.347 & Surface & 2011 & 7372.395 & 218.910 & Surface & 2011 \\
\hline 1998.731 & 228.345 & Surface & 2011 & 7375.249 & 218.877 & Surface & 2011 \\
\hline 2001.678 & 228.343 & Surface & 2011 & 7378.104 & 218.855 & Surface & 2011 \\
\hline 2004.624 & 228.340 & Surface & 2011 & 7380.959 & 218.814 & Surface & 2011 \\
\hline 2007.571 & 228.323 & Surface & 2011 & 7383.814 & 218.712 & Surface & 2011 \\
\hline 2010.517 & 228.311 & Surface & 2011 & 7386.669 & 218.715 & Surface & 2011 \\
\hline 2013.463 & 228.320 & Surface & 2011 & 7389.524 & 218.765 & Surface & 2011 \\
\hline 2016.410 & 228.331 & Surface & 2011 & 7392.511 & 218.823 & Surface & 2011 \\
\hline 2019.356 & 228.330 & Surface & 2011 & 7395.497 & 218.811 & Surface & 2011 \\
\hline 2022.302 & 228.332 & Surface & 2011 & 7398.483 & 218.786 & Surface & 2011 \\
\hline 2025.249 & 228.330 & Surface & 2011 & 7401.470 & 218.754 & Surface & 2011 \\
\hline 2028.195 & 228.329 & Surface & 2011 & 7404.456 & 218.673 & Surface & 2011 \\
\hline 2031.142 & 228.333 & Surface & 2011 & 7407.443 & 218.651 & Surface & 2011 \\
\hline 2034.088 & 228.333 & Surface & 2011 & 7410.429 & 218.677 & Surface & 2011 \\
\hline 2037.034 & 228.321 & Surface & 2011 & 7413.416 & 218.681 & Surface & 2011 \\
\hline 2039.981 & 228.310 & Surface & 2011 & 7416.402 & 218.694 & Surface & 2011 \\
\hline 2042.927 & 228.299 & Surface & 2011 & 7419.389 & 218.705 & Surface & 2011 \\
\hline 2045.873 & 228.317 & Surface & 2011 & 7422.375 & 218.696 & Surface & 2011 \\
\hline 2048.820 & 228.330 & Surface & 2011 & 7425.362 & 218.705 & Surface & 2011 \\
\hline 2051.766 & 228.315 & Surface & 2011 & 7428.348 & 218.716 & Surface & 2011 \\
\hline 2054.713 & 228.301 & Surface & 2011 & 7431.335 & 218.694 & Surface & 2011 \\
\hline 2057.659 & 228.289 & Surface & 2011 & 7434.321 & 218.687 & Surface & 2011 \\
\hline 2060.575 & 228.275 & Surface & 2011 & 7437.308 & 218.705 & Surface & 2011 \\
\hline 2063.491 & 228.285 & Surface & 2011 & 7440.295 & 218.722 & Surface & 2011 \\
\hline 2066.408 & 228.305 & Surface & 2011 & 7443.281 & 218.739 & Surface & 2011 \\
\hline 2069.324 & 228.313 & Surface & 2011 & 7446.268 & 218.739 & Surface & 2011 \\
\hline 2072.240 & 228.313 & Surface & 2011 & 7449.254 & 218.755 & Surface & 2011 \\
\hline 2075.156 & 228.308 & Surface & 2011 & 7452.240 & 218.751 & Surface & 2011 \\
\hline 2078.072 & 228.299 & Surface & 2011 & 7455.193 & 218.749 & Surface & 2011 \\
\hline 2080.989 & 228.302 & Surface & 2011 & 7458.146 & 218.754 & Surface & 2011 \\
\hline 2083.905 & 228.317 & Surface & 2011 & 7461.098 & 218.750 & Surface & 2011 \\
\hline 2086.821 & 228.327 & Surface & 2011 & 7464.051 & 218.740 & Surface & 2011 \\
\hline 2089.737 & 228.324 & Surface & 2011 & 7467.003 & 218.729 & Surface & 2011 \\
\hline 2092.653 & 228.306 & Surface & 2011 & 7469.956 & 218.706 & Surface & 2011 \\
\hline 2095.570 & 228.294 & Surface & 2011 & 7472.908 & 218.676 & Surface & 2011 \\
\hline 2098.486 & 228.297 & Surface & 2011 & 7475.861 & 218.689 & Surface & 2011 \\
\hline 2101.402 & 228.295 & Surface & 2011 & 7478.813 & 218.707 & Surface & 2011 \\
\hline 2104.318 & 228.306 & Surface & 2011 & 7481.766 & 218.719 & Surface & 2011 \\
\hline 2107.234 & 228.283 & Surface & 2011 & 7484.718 & 218.730 & Surface & 2011 \\
\hline 2110.151 & 228.265 & Surface & 2011 & 7487.671 & 218.734 & Surface & 2011 \\
\hline 2113.067 & 228.272 & Surface & 2011 & 7490.623 & 218.744 & Surface & 2011 \\
\hline 2115.983 & 228.306 & Surface & 2011 & 7493.576 & 218.757 & Surface & 2011 \\
\hline 2118.899 & 228.309 & Surface & 2011 & 7496.529 & 218.743 & Surface & 2011 \\
\hline
\end{tabular}




\begin{tabular}{|c|c|c|c|c|c|c|c|}
\hline 2121.815 & 228.305 & Surface & 2011 & 7499.481 & 218.706 & Surface & 2011 \\
\hline 2124.776 & 228.300 & Surface & 2011 & 7502.434 & 218.693 & Surface & 2011 \\
\hline 2127.738 & 228.303 & Surface & 2011 & 7505.386 & 218.723 & Surface & 2011 \\
\hline 2130.699 & 228.324 & Surface & 2011 & 7508.339 & 218.733 & Surface & 2011 \\
\hline 2133.660 & 228.319 & Surface & 2011 & 7511.291 & 218.727 & Surface & 2011 \\
\hline 2136.621 & 228.311 & Surface & 2011 & 7514.244 & 218.719 & Surface & 2011 \\
\hline 2139.582 & 228.302 & Surface & 2011 & 7517.196 & 218.705 & Surface & 2011 \\
\hline 2142.543 & 228.295 & Surface & 2011 & 7520.149 & 218.692 & Surface & 2011 \\
\hline 2145.504 & 228.301 & Surface & 2011 & 7523.010 & 218.687 & Surface & 2011 \\
\hline 2148.465 & 228.278 & Surface & 2011 & 7525.872 & 218.687 & Surface & 2011 \\
\hline 2151.426 & 228.257 & Surface & 2011 & 7528.733 & 218.700 & Surface & 2011 \\
\hline 2154.387 & 228.278 & Surface & 2011 & 7531.594 & 218.709 & Surface & 2011 \\
\hline 2157.348 & 228.283 & Surface & 2011 & 7534.455 & 218.687 & Surface & 2011 \\
\hline 2160.309 & 228.279 & Surface & 2011 & 7537.317 & 218.641 & Surface & 2011 \\
\hline 2163.270 & 228.244 & Surface & 2011 & 7540.178 & 218.652 & Surface & 2011 \\
\hline 2166.231 & 228.255 & Surface & 2011 & 7543.039 & 218.656 & Surface & 2011 \\
\hline 2169.192 & 228.300 & Surface & 2011 & 7545.901 & 218.653 & Surface & 2011 \\
\hline 2172.153 & 228.304 & Surface & 2011 & 7548.762 & 218.641 & Surface & 2011 \\
\hline 2175.114 & 228.296 & Surface & 2011 & 7551.623 & 218.636 & Surface & 2011 \\
\hline 2178.076 & 228.296 & Surface & 2011 & 7554.485 & 218.620 & Surface & 2011 \\
\hline 2181.037 & 228.306 & Surface & 2011 & 7557.346 & 218.573 & Surface & 2011 \\
\hline 2183.894 & 228.292 & Surface & 2011 & 7560.207 & 218.546 & Surface & 2011 \\
\hline 2186.751 & 228.288 & Surface & 2011 & 7563.068 & 218.568 & Surface & 2011 \\
\hline 2189.608 & 228.296 & Surface & 2011 & 7565.930 & 218.586 & Surface & 2011 \\
\hline 2192.465 & 228.294 & Surface & 2011 & 7568.791 & 218.590 & Surface & 2011 \\
\hline 2195.322 & 228.290 & Surface & 2011 & 7571.754 & 218.575 & Surface & 2011 \\
\hline 2198.179 & 228.295 & Surface & 2011 & 7574.717 & 218.524 & Surface & 2011 \\
\hline 2201.036 & 228.306 & Surface & 2011 & 7577.679 & 218.502 & Surface & 2011 \\
\hline 2203.893 & 228.315 & Surface & 2011 & 7580.642 & 218.512 & Surface & 2011 \\
\hline 2206.751 & 228.312 & Surface & 2011 & 7583.605 & 218.531 & Surface & 2011 \\
\hline 2209.608 & 228.297 & Surface & 2011 & 7586.568 & 218.521 & Surface & 2011 \\
\hline 2212.465 & 228.289 & Surface & 2011 & 7589.531 & 218.518 & Surface & 2011 \\
\hline 2215.386 & 228.291 & Surface & 2011 & 7592.494 & 218.516 & Surface & 2011 \\
\hline 2218.308 & 228.281 & Surface & 2011 & 7595.456 & 218.520 & Surface & 2011 \\
\hline 2221.229 & 228.284 & Surface & 2011 & 7598.419 & 218.501 & Surface & 2011 \\
\hline 2224.151 & 228.287 & Surface & 2011 & 7601.382 & 218.485 & Surface & 2011 \\
\hline 2227.072 & 228.305 & Surface & 2011 & 7604.345 & 218.487 & Surface & 2011 \\
\hline 2229.993 & 228.304 & Surface & 2011 & 7607.308 & 218.489 & Surface & 2011 \\
\hline 2232.915 & 228.278 & Surface & 2011 & 7610.271 & 218.486 & Surface & 2011 \\
\hline 2235.837 & 228.289 & Surface & 2011 & 7613.233 & 218.465 & Surface & 2011 \\
\hline 2238.758 & 228.289 & Surface & 2011 & 7616.196 & 218.439 & Surface & 2011 \\
\hline 2241.679 & 228.286 & Surface & 2011 & 7619.159 & 218.448 & Surface & 2011 \\
\hline 2244.601 & 228.297 & Surface & 2011 & 7622.122 & 218.469 & Surface & 2011 \\
\hline 2247.522 & 228.303 & Surface & 2011 & 7625.085 & 218.470 & Surface & 2011 \\
\hline 2250.444 & 228.292 & Surface & 2011 & 7628.048 & 218.465 & Surface & 2011 \\
\hline 2253.346 & 228.279 & Surface & 2011 & 7630.863 & 218.450 & Surface & 2011 \\
\hline 2256.250 & 228.267 & Surface & 2011 & 7633.678 & 218.415 & Surface & 2011 \\
\hline 2259.153 & 228.274 & Surface & 2011 & 7636.493 & 218.420 & Surface & 2011 \\
\hline 2262.055 & 228.276 & Surface & 2011 & 7639.308 & 218.452 & Surface & 2011 \\
\hline
\end{tabular}




\begin{tabular}{|c|c|c|c|c|c|c|c|}
\hline 2264.959 & 228.277 & Surface & 2011 & 7642.123 & 218.456 & Surface & 2011 \\
\hline 2267.862 & 228.281 & Surface & 2011 & 7644.937 & 218.441 & Surface & 2011 \\
\hline 2270.764 & 228.280 & Surface & 2011 & 7647.752 & 218.425 & Surface & 2011 \\
\hline 2273.668 & 228.282 & Surface & 2011 & 7650.567 & 218.405 & Surface & 2011 \\
\hline 2276.571 & 228.278 & Surface & 2011 & 7653.382 & 218.385 & Surface & 2011 \\
\hline 2279.474 & 228.272 & Surface & 2011 & 7656.197 & 218.388 & Surface & 2011 \\
\hline 2282.377 & 228.272 & Surface & 2011 & 7659.012 & 218.396 & Surface & 2011 \\
\hline 2285.280 & 228.288 & Surface & 2011 & 7661.827 & 218.391 & Surface & 2011 \\
\hline 2288.183 & 228.315 & Surface & 2011 & 7664.642 & 218.385 & Surface & 2011 \\
\hline 2291.086 & 228.326 & Surface & 2011 & 7667.457 & 218.379 & Surface & 2011 \\
\hline 2293.989 & 228.314 & Surface & 2011 & 7670.272 & 218.386 & Surface & 2011 \\
\hline 2296.892 & 228.301 & Surface & 2011 & 7673.087 & 218.390 & Surface & 2011 \\
\hline 2299.795 & 228.301 & Surface & 2011 & 7675.998 & 218.397 & Surface & 2011 \\
\hline 2302.759 & 228.330 & Surface & 2011 & 7678.910 & 218.381 & Surface & 2011 \\
\hline 2305.723 & 228.345 & Surface & 2011 & 7681.821 & 218.383 & Surface & 2011 \\
\hline 2308.687 & 228.301 & Surface & 2011 & 7684.733 & 218.389 & Surface & 2011 \\
\hline 2311.651 & 228.284 & Surface & 2011 & 7687.644 & 218.401 & Surface & 2011 \\
\hline 2314.615 & 228.283 & Surface & 2011 & 7690.556 & 218.410 & Surface & 2011 \\
\hline 2317.579 & 228.278 & Surface & 2011 & 7693.467 & 218.384 & Surface & 2011 \\
\hline 2320.543 & 228.284 & Surface & 2011 & 7696.379 & 218.349 & Surface & 2011 \\
\hline 2323.507 & 228.308 & Surface & 2011 & 7699.291 & 218.320 & Surface & 2011 \\
\hline 2326.471 & 228.386 & Surface & 2011 & 7702.202 & 218.294 & Surface & 2011 \\
\hline 2329.435 & 228.479 & Surface & 2011 & 7705.114 & 218.286 & Surface & 2011 \\
\hline 2332.399 & 228.529 & Surface & 2011 & 7708.025 & 218.328 & Surface & 2011 \\
\hline 2335.363 & 228.520 & Surface & 2011 & 7710.833 & 218.364 & Surface & 2011 \\
\hline 2338.327 & 228.501 & Surface & 2011 & 7713.641 & 218.366 & Surface & 2011 \\
\hline 2341.291 & 228.491 & Surface & 2011 & 7716.448 & 218.368 & Surface & 2011 \\
\hline 2344.255 & 228.545 & Surface & 2011 & 7719.256 & 218.391 & Surface & 2011 \\
\hline 2347.219 & 228.529 & Surface & 2011 & 7722.064 & 218.398 & Surface & 2011 \\
\hline 2350.183 & 228.462 & Surface & 2011 & 7724.871 & 218.375 & Surface & 2011 \\
\hline 2353.147 & 228.430 & Surface & 2011 & 7727.679 & 218.348 & Surface & 2011 \\
\hline 2356.112 & 228.380 & Surface & 2011 & 7730.486 & 218.325 & Surface & 2011 \\
\hline 2358.958 & 228.319 & Surface & 2011 & 7733.294 & 218.289 & Surface & 2011 \\
\hline 2361.804 & 228.287 & Surface & 2011 & 7736.102 & 218.264 & Surface & 2011 \\
\hline 2364.651 & 228.294 & Surface & 2011 & 7738.909 & 218.275 & Surface & 2011 \\
\hline 2367.497 & 228.306 & Surface & 2011 & 7741.717 & 218.292 & Surface & 2011 \\
\hline 2370.343 & 228.318 & Surface & 2011 & 7744.525 & 218.291 & Surface & 2011 \\
\hline 2373.190 & 228.328 & Surface & 2011 & 7747.348 & 218.279 & Surface & 2011 \\
\hline 2376.036 & 228.337 & Surface & 2011 & 7750.171 & 218.276 & Surface & 2011 \\
\hline 2378.882 & 228.338 & Surface & 2011 & 7752.995 & 218.259 & Surface & 2011 \\
\hline 2381.729 & 228.321 & Surface & 2011 & 7755.818 & 218.243 & Surface & 2011 \\
\hline 2384.575 & 228.290 & Surface & 2011 & 7758.641 & 218.234 & Surface & 2011 \\
\hline 2387.421 & 228.249 & Surface & 2011 & 7761.465 & 218.236 & Surface & 2011 \\
\hline 2390.268 & 228.220 & Surface & 2011 & 7764.288 & 218.235 & Surface & 2011 \\
\hline 2393.114 & 228.239 & Surface & 2011 & 7767.111 & 218.233 & Surface & 2011 \\
\hline 2395.961 & 228.249 & Surface & 2011 & 7769.935 & 218.242 & Surface & 2011 \\
\hline 2398.807 & 228.239 & Surface & 2011 & 7772.758 & 218.235 & Surface & 2011 \\
\hline 2401.653 & 228.232 & Surface & 2011 & 7775.581 & 218.236 & Surface & 2011 \\
\hline 2404.500 & 228.239 & Surface & 2011 & 7778.571 & 218.233 & Surface & 2011 \\
\hline
\end{tabular}




\begin{tabular}{|c|c|c|c|c|c|c|c|}
\hline 2407.346 & 228.239 & Surface & 2011 & 7781.561 & 218.231 & Surface & 2011 \\
\hline 2410.263 & 228.213 & Surface & 2011 & 7784.550 & 218.230 & Surface & 2011 \\
\hline 2413.181 & 228.239 & Surface & 2011 & 7787.540 & 218.224 & Surface & 2011 \\
\hline 2416.098 & 228.243 & Surface & 2011 & 7790.530 & 218.220 & Surface & 2011 \\
\hline 2419.016 & 228.213 & Surface & 2011 & 7793.519 & 218.226 & Surface & 2011 \\
\hline 2421.933 & 228.236 & Surface & 2011 & 7796.509 & 218.232 & Surface & 2011 \\
\hline 2424.851 & 228.244 & Surface & 2011 & 7799.499 & 218.212 & Surface & 2011 \\
\hline 2427.768 & 228.241 & Surface & 2011 & 7802.488 & 218.183 & Surface & 2011 \\
\hline 2430.686 & 228.239 & Surface & 2011 & 7805.477 & 218.180 & Surface & 2011 \\
\hline 2433.603 & 228.234 & Surface & 2011 & 7808.467 & 218.179 & Surface & 2011 \\
\hline 2436.521 & 228.233 & Surface & 2011 & 7811.457 & 218.166 & Surface & 2011 \\
\hline 2439.438 & 228.228 & Surface & 2011 & 7814.446 & 218.152 & Surface & 2011 \\
\hline 2442.356 & 228.222 & Surface & 2011 & 7817.436 & 218.173 & Surface & 2011 \\
\hline 2445.273 & 228.216 & Surface & 2011 & 7820.426 & 218.193 & Surface & 2011 \\
\hline 2448.191 & 228.209 & Surface & 2011 & 7823.415 & 218.175 & Surface & 2011 \\
\hline 2451.108 & 228.206 & Surface & 2011 & 7826.405 & 218.154 & Surface & 2011 \\
\hline 2454.026 & 228.203 & Surface & 2011 & 7829.368 & 218.126 & Surface & 2011 \\
\hline 2456.943 & 228.190 & Surface & 2011 & 7832.332 & 218.115 & Surface & 2011 \\
\hline 2459.776 & 228.169 & Surface & 2011 & 7835.295 & 218.147 & Surface & 2011 \\
\hline 2462.609 & 228.151 & Surface & 2011 & 7838.259 & 218.164 & Surface & 2011 \\
\hline 2465.441 & 228.165 & Surface & 2011 & 7841.222 & 218.154 & Surface & 2011 \\
\hline 2468.274 & 228.188 & Surface & 2011 & 7844.185 & 218.136 & Surface & 2011 \\
\hline 2471.107 & 228.174 & Surface & 2011 & 7847.149 & 218.117 & Surface & 2011 \\
\hline 2473.940 & 228.144 & Surface & 2011 & 7850.112 & 218.132 & Surface & 2011 \\
\hline 2476.773 & 228.109 & Surface & 2011 & 7853.075 & 218.126 & Surface & 2011 \\
\hline 2479.605 & 228.106 & Surface & 2011 & 7856.039 & 218.111 & Surface & 2011 \\
\hline 2482.438 & 228.114 & Surface & 2011 & 7859.002 & 218.087 & Surface & 2011 \\
\hline 2485.271 & 228.118 & Surface & 2011 & 7861.966 & 218.076 & Surface & 2011 \\
\hline 2488.104 & 228.125 & Surface & 2011 & 7864.929 & 218.101 & Surface & 2011 \\
\hline 2490.937 & 228.135 & Surface & 2011 & 7867.892 & 218.104 & Surface & 2011 \\
\hline 2493.769 & 228.148 & Surface & 2011 & 7870.856 & 218.107 & Surface & 2011 \\
\hline 2496.602 & 228.152 & Surface & 2011 & 7873.819 & 218.114 & Surface & 2011 \\
\hline 2499.435 & 228.133 & Surface & 2011 & 7876.782 & 218.113 & Surface & 2011 \\
\hline 2502.268 & 228.104 & Surface & 2011 & 7879.733 & 218.123 & Surface & 2011 \\
\hline 2505.101 & 228.082 & Surface & 2011 & 7882.684 & 218.132 & Surface & 2011 \\
\hline 2507.954 & 228.081 & Surface & 2011 & 7885.635 & 218.131 & Surface & 2011 \\
\hline 2510.808 & 228.104 & Surface & 2011 & 7888.586 & 218.146 & Surface & 2011 \\
\hline 2513.661 & 228.104 & Surface & 2011 & 7891.537 & 218.141 & Surface & 2011 \\
\hline 2516.515 & 228.078 & Surface & 2011 & 7894.487 & 218.150 & Surface & 2011 \\
\hline 2519.368 & 228.047 & Surface & 2011 & 7897.438 & 218.148 & Surface & 2011 \\
\hline 2522.222 & 228.014 & Surface & 2011 & 7900.389 & 218.126 & Surface & 2011 \\
\hline 2525.075 & 228.014 & Surface & 2011 & 7903.340 & 218.143 & Surface & 2011 \\
\hline 2527.929 & 228.026 & Surface & 2011 & 7906.291 & 218.165 & Surface & 2011 \\
\hline 2530.782 & 228.050 & Surface & 2011 & 7909.241 & 218.162 & Surface & 2011 \\
\hline 2533.636 & 228.047 & Surface & 2011 & 7912.192 & 218.144 & Surface & 2011 \\
\hline 2536.489 & 228.030 & Surface & 2011 & 7915.143 & 218.154 & Surface & 2011 \\
\hline 2539.343 & 228.024 & Surface & 2011 & 7918.094 & 218.164 & Surface & 2011 \\
\hline 2542.196 & 227.990 & Surface & 2011 & 7921.045 & 218.164 & Surface & 2011 \\
\hline 2545.049 & 227.967 & Surface & 2011 & 7923.953 & 218.161 & Surface & 2011 \\
\hline
\end{tabular}




\begin{tabular}{|c|c|c|c|c|c|c|c|}
\hline 2547.903 & 227.946 & Surface & 2011 & 7926.861 & 218.152 & Surface & 2011 \\
\hline 2550.864 & 227.911 & Surface & 2011 & 7929.769 & 218.139 & Surface & 2011 \\
\hline 2553.825 & 227.877 & Surface & 2011 & 7932.678 & 218.135 & Surface & 2011 \\
\hline 2556.786 & 227.881 & Surface & 2011 & 7935.586 & 218.140 & Surface & 2011 \\
\hline 2559.747 & 227.909 & Surface & 2011 & 7938.494 & 218.142 & Surface & 2011 \\
\hline 2562.708 & 227.921 & Surface & 2011 & 7941.402 & 218.144 & Surface & 2011 \\
\hline 2565.669 & 227.919 & Surface & 2011 & 7944.310 & 218.140 & Surface & 2011 \\
\hline 2568.630 & 227.909 & Surface & 2011 & 7947.219 & 218.140 & Surface & 2011 \\
\hline 2571.592 & 227.904 & Surface & 2011 & 7950.127 & 218.144 & Surface & 2011 \\
\hline 2574.553 & 227.908 & Surface & 2011 & 7953.035 & 218.133 & Surface & 2011 \\
\hline 2577.514 & 227.903 & Surface & 2011 & 7955.943 & 218.141 & Surface & 2011 \\
\hline 2580.475 & 227.896 & Surface & 2011 & 7958.852 & 218.144 & Surface & 2011 \\
\hline 2583.436 & 227.886 & Surface & 2011 & 7961.760 & 218.151 & Surface & 2011 \\
\hline 2586.397 & 227.861 & Surface & 2011 & 7964.668 & 218.157 & Surface & 2011 \\
\hline 2589.358 & 227.841 & Surface & 2011 & 7967.576 & 218.160 & Surface & 2011 \\
\hline 2592.319 & 227.826 & Surface & 2011 & 7970.485 & 218.147 & Surface & 2011 \\
\hline 2595.280 & 227.823 & Surface & 2011 & 7973.393 & 218.108 & Surface & 2011 \\
\hline 2598.241 & 227.842 & Surface & 2011 & 7976.301 & 218.085 & Surface & 2011 \\
\hline 2601.202 & 227.847 & Surface & 2011 & 7979.260 & 218.079 & Surface & 2011 \\
\hline 2604.163 & 227.828 & Surface & 2011 & 7982.218 & 218.081 & Surface & 2011 \\
\hline 2607.124 & 227.836 & Surface & 2011 & 7985.177 & 218.086 & Surface & 2011 \\
\hline 2609.921 & 227.865 & Surface & 2011 & 7988.136 & 218.091 & Surface & 2011 \\
\hline 2612.716 & 227.868 & Surface & 2011 & 7991.094 & 218.095 & Surface & 2011 \\
\hline 2615.512 & 227.851 & Surface & 2011 & 7994.053 & 218.099 & Surface & 2011 \\
\hline 2618.309 & 227.830 & Surface & 2011 & 7997.012 & 218.102 & Surface & 2011 \\
\hline 2621.105 & 227.813 & Surface & 2011 & 7999.971 & 218.106 & Surface & 2011 \\
\hline 2623.900 & 227.799 & Surface & 2011 & 8002.929 & 218.111 & Surface & 2011 \\
\hline 2626.696 & 227.789 & Surface & 2011 & 8005.888 & 218.105 & Surface & 2011 \\
\hline 2629.493 & 227.787 & Surface & 2011 & 8008.847 & 218.132 & Surface & 2011 \\
\hline 2632.289 & 227.787 & Surface & 2011 & 8011.805 & 218.146 & Surface & 2011 \\
\hline 2635.084 & 227.782 & Surface & 2011 & 8014.764 & 218.138 & Surface & 2011 \\
\hline 2637.881 & 227.771 & Surface & 2011 & 8017.723 & 218.139 & Surface & 2011 \\
\hline 2640.677 & 227.754 & Surface & 2011 & 8020.681 & 218.147 & Surface & 2011 \\
\hline 2643.586 & 227.741 & Surface & 2011 & 8023.640 & 218.150 & Surface & 2011 \\
\hline 2646.496 & 227.734 & Surface & 2011 & 8026.599 & 218.154 & Surface & 2011 \\
\hline 2649.405 & 227.736 & Surface & 2011 & 8029.558 & 218.159 & Surface & 2011 \\
\hline 2652.314 & 227.730 & Surface & 2011 & 8032.523 & 218.149 & Surface & 2011 \\
\hline 2655.224 & 227.711 & Surface & 2011 & 8035.488 & 218.183 & Surface & 2011 \\
\hline 2658.133 & 227.699 & Surface & 2011 & 8038.453 & 218.196 & Surface & 2011 \\
\hline 2661.043 & 227.709 & Surface & 2011 & 8041.418 & 218.189 & Surface & 2011 \\
\hline 2663.952 & 227.707 & Surface & 2011 & 8044.383 & 218.176 & Surface & 2011 \\
\hline 2666.862 & 227.688 & Surface & 2011 & 8047.349 & 218.166 & Surface & 2011 \\
\hline 2669.771 & 227.696 & Surface & 2011 & 8050.314 & 218.165 & Surface & 2011 \\
\hline 2672.681 & 227.682 & Surface & 2011 & 8053.279 & 218.152 & Surface & 2011 \\
\hline 2675.590 & 227.667 & Surface & 2011 & 8056.245 & 218.163 & Surface & 2011 \\
\hline 2678.500 & 227.670 & Surface & 2011 & 8059.210 & 218.172 & Surface & 2011 \\
\hline 2681.409 & 227.655 & Surface & 2011 & 8062.175 & 218.173 & Surface & 2011 \\
\hline 2684.318 & 227.647 & Surface & 2011 & 8065.140 & 218.161 & Surface & 2011 \\
\hline 2687.228 & 227.666 & Surface & 2011 & 8068.105 & 218.150 & Surface & 2011 \\
\hline
\end{tabular}




\begin{tabular}{|c|c|c|c|c|c|c|c|}
\hline 2690.137 & 227.688 & Surface & 2011 & 8071.009 & 218.151 & Surface & 2011 \\
\hline 2693.131 & 227.674 & Surface & 2011 & 8073.913 & 218.150 & Surface & 2011 \\
\hline 2696.124 & 227.650 & Surface & 2011 & 8076.817 & 218.149 & Surface & 2011 \\
\hline 2699.117 & 227.641 & Surface & 2011 & 8079.721 & 218.154 & Surface & 2011 \\
\hline 2702.111 & 227.622 & Surface & 2011 & 8082.625 & 218.167 & Surface & 2011 \\
\hline 2705.104 & 227.616 & Surface & 2011 & 8085.529 & 218.180 & Surface & 2011 \\
\hline 2708.098 & 227.609 & Surface & 2011 & 8088.433 & 218.188 & Surface & 2011 \\
\hline 2711.091 & 227.601 & Surface & 2011 & 8091.337 & 218.187 & Surface & 2011 \\
\hline 2714.084 & 227.585 & Surface & 2011 & 8094.241 & 218.184 & Surface & 2011 \\
\hline 2717.078 & 227.555 & Surface & 2011 & 8097.145 & 218.182 & Surface & 2011 \\
\hline 2720.071 & 227.524 & Surface & 2011 & 8100.049 & 218.181 & Surface & 2011 \\
\hline 2723.065 & 227.522 & Surface & 2011 & 8102.952 & 218.183 & Surface & 2011 \\
\hline 2726.058 & 227.541 & Surface & 2011 & 8105.856 & 218.182 & Surface & 2011 \\
\hline 2729.051 & 227.551 & Surface & 2011 & 8108.760 & 218.178 & Surface & 2011 \\
\hline 2732.045 & 227.533 & Surface & 2011 & 8111.664 & 218.175 & Surface & 2011 \\
\hline 2735.038 & 227.523 & Surface & 2011 & 8114.568 & 218.170 & Surface & 2011 \\
\hline 2737.767 & 227.527 & Surface & 2011 & 8117.472 & 218.158 & Surface & 2011 \\
\hline 2740.496 & 227.535 & Surface & 2011 & 8120.376 & 218.149 & Surface & 2011 \\
\hline 2743.225 & 227.517 & Surface & 2011 & 8123.280 & 218.139 & Surface & 2011 \\
\hline 2745.954 & 227.487 & Surface & 2011 & 8126.197 & 218.127 & Surface & 2011 \\
\hline 2748.683 & 227.465 & Surface & 2011 & 8129.113 & 218.127 & Surface & 2011 \\
\hline 2751.412 & 227.452 & Surface & 2011 & 8132.030 & 218.143 & Surface & 2011 \\
\hline 2754.141 & 227.451 & Surface & 2011 & 8134.947 & 218.160 & Surface & 2011 \\
\hline 2756.870 & 227.439 & Surface & 2011 & 8137.863 & 218.162 & Surface & 2011 \\
\hline 2759.599 & 227.419 & Surface & 2011 & 8140.780 & 218.157 & Surface & 2011 \\
\hline 2762.328 & 227.408 & Surface & 2011 & 8143.697 & 218.152 & Surface & 2011 \\
\hline 2765.057 & 227.405 & Surface & 2011 & 8146.614 & 218.143 & Surface & 2011 \\
\hline 2767.920 & 227.399 & Surface & 2011 & 8149.530 & 218.133 & Surface & 2011 \\
\hline 2770.782 & 227.393 & Surface & 2011 & 8152.447 & 218.128 & Surface & 2011 \\
\hline 2773.644 & 227.357 & Surface & 2011 & 8155.364 & 218.127 & Surface & 2011 \\
\hline 2776.507 & 227.333 & Surface & 2011 & 8158.280 & 218.100 & Surface & 2011 \\
\hline 2779.369 & 227.337 & Surface & 2011 & 8161.197 & 218.022 & Surface & 2011 \\
\hline 2782.231 & 227.323 & Surface & 2011 & 8164.080 & 218.016 & Surface & 2011 \\
\hline 2785.094 & 227.285 & Surface & 2011 & 8166.963 & 218.023 & Surface & 2011 \\
\hline 2787.957 & 227.271 & Surface & 2011 & 8169.847 & 218.035 & Surface & 2011 \\
\hline 2790.819 & 227.244 & Surface & 2011 & 8172.730 & 218.013 & Surface & 2011 \\
\hline 2793.681 & 227.204 & Surface & 2011 & 8175.613 & 217.981 & Surface & 2011 \\
\hline 2796.544 & 227.157 & Surface & 2011 & 8178.496 & 217.950 & Surface & 2011 \\
\hline 2799.406 & 227.102 & Surface & 2011 & 8181.379 & 217.908 & Surface & 2011 \\
\hline 2802.252 & 227.091 & Surface & 2011 & 8184.262 & 217.871 & Surface & 2011 \\
\hline 2805.098 & 227.128 & Surface & 2011 & 8187.145 & 217.785 & Surface & 2011 \\
\hline 2807.944 & 227.154 & Surface & 2011 & 8190.028 & 217.711 & Surface & 2011 \\
\hline 2810.790 & 227.135 & Surface & 2011 & 8192.911 & 217.642 & Surface & 2011 \\
\hline 2813.636 & 227.126 & Surface & 2011 & 8195.794 & 217.588 & Surface & 2011 \\
\hline 2816.482 & 227.119 & Surface & 2011 & 8198.677 & 217.545 & Surface & 2011 \\
\hline 2819.328 & 227.099 & Surface & 2011 & 8201.560 & 217.515 & Surface & 2011 \\
\hline 2822.174 & 227.100 & Surface & 2011 & 8204.444 & 217.468 & Surface & 2011 \\
\hline 2825.020 & 227.123 & Surface & 2011 & 8207.327 & 217.384 & Surface & 2011 \\
\hline 2827.866 & 227.142 & Surface & 2011 & 8210.292 & 217.295 & Surface & 2011 \\
\hline
\end{tabular}




\begin{tabular}{|c|c|c|c|c|c|c|c|}
\hline 2830.712 & 227.140 & Surface & 2011 & 8213.257 & 217.245 & Surface & 2011 \\
\hline 2833.558 & 227.130 & Surface & 2011 & 8216.223 & 217.185 & Surface & 2011 \\
\hline 2836.404 & 227.129 & Surface & 2011 & 8219.188 & 217.135 & Surface & 2011 \\
\hline 2839.250 & 227.111 & Surface & 2011 & 8222.153 & 217.073 & Surface & 2011 \\
\hline 2842.096 & 227.107 & Surface & 2011 & 8225.118 & 217.024 & Surface & 2011 \\
\hline 2844.942 & 227.128 & Surface & 2011 & 8228.083 & 216.997 & Surface & 2011 \\
\hline 2847.788 & 227.127 & Surface & 2011 & 8231.048 & 217.020 & Surface & 2011 \\
\hline 2850.651 & 227.101 & Surface & 2011 & 8234.014 & 217.009 & Surface & 2011 \\
\hline 2853.513 & 227.098 & Surface & 2011 & 8236.979 & 216.985 & Surface & 2011 \\
\hline 2856.375 & 227.102 & Surface & 2011 & 8239.944 & 216.958 & Surface & 2011 \\
\hline 2859.238 & 227.101 & Surface & 2011 & 8242.910 & 216.941 & Surface & 2011 \\
\hline 2862.100 & 227.098 & Surface & 2011 & 8245.875 & 216.943 & Surface & 2011 \\
\hline 2864.963 & 227.093 & Surface & 2011 & 8248.840 & 216.941 & Surface & 2011 \\
\hline 2867.825 & 227.091 & Surface & 2011 & 8251.805 & 216.932 & Surface & 2011 \\
\hline 2870.688 & 227.079 & Surface & 2011 & 8254.770 & 216.877 & Surface & 2011 \\
\hline 2873.550 & 227.072 & Surface & 2011 & 8257.736 & 216.768 & Surface & 2011 \\
\hline 2876.412 & 227.057 & Surface & 2011 & 8260.701 & 216.609 & Surface & 2011 \\
\hline 2879.275 & 227.059 & Surface & 2011 & 8263.666 & 216.462 & Surface & 2011 \\
\hline 2882.137 & 227.065 & Surface & 2011 & 8266.631 & 216.512 & Surface & 2011 \\
\hline 2885.005 & 227.061 & Surface & 2011 & 8269.596 & 216.598 & Surface & 2011 \\
\hline 2887.873 & 227.054 & Surface & 2011 & 8272.562 & 216.651 & Surface & 2011 \\
\hline 2890.741 & 227.044 & Surface & 2011 & 8275.527 & 216.659 & Surface & 2011 \\
\hline 2893.609 & 227.040 & Surface & 2011 & 8278.492 & 216.664 & Surface & 2011 \\
\hline 2896.476 & 227.042 & Surface & 2011 & 8281.457 & 216.662 & Surface & 2011 \\
\hline 2899.344 & 227.034 & Surface & 2011 & 8284.423 & 216.633 & Surface & 2011 \\
\hline 2902.212 & 226.992 & Surface & 2011 & 8287.281 & 216.610 & Surface & 2011 \\
\hline 2905.080 & 226.965 & Surface & 2011 & 8290.139 & 216.604 & Surface & 2011 \\
\hline 2907.947 & 226.949 & Surface & 2011 & 8292.997 & 216.595 & Surface & 2011 \\
\hline 2910.815 & 226.919 & Surface & 2011 & 8295.855 & 216.596 & Surface & 2011 \\
\hline 2913.683 & 226.889 & Surface & 2011 & 8298.713 & 216.579 & Surface & 2011 \\
\hline 2916.551 & 226.850 & Surface & 2011 & 8301.571 & 216.583 & Surface & 2011 \\
\hline 2919.418 & 226.811 & Surface & 2011 & 8304.429 & 216.609 & Surface & 2011 \\
\hline 2922.286 & 226.772 & Surface & 2011 & 8307.287 & 216.629 & Surface & 2011 \\
\hline 2925.154 & 226.723 & Surface & 2011 & 8310.145 & 216.627 & Surface & 2011 \\
\hline 2928.022 & 226.685 & Surface & 2011 & 8313.003 & 216.636 & Surface & 2011 \\
\hline 2930.981 & 226.665 & Surface & 2011 & 8315.861 & 216.631 & Surface & 2011 \\
\hline 2933.940 & 226.665 & Surface & 2011 & 8318.719 & 216.611 & Surface & 2011 \\
\hline 2936.900 & 226.698 & Surface & 2011 & 8321.577 & 216.594 & Surface & 2011 \\
\hline 2939.859 & 226.712 & Surface & 2011 & 8324.447 & 216.583 & Surface & 2011 \\
\hline 2942.818 & 226.720 & Surface & 2011 & 8327.317 & 216.534 & Surface & 2011 \\
\hline 2945.777 & 226.749 & Surface & 2011 & 8330.186 & 216.470 & Surface & 2011 \\
\hline 2948.737 & 226.746 & Surface & 2011 & 8333.056 & 216.424 & Surface & 2011 \\
\hline 2951.696 & 226.728 & Surface & 2011 & 8335.926 & 216.404 & Surface & 2011 \\
\hline 2954.655 & 226.723 & Surface & 2011 & 8338.796 & 216.373 & Surface & 2011 \\
\hline 2957.615 & 226.735 & Surface & 2011 & 8341.666 & 216.343 & Surface & 2011 \\
\hline 2960.574 & 226.747 & Surface & 2011 & 8344.535 & 216.348 & Surface & 2011 \\
\hline 2963.533 & 226.734 & Surface & 2011 & 8347.405 & 216.375 & Surface & 2011 \\
\hline 2966.262 & 226.711 & Surface & 2011 & 8350.275 & 216.387 & Surface & 2011 \\
\hline 2968.991 & 226.711 & Surface & 2011 & 8353.145 & 216.389 & Surface & 2011 \\
\hline
\end{tabular}




\begin{tabular}{|c|c|c|c|c|c|c|c|}
\hline 2971.720 & 226.729 & Surface & 2011 & 8356.015 & 216.367 & Surface & 2011 \\
\hline 2974.449 & 226.759 & Surface & 2011 & 8358.884 & 216.316 & Surface & 2011 \\
\hline 2977.179 & 226.774 & Surface & 2011 & 8361.754 & 216.300 & Surface & 2011 \\
\hline 2979.907 & 226.778 & Surface & 2011 & 8364.624 & 216.314 & Surface & 2011 \\
\hline 2982.636 & 226.782 & Surface & 2011 & 8367.494 & 216.330 & Surface & 2011 \\
\hline 2985.365 & 226.770 & Surface & 2011 & 8370.363 & 216.366 & Surface & 2011 \\
\hline 2988.094 & 226.737 & Surface & 2011 & 8373.233 & 216.361 & Surface & 2011 \\
\hline 2990.823 & 226.758 & Surface & 2011 & 8376.103 & 216.349 & Surface & 2011 \\
\hline 2993.553 & 226.763 & Surface & 2011 & 8378.973 & 216.334 & Surface & 2011 \\
\hline 2996.456 & 226.760 & Surface & 2011 & 8381.843 & 216.317 & Surface & 2011 \\
\hline 2999.360 & 226.765 & Surface & 2011 & 8384.706 & 216.315 & Surface & 2011 \\
\hline 3002.264 & 226.765 & Surface & 2011 & 8387.569 & 216.326 & Surface & 2011 \\
\hline 3005.167 & 226.766 & Surface & 2011 & 8390.432 & 216.339 & Surface & 2011 \\
\hline 3008.071 & 226.771 & Surface & 2011 & 8393.296 & 216.351 & Surface & 2011 \\
\hline 3010.975 & 226.773 & Surface & 2011 & 8396.159 & 216.351 & Surface & 2011 \\
\hline 3013.878 & 226.767 & Surface & 2011 & 8399.022 & 216.332 & Surface & 2011 \\
\hline 3016.782 & 226.762 & Surface & 2011 & 8401.886 & 216.327 & Surface & 2011 \\
\hline 3019.686 & 226.752 & Surface & 2011 & 8404.749 & 216.333 & Surface & 2011 \\
\hline 3022.589 & 226.744 & Surface & 2011 & 8407.612 & 216.359 & Surface & 2011 \\
\hline 3025.493 & 226.743 & Surface & 2011 & 8410.475 & 216.334 & Surface & 2011 \\
\hline 3028.453 & 226.745 & Surface & 2011 & 8413.339 & 216.314 & Surface & 2011 \\
\hline 3031.414 & 226.731 & Surface & 2011 & 8416.202 & 216.302 & Surface & 2011 \\
\hline 3034.374 & 226.718 & Surface & 2011 & 8419.065 & 216.290 & Surface & 2011 \\
\hline 3037.334 & 226.703 & Surface & 2011 & 8421.929 & 216.269 & Surface & 2011 \\
\hline 3040.295 & 226.688 & Surface & 2011 & 8424.792 & 216.251 & Surface & 2011 \\
\hline 3043.255 & 226.693 & Surface & 2011 & 8427.655 & 216.261 & Surface & 2011 \\
\hline 3046.215 & 226.700 & Surface & 2011 & 8430.518 & 216.283 & Surface & 2011 \\
\hline 3049.175 & 226.702 & Surface & 2011 & 8433.382 & 216.298 & Surface & 2011 \\
\hline 3052.136 & 226.702 & Surface & 2011 & 8436.245 & 216.311 & Surface & 2011 \\
\hline 3055.096 & 226.711 & Surface & 2011 & 8439.108 & 216.308 & Surface & 2011 \\
\hline 3058.056 & 226.718 & Surface & 2011 & 8442.046 & 216.313 & Surface & 2011 \\
\hline 3061.017 & 226.710 & Surface & 2011 & 8444.984 & 216.306 & Surface & 2011 \\
\hline 3063.977 & 226.710 & Surface & 2011 & 8447.922 & 216.296 & Surface & 2011 \\
\hline 3066.937 & 226.715 & Surface & 2011 & 8450.860 & 216.292 & Surface & 2011 \\
\hline 3069.897 & 226.696 & Surface & 2011 & 8453.798 & 216.304 & Surface & 2011 \\
\hline 3072.858 & 226.683 & Surface & 2011 & 8456.736 & 216.299 & Surface & 2011 \\
\hline 3075.843 & 226.675 & Surface & 2011 & 8459.674 & 216.289 & Surface & 2011 \\
\hline 3078.829 & 226.662 & Surface & 2011 & 8462.612 & 216.282 & Surface & 2011 \\
\hline 3081.814 & 226.655 & Surface & 2011 & 8465.550 & 216.278 & Surface & 2011 \\
\hline 3084.800 & 226.641 & Surface & 2011 & 8468.488 & 216.302 & Surface & 2011 \\
\hline 3087.785 & 226.657 & Surface & 2011 & 8471.426 & 216.280 & Surface & 2011 \\
\hline 3090.771 & 226.670 & Surface & 2011 & 8474.364 & 216.318 & Surface & 2011 \\
\hline 3093.756 & 226.667 & Surface & 2011 & 8477.302 & 216.341 & Surface & 2011 \\
\hline 3096.741 & 226.637 & Surface & 2011 & 8480.091 & 216.396 & Surface & 2011 \\
\hline 3099.727 & 226.624 & Surface & 2011 & 8482.880 & 216.402 & Surface & 2011 \\
\hline 3102.712 & 226.619 & Surface & 2011 & 8485.668 & 216.359 & Surface & 2011 \\
\hline 3105.698 & 226.605 & Surface & 2011 & 8488.457 & 216.318 & Surface & 2011 \\
\hline 3108.683 & 226.571 & Surface & 2011 & 8491.245 & 216.280 & Surface & 2011 \\
\hline 3111.669 & 226.566 & Surface & 2011 & 8494.034 & 216.263 & Surface & 2011 \\
\hline
\end{tabular}




\begin{tabular}{|c|c|c|c|c|c|c|c|}
\hline 3114.654 & 226.588 & Surface & 2011 & 8496.822 & 216.277 & Surface & 2011 \\
\hline 3117.640 & 226.597 & Surface & 2011 & 8499.611 & 216.281 & Surface & 2011 \\
\hline 3120.625 & 226.593 & Surface & 2011 & 8502.400 & 216.298 & Surface & 2011 \\
\hline 3123.611 & 226.574 & Surface & 2011 & 8505.188 & 216.293 & Surface & 2011 \\
\hline 3126.596 & 226.552 & Surface & 2011 & 8507.977 & 216.300 & Surface & 2011 \\
\hline 3129.582 & 226.538 & Surface & 2011 & 8510.765 & 216.306 & Surface & 2011 \\
\hline 3132.536 & 226.549 & Surface & 2011 & 8513.554 & 216.299 & Surface & 2011 \\
\hline 3135.490 & 226.540 & Surface & 2011 & 8516.412 & 216.277 & Surface & 2011 \\
\hline 3138.444 & 226.552 & Surface & 2011 & 8519.271 & 216.255 & Surface & 2011 \\
\hline 3141.398 & 226.540 & Surface & 2011 & 8522.130 & 216.268 & Surface & 2011 \\
\hline 3144.352 & 226.511 & Surface & 2011 & 8524.988 & 216.303 & Surface & 2011 \\
\hline 3147.306 & 226.508 & Surface & 2011 & 8527.847 & 216.324 & Surface & 2011 \\
\hline 3150.261 & 226.515 & Surface & 2011 & 8530.705 & 216.329 & Surface & 2011 \\
\hline 3153.215 & 226.501 & Surface & 2011 & 8533.564 & 216.340 & Surface & 2011 \\
\hline 3156.169 & 226.505 & Surface & 2011 & 8536.422 & 216.346 & Surface & 2011 \\
\hline 3159.123 & 226.501 & Surface & 2011 & 8539.281 & 216.341 & Surface & 2011 \\
\hline 3162.077 & 226.477 & Surface & 2011 & 8542.140 & 216.338 & Surface & 2011 \\
\hline 3165.031 & 226.431 & Surface & 2011 & 8544.998 & 216.336 & Surface & 2011 \\
\hline 3167.986 & 226.415 & Surface & 2011 & 8547.857 & 216.338 & Surface & 2011 \\
\hline 3170.940 & 226.427 & Surface & 2011 & 8550.715 & 216.333 & Surface & 2011 \\
\hline 3173.894 & 226.438 & Surface & 2011 & 8553.574 & 216.290 & Surface & 2011 \\
\hline 3176.848 & 226.458 & Surface & 2011 & 8556.432 & 216.223 & Surface & 2011 \\
\hline 3179.802 & 226.464 & Surface & 2011 & 8559.287 & 216.180 & Surface & 2011 \\
\hline 3182.595 & 226.427 & Surface & 2011 & 8562.141 & 216.107 & Surface & 2011 \\
\hline 3185.388 & 226.352 & Surface & 2011 & 8564.996 & 216.046 & Surface & 2011 \\
\hline 3188.181 & 226.326 & Surface & 2011 & 8567.850 & 216.024 & Surface & 2011 \\
\hline 3190.974 & 226.325 & Surface & 2011 & 8570.704 & 216.019 & Surface & 2011 \\
\hline 3193.767 & 226.321 & Surface & 2011 & 8573.559 & 216.024 & Surface & 2011 \\
\hline 3196.560 & 226.319 & Surface & 2011 & 8576.413 & 216.034 & Surface & 2011 \\
\hline 3199.353 & 226.309 & Surface & 2011 & 8579.267 & 216.055 & Surface & 2011 \\
\hline 3202.146 & 226.295 & Surface & 2011 & 8582.122 & 216.056 & Surface & 2011 \\
\hline 3204.939 & 226.286 & Surface & 2011 & 8584.976 & 216.068 & Surface & 2011 \\
\hline 3207.732 & 226.283 & Surface & 2011 & 8587.830 & 216.088 & Surface & 2011 \\
\hline 3210.525 & 226.263 & Surface & 2011 & 8590.685 & 216.107 & Surface & 2011 \\
\hline 3213.318 & 226.239 & Surface & 2011 & 8593.539 & 216.092 & Surface & 2011 \\
\hline 3216.111 & 226.219 & Surface & 2011 & 8596.393 & 216.103 & Surface & 2011 \\
\hline 3218.904 & 226.196 & Surface & 2011 & 8599.248 & 216.121 & Surface & 2011 \\
\hline 3221.761 & 226.170 & Surface & 2011 & 8602.102 & 216.125 & Surface & 2011 \\
\hline 3224.618 & 226.158 & Surface & 2011 & 8604.928 & 216.126 & Surface & 2011 \\
\hline 3227.474 & 226.149 & Surface & 2011 & 8607.753 & 216.119 & Surface & 2011 \\
\hline 3230.331 & 226.105 & Surface & 2011 & 8610.579 & 216.111 & Surface & 2011 \\
\hline 3233.188 & 226.058 & Surface & 2011 & 8613.405 & 216.105 & Surface & 2011 \\
\hline 3236.044 & 226.019 & Surface & 2011 & 8616.230 & 216.091 & Surface & 2011 \\
\hline 3238.901 & 225.981 & Surface & 2011 & 8619.056 & 216.094 & Surface & 2011 \\
\hline 3241.758 & 225.937 & Surface & 2011 & 8621.882 & 216.091 & Surface & 2011 \\
\hline 3244.615 & 225.908 & Surface & 2011 & 8624.707 & 216.083 & Surface & 2011 \\
\hline 3247.471 & 225.912 & Surface & 2011 & 8627.533 & 216.063 & Surface & 2011 \\
\hline 3250.328 & 225.906 & Surface & 2011 & 8630.358 & 216.054 & Surface & 2011 \\
\hline 3253.185 & 225.874 & Surface & 2011 & 8633.184 & 216.052 & Surface & 2011 \\
\hline
\end{tabular}




\begin{tabular}{|c|c|c|c|c|c|c|c|}
\hline 3256.041 & 225.858 & Surface & 2011 & 8636.009 & 216.057 & Surface & 2011 \\
\hline 3258.898 & 225.879 & Surface & 2011 & 8638.835 & 216.059 & Surface & 2011 \\
\hline 3261.755 & 225.889 & Surface & 2011 & 8641.660 & 216.054 & Surface & 2011 \\
\hline 3264.611 & 225.885 & Surface & 2011 & 8644.486 & 216.059 & Surface & 2011 \\
\hline 3267.554 & 225.858 & Surface & 2011 & 8647.469 & 216.064 & Surface & 2011 \\
\hline 3270.497 & 225.795 & Surface & 2011 & 8650.451 & 216.055 & Surface & 2011 \\
\hline 3273.439 & 225.713 & Surface & 2011 & 8653.434 & 216.049 & Surface & 2011 \\
\hline 3276.382 & 225.612 & Surface & 2011 & 8656.416 & 216.065 & Surface & 2011 \\
\hline 3279.325 & 225.565 & Surface & 2011 & 8659.399 & 216.079 & Surface & 2011 \\
\hline 3282.268 & 225.619 & Surface & 2011 & 8662.382 & 216.104 & Surface & 2011 \\
\hline 3285.210 & 225.635 & Surface & 2011 & 8665.364 & 216.118 & Surface & 2011 \\
\hline 3288.153 & 225.629 & Surface & 2011 & 8668.347 & 216.125 & Surface & 2011 \\
\hline 3291.096 & 225.611 & Surface & 2011 & 8671.329 & 216.117 & Surface & 2011 \\
\hline 3294.038 & 225.616 & Surface & 2011 & 8674.312 & 216.103 & Surface & 2011 \\
\hline 3296.983 & 225.582 & Surface & 2011 & 8677.294 & 216.115 & Surface & 2011 \\
\hline 3299.927 & 225.519 & Surface & 2011 & 8680.277 & 216.115 & Surface & 2011 \\
\hline 3302.871 & 225.513 & Surface & 2011 & 8683.210 & 216.088 & Surface & 2011 \\
\hline 3305.815 & 225.519 & Surface & 2011 & 8686.144 & 216.114 & Surface & 2011 \\
\hline 3308.759 & 225.473 & Surface & 2011 & 8689.077 & 216.131 & Surface & 2011 \\
\hline 3311.703 & 225.407 & Surface & 2011 & 8692.011 & 216.133 & Surface & 2011 \\
\hline 3314.648 & 225.380 & Surface & 2011 & 8694.944 & 216.134 & Surface & 2011 \\
\hline 3317.592 & 225.386 & Surface & 2011 & 8697.878 & 216.136 & Surface & 2011 \\
\hline 3320.536 & 225.410 & Surface & 2011 & 8700.811 & 216.136 & Surface & 2011 \\
\hline 3323.480 & 225.432 & Surface & 2011 & 8703.744 & 216.140 & Surface & 2011 \\
\hline 3326.424 & 225.435 & Surface & 2011 & 8706.678 & 216.137 & Surface & 2011 \\
\hline 3329.368 & 225.421 & Surface & 2011 & 8709.611 & 216.141 & Surface & 2011 \\
\hline 3332.313 & 225.414 & Surface & 2011 & 8712.545 & 216.140 & Surface & 2011 \\
\hline 3335.257 & 225.397 & Surface & 2011 & 8715.478 & 216.129 & Surface & 2011 \\
\hline 3338.201 & 225.419 & Surface & 2011 & 8718.411 & 216.104 & Surface & 2011 \\
\hline 3341.145 & 225.434 & Surface & 2011 & 8721.345 & 216.078 & Surface & 2011 \\
\hline 3343.939 & 225.435 & Surface & 2011 & 8724.278 & 216.058 & Surface & 2011 \\
\hline 3346.733 & 225.421 & Surface & 2011 & 8727.212 & 216.057 & Surface & 2011 \\
\hline 3349.526 & 225.387 & Surface & 2011 & 8730.145 & 216.062 & Surface & 2011 \\
\hline 3352.320 & 225.376 & Surface & 2011 & 8733.079 & 216.065 & Surface & 2011 \\
\hline 3355.114 & 225.406 & Surface & 2011 & 8736.023 & 216.069 & Surface & 2011 \\
\hline 3357.908 & 225.451 & Surface & 2011 & 8738.968 & 216.074 & Surface & 2011 \\
\hline 3360.702 & 225.475 & Surface & 2011 & 8741.912 & 216.079 & Surface & 2011 \\
\hline 3363.496 & 225.437 & Surface & 2011 & 8744.857 & 216.084 & Surface & 2011 \\
\hline 3366.289 & 225.411 & Surface & 2011 & 8747.801 & 216.089 & Surface & 2011 \\
\hline 3369.083 & 225.421 & Surface & 2011 & 8750.746 & 216.094 & Surface & 2011 \\
\hline 3371.877 & 225.426 & Surface & 2011 & 8753.690 & 216.102 & Surface & 2011 \\
\hline 3374.824 & 225.413 & Surface & 2011 & 8756.635 & 216.110 & Surface & 2011 \\
\hline 3377.771 & 225.401 & Surface & 2011 & 8759.579 & 216.118 & Surface & 2011 \\
\hline 3380.718 & 225.406 & Surface & 2011 & 8762.524 & 216.123 & Surface & 2011 \\
\hline 3383.665 & 225.411 & Surface & 2011 & 8765.468 & 216.123 & Surface & 2011 \\
\hline 3386.612 & 225.377 & Surface & 2011 & 8768.413 & 216.121 & Surface & 2011 \\
\hline 3389.559 & 225.383 & Surface & 2011 & 8771.357 & 216.112 & Surface & 2011 \\
\hline 3392.506 & 225.404 & Surface & 2011 & 8774.302 & 216.086 & Surface & 2011 \\
\hline 3395.452 & 225.418 & Surface & 2011 & 8777.246 & 216.060 & Surface & 2011 \\
\hline
\end{tabular}




\begin{tabular}{|c|c|c|c|c|c|c|c|}
\hline 3398.400 & 225.394 & Surface & 2011 & 8780.191 & 216.042 & Surface & 2011 \\
\hline 3401.346 & 225.372 & Surface & 2011 & 8783.135 & 216.007 & Surface & 2011 \\
\hline 3404.293 & 225.372 & Surface & 2011 & 8786.080 & 215.971 & Surface & 2011 \\
\hline 3407.240 & 225.369 & Surface & 2011 & 8789.009 & 215.957 & Surface & 2011 \\
\hline 3410.187 & 225.344 & Surface & 2011 & 8791.939 & 215.940 & Surface & 2011 \\
\hline 3413.134 & 225.311 & Surface & 2011 & 8794.868 & 215.932 & Surface & 2011 \\
\hline 3416.121 & 225.266 & Surface & 2011 & 8797.798 & 215.921 & Surface & 2011 \\
\hline 3419.109 & 225.217 & Surface & 2011 & 8800.727 & 215.916 & Surface & 2011 \\
\hline 3422.096 & 225.185 & Surface & 2011 & 8803.657 & 215.931 & Surface & 2011 \\
\hline 3425.083 & 225.173 & Surface & 2011 & 8806.586 & 215.938 & Surface & 2011 \\
\hline 3428.070 & 225.162 & Surface & 2011 & 8809.516 & 215.895 & Surface & 2011 \\
\hline 3431.058 & 225.164 & Surface & 2011 & 8812.445 & 215.855 & Surface & 2011 \\
\hline 3434.045 & 225.130 & Surface & 2011 & 8815.375 & 215.845 & Surface & 2011 \\
\hline 3437.032 & 225.069 & Surface & 2011 & 8818.304 & 215.830 & Surface & 2011 \\
\hline 3440.020 & 225.045 & Surface & 2011 & 8821.234 & 215.815 & Surface & 2011 \\
\hline 3443.007 & 225.060 & Surface & 2011 & 8824.124 & 215.810 & Surface & 2011 \\
\hline 3445.994 & 225.078 & Surface & 2011 & 8827.014 & 215.808 & Surface & 2011 \\
\hline 3448.982 & 225.053 & Surface & 2011 & 8829.904 & 215.790 & Surface & 2011 \\
\hline 3451.969 & 225.027 & Surface & 2011 & 8832.795 & 215.829 & Surface & 2011 \\
\hline 3454.956 & 225.011 & Surface & 2011 & 8835.685 & 215.834 & Surface & 2011 \\
\hline 3457.943 & 224.981 & Surface & 2011 & 8838.575 & 215.838 & Surface & 2011 \\
\hline 3460.931 & 224.969 & Surface & 2011 & 8841.466 & 215.845 & Surface & 2011 \\
\hline 3463.918 & 224.945 & Surface & 2011 & 8844.356 & 215.825 & Surface & 2011 \\
\hline 3466.862 & 224.934 & Surface & 2011 & 8847.246 & 215.813 & Surface & 2011 \\
\hline 3469.807 & 224.976 & Surface & 2011 & 8850.137 & 215.829 & Surface & 2011 \\
\hline 3472.752 & 224.999 & Surface & 2011 & 8853.027 & 215.841 & Surface & 2011 \\
\hline 3475.697 & 224.940 & Surface & 2011 & 8855.917 & 215.832 & Surface & 2011 \\
\hline 3478.641 & 224.963 & Surface & 2011 & 8858.807 & 215.822 & Surface & 2011 \\
\hline 3481.586 & 224.999 & Surface & 2011 & 8861.698 & 215.829 & Surface & 2011 \\
\hline 3484.531 & 225.024 & Surface & 2011 & 8864.588 & 215.847 & Surface & 2011 \\
\hline 3487.476 & 225.028 & Surface & 2011 & 8867.478 & 215.845 & Surface & 2011 \\
\hline 3490.421 & 225.010 & Surface & 2011 & 8870.369 & 215.811 & Surface & 2011 \\
\hline 3493.365 & 225.007 & Surface & 2011 & 8873.259 & 215.800 & Surface & 2011 \\
\hline 3496.310 & 225.021 & Surface & 2011 & 8876.053 & 215.793 & Surface & 2011 \\
\hline 3499.255 & 225.038 & Surface & 2011 & 8878.847 & 215.804 & Surface & 2011 \\
\hline 3502.199 & 225.038 & Surface & 2011 & 8881.641 & 215.833 & Surface & 2011 \\
\hline 3505.144 & 225.028 & Surface & 2011 & 8884.436 & 215.822 & Surface & 2011 \\
\hline 3508.089 & 225.001 & Surface & 2011 & 8887.230 & 215.800 & Surface & 2011 \\
\hline 3511.034 & 225.015 & Surface & 2011 & 8890.024 & 215.812 & Surface & 2011 \\
\hline 3513.978 & 225.022 & Surface & 2011 & 8892.818 & 215.843 & Surface & 2011 \\
\hline 3516.923 & 225.038 & Surface & 2011 & 8895.612 & 215.854 & Surface & 2011 \\
\hline 3519.868 & 225.040 & Surface & 2011 & 8898.406 & 215.846 & Surface & 2011 \\
\hline 3522.812 & 225.012 & Surface & 2011 & 8901.201 & 215.838 & Surface & 2011 \\
\hline 3525.758 & 224.967 & Surface & 2011 & 8903.995 & 215.824 & Surface & 2011 \\
\hline 3528.702 & 224.940 & Surface & 2011 & 8906.789 & 215.830 & Surface & 2011 \\
\hline 3531.647 & 224.913 & Surface & 2011 & 8909.684 & 215.831 & Surface & 2011 \\
\hline 3534.592 & 224.904 & Surface & 2011 & 8912.579 & 215.840 & Surface & 2011 \\
\hline 3537.536 & 224.901 & Surface & 2011 & 8915.474 & 215.839 & Surface & 2011 \\
\hline 3540.481 & 224.904 & Surface & 2011 & 8918.369 & 215.839 & Surface & 2011 \\
\hline
\end{tabular}




\begin{tabular}{|c|c|c|c|c|c|c|c|}
\hline 3543.426 & 224.932 & Surface & 2011 & 8921.264 & 215.845 & Surface & 2011 \\
\hline 3546.317 & 224.966 & Surface & 2011 & 8924.159 & 215.821 & Surface & 2011 \\
\hline 3549.208 & 224.979 & Surface & 2011 & 8927.054 & 215.817 & Surface & 2011 \\
\hline 3552.099 & 224.972 & Surface & 2011 & 8929.949 & 215.832 & Surface & 2011 \\
\hline 3554.990 & 224.971 & Surface & 2011 & 8932.844 & 215.860 & Surface & 2011 \\
\hline 3557.881 & 224.995 & Surface & 2011 & 8935.739 & 215.845 & Surface & 2011 \\
\hline 3560.773 & 225.014 & Surface & 2011 & 8938.634 & 215.826 & Surface & 2011 \\
\hline 3563.664 & 224.991 & Surface & 2011 & 8941.529 & 215.814 & Surface & 2011 \\
\hline 3566.555 & 224.971 & Surface & 2011 & 8944.424 & 215.827 & Surface & 2011 \\
\hline 3569.446 & 224.972 & Surface & 2011 & 8947.319 & 215.834 & Surface & 2011 \\
\hline 3572.337 & 224.968 & Surface & 2011 & 8950.186 & 215.839 & Surface & 2011 \\
\hline 3575.228 & 224.967 & Surface & 2011 & 8953.052 & 215.855 & Surface & 2011 \\
\hline 3578.119 & 224.988 & Surface & 2011 & 8955.919 & 215.875 & Surface & 2011 \\
\hline 3581.010 & 224.983 & Surface & 2011 & 8958.786 & 215.882 & Surface & 2011 \\
\hline 3583.902 & 224.979 & Surface & 2011 & 8961.653 & 215.880 & Surface & 2011 \\
\hline 3586.793 & 224.983 & Surface & 2011 & 8964.519 & 215.880 & Surface & 2011 \\
\hline 3589.684 & 224.975 & Surface & 2011 & 8967.386 & 215.881 & Surface & 2011 \\
\hline 3592.575 & 224.955 & Surface & 2011 & 8970.253 & 215.875 & Surface & 2011 \\
\hline 3595.466 & 224.950 & Surface & 2011 & 8973.120 & 215.869 & Surface & 2011 \\
\hline 3598.357 & 224.959 & Surface & 2011 & 8975.986 & 215.868 & Surface & 2011 \\
\hline 3601.248 & 224.971 & Surface & 2011 & 8978.853 & 215.847 & Surface & 2011 \\
\hline 3604.139 & 224.980 & Surface & 2011 & 8981.720 & 215.836 & Surface & 2011 \\
\hline 3607.031 & 224.979 & Surface & 2011 & 8984.587 & 215.836 & Surface & 2011 \\
\hline 3609.921 & 224.976 & Surface & 2011 & 8987.454 & 215.835 & Surface & 2011 \\
\hline 3612.813 & 224.966 & Surface & 2011 & 8990.320 & 215.835 & Surface & 2011 \\
\hline 3615.704 & 224.968 & Surface & 2011 & 8993.187 & 215.835 & Surface & 2011 \\
\hline 3618.595 & 224.982 & Surface & 2011 & 8996.054 & 215.834 & Surface & 2011 \\
\hline 3621.452 & 224.985 & Surface & 2011 & 8998.948 & 215.834 & Surface & 2011 \\
\hline 3624.308 & 224.985 & Surface & 2011 & 9001.842 & 215.836 & Surface & 2011 \\
\hline 3627.165 & 224.989 & Surface & 2011 & 9004.736 & 215.836 & Surface & 2011 \\
\hline 3630.022 & 224.983 & Surface & 2011 & 9007.631 & 215.836 & Surface & 2011 \\
\hline 3632.879 & 224.940 & Surface & 2011 & 9010.525 & 215.837 & Surface & 2011 \\
\hline 3635.735 & 224.936 & Surface & 2011 & 9013.419 & 215.837 & Surface & 2011 \\
\hline 3638.592 & 224.954 & Surface & 2011 & 9016.313 & 215.837 & Surface & 2011 \\
\hline 3641.448 & 224.961 & Surface & 2011 & 9019.208 & 215.837 & Surface & 2011 \\
\hline 3644.305 & 224.941 & Surface & 2011 & 9022.102 & 215.829 & Surface & 2011 \\
\hline 3647.162 & 224.900 & Surface & 2011 & 9024.996 & 215.814 & Surface & 2011 \\
\hline 3650.018 & 224.912 & Surface & 2011 & 9027.890 & 215.799 & Surface & 2011 \\
\hline 3652.875 & 224.905 & Surface & 2011 & 9030.784 & 215.799 & Surface & 2011 \\
\hline 3655.732 & 224.887 & Surface & 2011 & 9033.679 & 215.806 & Surface & 2011 \\
\hline 3658.589 & 224.893 & Surface & 2011 & 9036.573 & 215.809 & Surface & 2011 \\
\hline 3661.446 & 224.906 & Surface & 2011 & 9039.467 & 215.813 & Surface & 2011 \\
\hline 3664.302 & 224.919 & Surface & 2011 & 9042.361 & 215.816 & Surface & 2011 \\
\hline 3667.227 & 224.923 & Surface & 2011 & 9045.256 & 215.816 & Surface & 2011 \\
\hline 3670.151 & 224.900 & Surface & 2011 & 9048.150 & 215.818 & Surface & 2011 \\
\hline 3673.075 & 224.891 & Surface & 2011 & 9051.044 & 215.826 & Surface & 2011 \\
\hline 3675.999 & 224.894 & Surface & 2011 & 9053.939 & 215.828 & Surface & 2011 \\
\hline 3678.924 & 224.887 & Surface & 2011 & 9056.833 & 215.825 & Surface & 2011 \\
\hline 3681.848 & 224.875 & Surface & 2011 & 9059.727 & 215.814 & Surface & 2011 \\
\hline
\end{tabular}




\begin{tabular}{|c|c|c|c|c|c|c|c|}
\hline 3684.772 & 224.878 & Surface & 2011 & 9062.621 & 215.779 & Surface & 2011 \\
\hline 3687.697 & 224.920 & Surface & 2011 & 9065.516 & 215.779 & Surface & 2011 \\
\hline 3690.621 & 224.920 & Surface & 2011 & 9068.410 & 215.777 & Surface & 2011 \\
\hline 3693.546 & 224.879 & Surface & 2011 & 9071.304 & 215.778 & Surface & 2011 \\
\hline 3696.470 & 224.897 & Surface & 2011 & 9074.198 & 215.769 & Surface & 2011 \\
\hline 3699.394 & 224.907 & Surface & 2011 & 9077.075 & 215.744 & Surface & 2011 \\
\hline 3702.319 & 224.911 & Surface & 2011 & 9079.952 & 215.732 & Surface & 2011 \\
\hline 3705.243 & 224.916 & Surface & 2011 & 9082.830 & 215.731 & Surface & 2011 \\
\hline 3708.167 & 224.922 & Surface & 2011 & 9085.707 & 215.724 & Surface & 2011 \\
\hline 3711.092 & 224.887 & Surface & 2011 & 9088.585 & 215.718 & Surface & 2011 \\
\hline 3713.947 & 224.916 & Surface & 2011 & 9091.462 & 215.711 & Surface & 2011 \\
\hline 3716.802 & 224.920 & Surface & 2011 & 9094.339 & 215.688 & Surface & 2011 \\
\hline 3719.658 & 224.921 & Surface & 2011 & 9097.216 & 215.670 & Surface & 2011 \\
\hline 3722.513 & 224.897 & Surface & 2011 & 9100.094 & 215.659 & Surface & 2011 \\
\hline 3725.368 & 224.874 & Surface & 2011 & 9102.971 & 215.646 & Surface & 2011 \\
\hline 3728.223 & 224.874 & Surface & 2011 & 9105.848 & 215.623 & Surface & 2011 \\
\hline 3731.078 & 224.900 & Surface & 2011 & 9108.725 & 215.599 & Surface & 2011 \\
\hline 3733.933 & 224.894 & Surface & 2011 & 9111.603 & 215.582 & Surface & 2011 \\
\hline 3736.789 & 224.890 & Surface & 2011 & 9114.480 & 215.565 & Surface & 2011 \\
\hline 3739.644 & 224.871 & Surface & 2011 & 9117.357 & 215.539 & Surface & 2011 \\
\hline 3742.499 & 224.871 & Surface & 2011 & 9120.235 & 215.509 & Surface & 2011 \\
\hline 3745.354 & 224.911 & Surface & 2011 & 9123.112 & 215.472 & Surface & 2011 \\
\hline 3748.210 & 224.909 & Surface & 2011 & 9125.989 & 215.436 & Surface & 2011 \\
\hline 3751.065 & 224.896 & Surface & 2011 & 9128.866 & 215.399 & Surface & 2011 \\
\hline 3753.920 & 224.896 & Surface & 2011 & 9131.744 & 215.357 & Surface & 2011 \\
\hline 3756.775 & 224.918 & Surface & 2011 & 9134.621 & 215.313 & Surface & 2011 \\
\hline 3759.630 & 224.918 & Surface & 2011 & 9137.498 & 215.278 & Surface & 2011 \\
\hline 3762.485 & 224.901 & Surface & 2011 & 9140.375 & 215.297 & Surface & 2011 \\
\hline 3765.341 & 224.925 & Surface & 2011 & 9143.336 & 215.320 & Surface & 2011 \\
\hline 3768.196 & 224.936 & Surface & 2011 & 9146.296 & 215.337 & Surface & 2011 \\
\hline 3771.156 & 224.913 & Surface & 2011 & 9149.256 & 215.358 & Surface & 2011 \\
\hline 3774.116 & 224.875 & Surface & 2011 & 9152.217 & 215.377 & Surface & 2011 \\
\hline 3777.076 & 224.860 & Surface & 2011 & 9155.177 & 215.392 & Surface & 2011 \\
\hline 3780.036 & 224.876 & Surface & 2011 & 9158.137 & 215.405 & Surface & 2011 \\
\hline 3782.996 & 224.912 & Surface & 2011 & 9161.097 & 215.413 & Surface & 2011 \\
\hline 3785.956 & 224.932 & Surface & 2011 & 9164.058 & 215.435 & Surface & 2011 \\
\hline 3788.916 & 224.928 & Surface & 2011 & 9167.018 & 215.440 & Surface & 2011 \\
\hline 3791.876 & 224.921 & Surface & 2011 & 9169.978 & 215.404 & Surface & 2011 \\
\hline 3794.836 & 224.925 & Surface & 2011 & 9172.938 & 215.371 & Surface & 2011 \\
\hline 3797.796 & 224.923 & Surface & 2011 & 9175.898 & 215.384 & Surface & 2011 \\
\hline 3800.756 & 224.910 & Surface & 2011 & 9178.859 & 215.402 & Surface & 2011 \\
\hline 3803.716 & 224.913 & Surface & 2011 & 9181.819 & 215.391 & Surface & 2011 \\
\hline 3806.676 & 224.925 & Surface & 2011 & 9184.779 & 215.397 & Surface & 2011 \\
\hline 3809.636 & 224.912 & Surface & 2011 & 9187.739 & 215.386 & Surface & 2011 \\
\hline 3812.596 & 224.901 & Surface & 2011 & 9190.700 & 215.368 & Surface & 2011 \\
\hline 3815.556 & 224.902 & Surface & 2011 & 9193.660 & 215.348 & Surface & 2011 \\
\hline 3818.516 & 224.898 & Surface & 2011 & 9196.620 & 215.330 & Surface & 2011 \\
\hline 3821.476 & 224.893 & Surface & 2011 & 9199.580 & 215.318 & Surface & 2011 \\
\hline 3824.379 & 224.887 & Surface & 2011 & 9202.541 & 215.317 & Surface & 2011 \\
\hline
\end{tabular}




\begin{tabular}{|c|c|c|c|c|c|c|c|}
\hline 3827.281 & 224.894 & Surface & 2011 & 9205.501 & 215.333 & Surface & 2011 \\
\hline 3830.184 & 224.902 & Surface & 2011 & 9208.461 & 215.336 & Surface & 2011 \\
\hline 3833.087 & 224.912 & Surface & 2011 & 9211.442 & 215.337 & Surface & 2011 \\
\hline 3835.989 & 224.909 & Surface & 2011 & 9214.423 & 215.333 & Surface & 2011 \\
\hline 3838.892 & 224.920 & Surface & 2011 & 9217.404 & 215.265 & Surface & 2011 \\
\hline 3841.795 & 224.923 & Surface & 2011 & 9220.384 & 215.244 & Surface & 2011 \\
\hline 3844.698 & 224.913 & Surface & 2011 & 9223.365 & 215.241 & Surface & 2011 \\
\hline 3847.600 & 224.908 & Surface & 2011 & 9226.346 & 215.221 & Surface & 2011 \\
\hline 3850.503 & 224.903 & Surface & 2011 & 9229.327 & 215.186 & Surface & 2011 \\
\hline 3853.406 & 224.886 & Surface & 2011 & 9232.307 & 215.164 & Surface & 2011 \\
\hline 3856.308 & 224.866 & Surface & 2011 & 9235.288 & 215.157 & Surface & 2011 \\
\hline 3859.211 & 224.880 & Surface & 2011 & 9238.269 & 215.125 & Surface & 2011 \\
\hline 3862.114 & 224.908 & Surface & 2011 & 9241.250 & 215.089 & Surface & 2011 \\
\hline 3865.017 & 224.910 & Surface & 2011 & 9244.231 & 215.062 & Surface & 2011 \\
\hline 3867.919 & 224.903 & Surface & 2011 & 9247.211 & 215.039 & Surface & 2011 \\
\hline 3870.822 & 224.889 & Surface & 2011 & 9250.192 & 215.003 & Surface & 2011 \\
\hline 3873.725 & 224.916 & Surface & 2011 & 9253.173 & 214.975 & Surface & 2011 \\
\hline 3876.627 & 224.910 & Surface & 2011 & 9256.154 & 214.970 & Surface & 2011 \\
\hline 3879.500 & 224.907 & Surface & 2011 & 9259.135 & 214.965 & Surface & 2011 \\
\hline 3882.373 & 224.921 & Surface & 2011 & 9262.115 & 214.970 & Surface & 2011 \\
\hline 3885.246 & 224.929 & Surface & 2011 & 9265.096 & 214.986 & Surface & 2011 \\
\hline 3888.119 & 224.934 & Surface & 2011 & 9268.077 & 214.989 & Surface & 2011 \\
\hline 3890.992 & 224.936 & Surface & 2011 & 9271.058 & 214.941 & Surface & 2011 \\
\hline 3893.865 & 224.934 & Surface & 2011 & 9274.051 & 214.896 & Surface & 2011 \\
\hline 3896.738 & 224.925 & Surface & 2011 & 9277.044 & 214.883 & Surface & 2011 \\
\hline 3899.611 & 224.901 & Surface & 2011 & 9280.037 & 214.882 & Surface & 2011 \\
\hline 3902.484 & 224.879 & Surface & 2011 & 9283.030 & 214.881 & Surface & 2011 \\
\hline 3905.357 & 224.883 & Surface & 2011 & 9286.023 & 214.877 & Surface & 2011 \\
\hline 3908.229 & 224.895 & Surface & 2011 & 9289.016 & 214.876 & Surface & 2011 \\
\hline 3911.103 & 224.918 & Surface & 2011 & 9292.009 & 214.873 & Surface & 2011 \\
\hline 3913.975 & 224.936 & Surface & 2011 & 9295.003 & 214.872 & Surface & 2011 \\
\hline 3916.848 & 224.936 & Surface & 2011 & 9297.996 & 214.869 & Surface & 2011 \\
\hline 3919.721 & 224.930 & Surface & 2011 & 9300.989 & 214.859 & Surface & 2011 \\
\hline 3922.594 & 224.924 & Surface & 2011 & 9303.982 & 214.853 & Surface & 2011 \\
\hline 3925.467 & 224.926 & Surface & 2011 & 9306.975 & 214.876 & Surface & 2011 \\
\hline 3928.340 & 224.912 & Surface & 2011 & 9309.968 & 214.897 & Surface & 2011 \\
\hline 3931.213 & 224.904 & Surface & 2011 & 9312.961 & 214.865 & Surface & 2011 \\
\hline 3934.086 & 224.901 & Surface & 2011 & 9315.954 & 214.847 & Surface & 2011 \\
\hline 3937.083 & 224.887 & Surface & 2011 & 9318.947 & 214.845 & Surface & 2011 \\
\hline 3940.081 & 224.913 & Surface & 2011 & 9321.941 & 214.836 & Surface & 2011 \\
\hline 3943.078 & 224.928 & Surface & 2011 & 9324.934 & 214.837 & Surface & 2011 \\
\hline 3946.076 & 224.917 & Surface & 2011 & 9327.927 & 214.829 & Surface & 2011 \\
\hline 3949.073 & 224.909 & Surface & 2011 & 9330.733 & 214.837 & Surface & 2011 \\
\hline 3952.070 & 224.913 & Surface & 2011 & 9333.539 & 214.840 & Surface & 2011 \\
\hline 3955.068 & 224.919 & Surface & 2011 & 9336.345 & 214.837 & Surface & 2011 \\
\hline 3958.065 & 224.923 & Surface & 2011 & 9339.151 & 214.831 & Surface & 2011 \\
\hline 3961.063 & 224.917 & Surface & 2011 & 9341.957 & 214.834 & Surface & 2011 \\
\hline 3964.060 & 224.909 & Surface & 2011 & 9344.763 & 214.830 & Surface & 2011 \\
\hline 3967.058 & 224.901 & Surface & 2011 & 9347.569 & 214.828 & Surface & 2011 \\
\hline
\end{tabular}




\begin{tabular}{|c|c|c|c|c|c|c|c|}
\hline 3970.055 & 224.882 & Surface & 2011 & 9350.375 & 214.831 & Surface & 2011 \\
\hline 3973.052 & 224.873 & Surface & 2011 & 9353.181 & 214.832 & Surface & 2011 \\
\hline 3976.050 & 224.871 & Surface & 2011 & 9355.986 & 214.833 & Surface & 2011 \\
\hline 3979.047 & 224.876 & Surface & 2011 & 9358.793 & 214.836 & Surface & 2011 \\
\hline 3982.045 & 224.860 & Surface & 2011 & 9361.599 & 214.844 & Surface & 2011 \\
\hline 3985.042 & 224.817 & Surface & 2011 & 9364.404 & 214.857 & Surface & 2011 \\
\hline 3988.039 & 224.799 & Surface & 2011 & 9367.211 & 214.869 & Surface & 2011 \\
\hline 3991.037 & 224.810 & Surface & 2011 & 9370.103 & 214.866 & Surface & 2011 \\
\hline 3994.034 & 224.814 & Surface & 2011 & 9372.995 & 214.845 & Surface & 2011 \\
\hline 3997.032 & 224.815 & Surface & 2011 & 9375.887 & 214.830 & Surface & 2011 \\
\hline 4000.029 & 224.838 & Surface & 2011 & 9378.779 & 214.876 & Surface & 2011 \\
\hline 4003.027 & 224.850 & Surface & 2011 & 9381.671 & 214.904 & Surface & 2011 \\
\hline 4006.024 & 224.856 & Surface & 2011 & 9384.563 & 214.904 & Surface & 2011 \\
\hline 4009.021 & 224.855 & Surface & 2011 & 9387.455 & 214.891 & Surface & 2011 \\
\hline 4012.019 & 224.867 & Surface & 2011 & 9390.347 & 214.865 & Surface & 2011 \\
\hline 4015.016 & 224.872 & Surface & 2011 & 9393.240 & 214.868 & Surface & 2011 \\
\hline 4018.014 & 224.872 & Surface & 2011 & 9396.132 & 214.896 & Surface & 2011 \\
\hline 4021.011 & 224.862 & Surface & 2011 & 9399.024 & 214.905 & Surface & 2011 \\
\hline 4024.008 & 224.870 & Surface & 2011 & 9401.916 & 214.883 & Surface & 2011 \\
\hline 4027.006 & 224.872 & Surface & 2011 & 9404.808 & 214.829 & Surface & 2011 \\
\hline 4030.003 & 224.872 & Surface & 2011 & 9407.700 & 214.873 & Surface & 2011 \\
\hline 4033.001 & 224.864 & Surface & 2011 & 9410.592 & 214.920 & Surface & 2011 \\
\hline 4035.998 & 224.855 & Surface & 2011 & 9413.484 & 214.928 & Surface & 2011 \\
\hline 4038.996 & 224.862 & Surface & 2011 & 9416.377 & 214.934 & Surface & 2011 \\
\hline 4041.993 & 224.875 & Surface & 2011 & 9419.310 & 214.916 & Surface & 2011 \\
\hline 4044.990 & 224.886 & Surface & 2011 & 9422.243 & 214.914 & Surface & 2011 \\
\hline 4047.988 & 224.882 & Surface & 2011 & 9425.177 & 214.919 & Surface & 2011 \\
\hline 4050.985 & 224.871 & Surface & 2011 & 9428.110 & 214.929 & Surface & 2011 \\
\hline 4053.983 & 224.857 & Surface & 2011 & 9431.044 & 214.935 & Surface & 2011 \\
\hline 4056.980 & 224.858 & Surface & 2011 & 9433.977 & 214.911 & Surface & 2011 \\
\hline 4059.975 & 224.856 & Surface & 2011 & 9436.910 & 214.886 & Surface & 2011 \\
\hline 4062.970 & 224.852 & Surface & 2011 & 9439.844 & 214.894 & Surface & 2011 \\
\hline 4065.964 & 224.850 & Surface & 2011 & 9442.777 & 214.870 & Surface & 2011 \\
\hline 4068.959 & 224.869 & Surface & 2011 & 9445.711 & 214.848 & Surface & 2011 \\
\hline 4071.954 & 224.882 & Surface & 2011 & 9448.644 & 214.873 & Surface & 2011 \\
\hline 4074.949 & 224.887 & Surface & 2011 & 9451.578 & 214.895 & Surface & 2011 \\
\hline 4077.944 & 224.886 & Surface & 2011 & 9454.511 & 214.861 & Surface & 2011 \\
\hline 4080.938 & 224.879 & Surface & 2011 & 9457.445 & 214.877 & Surface & 2011 \\
\hline 4083.933 & 224.868 & Surface & 2011 & 9460.378 & 214.900 & Surface & 2011 \\
\hline 4086.928 & 224.858 & Surface & 2011 & 9463.312 & 214.905 & Surface & 2011 \\
\hline 4089.923 & 224.844 & Surface & 2011 & 9466.245 & 214.923 & Surface & 2011 \\
\hline 4092.918 & 224.832 & Surface & 2011 & 9469.178 & 214.951 & Surface & 2011 \\
\hline 4095.912 & 224.843 & Surface & 2011 & 9472.104 & 214.956 & Surface & 2011 \\
\hline 4098.907 & 224.856 & Surface & 2011 & 9475.029 & 214.920 & Surface & 2011 \\
\hline 4101.902 & 224.861 & Surface & 2011 & 9477.954 & 214.916 & Surface & 2011 \\
\hline 4104.897 & 224.865 & Surface & 2011 & 9480.879 & 214.916 & Surface & 2011 \\
\hline 4107.892 & 224.847 & Surface & 2011 & 9483.804 & 214.918 & Surface & 2011 \\
\hline 4110.887 & 224.823 & Surface & 2011 & 9486.730 & 214.918 & Surface & 2011 \\
\hline 4113.881 & 224.823 & Surface & 2011 & 9489.655 & 214.918 & Surface & 2011 \\
\hline
\end{tabular}




\begin{tabular}{|c|c|c|c|c|c|c|c|}
\hline 4116.876 & 224.849 & Surface & 2011 & 9492.580 & 214.924 & Surface & 2011 \\
\hline 4119.871 & 224.854 & Surface & 2011 & 9495.505 & 214.927 & Surface & 2011 \\
\hline 4122.866 & 224.854 & Surface & 2011 & 9498.431 & 214.932 & Surface & 2011 \\
\hline 4125.860 & 224.839 & Surface & 2011 & 9501.356 & 214.936 & Surface & 2011 \\
\hline 4128.855 & 224.834 & Surface & 2011 & 9504.281 & 214.941 & Surface & 2011 \\
\hline 4131.850 & 224.832 & Surface & 2011 & 9507.206 & 214.947 & Surface & 2011 \\
\hline 4134.845 & 224.819 & Surface & 2011 & 9510.132 & 214.951 & Surface & 2011 \\
\hline 4137.840 & 224.810 & Surface & 2011 & 9513.057 & 214.954 & Surface & 2011 \\
\hline 4140.835 & 224.816 & Surface & 2011 & 9515.982 & 214.949 & Surface & 2011 \\
\hline 4143.829 & 224.810 & Surface & 2011 & 9518.907 & 214.935 & Surface & 2011 \\
\hline 4146.824 & 224.789 & Surface & 2011 & 9521.832 & 214.922 & Surface & 2011 \\
\hline 4149.819 & 224.792 & Surface & 2011 & 9524.758 & 214.909 & Surface & 2011 \\
\hline 4152.785 & 224.803 & Surface & 2011 & 9527.683 & 214.905 & Surface & 2011 \\
\hline 4155.751 & 224.815 & Surface & 2011 & 9530.608 & 214.907 & Surface & 2011 \\
\hline 4158.717 & 224.824 & Surface & 2011 & 9533.553 & 214.908 & Surface & 2011 \\
\hline 4161.683 & 224.806 & Surface & 2011 & 9536.497 & 214.905 & Surface & 2011 \\
\hline 4164.649 & 224.779 & Surface & 2011 & 9539.441 & 214.903 & Surface & 2011 \\
\hline 4167.616 & 224.757 & Surface & 2011 & 9542.386 & 214.901 & Surface & 2011 \\
\hline 4170.582 & 224.751 & Surface & 2011 & 9545.331 & 214.902 & Surface & 2011 \\
\hline 4173.548 & 224.747 & Surface & 2011 & 9548.275 & 214.903 & Surface & 2011 \\
\hline 4176.514 & 224.768 & Surface & 2011 & 9551.219 & 214.905 & Surface & 2011 \\
\hline 4179.480 & 224.769 & Surface & 2011 & 9554.164 & 214.906 & Surface & 2011 \\
\hline 4182.446 & 224.720 & Surface & 2011 & 9557.108 & 214.911 & Surface & 2011 \\
\hline 4185.412 & 224.675 & Surface & 2011 & 9560.053 & 214.925 & Surface & 2011 \\
\hline 4188.378 & 224.645 & Surface & 2011 & 9562.998 & 214.936 & Surface & 2011 \\
\hline 4191.344 & 224.636 & Surface & 2011 & 9565.942 & 214.939 & Surface & 2011 \\
\hline 4194.310 & 224.667 & Surface & 2011 & 9568.886 & 214.932 & Surface & 2011 \\
\hline 4197.277 & 224.688 & Surface & 2011 & 9571.831 & 214.917 & Surface & 2011 \\
\hline 4200.243 & 224.672 & Surface & 2011 & 9574.776 & 214.890 & Surface & 2011 \\
\hline 4203.209 & 224.624 & Surface & 2011 & 9577.720 & 214.865 & Surface & 2011 \\
\hline 4206.175 & 224.600 & Surface & 2011 & 9580.665 & 214.875 & Surface & 2011 \\
\hline 4209.141 & 224.588 & Surface & 2011 & 9583.609 & 214.902 & Surface & 2011 \\
\hline 4212.107 & 224.612 & Surface & 2011 & 9586.535 & 214.904 & Surface & 2011 \\
\hline 4215.073 & 224.638 & Surface & 2011 & 9589.460 & 214.876 & Surface & 2011 \\
\hline 4218.039 & 224.646 & Surface & 2011 & 9592.385 & 214.853 & Surface & 2011 \\
\hline 4221.005 & 224.641 & Surface & 2011 & 9595.311 & 214.845 & Surface & 2011 \\
\hline 4223.972 & 224.620 & Surface & 2011 & 9598.236 & 214.835 & Surface & 2011 \\
\hline 4226.938 & 224.609 & Surface & 2011 & 9601.162 & 214.836 & Surface & 2011 \\
\hline 4229.904 & 224.607 & Surface & 2011 & 9604.087 & 214.832 & Surface & 2011 \\
\hline 4232.870 & 224.605 & Surface & 2011 & 9607.012 & 214.828 & Surface & 2011 \\
\hline 4235.836 & 224.608 & Surface & 2011 & 9609.938 & 214.818 & Surface & 2011 \\
\hline 4238.802 & 224.607 & Surface & 2011 & 9612.863 & 214.811 & Surface & 2011 \\
\hline 4241.768 & 224.573 & Surface & 2011 & 9615.789 & 214.799 & Surface & 2011 \\
\hline 4244.734 & 224.529 & Surface & 2011 & 9618.714 & 214.814 & Surface & 2011 \\
\hline 4247.700 & 224.546 & Surface & 2011 & 9621.640 & 214.836 & Surface & 2011 \\
\hline 4250.666 & 224.564 & Surface & 2011 & 9624.565 & 214.864 & Surface & 2011 \\
\hline 4253.633 & 224.563 & Surface & 2011 & 9627.490 & 214.866 & Surface & 2011 \\
\hline 4256.599 & 224.546 & Surface & 2011 & 9630.416 & 214.861 & Surface & 2011 \\
\hline 4259.565 & 224.541 & Surface & 2011 & 9633.341 & 214.859 & Surface & 2011 \\
\hline
\end{tabular}




\begin{tabular}{|c|c|c|c|c|c|c|c|}
\hline 4262.531 & 224.547 & Surface & 2011 & 9636.266 & 214.886 & Surface & 2011 \\
\hline 4265.497 & 224.546 & Surface & 2011 & 9639.192 & 214.911 & Surface & 2011 \\
\hline 4268.463 & 224.513 & Surface & 2011 & 9642.046 & 214.916 & Surface & 2011 \\
\hline 4271.429 & 224.506 & Surface & 2011 & 9644.901 & 214.906 & Surface & 2011 \\
\hline 4274.377 & 224.518 & Surface & 2011 & 9647.756 & 214.913 & Surface & 2011 \\
\hline 4277.325 & 224.514 & Surface & 2011 & 9650.610 & 214.914 & Surface & 2011 \\
\hline 4280.272 & 224.477 & Surface & 2011 & 9653.465 & 214.903 & Surface & 2011 \\
\hline 4283.220 & 224.468 & Surface & 2011 & 9656.320 & 214.873 & Surface & 2011 \\
\hline 4286.168 & 224.502 & Surface & 2011 & 9659.174 & 214.857 & Surface & 2011 \\
\hline 4289.116 & 224.496 & Surface & 2011 & 9662.029 & 214.853 & Surface & 2011 \\
\hline 4292.064 & 224.464 & Surface & 2011 & 9664.883 & 214.843 & Surface & 2011 \\
\hline 4295.011 & 224.434 & Surface & 2011 & 9667.738 & 214.840 & Surface & 2011 \\
\hline 4297.959 & 224.430 & Surface & 2011 & 9670.593 & 214.810 & Surface & 2011 \\
\hline 4300.907 & 224.423 & Surface & 2011 & 9673.447 & 214.729 & Surface & 2011 \\
\hline 4303.855 & 224.401 & Surface & 2011 & 9676.302 & 214.656 & Surface & 2011 \\
\hline 4306.802 & 224.335 & Surface & 2011 & 9679.157 & 214.577 & Surface & 2011 \\
\hline 4309.750 & 224.309 & Surface & 2011 & 9682.011 & 214.491 & Surface & 2011 \\
\hline 4312.698 & 224.315 & Surface & 2011 & 9684.866 & 214.429 & Surface & 2011 \\
\hline 4315.646 & 224.318 & Surface & 2011 & 9687.721 & 214.379 & Surface & 2011 \\
\hline 4318.593 & 224.296 & Surface & 2011 & 9690.575 & 214.338 & Surface & 2011 \\
\hline 4321.541 & 224.243 & Surface & 2011 & 9693.430 & 214.325 & Surface & 2011 \\
\hline 4324.489 & 224.226 & Surface & 2011 & 9696.346 & 214.332 & Surface & 2011 \\
\hline 4327.437 & 224.249 & Surface & 2011 & 9699.263 & 214.334 & Surface & 2011 \\
\hline 4330.384 & 224.279 & Surface & 2011 & 9702.180 & 214.329 & Surface & 2011 \\
\hline 4333.332 & 224.299 & Surface & 2011 & 9705.097 & 214.337 & Surface & 2011 \\
\hline 4336.280 & 224.306 & Surface & 2011 & 9708.013 & 214.341 & Surface & 2011 \\
\hline 4339.227 & 224.310 & Surface & 2011 & 9710.930 & 214.331 & Surface & 2011 \\
\hline 4342.175 & 224.290 & Surface & 2011 & 9713.847 & 214.326 & Surface & 2011 \\
\hline 4345.123 & 224.271 & Surface & 2011 & 9716.763 & 214.307 & Surface & 2011 \\
\hline 4348.071 & 224.262 & Surface & 2011 & 9719.680 & 214.353 & Surface & 2011 \\
\hline 4351.019 & 224.267 & Surface & 2011 & 9722.597 & 214.356 & Surface & 2011 \\
\hline 4353.966 & 224.273 & Surface & 2011 & 9725.513 & 214.355 & Surface & 2011 \\
\hline 4356.914 & 224.278 & Surface & 2011 & 9728.430 & 214.356 & Surface & 2011 \\
\hline 4359.862 & 224.287 & Surface & 2011 & 9731.347 & 214.354 & Surface & 2011 \\
\hline 4362.809 & 224.307 & Surface & 2011 & 9734.264 & 214.341 & Surface & 2011 \\
\hline 4365.757 & 224.300 & Surface & 2011 & 9737.180 & 214.336 & Surface & 2011 \\
\hline 4368.705 & 224.299 & Surface & 2011 & 9740.097 & 214.339 & Surface & 2011 \\
\hline 4371.653 & 224.304 & Surface & 2011 & 9743.014 & 214.306 & Surface & 2011 \\
\hline 4374.601 & 224.285 & Surface & 2011 & 9745.930 & 214.303 & Surface & 2011 \\
\hline 4377.548 & 224.258 & Surface & 2011 & 9748.847 & 214.326 & Surface & 2011 \\
\hline 4380.496 & 224.258 & Surface & 2011 & 9751.764 & 214.344 & Surface & 2011 \\
\hline 4383.444 & 224.272 & Surface & 2011 & 9754.611 & 214.342 & Surface & 2011 \\
\hline 4386.392 & 224.276 & Surface & 2011 & 9757.458 & 214.324 & Surface & 2011 \\
\hline 4389.339 & 224.264 & Surface & 2011 & 9760.304 & 214.313 & Surface & 2011 \\
\hline 4392.287 & 224.280 & Surface & 2011 & 9763.151 & 214.328 & Surface & 2011 \\
\hline 4395.235 & 224.299 & Surface & 2011 & 9765.998 & 214.330 & Surface & 2011 \\
\hline 4398.183 & 224.295 & Surface & 2011 & 9768.845 & 214.347 & Surface & 2011 \\
\hline 4401.131 & 224.270 & Surface & 2011 & 9771.692 & 214.352 & Surface & 2011 \\
\hline 4404.078 & 224.297 & Surface & 2011 & 9774.539 & 214.337 & Surface & 2011 \\
\hline
\end{tabular}




\begin{tabular}{|c|c|c|c|c|c|c|c|}
\hline 4407.026 & 224.296 & Surface & 2011 & 9777.386 & 214.332 & Surface & 2011 \\
\hline 4409.974 & 224.281 & Surface & 2011 & 9780.232 & 214.336 & Surface & 2011 \\
\hline 4412.935 & 224.275 & Surface & 2011 & 9783.079 & 214.348 & Surface & 2011 \\
\hline 4415.897 & 224.286 & Surface & 2011 & 9785.926 & 214.359 & Surface & 2011 \\
\hline 4418.859 & 224.278 & Surface & 2011 & 9788.773 & 214.364 & Surface & 2011 \\
\hline 4421.821 & 224.266 & Surface & 2011 & 9791.620 & 214.368 & Surface & 2011 \\
\hline 4424.783 & 224.267 & Surface & 2011 & 9794.467 & 214.362 & Surface & 2011 \\
\hline 4427.745 & 224.262 & Surface & 2011 & 9797.255 & 214.335 & Surface & 2011 \\
\hline 4430.706 & 224.265 & Surface & 2011 & 9800.044 & 214.315 & Surface & 2011 \\
\hline 4433.668 & 224.265 & Surface & 2011 & 9802.832 & 214.324 & Surface & 2011 \\
\hline 4436.630 & 224.261 & Surface & 2011 & 9805.621 & 214.321 & Surface & 2011 \\
\hline 4439.592 & 224.251 & Surface & 2011 & 9808.410 & 214.323 & Surface & 2011 \\
\hline 4442.553 & 224.261 & Surface & 2011 & 9811.198 & 214.346 & Surface & 2011 \\
\hline 4445.515 & 224.272 & Surface & 2011 & 9813.987 & 214.376 & Surface & 2011 \\
\hline 4448.477 & 224.284 & Surface & 2011 & 9816.775 & 214.383 & Surface & 2011 \\
\hline 4451.439 & 224.282 & Surface & 2011 & 9819.564 & 214.359 & Surface & 2011 \\
\hline 4454.401 & 224.259 & Surface & 2011 & 9822.352 & 214.319 & Surface & 2011 \\
\hline 4457.362 & 224.276 & Surface & 2011 & 9825.141 & 214.342 & Surface & 2011 \\
\hline 4460.324 & 224.268 & Surface & 2011 & 9827.929 & 214.337 & Surface & 2011 \\
\hline 4463.286 & 224.286 & Surface & 2011 & 9830.718 & 214.320 & Surface & 2011 \\
\hline 4466.248 & 224.301 & Surface & 2011 & 9833.615 & 214.306 & Surface & 2011 \\
\hline 4469.210 & 224.296 & Surface & 2011 & 9836.512 & 214.328 & Surface & 2011 \\
\hline 4472.172 & 224.275 & Surface & 2011 & 9839.409 & 214.354 & Surface & 2011 \\
\hline 4475.133 & 224.267 & Surface & 2011 & 9842.306 & 214.363 & Surface & 2011 \\
\hline 4478.095 & 224.260 & Surface & 2011 & 9845.203 & 214.358 & Surface & 2011 \\
\hline 4481.057 & 224.284 & Surface & 2011 & 9848.101 & 214.356 & Surface & 2011 \\
\hline 4484.019 & 224.287 & Surface & 2011 & 9850.998 & 214.361 & Surface & 2011 \\
\hline 4486.981 & 224.274 & Surface & 2011 & 9853.895 & 214.369 & Surface & 2011 \\
\hline 4489.942 & 224.269 & Surface & 2011 & 9856.792 & 214.371 & Surface & 2011 \\
\hline 4492.904 & 224.255 & Surface & 2011 & 9859.689 & 214.363 & Surface & 2011 \\
\hline 4495.866 & 224.256 & Surface & 2011 & 9862.586 & 214.362 & Surface & 2011 \\
\hline 4498.828 & 224.263 & Surface & 2011 & 9865.483 & 214.363 & Surface & 2011 \\
\hline 4501.790 & 224.271 & Surface & 2011 & 9868.381 & 214.348 & Surface & 2011 \\
\hline 4504.751 & 224.272 & Surface & 2011 & 9871.278 & 214.319 & Surface & 2011 \\
\hline 4507.713 & 224.261 & Surface & 2011 & 9874.175 & 214.328 & Surface & 2011 \\
\hline 4510.675 & 224.270 & Surface & 2011 & 9877.072 & 214.347 & Surface & 2011 \\
\hline 4513.637 & 224.277 & Surface & 2011 & 9879.969 & 214.359 & Surface & 2011 \\
\hline 4516.599 & 224.272 & Surface & 2011 & 9882.866 & 214.354 & Surface & 2011 \\
\hline 4519.560 & 224.262 & Surface & 2011 & 9885.763 & 214.350 & Surface & 2011 \\
\hline 4522.522 & 224.261 & Surface & 2011 & 9888.660 & 214.324 & Surface & 2011 \\
\hline 4525.484 & 224.268 & Surface & 2011 & 9891.558 & 214.336 & Surface & 2011 \\
\hline 4528.446 & 224.277 & Surface & 2011 & 9894.455 & 214.342 & Surface & 2011 \\
\hline 4531.408 & 224.269 & Surface & 2011 & 9897.352 & 214.347 & Surface & 2011 \\
\hline 4534.369 & 224.264 & Surface & 2011 & 9900.249 & 214.330 & Surface & 2011 \\
\hline 4537.331 & 224.256 & Surface & 2011 & 9903.179 & 214.337 & Surface & 2011 \\
\hline 4540.293 & 224.269 & Surface & 2011 & 9906.108 & 214.350 & Surface & 2011 \\
\hline 4543.255 & 224.267 & Surface & 2011 & 9909.038 & 214.382 & Surface & 2011 \\
\hline 4546.217 & 224.268 & Surface & 2011 & 9911.968 & 214.385 & Surface & 2011 \\
\hline 4549.178 & 224.261 & Surface & 2011 & 9914.897 & 214.360 & Surface & 2011 \\
\hline
\end{tabular}




\begin{tabular}{|c|c|c|c|c|c|c|c|}
\hline 4552.140 & 224.251 & Surface & 2011 & 9917.827 & 214.340 & Surface & 2011 \\
\hline 4555.102 & 224.237 & Surface & 2011 & 9920.757 & 214.334 & Surface & 2011 \\
\hline 4558.064 & 224.226 & Surface & 2011 & 9923.686 & 214.317 & Surface & 2011 \\
\hline 4561.025 & 224.234 & Surface & 2011 & 9926.616 & 214.294 & Surface & 2011 \\
\hline 4563.987 & 224.240 & Surface & 2011 & 9929.546 & 214.280 & Surface & 2011 \\
\hline 4566.949 & 224.226 & Surface & 2011 & 9932.476 & 214.298 & Surface & 2011 \\
\hline 4569.911 & 224.220 & Surface & 2011 & 9935.405 & 214.320 & Surface & 2011 \\
\hline 4572.873 & 224.216 & Surface & 2011 & 9938.335 & 214.332 & Surface & 2011 \\
\hline 4575.835 & 224.184 & Surface & 2011 & 9941.265 & 214.351 & Surface & 2011 \\
\hline 4578.796 & 224.159 & Surface & 2011 & 9944.194 & 214.363 & Surface & 2011 \\
\hline 4581.707 & 224.147 & Surface & 2011 & 9947.124 & 214.355 & Surface & 2011 \\
\hline 4584.617 & 224.168 & Surface & 2011 & 9950.010 & 214.351 & Surface & 2011 \\
\hline 4587.527 & 224.191 & Surface & 2011 & 9952.895 & 214.354 & Surface & 2011 \\
\hline 4590.438 & 224.214 & Surface & 2011 & 9955.781 & 214.353 & Surface & 2011 \\
\hline 4593.348 & 224.218 & Surface & 2011 & 9958.666 & 214.350 & Surface & 2011 \\
\hline 4596.258 & 224.213 & Surface & 2011 & 9961.552 & 214.347 & Surface & 2011 \\
\hline 4599.168 & 224.213 & Surface & 2011 & 9964.438 & 214.357 & Surface & 2011 \\
\hline 4602.079 & 224.211 & Surface & 2011 & 9967.323 & 214.372 & Surface & 2011 \\
\hline 4604.989 & 224.205 & Surface & 2011 & 9970.209 & 214.368 & Surface & 2011 \\
\hline 4607.899 & 224.204 & Surface & 2011 & 9973.094 & 214.360 & Surface & 2011 \\
\hline 4610.810 & 224.201 & Surface & 2011 & 9975.980 & 214.355 & Surface & 2011 \\
\hline 4613.720 & 224.192 & Surface & 2011 & 9978.865 & 214.353 & Surface & 2011 \\
\hline 4616.630 & 224.182 & Surface & 2011 & 9981.751 & 214.354 & Surface & 2011 \\
\hline 4619.541 & 224.180 & Surface & 2011 & 9984.636 & 214.372 & Surface & 2011 \\
\hline 4622.451 & 224.193 & Surface & 2011 & 9987.522 & 214.348 & Surface & 2011 \\
\hline 4625.361 & 224.209 & Surface & 2011 & 9990.407 & 214.329 & Surface & 2011 \\
\hline 4628.272 & 224.223 & Surface & 2011 & 9993.293 & 214.318 & Surface & 2011 \\
\hline 4631.182 & 224.215 & Surface & 2011 & 9996.178 & 214.311 & Surface & 2011 \\
\hline 4634.092 & 224.206 & Surface & 2011 & 9999.064 & 214.318 & Surface & 2011 \\
\hline 4637.002 & 224.181 & Surface & 2011 & 10001.950 & 214.332 & Surface & 2011 \\
\hline 4639.913 & 224.177 & Surface & 2011 & 10004.835 & 214.345 & Surface & 2011 \\
\hline 4642.823 & 224.191 & Surface & 2011 & 10007.704 & 214.366 & Surface & 2011 \\
\hline 4645.734 & 224.197 & Surface & 2011 & 10010.574 & 214.371 & Surface & 2011 \\
\hline 4648.644 & 224.190 & Surface & 2011 & 10013.443 & 214.360 & Surface & 2011 \\
\hline 4651.554 & 224.174 & Surface & 2011 & 10016.312 & 214.368 & Surface & 2011 \\
\hline 4654.464 & 224.160 & Surface & 2011 & 10019.181 & 214.389 & Surface & 2011 \\
\hline 4657.375 & 224.155 & Surface & 2011 & 10022.050 & 214.388 & Surface & 2011 \\
\hline 4660.285 & 224.151 & Surface & 2011 & 10024.920 & 214.350 & Surface & 2011 \\
\hline 4663.195 & 224.155 & Surface & 2011 & 10027.789 & 214.328 & Surface & 2011 \\
\hline 4666.106 & 224.158 & Surface & 2011 & 10030.658 & 214.344 & Surface & 2011 \\
\hline 4669.016 & 224.164 & Surface & 2011 & 10033.527 & 214.368 & Surface & 2011 \\
\hline 4671.926 & 224.170 & Surface & 2011 & 10036.397 & 214.387 & Surface & 2011 \\
\hline 4674.854 & 224.138 & Surface & 2011 & 10039.266 & 214.386 & Surface & 2011 \\
\hline 4677.783 & 224.122 & Surface & 2011 & 10042.135 & 214.376 & Surface & 2011 \\
\hline 4680.711 & 224.124 & Surface & 2011 & 10045.004 & 214.375 & Surface & 2011 \\
\hline 4683.640 & 224.128 & Surface & 2011 & 10047.873 & 214.379 & Surface & 2011 \\
\hline 4686.568 & 224.126 & Surface & 2011 & 10050.743 & 214.401 & Surface & 2011 \\
\hline 4689.496 & 224.115 & Surface & 2011 & 10053.612 & 214.421 & Surface & 2011 \\
\hline 4692.425 & 224.106 & Surface & 2011 & 10056.481 & 214.425 & Surface & 2011 \\
\hline
\end{tabular}




\begin{tabular}{|c|c|c|c|c|c|c|c|}
\hline 4695.353 & 224.118 & Surface & 2011 & 10059.350 & 214.422 & Surface & 2011 \\
\hline 4698.281 & 224.122 & Surface & 2011 & 10062.327 & 214.419 & Surface & 2011 \\
\hline 4701.209 & 224.114 & Surface & 2011 & 10065.304 & 214.414 & Surface & 2011 \\
\hline 4704.138 & 224.099 & Surface & 2011 & 10068.282 & 214.410 & Surface & 2011 \\
\hline 4707.066 & 224.083 & Surface & 2011 & 10071.259 & 214.405 & Surface & 2011 \\
\hline 4709.994 & 224.074 & Surface & 2011 & 10074.236 & 214.400 & Surface & 2011 \\
\hline 4712.923 & 224.089 & Surface & 2011 & 10077.213 & 214.395 & Surface & 2011 \\
\hline 4715.851 & 224.086 & Surface & 2011 & 10080.190 & 214.397 & Surface & 2011 \\
\hline 4718.779 & 224.060 & Surface & 2011 & 10083.167 & 214.397 & Surface & 2011 \\
\hline 4721.708 & 224.040 & Surface & 2011 & 10086.145 & 214.398 & Surface & 2011 \\
\hline 4724.636 & 224.047 & Surface & 2011 & 10089.122 & 214.398 & Surface & 2011 \\
\hline 4727.564 & 224.056 & Surface & 2011 & 10092.099 & 214.399 & Surface & 2011 \\
\hline 4730.493 & 224.053 & Surface & 2011 & 10095.076 & 214.399 & Surface & 2011 \\
\hline 4733.421 & 224.036 & Surface & 2011 & 10098.053 & 214.399 & Surface & 2011 \\
\hline 4736.349 & 224.009 & Surface & 2011 & 10101.030 & 214.399 & Surface & 2011 \\
\hline 4739.278 & 223.993 & Surface & 2011 & 10104.008 & 214.399 & Surface & 2011 \\
\hline 4742.206 & 223.985 & Surface & 2011 & 10106.985 & 214.399 & Surface & 2011 \\
\hline 4745.134 & 223.978 & Surface & 2011 & 10109.962 & 214.400 & Surface & 2011 \\
\hline 4748.063 & 223.961 & Surface & 2011 & 10112.939 & 214.400 & Surface & 2011 \\
\hline 4750.991 & 223.983 & Surface & 2011 & 10115.916 & 214.401 & Surface & 2011 \\
\hline 4753.919 & 223.988 & Surface & 2011 & 10118.893 & 214.401 & Surface & 2011 \\
\hline 4756.847 & 223.976 & Surface & 2011 & 10121.871 & 214.401 & Surface & 2011 \\
\hline 4759.776 & 223.972 & Surface & 2011 & 10124.848 & 214.402 & Surface & 2011 \\
\hline 4762.704 & 223.960 & Surface & 2011 & 10127.825 & 214.402 & Surface & 2011 \\
\hline 4765.632 & 223.891 & Surface & 2011 & 10130.802 & 214.401 & Surface & 2011 \\
\hline 4768.561 & 223.852 & Surface & 2011 & 10133.779 & 214.401 & Surface & 2011 \\
\hline 4771.489 & 223.847 & Surface & 2011 & 10136.756 & 214.395 & Surface & 2011 \\
\hline 4774.417 & 223.849 & Surface & 2011 & 10139.733 & 214.390 & Surface & 2011 \\
\hline 4777.346 & 223.853 & Surface & 2011 & 10142.711 & 214.387 & Surface & 2011 \\
\hline 4780.274 & 223.858 & Surface & 2011 & 10145.688 & 214.388 & Surface & 2011 \\
\hline 4783.199 & 223.854 & Surface & 2011 & 10148.665 & 214.389 & Surface & 2011 \\
\hline 4786.123 & 223.836 & Surface & 2011 & 10151.642 & 214.390 & Surface & 2011 \\
\hline 4789.048 & 223.835 & Surface & 2011 & 10154.642 & 214.382 & Surface & 2011 \\
\hline 4791.973 & 223.838 & Surface & 2011 & 10157.642 & 214.375 & Surface & 2011 \\
\hline 4794.898 & 223.837 & Surface & 2011 & 10160.642 & 214.366 & Surface & 2011 \\
\hline 4797.823 & 223.838 & Surface & 2011 & 10163.642 & 214.353 & Surface & 2011 \\
\hline 4800.748 & 223.831 & Surface & 2011 & 10166.642 & 214.344 & Surface & 2011 \\
\hline 4803.672 & 223.815 & Surface & 2011 & 10169.642 & 214.355 & Surface & 2011 \\
\hline 4806.597 & 223.829 & Surface & 2011 & 10172.642 & 214.346 & Surface & 2011 \\
\hline 4809.522 & 223.831 & Surface & 2011 & 10175.642 & 214.329 & Surface & 2011 \\
\hline 4812.447 & 223.821 & Surface & 2011 & 10178.642 & 214.330 & Surface & 2011 \\
\hline 4815.372 & 223.766 & Surface & 2011 & 10181.642 & 214.319 & Surface & 2011 \\
\hline 4818.296 & 223.696 & Surface & 2011 & 10184.642 & 214.306 & Surface & 2011 \\
\hline 4821.221 & 223.732 & Surface & 2011 & 10187.642 & 214.321 & Surface & 2011 \\
\hline 4824.146 & 223.748 & Surface & 2011 & 10190.642 & 214.325 & Surface & 2011 \\
\hline 4827.071 & 223.751 & Surface & 2011 & 10193.642 & 214.314 & Surface & 2011 \\
\hline 4829.995 & 223.734 & Surface & 2011 & 10196.642 & 214.304 & Surface & 2011 \\
\hline 4832.920 & 223.717 & Surface & 2011 & 10199.642 & 214.298 & Surface & 2011 \\
\hline 4835.845 & 223.701 & Surface & 2011 & 10202.642 & 214.292 & Surface & 2011 \\
\hline
\end{tabular}




\begin{tabular}{|c|c|c|c|c|c|c|c|}
\hline 4838.770 & 223.712 & Surface & 2011 & 10205.642 & 214.288 & Surface & 2011 \\
\hline 4841.695 & 223.670 & Surface & 2011 & 10208.642 & 214.288 & Surface & 2011 \\
\hline 4844.619 & 223.629 & Surface & 2011 & 10211.642 & 214.295 & Surface & 2011 \\
\hline 4847.544 & 223.622 & Surface & 2011 & 10214.642 & 214.308 & Surface & 2011 \\
\hline 4850.469 & 223.644 & Surface & 2011 & 10217.642 & 214.310 & Surface & 2011 \\
\hline 4853.394 & 223.656 & Surface & 2011 & 10220.642 & 214.303 & Surface & 2011 \\
\hline 4856.319 & 223.635 & Surface & 2011 & 10223.642 & 214.287 & Surface & 2011 \\
\hline 4859.243 & 223.590 & Surface & 2011 & 10226.642 & 214.286 & Surface & 2011 \\
\hline 4862.168 & 223.576 & Surface & 2011 & 10229.642 & 214.289 & Surface & 2011 \\
\hline 4865.093 & 223.571 & Surface & 2011 & 10232.642 & 214.262 & Surface & 2011 \\
\hline 4868.018 & 223.549 & Surface & 2011 & 10235.642 & 214.271 & Surface & 2011 \\
\hline 4870.924 & 223.517 & Surface & 2011 & 10238.553 & 214.271 & Surface & 2011 \\
\hline 4873.830 & 223.524 & Surface & 2011 & 10241.464 & 214.266 & Surface & 2011 \\
\hline 4876.736 & 223.545 & Surface & 2011 & 10244.375 & 214.267 & Surface & 2011 \\
\hline 4879.643 & 223.536 & Surface & 2011 & 10247.287 & 214.274 & Surface & 2011 \\
\hline 4882.549 & 223.513 & Surface & 2011 & 10250.198 & 214.279 & Surface & 2011 \\
\hline 4885.455 & 223.484 & Surface & 2011 & 10253.110 & 214.269 & Surface & 2011 \\
\hline 4888.362 & 223.458 & Surface & 2011 & 10256.021 & 214.259 & Surface & 2011 \\
\hline 4891.268 & 223.447 & Surface & 2011 & 10258.932 & 214.244 & Surface & 2011 \\
\hline 4894.174 & 223.426 & Surface & 2011 & 10261.843 & 214.218 & Surface & 2011 \\
\hline 4897.080 & 223.410 & Surface & 2011 & 10264.755 & 214.220 & Surface & 2011 \\
\hline 4899.986 & 223.411 & Surface & 2011 & 10267.666 & 214.235 & Surface & 2011 \\
\hline 4902.893 & 223.416 & Surface & 2011 & 10270.577 & 214.245 & Surface & 2011 \\
\hline 4905.799 & 223.409 & Surface & 2011 & 10273.488 & 214.227 & Surface & 2011 \\
\hline 4908.705 & 223.367 & Surface & 2011 & 10276.399 & 214.200 & Surface & 2011 \\
\hline 4911.611 & 223.350 & Surface & 2011 & 10279.311 & 214.225 & Surface & 2011 \\
\hline 4914.518 & 223.330 & Surface & 2011 & 10282.222 & 214.203 & Surface & 2011 \\
\hline 4917.424 & 223.303 & Surface & 2011 & 10285.134 & 214.178 & Surface & 2011 \\
\hline 4920.330 & 223.276 & Surface & 2011 & 10288.045 & 214.182 & Surface & 2011 \\
\hline 4923.236 & 223.248 & Surface & 2011 & 10290.956 & 214.205 & Surface & 2011 \\
\hline 4926.142 & 223.233 & Surface & 2011 & 10293.867 & 214.210 & Surface & 2011 \\
\hline 4929.049 & 223.219 & Surface & 2011 & 10296.779 & 214.205 & Surface & 2011 \\
\hline 4931.955 & 223.195 & Surface & 2011 & 10299.690 & 214.194 & Surface & 2011 \\
\hline 4934.861 & 223.177 & Surface & 2011 & 10302.580 & 214.174 & Surface & 2011 \\
\hline 4937.767 & 223.172 & Surface & 2011 & 10305.471 & 214.141 & Surface & 2011 \\
\hline 4940.673 & 223.161 & Surface & 2011 & 10308.361 & 214.131 & Surface & 2011 \\
\hline 4943.580 & 223.170 & Surface & 2011 & 10311.251 & 214.109 & Surface & 2011 \\
\hline 4946.486 & 223.171 & Surface & 2011 & 10314.142 & 214.079 & Surface & 2011 \\
\hline 4949.392 & 223.167 & Surface & 2011 & 10317.032 & 214.052 & Surface & 2011 \\
\hline 4952.298 & 223.171 & Surface & 2011 & 10319.922 & 214.042 & Surface & 2011 \\
\hline 4955.205 & 223.176 & Surface & 2011 & 10322.812 & 214.041 & Surface & 2011 \\
\hline 4958.095 & 223.176 & Surface & 2011 & 10325.703 & 214.038 & Surface & 2011 \\
\hline 4960.984 & 223.179 & Surface & 2011 & 10328.593 & 214.002 & Surface & 2011 \\
\hline 4963.874 & 223.153 & Surface & 2011 & 10331.483 & 213.945 & Surface & 2011 \\
\hline 4966.764 & 223.130 & Surface & 2011 & 10334.373 & 213.914 & Surface & 2011 \\
\hline 4969.654 & 223.125 & Surface & 2011 & 10337.264 & 213.909 & Surface & 2011 \\
\hline 4972.544 & 223.122 & Surface & 2011 & 10340.154 & 213.912 & Surface & 2011 \\
\hline 4975.434 & 223.111 & Surface & 2011 & 10343.045 & 213.917 & Surface & 2011 \\
\hline 4978.324 & 223.098 & Surface & 2011 & 10345.935 & 213.907 & Surface & 2011 \\
\hline
\end{tabular}




\begin{tabular}{|c|c|c|c|c|c|c|c|}
\hline 4981.214 & 223.072 & Surface & 2011 & 10348.825 & 213.885 & Surface & 2011 \\
\hline 4984.104 & 223.062 & Surface & 2011 & 10351.715 & 213.859 & Surface & 2011 \\
\hline 4986.994 & 223.069 & Surface & 2011 & 10354.620 & 213.836 & Surface & 2011 \\
\hline 4989.884 & 223.081 & Surface & 2011 & 10357.525 & 213.797 & Surface & 2011 \\
\hline 4992.773 & 223.085 & Surface & 2011 & 10360.430 & 213.770 & Surface & 2011 \\
\hline 4995.663 & 223.062 & Surface & 2011 & 10363.335 & 213.733 & Surface & 2011 \\
\hline 4998.553 & 223.032 & Surface & 2011 & 10366.240 & 213.710 & Surface & 2011 \\
\hline 5001.443 & 223.014 & Surface & 2011 & 10369.145 & 213.693 & Surface & 2011 \\
\hline 5004.333 & 223.050 & Surface & 2011 & 10372.050 & 213.677 & Surface & 2011 \\
\hline 5007.223 & 223.058 & Surface & 2011 & 10374.954 & 213.660 & Surface & 2011 \\
\hline 5010.113 & 223.044 & Surface & 2011 & 10377.859 & 213.626 & Surface & 2011 \\
\hline 5013.003 & 223.031 & Surface & 2011 & 10380.764 & 213.603 & Surface & 2011 \\
\hline 5015.893 & 223.019 & Surface & 2011 & 10383.669 & 213.571 & Surface & 2011 \\
\hline 5018.878 & 223.010 & Surface & 2011 & 10386.574 & 213.562 & Surface & 2011 \\
\hline 5021.864 & 223.020 & Surface & 2011 & 10389.479 & 213.548 & Surface & 2011 \\
\hline 5024.849 & 223.056 & Surface & 2011 & 10392.383 & 213.537 & Surface & 2011 \\
\hline 5027.835 & 223.067 & Surface & 2011 & 10395.289 & 213.538 & Surface & 2011 \\
\hline 5030.820 & 223.039 & Surface & 2011 & 10398.193 & 213.548 & Surface & 2011 \\
\hline 5033.806 & 223.011 & Surface & 2011 & 10401.098 & 213.549 & Surface & 2011 \\
\hline 5036.791 & 222.997 & Surface & 2011 & 10404.003 & 213.537 & Surface & 2011 \\
\hline 5039.777 & 223.027 & Surface & 2011 & 10406.908 & 213.544 & Surface & 2011 \\
\hline 5042.762 & 223.013 & Surface & 2011 & 10409.813 & 213.553 & Surface & 2011 \\
\hline 5045.748 & 222.985 & Surface & 2011 & 10412.718 & 213.537 & Surface & 2011 \\
\hline 5048.733 & 222.991 & Surface & 2011 & 10415.622 & 213.531 & Surface & 2011 \\
\hline 5051.719 & 223.001 & Surface & 2011 & 10418.527 & 213.515 & Surface & 2011 \\
\hline 5054.704 & 223.009 & Surface & 2011 & 10421.433 & 213.499 & Surface & 2011 \\
\hline 5057.690 & 223.016 & Surface & 2011 & 10424.338 & 213.492 & Surface & 2011 \\
\hline 5060.675 & 223.036 & Surface & 2011 & 10427.243 & 213.493 & Surface & 2011 \\
\hline 5063.661 & 223.027 & Surface & 2011 & 10430.148 & 213.485 & Surface & 2011 \\
\hline 5066.646 & 223.002 & Surface & 2011 & 10433.054 & 213.457 & Surface & 2011 \\
\hline 5069.632 & 222.962 & Surface & 2011 & 10435.959 & 213.433 & Surface & 2011 \\
\hline 5072.617 & 222.978 & Surface & 2011 & 10438.864 & 213.389 & Surface & 2011 \\
\hline 5075.603 & 222.978 & Surface & 2011 & 10441.769 & 213.342 & Surface & 2011 \\
\hline 5078.588 & 222.951 & Surface & 2011 & 10444.675 & 213.303 & Surface & 2011 \\
\hline 5081.573 & 222.957 & Surface & 2011 & 10447.580 & 213.251 & Surface & 2011 \\
\hline 5084.559 & 222.969 & Surface & 2011 & 10450.485 & 213.213 & Surface & 2011 \\
\hline 5087.545 & 222.967 & Surface & 2011 & 10453.390 & 213.209 & Surface & 2011 \\
\hline 5090.530 & 222.960 & Surface & 2011 & 10456.296 & 213.215 & Surface & 2011 \\
\hline 5093.516 & 222.933 & Surface & 2011 & 10459.201 & 213.207 & Surface & 2011 \\
\hline 5096.501 & 222.911 & Surface & 2011 & 10462.106 & 213.191 & Surface & 2011 \\
\hline 5099.486 & 222.930 & Surface & 2011 & 10465.012 & 213.114 & Surface & 2011 \\
\hline 5102.472 & 222.933 & Surface & 2011 & 10467.917 & 213.029 & Surface & 2011 \\
\hline 5105.319 & 222.928 & Surface & 2011 & 10470.822 & 212.946 & Surface & 2011 \\
\hline 5108.165 & 222.924 & Surface & 2011 & 10473.727 & 212.938 & Surface & 2011 \\
\hline 5111.011 & 222.931 & Surface & 2011 & 10476.633 & 212.915 & Surface & 2011 \\
\hline 5113.858 & 222.933 & Surface & 2011 & 10479.538 & 212.897 & Surface & 2011 \\
\hline 5116.704 & 222.927 & Surface & 2011 & 10482.443 & 212.878 & Surface & 2011 \\
\hline 5119.550 & 222.921 & Surface & 2011 & 10485.348 & 212.857 & Surface & 2011 \\
\hline 5122.396 & 222.904 & Surface & 2011 & 10488.254 & 212.838 & Surface & 2011 \\
\hline
\end{tabular}




\begin{tabular}{|c|c|c|c|c|c|c|c|}
\hline 5125.243 & 222.899 & Surface & 2011 & 10491.201 & 212.803 & Surface & 2011 \\
\hline 5128.089 & 222.881 & Surface & 2011 & 10494.148 & 212.752 & Surface & 2011 \\
\hline 5130.936 & 222.900 & Surface & 2011 & 10497.096 & 212.707 & Surface & 2011 \\
\hline 5133.782 & 222.911 & Surface & 2011 & 10500.044 & 212.643 & Surface & 2011 \\
\hline 5136.628 & 222.922 & Surface & 2011 & 10502.991 & 212.589 & Surface & 2011 \\
\hline 5139.475 & 222.911 & Surface & 2011 & 10505.938 & 212.575 & Surface & 2011 \\
\hline 5142.321 & 222.878 & Surface & 2011 & 10508.886 & 212.547 & Surface & 2011 \\
\hline 5145.167 & 222.869 & Surface & 2011 & 10511.833 & 212.564 & Surface & 2011 \\
\hline 5148.014 & 222.893 & Surface & 2011 & 10514.781 & 212.581 & Surface & 2011 \\
\hline 5150.860 & 222.895 & Surface & 2011 & 10517.728 & 212.579 & Surface & 2011 \\
\hline 5153.706 & 222.887 & Surface & 2011 & 10520.676 & 212.551 & Surface & 2011 \\
\hline 5156.647 & 222.879 & Surface & 2011 & 10523.623 & 212.533 & Surface & 2011 \\
\hline 5159.587 & 222.890 & Surface & 2011 & 10526.570 & 212.510 & Surface & 2011 \\
\hline 5162.528 & 222.898 & Surface & 2011 & 10529.518 & 212.483 & Surface & 2011 \\
\hline 5165.468 & 222.905 & Surface & 2011 & 10532.466 & 212.441 & Surface & 2011 \\
\hline 5168.409 & 222.898 & Surface & 2011 & 10535.413 & 212.398 & Surface & 2011 \\
\hline 5171.349 & 222.896 & Surface & 2011 & 10538.361 & 212.354 & Surface & 2011 \\
\hline 5174.289 & 222.900 & Surface & 2011 & 10541.249 & 212.339 & Surface & 2011 \\
\hline 5177.230 & 222.893 & Surface & 2011 & 10544.137 & 212.321 & Surface & 2011 \\
\hline 5180.170 & 222.874 & Surface & 2011 & 10547.026 & 212.277 & Surface & 2011 \\
\hline 5183.111 & 222.861 & Surface & 2011 & 10549.915 & 212.224 & Surface & 2011 \\
\hline 5186.051 & 222.867 & Surface & 2011 & 10552.803 & 212.183 & Surface & 2011 \\
\hline 5188.992 & 222.857 & Surface & 2011 & 10555.692 & 212.182 & Surface & 2011 \\
\hline 5191.932 & 222.896 & Surface & 2011 & 10558.580 & 212.208 & Surface & 2011 \\
\hline 5194.873 & 222.903 & Surface & 2011 & 10561.469 & 212.208 & Surface & 2011 \\
\hline 5197.813 & 222.892 & Surface & 2011 & 10564.357 & 212.214 & Surface & 2011 \\
\hline 5200.753 & 222.891 & Surface & 2011 & 10567.246 & 212.200 & Surface & 2011 \\
\hline 5203.694 & 222.897 & Surface & 2011 & 10570.134 & 212.160 & Surface & 2011 \\
\hline 5206.634 & 222.901 & Surface & 2011 & 10573.023 & 212.173 & Surface & 2011 \\
\hline 5209.575 & 222.905 & Surface & 2011 & 10575.911 & 212.157 & Surface & 2011 \\
\hline 5212.515 & 222.899 & Surface & 2011 & 10578.800 & 212.141 & Surface & 2011 \\
\hline 5215.456 & 222.892 & Surface & 2011 & 10581.688 & 212.084 & Surface & 2011 \\
\hline 5218.333 & 222.901 & Surface & 2011 & 10584.577 & 212.067 & Surface & 2011 \\
\hline 5221.209 & 222.905 & Surface & 2011 & 10587.465 & 212.037 & Surface & 2011 \\
\hline 5224.086 & 222.901 & Surface & 2011 & 10590.351 & 212.013 & Surface & 2011 \\
\hline 5226.963 & 222.895 & Surface & 2011 & 10593.237 & 212.014 & Surface & 2011 \\
\hline 5229.840 & 222.889 & Surface & 2011 & 10596.122 & 212.027 & Surface & 2011 \\
\hline 5232.718 & 222.890 & Surface & 2011 & 10599.007 & 212.042 & Surface & 2011 \\
\hline 5235.594 & 222.923 & Surface & 2011 & 10601.893 & 212.047 & Surface & 2011 \\
\hline 5238.471 & 222.913 & Surface & 2011 & 10604.779 & 212.019 & Surface & 2011 \\
\hline 5241.348 & 222.898 & Surface & 2011 & 10607.664 & 211.996 & Surface & 2011 \\
\hline 5244.225 & 222.892 & Surface & 2011 & 10610.550 & 211.995 & Surface & 2011 \\
\hline 5247.102 & 222.903 & Surface & 2011 & 10613.435 & 211.991 & Surface & 2011 \\
\hline 5249.979 & 222.910 & Surface & 2011 & 10616.321 & 211.962 & Surface & 2011 \\
\hline 5252.856 & 222.920 & Surface & 2011 & 10619.206 & 211.932 & Surface & 2011 \\
\hline 5255.733 & 222.920 & Surface & 2011 & 10622.092 & 211.943 & Surface & 2011 \\
\hline 5258.610 & 222.903 & Surface & 2011 & 10624.977 & 211.994 & Surface & 2011 \\
\hline 5261.487 & 222.893 & Surface & 2011 & 10627.863 & 212.045 & Surface & 2011 \\
\hline 5264.364 & 222.895 & Surface & 2011 & 10630.749 & 212.040 & Surface & 2011 \\
\hline
\end{tabular}




\begin{tabular}{|c|c|c|c|c|c|c|c|}
\hline 5267.241 & 222.896 & Surface & 2011 & 10633.634 & 212.029 & Surface & 2011 \\
\hline 5270.118 & 222.890 & Surface & 2011 & 10636.520 & 212.013 & Surface & 2011 \\
\hline 5272.995 & 222.882 & Surface & 2011 & 10639.405 & 211.995 & Surface & 2011 \\
\hline 5275.872 & 222.887 & Surface & 2011 & 10642.291 & 211.965 & Surface & 2011 \\
\hline 5278.749 & 222.894 & Surface & 2011 & 10645.176 & 211.939 & Surface & 2011 \\
\hline 5281.626 & 222.897 & Surface & 2011 & 10648.171 & 211.961 & Surface & 2011 \\
\hline 5284.602 & 222.900 & Surface & 2011 & 10651.165 & 211.969 & Surface & 2011 \\
\hline 5287.578 & 222.881 & Surface & 2011 & 10654.160 & 211.975 & Surface & 2011 \\
\hline 5290.554 & 222.876 & Surface & 2011 & 10657.154 & 211.970 & Surface & 2011 \\
\hline 5293.530 & 222.878 & Surface & 2011 & 10660.149 & 211.942 & Surface & 2011 \\
\hline 5296.505 & 222.875 & Surface & 2011 & 10663.143 & 211.918 & Surface & 2011 \\
\hline 5299.481 & 222.863 & Surface & 2011 & 10666.138 & 211.911 & Surface & 2011 \\
\hline 5302.457 & 222.848 & Surface & 2011 & 10669.132 & 211.913 & Surface & 2011 \\
\hline 5305.433 & 222.834 & Surface & 2011 & 10672.127 & 211.901 & Surface & 2011 \\
\hline 5308.409 & 222.831 & Surface & 2011 & 10675.121 & 211.922 & Surface & 2011 \\
\hline 5311.385 & 222.845 & Surface & 2011 & 10678.116 & 211.931 & Surface & 2011 \\
\hline 5314.361 & 222.869 & Surface & 2011 & 10681.110 & 211.877 & Surface & 2011 \\
\hline 5317.337 & 222.879 & Surface & 2011 & 10684.105 & 211.815 & Surface & 2011 \\
\hline 5320.313 & 222.892 & Surface & 2011 & 10687.099 & 211.818 & Surface & 2011 \\
\hline 5323.289 & 222.900 & Surface & 2011 & 10690.094 & 211.828 & Surface & 2011 \\
\hline 5326.265 & 222.886 & Surface & 2011 & 10693.088 & 211.836 & Surface & 2011 \\
\hline 5329.240 & 222.876 & Surface & 2011 & 10696.083 & 211.840 & Surface & 2011 \\
\hline 5332.216 & 222.887 & Surface & 2011 & 10699.077 & 211.843 & Surface & 2011 \\
\hline 5335.192 & 222.888 & Surface & 2011 & 10702.072 & 211.859 & Surface & 2011 \\
\hline 5338.168 & 222.869 & Surface & 2011 & 10705.067 & 211.871 & Surface & 2011 \\
\hline 5341.144 & 222.846 & Surface & 2011 & 10708.061 & 211.866 & Surface & 2011 \\
\hline 5344.120 & 222.812 & Surface & 2011 & 10710.644 & 211.862 & Surface & 2011 \\
\hline 5347.096 & 222.798 & Surface & 2011 & 10713.228 & 211.860 & Surface & 2011 \\
\hline 5350.072 & 222.782 & Surface & 2011 & 10715.811 & 211.851 & Surface & 2011 \\
\hline 5353.048 & 222.769 & Surface & 2011 & 10718.394 & 211.829 & Surface & 2011 \\
\hline
\end{tabular}


11.4 Stream Gradient - 2012 Lidar:

\begin{tabular}{|c|c|c|c|c|c|c|c|}
\hline Distance & $\mathrm{Z}$ & DEM & Year & Distance & $\mathrm{Z}$ & DEM & Year \\
\hline 0.000 & 233.208 & Surface & 2012 & 5336.472 & 222.577 & Surface & 2012 \\
\hline 3.183 & 233.195 & Surface & 2012 & 5339.502 & 222.587 & Surface & 2012 \\
\hline 6.366 & 233.191 & Surface & 2012 & 5342.531 & 222.571 & Surface & 2012 \\
\hline 9.548 & 233.187 & Surface & 2012 & 5345.561 & 222.565 & Surface & 2012 \\
\hline 12.731 & 233.179 & Surface & 2012 & 5348.590 & 222.566 & Surface & 2012 \\
\hline 15.914 & 233.185 & Surface & 2012 & 5351.620 & 222.552 & Surface & 2012 \\
\hline 19.096 & 233.190 & Surface & 2012 & 5354.650 & 222.519 & Surface & 2012 \\
\hline 22.279 & 233.150 & Surface & 2012 & 5357.680 & 222.502 & Surface & 2012 \\
\hline 25.462 & 233.157 & Surface & 2012 & 5360.710 & 222.514 & Surface & 2012 \\
\hline 28.645 & 233.165 & Surface & 2012 & 5363.739 & 222.510 & Surface & 2012 \\
\hline 31.827 & 233.163 & Surface & 2012 & 5366.769 & 222.515 & Surface & 2012 \\
\hline 35.010 & 233.160 & Surface & 2012 & 5369.798 & 222.521 & Surface & 2012 \\
\hline 38.193 & 233.141 & Surface & 2012 & 5372.828 & 222.510 & Surface & 2012 \\
\hline 41.375 & 233.117 & Surface & 2012 & 5375.963 & 222.489 & Surface & 2012 \\
\hline 44.558 & 233.117 & Surface & 2012 & 5379.098 & 222.474 & Surface & 2012 \\
\hline 47.741 & 233.121 & Surface & 2012 & 5382.232 & 222.461 & Surface & 2012 \\
\hline 50.924 & 233.116 & Surface & 2012 & 5385.367 & 222.459 & Surface & 2012 \\
\hline 54.107 & 233.109 & Surface & 2012 & 5388.501 & 222.452 & Surface & 2012 \\
\hline 57.343 & 233.110 & Surface & 2012 & 5391.636 & 222.446 & Surface & 2012 \\
\hline 60.579 & 233.110 & Surface & 2012 & 5394.771 & 222.414 & Surface & 2012 \\
\hline 63.815 & 233.103 & Surface & 2012 & 5397.905 & 222.395 & Surface & 2012 \\
\hline 67.051 & 233.083 & Surface & 2012 & 5401.040 & 222.402 & Surface & 2012 \\
\hline 70.287 & 233.087 & Surface & 2012 & 5404.175 & 222.387 & Surface & 2012 \\
\hline 73.523 & 233.115 & Surface & 2012 & 5407.309 & 222.375 & Surface & 2012 \\
\hline 76.759 & 233.099 & Surface & 2012 & 5410.444 & 222.386 & Surface & 2012 \\
\hline 79.995 & 233.073 & Surface & 2012 & 5413.579 & 222.392 & Surface & 2012 \\
\hline 83.231 & 233.080 & Surface & 2012 & 5416.770 & 222.380 & Surface & 2012 \\
\hline 86.467 & 233.067 & Surface & 2012 & 5419.961 & 222.395 & Surface & 2012 \\
\hline 89.703 & 233.079 & Surface & 2012 & 5423.152 & 222.382 & Surface & 2012 \\
\hline 92.940 & 233.082 & Surface & 2012 & 5426.343 & 222.374 & Surface & 2012 \\
\hline 96.176 & 233.084 & Surface & 2012 & 5429.534 & 222.393 & Surface & 2012 \\
\hline 99.412 & 233.068 & Surface & 2012 & 5432.725 & 222.364 & Surface & 2012 \\
\hline 102.648 & 233.082 & Surface & 2012 & 5435.917 & 222.369 & Surface & 2012 \\
\hline 105.884 & 233.104 & Surface & 2012 & 5439.108 & 222.400 & Surface & 2012 \\
\hline 109.120 & 233.076 & Surface & 2012 & 5442.299 & 222.382 & Surface & 2012 \\
\hline 112.356 & 233.052 & Surface & 2012 & 5445.490 & 222.390 & Surface & 2012 \\
\hline 115.592 & 233.046 & Surface & 2012 & 5448.681 & 222.393 & Surface & 2012 \\
\hline 118.828 & 233.065 & Surface & 2012 & 5451.872 & 222.391 & Surface & 2012 \\
\hline 122.064 & 233.073 & Surface & 2012 & 5455.063 & 222.390 & Surface & 2012 \\
\hline 125.300 & 233.060 & Surface & 2012 & 5458.254 & 222.369 & Surface & 2012 \\
\hline 128.537 & 233.047 & Surface & 2012 & 5461.445 & 222.381 & Surface & 2012 \\
\hline 131.773 & 233.022 & Surface & 2012 & 5464.637 & 222.382 & Surface & 2012 \\
\hline 135.009 & 233.005 & Surface & 2012 & 5467.828 & 222.374 & Surface & 2012 \\
\hline 138.245 & 232.996 & Surface & 2012 & 5471.029 & 222.382 & Surface & 2012 \\
\hline 141.481 & 233.002 & Surface & 2012 & 5474.230 & 222.360 & Surface & 2012 \\
\hline
\end{tabular}




\begin{tabular}{|c|c|c|c|c|c|c|c|}
\hline 144.717 & 232.990 & Surface & 2012 & 5477.432 & 222.368 & Surface & 2012 \\
\hline 147.953 & 232.970 & Surface & 2012 & 5480.634 & 222.367 & Surface & 2012 \\
\hline 151.189 & 232.970 & Surface & 2012 & 5483.835 & 222.376 & Surface & 2012 \\
\hline 154.425 & 232.989 & Surface & 2012 & 5487.036 & 222.363 & Surface & 2012 \\
\hline 157.661 & 232.970 & Surface & 2012 & 5490.238 & 222.337 & Surface & 2012 \\
\hline 160.735 & 232.962 & Surface & 2012 & 5493.439 & 222.353 & Surface & 2012 \\
\hline 163.809 & 232.956 & Surface & 2012 & 5496.641 & 222.350 & Surface & 2012 \\
\hline 166.883 & 232.938 & Surface & 2012 & 5499.842 & 222.352 & Surface & 2012 \\
\hline 169.956 & 232.923 & Surface & 2012 & 5503.044 & 222.346 & Surface & 2012 \\
\hline 173.030 & 232.916 & Surface & 2012 & 5506.245 & 222.341 & Surface & 2012 \\
\hline 176.104 & 232.910 & Surface & 2012 & 5509.446 & 222.320 & Surface & 2012 \\
\hline 179.177 & 232.928 & Surface & 2012 & 5512.648 & 222.335 & Surface & 2012 \\
\hline 182.251 & 232.938 & Surface & 2012 & 5515.849 & 222.341 & Surface & 2012 \\
\hline 185.325 & 232.929 & Surface & 2012 & 5519.051 & 222.336 & Surface & 2012 \\
\hline 188.398 & 232.915 & Surface & 2012 & 5522.252 & 222.324 & Surface & 2012 \\
\hline 191.472 & 232.936 & Surface & 2012 & 5525.454 & 222.314 & Surface & 2012 \\
\hline 194.604 & 232.927 & Surface & 2012 & 5528.655 & 222.317 & Surface & 2012 \\
\hline 197.734 & 232.946 & Surface & 2012 & 5531.857 & 222.304 & Surface & 2012 \\
\hline 200.866 & 232.940 & Surface & 2012 & 5535.028 & 222.298 & Surface & 2012 \\
\hline 203.997 & 232.927 & Surface & 2012 & 5538.199 & 222.295 & Surface & 2012 \\
\hline 207.128 & 232.939 & Surface & 2012 & 5541.370 & 222.295 & Surface & 2012 \\
\hline 210.259 & 232.956 & Surface & 2012 & 5544.541 & 222.288 & Surface & 2012 \\
\hline 213.390 & 232.932 & Surface & 2012 & 5547.712 & 222.280 & Surface & 2012 \\
\hline 216.522 & 232.928 & Surface & 2012 & 5550.883 & 222.266 & Surface & 2012 \\
\hline 219.653 & 232.915 & Surface & 2012 & 5554.055 & 222.273 & Surface & 2012 \\
\hline 222.784 & 232.917 & Surface & 2012 & 5557.226 & 222.279 & Surface & 2012 \\
\hline 225.915 & 232.932 & Surface & 2012 & 5560.397 & 222.274 & Surface & 2012 \\
\hline 229.046 & 232.910 & Surface & 2012 & 5563.568 & 222.267 & Surface & 2012 \\
\hline 232.177 & 232.906 & Surface & 2012 & 5566.739 & 222.250 & Surface & 2012 \\
\hline 235.406 & 232.891 & Surface & 2012 & 5569.911 & 222.239 & Surface & 2012 \\
\hline 238.635 & 232.896 & Surface & 2012 & 5573.082 & 222.231 & Surface & 2012 \\
\hline 241.864 & 232.896 & Surface & 2012 & 5576.253 & 222.221 & Surface & 2012 \\
\hline 245.093 & 232.879 & Surface & 2012 & 5579.499 & 222.194 & Surface & 2012 \\
\hline 248.322 & 232.875 & Surface & 2012 & 5582.745 & 222.216 & Surface & 2012 \\
\hline 251.551 & 232.855 & Surface & 2012 & 5585.991 & 222.217 & Surface & 2012 \\
\hline 254.780 & 232.848 & Surface & 2012 & 5589.238 & 222.216 & Surface & 2012 \\
\hline 258.009 & 232.835 & Surface & 2012 & 5592.483 & 222.209 & Surface & 2012 \\
\hline 261.238 & 232.832 & Surface & 2012 & 5595.730 & 222.207 & Surface & 2012 \\
\hline 264.468 & 232.831 & Surface & 2012 & 5598.976 & 222.191 & Surface & 2012 \\
\hline 267.697 & 232.835 & Surface & 2012 & 5602.222 & 222.197 & Surface & 2012 \\
\hline 270.926 & 232.820 & Surface & 2012 & 5605.468 & 222.172 & Surface & 2012 \\
\hline 274.155 & 232.819 & Surface & 2012 & 5608.715 & 222.144 & Surface & 2012 \\
\hline 277.384 & 232.824 & Surface & 2012 & 5611.961 & 222.103 & Surface & 2012 \\
\hline 280.613 & 232.794 & Surface & 2012 & 5615.207 & 222.053 & Surface & 2012 \\
\hline 283.842 & 232.748 & Surface & 2012 & 5618.453 & 222.048 & Surface & 2012 \\
\hline 287.071 & 232.710 & Surface & 2012 & 5621.699 & 222.060 & Surface & 2012 \\
\hline 290.300 & 232.693 & Surface & 2012 & 5624.945 & 222.054 & Surface & 2012 \\
\hline 293.529 & 232.693 & Surface & 2012 & 5628.192 & 222.063 & Surface & 2012 \\
\hline 296.758 & 232.702 & Surface & 2012 & 5631.438 & 222.081 & Surface & 2012 \\
\hline
\end{tabular}




\begin{tabular}{|c|c|c|c|c|c|c|c|}
\hline 299.987 & 232.717 & Surface & 2012 & 5634.684 & 222.031 & Surface & 2012 \\
\hline 303.216 & 232.704 & Surface & 2012 & 5637.930 & 222.006 & Surface & 2012 \\
\hline 306.445 & 232.677 & Surface & 2012 & 5641.046 & 221.989 & Surface & 2012 \\
\hline 309.674 & 232.646 & Surface & 2012 & 5644.162 & 221.974 & Surface & 2012 \\
\hline 312.903 & 232.627 & Surface & 2012 & 5647.278 & 221.973 & Surface & 2012 \\
\hline 316.049 & 232.658 & Surface & 2012 & 5650.395 & 221.960 & Surface & 2012 \\
\hline 319.195 & 232.604 & Surface & 2012 & 5653.511 & 221.951 & Surface & 2012 \\
\hline 322.342 & 232.574 & Surface & 2012 & 5656.627 & 221.948 & Surface & 2012 \\
\hline 325.488 & 232.546 & Surface & 2012 & 5659.743 & 221.924 & Surface & 2012 \\
\hline 328.634 & 232.501 & Surface & 2012 & 5662.859 & 221.895 & Surface & 2012 \\
\hline 331.780 & 232.456 & Surface & 2012 & 5665.975 & 221.893 & Surface & 2012 \\
\hline 334.926 & 232.441 & Surface & 2012 & 5669.092 & 221.889 & Surface & 2012 \\
\hline 338.073 & 232.456 & Surface & 2012 & 5672.208 & 221.860 & Surface & 2012 \\
\hline 341.219 & 232.466 & Surface & 2012 & 5675.324 & 221.845 & Surface & 2012 \\
\hline 344.365 & 232.462 & Surface & 2012 & 5678.440 & 221.849 & Surface & 2012 \\
\hline 347.511 & 232.461 & Surface & 2012 & 5681.556 & 221.850 & Surface & 2012 \\
\hline 350.657 & 232.521 & Surface & 2012 & 5684.673 & 221.841 & Surface & 2012 \\
\hline 353.803 & 232.429 & Surface & 2012 & 5687.789 & 221.816 & Surface & 2012 \\
\hline 356.950 & 232.422 & Surface & 2012 & 5690.905 & 221.780 & Surface & 2012 \\
\hline 360.096 & 232.425 & Surface & 2012 & 5694.021 & 221.781 & Surface & 2012 \\
\hline 363.242 & 232.428 & Surface & 2012 & 5697.257 & 221.792 & Surface & 2012 \\
\hline 366.523 & 232.415 & Surface & 2012 & 5700.493 & 221.788 & Surface & 2012 \\
\hline 369.804 & 232.330 & Surface & 2012 & 5703.729 & 221.763 & Surface & 2012 \\
\hline 373.084 & 232.322 & Surface & 2012 & 5706.965 & 221.741 & Surface & 2012 \\
\hline 376.365 & 232.342 & Surface & 2012 & 5710.200 & 221.732 & Surface & 2012 \\
\hline 379.646 & 232.318 & Surface & 2012 & 5713.436 & 221.725 & Surface & 2012 \\
\hline 382.927 & 232.315 & Surface & 2012 & 5716.672 & 221.721 & Surface & 2012 \\
\hline 386.208 & 232.325 & Surface & 2012 & 5719.908 & 221.730 & Surface & 2012 \\
\hline 389.489 & 232.317 & Surface & 2012 & 5723.144 & 221.738 & Surface & 2012 \\
\hline 392.770 & 232.308 & Surface & 2012 & 5726.380 & 221.729 & Surface & 2012 \\
\hline 396.051 & 232.300 & Surface & 2012 & 5729.616 & 221.722 & Surface & 2012 \\
\hline 399.332 & 232.262 & Surface & 2012 & 5732.852 & 221.701 & Surface & 2012 \\
\hline 402.613 & 232.225 & Surface & 2012 & 5736.088 & 221.687 & Surface & 2012 \\
\hline 405.894 & 232.206 & Surface & 2012 & 5739.323 & 221.668 & Surface & 2012 \\
\hline 409.175 & 232.232 & Surface & 2012 & 5742.559 & 221.681 & Surface & 2012 \\
\hline 412.456 & 232.208 & Surface & 2012 & 5745.795 & 221.682 & Surface & 2012 \\
\hline 415.737 & 232.215 & Surface & 2012 & 5748.930 & 221.689 & Surface & 2012 \\
\hline 418.865 & 232.170 & Surface & 2012 & 5752.065 & 221.696 & Surface & 2012 \\
\hline 421.994 & 232.121 & Surface & 2012 & 5755.199 & 221.684 & Surface & 2012 \\
\hline 425.122 & 232.040 & Surface & 2012 & 5758.334 & 221.654 & Surface & 2012 \\
\hline 428.251 & 232.003 & Surface & 2012 & 5761.469 & 221.642 & Surface & 2012 \\
\hline 431.379 & 231.996 & Surface & 2012 & 5764.603 & 221.651 & Surface & 2012 \\
\hline 434.508 & 231.985 & Surface & 2012 & 5767.738 & 221.660 & Surface & 2012 \\
\hline 437.637 & 231.942 & Surface & 2012 & 5770.873 & 221.651 & Surface & 2012 \\
\hline 440.765 & 231.875 & Surface & 2012 & 5774.007 & 221.655 & Surface & 2012 \\
\hline 443.894 & 231.763 & Surface & 2012 & 5777.142 & 221.658 & Surface & 2012 \\
\hline 447.022 & 231.666 & Surface & 2012 & 5780.277 & 221.653 & Surface & 2012 \\
\hline 450.151 & 231.572 & Surface & 2012 & 5783.411 & 221.637 & Surface & 2012 \\
\hline 453.279 & 231.465 & Surface & 2012 & 5786.546 & 221.631 & Surface & 2012 \\
\hline
\end{tabular}




\begin{tabular}{|c|c|c|c|c|c|c|c|}
\hline 456.408 & 231.373 & Surface & 2012 & 5789.681 & 221.618 & Surface & 2012 \\
\hline 459.536 & 231.285 & Surface & 2012 & 5792.816 & 221.630 & Surface & 2012 \\
\hline 462.665 & 231.126 & Surface & 2012 & 5795.950 & 221.629 & Surface & 2012 \\
\hline 465.793 & 230.915 & Surface & 2012 & 5799.001 & 221.624 & Surface & 2012 \\
\hline 469.047 & 230.769 & Surface & 2012 & 5802.052 & 221.596 & Surface & 2012 \\
\hline 472.301 & 230.728 & Surface & 2012 & 5805.103 & 221.597 & Surface & 2012 \\
\hline 475.555 & 230.719 & Surface & 2012 & 5808.154 & 221.593 & Surface & 2012 \\
\hline 478.809 & 230.727 & Surface & 2012 & 5811.205 & 221.580 & Surface & 2012 \\
\hline 482.063 & 230.711 & Surface & 2012 & 5814.256 & 221.563 & Surface & 2012 \\
\hline 485.317 & 230.661 & Surface & 2012 & 5817.307 & 221.500 & Surface & 2012 \\
\hline 488.571 & 230.659 & Surface & 2012 & 5820.358 & 221.489 & Surface & 2012 \\
\hline 491.825 & 230.659 & Surface & 2012 & 5823.409 & 221.447 & Surface & 2012 \\
\hline 495.079 & 230.659 & Surface & 2012 & 5826.460 & 221.409 & Surface & 2012 \\
\hline 498.333 & 230.656 & Surface & 2012 & 5829.511 & 221.375 & Surface & 2012 \\
\hline 501.587 & 230.642 & Surface & 2012 & 5832.562 & 221.346 & Surface & 2012 \\
\hline 504.841 & 230.650 & Surface & 2012 & 5835.613 & 221.326 & Surface & 2012 \\
\hline 508.095 & 230.662 & Surface & 2012 & 5838.858 & 221.310 & Surface & 2012 \\
\hline 511.349 & 230.655 & Surface & 2012 & 5842.104 & 221.263 & Surface & 2012 \\
\hline 514.603 & 230.653 & Surface & 2012 & 5845.350 & 221.208 & Surface & 2012 \\
\hline 517.857 & 230.666 & Surface & 2012 & 5848.595 & 221.112 & Surface & 2012 \\
\hline 521.111 & 230.645 & Surface & 2012 & 5851.841 & 221.074 & Surface & 2012 \\
\hline 524.365 & 230.611 & Surface & 2012 & 5855.086 & 221.081 & Surface & 2012 \\
\hline 527.619 & 230.633 & Surface & 2012 & 5858.332 & 221.045 & Surface & 2012 \\
\hline 530.873 & 230.640 & Surface & 2012 & 5861.578 & 221.022 & Surface & 2012 \\
\hline 534.127 & 230.639 & Surface & 2012 & 5864.823 & 221.046 & Surface & 2012 \\
\hline 537.381 & 230.640 & Surface & 2012 & 5868.069 & 221.067 & Surface & 2012 \\
\hline 540.635 & 230.671 & Surface & 2012 & 5871.315 & 221.049 & Surface & 2012 \\
\hline 543.889 & 230.633 & Surface & 2012 & 5874.560 & 221.048 & Surface & 2012 \\
\hline 547.143 & 230.603 & Surface & 2012 & 5877.806 & 221.030 & Surface & 2012 \\
\hline 550.397 & 230.599 & Surface & 2012 & 5881.051 & 221.033 & Surface & 2012 \\
\hline 553.651 & 230.594 & Surface & 2012 & 5884.297 & 221.042 & Surface & 2012 \\
\hline 556.932 & 230.610 & Surface & 2012 & 5887.485 & 221.054 & Surface & 2012 \\
\hline 560.212 & 230.600 & Surface & 2012 & 5890.672 & 221.050 & Surface & 2012 \\
\hline 563.493 & 230.586 & Surface & 2012 & 5893.860 & 221.037 & Surface & 2012 \\
\hline 566.774 & 230.577 & Surface & 2012 & 5897.048 & 221.025 & Surface & 2012 \\
\hline 570.055 & 230.524 & Surface & 2012 & 5900.235 & 221.013 & Surface & 2012 \\
\hline 573.335 & 230.514 & Surface & 2012 & 5903.423 & 221.067 & Surface & 2012 \\
\hline 576.616 & 230.518 & Surface & 2012 & 5906.611 & 221.054 & Surface & 2012 \\
\hline 579.897 & 230.508 & Surface & 2012 & 5909.798 & 221.064 & Surface & 2012 \\
\hline 583.177 & 230.500 & Surface & 2012 & 5912.986 & 221.083 & Surface & 2012 \\
\hline 586.458 & 230.510 & Surface & 2012 & 5916.174 & 221.071 & Surface & 2012 \\
\hline 589.739 & 230.484 & Surface & 2012 & 5919.361 & 221.066 & Surface & 2012 \\
\hline 593.020 & 230.466 & Surface & 2012 & 5922.549 & 221.080 & Surface & 2012 \\
\hline 596.300 & 230.482 & Surface & 2012 & 5925.737 & 221.058 & Surface & 2012 \\
\hline 599.581 & 230.452 & Surface & 2012 & 5928.981 & 221.046 & Surface & 2012 \\
\hline 602.862 & 230.399 & Surface & 2012 & 5932.226 & 221.068 & Surface & 2012 \\
\hline 606.142 & 230.423 & Surface & 2012 & 5935.470 & 221.051 & Surface & 2012 \\
\hline 609.423 & 230.397 & Surface & 2012 & 5938.715 & 221.058 & Surface & 2012 \\
\hline 612.539 & 230.378 & Surface & 2012 & 5941.959 & 221.054 & Surface & 2012 \\
\hline
\end{tabular}




\begin{tabular}{|c|c|c|c|c|c|c|c|}
\hline 615.655 & 230.352 & Surface & 2012 & 5945.204 & 221.054 & Surface & 2012 \\
\hline 618.770 & 230.353 & Surface & 2012 & 5948.449 & 221.028 & Surface & 2012 \\
\hline 621.886 & 230.367 & Surface & 2012 & 5951.693 & 221.030 & Surface & 2012 \\
\hline 625.001 & 230.344 & Surface & 2012 & 5954.938 & 221.041 & Surface & 2012 \\
\hline 628.117 & 230.323 & Surface & 2012 & 5958.182 & 221.034 & Surface & 2012 \\
\hline 631.232 & 230.303 & Surface & 2012 & 5961.427 & 221.029 & Surface & 2012 \\
\hline 634.348 & 230.283 & Surface & 2012 & 5964.671 & 221.030 & Surface & 2012 \\
\hline 637.464 & 230.299 & Surface & 2012 & 5967.916 & 221.037 & Surface & 2012 \\
\hline 640.579 & 230.333 & Surface & 2012 & 5971.161 & 221.023 & Surface & 2012 \\
\hline 643.695 & 230.338 & Surface & 2012 & 5974.405 & 221.015 & Surface & 2012 \\
\hline 646.811 & 230.307 & Surface & 2012 & 5977.650 & 221.020 & Surface & 2012 \\
\hline 649.926 & 230.281 & Surface & 2012 & 5980.894 & 221.010 & Surface & 2012 \\
\hline 653.042 & 230.281 & Surface & 2012 & 5984.139 & 220.994 & Surface & 2012 \\
\hline 656.158 & 230.278 & Surface & 2012 & 5987.384 & 220.986 & Surface & 2012 \\
\hline 659.423 & 230.271 & Surface & 2012 & 5990.607 & 220.974 & Surface & 2012 \\
\hline 662.689 & 230.282 & Surface & 2012 & 5993.831 & 220.963 & Surface & 2012 \\
\hline 665.954 & 230.293 & Surface & 2012 & 5997.055 & 220.953 & Surface & 2012 \\
\hline 669.220 & 230.261 & Surface & 2012 & 6000.278 & 220.930 & Surface & 2012 \\
\hline 672.485 & 230.271 & Surface & 2012 & 6003.502 & 220.916 & Surface & 2012 \\
\hline 675.751 & 230.280 & Surface & 2012 & 6006.725 & 220.924 & Surface & 2012 \\
\hline 679.016 & 230.280 & Surface & 2012 & 6009.949 & 220.916 & Surface & 2012 \\
\hline 682.282 & 230.280 & Surface & 2012 & 6013.172 & 220.914 & Surface & 2012 \\
\hline 685.284 & 230.295 & Surface & 2012 & 6016.396 & 220.917 & Surface & 2012 \\
\hline 688.288 & 230.292 & Surface & 2012 & 6019.620 & 220.885 & Surface & 2012 \\
\hline 691.291 & 230.291 & Surface & 2012 & 6022.843 & 220.881 & Surface & 2012 \\
\hline 694.294 & 230.302 & Surface & 2012 & 6026.067 & 220.842 & Surface & 2012 \\
\hline 697.297 & 230.306 & Surface & 2012 & 6029.290 & 220.846 & Surface & 2012 \\
\hline 700.300 & 230.293 & Surface & 2012 & 6032.514 & 220.837 & Surface & 2012 \\
\hline 703.303 & 230.270 & Surface & 2012 & 6035.738 & 220.834 & Surface & 2012 \\
\hline 706.307 & 230.267 & Surface & 2012 & 6038.862 & 220.857 & Surface & 2012 \\
\hline 709.310 & 230.287 & Surface & 2012 & 6041.987 & 220.856 & Surface & 2012 \\
\hline 712.457 & 230.292 & Surface & 2012 & 6045.112 & 220.832 & Surface & 2012 \\
\hline 715.604 & 230.288 & Surface & 2012 & 6048.236 & 220.844 & Surface & 2012 \\
\hline 718.751 & 230.311 & Surface & 2012 & 6051.361 & 220.813 & Surface & 2012 \\
\hline 721.899 & 230.297 & Surface & 2012 & 6054.486 & 220.827 & Surface & 2012 \\
\hline 725.046 & 230.289 & Surface & 2012 & 6057.611 & 220.845 & Surface & 2012 \\
\hline 728.193 & 230.299 & Surface & 2012 & 6060.735 & 220.837 & Surface & 2012 \\
\hline 731.340 & 230.315 & Surface & 2012 & 6063.860 & 220.851 & Surface & 2012 \\
\hline 734.302 & 230.305 & Surface & 2012 & 6066.985 & 220.864 & Surface & 2012 \\
\hline 737.264 & 230.280 & Surface & 2012 & 6070.109 & 220.857 & Surface & 2012 \\
\hline 740.225 & 230.271 & Surface & 2012 & 6073.234 & 220.842 & Surface & 2012 \\
\hline 743.187 & 230.275 & Surface & 2012 & 6076.359 & 220.833 & Surface & 2012 \\
\hline 746.148 & 230.284 & Surface & 2012 & 6079.483 & 220.842 & Surface & 2012 \\
\hline 749.110 & 230.293 & Surface & 2012 & 6082.608 & 220.848 & Surface & 2012 \\
\hline 752.072 & 230.310 & Surface & 2012 & 6085.733 & 220.844 & Surface & 2012 \\
\hline 755.033 & 230.305 & Surface & 2012 & 6088.994 & 220.857 & Surface & 2012 \\
\hline 757.995 & 230.279 & Surface & 2012 & 6092.255 & 220.859 & Surface & 2012 \\
\hline 760.956 & 230.291 & Surface & 2012 & 6095.516 & 220.853 & Surface & 2012 \\
\hline 764.059 & 230.297 & Surface & 2012 & 6098.777 & 220.858 & Surface & 2012 \\
\hline
\end{tabular}




\begin{tabular}{|c|c|c|c|c|c|c|c|}
\hline 767.161 & 230.296 & Surface & 2012 & 6102.038 & 220.844 & Surface & 2012 \\
\hline 770.263 & 230.291 & Surface & 2012 & 6105.299 & 220.864 & Surface & 2012 \\
\hline 773.366 & 230.300 & Surface & 2012 & 6108.560 & 220.876 & Surface & 2012 \\
\hline 776.468 & 230.308 & Surface & 2012 & 6111.821 & 220.854 & Surface & 2012 \\
\hline 779.570 & 230.311 & Surface & 2012 & 6115.082 & 220.858 & Surface & 2012 \\
\hline 782.672 & 230.304 & Surface & 2012 & 6118.343 & 220.857 & Surface & 2012 \\
\hline 785.774 & 230.306 & Surface & 2012 & 6121.604 & 220.860 & Surface & 2012 \\
\hline 788.877 & 230.308 & Surface & 2012 & 6124.866 & 220.857 & Surface & 2012 \\
\hline 791.979 & 230.310 & Surface & 2012 & 6128.127 & 220.858 & Surface & 2012 \\
\hline 795.081 & 230.310 & Surface & 2012 & 6131.388 & 220.857 & Surface & 2012 \\
\hline 798.184 & 230.318 & Surface & 2012 & 6134.649 & 220.851 & Surface & 2012 \\
\hline 801.363 & 230.318 & Surface & 2012 & 6137.910 & 220.877 & Surface & 2012 \\
\hline 804.543 & 230.318 & Surface & 2012 & 6141.069 & 220.874 & Surface & 2012 \\
\hline 807.723 & 230.321 & Surface & 2012 & 6144.228 & 220.883 & Surface & 2012 \\
\hline 810.903 & 230.323 & Surface & 2012 & 6147.387 & 220.872 & Surface & 2012 \\
\hline 814.082 & 230.320 & Surface & 2012 & 6150.546 & 220.853 & Surface & 2012 \\
\hline 817.262 & 230.319 & Surface & 2012 & 6153.705 & 220.854 & Surface & 2012 \\
\hline 820.442 & 230.310 & Surface & 2012 & 6156.864 & 220.853 & Surface & 2012 \\
\hline 823.622 & 230.309 & Surface & 2012 & 6160.022 & 220.844 & Surface & 2012 \\
\hline 826.801 & 230.302 & Surface & 2012 & 6163.182 & 220.856 & Surface & 2012 \\
\hline 829.981 & 230.292 & Surface & 2012 & 6166.340 & 220.860 & Surface & 2012 \\
\hline 833.161 & 230.289 & Surface & 2012 & 6169.499 & 220.842 & Surface & 2012 \\
\hline 836.341 & 230.286 & Surface & 2012 & 6172.658 & 220.838 & Surface & 2012 \\
\hline 839.520 & 230.290 & Surface & 2012 & 6175.817 & 220.848 & Surface & 2012 \\
\hline 842.700 & 230.296 & Surface & 2012 & 6178.976 & 220.848 & Surface & 2012 \\
\hline 845.880 & 230.286 & Surface & 2012 & 6182.143 & 220.840 & Surface & 2012 \\
\hline 849.060 & 230.268 & Surface & 2012 & 6185.309 & 220.839 & Surface & 2012 \\
\hline 852.240 & 230.250 & Surface & 2012 & 6188.475 & 220.841 & Surface & 2012 \\
\hline 855.209 & 230.239 & Surface & 2012 & 6191.642 & 220.827 & Surface & 2012 \\
\hline 858.179 & 230.221 & Surface & 2012 & 6194.808 & 220.826 & Surface & 2012 \\
\hline 861.150 & 230.210 & Surface & 2012 & 6197.975 & 220.830 & Surface & 2012 \\
\hline 864.119 & 230.194 & Surface & 2012 & 6201.141 & 220.835 & Surface & 2012 \\
\hline 867.089 & 230.181 & Surface & 2012 & 6204.307 & 220.841 & Surface & 2012 \\
\hline 870.059 & 230.167 & Surface & 2012 & 6207.474 & 220.840 & Surface & 2012 \\
\hline 873.029 & 230.152 & Surface & 2012 & 6210.641 & 220.826 & Surface & 2012 \\
\hline 875.999 & 230.140 & Surface & 2012 & 6213.807 & 220.832 & Surface & 2012 \\
\hline 878.969 & 230.129 & Surface & 2012 & 6216.973 & 220.846 & Surface & 2012 \\
\hline 882.163 & 230.133 & Surface & 2012 & 6220.165 & 220.834 & Surface & 2012 \\
\hline 885.357 & 230.061 & Surface & 2012 & 6223.357 & 220.831 & Surface & 2012 \\
\hline 888.551 & 229.923 & Surface & 2012 & 6226.549 & 220.835 & Surface & 2012 \\
\hline 891.745 & 229.947 & Surface & 2012 & 6229.741 & 220.836 & Surface & 2012 \\
\hline 894.939 & 229.994 & Surface & 2012 & 6232.933 & 220.812 & Surface & 2012 \\
\hline 898.133 & 229.975 & Surface & 2012 & 6236.125 & 220.814 & Surface & 2012 \\
\hline 901.327 & 229.833 & Surface & 2012 & 6239.317 & 220.839 & Surface & 2012 \\
\hline 904.521 & 229.775 & Surface & 2012 & 6242.508 & 220.827 & Surface & 2012 \\
\hline 907.715 & 229.738 & Surface & 2012 & 6245.700 & 220.838 & Surface & 2012 \\
\hline 910.909 & 229.729 & Surface & 2012 & 6248.892 & 220.826 & Surface & 2012 \\
\hline 914.103 & 229.669 & Surface & 2012 & 6252.084 & 220.821 & Surface & 2012 \\
\hline 917.297 & 229.647 & Surface & 2012 & 6255.276 & 220.818 & Surface & 2012 \\
\hline
\end{tabular}




\begin{tabular}{|c|c|c|c|c|c|c|c|}
\hline 920.491 & 229.665 & Surface & 2012 & 6258.468 & 220.812 & Surface & 2012 \\
\hline 923.685 & 229.616 & Surface & 2012 & 6261.660 & 220.815 & Surface & 2012 \\
\hline 926.879 & 229.561 & Surface & 2012 & 6264.875 & 220.795 & Surface & 2012 \\
\hline 930.073 & 229.524 & Surface & 2012 & 6268.090 & 220.785 & Surface & 2012 \\
\hline 933.105 & 229.496 & Surface & 2012 & 6271.305 & 220.780 & Surface & 2012 \\
\hline 936.137 & 229.492 & Surface & 2012 & 6274.520 & 220.771 & Surface & 2012 \\
\hline 939.168 & 229.459 & Surface & 2012 & 6277.735 & 220.779 & Surface & 2012 \\
\hline 942.200 & 229.392 & Surface & 2012 & 6280.950 & 220.788 & Surface & 2012 \\
\hline 945.232 & 229.340 & Surface & 2012 & 6284.165 & 220.788 & Surface & 2012 \\
\hline 948.264 & 229.378 & Surface & 2012 & 6287.380 & 220.780 & Surface & 2012 \\
\hline 951.296 & 229.348 & Surface & 2012 & 6290.595 & 220.776 & Surface & 2012 \\
\hline 954.328 & 229.351 & Surface & 2012 & 6293.810 & 220.775 & Surface & 2012 \\
\hline 957.359 & 229.324 & Surface & 2012 & 6297.025 & 220.753 & Surface & 2012 \\
\hline 960.391 & 229.287 & Surface & 2012 & 6300.241 & 220.723 & Surface & 2012 \\
\hline 963.423 & 229.261 & Surface & 2012 & 6303.456 & 220.761 & Surface & 2012 \\
\hline 966.455 & 229.256 & Surface & 2012 & 6306.671 & 220.767 & Surface & 2012 \\
\hline 969.486 & 229.270 & Surface & 2012 & 6309.886 & 220.750 & Surface & 2012 \\
\hline 972.697 & 229.235 & Surface & 2012 & 6313.101 & 220.745 & Surface & 2012 \\
\hline 975.908 & 229.171 & Surface & 2012 & 6316.316 & 220.758 & Surface & 2012 \\
\hline 979.118 & 229.117 & Surface & 2012 & 6319.550 & 220.731 & Surface & 2012 \\
\hline 982.329 & 229.034 & Surface & 2012 & 6322.784 & 220.746 & Surface & 2012 \\
\hline 985.540 & 228.992 & Surface & 2012 & 6326.017 & 220.753 & Surface & 2012 \\
\hline 988.750 & 228.977 & Surface & 2012 & 6329.251 & 220.747 & Surface & 2012 \\
\hline 991.961 & 228.988 & Surface & 2012 & 6332.484 & 220.750 & Surface & 2012 \\
\hline 995.172 & 229.012 & Surface & 2012 & 6335.718 & 220.757 & Surface & 2012 \\
\hline 998.383 & 229.029 & Surface & 2012 & 6338.952 & 220.757 & Surface & 2012 \\
\hline 1001.593 & 229.027 & Surface & 2012 & 6342.185 & 220.754 & Surface & 2012 \\
\hline 1004.804 & 229.022 & Surface & 2012 & 6345.419 & 220.752 & Surface & 2012 \\
\hline 1008.014 & 229.007 & Surface & 2012 & 6348.653 & 220.743 & Surface & 2012 \\
\hline 1011.225 & 228.983 & Surface & 2012 & 6351.887 & 220.750 & Surface & 2012 \\
\hline 1014.436 & 229.009 & Surface & 2012 & 6355.120 & 220.762 & Surface & 2012 \\
\hline 1017.646 & 229.000 & Surface & 2012 & 6358.354 & 220.778 & Surface & 2012 \\
\hline 1020.857 & 228.999 & Surface & 2012 & 6361.587 & 220.747 & Surface & 2012 \\
\hline 1024.068 & 228.993 & Surface & 2012 & 6364.821 & 220.748 & Surface & 2012 \\
\hline 1027.279 & 228.970 & Surface & 2012 & 6368.055 & 220.770 & Surface & 2012 \\
\hline 1030.489 & 228.977 & Surface & 2012 & 6371.251 & 220.767 & Surface & 2012 \\
\hline 1033.700 & 228.988 & Surface & 2012 & 6374.446 & 220.771 & Surface & 2012 \\
\hline 1036.911 & 228.997 & Surface & 2012 & 6377.642 & 220.763 & Surface & 2012 \\
\hline 1040.122 & 228.999 & Surface & 2012 & 6380.838 & 220.755 & Surface & 2012 \\
\hline 1043.332 & 229.002 & Surface & 2012 & 6384.034 & 220.768 & Surface & 2012 \\
\hline 1046.543 & 229.002 & Surface & 2012 & 6387.230 & 220.772 & Surface & 2012 \\
\hline 1049.555 & 229.005 & Surface & 2012 & 6390.426 & 220.765 & Surface & 2012 \\
\hline 1052.568 & 228.991 & Surface & 2012 & 6393.622 & 220.758 & Surface & 2012 \\
\hline 1055.581 & 228.991 & Surface & 2012 & 6396.817 & 220.768 & Surface & 2012 \\
\hline 1058.594 & 228.985 & Surface & 2012 & 6400.013 & 220.762 & Surface & 2012 \\
\hline 1061.607 & 228.981 & Surface & 2012 & 6403.209 & 220.769 & Surface & 2012 \\
\hline 1064.619 & 229.007 & Surface & 2012 & 6406.405 & 220.768 & Surface & 2012 \\
\hline 1067.632 & 229.022 & Surface & 2012 & 6409.600 & 220.767 & Surface & 2012 \\
\hline 1070.645 & 229.017 & Surface & 2012 & 6412.796 & 220.760 & Surface & 2012 \\
\hline
\end{tabular}




\begin{tabular}{|c|c|c|c|c|c|c|c|}
\hline 1073.657 & 229.002 & Surface & 2012 & 6415.859 & 220.762 & Surface & 2012 \\
\hline 1076.670 & 228.990 & Surface & 2012 & 6418.921 & 220.767 & Surface & 2012 \\
\hline 1079.683 & 228.999 & Surface & 2012 & 6421.983 & 220.732 & Surface & 2012 \\
\hline 1082.696 & 229.003 & Surface & 2012 & 6425.045 & 220.770 & Surface & 2012 \\
\hline 1085.926 & 228.998 & Surface & 2012 & 6428.107 & 220.759 & Surface & 2012 \\
\hline 1089.156 & 228.985 & Surface & 2012 & 6431.170 & 220.752 & Surface & 2012 \\
\hline 1092.387 & 228.977 & Surface & 2012 & 6434.232 & 220.754 & Surface & 2012 \\
\hline 1095.617 & 228.983 & Surface & 2012 & 6437.294 & 220.757 & Surface & 2012 \\
\hline 1098.848 & 228.990 & Surface & 2012 & 6440.357 & 220.750 & Surface & 2012 \\
\hline 1102.078 & 228.976 & Surface & 2012 & 6443.419 & 220.756 & Surface & 2012 \\
\hline 1105.308 & 228.987 & Surface & 2012 & 6446.481 & 220.745 & Surface & 2012 \\
\hline 1108.539 & 228.982 & Surface & 2012 & 6449.543 & 220.750 & Surface & 2012 \\
\hline 1111.769 & 228.990 & Surface & 2012 & 6452.606 & 220.770 & Surface & 2012 \\
\hline 1115.000 & 228.998 & Surface & 2012 & 6455.668 & 220.762 & Surface & 2012 \\
\hline 1118.230 & 228.999 & Surface & 2012 & 6458.774 & 220.758 & Surface & 2012 \\
\hline 1121.460 & 228.998 & Surface & 2012 & 6461.880 & 220.752 & Surface & 2012 \\
\hline 1124.691 & 228.994 & Surface & 2012 & 6464.986 & 220.752 & Surface & 2012 \\
\hline 1127.921 & 228.990 & Surface & 2012 & 6468.092 & 220.763 & Surface & 2012 \\
\hline 1131.152 & 228.983 & Surface & 2012 & 6471.199 & 220.750 & Surface & 2012 \\
\hline 1134.334 & 228.973 & Surface & 2012 & 6474.305 & 220.745 & Surface & 2012 \\
\hline 1137.517 & 228.955 & Surface & 2012 & 6477.411 & 220.735 & Surface & 2012 \\
\hline 1140.700 & 228.978 & Surface & 2012 & 6480.517 & 220.741 & Surface & 2012 \\
\hline 1143.882 & 228.980 & Surface & 2012 & 6483.623 & 220.747 & Surface & 2012 \\
\hline 1147.065 & 228.970 & Surface & 2012 & 6486.729 & 220.754 & Surface & 2012 \\
\hline 1150.247 & 228.977 & Surface & 2012 & 6489.836 & 220.744 & Surface & 2012 \\
\hline 1153.430 & 228.982 & Surface & 2012 & 6492.942 & 220.725 & Surface & 2012 \\
\hline 1156.613 & 228.964 & Surface & 2012 & 6496.048 & 220.721 & Surface & 2012 \\
\hline 1159.796 & 228.976 & Surface & 2012 & 6499.154 & 220.730 & Surface & 2012 \\
\hline 1162.978 & 228.973 & Surface & 2012 & 6502.260 & 220.752 & Surface & 2012 \\
\hline 1166.161 & 228.961 & Surface & 2012 & 6505.366 & 220.746 & Surface & 2012 \\
\hline 1169.344 & 228.970 & Surface & 2012 & 6508.475 & 220.730 & Surface & 2012 \\
\hline 1172.526 & 228.965 & Surface & 2012 & 6511.585 & 220.732 & Surface & 2012 \\
\hline 1175.709 & 228.967 & Surface & 2012 & 6514.694 & 220.753 & Surface & 2012 \\
\hline 1178.891 & 228.937 & Surface & 2012 & 6517.803 & 220.747 & Surface & 2012 \\
\hline 1182.074 & 228.933 & Surface & 2012 & 6520.912 & 220.759 & Surface & 2012 \\
\hline 1185.269 & 228.945 & Surface & 2012 & 6524.022 & 220.747 & Surface & 2012 \\
\hline 1188.463 & 228.954 & Surface & 2012 & 6527.131 & 220.732 & Surface & 2012 \\
\hline 1191.658 & 228.931 & Surface & 2012 & 6530.240 & 220.772 & Surface & 2012 \\
\hline 1194.852 & 228.924 & Surface & 2012 & 6533.349 & 220.763 & Surface & 2012 \\
\hline 1198.047 & 228.914 & Surface & 2012 & 6536.459 & 220.734 & Surface & 2012 \\
\hline 1201.242 & 228.896 & Surface & 2012 & 6539.568 & 220.738 & Surface & 2012 \\
\hline 1204.436 & 228.885 & Surface & 2012 & 6542.677 & 220.735 & Surface & 2012 \\
\hline 1207.631 & 228.878 & Surface & 2012 & 6545.787 & 220.721 & Surface & 2012 \\
\hline 1210.826 & 228.889 & Surface & 2012 & 6548.896 & 220.817 & Surface & 2012 \\
\hline 1214.020 & 228.893 & Surface & 2012 & 6552.005 & 220.849 & Surface & 2012 \\
\hline 1217.215 & 228.893 & Surface & 2012 & 6555.114 & 220.854 & Surface & 2012 \\
\hline 1220.409 & 228.885 & Surface & 2012 & 6558.224 & 220.893 & Surface & 2012 \\
\hline 1223.604 & 228.857 & Surface & 2012 & 6561.333 & 220.908 & Surface & 2012 \\
\hline 1226.799 & 228.840 & Surface & 2012 & 6564.442 & 220.855 & Surface & 2012 \\
\hline
\end{tabular}




\begin{tabular}{|c|c|c|c|c|c|c|c|}
\hline 1229.993 & 228.845 & Surface & 2012 & 6567.722 & 220.790 & Surface & 2012 \\
\hline 1233.208 & 228.813 & Surface & 2012 & 6571.003 & 220.746 & Surface & 2012 \\
\hline 1236.423 & 228.775 & Surface & 2012 & 6574.283 & 220.716 & Surface & 2012 \\
\hline 1239.638 & 228.722 & Surface & 2012 & 6577.563 & 220.735 & Surface & 2012 \\
\hline 1242.853 & 228.738 & Surface & 2012 & 6580.844 & 220.751 & Surface & 2012 \\
\hline 1246.068 & 228.647 & Surface & 2012 & 6584.124 & 220.758 & Surface & 2012 \\
\hline 1249.283 & 228.592 & Surface & 2012 & 6587.405 & 220.780 & Surface & 2012 \\
\hline 1252.498 & 228.601 & Surface & 2012 & 6590.685 & 220.777 & Surface & 2012 \\
\hline 1255.713 & 228.603 & Surface & 2012 & 6593.965 & 220.768 & Surface & 2012 \\
\hline 1258.928 & 228.597 & Surface & 2012 & 6597.246 & 220.733 & Surface & 2012 \\
\hline 1262.143 & 228.607 & Surface & 2012 & 6600.526 & 220.721 & Surface & 2012 \\
\hline 1265.358 & 228.612 & Surface & 2012 & 6603.807 & 220.794 & Surface & 2012 \\
\hline 1268.573 & 228.606 & Surface & 2012 & 6607.087 & 220.815 & Surface & 2012 \\
\hline 1271.788 & 228.590 & Surface & 2012 & 6610.367 & 220.761 & Surface & 2012 \\
\hline 1275.023 & 228.585 & Surface & 2012 & 6613.648 & 220.712 & Surface & 2012 \\
\hline 1278.258 & 228.599 & Surface & 2012 & 6616.928 & 220.694 & Surface & 2012 \\
\hline 1281.493 & 228.594 & Surface & 2012 & 6620.208 & 220.690 & Surface & 2012 \\
\hline 1284.728 & 228.614 & Surface & 2012 & 6623.489 & 220.708 & Surface & 2012 \\
\hline 1287.963 & 228.610 & Surface & 2012 & 6626.769 & 220.718 & Surface & 2012 \\
\hline 1291.198 & 228.620 & Surface & 2012 & 6630.050 & 220.721 & Surface & 2012 \\
\hline 1294.433 & 228.615 & Surface & 2012 & 6633.330 & 220.700 & Surface & 2012 \\
\hline 1297.668 & 228.622 & Surface & 2012 & 6636.610 & 220.709 & Surface & 2012 \\
\hline 1300.902 & 228.623 & Surface & 2012 & 6639.891 & 220.717 & Surface & 2012 \\
\hline 1304.137 & 228.624 & Surface & 2012 & 6643.131 & 220.702 & Surface & 2012 \\
\hline 1307.372 & 228.620 & Surface & 2012 & 6646.371 & 220.721 & Surface & 2012 \\
\hline 1310.607 & 228.625 & Surface & 2012 & 6649.610 & 220.729 & Surface & 2012 \\
\hline 1313.842 & 228.632 & Surface & 2012 & 6652.850 & 220.712 & Surface & 2012 \\
\hline 1317.077 & 228.628 & Surface & 2012 & 6656.090 & 220.718 & Surface & 2012 \\
\hline 1320.237 & 228.629 & Surface & 2012 & 6659.330 & 220.706 & Surface & 2012 \\
\hline 1323.398 & 228.633 & Surface & 2012 & 6662.570 & 220.709 & Surface & 2012 \\
\hline 1326.558 & 228.635 & Surface & 2012 & 6665.810 & 220.713 & Surface & 2012 \\
\hline 1329.718 & 228.632 & Surface & 2012 & 6669.049 & 220.714 & Surface & 2012 \\
\hline 1332.879 & 228.630 & Surface & 2012 & 6672.289 & 220.713 & Surface & 2012 \\
\hline 1336.039 & 228.608 & Surface & 2012 & 6675.529 & 220.705 & Surface & 2012 \\
\hline 1339.199 & 228.599 & Surface & 2012 & 6678.769 & 220.681 & Surface & 2012 \\
\hline 1342.359 & 228.615 & Surface & 2012 & 6682.009 & 220.697 & Surface & 2012 \\
\hline 1345.520 & 228.613 & Surface & 2012 & 6685.249 & 220.697 & Surface & 2012 \\
\hline 1348.680 & 228.605 & Surface & 2012 & 6688.489 & 220.705 & Surface & 2012 \\
\hline 1351.840 & 228.613 & Surface & 2012 & 6691.728 & 220.695 & Surface & 2012 \\
\hline 1355.000 & 228.625 & Surface & 2012 & 6694.968 & 220.692 & Surface & 2012 \\
\hline 1358.161 & 228.619 & Surface & 2012 & 6698.208 & 220.693 & Surface & 2012 \\
\hline 1361.321 & 228.620 & Surface & 2012 & 6701.448 & 220.678 & Surface & 2012 \\
\hline 1364.481 & 228.630 & Surface & 2012 & 6704.725 & 220.687 & Surface & 2012 \\
\hline 1367.642 & 228.615 & Surface & 2012 & 6708.003 & 220.687 & Surface & 2012 \\
\hline 1370.802 & 228.618 & Surface & 2012 & 6711.280 & 220.680 & Surface & 2012 \\
\hline 1373.962 & 228.619 & Surface & 2012 & 6714.558 & 220.688 & Surface & 2012 \\
\hline 1377.122 & 228.621 & Surface & 2012 & 6717.836 & 220.664 & Surface & 2012 \\
\hline 1380.283 & 228.610 & Surface & 2012 & 6721.113 & 220.669 & Surface & 2012 \\
\hline 1383.443 & 228.606 & Surface & 2012 & 6724.391 & 220.674 & Surface & 2012 \\
\hline
\end{tabular}




\begin{tabular}{|c|c|c|c|c|c|c|c|}
\hline 1386.603 & 228.620 & Surface & 2012 & 6727.668 & 220.660 & Surface & 2012 \\
\hline 1389.784 & 228.623 & Surface & 2012 & 6730.945 & 220.660 & Surface & 2012 \\
\hline 1392.964 & 228.620 & Surface & 2012 & 6734.223 & 220.665 & Surface & 2012 \\
\hline 1396.145 & 228.602 & Surface & 2012 & 6737.500 & 220.647 & Surface & 2012 \\
\hline 1399.325 & 228.602 & Surface & 2012 & 6740.778 & 220.652 & Surface & 2012 \\
\hline 1402.506 & 228.597 & Surface & 2012 & 6744.055 & 220.642 & Surface & 2012 \\
\hline 1405.686 & 228.598 & Surface & 2012 & 6747.333 & 220.658 & Surface & 2012 \\
\hline 1408.866 & 228.590 & Surface & 2012 & 6750.611 & 220.665 & Surface & 2012 \\
\hline 1412.047 & 228.594 & Surface & 2012 & 6753.891 & 220.655 & Surface & 2012 \\
\hline 1415.228 & 228.608 & Surface & 2012 & 6757.172 & 220.637 & Surface & 2012 \\
\hline 1418.408 & 228.602 & Surface & 2012 & 6760.453 & 220.649 & Surface & 2012 \\
\hline 1421.588 & 228.590 & Surface & 2012 & 6763.733 & 220.646 & Surface & 2012 \\
\hline 1424.769 & 228.585 & Surface & 2012 & 6767.014 & 220.630 & Surface & 2012 \\
\hline 1427.949 & 228.577 & Surface & 2012 & 6770.295 & 220.588 & Surface & 2012 \\
\hline 1431.130 & 228.580 & Surface & 2012 & 6773.576 & 220.629 & Surface & 2012 \\
\hline 1434.310 & 228.580 & Surface & 2012 & 6776.856 & 220.630 & Surface & 2012 \\
\hline 1437.491 & 228.570 & Surface & 2012 & 6780.137 & 220.623 & Surface & 2012 \\
\hline 1440.671 & 228.547 & Surface & 2012 & 6783.417 & 220.617 & Surface & 2012 \\
\hline 1443.851 & 228.535 & Surface & 2012 & 6786.698 & 220.621 & Surface & 2012 \\
\hline 1447.032 & 228.547 & Surface & 2012 & 6789.979 & 220.620 & Surface & 2012 \\
\hline 1450.213 & 228.528 & Surface & 2012 & 6793.260 & 220.615 & Surface & 2012 \\
\hline 1453.393 & 228.538 & Surface & 2012 & 6796.540 & 220.594 & Surface & 2012 \\
\hline 1456.573 & 228.527 & Surface & 2012 & 6799.821 & 220.595 & Surface & 2012 \\
\hline 1459.754 & 228.521 & Surface & 2012 & 6803.102 & 220.569 & Surface & 2012 \\
\hline 1462.934 & 228.520 & Surface & 2012 & 6806.382 & 220.579 & Surface & 2012 \\
\hline 1466.115 & 228.522 & Surface & 2012 & 6809.597 & 220.570 & Surface & 2012 \\
\hline 1469.295 & 228.504 & Surface & 2012 & 6812.810 & 220.577 & Surface & 2012 \\
\hline 1472.476 & 228.497 & Surface & 2012 & 6816.024 & 220.577 & Surface & 2012 \\
\hline 1475.616 & 228.483 & Surface & 2012 & 6819.238 & 220.585 & Surface & 2012 \\
\hline 1478.756 & 228.477 & Surface & 2012 & 6822.452 & 220.590 & Surface & 2012 \\
\hline 1481.897 & 228.471 & Surface & 2012 & 6825.666 & 220.595 & Surface & 2012 \\
\hline 1485.037 & 228.476 & Surface & 2012 & 6828.879 & 220.574 & Surface & 2012 \\
\hline 1488.178 & 228.494 & Surface & 2012 & 6832.093 & 220.573 & Surface & 2012 \\
\hline 1491.318 & 228.485 & Surface & 2012 & 6835.307 & 220.577 & Surface & 2012 \\
\hline 1494.458 & 228.478 & Surface & 2012 & 6838.521 & 220.558 & Surface & 2012 \\
\hline 1497.599 & 228.470 & Surface & 2012 & 6841.735 & 220.557 & Surface & 2012 \\
\hline 1500.739 & 228.471 & Surface & 2012 & 6844.948 & 220.534 & Surface & 2012 \\
\hline 1503.879 & 228.458 & Surface & 2012 & 6848.163 & 220.543 & Surface & 2012 \\
\hline 1507.020 & 228.463 & Surface & 2012 & 6851.345 & 220.549 & Surface & 2012 \\
\hline 1510.160 & 228.484 & Surface & 2012 & 6854.528 & 220.539 & Surface & 2012 \\
\hline 1513.301 & 228.498 & Surface & 2012 & 6857.710 & 220.532 & Surface & 2012 \\
\hline 1516.441 & 228.474 & Surface & 2012 & 6860.893 & 220.519 & Surface & 2012 \\
\hline 1519.581 & 228.464 & Surface & 2012 & 6864.075 & 220.530 & Surface & 2012 \\
\hline 1522.722 & 228.456 & Surface & 2012 & 6867.258 & 220.516 & Surface & 2012 \\
\hline 1525.862 & 228.441 & Surface & 2012 & 6870.440 & 220.511 & Surface & 2012 \\
\hline 1529.002 & 228.455 & Surface & 2012 & 6873.623 & 220.516 & Surface & 2012 \\
\hline 1532.143 & 228.469 & Surface & 2012 & 6876.806 & 220.523 & Surface & 2012 \\
\hline 1535.131 & 228.448 & Surface & 2012 & 6879.988 & 220.513 & Surface & 2012 \\
\hline 1538.119 & 228.429 & Surface & 2012 & 6883.171 & 220.513 & Surface & 2012 \\
\hline
\end{tabular}




\begin{tabular}{|c|c|c|c|c|c|c|c|}
\hline 1541.107 & 228.428 & Surface & 2012 & 6886.354 & 220.490 & Surface & 2012 \\
\hline 1544.095 & 228.401 & Surface & 2012 & 6889.536 & 220.503 & Surface & 2012 \\
\hline 1547.083 & 228.390 & Surface & 2012 & 6892.719 & 220.510 & Surface & 2012 \\
\hline 1550.071 & 228.387 & Surface & 2012 & 6895.901 & 220.510 & Surface & 2012 \\
\hline 1553.059 & 228.371 & Surface & 2012 & 6899.084 & 220.510 & Surface & 2012 \\
\hline 1556.047 & 228.378 & Surface & 2012 & 6902.266 & 220.513 & Surface & 2012 \\
\hline 1559.035 & 228.355 & Surface & 2012 & 6905.449 & 220.500 & Surface & 2012 \\
\hline 1562.023 & 228.336 & Surface & 2012 & 6908.632 & 220.491 & Surface & 2012 \\
\hline 1565.011 & 228.357 & Surface & 2012 & 6911.814 & 220.493 & Surface & 2012 \\
\hline 1568.047 & 228.361 & Surface & 2012 & 6914.997 & 220.494 & Surface & 2012 \\
\hline 1571.082 & 228.331 & Surface & 2012 & 6918.179 & 220.500 & Surface & 2012 \\
\hline 1574.117 & 228.314 & Surface & 2012 & 6921.362 & 220.522 & Surface & 2012 \\
\hline 1577.153 & 228.230 & Surface & 2012 & 6924.544 & 220.503 & Surface & 2012 \\
\hline 1580.188 & 228.218 & Surface & 2012 & 6927.727 & 220.492 & Surface & 2012 \\
\hline 1583.224 & 228.236 & Surface & 2012 & 6930.910 & 220.488 & Surface & 2012 \\
\hline 1586.259 & 228.207 & Surface & 2012 & 6934.092 & 220.501 & Surface & 2012 \\
\hline 1589.295 & 228.167 & Surface & 2012 & 6937.213 & 220.506 & Surface & 2012 \\
\hline 1592.330 & 228.169 & Surface & 2012 & 6940.333 & 220.515 & Surface & 2012 \\
\hline 1595.365 & 228.194 & Surface & 2012 & 6943.454 & 220.486 & Surface & 2012 \\
\hline 1598.401 & 228.180 & Surface & 2012 & 6946.574 & 220.479 & Surface & 2012 \\
\hline 1601.665 & 228.176 & Surface & 2012 & 6949.694 & 220.506 & Surface & 2012 \\
\hline 1604.930 & 228.155 & Surface & 2012 & 6952.815 & 220.478 & Surface & 2012 \\
\hline 1608.194 & 228.163 & Surface & 2012 & 6955.935 & 220.491 & Surface & 2012 \\
\hline 1611.458 & 228.169 & Surface & 2012 & 6959.056 & 220.489 & Surface & 2012 \\
\hline 1614.722 & 228.171 & Surface & 2012 & 6962.176 & 220.477 & Surface & 2012 \\
\hline 1617.987 & 228.178 & Surface & 2012 & 6965.297 & 220.475 & Surface & 2012 \\
\hline 1621.251 & 228.180 & Surface & 2012 & 6968.417 & 220.487 & Surface & 2012 \\
\hline 1624.515 & 228.190 & Surface & 2012 & 6971.538 & 220.492 & Surface & 2012 \\
\hline 1627.780 & 228.218 & Surface & 2012 & 6974.658 & 220.497 & Surface & 2012 \\
\hline 1631.044 & 228.219 & Surface & 2012 & 6977.779 & 220.506 & Surface & 2012 \\
\hline 1634.313 & 228.204 & Surface & 2012 & 6980.899 & 220.473 & Surface & 2012 \\
\hline 1637.581 & 228.189 & Surface & 2012 & 6984.019 & 220.485 & Surface & 2012 \\
\hline 1640.850 & 228.194 & Surface & 2012 & 6987.140 & 220.491 & Surface & 2012 \\
\hline 1644.119 & 228.192 & Surface & 2012 & 6990.260 & 220.481 & Surface & 2012 \\
\hline 1647.388 & 228.187 & Surface & 2012 & 6993.522 & 220.470 & Surface & 2012 \\
\hline 1650.656 & 228.185 & Surface & 2012 & 6996.784 & 220.463 & Surface & 2012 \\
\hline 1653.925 & 228.210 & Surface & 2012 & 7000.046 & 220.472 & Surface & 2012 \\
\hline 1657.194 & 228.211 & Surface & 2012 & 7003.308 & 220.483 & Surface & 2012 \\
\hline 1660.463 & 228.193 & Surface & 2012 & 7006.570 & 220.500 & Surface & 2012 \\
\hline 1663.732 & 228.213 & Surface & 2012 & 7009.832 & 220.468 & Surface & 2012 \\
\hline 1667.000 & 228.219 & Surface & 2012 & 7013.094 & 220.463 & Surface & 2012 \\
\hline 1670.269 & 228.204 & Surface & 2012 & 7016.355 & 220.473 & Surface & 2012 \\
\hline 1673.538 & 228.192 & Surface & 2012 & 7019.618 & 220.466 & Surface & 2012 \\
\hline 1676.806 & 228.201 & Surface & 2012 & 7022.879 & 220.482 & Surface & 2012 \\
\hline 1680.075 & 228.198 & Surface & 2012 & 7026.141 & 220.486 & Surface & 2012 \\
\hline 1683.344 & 228.191 & Surface & 2012 & 7029.403 & 220.478 & Surface & 2012 \\
\hline 1686.613 & 228.202 & Surface & 2012 & 7032.665 & 220.469 & Surface & 2012 \\
\hline 1689.881 & 228.204 & Surface & 2012 & 7035.927 & 220.454 & Surface & 2012 \\
\hline 1693.150 & 228.203 & Surface & 2012 & 7039.189 & 220.443 & Surface & 2012 \\
\hline
\end{tabular}




\begin{tabular}{|c|c|c|c|c|c|c|c|}
\hline 1696.419 & 228.192 & Surface & 2012 & 7042.451 & 220.452 & Surface & 2012 \\
\hline 1699.688 & 228.198 & Surface & 2012 & 7045.713 & 220.466 & Surface & 2012 \\
\hline 1702.757 & 228.212 & Surface & 2012 & 7048.975 & 220.464 & Surface & 2012 \\
\hline 1705.827 & 228.220 & Surface & 2012 & 7052.236 & 220.482 & Surface & 2012 \\
\hline 1708.897 & 228.209 & Surface & 2012 & 7055.499 & 220.462 & Surface & 2012 \\
\hline 1711.966 & 228.190 & Surface & 2012 & 7058.760 & 220.447 & Surface & 2012 \\
\hline 1715.036 & 228.208 & Surface & 2012 & 7061.887 & 220.442 & Surface & 2012 \\
\hline 1718.105 & 228.200 & Surface & 2012 & 7065.014 & 220.441 & Surface & 2012 \\
\hline 1721.175 & 228.206 & Surface & 2012 & 7068.140 & 220.461 & Surface & 2012 \\
\hline 1724.245 & 228.195 & Surface & 2012 & 7071.266 & 220.470 & Surface & 2012 \\
\hline 1727.314 & 228.189 & Surface & 2012 & 7074.393 & 220.463 & Surface & 2012 \\
\hline 1730.384 & 228.199 & Surface & 2012 & 7077.519 & 220.470 & Surface & 2012 \\
\hline 1733.454 & 228.200 & Surface & 2012 & 7080.646 & 220.470 & Surface & 2012 \\
\hline 1736.523 & 228.196 & Surface & 2012 & 7083.772 & 220.462 & Surface & 2012 \\
\hline 1739.593 & 228.170 & Surface & 2012 & 7086.899 & 220.458 & Surface & 2012 \\
\hline 1742.663 & 228.170 & Surface & 2012 & 7090.025 & 220.449 & Surface & 2012 \\
\hline 1745.732 & 228.185 & Surface & 2012 & 7093.151 & 220.443 & Surface & 2012 \\
\hline 1748.886 & 228.185 & Surface & 2012 & 7096.278 & 220.452 & Surface & 2012 \\
\hline 1752.040 & 228.187 & Surface & 2012 & 7099.404 & 220.438 & Surface & 2012 \\
\hline 1755.193 & 228.177 & Surface & 2012 & 7102.512 & 220.428 & Surface & 2012 \\
\hline 1758.347 & 228.171 & Surface & 2012 & 7105.620 & 220.436 & Surface & 2012 \\
\hline 1761.501 & 228.174 & Surface & 2012 & 7108.728 & 220.452 & Surface & 2012 \\
\hline 1764.655 & 228.175 & Surface & 2012 & 7111.836 & 220.434 & Surface & 2012 \\
\hline 1767.809 & 228.183 & Surface & 2012 & 7114.944 & 220.422 & Surface & 2012 \\
\hline 1770.963 & 228.191 & Surface & 2012 & 7118.051 & 220.424 & Surface & 2012 \\
\hline 1774.116 & 228.177 & Surface & 2012 & 7121.159 & 220.425 & Surface & 2012 \\
\hline 1777.270 & 228.173 & Surface & 2012 & 7124.267 & 220.428 & Surface & 2012 \\
\hline 1780.424 & 228.177 & Surface & 2012 & 7127.375 & 220.432 & Surface & 2012 \\
\hline 1783.588 & 228.181 & Surface & 2012 & 7130.482 & 220.428 & Surface & 2012 \\
\hline 1786.752 & 228.185 & Surface & 2012 & 7133.590 & 220.418 & Surface & 2012 \\
\hline 1789.915 & 228.174 & Surface & 2012 & 7136.698 & 220.406 & Surface & 2012 \\
\hline 1793.079 & 228.188 & Surface & 2012 & 7139.943 & 220.405 & Surface & 2012 \\
\hline 1796.242 & 228.200 & Surface & 2012 & 7143.189 & 220.405 & Surface & 2012 \\
\hline 1799.406 & 228.177 & Surface & 2012 & 7146.434 & 220.401 & Surface & 2012 \\
\hline 1802.570 & 228.164 & Surface & 2012 & 7149.680 & 220.397 & Surface & 2012 \\
\hline 1805.733 & 228.162 & Surface & 2012 & 7152.925 & 220.397 & Surface & 2012 \\
\hline 1808.897 & 228.175 & Surface & 2012 & 7156.170 & 220.410 & Surface & 2012 \\
\hline 1812.060 & 228.171 & Surface & 2012 & 7159.416 & 220.383 & Surface & 2012 \\
\hline 1815.224 & 228.173 & Surface & 2012 & 7162.662 & 220.386 & Surface & 2012 \\
\hline 1818.388 & 228.167 & Surface & 2012 & 7165.907 & 220.378 & Surface & 2012 \\
\hline 1821.551 & 228.170 & Surface & 2012 & 7169.153 & 220.389 & Surface & 2012 \\
\hline 1824.715 & 228.174 & Surface & 2012 & 7172.398 & 220.391 & Surface & 2012 \\
\hline 1827.878 & 228.167 & Surface & 2012 & 7175.643 & 220.395 & Surface & 2012 \\
\hline 1831.125 & 228.168 & Surface & 2012 & 7178.889 & 220.386 & Surface & 2012 \\
\hline 1834.372 & 228.178 & Surface & 2012 & 7181.997 & 220.394 & Surface & 2012 \\
\hline 1837.619 & 228.186 & Surface & 2012 & 7185.105 & 220.388 & Surface & 2012 \\
\hline 1840.865 & 228.179 & Surface & 2012 & 7188.213 & 220.380 & Surface & 2012 \\
\hline 1844.112 & 228.171 & Surface & 2012 & 7191.321 & 220.375 & Surface & 2012 \\
\hline 1847.359 & 228.164 & Surface & 2012 & 7194.429 & 220.365 & Surface & 2012 \\
\hline
\end{tabular}




\begin{tabular}{|c|c|c|c|c|c|c|c|}
\hline 1850.605 & 228.174 & Surface & 2012 & 7197.537 & 220.361 & Surface & 2012 \\
\hline 1853.852 & 228.172 & Surface & 2012 & 7200.646 & 220.342 & Surface & 2012 \\
\hline 1857.099 & 228.171 & Surface & 2012 & 7203.754 & 220.346 & Surface & 2012 \\
\hline 1860.345 & 228.179 & Surface & 2012 & 7206.862 & 220.337 & Surface & 2012 \\
\hline 1863.592 & 228.181 & Surface & 2012 & 7209.970 & 220.330 & Surface & 2012 \\
\hline 1866.839 & 228.161 & Surface & 2012 & 7213.078 & 220.341 & Surface & 2012 \\
\hline 1870.085 & 228.182 & Surface & 2012 & 7216.186 & 220.340 & Surface & 2012 \\
\hline 1873.332 & 228.168 & Surface & 2012 & 7219.294 & 220.329 & Surface & 2012 \\
\hline 1876.579 & 228.150 & Surface & 2012 & 7222.402 & 220.335 & Surface & 2012 \\
\hline 1879.825 & 228.176 & Surface & 2012 & 7225.658 & 220.324 & Surface & 2012 \\
\hline 1883.072 & 228.164 & Surface & 2012 & 7228.914 & 220.304 & Surface & 2012 \\
\hline 1886.319 & 228.174 & Surface & 2012 & 7232.170 & 220.301 & Surface & 2012 \\
\hline 1889.565 & 228.176 & Surface & 2012 & 7235.425 & 220.299 & Surface & 2012 \\
\hline 1892.699 & 228.163 & Surface & 2012 & 7238.681 & 220.289 & Surface & 2012 \\
\hline 1895.833 & 228.159 & Surface & 2012 & 7241.937 & 220.284 & Surface & 2012 \\
\hline 1898.967 & 228.167 & Surface & 2012 & 7245.193 & 220.280 & Surface & 2012 \\
\hline 1902.101 & 228.164 & Surface & 2012 & 7248.449 & 220.260 & Surface & 2012 \\
\hline 1905.235 & 228.167 & Surface & 2012 & 7251.705 & 220.239 & Surface & 2012 \\
\hline 1908.369 & 228.174 & Surface & 2012 & 7254.960 & 220.256 & Surface & 2012 \\
\hline 1911.503 & 228.173 & Surface & 2012 & 7258.216 & 220.255 & Surface & 2012 \\
\hline 1914.637 & 228.168 & Surface & 2012 & 7261.472 & 220.237 & Surface & 2012 \\
\hline 1917.771 & 228.165 & Surface & 2012 & 7264.728 & 220.231 & Surface & 2012 \\
\hline 1920.904 & 228.163 & Surface & 2012 & 7267.984 & 220.244 & Surface & 2012 \\
\hline 1924.038 & 228.163 & Surface & 2012 & 7271.239 & 220.232 & Surface & 2012 \\
\hline 1927.172 & 228.168 & Surface & 2012 & 7274.495 & 220.212 & Surface & 2012 \\
\hline 1930.306 & 228.161 & Surface & 2012 & 7277.751 & 220.180 & Surface & 2012 \\
\hline 1933.440 & 228.164 & Surface & 2012 & 7281.007 & 220.162 & Surface & 2012 \\
\hline 1936.574 & 228.174 & Surface & 2012 & 7284.244 & 220.183 & Surface & 2012 \\
\hline 1939.708 & 228.151 & Surface & 2012 & 7287.481 & 220.163 & Surface & 2012 \\
\hline 1942.804 & 228.166 & Surface & 2012 & 7290.717 & 220.169 & Surface & 2012 \\
\hline 1945.900 & 228.188 & Surface & 2012 & 7293.954 & 220.157 & Surface & 2012 \\
\hline 1948.996 & 228.168 & Surface & 2012 & 7297.191 & 220.145 & Surface & 2012 \\
\hline 1952.092 & 228.151 & Surface & 2012 & 7300.428 & 220.122 & Surface & 2012 \\
\hline 1955.188 & 228.156 & Surface & 2012 & 7303.665 & 220.120 & Surface & 2012 \\
\hline 1958.283 & 228.153 & Surface & 2012 & 7306.902 & 220.134 & Surface & 2012 \\
\hline 1961.379 & 228.163 & Surface & 2012 & 7310.139 & 220.114 & Surface & 2012 \\
\hline 1964.475 & 228.164 & Surface & 2012 & 7313.375 & 220.093 & Surface & 2012 \\
\hline 1967.571 & 228.163 & Surface & 2012 & 7316.612 & 220.094 & Surface & 2012 \\
\hline 1970.667 & 228.164 & Surface & 2012 & 7319.849 & 220.109 & Surface & 2012 \\
\hline 1973.763 & 228.166 & Surface & 2012 & 7323.086 & 219.990 & Surface & 2012 \\
\hline 1976.859 & 228.169 & Surface & 2012 & 7326.323 & 220.019 & Surface & 2012 \\
\hline 1979.955 & 228.158 & Surface & 2012 & 7329.560 & 220.038 & Surface & 2012 \\
\hline 1983.051 & 228.143 & Surface & 2012 & 7332.797 & 219.980 & Surface & 2012 \\
\hline 1986.302 & 228.149 & Surface & 2012 & 7336.034 & 219.940 & Surface & 2012 \\
\hline 1989.554 & 228.142 & Surface & 2012 & 7339.130 & 219.867 & Surface & 2012 \\
\hline 1992.805 & 228.149 & Surface & 2012 & 7342.226 & 219.883 & Surface & 2012 \\
\hline 1996.057 & 228.140 & Surface & 2012 & 7345.322 & 219.892 & Surface & 2012 \\
\hline 1999.308 & 228.139 & Surface & 2012 & 7348.417 & 219.881 & Surface & 2012 \\
\hline 2002.560 & 228.121 & Surface & 2012 & 7351.513 & 219.818 & Surface & 2012 \\
\hline
\end{tabular}




\begin{tabular}{|c|c|c|c|c|c|c|c|}
\hline 2005.811 & 228.130 & Surface & 2012 & 7354.609 & 219.800 & Surface & 2012 \\
\hline 2009.063 & 228.127 & Surface & 2012 & 7357.705 & 219.751 & Surface & 2012 \\
\hline 2012.314 & 228.139 & Surface & 2012 & 7360.801 & 219.748 & Surface & 2012 \\
\hline 2015.566 & 228.129 & Surface & 2012 & 7363.897 & 219.727 & Surface & 2012 \\
\hline 2018.817 & 228.130 & Surface & 2012 & 7366.993 & 219.660 & Surface & 2012 \\
\hline 2022.069 & 228.107 & Surface & 2012 & 7370.089 & 219.557 & Surface & 2012 \\
\hline 2025.320 & 228.110 & Surface & 2012 & 7373.185 & 219.540 & Surface & 2012 \\
\hline 2028.572 & 228.122 & Surface & 2012 & 7376.281 & 219.529 & Surface & 2012 \\
\hline 2031.823 & 228.128 & Surface & 2012 & 7379.377 & 219.473 & Surface & 2012 \\
\hline 2035.075 & 228.117 & Surface & 2012 & 7382.516 & 219.414 & Surface & 2012 \\
\hline 2038.177 & 228.109 & Surface & 2012 & 7385.655 & 219.387 & Surface & 2012 \\
\hline 2041.279 & 228.115 & Surface & 2012 & 7388.795 & 219.347 & Surface & 2012 \\
\hline 2044.381 & 228.125 & Surface & 2012 & 7391.934 & 219.299 & Surface & 2012 \\
\hline 2047.484 & 228.115 & Surface & 2012 & 7395.073 & 219.253 & Surface & 2012 \\
\hline 2050.586 & 228.097 & Surface & 2012 & 7398.212 & 219.202 & Surface & 2012 \\
\hline 2053.688 & 228.107 & Surface & 2012 & 7401.352 & 219.173 & Surface & 2012 \\
\hline 2056.790 & 228.100 & Surface & 2012 & 7404.491 & 219.173 & Surface & 2012 \\
\hline 2059.893 & 228.101 & Surface & 2012 & 7407.630 & 219.155 & Surface & 2012 \\
\hline 2062.995 & 228.110 & Surface & 2012 & 7410.770 & 219.089 & Surface & 2012 \\
\hline 2066.097 & 228.100 & Surface & 2012 & 7413.909 & 219.031 & Surface & 2012 \\
\hline 2069.200 & 228.100 & Surface & 2012 & 7417.048 & 218.950 & Surface & 2012 \\
\hline 2072.302 & 228.112 & Surface & 2012 & 7420.260 & 218.935 & Surface & 2012 \\
\hline 2075.325 & 228.104 & Surface & 2012 & 7423.471 & 218.888 & Surface & 2012 \\
\hline 2078.349 & 228.110 & Surface & 2012 & 7426.683 & 218.837 & Surface & 2012 \\
\hline 2081.372 & 228.116 & Surface & 2012 & 7429.894 & 218.828 & Surface & 2012 \\
\hline 2084.395 & 228.109 & Surface & 2012 & 7433.105 & 218.812 & Surface & 2012 \\
\hline 2087.418 & 228.114 & Surface & 2012 & 7436.316 & 218.784 & Surface & 2012 \\
\hline 2090.442 & 228.106 & Surface & 2012 & 7439.528 & 218.798 & Surface & 2012 \\
\hline 2093.465 & 228.110 & Surface & 2012 & 7442.739 & 218.810 & Surface & 2012 \\
\hline 2096.488 & 228.106 & Surface & 2012 & 7445.951 & 218.814 & Surface & 2012 \\
\hline 2099.511 & 228.109 & Surface & 2012 & 7449.162 & 218.811 & Surface & 2012 \\
\hline 2102.760 & 228.122 & Surface & 2012 & 7452.374 & 218.806 & Surface & 2012 \\
\hline 2106.009 & 228.134 & Surface & 2012 & 7455.457 & 218.805 & Surface & 2012 \\
\hline 2109.258 & 228.129 & Surface & 2012 & 7458.541 & 218.807 & Surface & 2012 \\
\hline 2112.507 & 228.120 & Surface & 2012 & 7461.625 & 218.794 & Surface & 2012 \\
\hline 2115.755 & 228.122 & Surface & 2012 & 7464.709 & 218.801 & Surface & 2012 \\
\hline 2119.004 & 228.128 & Surface & 2012 & 7467.792 & 218.794 & Surface & 2012 \\
\hline 2122.253 & 228.108 & Surface & 2012 & 7470.876 & 218.801 & Surface & 2012 \\
\hline 2125.502 & 228.093 & Surface & 2012 & 7473.960 & 218.788 & Surface & 2012 \\
\hline 2128.751 & 228.098 & Surface & 2012 & 7477.043 & 218.776 & Surface & 2012 \\
\hline 2132.000 & 228.104 & Surface & 2012 & 7480.127 & 218.767 & Surface & 2012 \\
\hline 2135.249 & 228.102 & Surface & 2012 & 7483.211 & 218.732 & Surface & 2012 \\
\hline 2138.497 & 228.105 & Surface & 2012 & 7486.295 & 218.745 & Surface & 2012 \\
\hline 2141.746 & 228.112 & Surface & 2012 & 7489.378 & 218.751 & Surface & 2012 \\
\hline 2144.896 & 228.114 & Surface & 2012 & 7492.462 & 218.731 & Surface & 2012 \\
\hline 2148.046 & 228.111 & Surface & 2012 & 7495.546 & 218.740 & Surface & 2012 \\
\hline 2151.196 & 228.110 & Surface & 2012 & 7498.743 & 218.723 & Surface & 2012 \\
\hline 2154.347 & 228.110 & Surface & 2012 & 7501.940 & 218.735 & Surface & 2012 \\
\hline 2157.497 & 228.110 & Surface & 2012 & 7505.136 & 218.737 & Surface & 2012 \\
\hline
\end{tabular}




\begin{tabular}{|c|c|c|c|c|c|c|c|}
\hline 2160.647 & 228.114 & Surface & 2012 & 7508.333 & 218.725 & Surface & 2012 \\
\hline 2163.797 & 228.120 & Surface & 2012 & 7511.530 & 218.704 & Surface & 2012 \\
\hline 2166.947 & 228.118 & Surface & 2012 & 7514.727 & 218.680 & Surface & 2012 \\
\hline 2170.097 & 228.107 & Surface & 2012 & 7517.924 & 218.704 & Surface & 2012 \\
\hline 2173.248 & 228.095 & Surface & 2012 & 7521.120 & 218.679 & Surface & 2012 \\
\hline 2176.398 & 228.090 & Surface & 2012 & 7524.317 & 218.671 & Surface & 2012 \\
\hline 2179.548 & 228.090 & Surface & 2012 & 7527.514 & 218.671 & Surface & 2012 \\
\hline 2182.708 & 228.090 & Surface & 2012 & 7530.711 & 218.658 & Surface & 2012 \\
\hline 2185.868 & 228.090 & Surface & 2012 & 7533.908 & 218.670 & Surface & 2012 \\
\hline 2189.028 & 228.090 & Surface & 2012 & 7537.105 & 218.651 & Surface & 2012 \\
\hline 2192.188 & 228.090 & Surface & 2012 & 7540.183 & 218.625 & Surface & 2012 \\
\hline 2195.349 & 228.090 & Surface & 2012 & 7543.262 & 218.604 & Surface & 2012 \\
\hline 2198.508 & 228.090 & Surface & 2012 & 7546.340 & 218.587 & Surface & 2012 \\
\hline 2201.669 & 228.090 & Surface & 2012 & 7549.419 & 218.621 & Surface & 2012 \\
\hline 2204.829 & 228.090 & Surface & 2012 & 7552.498 & 218.612 & Surface & 2012 \\
\hline 2207.989 & 228.090 & Surface & 2012 & 7555.576 & 218.582 & Surface & 2012 \\
\hline 2211.149 & 228.090 & Surface & 2012 & 7558.655 & 218.585 & Surface & 2012 \\
\hline 2214.309 & 228.090 & Surface & 2012 & 7561.734 & 218.577 & Surface & 2012 \\
\hline 2217.469 & 228.090 & Surface & 2012 & 7564.812 & 218.570 & Surface & 2012 \\
\hline 2220.629 & 228.090 & Surface & 2012 & 7567.891 & 218.565 & Surface & 2012 \\
\hline 2223.683 & 228.090 & Surface & 2012 & 7570.970 & 218.552 & Surface & 2012 \\
\hline 2226.737 & 228.085 & Surface & 2012 & 7574.048 & 218.508 & Surface & 2012 \\
\hline 2229.790 & 228.080 & Surface & 2012 & 7577.127 & 218.461 & Surface & 2012 \\
\hline 2232.844 & 228.080 & Surface & 2012 & 7580.205 & 218.469 & Surface & 2012 \\
\hline 2235.898 & 228.080 & Surface & 2012 & 7583.432 & 218.487 & Surface & 2012 \\
\hline 2238.951 & 228.078 & Surface & 2012 & 7586.659 & 218.474 & Surface & 2012 \\
\hline 2242.005 & 228.070 & Surface & 2012 & 7589.886 & 218.444 & Surface & 2012 \\
\hline 2245.058 & 228.071 & Surface & 2012 & 7593.113 & 218.441 & Surface & 2012 \\
\hline 2248.112 & 228.080 & Surface & 2012 & 7596.340 & 218.395 & Surface & 2012 \\
\hline 2251.166 & 228.079 & Surface & 2012 & 7599.567 & 218.356 & Surface & 2012 \\
\hline 2254.219 & 228.079 & Surface & 2012 & 7602.794 & 218.347 & Surface & 2012 \\
\hline 2257.273 & 228.083 & Surface & 2012 & 7606.021 & 218.376 & Surface & 2012 \\
\hline 2260.261 & 228.086 & Surface & 2012 & 7609.248 & 218.391 & Surface & 2012 \\
\hline 2263.249 & 228.082 & Surface & 2012 & 7612.475 & 218.355 & Surface & 2012 \\
\hline 2266.237 & 228.075 & Surface & 2012 & 7615.702 & 218.333 & Surface & 2012 \\
\hline 2269.225 & 228.069 & Surface & 2012 & 7618.929 & 218.324 & Surface & 2012 \\
\hline 2272.213 & 228.065 & Surface & 2012 & 7622.156 & 218.304 & Surface & 2012 \\
\hline 2275.201 & 228.065 & Surface & 2012 & 7625.383 & 218.300 & Surface & 2012 \\
\hline 2278.190 & 228.070 & Surface & 2012 & 7628.610 & 218.281 & Surface & 2012 \\
\hline 2281.178 & 228.070 & Surface & 2012 & 7631.836 & 218.253 & Surface & 2012 \\
\hline 2284.166 & 228.061 & Surface & 2012 & 7635.080 & 218.246 & Surface & 2012 \\
\hline 2287.154 & 228.060 & Surface & 2012 & 7638.323 & 218.246 & Surface & 2012 \\
\hline 2290.142 & 228.061 & Surface & 2012 & 7641.566 & 218.198 & Surface & 2012 \\
\hline 2293.404 & 228.061 & Surface & 2012 & 7644.809 & 218.201 & Surface & 2012 \\
\hline 2296.666 & 228.060 & Surface & 2012 & 7648.052 & 218.187 & Surface & 2012 \\
\hline 2299.929 & 228.060 & Surface & 2012 & 7651.295 & 218.182 & Surface & 2012 \\
\hline 2303.191 & 228.057 & Surface & 2012 & 7654.538 & 218.181 & Surface & 2012 \\
\hline 2306.453 & 228.052 & Surface & 2012 & 7657.781 & 218.190 & Surface & 2012 \\
\hline 2309.715 & 228.050 & Surface & 2012 & 7661.024 & 218.196 & Surface & 2012 \\
\hline
\end{tabular}




\begin{tabular}{|c|c|c|c|c|c|c|c|}
\hline 2312.978 & 228.050 & Surface & 2012 & 7664.267 & 218.194 & Surface & 2012 \\
\hline 2316.240 & 228.050 & Surface & 2012 & 7667.510 & 218.159 & Surface & 2012 \\
\hline 2319.502 & 228.044 & Surface & 2012 & 7670.754 & 218.178 & Surface & 2012 \\
\hline 2322.764 & 228.040 & Surface & 2012 & 7673.997 & 218.146 & Surface & 2012 \\
\hline 2326.027 & 228.040 & Surface & 2012 & 7677.240 & 218.125 & Surface & 2012 \\
\hline 2329.289 & 228.040 & Surface & 2012 & 7680.483 & 218.149 & Surface & 2012 \\
\hline 2332.551 & 228.040 & Surface & 2012 & 7683.647 & 218.131 & Surface & 2012 \\
\hline 2335.539 & 228.040 & Surface & 2012 & 7686.811 & 218.114 & Surface & 2012 \\
\hline 2338.527 & 228.040 & Surface & 2012 & 7689.976 & 218.095 & Surface & 2012 \\
\hline 2341.515 & 228.040 & Surface & 2012 & 7693.140 & 218.076 & Surface & 2012 \\
\hline 2344.503 & 228.038 & Surface & 2012 & 7696.304 & 218.090 & Surface & 2012 \\
\hline 2347.491 & 228.030 & Surface & 2012 & 7699.469 & 218.126 & Surface & 2012 \\
\hline 2350.480 & 228.030 & Surface & 2012 & 7702.633 & 218.115 & Surface & 2012 \\
\hline 2353.468 & 228.030 & Surface & 2012 & 7705.797 & 218.066 & Surface & 2012 \\
\hline 2356.456 & 228.030 & Surface & 2012 & 7708.961 & 218.058 & Surface & 2012 \\
\hline 2359.444 & 228.030 & Surface & 2012 & 7712.126 & 218.029 & Surface & 2012 \\
\hline 2362.432 & 228.030 & Surface & 2012 & 7715.290 & 218.037 & Surface & 2012 \\
\hline 2365.420 & 228.030 & Surface & 2012 & 7718.454 & 218.067 & Surface & 2012 \\
\hline 2368.614 & 228.026 & Surface & 2012 & 7721.619 & 218.041 & Surface & 2012 \\
\hline 2371.808 & 228.020 & Surface & 2012 & 7724.783 & 218.026 & Surface & 2012 \\
\hline 2375.002 & 228.020 & Surface & 2012 & 7727.947 & 218.036 & Surface & 2012 \\
\hline 2378.196 & 228.020 & Surface & 2012 & 7731.112 & 218.009 & Surface & 2012 \\
\hline 2381.390 & 228.020 & Surface & 2012 & 7734.276 & 217.992 & Surface & 2012 \\
\hline 2384.584 & 228.020 & Surface & 2012 & 7737.417 & 217.972 & Surface & 2012 \\
\hline 2387.778 & 228.020 & Surface & 2012 & 7740.558 & 218.013 & Surface & 2012 \\
\hline 2390.972 & 228.020 & Surface & 2012 & 7743.699 & 218.018 & Surface & 2012 \\
\hline 2394.166 & 228.022 & Surface & 2012 & 7746.840 & 218.026 & Surface & 2012 \\
\hline 2397.360 & 228.030 & Surface & 2012 & 7749.981 & 218.030 & Surface & 2012 \\
\hline 2400.554 & 228.031 & Surface & 2012 & 7753.122 & 218.011 & Surface & 2012 \\
\hline 2403.748 & 228.041 & Surface & 2012 & 7756.263 & 218.002 & Surface & 2012 \\
\hline 2406.942 & 228.050 & Surface & 2012 & 7759.404 & 217.989 & Surface & 2012 \\
\hline 2410.136 & 228.050 & Surface & 2012 & 7762.545 & 217.986 & Surface & 2012 \\
\hline 2413.330 & 228.049 & Surface & 2012 & 7765.686 & 218.002 & Surface & 2012 \\
\hline 2416.524 & 228.030 & Surface & 2012 & 7768.827 & 217.996 & Surface & 2012 \\
\hline 2419.450 & 228.020 & Surface & 2012 & 7771.968 & 217.988 & Surface & 2012 \\
\hline 2422.377 & 228.003 & Surface & 2012 & 7775.109 & 218.000 & Surface & 2012 \\
\hline 2425.304 & 227.999 & Surface & 2012 & 7778.250 & 218.008 & Surface & 2012 \\
\hline 2428.230 & 228.006 & Surface & 2012 & 7781.391 & 217.993 & Surface & 2012 \\
\hline 2431.157 & 228.007 & Surface & 2012 & 7784.445 & 217.983 & Surface & 2012 \\
\hline 2434.084 & 228.000 & Surface & 2012 & 7787.499 & 217.984 & Surface & 2012 \\
\hline 2437.011 & 227.996 & Surface & 2012 & 7790.553 & 217.981 & Surface & 2012 \\
\hline 2439.937 & 228.001 & Surface & 2012 & 7793.606 & 217.988 & Surface & 2012 \\
\hline 2443.042 & 228.004 & Surface & 2012 & 7796.660 & 217.991 & Surface & 2012 \\
\hline 2446.148 & 227.987 & Surface & 2012 & 7799.714 & 217.991 & Surface & 2012 \\
\hline 2449.253 & 227.969 & Surface & 2012 & 7802.768 & 217.986 & Surface & 2012 \\
\hline 2452.358 & 227.955 & Surface & 2012 & 7805.822 & 217.980 & Surface & 2012 \\
\hline 2455.463 & 227.975 & Surface & 2012 & 7808.875 & 217.976 & Surface & 2012 \\
\hline 2458.568 & 227.980 & Surface & 2012 & 7811.929 & 217.971 & Surface & 2012 \\
\hline 2461.673 & 227.973 & Surface & 2012 & 7814.983 & 217.966 & Surface & 2012 \\
\hline
\end{tabular}




\begin{tabular}{|c|c|c|c|c|c|c|c|}
\hline 2464.779 & 227.989 & Surface & 2012 & 7818.212 & 217.967 & Surface & 2012 \\
\hline 2467.884 & 227.984 & Surface & 2012 & 7821.441 & 217.981 & Surface & 2012 \\
\hline 2471.149 & 227.967 & Surface & 2012 & 7824.669 & 217.969 & Surface & 2012 \\
\hline 2474.414 & 227.963 & Surface & 2012 & 7827.898 & 217.974 & Surface & 2012 \\
\hline 2477.679 & 227.955 & Surface & 2012 & 7831.127 & 217.985 & Surface & 2012 \\
\hline 2480.944 & 227.939 & Surface & 2012 & 7834.356 & 217.957 & Surface & 2012 \\
\hline 2484.210 & 227.924 & Surface & 2012 & 7837.585 & 217.990 & Surface & 2012 \\
\hline 2487.475 & 227.914 & Surface & 2012 & 7840.813 & 217.988 & Surface & 2012 \\
\hline 2490.740 & 227.897 & Surface & 2012 & 7844.042 & 217.973 & Surface & 2012 \\
\hline 2494.005 & 227.894 & Surface & 2012 & 7847.271 & 217.999 & Surface & 2012 \\
\hline 2497.270 & 227.907 & Surface & 2012 & 7850.500 & 217.988 & Surface & 2012 \\
\hline 2500.470 & 227.905 & Surface & 2012 & 7853.644 & 217.987 & Surface & 2012 \\
\hline 2503.671 & 227.893 & Surface & 2012 & 7856.788 & 217.990 & Surface & 2012 \\
\hline 2506.871 & 227.874 & Surface & 2012 & 7859.932 & 217.993 & Surface & 2012 \\
\hline 2510.071 & 227.860 & Surface & 2012 & 7863.076 & 218.006 & Surface & 2012 \\
\hline 2513.271 & 227.841 & Surface & 2012 & 7866.220 & 218.001 & Surface & 2012 \\
\hline 2516.471 & 227.821 & Surface & 2012 & 7869.365 & 218.000 & Surface & 2012 \\
\hline 2519.672 & 227.822 & Surface & 2012 & 7872.509 & 218.002 & Surface & 2012 \\
\hline 2522.872 & 227.807 & Surface & 2012 & 7875.653 & 217.996 & Surface & 2012 \\
\hline 2526.072 & 227.778 & Surface & 2012 & 7878.797 & 217.999 & Surface & 2012 \\
\hline 2529.272 & 227.782 & Surface & 2012 & 7882.024 & 217.995 & Surface & 2012 \\
\hline 2532.472 & 227.788 & Surface & 2012 & 7885.251 & 218.000 & Surface & 2012 \\
\hline 2535.673 & 227.779 & Surface & 2012 & 7888.478 & 218.004 & Surface & 2012 \\
\hline 2538.873 & 227.783 & Surface & 2012 & 7891.705 & 217.996 & Surface & 2012 \\
\hline 2542.037 & 227.769 & Surface & 2012 & 7894.932 & 217.993 & Surface & 2012 \\
\hline 2545.200 & 227.763 & Surface & 2012 & 7898.159 & 217.989 & Surface & 2012 \\
\hline 2548.364 & 227.757 & Surface & 2012 & 7901.386 & 217.993 & Surface & 2012 \\
\hline 2551.527 & 227.751 & Surface & 2012 & 7904.613 & 218.001 & Surface & 2012 \\
\hline 2554.691 & 227.754 & Surface & 2012 & 7907.704 & 218.017 & Surface & 2012 \\
\hline 2557.854 & 227.747 & Surface & 2012 & 7910.795 & 218.017 & Surface & 2012 \\
\hline 2561.018 & 227.714 & Surface & 2012 & 7913.886 & 218.008 & Surface & 2012 \\
\hline 2564.181 & 227.716 & Surface & 2012 & 7916.976 & 217.991 & Surface & 2012 \\
\hline 2567.345 & 227.709 & Surface & 2012 & 7920.068 & 217.982 & Surface & 2012 \\
\hline 2570.509 & 227.681 & Surface & 2012 & 7923.158 & 217.984 & Surface & 2012 \\
\hline 2573.672 & 227.674 & Surface & 2012 & 7926.249 & 217.990 & Surface & 2012 \\
\hline 2576.836 & 227.694 & Surface & 2012 & 7929.340 & 217.989 & Surface & 2012 \\
\hline 2579.999 & 227.703 & Surface & 2012 & 7932.431 & 217.989 & Surface & 2012 \\
\hline 2583.279 & 227.691 & Surface & 2012 & 7935.522 & 217.995 & Surface & 2012 \\
\hline 2586.559 & 227.674 & Surface & 2012 & 7938.613 & 217.999 & Surface & 2012 \\
\hline 2589.838 & 227.661 & Surface & 2012 & 7941.704 & 217.992 & Surface & 2012 \\
\hline 2593.118 & 227.655 & Surface & 2012 & 7944.795 & 217.983 & Surface & 2012 \\
\hline 2596.397 & 227.658 & Surface & 2012 & 7947.886 & 217.957 & Surface & 2012 \\
\hline 2599.677 & 227.656 & Surface & 2012 & 7950.977 & 217.961 & Surface & 2012 \\
\hline 2602.957 & 227.659 & Surface & 2012 & 7954.087 & 217.967 & Surface & 2012 \\
\hline 2606.236 & 227.658 & Surface & 2012 & 7957.197 & 217.984 & Surface & 2012 \\
\hline 2609.516 & 227.634 & Surface & 2012 & 7960.306 & 217.974 & Surface & 2012 \\
\hline 2612.796 & 227.625 & Surface & 2012 & 7963.416 & 217.977 & Surface & 2012 \\
\hline 2616.075 & 227.612 & Surface & 2012 & 7966.525 & 217.985 & Surface & 2012 \\
\hline 2619.355 & 227.577 & Surface & 2012 & 7969.635 & 217.980 & Surface & 2012 \\
\hline
\end{tabular}




\begin{tabular}{|c|c|c|c|c|c|c|c|}
\hline 2622.635 & 227.541 & Surface & 2012 & 7972.745 & 217.979 & Surface & 2012 \\
\hline 2625.914 & 227.534 & Surface & 2012 & 7975.854 & 217.968 & Surface & 2012 \\
\hline 2629.194 & 227.548 & Surface & 2012 & 7978.964 & 217.974 & Surface & 2012 \\
\hline 2632.473 & 227.540 & Surface & 2012 & 7982.074 & 217.984 & Surface & 2012 \\
\hline 2635.753 & 227.522 & Surface & 2012 & 7985.276 & 217.970 & Surface & 2012 \\
\hline 2639.033 & 227.509 & Surface & 2012 & 7988.477 & 217.969 & Surface & 2012 \\
\hline 2642.312 & 227.492 & Surface & 2012 & 7991.679 & 217.967 & Surface & 2012 \\
\hline 2645.405 & 227.478 & Surface & 2012 & 7994.881 & 217.965 & Surface & 2012 \\
\hline 2648.497 & 227.471 & Surface & 2012 & 7998.082 & 217.952 & Surface & 2012 \\
\hline 2651.590 & 227.462 & Surface & 2012 & 8001.284 & 217.970 & Surface & 2012 \\
\hline 2654.682 & 227.455 & Surface & 2012 & 8004.486 & 217.982 & Surface & 2012 \\
\hline 2657.775 & 227.450 & Surface & 2012 & 8007.687 & 218.005 & Surface & 2012 \\
\hline 2660.867 & 227.426 & Surface & 2012 & 8010.889 & 217.982 & Surface & 2012 \\
\hline 2663.960 & 227.412 & Surface & 2012 & 8014.131 & 217.957 & Surface & 2012 \\
\hline 2667.052 & 227.390 & Surface & 2012 & 8017.374 & 217.969 & Surface & 2012 \\
\hline 2670.145 & 227.379 & Surface & 2012 & 8020.616 & 217.972 & Surface & 2012 \\
\hline 2673.237 & 227.360 & Surface & 2012 & 8023.859 & 217.979 & Surface & 2012 \\
\hline 2676.330 & 227.379 & Surface & 2012 & 8027.101 & 217.980 & Surface & 2012 \\
\hline 2679.422 & 227.389 & Surface & 2012 & 8030.344 & 217.988 & Surface & 2012 \\
\hline 2682.515 & 227.364 & Surface & 2012 & 8033.586 & 217.983 & Surface & 2012 \\
\hline 2685.644 & 227.342 & Surface & 2012 & 8036.829 & 217.975 & Surface & 2012 \\
\hline 2688.774 & 227.339 & Surface & 2012 & 8040.071 & 217.972 & Surface & 2012 \\
\hline 2691.904 & 227.343 & Surface & 2012 & 8043.314 & 217.971 & Surface & 2012 \\
\hline 2695.033 & 227.333 & Surface & 2012 & 8046.556 & 217.964 & Surface & 2012 \\
\hline 2698.163 & 227.313 & Surface & 2012 & 8049.799 & 217.962 & Surface & 2012 \\
\hline 2701.293 & 227.291 & Surface & 2012 & 8052.894 & 217.965 & Surface & 2012 \\
\hline 2704.422 & 227.263 & Surface & 2012 & 8055.989 & 217.967 & Surface & 2012 \\
\hline 2707.552 & 227.260 & Surface & 2012 & 8059.084 & 217.966 & Surface & 2012 \\
\hline 2710.682 & 227.284 & Surface & 2012 & 8062.179 & 217.960 & Surface & 2012 \\
\hline 2713.811 & 227.269 & Surface & 2012 & 8065.275 & 217.960 & Surface & 2012 \\
\hline 2716.941 & 227.218 & Surface & 2012 & 8068.370 & 217.960 & Surface & 2012 \\
\hline 2720.071 & 227.201 & Surface & 2012 & 8071.465 & 217.960 & Surface & 2012 \\
\hline 2723.200 & 227.169 & Surface & 2012 & 8074.560 & 217.955 & Surface & 2012 \\
\hline 2726.330 & 227.145 & Surface & 2012 & 8077.655 & 217.954 & Surface & 2012 \\
\hline 2729.459 & 227.150 & Surface & 2012 & 8080.751 & 217.957 & Surface & 2012 \\
\hline 2732.610 & 227.110 & Surface & 2012 & 8083.846 & 217.962 & Surface & 2012 \\
\hline 2735.761 & 227.090 & Surface & 2012 & 8087.019 & 217.971 & Surface & 2012 \\
\hline 2738.911 & 227.057 & Surface & 2012 & 8090.192 & 217.980 & Surface & 2012 \\
\hline 2742.062 & 227.057 & Surface & 2012 & 8093.364 & 217.973 & Surface & 2012 \\
\hline 2745.213 & 227.073 & Surface & 2012 & 8096.537 & 217.963 & Surface & 2012 \\
\hline 2748.363 & 227.036 & Surface & 2012 & 8099.710 & 217.960 & Surface & 2012 \\
\hline 2751.514 & 227.005 & Surface & 2012 & 8102.883 & 217.960 & Surface & 2012 \\
\hline 2754.664 & 226.984 & Surface & 2012 & 8106.055 & 217.960 & Surface & 2012 \\
\hline 2757.815 & 226.971 & Surface & 2012 & 8109.228 & 217.960 & Surface & 2012 \\
\hline 2760.966 & 226.976 & Surface & 2012 & 8112.401 & 217.960 & Surface & 2012 \\
\hline 2764.116 & 226.975 & Surface & 2012 & 8115.574 & 217.960 & Surface & 2012 \\
\hline 2767.267 & 226.977 & Surface & 2012 & 8118.746 & 217.960 & Surface & 2012 \\
\hline 2770.417 & 226.978 & Surface & 2012 & 8121.919 & 217.960 & Surface & 2012 \\
\hline 2773.568 & 226.933 & Surface & 2012 & 8125.092 & 217.960 & Surface & 2012 \\
\hline
\end{tabular}




\begin{tabular}{|c|c|c|c|c|c|c|c|}
\hline 2776.719 & 226.870 & Surface & 2012 & 8128.293 & 217.961 & Surface & 2012 \\
\hline 2779.843 & 226.829 & Surface & 2012 & 8131.495 & 217.960 & Surface & 2012 \\
\hline 2782.967 & 226.803 & Surface & 2012 & 8134.696 & 217.964 & Surface & 2012 \\
\hline 2786.092 & 226.739 & Surface & 2012 & 8137.898 & 217.970 & Surface & 2012 \\
\hline 2789.216 & 226.722 & Surface & 2012 & 8141.099 & 217.977 & Surface & 2012 \\
\hline 2792.340 & 226.705 & Surface & 2012 & 8144.300 & 217.980 & Surface & 2012 \\
\hline 2795.465 & 226.677 & Surface & 2012 & 8147.502 & 217.967 & Surface & 2012 \\
\hline 2798.589 & 226.686 & Surface & 2012 & 8150.703 & 217.951 & Surface & 2012 \\
\hline 2801.713 & 226.673 & Surface & 2012 & 8153.905 & 217.950 & Surface & 2012 \\
\hline 2804.837 & 226.676 & Surface & 2012 & 8157.106 & 217.944 & Surface & 2012 \\
\hline 2807.961 & 226.680 & Surface & 2012 & 8160.117 & 217.938 & Surface & 2012 \\
\hline 2811.086 & 226.697 & Surface & 2012 & 8163.127 & 217.930 & Surface & 2012 \\
\hline 2814.210 & 226.708 & Surface & 2012 & 8166.137 & 217.924 & Surface & 2012 \\
\hline 2817.334 & 226.697 & Surface & 2012 & 8169.147 & 217.908 & Surface & 2012 \\
\hline 2820.459 & 226.689 & Surface & 2012 & 8172.157 & 217.889 & Surface & 2012 \\
\hline 2823.583 & 226.693 & Surface & 2012 & 8175.167 & 217.886 & Surface & 2012 \\
\hline 2826.707 & 226.695 & Surface & 2012 & 8178.178 & 217.891 & Surface & 2012 \\
\hline 2829.832 & 226.708 & Surface & 2012 & 8181.188 & 217.896 & Surface & 2012 \\
\hline 2833.062 & 226.696 & Surface & 2012 & 8184.198 & 217.897 & Surface & 2012 \\
\hline 2836.293 & 226.710 & Surface & 2012 & 8187.208 & 217.876 & Surface & 2012 \\
\hline 2839.523 & 226.709 & Surface & 2012 & 8190.218 & 217.871 & Surface & 2012 \\
\hline 2842.753 & 226.699 & Surface & 2012 & 8193.359 & 217.863 & Surface & 2012 \\
\hline 2845.984 & 226.690 & Surface & 2012 & 8196.501 & 217.801 & Surface & 2012 \\
\hline 2849.214 & 226.680 & Surface & 2012 & 8199.642 & 217.787 & Surface & 2012 \\
\hline 2852.444 & 226.689 & Surface & 2012 & 8202.783 & 217.754 & Surface & 2012 \\
\hline 2855.675 & 226.699 & Surface & 2012 & 8205.924 & 217.683 & Surface & 2012 \\
\hline 2858.905 & 226.692 & Surface & 2012 & 8209.066 & 217.572 & Surface & 2012 \\
\hline 2862.135 & 226.696 & Surface & 2012 & 8212.207 & 217.504 & Surface & 2012 \\
\hline 2865.366 & 226.704 & Surface & 2012 & 8215.348 & 217.491 & Surface & 2012 \\
\hline 2868.489 & 226.704 & Surface & 2012 & 8218.490 & 217.539 & Surface & 2012 \\
\hline 2871.612 & 226.705 & Surface & 2012 & 8221.631 & 217.420 & Surface & 2012 \\
\hline 2874.735 & 226.701 & Surface & 2012 & 8224.772 & 217.314 & Surface & 2012 \\
\hline 2877.859 & 226.703 & Surface & 2012 & 8227.913 & 217.226 & Surface & 2012 \\
\hline 2880.982 & 226.702 & Surface & 2012 & 8231.054 & 217.223 & Surface & 2012 \\
\hline 2884.105 & 226.703 & Surface & 2012 & 8234.196 & 217.206 & Surface & 2012 \\
\hline 2887.228 & 226.690 & Surface & 2012 & 8237.282 & 217.175 & Surface & 2012 \\
\hline 2890.388 & 226.694 & Surface & 2012 & 8240.367 & 217.150 & Surface & 2012 \\
\hline 2893.547 & 226.693 & Surface & 2012 & 8243.453 & 217.140 & Surface & 2012 \\
\hline 2896.706 & 226.702 & Surface & 2012 & 8246.539 & 217.121 & Surface & 2012 \\
\hline 2899.866 & 226.709 & Surface & 2012 & 8249.625 & 217.033 & Surface & 2012 \\
\hline 2903.025 & 226.705 & Surface & 2012 & 8252.711 & 216.997 & Surface & 2012 \\
\hline 2906.184 & 226.715 & Surface & 2012 & 8255.797 & 217.000 & Surface & 2012 \\
\hline 2909.343 & 226.728 & Surface & 2012 & 8258.883 & 217.011 & Surface & 2012 \\
\hline 2912.348 & 226.716 & Surface & 2012 & 8261.968 & 217.020 & Surface & 2012 \\
\hline 2915.353 & 226.707 & Surface & 2012 & 8265.054 & 216.974 & Surface & 2012 \\
\hline 2918.358 & 226.698 & Surface & 2012 & 8268.123 & 216.899 & Surface & 2012 \\
\hline 2921.363 & 226.691 & Surface & 2012 & 8271.192 & 216.887 & Surface & 2012 \\
\hline 2924.368 & 226.700 & Surface & 2012 & 8274.261 & 216.913 & Surface & 2012 \\
\hline 2927.373 & 226.683 & Surface & 2012 & 8277.330 & 216.875 & Surface & 2012 \\
\hline
\end{tabular}




\begin{tabular}{|c|c|c|c|c|c|c|c|}
\hline 2930.378 & 226.707 & Surface & 2012 & 8280.398 & 216.880 & Surface & 2012 \\
\hline 2933.383 & 226.705 & Surface & 2012 & 8283.467 & 216.854 & Surface & 2012 \\
\hline 2936.388 & 226.689 & Surface & 2012 & 8286.536 & 216.798 & Surface & 2012 \\
\hline 2939.393 & 226.687 & Surface & 2012 & 8289.604 & 216.793 & Surface & 2012 \\
\hline 2942.389 & 226.685 & Surface & 2012 & 8292.673 & 216.779 & Surface & 2012 \\
\hline 2945.386 & 226.693 & Surface & 2012 & 8295.742 & 216.772 & Surface & 2012 \\
\hline 2948.383 & 226.711 & Surface & 2012 & 8298.811 & 216.791 & Surface & 2012 \\
\hline 2951.379 & 226.711 & Surface & 2012 & 8301.955 & 216.770 & Surface & 2012 \\
\hline 2954.376 & 226.718 & Surface & 2012 & 8305.099 & 216.751 & Surface & 2012 \\
\hline 2957.373 & 226.710 & Surface & 2012 & 8308.243 & 216.677 & Surface & 2012 \\
\hline 2960.370 & 226.728 & Surface & 2012 & 8311.387 & 216.691 & Surface & 2012 \\
\hline 2963.367 & 226.730 & Surface & 2012 & 8314.531 & 216.711 & Surface & 2012 \\
\hline 2966.363 & 226.706 & Surface & 2012 & 8317.676 & 216.697 & Surface & 2012 \\
\hline 2969.360 & 226.685 & Surface & 2012 & 8320.819 & 216.700 & Surface & 2012 \\
\hline 2972.303 & 226.692 & Surface & 2012 & 8323.964 & 216.700 & Surface & 2012 \\
\hline 2975.246 & 226.695 & Surface & 2012 & 8327.108 & 216.688 & Surface & 2012 \\
\hline 2978.189 & 226.696 & Surface & 2012 & 8330.069 & 216.679 & Surface & 2012 \\
\hline 2981.132 & 226.705 & Surface & 2012 & 8333.031 & 216.669 & Surface & 2012 \\
\hline 2984.076 & 226.712 & Surface & 2012 & 8335.992 & 216.627 & Surface & 2012 \\
\hline 2987.019 & 226.715 & Surface & 2012 & 8338.954 & 216.562 & Surface & 2012 \\
\hline 2989.962 & 226.720 & Surface & 2012 & 8341.916 & 216.537 & Surface & 2012 \\
\hline 2992.905 & 226.724 & Surface & 2012 & 8344.877 & 216.591 & Surface & 2012 \\
\hline 2996.170 & 226.728 & Surface & 2012 & 8347.839 & 216.559 & Surface & 2012 \\
\hline 2999.436 & 226.720 & Surface & 2012 & 8350.800 & 216.534 & Surface & 2012 \\
\hline 3002.701 & 226.719 & Surface & 2012 & 8353.762 & 216.517 & Surface & 2012 \\
\hline 3005.967 & 226.697 & Surface & 2012 & 8356.724 & 216.460 & Surface & 2012 \\
\hline 3009.232 & 226.691 & Surface & 2012 & 8359.757 & 216.428 & Surface & 2012 \\
\hline 3012.498 & 226.696 & Surface & 2012 & 8362.791 & 216.447 & Surface & 2012 \\
\hline 3015.763 & 226.707 & Surface & 2012 & 8365.825 & 216.469 & Surface & 2012 \\
\hline 3019.029 & 226.719 & Surface & 2012 & 8368.859 & 216.461 & Surface & 2012 \\
\hline 3022.305 & 226.716 & Surface & 2012 & 8371.892 & 216.462 & Surface & 2012 \\
\hline 3025.582 & 226.710 & Surface & 2012 & 8374.926 & 216.453 & Surface & 2012 \\
\hline 3028.859 & 226.704 & Surface & 2012 & 8377.960 & 216.438 & Surface & 2012 \\
\hline 3032.136 & 226.703 & Surface & 2012 & 8380.993 & 216.397 & Surface & 2012 \\
\hline 3035.412 & 226.701 & Surface & 2012 & 8384.027 & 216.405 & Surface & 2012 \\
\hline 3038.689 & 226.700 & Surface & 2012 & 8387.061 & 216.439 & Surface & 2012 \\
\hline 3041.966 & 226.701 & Surface & 2012 & 8390.095 & 216.461 & Surface & 2012 \\
\hline 3045.243 & 226.694 & Surface & 2012 & 8393.210 & 216.451 & Surface & 2012 \\
\hline 3048.520 & 226.690 & Surface & 2012 & 8396.325 & 216.434 & Surface & 2012 \\
\hline 3051.652 & 226.692 & Surface & 2012 & 8399.440 & 216.430 & Surface & 2012 \\
\hline 3054.785 & 226.700 & Surface & 2012 & 8402.555 & 216.424 & Surface & 2012 \\
\hline 3057.918 & 226.703 & Surface & 2012 & 8405.670 & 216.389 & Surface & 2012 \\
\hline 3061.051 & 226.699 & Surface & 2012 & 8408.786 & 216.448 & Surface & 2012 \\
\hline 3064.183 & 226.664 & Surface & 2012 & 8411.901 & 216.476 & Surface & 2012 \\
\hline 3067.316 & 226.651 & Surface & 2012 & 8415.175 & 216.479 & Surface & 2012 \\
\hline 3070.449 & 226.650 & Surface & 2012 & 8418.449 & 216.457 & Surface & 2012 \\
\hline 3073.582 & 226.650 & Surface & 2012 & 8421.724 & 216.446 & Surface & 2012 \\
\hline 3076.714 & 226.650 & Surface & 2012 & 8424.998 & 216.452 & Surface & 2012 \\
\hline 3079.847 & 226.656 & Surface & 2012 & 8428.272 & 216.467 & Surface & 2012 \\
\hline
\end{tabular}




\begin{tabular}{|c|c|c|c|c|c|c|c|}
\hline 3082.980 & 226.663 & Surface & 2012 & 8431.547 & 216.446 & Surface & 2012 \\
\hline 3086.058 & 226.660 & Surface & 2012 & 8434.821 & 216.443 & Surface & 2012 \\
\hline 3089.137 & 226.653 & Surface & 2012 & 8438.096 & 216.449 & Surface & 2012 \\
\hline 3092.215 & 226.661 & Surface & 2012 & 8441.276 & 216.435 & Surface & 2012 \\
\hline 3095.293 & 226.665 & Surface & 2012 & 8444.456 & 216.405 & Surface & 2012 \\
\hline 3098.372 & 226.660 & Surface & 2012 & 8447.636 & 216.417 & Surface & 2012 \\
\hline 3101.450 & 226.659 & Surface & 2012 & 8450.817 & 216.446 & Surface & 2012 \\
\hline 3104.528 & 226.656 & Surface & 2012 & 8453.997 & 216.435 & Surface & 2012 \\
\hline 3107.607 & 226.647 & Surface & 2012 & 8457.177 & 216.438 & Surface & 2012 \\
\hline 3110.685 & 226.640 & Surface & 2012 & 8460.357 & 216.441 & Surface & 2012 \\
\hline 3113.763 & 226.635 & Surface & 2012 & 8463.537 & 216.447 & Surface & 2012 \\
\hline 3116.842 & 226.626 & Surface & 2012 & 8466.718 & 216.455 & Surface & 2012 \\
\hline 3119.920 & 226.615 & Surface & 2012 & 8469.898 & 216.450 & Surface & 2012 \\
\hline 3122.998 & 226.630 & Surface & 2012 & 8472.981 & 216.446 & Surface & 2012 \\
\hline 3126.041 & 226.626 & Surface & 2012 & 8476.064 & 216.444 & Surface & 2012 \\
\hline 3129.083 & 226.618 & Surface & 2012 & 8479.147 & 216.463 & Surface & 2012 \\
\hline 3132.125 & 226.604 & Surface & 2012 & 8482.230 & 216.450 & Surface & 2012 \\
\hline 3135.168 & 226.607 & Surface & 2012 & 8485.313 & 216.465 & Surface & 2012 \\
\hline 3138.210 & 226.615 & Surface & 2012 & 8488.396 & 216.462 & Surface & 2012 \\
\hline 3141.253 & 226.620 & Surface & 2012 & 8491.479 & 216.452 & Surface & 2012 \\
\hline 3144.295 & 226.615 & Surface & 2012 & 8494.562 & 216.450 & Surface & 2012 \\
\hline 3147.338 & 226.602 & Surface & 2012 & 8497.645 & 216.457 & Surface & 2012 \\
\hline 3150.380 & 226.600 & Surface & 2012 & 8500.901 & 216.452 & Surface & 2012 \\
\hline 3153.422 & 226.610 & Surface & 2012 & 8504.157 & 216.453 & Surface & 2012 \\
\hline 3156.465 & 226.606 & Surface & 2012 & 8507.413 & 216.456 & Surface & 2012 \\
\hline 3159.507 & 226.597 & Surface & 2012 & 8510.668 & 216.466 & Surface & 2012 \\
\hline 3162.703 & 226.586 & Surface & 2012 & 8513.925 & 216.471 & Surface & 2012 \\
\hline 3165.898 & 226.581 & Surface & 2012 & 8517.180 & 216.475 & Surface & 2012 \\
\hline 3169.094 & 226.579 & Surface & 2012 & 8520.436 & 216.471 & Surface & 2012 \\
\hline 3172.290 & 226.584 & Surface & 2012 & 8523.692 & 216.470 & Surface & 2012 \\
\hline 3175.485 & 226.573 & Surface & 2012 & 8526.948 & 216.463 & Surface & 2012 \\
\hline 3178.681 & 226.580 & Surface & 2012 & 8530.056 & 216.456 & Surface & 2012 \\
\hline 3181.877 & 226.571 & Surface & 2012 & 8533.163 & 216.448 & Surface & 2012 \\
\hline 3185.073 & 226.560 & Surface & 2012 & 8536.271 & 216.440 & Surface & 2012 \\
\hline 3188.268 & 226.563 & Surface & 2012 & 8539.379 & 216.446 & Surface & 2012 \\
\hline 3191.464 & 226.562 & Surface & 2012 & 8542.487 & 216.441 & Surface & 2012 \\
\hline 3194.681 & 226.559 & Surface & 2012 & 8545.594 & 216.440 & Surface & 2012 \\
\hline 3197.898 & 226.560 & Surface & 2012 & 8548.702 & 216.432 & Surface & 2012 \\
\hline 3201.116 & 226.552 & Surface & 2012 & 8551.810 & 216.440 & Surface & 2012 \\
\hline 3204.333 & 226.553 & Surface & 2012 & 8554.918 & 216.443 & Surface & 2012 \\
\hline 3207.551 & 226.543 & Surface & 2012 & 8558.026 & 216.448 & Surface & 2012 \\
\hline 3210.768 & 226.527 & Surface & 2012 & 8561.133 & 216.441 & Surface & 2012 \\
\hline 3213.985 & 226.505 & Surface & 2012 & 8564.241 & 216.440 & Surface & 2012 \\
\hline 3217.202 & 226.485 & Surface & 2012 & 8567.478 & 216.445 & Surface & 2012 \\
\hline 3220.420 & 226.490 & Surface & 2012 & 8570.715 & 216.437 & Surface & 2012 \\
\hline 3223.637 & 226.503 & Surface & 2012 & 8573.952 & 216.435 & Surface & 2012 \\
\hline 3226.855 & 226.499 & Surface & 2012 & 8577.189 & 216.440 & Surface & 2012 \\
\hline 3230.072 & 226.477 & Surface & 2012 & 8580.427 & 216.430 & Surface & 2012 \\
\hline 3233.282 & 226.459 & Surface & 2012 & 8583.664 & 216.431 & Surface & 2012 \\
\hline
\end{tabular}




\begin{tabular}{|c|c|c|c|c|c|c|c|}
\hline 3236.493 & 226.461 & Surface & 2012 & 8586.901 & 216.454 & Surface & 2012 \\
\hline 3239.703 & 226.467 & Surface & 2012 & 8590.138 & 216.446 & Surface & 2012 \\
\hline 3242.914 & 226.481 & Surface & 2012 & 8593.375 & 216.442 & Surface & 2012 \\
\hline 3246.125 & 226.451 & Surface & 2012 & 8596.432 & 216.442 & Surface & 2012 \\
\hline 3249.335 & 226.428 & Surface & 2012 & 8599.489 & 216.440 & Surface & 2012 \\
\hline 3252.546 & 226.406 & Surface & 2012 & 8602.546 & 216.441 & Surface & 2012 \\
\hline 3255.756 & 226.403 & Surface & 2012 & 8605.602 & 216.446 & Surface & 2012 \\
\hline 3258.967 & 226.395 & Surface & 2012 & 8608.659 & 216.454 & Surface & 2012 \\
\hline 3262.177 & 226.385 & Surface & 2012 & 8611.716 & 216.460 & Surface & 2012 \\
\hline 3265.388 & 226.372 & Surface & 2012 & 8614.773 & 216.458 & Surface & 2012 \\
\hline 3268.598 & 226.349 & Surface & 2012 & 8617.830 & 216.450 & Surface & 2012 \\
\hline 3271.809 & 226.323 & Surface & 2012 & 8620.887 & 216.450 & Surface & 2012 \\
\hline 3275.020 & 226.301 & Surface & 2012 & 8623.944 & 216.450 & Surface & 2012 \\
\hline 3278.269 & 226.311 & Surface & 2012 & 8627.001 & 216.447 & Surface & 2012 \\
\hline 3281.518 & 226.320 & Surface & 2012 & 8630.058 & 216.445 & Surface & 2012 \\
\hline 3284.768 & 226.288 & Surface & 2012 & 8633.115 & 216.450 & Surface & 2012 \\
\hline 3288.017 & 226.270 & Surface & 2012 & 8636.223 & 216.450 & Surface & 2012 \\
\hline 3291.267 & 226.285 & Surface & 2012 & 8639.331 & 216.446 & Surface & 2012 \\
\hline 3294.516 & 226.274 & Surface & 2012 & 8642.440 & 216.440 & Surface & 2012 \\
\hline 3297.765 & 226.224 & Surface & 2012 & 8645.548 & 216.440 & Surface & 2012 \\
\hline 3301.015 & 226.190 & Surface & 2012 & 8648.656 & 216.440 & Surface & 2012 \\
\hline 3304.264 & 226.167 & Surface & 2012 & 8651.765 & 216.440 & Surface & 2012 \\
\hline 3307.513 & 226.145 & Surface & 2012 & 8654.873 & 216.440 & Surface & 2012 \\
\hline 3310.762 & 226.117 & Surface & 2012 & 8657.981 & 216.440 & Surface & 2012 \\
\hline 3314.012 & 226.098 & Surface & 2012 & 8661.090 & 216.440 & Surface & 2012 \\
\hline 3317.261 & 226.097 & Surface & 2012 & 8664.198 & 216.440 & Surface & 2012 \\
\hline 3320.511 & 226.121 & Surface & 2012 & 8667.306 & 216.435 & Surface & 2012 \\
\hline 3323.760 & 226.087 & Surface & 2012 & 8670.579 & 216.427 & Surface & 2012 \\
\hline 3327.009 & 226.063 & Surface & 2012 & 8673.851 & 216.409 & Surface & 2012 \\
\hline 3330.259 & 226.063 & Surface & 2012 & 8677.123 & 216.409 & Surface & 2012 \\
\hline 3333.373 & 226.066 & Surface & 2012 & 8680.395 & 216.404 & Surface & 2012 \\
\hline 3336.487 & 226.083 & Surface & 2012 & 8683.668 & 216.402 & Surface & 2012 \\
\hline 3339.601 & 226.062 & Surface & 2012 & 8686.940 & 216.453 & Surface & 2012 \\
\hline 3342.715 & 226.054 & Surface & 2012 & 8690.212 & 216.460 & Surface & 2012 \\
\hline 3345.830 & 226.070 & Surface & 2012 & 8693.484 & 216.460 & Surface & 2012 \\
\hline 3348.944 & 226.065 & Surface & 2012 & 8696.756 & 216.455 & Surface & 2012 \\
\hline 3352.058 & 226.054 & Surface & 2012 & 8700.029 & 216.446 & Surface & 2012 \\
\hline 3355.172 & 226.060 & Surface & 2012 & 8703.301 & 216.438 & Surface & 2012 \\
\hline 3358.286 & 226.072 & Surface & 2012 & 8706.573 & 216.429 & Surface & 2012 \\
\hline 3361.400 & 226.072 & Surface & 2012 & 8709.845 & 216.420 & Surface & 2012 \\
\hline 3364.515 & 226.064 & Surface & 2012 & 8713.118 & 216.402 & Surface & 2012 \\
\hline 3367.629 & 226.062 & Surface & 2012 & 8716.390 & 216.396 & Surface & 2012 \\
\hline 3370.743 & 226.065 & Surface & 2012 & 8719.662 & 216.407 & Surface & 2012 \\
\hline 3373.857 & 226.062 & Surface & 2012 & 8722.934 & 216.402 & Surface & 2012 \\
\hline 3376.972 & 226.059 & Surface & 2012 & 8726.134 & 216.400 & Surface & 2012 \\
\hline 3380.086 & 226.052 & Surface & 2012 & 8729.333 & 216.400 & Surface & 2012 \\
\hline 3383.200 & 226.068 & Surface & 2012 & 8732.532 & 216.400 & Surface & 2012 \\
\hline 3386.314 & 226.054 & Surface & 2012 & 8735.732 & 216.398 & Surface & 2012 \\
\hline 3389.428 & 226.037 & Surface & 2012 & 8738.931 & 216.390 & Surface & 2012 \\
\hline
\end{tabular}




\begin{tabular}{|c|c|c|c|c|c|c|c|}
\hline 3392.688 & 226.039 & Surface & 2012 & 8742.130 & 216.390 & Surface & 2012 \\
\hline 3395.947 & 226.042 & Surface & 2012 & 8745.329 & 216.387 & Surface & 2012 \\
\hline 3399.207 & 226.036 & Surface & 2012 & 8748.529 & 216.380 & Surface & 2012 \\
\hline 3402.466 & 226.030 & Surface & 2012 & 8751.728 & 216.379 & Surface & 2012 \\
\hline 3405.726 & 226.022 & Surface & 2012 & 8754.895 & 216.379 & Surface & 2012 \\
\hline 3408.985 & 226.011 & Surface & 2012 & 8758.061 & 216.375 & Surface & 2012 \\
\hline 3412.245 & 226.008 & Surface & 2012 & 8761.228 & 216.364 & Surface & 2012 \\
\hline 3415.504 & 226.007 & Surface & 2012 & 8764.395 & 216.346 & Surface & 2012 \\
\hline 3418.764 & 225.995 & Surface & 2012 & 8767.562 & 216.350 & Surface & 2012 \\
\hline 3422.023 & 225.990 & Surface & 2012 & 8770.728 & 216.341 & Surface & 2012 \\
\hline 3425.283 & 225.973 & Surface & 2012 & 8773.895 & 216.322 & Surface & 2012 \\
\hline 3428.542 & 225.971 & Surface & 2012 & 8777.062 & 216.328 & Surface & 2012 \\
\hline 3431.802 & 225.973 & Surface & 2012 & 8780.228 & 216.326 & Surface & 2012 \\
\hline 3435.061 & 225.957 & Surface & 2012 & 8783.395 & 216.310 & Surface & 2012 \\
\hline 3438.321 & 225.941 & Surface & 2012 & 8786.562 & 216.312 & Surface & 2012 \\
\hline 3441.580 & 225.946 & Surface & 2012 & 8789.596 & 216.304 & Surface & 2012 \\
\hline 3444.840 & 225.941 & Surface & 2012 & 8792.630 & 216.264 & Surface & 2012 \\
\hline 3448.099 & 225.918 & Surface & 2012 & 8795.663 & 216.276 & Surface & 2012 \\
\hline 3451.358 & 225.899 & Surface & 2012 & 8798.697 & 216.251 & Surface & 2012 \\
\hline 3454.618 & 225.911 & Surface & 2012 & 8801.732 & 216.222 & Surface & 2012 \\
\hline 3457.877 & 225.895 & Surface & 2012 & 8804.766 & 216.210 & Surface & 2012 \\
\hline 3461.137 & 225.867 & Surface & 2012 & 8807.799 & 216.201 & Surface & 2012 \\
\hline 3464.396 & 225.844 & Surface & 2012 & 8810.834 & 216.165 & Surface & 2012 \\
\hline 3467.656 & 225.836 & Surface & 2012 & 8813.867 & 216.107 & Surface & 2012 \\
\hline 3470.916 & 225.816 & Surface & 2012 & 8816.901 & 216.066 & Surface & 2012 \\
\hline 3474.175 & 225.822 & Surface & 2012 & 8819.935 & 216.031 & Surface & 2012 \\
\hline 3477.434 & 225.822 & Surface & 2012 & 8822.969 & 215.964 & Surface & 2012 \\
\hline 3480.694 & 225.822 & Surface & 2012 & 8826.155 & 215.851 & Surface & 2012 \\
\hline 3483.953 & 225.798 & Surface & 2012 & 8829.340 & 215.707 & Surface & 2012 \\
\hline 3487.213 & 225.780 & Surface & 2012 & 8832.525 & 215.560 & Surface & 2012 \\
\hline 3490.303 & 225.763 & Surface & 2012 & 8835.710 & 215.526 & Surface & 2012 \\
\hline 3493.394 & 225.774 & Surface & 2012 & 8838.896 & 215.430 & Surface & 2012 \\
\hline 3496.484 & 225.784 & Surface & 2012 & 8842.081 & 215.415 & Surface & 2012 \\
\hline 3499.575 & 225.778 & Surface & 2012 & 8845.266 & 215.395 & Surface & 2012 \\
\hline 3502.665 & 225.777 & Surface & 2012 & 8848.452 & 215.376 & Surface & 2012 \\
\hline 3505.756 & 225.765 & Surface & 2012 & 8851.637 & 215.350 & Surface & 2012 \\
\hline 3508.846 & 225.748 & Surface & 2012 & 8854.822 & 215.323 & Surface & 2012 \\
\hline 3511.937 & 225.736 & Surface & 2012 & 8858.007 & 215.353 & Surface & 2012 \\
\hline 3515.027 & 225.713 & Surface & 2012 & 8861.192 & 215.313 & Surface & 2012 \\
\hline 3518.263 & 225.697 & Surface & 2012 & 8864.158 & 215.266 & Surface & 2012 \\
\hline 3521.499 & 225.663 & Surface & 2012 & 8867.124 & 215.291 & Surface & 2012 \\
\hline 3524.735 & 225.677 & Surface & 2012 & 8870.090 & 215.295 & Surface & 2012 \\
\hline 3527.970 & 225.653 & Surface & 2012 & 8873.056 & 215.360 & Surface & 2012 \\
\hline 3531.206 & 225.630 & Surface & 2012 & 8876.022 & 215.394 & Surface & 2012 \\
\hline 3534.443 & 225.597 & Surface & 2012 & 8878.988 & 215.357 & Surface & 2012 \\
\hline 3537.678 & 225.613 & Surface & 2012 & 8881.954 & 215.346 & Surface & 2012 \\
\hline 3540.914 & 225.614 & Surface & 2012 & 8884.920 & 215.364 & Surface & 2012 \\
\hline 3544.150 & 225.627 & Surface & 2012 & 8887.916 & 215.347 & Surface & 2012 \\
\hline 3547.386 & 225.610 & Surface & 2012 & 8890.913 & 215.336 & Surface & 2012 \\
\hline
\end{tabular}




\begin{tabular}{|c|c|c|c|c|c|c|c|}
\hline 3550.631 & 225.598 & Surface & 2012 & 8893.910 & 215.309 & Surface & 2012 \\
\hline 3553.877 & 225.592 & Surface & 2012 & 8896.906 & 215.303 & Surface & 2012 \\
\hline 3557.122 & 225.598 & Surface & 2012 & 8899.903 & 215.304 & Surface & 2012 \\
\hline 3560.368 & 225.593 & Surface & 2012 & 8902.900 & 215.300 & Surface & 2012 \\
\hline 3563.613 & 225.558 & Surface & 2012 & 8905.897 & 215.290 & Surface & 2012 \\
\hline 3566.859 & 225.561 & Surface & 2012 & 8908.893 & 215.270 & Surface & 2012 \\
\hline 3570.104 & 225.562 & Surface & 2012 & 8911.890 & 215.258 & Surface & 2012 \\
\hline 3573.350 & 225.558 & Surface & 2012 & 8914.887 & 215.265 & Surface & 2012 \\
\hline 3576.595 & 225.532 & Surface & 2012 & 8918.046 & 215.267 & Surface & 2012 \\
\hline 3579.841 & 225.537 & Surface & 2012 & 8921.205 & 215.257 & Surface & 2012 \\
\hline 3583.086 & 225.535 & Surface & 2012 & 8924.364 & 215.251 & Surface & 2012 \\
\hline 3586.332 & 225.530 & Surface & 2012 & 8927.523 & 215.258 & Surface & 2012 \\
\hline 3589.577 & 225.514 & Surface & 2012 & 8930.682 & 215.250 & Surface & 2012 \\
\hline 3592.746 & 225.468 & Surface & 2012 & 8933.841 & 215.250 & Surface & 2012 \\
\hline 3595.914 & 225.434 & Surface & 2012 & 8937.000 & 215.250 & Surface & 2012 \\
\hline 3599.082 & 225.438 & Surface & 2012 & 8940.158 & 215.253 & Surface & 2012 \\
\hline 3602.250 & 225.446 & Surface & 2012 & 8943.318 & 215.242 & Surface & 2012 \\
\hline 3605.419 & 225.441 & Surface & 2012 & 8946.476 & 215.228 & Surface & 2012 \\
\hline 3608.587 & 225.421 & Surface & 2012 & 8949.635 & 215.235 & Surface & 2012 \\
\hline 3611.755 & 225.393 & Surface & 2012 & 8952.794 & 215.222 & Surface & 2012 \\
\hline 3614.924 & 225.364 & Surface & 2012 & 8955.953 & 215.214 & Surface & 2012 \\
\hline 3618.092 & 225.360 & Surface & 2012 & 8959.047 & 215.212 & Surface & 2012 \\
\hline 3621.260 & 225.358 & Surface & 2012 & 8962.140 & 215.219 & Surface & 2012 \\
\hline 3624.429 & 225.345 & Surface & 2012 & 8965.234 & 215.226 & Surface & 2012 \\
\hline 3627.512 & 225.305 & Surface & 2012 & 8968.327 & 215.206 & Surface & 2012 \\
\hline 3630.596 & 225.248 & Surface & 2012 & 8971.421 & 215.187 & Surface & 2012 \\
\hline 3633.680 & 225.180 & Surface & 2012 & 8974.514 & 215.196 & Surface & 2012 \\
\hline 3636.764 & 225.118 & Surface & 2012 & 8977.608 & 215.216 & Surface & 2012 \\
\hline 3639.848 & 225.093 & Surface & 2012 & 8980.701 & 215.223 & Surface & 2012 \\
\hline 3642.932 & 225.102 & Surface & 2012 & 8983.794 & 215.216 & Surface & 2012 \\
\hline 3646.015 & 225.053 & Surface & 2012 & 8986.888 & 215.201 & Surface & 2012 \\
\hline 3649.100 & 225.008 & Surface & 2012 & 8989.981 & 215.186 & Surface & 2012 \\
\hline 3652.183 & 224.972 & Surface & 2012 & 8993.075 & 215.218 & Surface & 2012 \\
\hline 3655.267 & 224.935 & Surface & 2012 & 8996.168 & 215.227 & Surface & 2012 \\
\hline 3658.126 & 224.914 & Surface & 2012 & 8999.262 & 215.211 & Surface & 2012 \\
\hline 3660.984 & 224.890 & Surface & 2012 & 9002.355 & 215.216 & Surface & 2012 \\
\hline 3663.842 & 224.826 & Surface & 2012 & 9005.449 & 215.189 & Surface & 2012 \\
\hline 3666.701 & 224.743 & Surface & 2012 & 9008.542 & 215.193 & Surface & 2012 \\
\hline 3669.559 & 224.707 & Surface & 2012 & 9011.801 & 215.169 & Surface & 2012 \\
\hline 3672.418 & 224.661 & Surface & 2012 & 9015.060 & 215.163 & Surface & 2012 \\
\hline 3675.276 & 224.629 & Surface & 2012 & 9018.319 & 215.155 & Surface & 2012 \\
\hline 3678.306 & 224.624 & Surface & 2012 & 9021.578 & 215.165 & Surface & 2012 \\
\hline 3681.336 & 224.640 & Surface & 2012 & 9024.838 & 215.126 & Surface & 2012 \\
\hline 3684.366 & 224.621 & Surface & 2012 & 9028.097 & 215.114 & Surface & 2012 \\
\hline 3687.396 & 224.606 & Surface & 2012 & 9031.356 & 215.130 & Surface & 2012 \\
\hline 3690.427 & 224.622 & Surface & 2012 & 9034.615 & 215.109 & Surface & 2012 \\
\hline 3693.457 & 224.621 & Surface & 2012 & 9037.874 & 215.100 & Surface & 2012 \\
\hline 3696.487 & 224.623 & Surface & 2012 & 9041.133 & 215.095 & Surface & 2012 \\
\hline 3699.517 & 224.626 & Surface & 2012 & 9044.392 & 215.101 & Surface & 2012 \\
\hline
\end{tabular}




\begin{tabular}{|c|c|c|c|c|c|c|c|}
\hline 3702.726 & 224.620 & Surface & 2012 & 9047.651 & 215.088 & Surface & 2012 \\
\hline 3705.934 & 224.628 & Surface & 2012 & 9050.911 & 215.073 & Surface & 2012 \\
\hline 3709.143 & 224.628 & Surface & 2012 & 9054.170 & 215.057 & Surface & 2012 \\
\hline 3712.352 & 224.619 & Surface & 2012 & 9057.333 & 215.027 & Surface & 2012 \\
\hline 3715.561 & 224.610 & Surface & 2012 & 9060.497 & 215.027 & Surface & 2012 \\
\hline 3718.770 & 224.630 & Surface & 2012 & 9063.660 & 215.009 & Surface & 2012 \\
\hline 3721.978 & 224.621 & Surface & 2012 & 9066.824 & 215.009 & Surface & 2012 \\
\hline 3725.187 & 224.649 & Surface & 2012 & 9069.988 & 215.002 & Surface & 2012 \\
\hline 3728.396 & 224.639 & Surface & 2012 & 9073.151 & 214.995 & Surface & 2012 \\
\hline 3731.378 & 224.631 & Surface & 2012 & 9076.315 & 215.010 & Surface & 2012 \\
\hline 3734.361 & 224.630 & Surface & 2012 & 9079.479 & 214.969 & Surface & 2012 \\
\hline 3737.344 & 224.633 & Surface & 2012 & 9082.642 & 214.996 & Surface & 2012 \\
\hline 3740.327 & 224.651 & Surface & 2012 & 9085.806 & 215.025 & Surface & 2012 \\
\hline 3743.309 & 224.646 & Surface & 2012 & 9088.969 & 215.022 & Surface & 2012 \\
\hline 3746.292 & 224.635 & Surface & 2012 & 9092.133 & 215.023 & Surface & 2012 \\
\hline 3749.275 & 224.634 & Surface & 2012 & 9095.297 & 215.026 & Surface & 2012 \\
\hline 3752.258 & 224.636 & Surface & 2012 & 9098.460 & 215.010 & Surface & 2012 \\
\hline 3755.241 & 224.641 & Surface & 2012 & 9101.624 & 215.041 & Surface & 2012 \\
\hline 3758.490 & 224.613 & Surface & 2012 & 9104.850 & 215.037 & Surface & 2012 \\
\hline 3761.739 & 224.617 & Surface & 2012 & 9108.077 & 215.058 & Surface & 2012 \\
\hline 3764.988 & 224.600 & Surface & 2012 & 9111.304 & 215.058 & Surface & 2012 \\
\hline 3768.237 & 224.612 & Surface & 2012 & 9114.530 & 215.069 & Surface & 2012 \\
\hline 3771.486 & 224.640 & Surface & 2012 & 9117.757 & 215.038 & Surface & 2012 \\
\hline 3774.735 & 224.630 & Surface & 2012 & 9120.984 & 215.046 & Surface & 2012 \\
\hline 3777.984 & 224.601 & Surface & 2012 & 9124.210 & 215.051 & Surface & 2012 \\
\hline 3781.233 & 224.645 & Surface & 2012 & 9127.437 & 215.036 & Surface & 2012 \\
\hline 3784.483 & 224.643 & Surface & 2012 & 9130.663 & 215.058 & Surface & 2012 \\
\hline 3787.732 & 224.633 & Surface & 2012 & 9133.890 & 215.036 & Surface & 2012 \\
\hline 3790.981 & 224.637 & Surface & 2012 & 9137.117 & 215.045 & Surface & 2012 \\
\hline 3794.230 & 224.624 & Surface & 2012 & 9140.343 & 215.043 & Surface & 2012 \\
\hline 3797.355 & 224.611 & Surface & 2012 & 9143.424 & 215.042 & Surface & 2012 \\
\hline 3800.481 & 224.623 & Surface & 2012 & 9146.504 & 215.037 & Surface & 2012 \\
\hline 3803.606 & 224.624 & Surface & 2012 & 9149.584 & 215.050 & Surface & 2012 \\
\hline 3806.732 & 224.626 & Surface & 2012 & 9152.664 & 215.059 & Surface & 2012 \\
\hline 3809.857 & 224.636 & Surface & 2012 & 9155.745 & 215.052 & Surface & 2012 \\
\hline 3812.983 & 224.645 & Surface & 2012 & 9158.825 & 215.047 & Surface & 2012 \\
\hline 3816.108 & 224.655 & Surface & 2012 & 9161.905 & 215.041 & Surface & 2012 \\
\hline 3819.234 & 224.659 & Surface & 2012 & 9164.986 & 215.039 & Surface & 2012 \\
\hline 3822.359 & 224.660 & Surface & 2012 & 9168.066 & 215.036 & Surface & 2012 \\
\hline 3825.485 & 224.654 & Surface & 2012 & 9171.147 & 215.043 & Surface & 2012 \\
\hline 3828.750 & 224.643 & Surface & 2012 & 9174.227 & 215.028 & Surface & 2012 \\
\hline 3832.015 & 224.633 & Surface & 2012 & 9177.307 & 215.020 & Surface & 2012 \\
\hline 3835.280 & 224.624 & Surface & 2012 & 9180.387 & 215.035 & Surface & 2012 \\
\hline 3838.545 & 224.624 & Surface & 2012 & 9183.468 & 215.037 & Surface & 2012 \\
\hline 3841.811 & 224.635 & Surface & 2012 & 9186.548 & 215.035 & Surface & 2012 \\
\hline 3845.076 & 224.632 & Surface & 2012 & 9189.828 & 215.026 & Surface & 2012 \\
\hline 3848.341 & 224.631 & Surface & 2012 & 9193.108 & 215.026 & Surface & 2012 \\
\hline 3851.606 & 224.631 & Surface & 2012 & 9196.388 & 215.046 & Surface & 2012 \\
\hline 3854.871 & 224.632 & Surface & 2012 & 9199.668 & 215.016 & Surface & 2012 \\
\hline
\end{tabular}




\begin{tabular}{|c|c|c|c|c|c|c|c|}
\hline 3857.986 & 224.623 & Surface & 2012 & 9202.948 & 215.031 & Surface & 2012 \\
\hline 3861.102 & 224.608 & Surface & 2012 & 9206.228 & 215.036 & Surface & 2012 \\
\hline 3864.217 & 224.602 & Surface & 2012 & 9209.509 & 215.003 & Surface & 2012 \\
\hline 3867.332 & 224.594 & Surface & 2012 & 9212.789 & 215.016 & Surface & 2012 \\
\hline 3870.447 & 224.564 & Surface & 2012 & 9216.069 & 215.010 & Surface & 2012 \\
\hline 3873.562 & 224.520 & Surface & 2012 & 9219.349 & 214.990 & Surface & 2012 \\
\hline 3876.677 & 224.537 & Surface & 2012 & 9222.629 & 214.990 & Surface & 2012 \\
\hline 3879.720 & 224.556 & Surface & 2012 & 9225.909 & 214.990 & Surface & 2012 \\
\hline 3882.763 & 224.573 & Surface & 2012 & 9229.189 & 214.988 & Surface & 2012 \\
\hline 3885.806 & 224.592 & Surface & 2012 & 9232.297 & 214.978 & Surface & 2012 \\
\hline 3888.848 & 224.578 & Surface & 2012 & 9235.404 & 214.992 & Surface & 2012 \\
\hline 3891.891 & 224.558 & Surface & 2012 & 9238.512 & 214.993 & Surface & 2012 \\
\hline 3894.934 & 224.571 & Surface & 2012 & 9241.620 & 214.989 & Surface & 2012 \\
\hline 3897.976 & 224.571 & Surface & 2012 & 9244.728 & 214.996 & Surface & 2012 \\
\hline 3901.019 & 224.575 & Surface & 2012 & 9247.836 & 214.985 & Surface & 2012 \\
\hline 3904.158 & 224.567 & Surface & 2012 & 9250.943 & 214.978 & Surface & 2012 \\
\hline 3907.298 & 224.568 & Surface & 2012 & 9254.051 & 214.974 & Surface & 2012 \\
\hline 3910.437 & 224.585 & Surface & 2012 & 9257.159 & 214.980 & Surface & 2012 \\
\hline 3913.576 & 224.583 & Surface & 2012 & 9260.267 & 214.986 & Surface & 2012 \\
\hline 3916.716 & 224.570 & Surface & 2012 & 9263.375 & 214.976 & Surface & 2012 \\
\hline 3919.855 & 224.555 & Surface & 2012 & 9266.482 & 214.980 & Surface & 2012 \\
\hline 3922.994 & 224.526 & Surface & 2012 & 9269.590 & 214.985 & Surface & 2012 \\
\hline 3926.133 & 224.515 & Surface & 2012 & 9272.665 & 214.982 & Surface & 2012 \\
\hline 3929.297 & 224.506 & Surface & 2012 & 9275.741 & 214.977 & Surface & 2012 \\
\hline 3932.461 & 224.517 & Surface & 2012 & 9278.816 & 214.969 & Surface & 2012 \\
\hline 3935.625 & 224.514 & Surface & 2012 & 9281.892 & 214.969 & Surface & 2012 \\
\hline 3938.788 & 224.499 & Surface & 2012 & 9284.967 & 214.978 & Surface & 2012 \\
\hline 3941.952 & 224.499 & Surface & 2012 & 9288.042 & 214.980 & Surface & 2012 \\
\hline 3945.116 & 224.487 & Surface & 2012 & 9291.118 & 214.975 & Surface & 2012 \\
\hline 3948.279 & 224.512 & Surface & 2012 & 9294.193 & 214.988 & Surface & 2012 \\
\hline 3951.443 & 224.531 & Surface & 2012 & 9297.268 & 214.965 & Surface & 2012 \\
\hline 3954.116 & 224.565 & Surface & 2012 & 9300.344 & 214.972 & Surface & 2012 \\
\hline 3956.789 & 224.544 & Surface & 2012 & 9303.419 & 215.019 & Surface & 2012 \\
\hline 3959.462 & 224.544 & Surface & 2012 & 9306.499 & 214.981 & Surface & 2012 \\
\hline 3962.135 & 224.556 & Surface & 2012 & 9309.578 & 214.981 & Surface & 2012 \\
\hline 3964.808 & 224.562 & Surface & 2012 & 9312.658 & 215.006 & Surface & 2012 \\
\hline 3968.044 & 224.548 & Surface & 2012 & 9315.737 & 214.972 & Surface & 2012 \\
\hline 3971.280 & 224.500 & Surface & 2012 & 9318.817 & 214.970 & Surface & 2012 \\
\hline 3974.516 & 224.483 & Surface & 2012 & 9321.896 & 214.975 & Surface & 2012 \\
\hline 3977.752 & 224.475 & Surface & 2012 & 9324.976 & 214.979 & Surface & 2012 \\
\hline 3980.988 & 224.475 & Surface & 2012 & 9328.055 & 214.970 & Surface & 2012 \\
\hline 3984.223 & 224.476 & Surface & 2012 & 9331.135 & 214.979 & Surface & 2012 \\
\hline 3987.243 & 224.477 & Surface & 2012 & 9334.214 & 214.989 & Surface & 2012 \\
\hline 3990.263 & 224.477 & Surface & 2012 & 9337.294 & 214.996 & Surface & 2012 \\
\hline 3993.283 & 224.451 & Surface & 2012 & 9340.373 & 214.990 & Surface & 2012 \\
\hline 3996.303 & 224.422 & Surface & 2012 & 9343.453 & 214.974 & Surface & 2012 \\
\hline 3999.323 & 224.427 & Surface & 2012 & 9346.569 & 214.989 & Surface & 2012 \\
\hline 4002.342 & 224.434 & Surface & 2012 & 9349.686 & 214.974 & Surface & 2012 \\
\hline 4005.362 & 224.441 & Surface & 2012 & 9352.802 & 214.969 & Surface & 2012 \\
\hline
\end{tabular}




\begin{tabular}{|c|c|c|c|c|c|c|c|}
\hline 4008.382 & 224.421 & Surface & 2012 & 9355.919 & 214.960 & Surface & 2012 \\
\hline 4011.402 & 224.392 & Surface & 2012 & 9359.035 & 214.979 & Surface & 2012 \\
\hline 4014.422 & 224.390 & Surface & 2012 & 9362.152 & 215.000 & Surface & 2012 \\
\hline 4017.441 & 224.393 & Surface & 2012 & 9365.268 & 214.995 & Surface & 2012 \\
\hline 4020.461 & 224.398 & Surface & 2012 & 9368.385 & 214.991 & Surface & 2012 \\
\hline 4023.639 & 224.402 & Surface & 2012 & 9371.502 & 214.989 & Surface & 2012 \\
\hline 4026.816 & 224.401 & Surface & 2012 & 9374.618 & 215.002 & Surface & 2012 \\
\hline 4029.994 & 224.406 & Surface & 2012 & 9377.734 & 215.013 & Surface & 2012 \\
\hline 4033.171 & 224.400 & Surface & 2012 & 9380.961 & 214.999 & Surface & 2012 \\
\hline 4036.348 & 224.400 & Surface & 2012 & 9384.187 & 214.991 & Surface & 2012 \\
\hline 4039.526 & 224.400 & Surface & 2012 & 9387.413 & 215.002 & Surface & 2012 \\
\hline 4042.703 & 224.401 & Surface & 2012 & 9390.640 & 214.993 & Surface & 2012 \\
\hline 4045.881 & 224.410 & Surface & 2012 & 9393.866 & 214.982 & Surface & 2012 \\
\hline 4049.058 & 224.407 & Surface & 2012 & 9397.093 & 214.988 & Surface & 2012 \\
\hline 4052.236 & 224.400 & Surface & 2012 & 9400.319 & 214.983 & Surface & 2012 \\
\hline 4055.413 & 224.400 & Surface & 2012 & 9403.545 & 214.972 & Surface & 2012 \\
\hline 4058.590 & 224.400 & Surface & 2012 & 9406.772 & 214.972 & Surface & 2012 \\
\hline 4061.768 & 224.400 & Surface & 2012 & 9409.998 & 214.984 & Surface & 2012 \\
\hline 4064.945 & 224.400 & Surface & 2012 & 9413.087 & 214.988 & Surface & 2012 \\
\hline 4068.123 & 224.400 & Surface & 2012 & 9416.175 & 215.004 & Surface & 2012 \\
\hline 4071.247 & 224.400 & Surface & 2012 & 9419.264 & 215.002 & Surface & 2012 \\
\hline 4074.371 & 224.401 & Surface & 2012 & 9422.352 & 215.007 & Surface & 2012 \\
\hline 4077.496 & 224.394 & Surface & 2012 & 9425.441 & 214.977 & Surface & 2012 \\
\hline 4080.620 & 224.390 & Surface & 2012 & 9428.530 & 214.975 & Surface & 2012 \\
\hline 4083.744 & 224.398 & Surface & 2012 & 9431.618 & 214.986 & Surface & 2012 \\
\hline 4086.869 & 224.400 & Surface & 2012 & 9434.707 & 214.999 & Surface & 2012 \\
\hline 4089.993 & 224.400 & Surface & 2012 & 9437.795 & 215.000 & Surface & 2012 \\
\hline 4093.117 & 224.400 & Surface & 2012 & 9440.884 & 214.998 & Surface & 2012 \\
\hline 4096.242 & 224.403 & Surface & 2012 & 9443.973 & 214.985 & Surface & 2012 \\
\hline 4099.366 & 224.411 & Surface & 2012 & 9447.116 & 214.980 & Surface & 2012 \\
\hline 4102.490 & 224.418 & Surface & 2012 & 9450.259 & 214.980 & Surface & 2012 \\
\hline 4105.615 & 224.420 & Surface & 2012 & 9453.402 & 214.980 & Surface & 2012 \\
\hline 4108.739 & 224.416 & Surface & 2012 & 9456.545 & 214.980 & Surface & 2012 \\
\hline 4111.863 & 224.395 & Surface & 2012 & 9459.688 & 214.980 & Surface & 2012 \\
\hline 4114.987 & 224.380 & Surface & 2012 & 9462.832 & 214.980 & Surface & 2012 \\
\hline 4118.112 & 224.372 & Surface & 2012 & 9465.975 & 214.980 & Surface & 2012 \\
\hline 4121.236 & 224.373 & Surface & 2012 & 9469.118 & 214.980 & Surface & 2012 \\
\hline 4124.442 & 224.379 & Surface & 2012 & 9472.262 & 214.980 & Surface & 2012 \\
\hline 4127.647 & 224.371 & Surface & 2012 & 9475.405 & 214.976 & Surface & 2012 \\
\hline 4130.852 & 224.362 & Surface & 2012 & 9478.546 & 214.970 & Surface & 2012 \\
\hline 4134.058 & 224.360 & Surface & 2012 & 9481.686 & 214.970 & Surface & 2012 \\
\hline 4137.263 & 224.355 & Surface & 2012 & 9484.827 & 214.970 & Surface & 2012 \\
\hline 4140.469 & 224.350 & Surface & 2012 & 9487.968 & 214.970 & Surface & 2012 \\
\hline 4143.674 & 224.350 & Surface & 2012 & 9491.109 & 214.970 & Surface & 2012 \\
\hline 4146.880 & 224.350 & Surface & 2012 & 9494.250 & 214.966 & Surface & 2012 \\
\hline 4150.085 & 224.354 & Surface & 2012 & 9497.390 & 214.960 & Surface & 2012 \\
\hline 4153.291 & 224.360 & Surface & 2012 & 9500.531 & 214.963 & Surface & 2012 \\
\hline 4156.496 & 224.358 & Surface & 2012 & 9503.672 & 214.968 & Surface & 2012 \\
\hline 4159.702 & 224.347 & Surface & 2012 & 9506.813 & 214.961 & Surface & 2012 \\
\hline
\end{tabular}




\begin{tabular}{|c|c|c|c|c|c|c|c|}
\hline 4162.907 & 224.337 & Surface & 2012 & 9509.953 & 214.963 & Surface & 2012 \\
\hline 4166.112 & 224.327 & Surface & 2012 & 9513.094 & 214.966 & Surface & 2012 \\
\hline 4169.318 & 224.307 & Surface & 2012 & 9516.235 & 214.965 & Surface & 2012 \\
\hline 4172.523 & 224.288 & Surface & 2012 & 9519.376 & 214.956 & Surface & 2012 \\
\hline 4175.729 & 224.295 & Surface & 2012 & 9522.517 & 214.935 & Surface & 2012 \\
\hline 4178.934 & 224.314 & Surface & 2012 & 9525.657 & 214.938 & Surface & 2012 \\
\hline 4182.140 & 224.318 & Surface & 2012 & 9528.877 & 214.953 & Surface & 2012 \\
\hline 4185.345 & 224.307 & Surface & 2012 & 9532.096 & 214.955 & Surface & 2012 \\
\hline 4188.551 & 224.300 & Surface & 2012 & 9535.316 & 214.936 & Surface & 2012 \\
\hline 4191.756 & 224.300 & Surface & 2012 & 9538.535 & 214.904 & Surface & 2012 \\
\hline 4194.961 & 224.291 & Surface & 2012 & 9541.754 & 214.880 & Surface & 2012 \\
\hline 4198.167 & 224.291 & Surface & 2012 & 9544.974 & 214.889 & Surface & 2012 \\
\hline 4201.372 & 224.289 & Surface & 2012 & 9548.193 & 214.865 & Surface & 2012 \\
\hline 4204.578 & 224.292 & Surface & 2012 & 9551.412 & 214.850 & Surface & 2012 \\
\hline 4207.785 & 224.291 & Surface & 2012 & 9554.632 & 214.841 & Surface & 2012 \\
\hline 4210.991 & 224.285 & Surface & 2012 & 9557.851 & 214.820 & Surface & 2012 \\
\hline 4214.197 & 224.283 & Surface & 2012 & 9561.070 & 214.803 & Surface & 2012 \\
\hline 4217.403 & 224.271 & Surface & 2012 & 9564.248 & 214.733 & Surface & 2012 \\
\hline 4220.610 & 224.268 & Surface & 2012 & 9567.425 & 214.722 & Surface & 2012 \\
\hline 4223.816 & 224.248 & Surface & 2012 & 9570.602 & 214.715 & Surface & 2012 \\
\hline 4227.022 & 224.235 & Surface & 2012 & 9573.780 & 214.654 & Surface & 2012 \\
\hline 4230.228 & 224.240 & Surface & 2012 & 9576.957 & 214.617 & Surface & 2012 \\
\hline 4233.434 & 224.242 & Surface & 2012 & 9580.134 & 214.582 & Surface & 2012 \\
\hline 4236.641 & 224.267 & Surface & 2012 & 9583.312 & 214.520 & Surface & 2012 \\
\hline 4239.847 & 224.237 & Surface & 2012 & 9586.489 & 214.506 & Surface & 2012 \\
\hline 4243.053 & 224.232 & Surface & 2012 & 9589.667 & 214.495 & Surface & 2012 \\
\hline 4246.259 & 224.230 & Surface & 2012 & 9592.844 & 214.498 & Surface & 2012 \\
\hline 4249.466 & 224.217 & Surface & 2012 & 9596.021 & 214.502 & Surface & 2012 \\
\hline 4252.672 & 224.209 & Surface & 2012 & 9599.199 & 214.495 & Surface & 2012 \\
\hline 4255.878 & 224.199 & Surface & 2012 & 9602.376 & 214.477 & Surface & 2012 \\
\hline 4259.084 & 224.169 & Surface & 2012 & 9605.433 & 214.459 & Surface & 2012 \\
\hline 4262.290 & 224.181 & Surface & 2012 & 9608.490 & 214.451 & Surface & 2012 \\
\hline 4265.497 & 224.173 & Surface & 2012 & 9611.547 & 214.433 & Surface & 2012 \\
\hline 4268.703 & 224.157 & Surface & 2012 & 9614.604 & 214.401 & Surface & 2012 \\
\hline 4271.909 & 224.164 & Surface & 2012 & 9617.661 & 214.398 & Surface & 2012 \\
\hline 4275.115 & 224.166 & Surface & 2012 & 9620.718 & 214.376 & Surface & 2012 \\
\hline 4278.322 & 224.157 & Surface & 2012 & 9623.775 & 214.351 & Surface & 2012 \\
\hline 4281.528 & 224.140 & Surface & 2012 & 9626.832 & 214.350 & Surface & 2012 \\
\hline 4284.734 & 224.140 & Surface & 2012 & 9629.888 & 214.340 & Surface & 2012 \\
\hline 4287.940 & 224.136 & Surface & 2012 & 9632.945 & 214.317 & Surface & 2012 \\
\hline 4291.147 & 224.137 & Surface & 2012 & 9636.002 & 214.314 & Surface & 2012 \\
\hline 4294.353 & 224.132 & Surface & 2012 & 9639.059 & 214.286 & Surface & 2012 \\
\hline 4297.559 & 224.133 & Surface & 2012 & 9642.116 & 214.230 & Surface & 2012 \\
\hline 4300.765 & 224.132 & Surface & 2012 & 9645.103 & 214.243 & Surface & 2012 \\
\hline 4303.971 & 224.126 & Surface & 2012 & 9648.089 & 214.222 & Surface & 2012 \\
\hline 4307.178 & 224.130 & Surface & 2012 & 9651.076 & 214.191 & Surface & 2012 \\
\hline 4310.384 & 224.124 & Surface & 2012 & 9654.062 & 214.167 & Surface & 2012 \\
\hline 4313.590 & 224.117 & Surface & 2012 & 9657.048 & 214.148 & Surface & 2012 \\
\hline 4316.796 & 224.121 & Surface & 2012 & 9660.035 & 214.138 & Surface & 2012 \\
\hline
\end{tabular}




\begin{tabular}{|c|c|c|c|c|c|c|c|}
\hline 4320.003 & 224.124 & Surface & 2012 & 9663.021 & 214.140 & Surface & 2012 \\
\hline 4323.209 & 224.137 & Surface & 2012 & 9666.007 & 214.135 & Surface & 2012 \\
\hline 4326.359 & 224.122 & Surface & 2012 & 9668.994 & 214.120 & Surface & 2012 \\
\hline 4329.509 & 224.124 & Surface & 2012 & 9671.980 & 214.106 & Surface & 2012 \\
\hline 4332.658 & 224.118 & Surface & 2012 & 9675.124 & 214.115 & Surface & 2012 \\
\hline 4335.808 & 224.116 & Surface & 2012 & 9678.269 & 214.143 & Surface & 2012 \\
\hline 4338.958 & 224.128 & Surface & 2012 & 9681.413 & 214.177 & Surface & 2012 \\
\hline 4342.108 & 224.130 & Surface & 2012 & 9684.557 & 214.183 & Surface & 2012 \\
\hline 4345.257 & 224.138 & Surface & 2012 & 9687.701 & 214.181 & Surface & 2012 \\
\hline 4348.407 & 224.119 & Surface & 2012 & 9690.845 & 214.170 & Surface & 2012 \\
\hline 4351.557 & 224.116 & Surface & 2012 & 9693.989 & 214.196 & Surface & 2012 \\
\hline 4354.707 & 224.122 & Surface & 2012 & 9697.133 & 214.208 & Surface & 2012 \\
\hline 4357.857 & 224.126 & Surface & 2012 & 9700.278 & 214.192 & Surface & 2012 \\
\hline 4361.007 & 224.124 & Surface & 2012 & 9703.433 & 214.193 & Surface & 2012 \\
\hline 4364.156 & 224.122 & Surface & 2012 & 9706.587 & 214.197 & Surface & 2012 \\
\hline 4367.306 & 224.123 & Surface & 2012 & 9709.742 & 214.203 & Surface & 2012 \\
\hline 4370.456 & 224.116 & Surface & 2012 & 9712.897 & 214.188 & Surface & 2012 \\
\hline 4373.606 & 224.128 & Surface & 2012 & 9716.052 & 214.192 & Surface & 2012 \\
\hline 4376.755 & 224.137 & Surface & 2012 & 9719.207 & 214.207 & Surface & 2012 \\
\hline 4379.905 & 224.126 & Surface & 2012 & 9722.362 & 214.216 & Surface & 2012 \\
\hline 4383.055 & 224.130 & Surface & 2012 & 9725.517 & 214.189 & Surface & 2012 \\
\hline 4386.205 & 224.123 & Surface & 2012 & 9728.672 & 214.195 & Surface & 2012 \\
\hline 4389.355 & 224.124 & Surface & 2012 & 9731.827 & 214.198 & Surface & 2012 \\
\hline 4392.505 & 224.117 & Surface & 2012 & 9734.937 & 214.199 & Surface & 2012 \\
\hline 4395.730 & 224.126 & Surface & 2012 & 9738.046 & 214.197 & Surface & 2012 \\
\hline 4398.956 & 224.119 & Surface & 2012 & 9741.156 & 214.199 & Surface & 2012 \\
\hline 4402.182 & 224.121 & Surface & 2012 & 9744.266 & 214.191 & Surface & 2012 \\
\hline 4405.407 & 224.120 & Surface & 2012 & 9747.375 & 214.199 & Surface & 2012 \\
\hline 4408.633 & 224.126 & Surface & 2012 & 9750.485 & 214.202 & Surface & 2012 \\
\hline 4411.859 & 224.122 & Surface & 2012 & 9753.595 & 214.206 & Surface & 2012 \\
\hline 4415.084 & 224.098 & Surface & 2012 & 9756.704 & 214.201 & Surface & 2012 \\
\hline 4418.310 & 224.126 & Surface & 2012 & 9759.814 & 214.200 & Surface & 2012 \\
\hline 4421.536 & 224.140 & Surface & 2012 & 9762.924 & 214.190 & Surface & 2012 \\
\hline 4424.761 & 224.138 & Surface & 2012 & 9766.203 & 214.176 & Surface & 2012 \\
\hline 4427.987 & 224.135 & Surface & 2012 & 9769.483 & 214.174 & Surface & 2012 \\
\hline 4431.213 & 224.138 & Surface & 2012 & 9772.762 & 214.210 & Surface & 2012 \\
\hline 4434.438 & 224.141 & Surface & 2012 & 9776.041 & 214.200 & Surface & 2012 \\
\hline 4437.664 & 224.122 & Surface & 2012 & 9779.321 & 214.188 & Surface & 2012 \\
\hline 4440.890 & 224.136 & Surface & 2012 & 9782.600 & 214.189 & Surface & 2012 \\
\hline 4444.115 & 224.139 & Surface & 2012 & 9785.880 & 214.206 & Surface & 2012 \\
\hline 4447.341 & 224.132 & Surface & 2012 & 9789.159 & 214.210 & Surface & 2012 \\
\hline 4450.485 & 224.130 & Surface & 2012 & 9792.438 & 214.202 & Surface & 2012 \\
\hline 4453.628 & 224.143 & Surface & 2012 & 9795.718 & 214.196 & Surface & 2012 \\
\hline 4456.772 & 224.133 & Surface & 2012 & 9798.877 & 214.199 & Surface & 2012 \\
\hline 4459.916 & 224.134 & Surface & 2012 & 9802.035 & 214.205 & Surface & 2012 \\
\hline 4463.059 & 224.120 & Surface & 2012 & 9805.194 & 214.205 & Surface & 2012 \\
\hline 4466.203 & 224.129 & Surface & 2012 & 9808.353 & 214.211 & Surface & 2012 \\
\hline 4469.346 & 224.130 & Surface & 2012 & 9811.512 & 214.200 & Surface & 2012 \\
\hline 4472.490 & 224.128 & Surface & 2012 & 9814.671 & 214.198 & Surface & 2012 \\
\hline
\end{tabular}




\begin{tabular}{|c|c|c|c|c|c|c|c|}
\hline 4475.634 & 224.140 & Surface & 2012 & 9817.830 & 214.204 & Surface & 2012 \\
\hline 4478.777 & 224.143 & Surface & 2012 & 9820.988 & 214.194 & Surface & 2012 \\
\hline 4481.921 & 224.129 & Surface & 2012 & 9824.147 & 214.192 & Surface & 2012 \\
\hline 4485.065 & 224.131 & Surface & 2012 & 9827.306 & 214.209 & Surface & 2012 \\
\hline 4488.208 & 224.125 & Surface & 2012 & 9830.564 & 214.208 & Surface & 2012 \\
\hline 4491.352 & 224.114 & Surface & 2012 & 9833.823 & 214.201 & Surface & 2012 \\
\hline 4494.495 & 224.134 & Surface & 2012 & 9837.081 & 214.199 & Surface & 2012 \\
\hline 4497.639 & 224.130 & Surface & 2012 & 9840.339 & 214.205 & Surface & 2012 \\
\hline 4500.783 & 224.122 & Surface & 2012 & 9843.597 & 214.203 & Surface & 2012 \\
\hline 4503.926 & 224.120 & Surface & 2012 & 9846.856 & 214.213 & Surface & 2012 \\
\hline 4507.070 & 224.123 & Surface & 2012 & 9850.114 & 214.209 & Surface & 2012 \\
\hline 4510.286 & 224.136 & Surface & 2012 & 9853.373 & 214.175 & Surface & 2012 \\
\hline 4513.502 & 224.112 & Surface & 2012 & 9856.631 & 214.181 & Surface & 2012 \\
\hline 4516.718 & 224.113 & Surface & 2012 & 9859.889 & 214.199 & Surface & 2012 \\
\hline 4519.933 & 224.109 & Surface & 2012 & 9863.147 & 214.207 & Surface & 2012 \\
\hline 4523.149 & 224.107 & Surface & 2012 & 9866.406 & 214.208 & Surface & 2012 \\
\hline 4526.365 & 224.114 & Surface & 2012 & 9869.633 & 214.199 & Surface & 2012 \\
\hline 4529.581 & 224.125 & Surface & 2012 & 9872.861 & 214.205 & Surface & 2012 \\
\hline 4532.797 & 224.113 & Surface & 2012 & 9876.089 & 214.210 & Surface & 2012 \\
\hline 4536.013 & 224.111 & Surface & 2012 & 9879.316 & 214.204 & Surface & 2012 \\
\hline 4539.228 & 224.121 & Surface & 2012 & 9882.544 & 214.204 & Surface & 2012 \\
\hline 4542.444 & 224.113 & Surface & 2012 & 9885.772 & 214.204 & Surface & 2012 \\
\hline 4545.660 & 224.097 & Surface & 2012 & 9888.999 & 214.202 & Surface & 2012 \\
\hline 4548.876 & 224.109 & Surface & 2012 & 9892.227 & 214.188 & Surface & 2012 \\
\hline 4552.092 & 224.119 & Surface & 2012 & 9895.454 & 214.185 & Surface & 2012 \\
\hline 4555.308 & 224.115 & Surface & 2012 & 9898.599 & 214.191 & Surface & 2012 \\
\hline 4558.524 & 224.104 & Surface & 2012 & 9901.743 & 214.193 & Surface & 2012 \\
\hline 4561.740 & 224.084 & Surface & 2012 & 9904.887 & 214.202 & Surface & 2012 \\
\hline 4564.955 & 224.083 & Surface & 2012 & 9908.031 & 214.195 & Surface & 2012 \\
\hline 4568.175 & 224.099 & Surface & 2012 & 9911.175 & 214.190 & Surface & 2012 \\
\hline 4571.394 & 224.106 & Surface & 2012 & 9914.319 & 214.192 & Surface & 2012 \\
\hline 4574.613 & 224.098 & Surface & 2012 & 9917.464 & 214.182 & Surface & 2012 \\
\hline 4577.833 & 224.082 & Surface & 2012 & 9920.608 & 214.199 & Surface & 2012 \\
\hline 4581.052 & 224.094 & Surface & 2012 & 9923.752 & 214.215 & Surface & 2012 \\
\hline 4584.271 & 224.092 & Surface & 2012 & 9926.840 & 214.209 & Surface & 2012 \\
\hline 4587.491 & 224.096 & Surface & 2012 & 9929.927 & 214.202 & Surface & 2012 \\
\hline 4590.710 & 224.102 & Surface & 2012 & 9933.015 & 214.195 & Surface & 2012 \\
\hline 4593.929 & 224.090 & Surface & 2012 & 9936.103 & 214.190 & Surface & 2012 \\
\hline 4597.149 & 224.115 & Surface & 2012 & 9939.191 & 214.201 & Surface & 2012 \\
\hline 4600.368 & 224.092 & Surface & 2012 & 9942.279 & 214.205 & Surface & 2012 \\
\hline 4603.400 & 224.089 & Surface & 2012 & 9945.366 & 214.199 & Surface & 2012 \\
\hline 4606.432 & 224.084 & Surface & 2012 & 9948.454 & 214.193 & Surface & 2012 \\
\hline 4609.464 & 224.084 & Surface & 2012 & 9951.542 & 214.192 & Surface & 2012 \\
\hline 4612.495 & 224.095 & Surface & 2012 & 9954.630 & 214.205 & Surface & 2012 \\
\hline 4615.527 & 224.077 & Surface & 2012 & 9957.718 & 214.202 & Surface & 2012 \\
\hline 4618.559 & 224.077 & Surface & 2012 & 9960.805 & 214.188 & Surface & 2012 \\
\hline 4621.590 & 224.074 & Surface & 2012 & 9963.893 & 214.184 & Surface & 2012 \\
\hline 4624.622 & 224.075 & Surface & 2012 & 9966.981 & 214.190 & Surface & 2012 \\
\hline 4627.654 & 224.079 & Surface & 2012 & 9970.121 & 214.192 & Surface & 2012 \\
\hline
\end{tabular}




\begin{tabular}{|c|c|c|c|c|c|c|c|}
\hline 4630.686 & 224.072 & Surface & 2012 & 9973.261 & 214.199 & Surface & 2012 \\
\hline 4633.717 & 224.064 & Surface & 2012 & 9976.401 & 214.201 & Surface & 2012 \\
\hline 4636.749 & 224.071 & Surface & 2012 & 9979.542 & 214.209 & Surface & 2012 \\
\hline 4639.781 & 224.079 & Surface & 2012 & 9982.682 & 214.210 & Surface & 2012 \\
\hline 4642.901 & 224.071 & Surface & 2012 & 9985.822 & 214.210 & Surface & 2012 \\
\hline 4646.022 & 224.068 & Surface & 2012 & 9988.962 & 214.216 & Surface & 2012 \\
\hline 4649.142 & 224.051 & Surface & 2012 & 9992.102 & 214.220 & Surface & 2012 \\
\hline 4652.262 & 224.050 & Surface & 2012 & 9995.242 & 214.227 & Surface & 2012 \\
\hline 4655.382 & 224.053 & Surface & 2012 & 9998.382 & 214.215 & Surface & 2012 \\
\hline 4658.502 & 224.038 & Surface & 2012 & 10001.522 & 214.205 & Surface & 2012 \\
\hline 4661.622 & 224.052 & Surface & 2012 & 10004.663 & 214.200 & Surface & 2012 \\
\hline 4664.743 & 224.040 & Surface & 2012 & 10007.803 & 214.200 & Surface & 2012 \\
\hline 4667.863 & 224.041 & Surface & 2012 & 10010.943 & 214.200 & Surface & 2012 \\
\hline 4670.983 & 224.012 & Surface & 2012 & 10014.083 & 214.200 & Surface & 2012 \\
\hline 4674.103 & 224.014 & Surface & 2012 & 10017.174 & 214.200 & Surface & 2012 \\
\hline 4677.224 & 224.019 & Surface & 2012 & 10020.265 & 214.196 & Surface & 2012 \\
\hline 4680.344 & 224.008 & Surface & 2012 & 10023.356 & 214.190 & Surface & 2012 \\
\hline 4683.464 & 224.004 & Surface & 2012 & 10026.447 & 214.190 & Surface & 2012 \\
\hline 4686.388 & 224.027 & Surface & 2012 & 10029.538 & 214.190 & Surface & 2012 \\
\hline 4689.311 & 223.987 & Surface & 2012 & 10032.629 & 214.190 & Surface & 2012 \\
\hline 4692.235 & 223.992 & Surface & 2012 & 10035.720 & 214.190 & Surface & 2012 \\
\hline 4695.158 & 224.002 & Surface & 2012 & 10038.811 & 214.190 & Surface & 2012 \\
\hline 4698.082 & 224.005 & Surface & 2012 & 10041.902 & 214.190 & Surface & 2012 \\
\hline 4701.005 & 223.993 & Surface & 2012 & 10044.993 & 214.182 & Surface & 2012 \\
\hline 4703.929 & 223.982 & Surface & 2012 & 10048.083 & 214.180 & Surface & 2012 \\
\hline 4706.852 & 223.993 & Surface & 2012 & 10051.175 & 214.180 & Surface & 2012 \\
\hline 4709.776 & 223.989 & Surface & 2012 & 10054.265 & 214.180 & Surface & 2012 \\
\hline 4712.836 & 223.973 & Surface & 2012 & 10057.357 & 214.180 & Surface & 2012 \\
\hline 4715.897 & 223.944 & Surface & 2012 & 10060.447 & 214.180 & Surface & 2012 \\
\hline 4718.957 & 223.946 & Surface & 2012 & 10063.593 & 214.183 & Surface & 2012 \\
\hline 4722.018 & 223.954 & Surface & 2012 & 10066.738 & 214.180 & Surface & 2012 \\
\hline 4725.079 & 223.945 & Surface & 2012 & 10069.884 & 214.180 & Surface & 2012 \\
\hline 4728.139 & 223.945 & Surface & 2012 & 10073.029 & 214.172 & Surface & 2012 \\
\hline 4731.200 & 223.921 & Surface & 2012 & 10076.174 & 214.167 & Surface & 2012 \\
\hline 4734.260 & 223.915 & Surface & 2012 & 10079.320 & 214.145 & Surface & 2012 \\
\hline 4737.320 & 223.917 & Surface & 2012 & 10082.465 & 214.126 & Surface & 2012 \\
\hline 4740.381 & 223.898 & Surface & 2012 & 10085.610 & 214.120 & Surface & 2012 \\
\hline 4743.442 & 223.855 & Surface & 2012 & 10088.672 & 214.120 & Surface & 2012 \\
\hline 4746.502 & 223.820 & Surface & 2012 & 10091.734 & 214.117 & Surface & 2012 \\
\hline 4749.563 & 223.827 & Surface & 2012 & 10094.796 & 214.101 & Surface & 2012 \\
\hline 4752.730 & 223.827 & Surface & 2012 & 10097.858 & 214.066 & Surface & 2012 \\
\hline 4755.896 & 223.815 & Surface & 2012 & 10100.920 & 214.046 & Surface & 2012 \\
\hline 4759.063 & 223.822 & Surface & 2012 & 10103.981 & 214.009 & Surface & 2012 \\
\hline 4762.230 & 223.842 & Surface & 2012 & 10107.043 & 213.936 & Surface & 2012 \\
\hline 4765.396 & 223.834 & Surface & 2012 & 10110.105 & 213.904 & Surface & 2012 \\
\hline 4768.563 & 223.820 & Surface & 2012 & 10113.167 & 213.876 & Surface & 2012 \\
\hline 4771.729 & 223.799 & Surface & 2012 & 10116.229 & 213.735 & Surface & 2012 \\
\hline 4774.896 & 223.778 & Surface & 2012 & 10119.230 & 213.628 & Surface & 2012 \\
\hline 4778.063 & 223.777 & Surface & 2012 & 10122.231 & 213.620 & Surface & 2012 \\
\hline
\end{tabular}




\begin{tabular}{|c|c|c|c|c|c|c|c|}
\hline 4781.230 & 223.794 & Surface & 2012 & 10125.233 & 213.614 & Surface & 2012 \\
\hline 4784.396 & 223.780 & Surface & 2012 & 10128.234 & 213.587 & Surface & 2012 \\
\hline 4787.573 & 223.754 & Surface & 2012 & 10131.016 & 213.558 & Surface & 2012 \\
\hline 4790.750 & 223.751 & Surface & 2012 & 10133.797 & 213.538 & Surface & 2012 \\
\hline 4793.926 & 223.745 & Surface & 2012 & 10136.579 & 213.526 & Surface & 2012 \\
\hline 4797.103 & 223.745 & Surface & 2012 & 10139.360 & 213.520 & Surface & 2012 \\
\hline 4800.279 & 223.727 & Surface & 2012 & 10142.141 & 213.504 & Surface & 2012 \\
\hline 4803.456 & 223.720 & Surface & 2012 & 10145.271 & 213.455 & Surface & 2012 \\
\hline 4806.633 & 223.702 & Surface & 2012 & 10148.400 & 213.411 & Surface & 2012 \\
\hline 4809.810 & 223.680 & Surface & 2012 & 10151.530 & 213.376 & Surface & 2012 \\
\hline 4812.986 & 223.691 & Surface & 2012 & 10154.659 & 213.348 & Surface & 2012 \\
\hline 4816.163 & 223.668 & Surface & 2012 & 10157.789 & 213.317 & Surface & 2012 \\
\hline 4819.339 & 223.668 & Surface & 2012 & 10160.918 & 213.305 & Surface & 2012 \\
\hline 4822.516 & 223.654 & Surface & 2012 & 10164.048 & 213.310 & Surface & 2012 \\
\hline 4825.693 & 223.630 & Surface & 2012 & 10167.177 & 213.308 & Surface & 2012 \\
\hline 4828.869 & 223.630 & Surface & 2012 & 10170.307 & 213.289 & Surface & 2012 \\
\hline 4832.046 & 223.629 & Surface & 2012 & 10173.436 & 213.229 & Surface & 2012 \\
\hline 4835.222 & 223.612 & Surface & 2012 & 10176.566 & 213.189 & Surface & 2012 \\
\hline 4838.272 & 223.604 & Surface & 2012 & 10179.532 & 213.176 & Surface & 2012 \\
\hline 4841.322 & 223.549 & Surface & 2012 & 10182.498 & 213.167 & Surface & 2012 \\
\hline 4844.372 & 223.552 & Surface & 2012 & 10185.464 & 213.155 & Surface & 2012 \\
\hline 4847.422 & 223.566 & Surface & 2012 & 10188.429 & 213.133 & Surface & 2012 \\
\hline 4850.472 & 223.528 & Surface & 2012 & 10191.395 & 213.109 & Surface & 2012 \\
\hline 4853.522 & 223.498 & Surface & 2012 & 10194.361 & 213.087 & Surface & 2012 \\
\hline 4856.572 & 223.487 & Surface & 2012 & 10197.327 & 213.061 & Surface & 2012 \\
\hline 4859.622 & 223.470 & Surface & 2012 & 10200.293 & 213.034 & Surface & 2012 \\
\hline 4862.672 & 223.480 & Surface & 2012 & 10203.376 & 213.005 & Surface & 2012 \\
\hline 4865.722 & 223.489 & Surface & 2012 & 10206.459 & 212.992 & Surface & 2012 \\
\hline 4868.771 & 223.429 & Surface & 2012 & 10209.542 & 213.020 & Surface & 2012 \\
\hline 4871.821 & 223.423 & Surface & 2012 & 10212.625 & 213.074 & Surface & 2012 \\
\hline 4874.871 & 223.423 & Surface & 2012 & 10215.708 & 213.120 & Surface & 2012 \\
\hline 4877.921 & 223.405 & Surface & 2012 & 10218.791 & 213.132 & Surface & 2012 \\
\hline 4881.164 & 223.378 & Surface & 2012 & 10221.875 & 213.125 & Surface & 2012 \\
\hline 4884.407 & 223.350 & Surface & 2012 & 10224.958 & 213.142 & Surface & 2012 \\
\hline 4887.650 & 223.329 & Surface & 2012 & 10228.041 & 213.187 & Surface & 2012 \\
\hline 4890.893 & 223.280 & Surface & 2012 & 10231.244 & 213.205 & Surface & 2012 \\
\hline 4894.137 & 223.264 & Surface & 2012 & 10234.446 & 213.203 & Surface & 2012 \\
\hline 4897.380 & 223.236 & Surface & 2012 & 10237.649 & 213.209 & Surface & 2012 \\
\hline 4900.623 & 223.245 & Surface & 2012 & 10240.852 & 213.230 & Surface & 2012 \\
\hline 4903.866 & 223.231 & Surface & 2012 & 10244.056 & 213.282 & Surface & 2012 \\
\hline 4907.109 & 223.209 & Surface & 2012 & 10247.259 & 213.321 & Surface & 2012 \\
\hline 4910.352 & 223.195 & Surface & 2012 & 10250.462 & 213.305 & Surface & 2012 \\
\hline 4913.595 & 223.183 & Surface & 2012 & 10253.665 & 213.264 & Surface & 2012 \\
\hline 4916.838 & 223.168 & Surface & 2012 & 10256.873 & 213.252 & Surface & 2012 \\
\hline 4920.081 & 223.160 & Surface & 2012 & 10260.082 & 213.242 & Surface & 2012 \\
\hline 4923.324 & 223.108 & Surface & 2012 & 10263.291 & 213.161 & Surface & 2012 \\
\hline 4926.567 & 223.086 & Surface & 2012 & 10266.500 & 213.034 & Surface & 2012 \\
\hline 4929.651 & 223.083 & Surface & 2012 & 10269.708 & 212.980 & Surface & 2012 \\
\hline 4932.735 & 223.053 & Surface & 2012 & 10272.917 & 212.936 & Surface & 2012 \\
\hline
\end{tabular}




\begin{tabular}{|c|c|c|c|c|c|c|c|}
\hline 4935.819 & 223.040 & Surface & 2012 & 10276.126 & 212.835 & Surface & 2012 \\
\hline 4938.903 & 223.014 & Surface & 2012 & 10279.335 & 212.808 & Surface & 2012 \\
\hline 4941.987 & 223.017 & Surface & 2012 & 10282.543 & 212.764 & Surface & 2012 \\
\hline 4945.071 & 223.015 & Surface & 2012 & 10285.770 & 212.767 & Surface & 2012 \\
\hline 4948.154 & 223.014 & Surface & 2012 & 10288.996 & 212.746 & Surface & 2012 \\
\hline 4951.238 & 223.006 & Surface & 2012 & 10292.222 & 212.731 & Surface & 2012 \\
\hline 4954.322 & 223.017 & Surface & 2012 & 10295.449 & 212.708 & Surface & 2012 \\
\hline 4957.406 & 223.001 & Surface & 2012 & 10298.675 & 212.715 & Surface & 2012 \\
\hline 4960.490 & 222.990 & Surface & 2012 & 10301.902 & 212.753 & Surface & 2012 \\
\hline 4963.574 & 222.974 & Surface & 2012 & 10305.128 & 212.764 & Surface & 2012 \\
\hline 4966.658 & 222.973 & Surface & 2012 & 10308.355 & 212.744 & Surface & 2012 \\
\hline 4969.741 & 222.962 & Surface & 2012 & 10311.581 & 212.738 & Surface & 2012 \\
\hline 4972.825 & 222.925 & Surface & 2012 & 10314.807 & 212.756 & Surface & 2012 \\
\hline 4975.884 & 222.911 & Surface & 2012 & 10318.028 & 212.746 & Surface & 2012 \\
\hline 4978.943 & 222.927 & Surface & 2012 & 10321.248 & 212.752 & Surface & 2012 \\
\hline 4982.003 & 222.905 & Surface & 2012 & 10324.469 & 212.779 & Surface & 2012 \\
\hline 4985.062 & 222.884 & Surface & 2012 & 10327.690 & 212.784 & Surface & 2012 \\
\hline 4988.121 & 222.877 & Surface & 2012 & 10330.910 & 212.773 & Surface & 2012 \\
\hline 4991.180 & 222.860 & Surface & 2012 & 10334.131 & 212.758 & Surface & 2012 \\
\hline 4994.239 & 222.864 & Surface & 2012 & 10337.352 & 212.757 & Surface & 2012 \\
\hline 4997.298 & 222.861 & Surface & 2012 & 10340.572 & 212.760 & Surface & 2012 \\
\hline 5000.358 & 222.860 & Surface & 2012 & 10343.793 & 212.761 & Surface & 2012 \\
\hline 5003.417 & 222.890 & Surface & 2012 & 10347.014 & 212.765 & Surface & 2012 \\
\hline 5006.476 & 222.880 & Surface & 2012 & 10350.201 & 212.767 & Surface & 2012 \\
\hline 5009.535 & 222.825 & Surface & 2012 & 10353.387 & 212.762 & Surface & 2012 \\
\hline 5012.594 & 222.836 & Surface & 2012 & 10356.574 & 212.768 & Surface & 2012 \\
\hline 5015.653 & 222.836 & Surface & 2012 & 10359.761 & 212.765 & Surface & 2012 \\
\hline 5018.917 & 222.832 & Surface & 2012 & 10362.948 & 212.727 & Surface & 2012 \\
\hline 5022.180 & 222.838 & Surface & 2012 & 10366.135 & 212.727 & Surface & 2012 \\
\hline 5025.443 & 222.820 & Surface & 2012 & 10369.322 & 212.737 & Surface & 2012 \\
\hline 5028.707 & 222.810 & Surface & 2012 & 10372.566 & 212.723 & Surface & 2012 \\
\hline 5031.970 & 222.801 & Surface & 2012 & 10375.810 & 212.695 & Surface & 2012 \\
\hline 5035.233 & 222.807 & Surface & 2012 & 10379.054 & 212.686 & Surface & 2012 \\
\hline 5038.496 & 222.811 & Surface & 2012 & 10382.298 & 212.698 & Surface & 2012 \\
\hline 5041.760 & 222.813 & Surface & 2012 & 10385.543 & 212.672 & Surface & 2012 \\
\hline 5045.023 & 222.797 & Surface & 2012 & 10388.787 & 212.648 & Surface & 2012 \\
\hline 5048.286 & 222.800 & Surface & 2012 & 10392.031 & 212.619 & Surface & 2012 \\
\hline 5051.550 & 222.800 & Surface & 2012 & 10395.275 & 212.575 & Surface & 2012 \\
\hline 5054.813 & 222.777 & Surface & 2012 & 10398.519 & 212.516 & Surface & 2012 \\
\hline 5058.076 & 222.762 & Surface & 2012 & 10401.624 & 212.538 & Surface & 2012 \\
\hline 5061.339 & 222.753 & Surface & 2012 & 10404.729 & 212.532 & Surface & 2012 \\
\hline 5064.603 & 222.770 & Surface & 2012 & 10407.835 & 212.517 & Surface & 2012 \\
\hline 5067.696 & 222.787 & Surface & 2012 & 10410.940 & 212.492 & Surface & 2012 \\
\hline 5070.790 & 222.773 & Surface & 2012 & 10414.045 & 212.468 & Surface & 2012 \\
\hline 5073.884 & 222.741 & Surface & 2012 & 10417.150 & 212.431 & Surface & 2012 \\
\hline 5076.978 & 222.717 & Surface & 2012 & 10420.255 & 212.383 & Surface & 2012 \\
\hline 5080.071 & 222.739 & Surface & 2012 & 10423.361 & 212.327 & Surface & 2012 \\
\hline 5083.165 & 222.742 & Surface & 2012 & 10426.466 & 212.297 & Surface & 2012 \\
\hline 5086.259 & 222.743 & Surface & 2012 & 10429.632 & 212.290 & Surface & 2012 \\
\hline
\end{tabular}




\begin{tabular}{|c|c|c|c|c|c|c|c|}
\hline 5089.352 & 222.732 & Surface & 2012 & 10432.798 & 212.272 & Surface & 2012 \\
\hline 5092.446 & 222.714 & Surface & 2012 & 10435.965 & 212.249 & Surface & 2012 \\
\hline 5095.540 & 222.716 & Surface & 2012 & 10439.132 & 212.236 & Surface & 2012 \\
\hline 5098.634 & 222.700 & Surface & 2012 & 10442.298 & 212.203 & Surface & 2012 \\
\hline 5101.727 & 222.698 & Surface & 2012 & 10445.464 & 212.152 & Surface & 2012 \\
\hline 5104.821 & 222.698 & Surface & 2012 & 10448.631 & 212.095 & Surface & 2012 \\
\hline 5107.915 & 222.686 & Surface & 2012 & 10451.797 & 212.018 & Surface & 2012 \\
\hline 5111.009 & 222.668 & Surface & 2012 & 10454.963 & 211.991 & Surface & 2012 \\
\hline 5114.102 & 222.660 & Surface & 2012 & 10458.130 & 211.955 & Surface & 2012 \\
\hline 5117.341 & 222.656 & Surface & 2012 & 10461.296 & 211.911 & Surface & 2012 \\
\hline 5120.580 & 222.658 & Surface & 2012 & 10464.463 & 211.912 & Surface & 2012 \\
\hline 5123.819 & 222.659 & Surface & 2012 & 10467.629 & 211.919 & Surface & 2012 \\
\hline 5127.058 & 222.651 & Surface & 2012 & 10470.796 & 211.887 & Surface & 2012 \\
\hline 5130.297 & 222.639 & Surface & 2012 & 10473.962 & 211.881 & Surface & 2012 \\
\hline 5133.537 & 222.642 & Surface & 2012 & 10477.129 & 211.879 & Surface & 2012 \\
\hline 5136.776 & 222.635 & Surface & 2012 & 10480.295 & 211.876 & Surface & 2012 \\
\hline 5140.015 & 222.620 & Surface & 2012 & 10483.461 & 211.877 & Surface & 2012 \\
\hline 5143.254 & 222.611 & Surface & 2012 & 10486.599 & 211.877 & Surface & 2012 \\
\hline 5146.493 & 222.625 & Surface & 2012 & 10489.736 & 211.860 & Surface & 2012 \\
\hline 5149.732 & 222.614 & Surface & 2012 & 10492.873 & 211.847 & Surface & 2012 \\
\hline 5152.971 & 222.595 & Surface & 2012 & 10496.010 & 211.850 & Surface & 2012 \\
\hline 5156.210 & 222.608 & Surface & 2012 & 10499.148 & 211.830 & Surface & 2012 \\
\hline 5159.449 & 222.611 & Surface & 2012 & 10502.285 & 211.784 & Surface & 2012 \\
\hline 5162.688 & 222.614 & Surface & 2012 & 10505.422 & 211.748 & Surface & 2012 \\
\hline 5165.927 & 222.620 & Surface & 2012 & 10508.559 & 211.736 & Surface & 2012 \\
\hline 5169.166 & 222.615 & Surface & 2012 & 10511.696 & 211.769 & Surface & 2012 \\
\hline 5172.405 & 222.619 & Surface & 2012 & 10514.834 & 211.779 & Surface & 2012 \\
\hline 5175.644 & 222.615 & Surface & 2012 & 10517.971 & 211.755 & Surface & 2012 \\
\hline 5178.883 & 222.620 & Surface & 2012 & 10521.108 & 211.738 & Surface & 2012 \\
\hline 5182.122 & 222.615 & Surface & 2012 & 10524.245 & 211.730 & Surface & 2012 \\
\hline 5185.361 & 222.618 & Surface & 2012 & 10527.383 & 211.723 & Surface & 2012 \\
\hline 5188.572 & 222.604 & Surface & 2012 & 10530.520 & 211.689 & Surface & 2012 \\
\hline 5191.782 & 222.620 & Surface & 2012 & 10533.657 & 211.619 & Surface & 2012 \\
\hline 5194.993 & 222.623 & Surface & 2012 & 10536.794 & 211.514 & Surface & 2012 \\
\hline 5198.203 & 222.601 & Surface & 2012 & 10539.982 & 211.494 & Surface & 2012 \\
\hline 5201.414 & 222.596 & Surface & 2012 & 10543.168 & 211.635 & Surface & 2012 \\
\hline 5204.624 & 222.605 & Surface & 2012 & 10546.355 & 211.703 & Surface & 2012 \\
\hline 5207.835 & 222.606 & Surface & 2012 & 10549.542 & 211.660 & Surface & 2012 \\
\hline 5211.046 & 222.625 & Surface & 2012 & 10552.729 & 211.667 & Surface & 2012 \\
\hline 5214.256 & 222.595 & Surface & 2012 & 10555.916 & 211.690 & Surface & 2012 \\
\hline 5217.467 & 222.593 & Surface & 2012 & 10559.103 & 211.677 & Surface & 2012 \\
\hline 5220.677 & 222.610 & Surface & 2012 & 10562.290 & 211.675 & Surface & 2012 \\
\hline 5223.888 & 222.607 & Surface & 2012 & 10565.477 & 211.682 & Surface & 2012 \\
\hline 5227.098 & 222.607 & Surface & 2012 & 10568.664 & 211.670 & Surface & 2012 \\
\hline 5230.309 & 222.621 & Surface & 2012 & 10571.851 & 211.690 & Surface & 2012 \\
\hline 5233.363 & 222.613 & Surface & 2012 & 10575.038 & 211.664 & Surface & 2012 \\
\hline 5236.416 & 222.616 & Surface & 2012 & 10578.225 & 211.695 & Surface & 2012 \\
\hline 5239.470 & 222.600 & Surface & 2012 & 10581.412 & 211.714 & Surface & 2012 \\
\hline 5242.524 & 222.587 & Surface & 2012 & 10584.536 & 211.692 & Surface & 2012 \\
\hline
\end{tabular}




\begin{tabular}{|c|c|c|c|c|c|c|c|}
\hline 5245.577 & 222.594 & Surface & 2012 & 10587.661 & 211.672 & Surface & 2012 \\
\hline 5248.631 & 222.596 & Surface & 2012 & 10590.785 & 211.674 & Surface & 2012 \\
\hline 5251.684 & 222.591 & Surface & 2012 & 10593.909 & 211.687 & Surface & 2012 \\
\hline 5254.738 & 222.609 & Surface & 2012 & 10597.033 & 211.685 & Surface & 2012 \\
\hline 5257.792 & 222.613 & Surface & 2012 & 10600.158 & 211.670 & Surface & 2012 \\
\hline 5260.845 & 222.599 & Surface & 2012 & 10603.282 & 211.656 & Surface & 2012 \\
\hline 5263.899 & 222.601 & Surface & 2012 & 10606.406 & 211.644 & Surface & 2012 \\
\hline 5266.952 & 222.607 & Surface & 2012 & 10609.530 & 211.650 & Surface & 2012 \\
\hline 5269.963 & 222.606 & Surface & 2012 & 10612.655 & 211.655 & Surface & 2012 \\
\hline 5272.973 & 222.587 & Surface & 2012 & 10615.779 & 211.660 & Surface & 2012 \\
\hline 5275.983 & 222.590 & Surface & 2012 & 10618.903 & 211.642 & Surface & 2012 \\
\hline 5278.993 & 222.598 & Surface & 2012 & 10622.028 & 211.640 & Surface & 2012 \\
\hline 5282.003 & 222.622 & Surface & 2012 & 10625.152 & 211.625 & Surface & 2012 \\
\hline 5285.013 & 222.598 & Surface & 2012 & 10628.276 & 211.670 & Surface & 2012 \\
\hline 5288.023 & 222.593 & Surface & 2012 & 10631.401 & 211.650 & Surface & 2012 \\
\hline 5291.033 & 222.613 & Surface & 2012 & 10634.525 & 211.651 & Surface & 2012 \\
\hline 5294.044 & 222.598 & Surface & 2012 & 10637.604 & 211.636 & Surface & 2012 \\
\hline 5297.054 & 222.593 & Surface & 2012 & 10640.683 & 211.647 & Surface & 2012 \\
\hline 5300.064 & 222.596 & Surface & 2012 & 10643.763 & 211.669 & Surface & 2012 \\
\hline 5303.098 & 222.588 & Surface & 2012 & 10646.842 & 211.674 & Surface & 2012 \\
\hline 5306.132 & 222.586 & Surface & 2012 & 10649.921 & 211.664 & Surface & 2012 \\
\hline 5309.166 & 222.584 & Surface & 2012 & 10653.000 & 211.657 & Surface & 2012 \\
\hline 5312.200 & 222.584 & Surface & 2012 & 10656.080 & 211.643 & Surface & 2012 \\
\hline 5315.234 & 222.588 & Surface & 2012 & 10659.159 & 211.650 & Surface & 2012 \\
\hline 5318.268 & 222.587 & Surface & 2012 & 10662.238 & 211.660 & Surface & 2012 \\
\hline 5321.302 & 222.593 & Surface & 2012 & 10665.317 & 211.684 & Surface & 2012 \\
\hline 5324.336 & 222.583 & Surface & 2012 & 10668.397 & 211.673 & Surface & 2012 \\
\hline 5327.370 & 222.589 & Surface & 2012 & 10671.476 & 211.653 & Surface & 2012 \\
\hline 5330.404 & 222.591 & Surface & 2012 & 10674.555 & 211.655 & Surface & 2012 \\
\hline 5333.438 & 222.578 & Surface & 2012 & 10677.634 & 211.659 & Surface & 2012 \\
\hline
\end{tabular}

